\title{
Common Indoor Sources of Volatile Organic Compounds: Emission Rates and Techniques for Reducing Consumer Exposures
}

\author{
Final Report \\ Contract Number 95-302 \\ Prepared for: \\ California Environmental Protection Agency \\ Air Resources Board \\ Research Division \\ 2020 L Street, P.O. Box 2815 \\ Sacramento, CA 95812 \\ Prepared by: \\ Alfred T. Hodgson \\ Environmental Energy Technologies Division \\ E.O. Lawrence Berkeley National Laboratory \\ 1 Cyclotron Road \\ Berkeley, CA 94720
}

January 1999 



\section{ACKNOWLEDGMENTS}

The author, Alfred Hodgson, gratefully acknowledges Douglas Sullivan, Glenn Morrison and Valerie Harris of Lawrence Berkeley National Laboratory and Dr. Jehuda Binenboym, a visiting scientist from Soreq Nuclear Research Center, Israel, for their invaluable assistance with all aspects of the experimental work. Dr. Lara Gundel of Lawrence Berkeley National Laboratory assisted with the statistical analysis of experimental uncertainty and provided valuable comments. The author is also grateful to Joyce Cordell and Richard Wilson of Lawrence Berkeley National Laboratory for their administrative support and to Dorothy Shimer of the California Air Resources Board for her advice and assistance through out the project. Larry Crevin of Wall to Wall, Inc., Emeryville, CA, generously assisted with the identification and procurement of representative samples of flooring materials.

This work was supported, in part, by the Assistant Secretary for Energy Efficiency and Renewable Energy, Office of Building Technologies of the U.S. Department of Energy under Contract Number DE-AC03-76SF00098.

This report was submitted in fulfillment of California Air Resources Board Contract Number 95-302, "Common Indoor Sources of Volatile Organic Compounds: Emission Rates and Techniques for Reducing Consumer Exposures", by E.O. Lawrence Berkeley National Laboratory under the sponsorship of the California Air Resources Board. Work was completed as of July 1998. 


\section{TABLE OF CONTENTS}

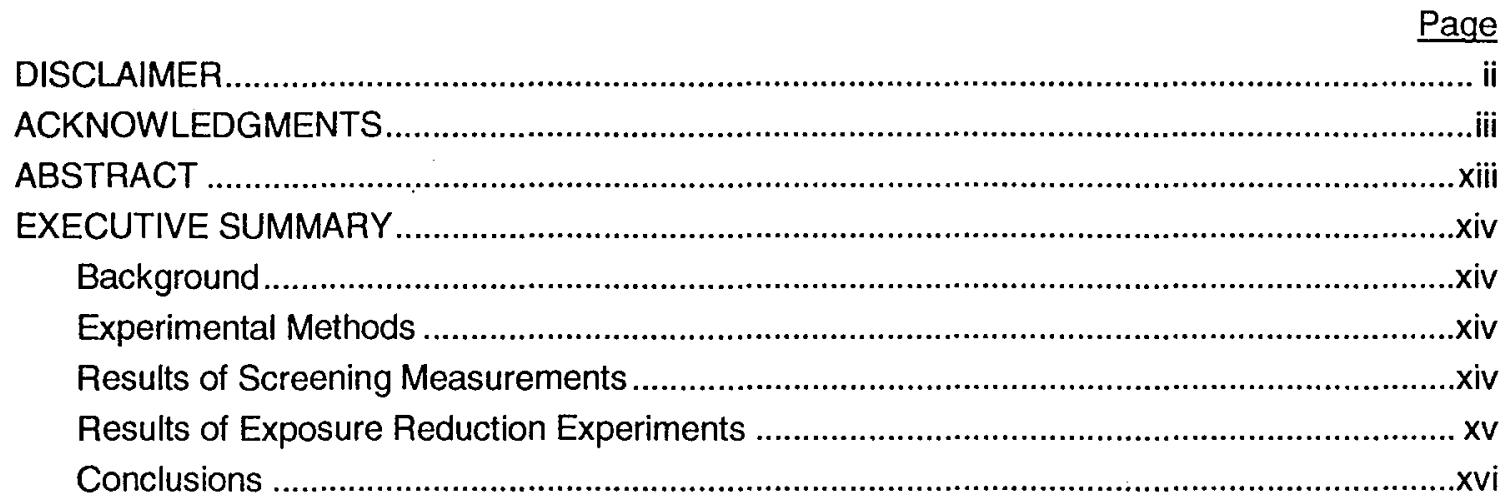

1.0 INTRODUCTION

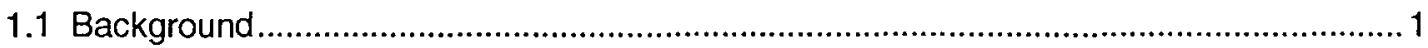

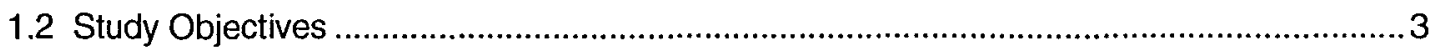

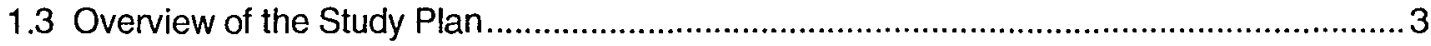

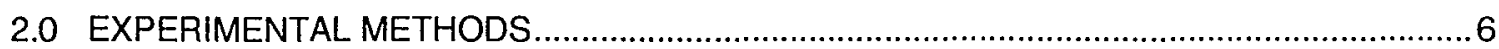

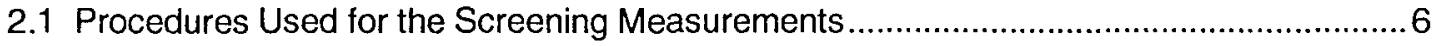

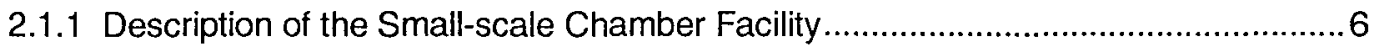

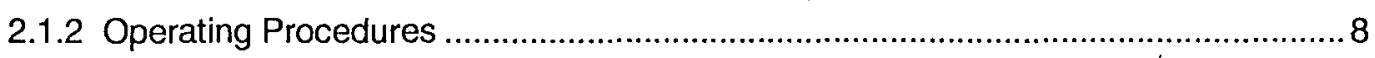

2.2 Procedures Used for the Exposure Reduction Experiments .......................................... 9

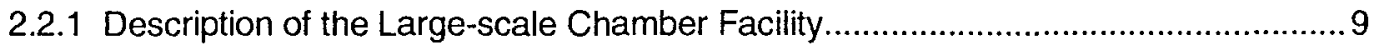

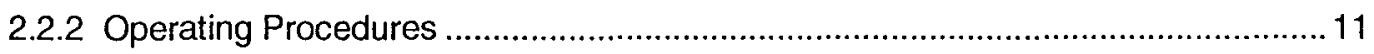

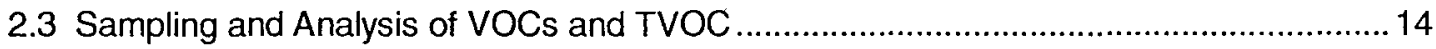

2.4 Sampling and Analysis of Formaldehyde and Acetaldehyde ...................................... 17

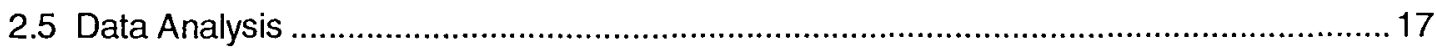

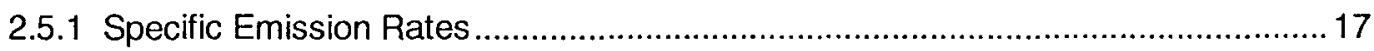

2.5.2 Cumulative Mass Emissions and Exposures …............................................... 18

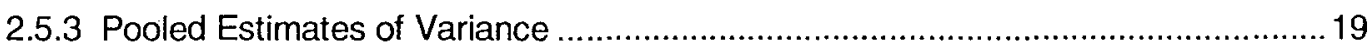

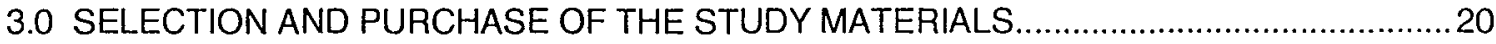

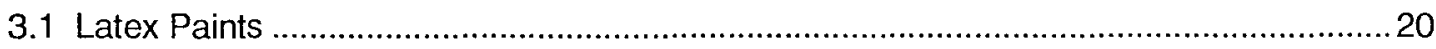

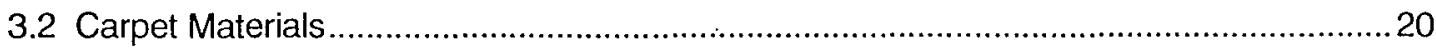

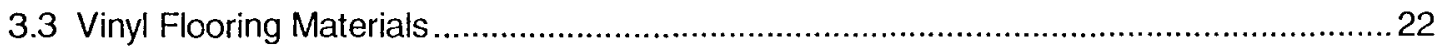

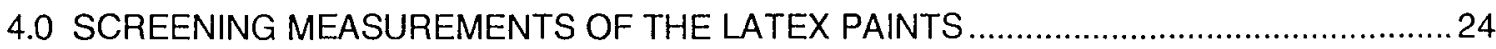

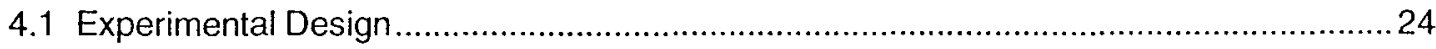

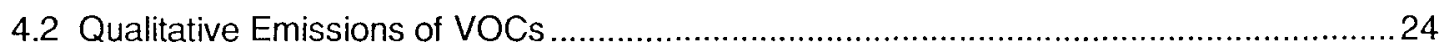

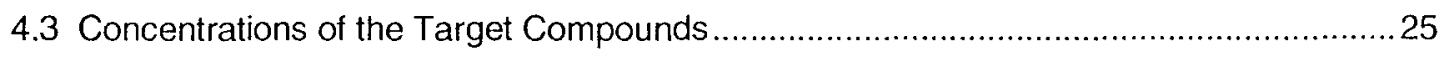

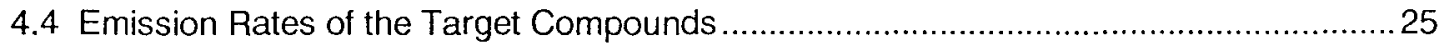

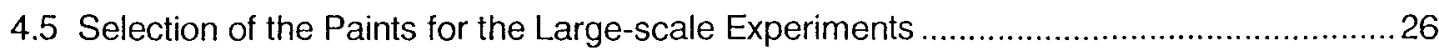

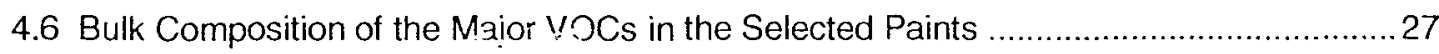




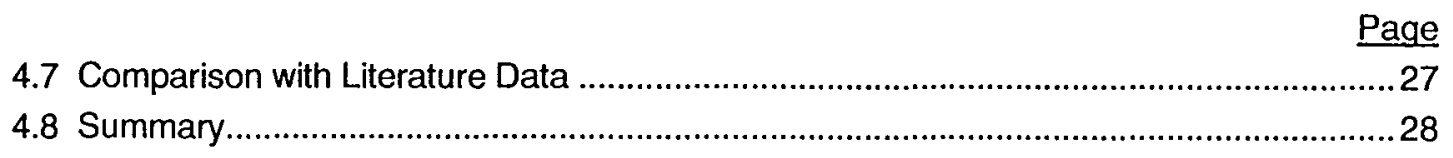

5.0 SCREENING MEASUREMENTS OF THE CARPET MATERIALS .....................................28

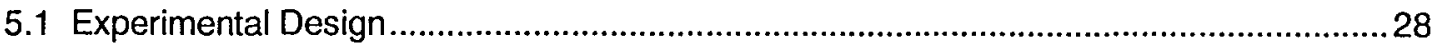

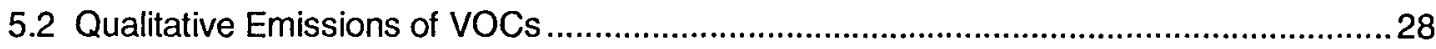

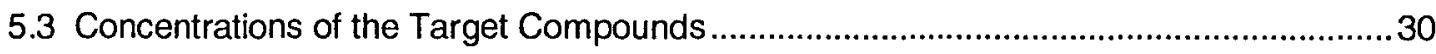

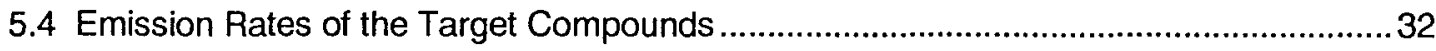

5.5 Selection of the Carpet Materials for the Large-scale Experiments..................................33

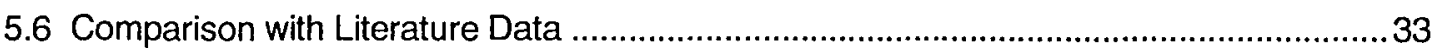

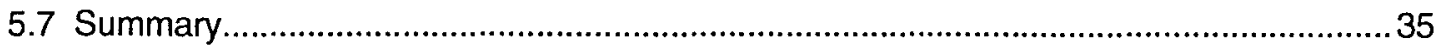

6.0 SCREENING MEASUREMENTS OF THE VINYL FLOORING MATERIALS.........................35

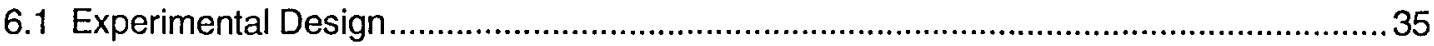

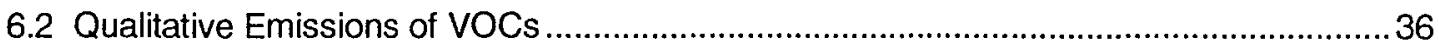

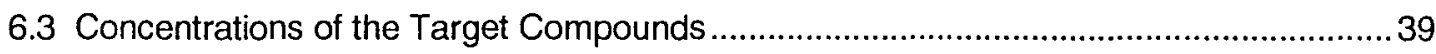

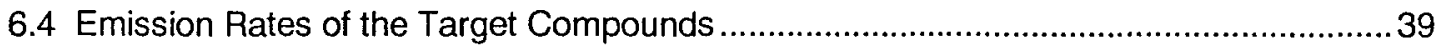

6.5 Selection of the Sheet Vinyl Material for the Large-scale Experiments ............................40

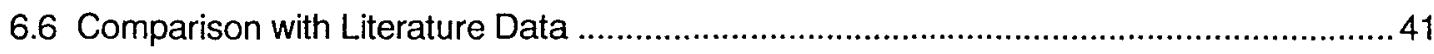

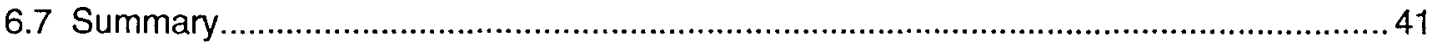

7.0 EXPOSURE REDUCTION EXPERIMENTS WITH THE LATEX PAINT COMBINATION ...... 42

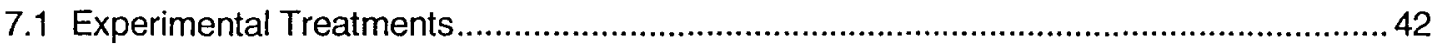

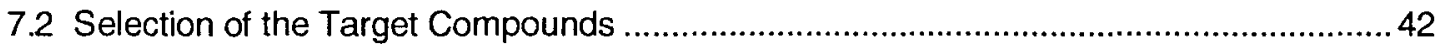

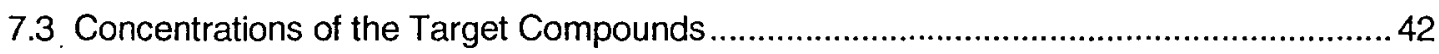

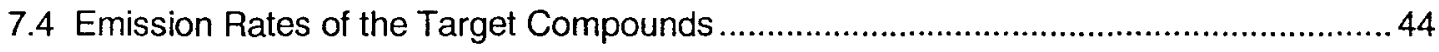

7.5 Cumulative Masses and Exposures for the Target Compounds ......................................4 44

7.6 Comparison with the Screening Measurement Results ................................................. 47

8.0 EXPOSURE REDUCTION EXPERIMENTS WITH THE CARPET ASSEMBLY ..................48

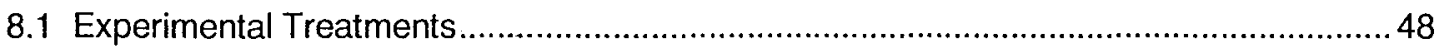

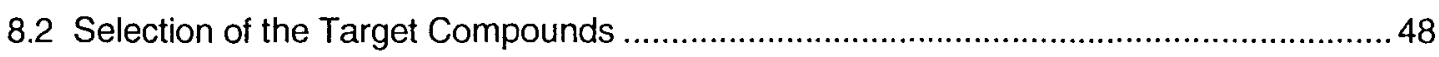

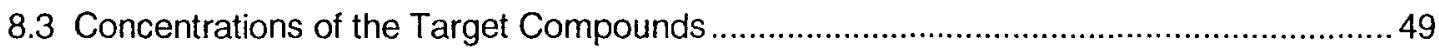

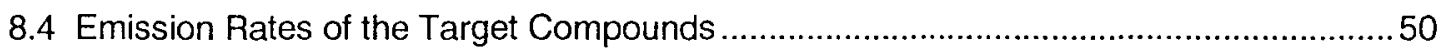

8.5 Cumulative Masses and Exposures for the Target Compounds ...................................51

8.6 Comparison with the Screening Measurement Results .................................................. 52

9.0 EXPOSURE REDUCTION EXPERIMENTS WITH THE VINYL FLOORING ASSEMBLY .....52

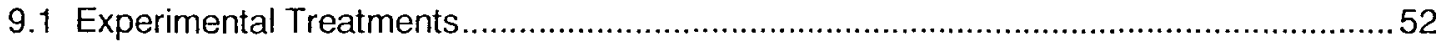

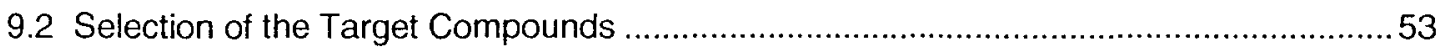

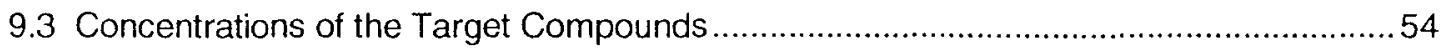

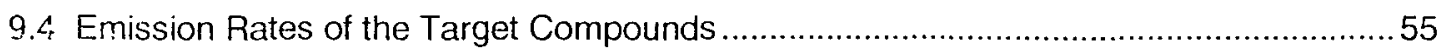


TABLE OF CONTENTS, CONTINUED

9.5 Cumulative Masses and Exposures for the Target Compounds .......................................57

9.6 Comparison with the Screening Measurement Results ...............................................58

10.0 EXPOSURE REDUCTION AND LONG-TERM EXPERIMENTS WITH THE COMBINED ASSEMBLIES

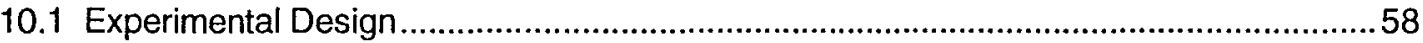

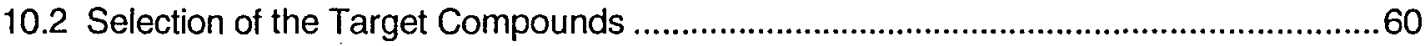

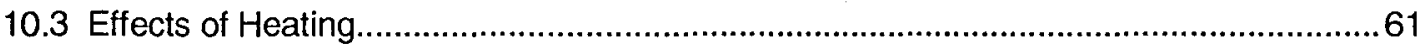

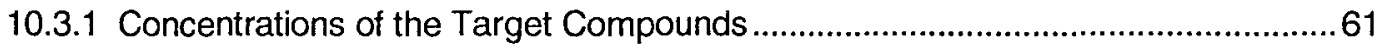

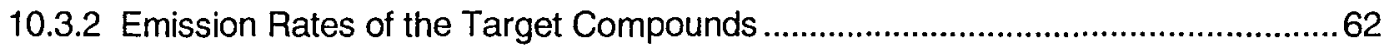

10.3.3 Cumulative Masses and Exposures for the Target Compounds ..........................63

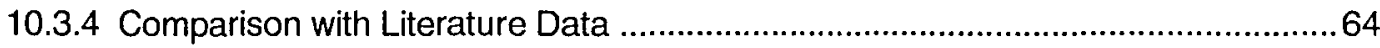

10.4 Comparison with Experiments using the Individual Source Assemblies ........................64

10.5 Long-term Concentrations, Emission Rates and Exposures ....................................64

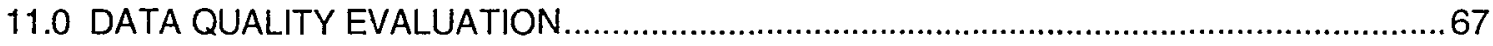

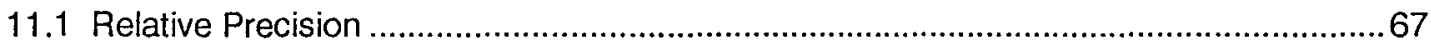

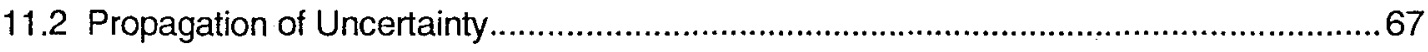

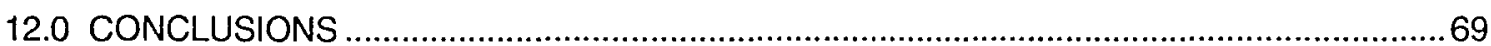

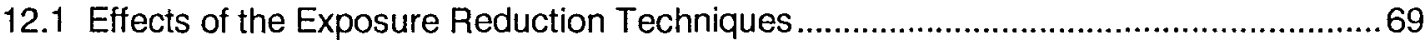

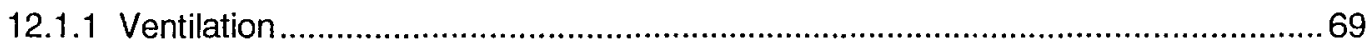

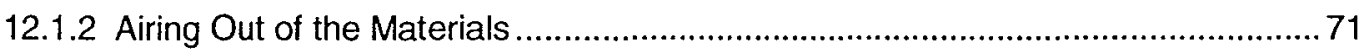

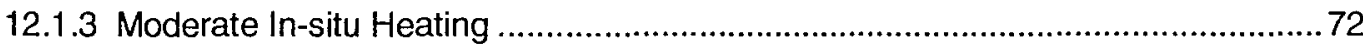

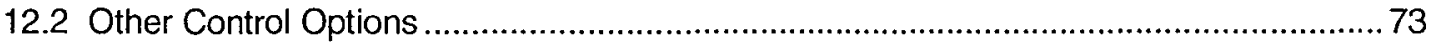

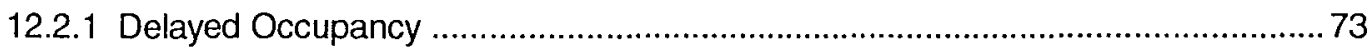

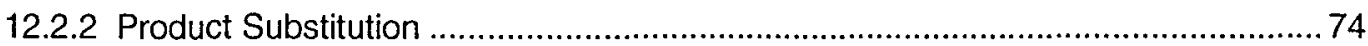

12.3 Recommended Practices to Reduce Occupant Exposures............................................. 75

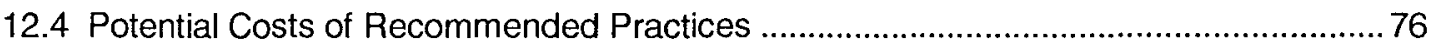

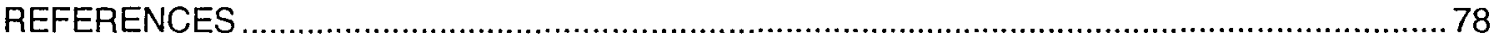

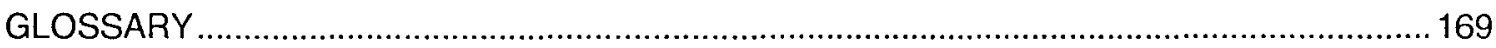

APPENDIX A SMALL-SCALE SCREENING MEASUREMENTS TO CHARACTERIZE EMISSIONS OF VOCS FROM INDOOR MATERIALS/PRODUCTS .................171

APPENDIX B SUMMARY OF ENVIRONMENTAL DATA FOR SCREENING

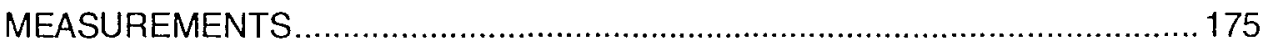

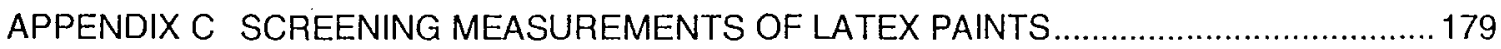

APPENDIX D SCREENING MEASUREMENTS OF CARPET MATERIALS …......................215

APPENDIX E SCREENING MEASUREMENTS OF VINYL FLOORING MATERIALS.............263

APPENDIX F VENTILATION RATE AND AIR MIXING STUDIES OF THE LARGE-SCALE CHAMBER FACILITY 
TABLE OF CONTENTS, CONTINUED

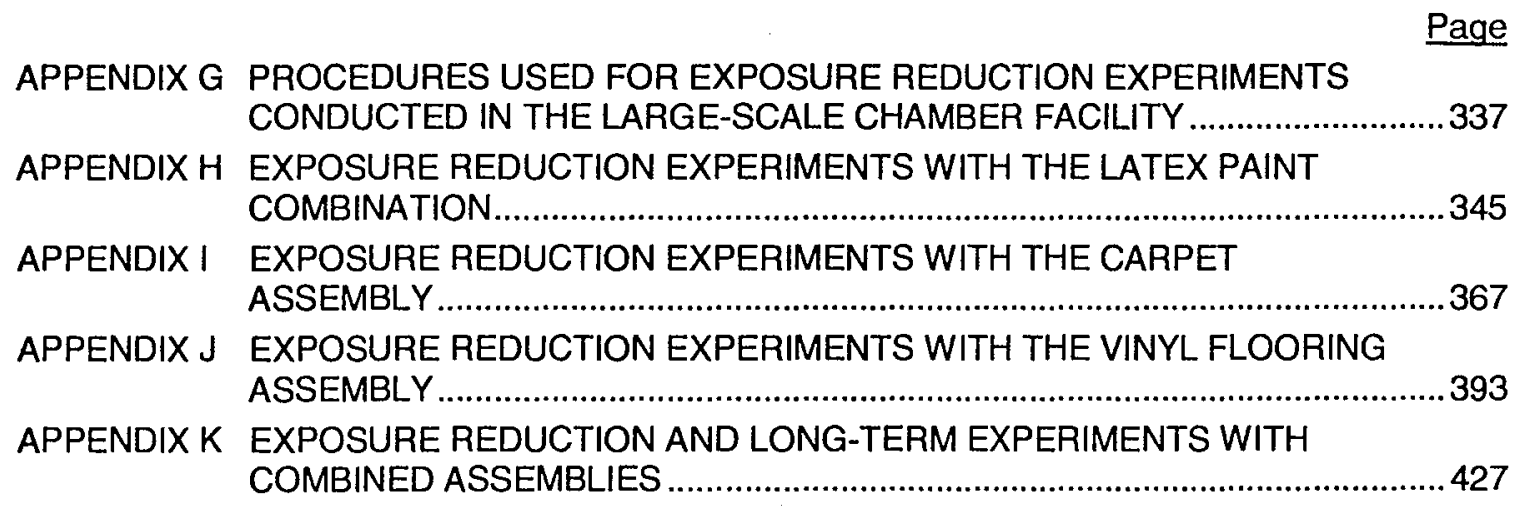




\section{LIST OF FIGURES}

Page

Figure 1. Schematic diagram of the dual-compartment, large-scale chamber facility ...............82

Figure 2. Area-specific emission rates $\left(\mathrm{mg} \mathrm{m}^{-2} \mathrm{~h}^{-1}\right)$ of ethylene glycol emitted at 96 hours in the screening measurements of the individual and combined conventional paints ....83

Figure 3. Mass-specific emission rates $\left(\mathrm{mg} \mathrm{kg} \mathrm{k}^{-1} \mathrm{~h}^{-1}\right)$ of ethylene glycol emitted at 96 hours in the screening measurements of the individual and conventional combined paints ....84

Figure 4. Area-specific emission rates $\left(\mathrm{mg} \mathrm{m}^{-2} \mathrm{~h}^{-1}\right)$ of DEGBE emitted at 96 hours in the screening measurements of the individual and combined conventional paints .......... 85

Figure 5. Mass-specific emission rates $\left(\mathrm{mg} \mathrm{kg}^{-1} \mathrm{~h}^{-1}\right)$ of DEGBE emitted at 96 hours in the screening measurements of the individual and combined conventional paints 86

Figure 6. Area-specific emission rates $\left(\mathrm{mg} \mathrm{m}^{-2} \mathrm{~h}^{-1}\right)$ of Texano® emitted at 96 hours in the screening measurements of the individual and combined conventional paints

Figure 7. Mass-specific emission rates $\left(\mathrm{mg} \mathrm{kg}^{-1} \mathrm{~h}^{-1}\right)$ of Texanol emitted at 96 hours in the screening measurements of the individual and combined conventional paints

Figure 8. Area-specific emission rates $\left(\mathrm{mg} \mathrm{m}^{-2} \mathrm{~h}^{-1}\right)$ of SigmaVOC emitted at 96 hours in the screening measurements of the individual and combined conventional paints

Figure 9. Mass-specific emission rates $\left(\mathrm{mg} \mathrm{kg}^{-1} \mathrm{~h}^{-1}\right)$ of SigmaVOC emitted at 96 hours in the screening measurements of the individual and combined conventional paints ..........90

Figure 10. Concentration ratios of SigmaVOC to TVOC at 24 and 48 hours in the screening measurements of the carpet and carpet cushion materials.

Figure 11. Specific emission rates $\left(\mu \mathrm{g} \mathrm{m}^{-2} \mathrm{~h}^{-1}\right)$ of $4-\mathrm{PCH}$ at 24 and 48 hours in the screening measurements of the carpet materials.

Figure 12. Specific emission rates $\left(\mu \mathrm{g} \mathrm{m}^{-2} \mathrm{~h}^{-1}\right)$ of TVOC at 24 and 48 hours in the screening measurements of the carpet materials

Figure 13. Specific emission rates $\left(\mu \mathrm{g} \mathrm{m}^{-2} \mathrm{~h}^{-1}\right)$ of BHT at 24 and 48 hours in the screening measurements of the carpet cushion materials

Figure 14. Specific emission rates $\left(\mu \mathrm{g} \mathrm{m}^{-2} \mathrm{~h}^{-1}\right)$ of TVOC at 24 and 48 hours in the screening measurements of the carpet cushion materials

Figure 15. Concentration ratios of SigmaVOC to TVOC at 24 and 48 hours in the screening measurements of the vinyl flooring materials.

Figure 16. Specific emission rates $\left(\mu \mathrm{g} \mathrm{m}^{-2} \mathrm{~h}^{-1}\right)$ of $n$-tridecane at 24 and 48 hours in the screening measurements of the sheet vinyl materials

Figure 17. Specific emission rates $\left(\mu \mathrm{g} \mathrm{m}^{-2} \mathrm{~h}^{-1}\right)$ of phenol at 24 and 48 hours in the screening measurements of the sheet vinyl materials

Figure 18. Specific emission rates $\left(\mu \mathrm{g} \mathrm{m}^{-2} \mathrm{~h}^{-1}\right)$ of TXIBQ at 24 and 48 hours in the screening measurements of the sheet vinyl materials

Figure 19. Specific emission rates $\left(\mu \mathrm{g} \mathrm{m}^{-2} \mathrm{~h}^{-1}\right)$ of TVOC at 24 and 48 hours in the screening measurements of the sheet vinyl materials

Figure 20a. Compartment concentrations $\left(\mu \mathrm{g} \mathrm{m}^{-3}\right)$ of ethylene glycol in Experiments P-1 - P-4

Figure 20b. Compartment concentrations $\left(\mu \mathrm{g} \mathrm{m}^{-3}\right)$ of ethylene glycol over 48 - 336 hours in Experiments P-1 - P-4.

Figure 21. Compartment concentrations $\left(\mu \mathrm{g} \mathrm{m}^{-3}\right)$ of Texanoß $\circledast$ in Experiments P-1 - P-4 ....... 102

Figure 22. Compartment air relative humidity over $48-336$ hours in Experiment $P-2$............ 103

Figure 23. Compartment concentrations $\left(\mu \mathrm{g} \mathrm{m}^{-3}\right)$ of acetaldehyde over $1-120$ hours in Experiments P-1 - P-4

Figure 24a. Specific emission rates $\left(\mathrm{mg} \mathrm{m}^{-2} \mathrm{~h}^{-1}\right)$ of ethylene glycol in Experiments P-1 - P-4 ... 105 


\section{LIST OF FIGURES, CONTINUED}

$\underline{\text { Page }}$

Figure 24b. Specific emission rates (mg m-2 $\left.\mathrm{h}^{-1}\right)$ of ethylene glycol over 48 - 336 hours

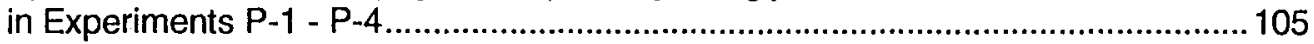

Figure 25. Specific emission rates $\left(\mathrm{mg} \mathrm{m}^{-2} \mathrm{~h}^{-1}\right)$ of TexanolQ in Experiments $\mathrm{P}-1$ - P-4 ......... 106

Figure 26. Effects of the treatments on the 0- to 240-h cumulative mass emissions of acetaldehyde, target VOCs and SigmaVOC from paint relative to averaged base-case Experiments $\mathrm{P}-1$ and $\mathrm{P}-2$ 107

Figure 27. Effects of the treatments on the 0- to 48-h cumulative exposures to formaldehyde, acetaldehyde and target VOCs from paint relative to averaged base-case Experiments $\mathrm{P}-1$ and $\mathrm{P}-2$. 108

Figure 28. Effects of the treatments on the 48- to 240-h cumulative exposures to acetaldehyde and target VOCs from paint relative to averaged base-case Experiments $P-1$ and $P-2$. 109

Figure 29. Cumulative exposures to target compounds over $0-48$ hours divided by total cumulative exposures over 0 - 240 hours for Experiments P-1 - P-4 110

Figure 30. Compartment concentrations $\left(\mu \mathrm{g} \mathrm{m}^{-3}\right)$ of styrene in Experiments $\mathrm{C} 1$ - $\mathrm{C} 3 \ldots \ldots \ldots \ldots . . . . .111$

Figure 31. Compartment concentrations $\left(\mu \mathrm{g} \mathrm{m}^{-3}\right)$ of 4-PCH in Experiments $\mathrm{C} 1-\mathrm{C} 3 \ldots \ldots \ldots \ldots 112$

Figure 32. Compartment concentrations $\left(\mu \mathrm{g} \mathrm{m}^{-3}\right)$ of DPGME in Experiments $\mathrm{C} 1-\mathrm{C} 3 \ldots \ldots \ldots . . .113$

Figure 33. Compartment concentrations $\left(\mu \mathrm{g} \mathrm{m}^{-3}\right)$ of BHT in Experiments $\mathrm{C} 1$ - C3.................114

Figure 34. Compartment concentrations $\left(\mu \mathrm{g} \mathrm{m}^{-3}\right)$ of TVOC in Experiments $\mathrm{C} 1-\mathrm{C} 3 \ldots \ldots \ldots \ldots . \ldots 115$

Figure 35. Specific emission rates $\left(\mu \mathrm{g} \mathrm{m}^{-2} \mathrm{~h}^{-1}\right)$ of styrene in Experiments $\mathrm{C} 1-\mathrm{C} 3 \ldots \ldots \ldots \ldots . . .116$

Figure 36. Specific emission rates $\left(\mu \mathrm{g} \mathrm{m}^{-2} \mathrm{~h}^{-1}\right)$ of 4-PCH in Experiments $\mathrm{C} 1-\mathrm{C} 3 \ldots \ldots \ldots \ldots \ldots . . . . .117$

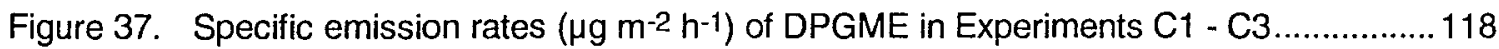

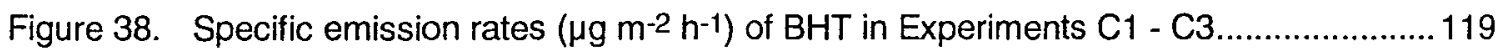

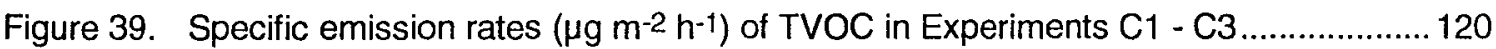

Figure 40. Effects of the treatments on the 0 - to 336-h cumulative mass emissions of target VOCs and TVOC from the carpet assembly relative to base-case Experiment C-1 121

Figure 41. Effects of the treatments on the 0- to 48-h cumulative exposures to target VOCs from the carpet assembly relative to base-case Experiment $\mathrm{C}-1$......................... 122

Figure 42. Effects of the treatments on the 48- to 336-h cumulative exposures to target VOCs from the carpet assembly relative to base-case Experiment $\mathrm{C}-1$.........................123

Figure 43. Cumulative exposures to target VOCs over $0-48$ hours divided by total cumulative exposures over 0 - 366 hours for Experiments C-1 - C-3 …................................. 124

Figure 44. Compartment concentrations $\left(\mu \mathrm{g} \mathrm{m}^{-3}\right)$ of $n$-tridecane in Experiments $\mathrm{V} 1$ - V4 ....... 125

Figure 45a. Compartment concentrations $\left(\mu \mathrm{g} \mathrm{m}^{-3}\right)$ of toluene in Experiments $\mathrm{V} 1-\mathrm{V} 4 \ldots \ldots \ldots \ldots . \ldots 126$

Figure 45b. Compartment concentrations $\left(\mu \mathrm{g} \mathrm{m}^{-3}\right)$ of toluene in Experiments $\mathrm{V} 2-\mathrm{V} 4 \ldots \ldots \ldots \ldots \ldots 126$

Figure 46. Compartment concentrations $\left(\mu \mathrm{g} \mathrm{m}^{-3}\right)$ of tetrahydrofuran in Experiments $\mathrm{V} 1-\mathrm{V} 4$.. 127

Figure 47. Compartment concentrations $\left(\mu \mathrm{g} \mathrm{m}^{-3}\right)$ of phenol in Experiments $\mathrm{V} 1-\mathrm{V} 4 \ldots \ldots \ldots \ldots \ldots . \ldots . \ldots . \ldots 128$

Figure 48. Compartment concentrations $\left(\mu \mathrm{g} \mathrm{m}^{-3}\right)$ of TXIB $\otimes$ in Experiments $\mathrm{V} 1-\mathrm{V} 4 \ldots \ldots \ldots \ldots \ldots 129$

Figure 49. Compartment concentrations $\left(\mu \mathrm{g} \mathrm{m}^{-3}\right)$ of benzothiazole in Experiments $\mathrm{V} 1-\mathrm{V} 4 \ldots 130$

Figure 50. Compartment concentrations $\left(\mu \mathrm{g} \mathrm{m}^{-3}\right)$ of TVOC in Experiments $\mathrm{V} 1-\mathrm{V} 4 \ldots \ldots \ldots \ldots . . . .131$

Figure 51. Specific emission rates $\left(\mu \mathrm{g} \mathrm{m}^{-2} \mathrm{~h}^{-1}\right)$ of $\mathrm{n}$-tridecane in Experiments $\mathrm{V} 1$ - V4 ......... 132

Figure 52. Specific emission rates $\left(\mu \mathrm{g} \mathrm{m}^{-2} \mathrm{~h}^{-1}\right)$ of toluene in Experiments $\mathrm{V} 1-\mathrm{V} 4 \ldots \ldots \ldots \ldots \ldots . . . .133$

Figure 53. Specific emission rates $\left(\mu \mathrm{g} \mathrm{m}^{-2} \mathrm{~h}^{-1}\right)$ of phenol in Experiments $\mathrm{V} 1-\mathrm{V} 4 \ldots \ldots \ldots \ldots \ldots \ldots . \ldots \ldots$

Figure 54. Specific emission rates $\left(\mu \mathrm{g} \mathrm{m}^{-2} \mathrm{~h}^{-1}\right)$ of TXIBQ in Experiments $\mathrm{V} 1-\mathrm{V} 4 \ldots \ldots \ldots \ldots \ldots \ldots$

Figure 55. Specific emission rates $\left(\mu \mathrm{g} \mathrm{m}^{-2} \mathrm{~h}^{-1}\right)$ of benzothiazole in Experiments $V 1-1 / 4 \ldots \ldots 136$ 


\section{LIST OF FIGURES, CONTINUED}

Page
Paxperiments $V 1-V 4.0 .137$

Figure 56a. Specific emission rates $\left(\mu \mathrm{g} \mathrm{m}^{-2} \mathrm{~h}^{-1}\right)$ of TVOC in Experiments V1 - V4 ................. 137

Figure 56b. Specific emission rates $\left(\mu \mathrm{g} \mathrm{m}^{-2} \mathrm{~h}^{-1}\right)$ of TVOC over $48-336$ hours in Experiments V1 - V4

Figure 57. Effects of the treatments on the 0 - to 336 -h cumulative mass emissions of target VOCs and TVOC from the vinyl flooring assembly relative to base-case Experiment $\mathrm{V}-4$

Figure 58. Effects of the treatments on the 0 - to 48-h cumulative exposures to target VOCs from the vinyl flooring assembly relative to averaged base-case Experiments $\mathrm{V}-1$ and $\mathrm{V}-4$

Figure 59. Effects of the treatments on the 48- to 336-h cumulative exposures to target VOCs from the vinyl flooring assembly relative to base-case Experiment $V-4$. 140

Figure 60. Cumulative exposures to target VOCs over $0-48$ hours divided by total cumulative exposures over $0-336$ hours for Experiments V-2, V-3 and V-4

Figure 61. Compartment concentrations $\left(\mu \mathrm{g} \mathrm{m}^{-3}\right)$ of $n$-tridecane in Experiments $\mathrm{A}-1$ and $\mathrm{A}-2.142$

Figure 62. Compartment concentrations $\left(\mu \mathrm{g} \mathrm{m}^{-3}\right)$ of toluene in Experiments $A-1$ and $A-2 \ldots \ldots .143$

Figure 63. Compartment concentrations $\left(\mu \mathrm{g} \mathrm{m}^{-3}\right.$ ) of phenol in Experiments $\mathrm{A}-1$ and $\mathrm{A}-2$....... 144

Figure 64. Compartment concentrations $\left(\mu \mathrm{g} \mathrm{m}^{-3}\right)$ of TXIB in Experiments $A-1$ and $A-2 \ldots \ldots \ldots .145$

Figure 65. Compartment concentrations $\left(\mu \mathrm{g} \mathrm{m}^{-3}\right)$ of benzothiazole in Experiments $A-1$

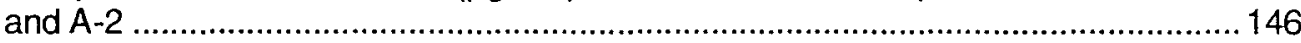

Figure 66. Compartment concentrations $\left(\mu \mathrm{g} \mathrm{m}^{-3}\right)$ of $4-\mathrm{PCH}$ in Experiments $\mathrm{A}-1$ and $\mathrm{A}-2$.......147

Figure 67. Compartment concentrations $\left(\mu \mathrm{g} \mathrm{m}^{-3}\right)$ of $B H T$ in Experiments $A-1$ and $A-2 \ldots \ldots \ldots . . .148$

Figure 68. Compartment concentrations $\left(\mu \mathrm{g} \mathrm{m}^{-3}\right)$ of ethylene glycol in Experiments $A-1$ and $A-2$

Figure 69. Compartment concentrations $\left(\mu \mathrm{g} \mathrm{m}^{-3}\right)$ of Texanol $\otimes$ in Experiments $A-1$ and $A-2 \ldots 150$

Figure 70. Compartment concentrations $\left(\mu \mathrm{g} \mathrm{m}^{-3}\right)$ of $T V O C_{R}$ in Experiments $A-1$ and $A-2 \ldots \ldots 151$

Figure 71. Specific emission rates $\left(\mu \mathrm{g} \mathrm{m}^{-2} \mathrm{~h}^{-1}\right)$ of $n$-tridecane in Experiments $A-1$ and $A-2 \ldots . .152$

Figure 72. Specific emission rates $\left(\mu \mathrm{g} \mathrm{m}^{-2} \mathrm{~h}^{-1}\right)$ of toluene in Experiments $A-1$ and $A-2 \ldots \ldots \ldots 153$

Figure 73. Specific emission rates $\left(\mu \mathrm{g} \mathrm{m}^{-2} \mathrm{~h}^{-1}\right)$ of phenol in Experiments $\mathrm{A}-1$ and $\mathrm{A}-2 \ldots \ldots \ldots . .154$

Figure 74. Specific emission rates $\left(\mu \mathrm{g} \mathrm{m}^{-2} \mathrm{~h}^{-1}\right)$ of TXIB® in Experiments $A-1$ and $A-2 \ldots \ldots \ldots . .155$

Figure 75. Specific emission rates $\left(\mu \mathrm{g} \mathrm{m}^{-2} \mathrm{~h}^{-1}\right)$ of benzothiazole in Experiments $A-1$

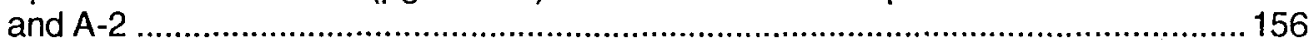

Figure 76. Specific emission rates $\left(\mu \mathrm{g} \mathrm{m}^{-2} \mathrm{~h}^{-1}\right)$ of $4-\mathrm{PCH}$ in Experiments $\mathrm{A}-1$ and $\mathrm{A}-2$...........157

Figure 77. Specific emission rates $\left(\mu \mathrm{g} \mathrm{m}^{-2} \mathrm{~h}^{-1}\right)$ of $B H T$ in Experiments $A-1$ and $A-2 \ldots \ldots \ldots \ldots . . .158$

Figure 78. Specific emission rates $\left(\mu \mathrm{g} \mathrm{m}^{-2} \mathrm{~h}^{-1}\right)$ of ethylene glycol in Experiments $A-1$ and $\mathrm{A}-2$

Figure 79. Specific emission rates $\left(\mu \mathrm{g} \mathrm{m}^{-2} \mathrm{~h}^{-1}\right)$ of Texanok in Experiments $A-1$ and $A-2 \ldots \ldots . .160$

Figure 80. Specific emission rates $\left(\mu \mathrm{g} \mathrm{m}^{-2} \mathrm{~h}^{-1}\right)$ of $\mathrm{TVOC}_{R}$ in Experiments $A-1$ and $A-2 \ldots \ldots \ldots 161$

Figure 81. Effects of the heating treatment in Experiment $A-1$ on the 0 - to 336 -h cumulative mass emissions of target VOCs and $T_{V O C}$ from the combined assemblies relative to base-case Experiment A-2 . .

Figure 82. Effects of the heating treatment in Experiment A-1 on the 96- to 336-h cumulative exposures to target VOCs from the combined assemblies relative to base-case Experiment A-2

Figure 83. Compartment concentrations $\left(\mu \mathrm{g} \mathrm{m}^{-3}\right)$ of $n$-tridecane and toluene over 336 - 2,016 hours in Experiment A-2 . 


\section{LIST OF FIGURES, CONTINUED}

Page

Figure 84. Compartment concentrations $\left(\mu \mathrm{g} \mathrm{m}^{-3}\right)$ of phenol, TXIB® and benzothiazole over 336 - 2,016 hours in Experiment A-2

Figure 85. Compartment concentrations $\left(\mu \mathrm{g} \mathrm{m}^{-3}\right)$ of 4-PCH and BHT over $336-2,016$ hours in Experiment A-2 166

Figure 86. Compartment concentrations $\left(\mu \mathrm{g} \mathrm{m}^{-3}\right)$ of ethylene glycol and Texanok over $336-2,016$ hours in Experiment A-2

Figure 87. Compartment concentrations $\left(\mu \mathrm{g} \mathrm{m}^{-3}\right)$ of $n-$ SigmaVOC $_{p}$ (paint) and $\mathrm{TVOC}_{R}$ (remainder) over 336 - 2,016 hours in Experiment A-2 168 


\section{LIST OF TABLES}

Page

Table 1. Summary of the exposure reduction experiments conducted in the large-scale chamber facility

Table 2. Specifications and operating parameters for the screening measurements conducted in small-scale chambers 6

Table 3. Specifications and operating parameters for the base-case experiments conducted in the large-scale chamber facility ................................................................................. 10

Table 4. Specifications for the first, UNACON-based system used for the analysis of VOCs ..... 16

Table 5. Specifications for the second, Chrompack-based system used for the analysis of VOCs

Table 6. Summary of the qualitative emissions of VOCs by chemical class for the screening measurements of the carpet and carpet cushion materials

Table 7. Most abundant VOCs emitted in the screening measurements of the carpet and carpet cushion materials

Table 8. Summary of the qualitative emissions of VOCs by chemical class for the screening measurements of the five sheet vinyl materials

Table 9. Most abundant VOCs emitted in the screening measurements of the five sheet vinyl materials, the cove base and the associated adhesives.

Table 10. Applied masses $(\mathrm{g})$ of ethylene glycol and Texanoß ${ }^{\circ}$ compared to the masses of these compounds emitted over $0-336 \mathrm{~h}$ in Experiments P-1 - P-4

Table 11. Area-specific emission rates $\left(\mathrm{mg} \mathrm{m}^{-2} \mathrm{~h}^{-1}\right)$ of ethylene glycol, propylene glycol, 2-(2-butoxyethoxy)ethanol, Texanok $\otimes$ and SigmaVOC at 96-h elapsed time in the three screening measurements of the LPS2 and FLP3 paint combination and in large-scale Experiments P-1 - P-4

Table 12. Area-specific emission rates $\left(\mu \mathrm{g} \mathrm{m}^{-2} \mathrm{~h}^{-1}\right)$ of target VOCs and TVOC at 48-h elapsed time in the screening measurements of carpet assembly materials CP4 and CC4 and in large-scale Experiments $\mathrm{C}-1$ and $\mathrm{C}-2$

Table 13. Area-specific emission rates $\left(\mu \mathrm{g} \mathrm{m}^{-2} \mathrm{~h}^{-1}\right)$ of target VOCs and TVOC at 48 -h elapsed time in the screening measurements of individual and assembled vinyl flooring materials and in large-scale Experiments V-1 - V-4.

Table 14. Area-specific 336-h emission rates $\left(\mu \mathrm{g} \mathrm{m}^{-2} \mathrm{~h}^{-1}\right)$ of target VOCs in the large-scale experiments with the individual source assemblies and in Experiments A-2 with the combined source assemblies.

Table 15. Relative precisions for the 18 target VOCs in large-scale experiments $A-1$ and $A-2 \ldots . .68$ 
This investigation characterized the emissions of volatile organic compounds (VOCs) from interior latex paints and newly manufactured carpet and vinyl flooring materials that are representative of materials widely used in California. Specimens of three or more materials in each major source category were obtained and screened for their emissions of VOCs over 48 or 96 hours using small-scale chambers. Forty-five experiments were conducted with 24 primary materials. Seventeen of the VOCs measured have been identified as toxic air contaminants by the California Air Resourses Board. Based on small-chamber results, a paint combination, a carpet assembly, and a vinyl flooring assembly were selected for investigation in large-scale chamber experiments designed to simulate conditions in a small residential room environment. Thirteen large-scale experiments were conducted to provide real-world emissions data and evaluate the effectiveness of several techniques that consumers might use to reduce concentrations of VOCs and their cumulative exposures to these compounds. The exposure reduction techniques included additional ventilation for three days following installation (all source assemblies), additional air mixing (paint combination), airing out of materials prior to installation (carpet and vinyl flooring assemblies), and room heating at $33^{\circ} \mathrm{C}$ subsequent to installation (all sources present together). The assemblies were installed following typical construction industry practice and concentrations of selected VOCs in chamber air were measured over the following two weeks. For each assembly, one or two experiments were performed using a base-case low ventilation rate scenario and two experiments were performed to evaluate the treatments. Two of the 13 experiments employed a combination of all three assemblies. One of these was extended for a period of three months to obtain information on the longer-term emission characteristics of the sources.

There were clear differences in VOC emissions among materials in each class. Therefore, the results demonstrated that there are opportunities to reduce exposures by selecting materials that emit lower amounts of toxic or odorous VOCs. A short period of additional ventilation following material installation generally increased the mass emissions of the compounds and also reduced the cumulative exposures for many compounds during the period of additional ventilation. After the period of additional ventilation, room concentrations were similar to concentrations in experiments without additional ventilation. This suggests that additional ventilation is likely to be a more effective treatment if it can be maintained for longer periods. Airing out carpet material for several days prior to installation effectively reduced the emissions of VOCs. After installing materials, applying heat to the chamber, in conjunction with additional ventilation, increased the emissions of VOCs during the heating period, but was relatively ineffective for reducing the subsequent concentrations and exposures for most compounds. 



\section{EXECUTIVE SUMMARY}

\section{BACKGROUND}

Paints and other materials used to finish the interiors of buildings can emit volatile organic compounds (VOCs), including some toxic pollutants, exposing building occupants to the resulting emissions. The Air Resources Board (ARB) is required to consider such indoor exposures in assessing the risks to public health posed by toxic air contaminants (TACs), as required by California Health and Safety Code, Section 39660.5. The ARB provides information and guidance to the public regarding exposures to TACs, including exposures that occur indoors. In order to perform these assessments, it is necessary to identify the potential sources for the compounds of interest and determine the emission characteristics of these sources. This study was designed to generate data that is needed by ARB to perform these functions.

\section{EXPERIMENTAL METHODS}

The three general categories of interior finish materials selected for this study were interior latex paints, carpet and associated materials, and vinyl floor installation materials. First, the brand names of materials in each category that are widely used by California consumers were identified. Then, newly manufactured specimens of three or more materials in each major category were obtained for testing. Forty-five screening measurements were conducted with specimens of 24 primary materials. These measurements were conducted over 48 or 96 hours at standardized conditions in small-scale environmental chambers. The VOCs emitted by the materials were identified and chamber concentrations and specific emission rates for selected individual compounds and total VOCs (TVOC) were determined. Formaldehyde and acetaldehyde were also quantified.

In the second phase of the study, 13 large-scale experiments were conducted to characterize the emissions from realistic assemblies of the sources and evaluate the effectiveness of treatments that consumers might employ in their residences to reduce their exposures to VOCs. These experiments were performed in a chamber facility designed and operated to simulate a residential room environment. The walls and ceiling of a chamber compartment were painted gypsum board, the floor was carpeted or covered with sheet vinyl, and typical furnishings were installed. These surfaces mimicked typical "sink" effects that may substantially alter the impacts of emissions from indoor sources. Consequently, the net results produced by these experiments should be more realistic than those obtained in an all stainlesssteel chamber with an abnormally low sink effect.

For each large-scale experiment, air samples for the analysis of VOCs and aldehydes were collected from the chamber throughout the first day following installation of the materials, then daily over the next nine days, with final samples taken on day 14 . One experiment with combined source assemblies (carpet and vinyl flooring assemblies and paint) was extended for a total period of 90 days, with samples collected weekly.

The exposure reduction techniques that were investigated included: additional ventilation for three days following installation (all source assemblies); additional air mixing (paint); airing out of materials prior to installation (carpet and vinyl flooring assemblies); and room heating to $33^{\circ} \mathrm{C}$ following installation (combined assemblies). The results of the exposure reduction experiments were compared with those from base-case experiments (experiments with no exposure reduction actions) to quantify the effects of the treatments. The parameters evaluated were VOC concentrations, total VOC mass emissions, and potential cumulative exposures for full-time occupants.

\section{RESULTS OF SCREENING MEASUREMENTS}

The screening measurements generated a large amount of quantitative data on the emissions of VOCs, including 17 identified TACs. This data can be used by ARB in exposure models to estimate indoor exposures. 
Twelve target compounds, including five TACs, were quantified for latex paints. The predominant $\mathrm{VOC}$ emissions from the conventional latex paints were a solvent component (typically ethylene glycol and propylene glycol) and the Texanol $\otimes$ coalescing aid. The emissions from two new generation, "non-VOC" paints did not contain glycol ethers. These paints also had much lower emission rates of SigmaVOC (i.e., the sum of the quantified components) than the conventional paints.

Forty-six target compounds, including 13 TACs, were quantified for the carpet materials. All of the carpets emitted 4-phenylcyclohexene (4-PCH), an unwanted byproduct in the production of styrene-butadiene rubber latex. Styrene was detected in the emissions from three carpets. The emission rates of styrene were near detection limits, demonstrating a significant reduction relative to previously reported results. The two intermediate-grade nylon pile carpets emitted fewer VOCs and had lower emission rates of individual VOC and TVOC than the olefin carpets. The nylon carpets met the emissions guidelines of the Carpet and Rug Institute's (CRI) Indoor Air Quality Testing Program. Olefin carpets did not meet the CRI guidelines; however, the testing conditions were altered somewhat from the CRI procedures. All of the bonded urethane carpet cushions emitted butylated hydroxytoluene (BHT). The TVOC emission rate for the synthetic fiber cushion was substantially lower than the TVOC emission rates for the bonded urethane cushions.

Forty target compounds, including ten TACs, were quantified for the vinyl flooring materials. All of the sheet vinyls were a source of phenol, an identified TAC. The residential sheet vinyls with a clear "no-wax" top coat emitted more compounds and had higher emission rates of individual VOCs and TVOC than the single commercial sheet vinyl. The new-generation "solvent free" or "low VOC content" adhesive products were the source of a number of VOCs. No experiments were conducted with conventional adhesives.

\section{RESULTS OF EXPOSURE REDUCTION EXPERIMENTS}

In general, the use of additional ventilation at two air changes per hour for the first three days of an experiment decreased the chamber concentrations of many target VOCs during the treatment period, relative to the base-case experiments. Consequently, this treatment often produced beneficial effects with respect to cumulative exposures calculated for the first 48 hours after material installation. However, upon termination of additional ventilation, the chamber concentrations frequently returned to values similar to those measured in the base-case experiments. Thus, the beneficial effects of the treatment, with respect to potential exposures calculated for the remainder of the experimental period beginning 48 hours after material installation, were limited to specific compounds and were often relatively minor.

The large-scale experiments with paint produced the highest VOC concentrations. The dominant compounds were ethylene glycol and Texanol 8 . The initial concentrations of these compounds frequently ranged up to several tens of milligrams per cubic meter. The concentrations of the dominant VOCs were somewhat lower during the initial three-day period of additional ventilation, compared to the base-case experiments. Following the termination of additional ventilation, the concentrations in the treatment and base-case experiments were similar. The use of fans to increase air velocities at the painted surfaces, in combination with additional ventilation, did not result in large-magnitude reductions in air concentrations of the target VOCs, relative to the experiment with only additional ventilation.

The room concentrations of the target VOCs emitted by the carpet assembly were generally low when compared to the concentrations of VOCs emitted by the other source assemblies. The use of additional ventilation temporarily reduced the concentrations of many of the target VOCs emitted from the carpet. However, after the ventilation rate was reduced, the concentrations typically increased to values similar to those measured in the base-case experiment. One exception was the BHT emitted by the carpet cushion that reached slightly higher values in the treatment experiment with additional ventilation. 
The vinyl flooring assembly emitted a large number of compounds. The compounds with the highest concentrations were tetrahydrofuran and cyclohexanone emitted by the seam sealer, toluene emitted by the sheet flooring adhesive, and n-tridecane and phenol emitted by the sheet vinyl flooring. The concentrations of tetrahydrofuran, cyclohexanone, and toluene, all volatile compounds, decreased rapidly over the first $24-48$ hours of the experiments. Additional ventilation substantially decreased the concentrations of these compounds compared to the basecase experiment. Additional ventilation, however, had relatively small effects on the concentrations of the less volatile VOCs emitted by the assembly, such as phenol, TXIB® (a vinyl plasticizer), and benzothiazole.

Airing out materials for two days prior to their installation was effective for reducing the concentrations of VOCs emitted by the carpet assembly materials relative to the base-case experiment. This translated into reduced exposures for these constituents. However, the fractional reductions in exposures for two of the dominant compounds, 4-PCH from the carpet and BHT from the carpet cushion, beginning 48 hours after installation of the carpet, were relatively small. Airing out the sheet vinyl and the cove base had only a short-lived effect on the concentrations of the target VOCs or none at all.

Moderate in-situ heating, in combination with additional ventilation, increased the concentrations of many of the individual VOCs and TVOC emitted by the three combined source assemblies (carpet and vinyl flooring assemblies and paint). The effects were generally highest at the onset of heating and diminished with time over the 72-hour (72-h) heating period. However, the effects were largely limited to the actual heating period. Once heating was discontinued, the concentrations of many of the compounds quickly returned to values that were similar to those measured in the experiment with additional ventilation but no heating. Texanol $\otimes$, one of the dominant compounds emitted by the latex paints, was an exception. Its longer-term concentrations decreased as a result of heating.

The long-term experiment was conducted at the base-case ventilation rate of 0.5 air changes per hour following a $72-\mathrm{h}$ period of additional ventilation. The concentrations of a number of the dominant target VOCs and TVOC emitted by the three combined source assemblies remained relatively constant or decreased by less than a factor of three over the period of two to 12 weeks.

\section{CONCLUSIONS}

This is the first study to characterize VOC emissions from vinyl flooring assemblies and to obtain long-term measurements from the combined sources. This study generated a large amount of quantitative data on the emissions of VOCs, including TACs, from indoor sources commonly used by California consumers Additionally, the study quantified the effects of several exposure reduction techniques that homeowners or building managers might use to reduce the concentrations of VOCs and occupant exposures to VOCs following construction and/or remodeling of their homes or businesses.

The exposure reduction experiments demonstrated that there are some general practices homeowners or building managers can follow that may reduce $\mathrm{VOC}$ concentrations and decrease cumulative exposures to VOC emissions in their environments.

1) Materials that emit lower amounts of toxic or odorous VOCs should be selected whenever possible. This should lower occupant exposures and decrease the need for subsequent control strategies.

2) If possible, carpets and carpet cushions should be aired out in a well-ventilated, clean, dry environment for several days prior to their installation.

3) Very high, mechanically induced ventilation rates should be used during the application or installation of materials to protect the installers and any occupants that cre present. This technique may also reduce subsequent occupant exposures. 
4) Additional ventilation should be maintained for periods longer than three days to more effectively lower occupant exposures.

5) The amount of additional ventilation should be maximized. In many cases, the beneficial effects of ventilation, with respect to concentrations and exposures, are not in direct proportion to the increase in the ventilation rate.

6) The most effective way to reduce occupant exposures to the dominant VOCs emitted by latex paints is to delay occupancy for several days following painting. 


\subsection{INTRODUCTION}

\subsection{BACKGROUND}

Many materials and products that are used to construct and finish the interiors of residences and office buildings are potential sources of volatile organic compound (VOCs), including some toxic air contaminants (TACs). The emissions of VOCs from these materials result in exposures to building occupants. The Air Resources Board (ARB) considers such indoor exposures in assessing the risks to public health posed by TACs as required by California Health and Safety Code, Section 39660.5. In order to perform this assessment, it is necessary to identify the potential indoor sources of the compounds of interest and to determine the emission characteristics of these sources. The ARB additionally provides information and guidance to the public regarding exposures to TACs including exposures that occur indoors. Unfortunately, the information that is needed by the ARB to perform these functions is quite limited.

Hodgson and Wooley (1991) conducted a literature review to evaluate the existing data on the potential indoor uses and sources of 47 candidate compounds that, at that time, were being reviewed for classification as TACs. A number of the compounds were found to be present as minor constituents in building materials, interior-finish materials, and various consumer products. Generally, the data on the source emissions of the reviewed compounds was very limited. In 1993, California adopted the Federal list of 189 TACs, thus greatly expanding the list of compounds for which data was needed.

In addition to the materials that are used to construct and finish the interiors of residences and office buildings, other indoor sources of VOCs include furnishings, maintenance and cleaning products, other expendable consumer products, and combustion processes. The emissions of VOCs from all of these sources have become a focus of concern because it is believed that they may contribute to adverse short- and long-term health effects sometimes experienced by building occupants. Such short-term health effects include eye irritation, upper respiratory tract irritation, headache, dizziness and fatigue (USEPA, 1993). Some compounds produce objectionable odors at low concentrations and have adverse effects on occupant comfort. Long-term health effects are associated with TACs that are either known or suspected carcinogens, reproductive toxicants, or developmental toxicants.

In response to the ARB's need for information, this study was designed to qualitatively and quantitatively characterize the emissions of VOCs, including TACs, from three common indoor sources in the general category of interior finish materials and products. These sources were interior latex paints, carpet assemblies and vinyl flooring assemblies. The emissions of a broad spectrum of VOCs from the sources were first characterized in screening measurements using small-scale chambers. They were further characterized under controlled conditions in large-scale chambers designed and operated to simulate a residential room environment. In addition, various practical techniques that consumers might be able to use to reduce their exposures to the VOCs emitted by the sources were investigated in the large-scale experiments. One of these experiments was conducted with all three sources over a period of three months to provide much needed data on the longer-term emission characteristics of the sources.

The emissions of VOCs from typical residential carpets were studied extensively in the early 1990's (Black et al., 1991; Hetes et al., 1992; Hodgson et al., 1992 and 1993). In general, the emissions of VOCs from these carpets were found to be low compared to many other common indoor sources. More recently, the emissions of VOCs from the major types of carpet cushions were investigated (Hodgson and Phan, 1994; Schaeffer et al., 1996). There were substantial differences among the different types of cushions with respect to the kinds and amounts of compounds that were emitted, and the emission rates of total VOCs (TVOC) from the cushions were frequently higher than the emission rates from carpets.

Consumer complaints related to carpet installations (Schachter, 1990) prompted the U.S. Consumer Product Commission to initiate a health hazard assessment of VOC emissions for carpets and associated carpet cushions. The Carpet and Rug Institute (CRI), an industry trade association, iesponded to complaints by instituting an Indoor Air Quality Carpet Testing Program 
for VOC emissions (Leukroth, 1991). This testing program established an acceptable limit for the emission rate of 4-phenylcyclohexene (4- $\mathrm{PCH}$ ), an unwanted byproduct in the styrene-butadienerubber (SBR) latex used on the secondary backing of the carpets, which is responsible for the new carpet odor. Limits were also set for the emission rates of TVOC, styrene and formaldehyde from these carpets. The testing program, which is now widely used by the major carpet manufacturers, may have resulted in a general reduction in the emissions of 4-PCH and other VOCs from SBR carpets. Consequently, it was desirable to obtain current qualitative and quantitative data on the emissions of VOCs from representative carpets and associated carpet cushions.

There have only been limited studies of the emissions of VOCs from vinyl flooring materials (Bremer et al., 1993; Clausen et al., 1993; Christiansson et al., 1993). These studies have generally shown that many VOCs originate in vinyl flooring and that the emissions of these compounds can be expected to persist over relatively long time periods. Since different grades of vinyl flooring may employ different processes in their production, it was anticipated that there would be substantial differences in emission characteristics among the available products. Therefore, it was desirable to include representative vinyl flooring materials as one of the source categories.

Latex paints contain organic solvents such as ethylene glycol or propylene glycol and semi-volatile coalescent agents. Among the various brands of latex paint, the VOC content varies with the type of vehicles used as a binder and thinner (Sheldon and Naugle, 1994). Also, the stabilizing agents, the gloss type and differences in the amounts of organic solvents can significantly affect the emissions. The quality of the paint (e.g., consumer versus professional grade) is also expected to effect emissions, since paints with a strong film and high covering powers require a thinner coat or fewer coats to achieve an acceptable finish. Until just recently, the studies of latex paints have focused on determining the VOC content of the bulk products or on measuring the emissions of VOCs from paints applied to impermeable surfaces (Sheldon and Naugle, 1994; Censullo, et al., 1996). Thus, at the outset of this study, there was very little emissions data for this important indoor source of VOCs that was obtained under realistic conditions. It is now known that the substrate has a dramatic effect on the emission rates of VOCs from latex paints. When latex paints are applied to gypsum board, the most common residential substrate, the VOCs are emitted slowly over very long time periods (Chang, et al., 1997 and 1998).

Paint manufacturers have also been reformulating their products with lower VOC contents to comply with requirements first developed by the ARB (ARB-CAPCOA, 1989) and also mandated by the Clean Air Act of 1990 (e.g., C\&EN, 1994). New generation products that contain reduced amounts of VOCs are continually being introduced. There is almost no emissions data for these "low- or no-VOC content" paints, and it is not known what impact widespread use of these new products will have on consumer exposures to VOCs.

There has been considerable interest in developing strategies to reduce occupant exposures to indoor sources of VOCs. In response to a California legislative mandate, the California Department of Health Services (DHS) developed non-binding guidelines for the reduction of exposures to VOCs from construction materials used in office buildings (Alevantis, 1996). The DHS document reviewed the available literature and discussed strategies for evaluating and selecting "low VOC-impact" materials. Various exposure reduction techniques were also reviewed including: material conditioning; modified installation protocols; modified ventilation protocols; and delayed occupancy. A modeling study of source management techniques was also recently conducted by Tichenor and Sparks (1996). They employed an indoor air quality model to predict occupant exposures for different indoor source scenarios and selected source management options. Both the DHS document and the modeling study provided valuable information regarding exposure reduction options. However, controlled field- and/or laboratory-based studies are needed in order to validate and compare the efficacies of the various techniques that were proposed. 


\subsection{STUDY OBJECTIVES}

This study was designed to produce a substantial amount of emissions data that could be used by the ARB in their efforts to identify the potential indoor sources of VOCs, including TACs, and to assess their impacts. This data was obtained in small- and large-scale environmental chambers following accepted practice so that it could be used in indoor air quality exposure models. Secondly, the study was designed to provide an experimental basis for the ARB's public information efforts regarding exposure reduction techniques that consumers might be able to use to reduce the concentrations of indoor air contaminants and their exposures to these compounds. So that the data would be relevant to California, a strong emphasis was placed on identifying and obtaining source materials that were representative of the products available in the California marketplace and widely used by California consumers.

The specific objectives of the screening measurement phase of the study were:

- To select and obtain representative study materials, including interior latex paints, carpets, carpet cushions and sheet vinyl flooring.

- To identify the VOCs emitted by newly manufactured specimens of the materials in shortterm experiments conducted in small-scale chambers.

- To quantify the chamber concentrations of TVOC and selected individual VOCs emitted by the specimens.

- To determine the specific emission rates of the selected components.

- To compare qualitatively and quantitatively the materials within each source category based on their emission characteristics.

The specific objectives of the second phase of the study conducted in large-scale chambers were:

- To select typical and appropriate study materials for use in the large-scale experiments based on the screening measurement results.

- To develop realistic and reproducible protocols for the installation or application of the sources (i.e., a paint combination, a carpet assembly, and a vinyl flooring assembly) in the large-scale chambers.

- To conduct one or more base-case experiments for each source assembly under typical indoor conditions over a period of two weeks following installation.

- To identify the VOCs emitted by the source assemblies.

- To measure the chamber concentrations and determine the specific emission rates of TVOC and selected individual VOCs as a function of time for the basecase experiments.

- To experimentally determine for each source assembly the effects of several exposure reduction techniques on the concentrations and emission rates of TVOC and selected VOCs.

- To estimate the potential impacts of the exposure reduction techniques on the cumulative exposures for full-time occupants.

- To characterize the emissions of TVOC and selected VOCs from the combined assemblies over a period of three months.

\subsection{OVERVIEW OF THE STUDY PLAN}

The three different types of sources included in this study were: combinations of interior flat and semi-gloss latex paints; new carpet assemblies; and new vinyl flooring assemblies. For each assembly, three or four different types and/or brands of each major material were selected for initial screening. 
The first task was to identify the individual materials for the study. Literature on the emissions of VOCs from similar materials was reviewed. Relevant and current published data on the market shares of the materials under consideration was largely unavailable. Therefore, industry trade associations, manufacturers, and a number of California wholesalers and retailers were contacted to obtain qualitative estimates of the market shares of the different types and brands of materials. This information from informed individuals was used to guide the selection. The final decision was made in consultation with the ARB staff. All of the materials that were selected were commonly available and were believed to have relatively high market shares in California.

The next task was to obtain representative samples of the materials. All of the paints were purchased from open stock at local retail outlets. Most of the major flooring materials were purchased through a local contract flooring dealer. The dealer contacted the manufacturers or primary distributors to obtain information on the production schedules for the selected materials. Orders were placed based on the future production dates so that recently manufactured materials of known age were obtained.

All of the materials were individually screened for emissions of VOCs in small-scale chambers. For each major type of material, a duplicate experiment was conducted to assess experimental reproducibility. There were also a number of blank chamber runs. The materials were maintained at standard conditions in 10.5-L chambers for a period of 48 hours, or 96 hours for paints. The inlet flow rate of nitrogen humidified to $50 \pm 10$ percent relative humidity was controlled, and the chambers were held at $23 \pm 1^{\circ} \mathrm{C}$ in an incubator. Samples for VOCs and formaldehyde plus acetaldehyde were periodically collected from the chambers during this time. The VOCs emitted by the materials were identified. Individual VOCs were selected for quantitative analysis based on the qualitative results. The concentrations of TVOC and the individual compounds in the chamber exhaust were measured as a function of time. Specific emission rates of the selected components were calculated based on the concentration data and the experimental parameters.

The materials for the large-scale chamber experiments were selected primarily based on the results of the screening measurements. The selected paint combination consisted of a latex primer sealer and flat and semi-gloss latex paints. The flat and semi-gloss paints were compatible materials from the same manufacturer. The carpet assembly consisted of an action back carpet with olefin face fiber laid over a bonded urethane carpet cushion. The vinyl flooring assembly consisted of a residential "no-wax" sheet vinyl applied to particleboard underlayment, a rubber cove base, and associated adhesives.

The exposure reduction experiments were conducted using a large-scale chamber facility operated to simulate a typical residential room environment and constructed with typical interiorfinish materials. The facility consisted of two adjacent, identical compartments with volumes of $25.5 \mathrm{~m}^{3}$. Inlet air was drawn from outside the building and was filtered through a bed of activated charcoal. The base-case operating temperature was $23 \pm 2^{\circ} \mathrm{C}$, and the ventilation rate was $0.5 \pm 0.03 \mathrm{~h}^{-1}$. The walls and ceiling of a chamber compartment were painted gypsum board; the floor was carpeted or covered with sheet vinyl; and typical furnishings were installed. These surfaces were included to mimic typical "sink" effects that may substantially alter the impacts of VOC emissions from indoor sources.

Thirteen large-scale experiments were conducted. These are summarized in Table 1. For each source assembly, there was a base-case experiment to measure the emissions of VOCs at typical indoor conditions and to serve as a reference for evaluating the effects of the treatments. Duplicate experiments were conducted at the base-case conditions with the paint combination and the vinyl floor assembly to assess experimental reproducibility. Two treatment experiments were conducted for each individual assembly. For the paint combination, the effects of additional ventilation during the first three days of the experiment and of additional ventilation in combination with increased air mixing at the painted surfaces were investigated. For both the carpet and the vinyl flooring assemblies, the effects of additional ventilation were investigated. In other experiments, these materials were pre conditioned by airing them out for 48 hours prior to installation. Two experiments were performed with a combination of all three source assemblies. 
One of these was conducted with additional ventilation. The other was conducted with additional ventilation in combination with mild heating of the compartment to about $33^{\circ} \mathrm{C}$ over the first three days after installing the carpet and the vinyl flooring.

The large-scale experiments were conducted over a period of 14 days. Samples for VOCs and aldehydes were collected daily during the first ten days and then finally on the fourteenth day. The experiment with the combined source assemblies that was not heated was extended for a total elapsed time of 90 days $(2,016$ hours). Samples were collected weekly after the first fourteen days.

The individual compounds emitted by the source assemblies were identified and individual VOCs were selected for quantitation based on these results. In selecting the target compounds, emphasis was placed on the most abundant compounds, on compounds with known health effects such as ARB TACs, and on compounds that were indicative of individual source materials.

The area-specific emission rates for TVOC and the individual target compounds were calculated based on the concentration data and the experimental parameters. Additionally, the cumulative mass emissions of these components were calculated, and potential cumulative exposures for full-time occupants were estimated based on an assumed occupancy scenario.

The efficacies of the exposure reduction techniques were evaluated by comparing the compartment VOC concentrations, emissions parameters and estimated exposures for the treatments with the corresponding results for the base-case experiments. 
Table 1. Summary of the exposure reduction experiments conducted in the large-scale chamber facility.

\begin{tabular}{|c|c|c|c|}
\hline $\begin{array}{l}\text { Assembly / } \\
\text { Experiment ID }\end{array}$ & Treatment & $\begin{array}{l}\text { ET } \\
(h)\end{array}$ & Materials Used ${ }^{*}$ \\
\hline \multicolumn{4}{|l|}{ Latex Paints } \\
\hline P-1 & Base-case & 336 & LPS2,FLP3,SGLP3 \\
\hline$P-2$ & Base-case & 336 & LPS2,FLP3,SGLP3 \\
\hline$P-3$ & Additional ventilation & 336 & LPS2,FLP3,SGLP3 \\
\hline$P-4$ & $\begin{array}{l}\text { Additional ventilation } \\
\text { \& air mixing }\end{array}$ & 240 & LPS2,FLP3,SGLP3 \\
\hline \multicolumn{4}{|c|}{ Carpet Assembly } \\
\hline C-1 & Base-case & 336 & $\mathrm{CP} 4, \mathrm{CC} 4, \mathrm{ST}$ \\
\hline $\mathrm{C}-2$ & Additional ventilation & 336 & $\mathrm{CP} 4, \mathrm{CC} 4, \mathrm{ST}$ \\
\hline C-3 & Airing out of materials & 336 & $\mathrm{CP} 4, \mathrm{CC} 4, \mathrm{ST}$ \\
\hline \multicolumn{4}{|c|}{ Vinyl Floor Assembly } \\
\hline$V-1$ & Base-case & 144 & SV5,CB,SFA,CBA,SS \\
\hline$V-4$ & Base-case & 336 & SV5,CB,SFA,CBA,SS \\
\hline$V-2$ & Additional ventilation & 336 & SV5,CB,SFA,CBA,SS \\
\hline$V-3$ & Airing out of materials & 336 & SV5,CB,SFA,CBA,SS \\
\hline \multicolumn{4}{|c|}{ Combined Sources } \\
\hline$A-2$ & Additional ventilation & 2,016 & All materials \\
\hline$A-1$ & $\begin{array}{l}\text { Additional ventilation } \\
\text { \& heating }\end{array}$ & 336 & All materials \\
\hline
\end{tabular}

*LPS2 = Latex Primer Sealer No. 2; FLP3 = Flat Latex Paint No. 3; SGLP3 = Semigloss Latex Paint No. 3; CP4 = Carpet No. 4; CC4 = Carpet Cushion No. 4; ST = Seaming Tape; SV $5=$ Sheet Vinyl No. $5 ; \mathrm{CB}=$ Cove Base; SFA = Sheet Flooring Adhesive; $\mathrm{CBA}=$ Cove Base Adhesive; $\mathrm{SS}=$ Seam Sealer.

\subsection{EXPERIMENTAL METHODS}

\subsection{PROCEDURES USED FOR THE SCREENING MEASUREMENTS}

\subsubsection{Description of the Small-Scale Chamber Facility}

The small-scale chamber facility and the individual chambers were constructed and operated following the American Society for Testing and Materials Standard Guide D-5116-90 (ASTM, 1990). The specifications and operating parameters for the small-scale chambers are described in Appendix A and summarized in Table 2. The chambers consisted of polished 316 stainless steel, cylindrical vessels. The chambers were equipied with stainless-steel lined lids 
that were sealed with Teflon-coated silicone gaskets. The internal volume of the chambers was $10.5 \mathrm{~L}$. The facility was designed so four chambers could be simultaneously operated. They were held in a single incubator with heating and refrigeration controls. The standard chamber operating temperature was $23 \pm 1^{\circ} \mathrm{C}$ (average \pm one standard deviation).

The inlet gas for each chamber was high-purity nitrogen supplied by gas cylinders. The flow rate of nitrogen was regulated at $1.0 \pm 0.05 \mathrm{~L} \mathrm{~min}^{-1}$ (average \pm one standard deviation) with a mechanical flow controller and was measured with an electronic mass-flow sensor. The gas stream was split into two streams with balanced flow rates. One of these passed through a bubbler containing distilled water held in the incubator. This stream was mixed with the dry gas stream to generate an inlet gas stream with $50 \pm 5$ percent relative humidity (average \pm one standard deviation) at $23^{\circ} \mathrm{C}$. The humidified gas stream was introduced into the chamber through a fitting on the lid with a tubing extension that terminated near the bottom of the chamber. Gas exited the chamber through another fitting on the lid. A sampling manifold constructed of stainless-steel tube fittings was attached to the outlet of this fitting. A combined temperature and humidity probe (Part No. HMD 30 YB, Vaisala, Finland) was inserted into the chamber through a third fitting. Chamber temperature, relative humidity and inlet gas flow rate were monitored and recorded throughout an experiment at five-minute intervals with a PC-based data system (Computer Boards, Inc.) and data acquisition software (Labtech Notebook, Version 7, Laboratory Technologies, Corp.).

Table 2. Specifications and operating parameters for the screening measurements conducted in small-scale chambers.

\begin{tabular}{|c|c|}
\hline Parameter & Value \\
\hline Chamber material & 316 Stainless stee \\
\hline Chamber volume, $\mathrm{m}^{3}$ & $10.5 \times 10^{-3}$ \\
\hline Gas & Humidified $\mathrm{N}_{2}$ \\
\hline Inlet gas flow rate, $m^{3} h^{-1}$ & $6.0 \pm 0.3 \times 10^{-2 *}$ \\
\hline Ventilation rate, $\mathrm{h}^{-1}$ & $5.7 \pm 0.3^{*}$ \\
\hline Temperature, ${ }^{\circ} \mathrm{C}$ & $23 \pm 1^{*}$ \\
\hline Relative humidity, \% & $50 \pm 5^{*}$ \\
\hline Air velocity ${ }^{\star \star}, \mathrm{m} \mathrm{s}^{-1}$ & $\sim 0.25$ \\
\hline Sample surface areat, $\mathrm{m}^{2}$ & $1.95 \times 10^{-2}$ \\
\hline Sample loading ratio, $\mathrm{m}^{2} \mathrm{~m}^{-3}$ & 1.86 \\
\hline Ventilation/loading ratio, $\mathrm{m} \mathrm{h}^{-1}$ & 3.07 \\
\hline Exposure period, $\mathrm{h}$ & 48 or 96 \\
\hline Sample collection times, $\mathrm{h}$ & $1,6,24,48,96$ \\
\hline
\end{tabular}




\subsubsection{Operating Procedures}

The screening measurement procedures are described in detail in Appendix A.

A clean, empty chamber was operated at the temperature, humidity and ventilation conditions of the experiment for at least one hour. The material specimen to be tested was prepared as described below. The chamber was opened, and the specimen was placed on a metal rack that held the specimen near the approximate center of the chamber. The chamber lid was quickly re-attached and the data system was re-started. This established the initial time point for the experiment. Samples for VOCs and aldehydes were simultaneously collected from the sampling manifold on the chamber outlet at the average elapsed times given below for each material category. Typically, duplicate VOC samples and a single aldehyde sample were collected at each sampling interval. The sample flow rates were regulated with electronic massflow controllers (Model FC 280, Tylan General). The flow rate for the VOC samples was typically $0.1 \mathrm{~L} \mathrm{~min}^{-1}$; however, this rate was varied depending upon the magnitude of the source. The flow rate for the aldehyde samples was $0.5 \mathrm{~L} \mathrm{~min}^{-1}$. The total sample flow rate was always substantially less than the inlet flow rate of gas to the chamber. Three-way solenoid values controlled by the data system were used to automate the collection of the samples.

The experimental data consisted of: temperature and relative humidity measured by the probe inserted into the chamber; incubator temperature; inlet gas flow rates measured by the mass-flow sensor; solenoid sampling valve positions; elapsed time and clock time. Manual adjustments were periodically made to the split between the dry and humidified inlet gas streams to keep the chamber relative humidity within the desired range.

Latex Paints - The substrate for the latex primer sealers and the flat latex paints was a 15.2 by $15.2-\mathrm{cm}$ piece of $1.3-\mathrm{cm}$ thick, unfinished gypsum board. These paints were applied by roller. The substrate for the latex semi-gloss paints was a 15.2 by $15.2-\mathrm{cm}$ piece of $1.3-\mathrm{cm}$ thick, smooth plywood. These paints were applied with a brush. An area 14.0 by $14.0 \mathrm{~cm}\left(0.0195 \mathrm{~m}^{2}\right)$ was painted. The coverage in grams of wet paint applied per square meter of substrate surface was determined by weighing a substrate before and immediately after painting. For the paint combinations, there was a one-hour drying period between the application of the primer sealer and the finish coat. The painted substrate was placed into a stainless-steel holder that covered the back and edges of the substrate. The substrate was sealed into the holder with strips of aluminized tape so that only the painted surface was exposed. Blank substrates were similarly prepared. A freshly prepared specimen was immediately inserted into a chamber, and the experiment was initiated. For these experiments only, a small 3.8-cm diameter axial fan (12 VDC) was mounted in a chamber. The air velocity near the painted surface was approximately $0.25 \mathrm{~m}$ $\mathrm{sec}^{-1}$. The screening measurements with the paints were conducted over a period of 96 hours. Air samples were collected at average elapsed times of 1, 6, 24, 48 and 96 hours.

The bulk paints that were selected for use in the large-scale experiments were solvent extracted and quantitatively analyzed for their principal VOC components. Approximately $2.5 \mathrm{~g}$ of paint was weighed into a 15-mL disposable centrifuge tube and diluted with $10 \mathrm{~mL}$ of methanol. The diluted paint was placed in an ultrasonic bath for ten minutes and then centrifuged. A small aliquot of the supernatant was further diluted in methanol. Several microliters of the dilute extract were transferred to a Tenax sorbent tube. The tube was purged with helium to remove the solvent, and the sample was analyzed for VOCs by thermal desorption GC/MS as described below.

Carpet Materials - The test specimens of the carpet materials were cut from the large pieces and placed into 15.2 by $15.2-\mathrm{cm}\left(0.0232-\mathrm{m}^{2}\right)$ stainless-steel holders that covered the bottom and cut edges of the specimens. For the experiment with the seaming tape, the carpet specimen was cut in half and seamed using a hot iron. It was then placed into a holder. The specimens were immediately inserted into the chambers for testing. The screening measurements were conducted over a period of 48 hours. Air samples were collected at average elapsed times of $1,6,24$ and 48 hours.

Vinyl Flooring Materials - The sheet vinyl specimens were cut from the large pieces and were attached to 15.2 by $15.2-\mathrm{cm}$ stainless-steel plates with aluminized tape so that the exposed area 
of the specimens was $0.0195 \mathrm{~m}^{2}$. The freshly prepared specimens were immediately inserted into the chambers for testing. The cove base was tested with both sides of the piece exposed. The adhesives were tested by applying them to their respective substrates and leaving the surfaces of the adhesives exposed. The sheet flooring adhesive was applied with a notched trowel with $1-\mathrm{mm}$ teeth. The substrates were taped into stainless-steel holders. The composite assemblies were prepared following the adhesive manufacturers' instructions. The substrates for these tests were also taped into stainless-steel holders. The screening measurements were conducted over a period of 48 hours. Air samples were collected at average elapsed times of $1,6,24$ and 48 hours.

Measured microliter quantities of the two liquid components of the seam sealer were individually injected into sealed 2-L flasks. A flask was heated and gas was withdrawn from the flask with a gas-tight syringe and transferred to a sorbent sampler. The sampler was analyzed for the dominant solvent components by thermal desorption GC/MS as described below.

\subsection{PROCEDURES FOR THE EXPOSURE REDUCTION EXPERIMENTS}

\subsubsection{Description of the Large-Scale Chamber Facility}

The chamber facility is described in detail in Appendix F. A schematic of the facility is shown in Figure 1.

The chamber facility was housed in a small building equipped with a heat pump heating ventilating and air conditioning (HVAC) system.

There were two adjacent, identical compartments. Both were designed and constructed to simulate a small room in a typical residence. The specifications and base-case operating parameters for the compartments are summarized in Table 3. The interior dimensions of each compartment were $2.26 \mathrm{~m}$ wide by $4.62 \mathrm{~m}$ long with a $2.44-\mathrm{m}$ high ceiling, yielding a volume of $25.5 \mathrm{~m}^{3}$. The walls and ceiling were finished with gypsum board and painted with a "no VOC" primer and flat latex paint combination. The plywood floor was covered with thin aluminum plates. Each compartment had a single, unfinished, engineered-wood door. The door was weatherstripped. During an experiment, the gaps around the door were sealed with duct tape. There were no windows.

Ventilation air for the compartments was supplied from the exterior of the building. This air was filtered for gaseous contaminants by passing through a box containing a $5-\mathrm{cm}$ thick horizontal bed of activated charcoal with dimensions of 0.61 by $0.61 \mathrm{~m}$. The filter box had two exits, each leading to one compartment. The components for each of these systems were identical. An in-line centrifugal blower was attached to the filter box. Downstream of the blower was a manifold leading to three different sized ducts that were selected with manual ball valves. They were used to provide chamber compartment air change rates of $0.5,2.0$ and $5.0 \mathrm{~h}^{-1}$. A pitot tube (Dwyer Instruments, Inc.) was installed in each duct to monitor the air velocity pressure. A manual gate valve or a drilled plate installed downstream of the pitot tube was used to set the desired air flow rate. The supply air entered the compartment $20 \mathrm{~cm}$ above the floor at a narrow end of the compartment.

For all experiments, compartment air was controlled at a typical room temperature of $23 \pm 2^{\circ} \mathrm{C}$ (average \pm one standard deviation) by regulating the building's HVAC system. Compartment air temperature was maintained within a range of approximately $19-27^{\circ} \mathrm{C}$.

The experimental design specified that compartment air relative humidity be maintained at $50 \pm 10$ percent (average \pm one standard deviation) and within a range of $30-70$ percent. $A$ humidification system was added beginning with the fourth experiment. This system automatically injected water into a heated section of the supply air duct upstream of the filter box when the relative humidity of this gas stream fell below 40 percent at room temperature. The water supply rate was regulated with a variable speed pump controlled by the data svstem.

Air exited each compartment on the same wall that air eritered the compartment. The center of the outlet was located $38 \mathrm{~cm}$ from the ceiling, about $2.2 \mathrm{~m}$ cilagonally across from the air 
inlet. The dimensions of the outlet were $15 \mathrm{~cm}$ by $25 \mathrm{~cm}$. Exterior to the compartment, the outlet was fitted with a manually adjusted damper. Air was exhausted to the exterior of the building.

Each compartment was fitted with six 7.6-cm diameter axial fans to provide adequate air mixing. The fans were attached to removable metal poles. The placement of the fans is described in Appendix $F$. The fan speed was regulated with a variable transformer to achieve an average air velocity near the floor of $0.10 \pm 0.05 \mathrm{~m} \mathrm{~s}^{-1}$. This is a typical near-floor velocity for occupied buildings (Kovanen et al., 1987).

Table 3. Specifications and operating parameters for the base-case experiments conducted in the large-scale chamber facility.

\begin{tabular}{lc}
\hline \hline Parameter & Value \\
\hline Wall and ceiling material & $\begin{array}{c}\text { Painted gypsum board } \\
\text { Carpet or sheet vinyl }\end{array}$ \\
Flooring material & 25.5 \\
Compartment volume, $\mathrm{m}^{3}$ & 10.4 \\
Floor area, $\mathrm{m}^{2}$ & 2.14 \\
Surface-to-volume ratio, $\mathrm{m}^{2} \mathrm{~m}^{-3}$ & Charcoal-filtered outdoor air \\
Inlet air & $12.8 \pm 0.6^{*}$ \\
Inlet air flow rate, $\mathrm{m}^{3} \mathrm{~h}^{-1}$ & $0.50 \pm 0.03^{*}$ \\
Ventilation rate, $\mathrm{h}^{-1}$ & $23 \pm 2^{\star}$ \\
Temperature, ${ }^{\circ} \mathrm{C}$ & $50 \pm 10^{\star}$ \\
Relative humidity, \% & $0.10 \pm 0.05$ \\
Air velocity at floor, $\mathrm{m} \mathrm{s}-1$ & 0.41 \\
Loading ratio of floor ${ }^{\star *}, \mathrm{~m}^{2} \mathrm{~m}^{-3}$ & 1.23 \\
Vent./loading ratio of floor, $\mathrm{m} \mathrm{h} \mathrm{h}^{-1}$ & 336 \\
Exposure period, $\mathrm{h}$ & \\
\hline
\end{tabular}

${ }^{*}$ Average \pm one standard deviation.

${ }^{\star \star}$ For experiments with paints, the loading ratio of the painted surface was $0.63 \mathrm{~m}^{2} \mathrm{~m}^{-3}$.

Air was sampled from the mid-point of each compartment. The inlet of a 0.4-cm I.D. stainless-steel tube was positioned $1.5 \mathrm{~m}$ above the floor at the midpoint. This tube ran directly to the exterior of the chamber and was connected to a stainless-steel sampling manifold with three ports to allow for the simultaneous collection of multiple samples. Air was continuously pulled through the sampling tube at a rate of $1 \mathrm{~L} \mathrm{~min}-1$ throughout an experiment. Sampling flow rates were regulated with electronic mass-flow controllers (Model FC 280, Tylan General) connected to vacuum pumps that ran continuously. For sampling, a three-way solenoid valve was switched by the data system from the open pusition to the position connected to a sampling device. At the end of a set time period, the data system switched the valve back to the open position and sampling was terminated. 
The instrumentation for each compartment consisted of: 1) a pressure transducer (Model 239, Setra Systems, Inc.) to measure the velocity pressure for the selected pitot tube; 2) a combined temperature and relative humidity probe (Model HMD 30 YB, Vaisala, Finland) positioned at the midpoint of the compartment $1.5 \mathrm{~m}$ above the floor; 3 ) four Type $\mathrm{T}$ thermocouples to measure floor temperature and air temperature stratification; and 4) an air velocity transducer (Model 8470-5AM-V-STD-NC, TSI, Inc.) with a range of 0 to $50 \mathrm{~cm} \mathrm{sec}^{-1}$ to monitor air velocity $5 \mathrm{~cm}$ above the floor. The voltage outputs from these devices were measured at one-minute intervals and averaged and recorded at five-minute intervals with a PC-based data system (Series 500, Keithly/Metrabyte) and data acquisition software (Labtech Notebook, Version 7, Laboratory Technologies, Corp.).

\subsubsection{Operating Procedures}

The operating procedures for the experiments conducted in the large-scale chamber facility are described in detail in Appendix $G$.

The primary compartment ventilation rate of $0.50 \pm 0.03 \mathrm{~h}^{-1}$ (average \pm one standard deviation) was selected, based on communications with knowledgeable researchers, to approximately simulate typical ventilation conditions in newer California houses. Ventilation data for a set of 66 post-1980 construction single-family houses in California has recently been published (Matson and Fuestel, 1998). The effective air change rate for these houses was $0.35 \pm 0.13 \mathrm{~h}^{-1}$ (average \pm one standard deviation). Thus, the selected rate was slightly higher than actual values determined for newer California houses. The $0.5 \mathrm{~h}^{-1}$ ventilation rate was used starting at 2-h elapsed time in the base-case experiments and starting at 72-h elapsed time in the experiments with additional ventilation as the treatment.

The scenario for the additional ventilation rate treatment was selected, in part, based on the recommendations of the $\mathrm{CRI}$ (1997). These recommendations are to maintain fresh air ventilation for $48-72 \mathrm{~h}$ following carpet installation. Ventilation is to be achieved by opening windows and doors and/or by the use of exhaust fans. It is also recommended that any ventilation systems be operated at full capacity. The maximum compartment ventilation rate was $5 h^{-1}$. This rate was maintained during material installation and for the following two hours in the experiments with additional ventilation as the treatment. This simulated a condition in a residence in which the ventilation rate was augmented by the use of a household fan to exhaust air at an open exterior window or door. A single fan with an airflow rate capacity of $2,040 \mathrm{~m}^{3} \mathrm{~h}^{-1}\left(1,200 \mathrm{ft}^{3} \mathrm{~min}^{-1}\right)$ might be able to approximately achieve this rate in a typical-sized residence. From 2- to $72-\mathrm{h}$ elapsed time, the ventilation rate was maintained at $2 \mathrm{~h}^{-1}$. This was intended to simulate passive ventilation in a residence that could easily be induced by leaving a few exterior windows and/or doors open or by operating a whole-house ventilation system at maximum capacity. In practice, the air change rates in the various zones of an actual house with mechanically or passively enhanced ventilation will depend upon the layout and configuration of the house and the pathways for air supply and exhaust.

Furnishings were installed in the compartments two hours after material installation to mimic typical "sink" effects that may substantially alter the impacts of VOC emissions from indoor sources. For this purpose, a set of eight identical used chairs was purchased. The chairs were fully upholstered, medium-sized, arm chairs. The fabric was a fleecy synthetic material. A set of used drapery panels was also purchased. The fabric was cotton or a cotton blend. The panels were cut down to dimensions of 1.5 by $2.1 \mathrm{~m}$. Two chairs and one drapery panel were used for each experiment. They were alternated among the experiments and were aired out between uses.

The compartments were thoroughly ventilated between experiments to reduce the background concentrations of VOCs. Immediately prior to an experiment, the compartment was operated at a ventilation rate of $0.5 \mathrm{~h}^{-1}$ for at least two days. During this period, the compartment was equipped with the mixing fans and furnished with two chairs and a single drapery panel. For the experiments with paints, the painting substrates and a carpet were also installed. At the end of this period, air samples for VOCs and aldehydes were collected from the compartment and from the supply air downstream of the charcoal filter. 
In preparation for the installation of a source assembly, the supply air flow rate was set to provide a ventilation rate of 2.0 or $5.0 \mathrm{~h}^{-1}$ as required. The compartment exhaust damper was adjusted accordingly, and the pressure transducer was connected to the selected pitot tube. The chairs, the drapery panel, and the sampling line were then removed. For experiments with floor coverings, the air mixing fans were also removed. The source assemblies were installed following the protocols described below. The fans, the temperature and humidity probe, the thermocouples, and the sampling line were re-installed. The data acquisition and control program was then terminated and re-started. This established the initial time for the experiment.

The first set of air samples was collected from the compartment and the supply air at an average elapsed time of one hour. At 2-h elapsed time, the two chairs, the drapery panel and the air velocity transducer were installed, and the door was taped closed. The door was not reopened after this time. The compartment ventilation rate was reduced to either 0.5 or $2.0 \mathrm{~h}^{-1}$ as required.

Additional air samples were collected from the compartment and the supply air at average elapsed times of three and six hours on the first day of the experiment. Subsequently, samples were collected daily for the next nine days at 24-h intervals from the time the experiment was initiated. The final set of air samples was collected on day 14 at 336-h elapsed time. Replicate samples for VOCs were collected at least twice during an experiment.

For experiments with elevated ventilation, the compartment ventilation rate was reduced to $0.5 \mathrm{~h}^{-1}$ at $72-\mathrm{h}$ elapsed time.

The experimental data consisted of: probe temperature and relative humidity $1.5 \mathrm{~m}$ from floor at the midpoint of the compartment; four thermocouple temperatures in the compartment; air velocity $5-\mathrm{cm}$ above the floor; flow velocity pressure; supply air relative humidity; building temperatures; mass-flow controller flow rates; sampling valve positions; elapsed time and clock time.

Experimental details that were specific to each source assembly are described below.

Latex Paint Combination - The substrate used for the application of the flat latex paint consisted of five panels of textured, but otherwise unfinished, gypsum wall board each with surface dimensions of 1.22 by $2.44 \mathrm{~m}$ yielding a total painting surface area of $14.9 \mathrm{~m}^{2}$. The substrate used for the application of the semi-gloss latex paint consisted of two plywood panels each with surface dimensions of 0.61 by $0.91 \mathrm{~m}$ yielding a total painting surface area of $1.1 \mathrm{~m}^{2}$. The panels were positioned and attached to the compartment walls as described in Appendix G. In order to mimic the surfaces in a room environment, the floor of the compartment was carpeted with an action back, tufted loop, 100 percent olefin fiber material. This carpet had been in the dealer's stock for a number of months and was aired out prior to its use. The emissions of VOCs from the carpet were very low relative to the expected emissions of VOCs from the paints.

The paints were applied by hand following industry practice and using typical painting tools. A systematic procedure was developed and carefully followed for each experiment. The latex primer sealer was applied to the gypsum board panels by roller. A brush was used to apply the primer to the borders of the gypsum panels and to the plywood panels. The flat latex paint was applied to the primered gypsum panels using a roller. A brush was used to finish the borders of the panels. Another brush was used to apply the semi-gloss paint to the plywood panels. All paint containers, tools and drop cloths were weighed immediately prior to and following an experiment. These weights were used to calculate the applied masses of the three paints.

The latex primer sealer was applied first. The primer was allowed to dry for exactly one hour, the minimum time recommended by the manufacturer. Then, the flat and semi-gloss latex paints were applied following the same sequence used for the primer. The duration of the entire painting procedure was approximately one hour, 50 minutes and was the same for all of the experiments. The experimental period was initiated when the technicians exited the chamber following the application of the finish paints. For the experiment with added air mixing and additional ventilation, two oscillating room fans were installed in the compartment at 2-h elapsed time. The fans were positioned so that they moved air approximately perpendicular to the long 
walls of the compartment. The fans were continuously operated at their maximum speed setting until 72-h elapsed time when they were turned off.

Carpet Assembly - The carpet assembly was installed to completely cover the floor of the compartment. For each experiment, there were three sections of action back, tufted textured loop, 100 percent olefin carpet. There were also three sections of bonded urethane carpet cushion.

Prior to an experiment, a tack strip was attached to the floor around the inside perimeter of the compartment. The sections of cushion were laid out on the floor with the smooth webbing side facing up. The sections were trimmed so that they fit inside of the tack strip. Next, the sections of carpet were laid out over the cushion. The two seams were bonded with thermal seaming tape applied with a hot iron. The edges of the carpet were then trimmed, and the carpet was attached to the tack strip. Installation of the carpet assembly required approximately 50 minutes. The experimental period was initiated after the installation was complete.

For one experiment, the carpet and cushion materials were aired out immediately prior to their installation. The materials were laid out for 48 hours on the floor of a room that was ventilated at over ten air changes per hour. The carpet was positioned with the backing facing up and the cushion was positioned with the webbing side facing up. Oscillating fans were used to increase the air velocity over the surfaces.

Vinyl Flooring Assembly - The vinyl flooring assembly was installed to completely cover the floor of the compartment. The materials used for these experiments were aged particleboard underlayment, a "no wax" residential sheet vinyl flooring, rubber cove base, sheet flooring adhesive, cove base adhesive, and vinyl flooring seam sealer.

At the beginning of an experiment, four panels of pre-cut particleboard were installed on top of the aluminum-clad compartment floor. Three pieces of sheet vinyl flooring were brought into the compartment with the other materials. The pieces were first trimmed to approximately the correct sizes. The sheet flooring adhesive was applied to the surface of the underlayment in six stages using a notched trowel. The vinyl flooring was glued down starting at one end of the compartment, and the edges were trimmed as required. Next, the cove base was installed in sections around the inside perimeter of the compartment using the cove base adhesive. Finally, the two-part liquid seam sealer was mixed and applied to the two seams in the vinyl flooring. Installation of the vinyl flooring assembly required approximately 90 minutes. The experimental period was initiated after the installation was complete.

The containers of the sheet vinyl and cove base adhesives and the installation tools were weighed immediately prior to and following an experiment. These weights were used to calculate the applied masses of the adhesives.

For one experiment, the vinyl flooring and cove base materials were aired out immediately prior to their installation in the compartment as described for the carpet materials. The materials were positioned with their finished surfaces facing up during the airing out procedure.

Combined Sources - The source materials and painting substrates used for the two experiments with the combined sources were the same as those used in the experiments with the separate sources. Two-thirds of the floor area of the compartment was covered with the carpet assembly, and the remaining one-third was covered with the vinyl flooring assembly.

The paints were applied first and were allowed to dry for three days prior to installing the flooring materials. This initial drying period allowed the VOC emissions from the paints to decay down to lower levels and made it more practical to collect and analyze VOC samples for compounds that were representative of the other sources. The aluminum-clad floor was left bare during painting and the initial drying period. There were also no furnishings in the compartment during this period. The ventilation rate during painting was $5.0 \mathrm{~h}^{-1}$. Two hours later, the ventilation rate was reduced to $2.0 \mathrm{~h}^{-1}$ and was maintained at this setting. No air samples were collected during this period. 
The installation of the carpet and vinyl flooring assemblies was initiated 72 hours after painting and with the chamber still operating at $2.0 \mathrm{~h}^{-1}$. The vinyl flooring assembly was installed first followed by the carpet assembly. Installation of the flooring materials required approximately 70 minutes.

One of the experiments was conducted using mild heating over approximately three days in combination with additional ventilation. Two hours after initiating the experimental period, two portable electric space heaters were placed in the compartment. The operation of one of the heaters was automatically regulated with a temperature controller. The other heater was manually controlled from the exterior of the compartment.

At six hours elapsed time, the two heaters were turned on. After several hours when the air and surface temperatures approached the $33^{\circ} \mathrm{C}$ set-point temperature, the manually controlled heater was switched off and the single temperature-controlled heater was used to supply heat on demand. The compartment was continuously heated to about $33^{\circ} \mathrm{C}$ until $72-\mathrm{h}$ elapsed time. Then, the heater was switched off, and the ventilation rate was reduced to $0.5 \mathrm{~h}^{-1}$.

The other experiment was conducted with additional ventilation of $2.0 \mathrm{~h}^{-1}$, but without heating, throughout the first 72 hours. Then, the ventilation rate was reduced to $0.5 h^{-1}$. This experiment was conducted over a period of three months (2,016 hours). Air samples were collected weekly following the initial two-week period.

\subsection{SAMPLING AND ANALYSIS OF VOCS AND TVOC}

Gas samples for the analysis of individual VOCs and TVOC were collected on sorbent samplers and were analyzed by thermal desorption gas chromatography/mass spectrometry (GC/MS). Two different thermal desorption systems (described below) were used during the course of the study. The first system was used for the screening measurements of most of the carpet assembly materials. This system was found to be unsuitable for the analysis of the dominant VOCs emitted by the latex paints. Therefore, a different thermal desorption system was used for the screening measurements of the paints. Since this system performed well and the first system was no longer supported because the manufacturer had recently gone out of business, the second system was used for the remainder of the experiments.

Multisorbent samplers were used with the first system (Part No. ST-032, Envirochem, Inc.). These samplers contained Tenax-TA®, a carbon molecular sieve and activated carbon, in series. The inclusion of the two carbonaceous sorbents in these samplers prevented the breakthrough of very volatile VOCs. The sorbent samplers used with the second system (Part No. 16251, Chrompack, The Netherlands) contained Tenax-TA $®$. Tenax by itself has relatively low retention volumes for very volatile VOCs. However, for the compounds in the volatility range of interest in this study (i.e., compounds with boiling points greater than approximately $80^{\circ} \mathrm{C}$ ), the breakthrough volumes on Tenax are typically at least $2 \mathrm{~L}$ and are substantially higher for most compounds (Hodgson and Girman, 1989). The air sample flow rates for both the small- and large-scale chambers were typically $0.1 \mathrm{~L} \mathrm{min-1}$. These rates were regulated with electronic mass flow controllers. Sample volumes were varied depending upon the analyte concentrations and ranged up to $3 \mathrm{~L}$. The sorbent samplers were cleaned and conditioned prior to use by heating them to $300^{\circ} \mathrm{C}$ for 30 minutes in a helium gas stream.

The analytical method for the analysis of VOCs collected on multisorbent samplers using the first thermal desorption system has previously been described (Hodgson and Girman, 1989). This method is a modification of U.S. EPA Method TO-1 (Winberry et al., 1988a). In brief, the sample components were thermally desorbed from a sampler with a sorbent-trap inletting system (Model UNACON 810A, Envirochem, Inc.). This system used dual sequential internal traps to concentrate the sample. The sample components were introduced into a capillary gas chromatograph (GC) equipped with liquid nitrogen sub-ambient cooling (Model 5980 Series II, Hewlett-Packard Corp.). The GC was connected via a direct capillary interface to an electronimpact quadrupole mass spectrometer (Model 5970B, Hewlett-Packard, Corp.). The mass spectrometer was operated in the scan mode. The specifications and operating conditions for this analyticai = jstem are given in Table 4. 
The second method used for the analysis of VOCs collected on the Tenax samplers was also a modification of U.S. EPA Method TO-1. The sample components were thermally desorbed from a sampler using a cryogenic inletting system (Model CP-4020 TCT, Chrompack, The Netherlands). A section of large-diameter, capillary GC column cooled with liquid nitrogen was used to concentrate the sample. The GC/MS system was the same as described above. The specifications and operating conditions for this second analytical system are given in Table 5.

An internal standard (ISTD) was added to all samplers, including standards, immediately prior to their analysis. The ISTD was $120 \mathrm{ng}$ of bromofluorobenzene. This was generated by a gravimetrically calibrated diffusion source and was transferred to the samplers with a gas-tight syringe.

For qualitative analysis, the spectra of the peaks in the total-ion-current (TIC) chromatograms were first compared to spectra contained in a database of commonly occurring VOCs created in the laboratory from analyses of pure compounds. These analyses were conducted using the same methods used for the analysis of the samples. If a spectrum of a compound matched a spectrum of a compound in the database and if the compounds' retention times also matched, then the identification was "confirmed." If no match was obtained, then the unknown spectrum was compared to the spectra contained in the electronic NIST/EPA/NIH Mass Spectral Database of approximately 75,000 entries (Heller and Milne, 1978). The analyst decided if the identification was "probable" based on the match quality and the reasonableness of the retention time. Less certain matches were described as "tentative." In some cases, no strong match was obtained, and the compound was "unidentified." Commonly occurring hydrocarbons (HCs) have many isomers for which no pure standards are available. Such compounds were only identified to class.

Individual compounds were selected for quantitative analysis based on a combination of several criteria. One criterion was the apparent relative abundance of a compound as determined by its peak area in a TIC chromatogram. Identified compounds with high relative abundances were selected for quantitative analysis if pure standards of these compounds were available for calibration. Another criterion was the potential health effects of an identified compound. Particular emphasis was placed on selecting ARB TACs that were above a lower limit of quantitation of approximately $1 \mu \mathrm{g} \mathrm{m}^{-3}$ for a $3-L$ sample. Other compounds that were known sensory irritants (e.g., aldehydes) or that had low odor thresholds were selected. Finally, compounds that were indicative of a particular source were selected. Originally, it was planned to quantify up to 15 compounds for each source investigated in the screening measurements. For a number of the sources, it was not possible to quantify this many compounds. In some cases, the emissions from sources only included a few compounds (e.g., most latex paints). In other cases, pure standards were not available for many of the compounds or there were complex mixtures of compounds that were not chromatographically resolved. The final selections of the target compounds for the major sources were made in consultation with the ARB.

For quantitative analysis, abundant and characteristic mass ions for the compounds of interest were extracted from the TIC chromatograms and their peak areas were integrated by the data system. Mass responses were determined using multi-point ISTD calibrations. These calibrations were prepared using the procedures described below and were periodically repeated during the study.

For compounds with high to intermediate volatility, standard gas mixtures were created by injecting a several microliter aliquot of a liquid mixture of the compounds into a 2- $L$ flask with a septum cap that was then heated and maintained at $65^{\circ} \mathrm{C}$ (Riggan, 1984). A sample was withdrawn from the flask with a gas-tight syringe and injected into a helium gas stream flowing through a clean sampler. Calibrations were prepared by analyzing a range of volumes of the gas mixture.

For compounds with lower vapor pressures, dilute liquid standards were prepared in a low-boiling solvent such as methanol or n-pentane, and microliter amounts of the standard were injected directly onto a Tenax sampler. The sampler was purged with helium to remove mosi of 
the solvent and analyzed using the normal procedures. Calibrations were prepared by analyzing different volumes or serial dilutions of the liquid standard.

Table 4. Specifications for the first, UNACON-based system used for the analysis of VOCs.

\begin{tabular}{|c|c|}
\hline Component & Specifications \& Operating Parameters \\
\hline Analytical column & $\begin{array}{l}\text { DB-1701 (J\&W Scientific, Inc.) } \\
30 \mathrm{~m} \times 0.25 \mathrm{~mm} \text { ID } \times 1.0 \mu \mathrm{m} \text { film }\end{array}$ \\
\hline Carrier gas & Helium @ - $\mathrm{cm}^{3} \mathrm{sec}^{-1}$ \\
\hline Desorber & $\begin{array}{l}\text { Model 8916, } 16 \text {-tube (Envirochem, Inc) } \\
\text { Tube desorb temp: } 275^{\circ} \mathrm{C}\end{array}$ \\
\hline Concentrator & $\begin{array}{l}\text { UNACON } 810 \mathrm{~A} \text {, (Envirochem, Inc) } \\
\text { Trap } 1 \text { heat: } 20 \mathrm{sec} @ 275^{\circ} \mathrm{C} \\
\text { Trap } 2 \text { heat: } 20 \mathrm{sec} @ 275^{\circ} \mathrm{C}\end{array}$ \\
\hline GC oven & $\begin{array}{l}\text { Model } 5890 \text { II (Hewlett-Packard Corp.) } \\
1^{\circ} \mathrm{C}(4.3 \mathrm{~min}) \\
1-120^{\circ} \mathrm{C} @ 5^{\circ} \mathrm{C} \mathrm{min}-1 \\
120-225^{\circ} \mathrm{C} @ 10^{\circ} \mathrm{C} \mathrm{min}-1 \\
225^{\circ} \mathrm{C}(2 \mathrm{~min})\end{array}$ \\
\hline Mass spectrometer & $\begin{array}{l}\text { Model } 5970 B \text { (Hewlett-Packard Corp.) } \\
\text { SCAN mode: } m / z 33-300 @ \\
1.6 \text { scans sec-1 }\end{array}$ \\
\hline
\end{tabular}

Table 5. Specifications for the second, Chrompack-based system used for the analysis of VOCs.

\begin{tabular}{|c|c|}
\hline Component & Specifications \& Operating Parameters \\
\hline Analytical column & $\begin{array}{l}\text { DB-1701 (J\&W Scientific, Inc.) } \\
30 \mathrm{~m} \times 0.25 \mathrm{~mm} \text { ID } \times 1.0 \mu \mathrm{m} \text { film }\end{array}$ \\
\hline Carrier gas & Helium @ $\sim \mathrm{cm}^{3} \mathrm{sec}^{-1}$ \\
\hline Concentrator & $\begin{array}{l}\text { CP-4020 TCT, (Chrompack) } \\
\text { Tube desorb temp: } 6.5 \mathrm{~min} @ 250^{\circ} \mathrm{C} \\
\text { Trap inject temp: } 200^{\circ} \mathrm{C}\end{array}$ \\
\hline GC oven & $\begin{array}{l}\text { Model } 5890 \text { II (Hewlett-Packard Corp.) } \\
40^{\circ} \mathrm{C}(5 \mathrm{~min}) \\
40-225^{\circ} \mathrm{C} @ 5^{\circ} \mathrm{C} \mathrm{min}-1 \\
225^{\circ} \mathrm{C}(7 \mathrm{~min})\end{array}$ \\
\hline Mass spectrometer & $\begin{array}{l}\text { Model 5970B (Hewlett-Packard Corp.) } \\
\text { SCAN mode: } \mathrm{m} / \mathrm{z} 30-350 @ \\
1.9 \text { scans sec-1 }\end{array}$ \\
\hline
\end{tabular}


For each analyte, the calibration data points were plotted as the analyte response ratios (relative to the ISTD response) versus the concentration response ratios (relative to the ISTD concentration). Calibration curves were generated using a linear least squares fit through the data points. For most compounds, the linear regression was forced through the origin.

The GC/MS TIC method used for the analysis of TVOC has previously been described (Wallace et al., 1991; Hodgson, 1995). The TIC chromatogram for a sample was integrated over a broad retention-time range approximately bounded by the retention times of $n$-hexane and $\mathrm{n}$-heptadecane. The integration parameters that were used captured almost all of the chromatographic area within this range. The integrated areas minus the area of the ISTD were summed. The mass of the compounds represented by the sum were calculated relative to the known amount of the ISTD. This calculation used an average relative TIC response factor determined for 10 commonly occurring normal alkane and aromatic hydrocarbons. These compounds were: $n$-heptane, n-octane, n-nonane, n-undecane, n-tridecane, benzene, toluene, ethylbenzene, $\mathrm{m}$-xylene, and 1,2,4-trimethylbenzene. Because of the variation in the relative TIC response of different classes of compounds, the uncertainty in the method is estimated to be approximately \pm 40 percent when applied to different compound mixtures (Wallace et al., 1991).

\subsection{SAMPLING AND ANALYSIS OF FORMALDEHYDE AND ACETALDEHYDE}

Air samples for the analysis of formaldehyde and acetaldehyde were collected on duallayer silica gel tubes impregnated with an acid solution of 2,4-dinitrophenylhydrazine (Part No. 226-119, SKC Inc.). As an air sample was pulled through a tube, the derivatizing reagent reacted with the carbonyl compounds to form the stable hydrazone derivatives that were retained by the tube. The air sample flow rates for both the small- and large-scale chambers were typically $0.5 \mathrm{~L}$ min-1. These flow rates were regulated with electronic mass-flow controllers. Sample volumes were typically $30 \mathrm{~L}$.

For analysis, a sampling tube was broken open and both sorbent layers were transferred to a $15-\mathrm{mL}$ disposable centrifuge tube with a screw cap. Four milliiters of high-purity acetonitrile were pipetted into the centrifuge tube, and the tube was placed in an ultrasonic bath for five minutes. Next, the tube was centrifuged for five minutes. Approximately $1 \mathrm{~mL}$ of the clear extract was transferred to a 2-mL glass auto sampler vial.

The sample extracts were analyzed for the hydrazone derivatives of formaldehyde and acetonitrile using a high-performance liquid chromatograph (HPLC) generally following U.S. EPA Method TO-11 (Winberry et al., 1988b). The compounds were separated on a microbore, reverse-phase $\mathrm{C}_{18}$ column (Symmetry C18, Part No. WATO56975, Waters Corp.) using a gradient mixture of water and acetonitrile as the mobile phase. The HPLC instrument (Model 1090 LC, Hewlett-Packard Corp.) was equipped with a dual-pump solvent delivery system and a diode-array UV detector. The absorbance of the derivatives was measured at $360 \mathrm{~nm}$. Five-point external calibrations were prepared periodically during the study from standard solutions of the formaldehyde and acetaldehyde hydrazone derivatives. A single check standard of the derivatives was analyzed at least once on each day of analysis. At least one sample tube collected as an experimental blank was also analyzed. Approximately every tenth sample extract was analyzed in duplicate. The lower limits of quantitation for formaldehyde and acetaldehyde in a 30-L sample were approximately $4 \mu \mathrm{g} \mathrm{m}^{-3}$.

\subsection{DATA ANALYSIS}

\subsubsection{Specific Emission Rates}

The emission rates of the target analytes were calculated for both the small- and largescale chamber experiments assuming that the chambers were ideal continuously-stirred tank reactors (CSTRs). Losses of the analytes due to factors other than ventilation (i.e., sink effects) were ignored; consequently, the calculated values were net rates.

A model has been developed to calculate emission rates for sources of VOCs that decay rapidly with time (ASTM, 1990). This model requires a sufficiently large number of samples at the 
beginning of an experiment to define the time and magnitude of the initial concentration peak. The model further requires that the change in the emission rate be approximated by a first-order decay. The model was not practical for use in this study because of the large number of experiments to be conducted and the requirement for significantly more samples in the initial time periods. Furthermore, the emphasis of the study was on exposures of residential occupants that would occur subsequent to the installation or application of the sources. Thus, increased sampling in the initial time periods could not be justified.

The steady-state form of the mass-balance model for CSTRs was selected (ASTM, 1990). Quasi-steady state, area-specific emission rates $(E R)$ in $\mathrm{mg} \mathrm{m}^{-2} \mathrm{~h}^{-1}$ were calculated as:

$$
E R=\frac{V a\left(C-C_{o}\right)}{A}
$$

Where $V$ is the chamber volume $\left(m^{3}\right)$; $a$ is the chamber air change or ventilation rate $\left(h^{-1}\right) ; C$ is the air concentration of the analyte in the chamber $\left(\mathrm{mg} \mathrm{m}^{-3}\right) ; C_{0}$ is the chamber background or inlet air concentration of the analyte $\left(\mathrm{mg} \mathrm{m}^{-3}\right)$; and $A$ is the exposed surface area of the source $\left(\mathrm{m}^{2}\right)$. Mass-specific emission rates $\left(\mathrm{mg} \mathrm{kg}^{-1} \mathrm{~h}^{-1}\right)$ were calculated using the mass of the source $(\mathrm{kg})$ rather than the surface area.

The emission rates determined with the steady-state model best reflect actual emission rates during periods in which the chamber concentrations of the analytes are not changing rapidly with time. An unknown amount of uncertainty is introduced into the calculation of emission rates when the concentrations are changing rapidly. In this study, the period of rapidly changing concentrations was generally limited to the first 24 hours in experiments with latex paints. For the small-scale experiments with paints, emission rates were only calculated for the 48- and 96-h time periods.

\subsubsection{Cumulative Mass Emissions and Exposures}

For the large-scale experiments, cumulative mass emissions were determined for each target VOC and the estimate of TVOC. This was accomplished by integrating and summing the trapezoidal areas resulting from a linear interpolation of the average specific emission rates $(\mathrm{mg}$ $\left.\mathrm{m}^{-2} \mathrm{~h}^{-1}\right)$ for each sampling interval beginning at time zero. The resulting specific mass emission value $(\mathrm{mg} \mathrm{m}-2)$ was multiplied by the area of the source $\left(\mathrm{m}^{2}\right)$ to generate the total mass emitted (mg) over the experimental period.

Although the study included a broad range of VOCs, the overall emphasis was on compounds that are classified by the ARB as TACs. For such compounds, there is generally most concern in air quality investigations regarding cumulative inhalation exposures (i.e., the product of a compound's air concentration and the exposure time). Other types of inhalation exposures that might be of concern as suggested by Tichenor and Sparks (1996) include the maximum concentration that a compound attains and the length of time an exposure occurs above a threshold level (e.g., an odor or sensory irritation threshold). Although the latter type of exposure is of considerable concern and importance in indoor environments, it is difficult to evaluate because of the general lack of knowledge regarding effect levels. In particular, the data on human sensory irritation thresholds is very limited. There is considerably more data on olfactory thresholds; but even this frequently does not include the major compounds emitted by common indoor sources. Consequently, the exposure assessment that was conducted for this study was restricted to the calculation of cumulative exposures.

Knowledge of occupant activity patterns is needed in order to determine the exposure times. For this study, the population of most concern was full-time occupants of residences. Two different time periods, or scenarios, were selected for evaluation. The first time period of intere:: was the initial two days ( 0 - 48 hours) following the installation of a source assembly. It was anticipated that the highest concentrations would occur over a relatively short period after 
installation and that a substantial portion of an occupant's exposure might also occur during this period. The second scenario was for a full-time occupant who chose to vacate the residence, or was not present in the residence, for the first two days following the installation of a source assembly. In this way, the occupant avoided exposure to the initial emissions of VOCs. It was assumed in this case that occupancy began at exactly 48-h elapsed time. For both scenarios, it was assumed that the occupant stayed in the residence for 20 hours each day (i.e., 83 percent of their time was spent at home). For simplicity, an occupancy factor of 0.83 was used rather than a specific time schedule.

Cumulative exposures in parts-per-million hour (ppm-h) or parts-per-billion hour (ppb-h) were calculated as the product of the concentration profile of a target VOC expressed in molarvolume concentration units (i.e., ppm or ppb), the exposure time in hours, and the occupancy factor. This was accomplished by calculating the exposure for each time interval between successive air samples following the linear trapezoidal procedure, summing these individual exposures, and multiplying the sum by the exposure factor.

\subsubsection{Pooled Estimates of Variance}

Pooled estimates of variance for the concentration measurements of individual VOCs were calculated according to standard analysis of variance techniques. In order to combine several estimates of a common variance into a single estimate of pooled variance, the computed variances are combined, weighted by their respective degrees of freedom. For the special case where there are $k$ sets of duplicate measurements, the formula for the calculation of pooled variance, $s^{2} p$, is:

$$
s_{p}^{2}=\frac{1}{2 k} \sum_{1}^{k} d_{i}^{2}
$$

Where $d_{i}=$ the difference of the duplicate measurements $(\mathrm{Ku}, 1969)$. The pooled estimate of the standard deviation, $s \rho$, is the square root of the pooled variance. The pooled standard deviation has $k$ degrees of freedom.

Relative precisions in percent were calculated for the individual VOCs by dividing their pooled standard deviations by their respective median concentrations. Median concentrations rather than the arithmetic average concentrations were used to reduce the influence of the high initial concentrations for some compounds.

This analysis of precision was performed for a limited, but representative, set of VOCs. This data was generated in the two large-scale experiments with the combined source assemblies. The target VOCs selected for these experiments were among the most abundant compounds emitted by the source assemblies and were also characteristic of the emissions from the individual major materials. The target VOCs spanned a broad range of volatility and included representatives of the major chemical classes of compounds detected in all experiments.

Therefore, these compounds can be considered to generally represent all of the VOCs included in the study. For these experiments, there were more than 20 pairs of co-located samples. Limited numbers of paired samples were collected in the other large-scale experiments. However, the numbers of pairs were insufficient to calculate pooled estimates of variance. 


\subsection{SELECTION AND PURCHASE OF THE STUDY MATERIALS}

\subsection{LATEX PAINTS}

There was no directly applicable published data for interior latex paints giving National or California market shares by manufacturer and/or product line. Therefore, the product selections were primarily based on information obtained through informal conversations with knowledgeable individuals. These individuals included other scientific researchers, a contact at the National Paint and Coatings Association, the head of environmental affairs for a national paint company, a regional paint sales representative and several local retailers.

Three combinations of conventional latex finish paints were selected. Each of these combinations consisted of compatible flat and semi-gloss paints. Two of the paired combinations were produced by the two largest national manufacturers, which also dominate the California market. Both of these combinations are generally categorized as high-volume, intermediategrade, consumer-oriented products. These paints were designated as FLP1 (flat latex paint) and SGLP1 (semi-gloss latex paint) for the first combination and as FLP2 and SGLP2 for the second combination (Appendix C, Table C-01). The third combination was from a regional and predominantly California-based manufacturer with a relatively high sales ranking. This combination was characteristic of professional-quality paints used by painting contractors. These paints were designated as FLP3 and SGLP3.

The fourth combination of flat and semi-gloss latex paints was a new generation "nonVOC" product line from a major national manufacturer. The manufacturer claimed that the product contained no petroleum-based solvents or other organic solvents. This combination was selected to provide information on potential improvements in indoor air quality that might be achieved by product substitution. The non-VOC paints were designated as FLP4 and SGLP4.

All of the finish paints were either pure or off white stock color formulations.

A decision was made to use a primer sealer as the base coat in the large-scale experiments even though the current trend in professional residential painting is not to use a primer coat. Two latex primer sealers were selected for the screening measurements. Both were from major national manufacturers. One of the products required a drying time of 24 hours before it could be painted over. This paint was designated as LPS1 (latex primer sealer). The other product, LPS2, was formulated so that it could be painted over after only one hour of drying.

All of the paints for the screening measurements were purchased from open stock at local retail outlets. The flat latex paints were obtained in 1-gal (3.8-L) containers, and the semi-gloss latex paints and the latex primer sealers were obtained in either 1-quart (0.95-L) or 1-gal (3.8-L) containers. All of the containers were mechanically shaken at the stores with the exception of the two non-VOC paints, which according to the manufacturer, were not to be shaken. Upon delivery to the laboratory, the paints were mixed by hand stirring. Each paint was then aliquoted into four, or more, new $240-\mathrm{mL}$ amber glass jars with Teflon-lined lids. Two 6-mm ball bearings were added to each jar. These were used for stirring. The jars were sealed and stored at room conditions in the laboratory.

The three paints selected for use in the large-scale experiments, LPS2, FLP3 and SGLP3, were purchased in 1-gal (3.8-L) containers from a local paint retailer. Sufficient quantities of paint were purchased for all six experiments. The containers were mechanically shaken at the store. Upon delivery to the laboratory, the paints were mixed by hand stirring and then aliquoted into new 1-quart $(0.95-\mathrm{L})$ paint cans. These were stored at room conditions. The paints were mixed by hand stirring prior to use.

\subsection{CARPET MATERIALS}

The study plan specified that two of the carpets were to be representative of the materials typically used in residential installations. The third carpet was to be representative of commercial installations. 
The 50 largest North-American carpet manufacturers were ranked according to 1994 dollar sales volume by an industry trade publication (Carpet \& Rug Industry Magazine, 1995). The top four manufacturers, which are large corporations, accounted for almost one-half of the approximately 12 billion dollars (U.S.) of 1994 carpet sales. These top manufacturers are also predominant in the California market. There was no published data for either the nation or California on the market shares of the numerous styles or grades of carpets that could be used to make the final selection of the materials. Therefore, this selection had to be made based on information provided by knowledgeable individuals. A representative of the Carpet and Rug Institute (K. McIntosh, CRI, personal communication) provided information on the typical residential carpet styles and grades sold in California by three of the four largest manufacturers. With the assistance of a local contract flooring dealer, two nearly equivalent products from two different manufacturers were selected from this list. These materials were intermediate grade, action back carpets with nylon pile face fiber and styrene-butadiene-rubber (SBR) latex on the secondary backing. Both of these carpets were from lines certified under the CRI Indoor Air Quality Carpet Testing Program and bore the "Green Tag" label (CRI, 1994). They were designated as CP1 and CP3 (Appendix D, Table D-01).

The commercial grade carpet was selected from among the styles and grades produced by one of the other two top manufacturers based on information provided by the contract flooring dealer. This material was believed to have a relatively high sales volume. It was an action back carpet with olefin textured loop face fibers and SBR latex on the secondary backing. It was designated as CP2. At the time of the first purchase of this carpet, it was not certified under the CRI Indoor Air Quality Carpet Testing Program. Subsequently, this carpet was selected for use in the large-scale experiments. By the time the order was placed for the material for these experiments, the previous product had been discontinued and was replaced by a nearly identical style that was certified under the CRI Program. This new carpet was ordered and was designated as CP4.

A number of different types of carpet cushions are manufactured. The five dominant product types are bonded urethane, prime urethane, synthetic fiber, rubberized jute and sponge rubber (Shaeffer et al., 1996). Bonded urethane and prime urethane cushions, together account for nearly $90 \%$ of the combined residential and commercial markets (Leukroth, 1991). Bonded urethane, by itself, has approximately $50-60 \%$ of the total market. Consequently, it was decided to select two bonded urethane cushions with relatively high California sales volumes. In a previous small-scale chamber study, it was shown that synthetic fiber cushions had low emissions of VOCs relative to the other product types (Shaeffer et al., 1996). A synthetic fiber cushion was selected as the third material.

Generally, carpet cushions are manufactured and distributed on a more regional basis than carpets. No published data was available on the California market shares of manufacturers of bonded urethane cushions. Therefore, a telephone survey of California carpet retailers was conducted to obtain approximate market share information. Twenty-seven major carpet retailers in Northern, Central and Southern California were contacted. Eighteen of these retailers identified the major brands of bonded cushion that they sold. Some provided information about the relative sales volumes of these brands and identified the highest selling grades. The phone survey data was tabulated, and three top manufacturers were identified based on this data. Bonded cushions produced by one manufacturer were sold by more than $60 \%$ of the responding retailers. Next, a local retailer that carried this manufacturer's products was contacted. The grade with the highest sales volume in this retailer's stores was selected as one of the materials. The other bonded urethane cushion was selected based on the recommendation of the local contract flooring dealer from among the materials produced by the other two top manufacturers. These bonded urethane cushions were designated as $\mathrm{CC} 1$ and $\mathrm{CC} 2$, respectively (Appendix D, Table D-01). They were both 0.5 inches $(1.3 \mathrm{~cm}$ ) thick. Their densities varied by about a factor of two. The second manufacturer also produced the synthetic fiber cushion that was selected for the study based on the recommendation of the contract flooring dealer. It was designated as CC3. Carpet Cushion $\mathrm{CC} 2$ was subsequently selected for use in the large-scale experiments. At the time of this purchase, a slightly different, but nearly equivalent, material was available from the same retailer. This material was $7 / 16$ inches $(1.1 \mathrm{~cm})$ thick and was designated as CC4. 
The final material selected for the screening measurements of carpet materials was a thermal seaming tape. This was a professional grade product suitable for heavy traffic in commercial installations. It was stocked at a local high-volume retail outlet. The seaming tape was designated as ST.

The three carpets for the screening measurements, CP1, CP2 and CP3, were purchased through the contract flooring dealer. Once the materials had been selected and the experimental schedule was established, the dealer contacted the carpet manufacturers or primary distributors to obtain information on their production schedules. The specific materials were selected based on their known future production dates regardless of pattern or color. Arrangements were made to have the materials shipped and delivered to the dealer within one week of these production dates. In this way, recently manufactured carpets of known age were obtained.

Each carpet was initially obtained as a 1.8 by $3.7-\mathrm{m}\left(6.7-\mathrm{m}^{2}\right)$ piece. The piece was rolled and wrapped in polyethylene sheet plastic for shipment. Upon receipt, the carpet was unwrapped and tightly re-rolled with the face fiber facing outward. The roll was then placed in a large 2-mil thick Tedlar bag and sealed. The packaged carpet was stored at room conditions.

Prior to the large-scale experiments with the carpet assembly, a large quantity of Carpet CP4 was purchased following the same procedure. Upon receipt, the material was rolled out in the dealer's warehouse and cut into pieces for six large-scale experiments. The pieces for each experiment were rolled together and stored in a sealed Tedlar bag as described above.

Carpet Cushions $\mathrm{CC} 1$ and $\mathrm{CC} 3$ were also purchased through the contract flooring dealer. They were ordered from the manufacturer or distributor and delivered to the dealer. For these materials, it was not possible to establish an exact production date. However, they were believed to be of recent manufacture. Carpet Cushion CC2 was purchased from a carpet retailer.

According to the retailer, shipments of the material were received approximately every two weeks. The piece for the screening measurements was cut from a recently received roll.

Each cushion for the screening measurements was obtained as a 1.8 by $3.7-\mathrm{m}\left(6.7-\mathrm{m}^{2}\right)$ piece. The piece was rolled and stored in a sealed Tedlar bag.

A sufficient quantity of Carpet Cushion $\mathrm{CC} 4$ for six large-scale experiments was purchased from the same retailer. The roll from which the material was taken had recently been received. The material was cut into the approximate sizes needed for the experiments. The pieces for each experiment were rolled together and stored in a sealed Tedlar bag.

The thermal seaming tape for both the screening measurements and the large-scale experiments was purchased from open stock at a large-volume retail outlet. The rolls of tape were stored in a sealed Tedlar bag.

\subsection{VINYL FLOORING MATERIALS}

There was no published market data that could serve as the basis for the selection of the sheet vinyl materials. However, there are only a few major manufacturers of sheet vinyl flooring. These manufacturers dominate both the National and the California markets. They are readily identifiable from "shelf surveys" of flooring retailers. Due to the lack of published information, the material selections were primarily based on information informally obtained from knowledgeable individuals. These individuals included a former industry representative, a regional sales representative and the contract flooring dealer.

Base- and intermediate-grade residential vinyl flooring materials have a clear vinyl top layer. This coating provides the "no-wax" feature. The backings of these materials are specifically designed for one of two different installation techniques. These techniques are perimeter adhesive application and full-spread adhesive application. Full-spread adhesive materials can be installed by both consumers and professionals. Professionals generally only install materials requiring perimeter adhesive application. Vinyl flooring materials used in commercial installations require harder surfaces. Therefore, they dc nc: have a soft vinyl top coat. Instead, they rely on periodic maintenance to maintain a shiny appsarance. 
Four different sheet vinyl materials were selected. There were three residential no-wax materials. Two of these were intermediate-grade materials since it was believed that the majority of consumers would select this grade because of cost and durability considerations. A sales representative from one of the manufacturers confirmed that intermediate-grade materials had the largest sales volumes. One of the intermediate-grade materials was designed for perimeter adhesive application. This was designated as SV2 (Appendix E, Table E-01). The other intermediate-grade material, designated as SV3, was produced by another top manufacturer and was equivalent in quality and price to SV2. It was designed for full-spread adhesive application. The third residential no-wax product was a base-grade material available at large-volume retail outlets and intended for the budget-minded "do-it-yourself" market. It was designed for full-spread adhesive application. It was designated as SV4. A single intermediate-grade commercial material produced by one of the top vinyl flooring manufacturers was selected. It was designated as SV1. Following the initial screening measurements, Sheet Vinyl SV3 was selected for use in the large-scale experiments. The identical style of material was purchased for these experiments. However, it varied from SV3 with respect to color, pattern and production date. It was designated as SV5. A two-part, liquid seam sealer formulated for use with this sheet vinyl was obtained from the same manufacturer.

Professional installers generally prefer rubber cove base since it is more flexible and has less shrinkage than vinyl cove base. A rubber cove base from a major manufacturer was selected for this study. It was designated as CB.

The sheet flooring adhesive selected for this study was produced by a large national manufacturer and was labeled as "solvent free." The cove base adhesive was produced by the same manufacturer and was labeled as "low VOC" content. These products were designated as SFA and CBA, respectively.

Three of the sheet vinyl materials (SV1, SV2 and SV3) for the screening measurements were purchased through the contract flooring dealer. As for the carpets, the dealer contacted the manufacturers or primary distributors to obtain information about the production dates for the selected materials. Specific materials were ordered based on this information regardless of pattern or color. The materials were delivered to the dealer within approximately one week of their production dates.

Sheet Vinyl SV4 was purchased from open stock at a large-volume retail outlet. The roll from which the piece was cut was previously unopened and, according to the sales manager, had recently been received.

Each sheet vinyl material was obtained as a 1.8 by $1.8-\mathrm{m}\left(3.2-\mathrm{m}^{2}\right)$ piece. Upon receipt, the piece was unpacked and tightly re-rolled with the finished surface facing outward. The roll was stored at room conditions in a sealed Tedlar bag.

Sheet Vinyl SV 5 for the large-scale experiments was purchased following the same procedure used for Sheet Vinyls SV1 - SV3. Upon receipt, the material was rolled out and cut into pieces for six large-scale experiments. The pieces for each experiment were rolled together and stored in a sealed Tedlar bag.

The rubber cove base, the sheet flooring adhesive, the cove base adhesive and the seam sealer were all purchased through the contract flooring dealer. The cove base had been received by the distributor from the manufacturer within one week of its delivery to the dealer. The cove base was left in its original packaging; these packages were stored in sealed Tedlar bags.

The particleboard underlayment was purchased from open stock at a large-volume retail outlet. 


\subsection{SCREENING MEASUREMENTS OF THE LATEX PAINTS}

\subsection{EXPERIMENTAL DESIGN}

The latex paints are listed in Appendix C, Table C-01. There were two latex primer sealers (LPS1 and LPS2) and four combinations of flat and semi-gloss paints. Three of these combinations (FLP1 and SGLP1; FLP2 and SGLP2; and FLP3 and SGLP3) were conventional latex paints. The fourth combination (FLP4 and SGLP4) was a new-generation non-VOC product.

The screening measurements conducted with the latex paints are summarized in Appendix C, Table C-02. The screening measurements conducted with the gypsum board (GB) and plywood (PW) substrates alone served as the respective blank experiments for the flat and semi-gloss paints. There were 12 experiments with individual paints including the duplicate experiments with flat paint FLP1 and semi-gloss paint SGLP3. In addition, there were four experiments with the combinations of paints selected for the large-scale experiments.

Experiments with the combination of LPS2 and FLP3 applied to the GB substrate were conducted at the standard room temperature $\left(23^{\circ} \mathrm{C}\right)$ and relative humidity $(50$ percent) and at reduced ( 30 percent) and elevated ( 70 percent) relative humidities. One experiment at standard conditions was conducted with the combination of LPS2 and SGLP3 applied to the PW substrate.

The procedures used for the application and testing of the paints are described in detail in Appendix A. The environmental parameters for all of the experiments are summarized in Appendix B. The coverages that were achieved in grams of paint applied per square meter of substrate surface are given in Table C-02. The coverages for the duplicate experiments with the individual paints were in good agreement. There were substantial differences between the coverages for LPS1 and LPS2 and among the flat paints that presumably were due to differences in the formulations of the paints.

\subsection{QUALITATIVE EMISSIONS OF VOCS}

The 6- and 96-h samples for each individual paint, paint combination and substrate were qualitatively analyzed to identify the VOCs emitted by the paints and the substrate blanks. These qualitative results are presented in Appendix C, Tables C-03 - C-16.

The qualitative emissions of VOCs from the individual conventional latex paints are summarized in Appendix C, Table C-17. Relatively few compounds, all of which were oxidized, dominated these emissions. The major solvent components of these paints were ethylene glycol, propylene glycol and, for SGLP3, hexylene glycol. All of the paints emitted relatively large amounts of the two isomers of 2,2,4-trimethyl-1,3-pentanediol monoisbutyrate (Texanol(a). This compound is frequently used as the coalescing aid in conventional latex paints. Other compounds that were found at relatively high abundance in several of the paints were 2-(2-butoxyethoxy)ethanol (di(ethylene glycol)butyl ether or DEGBE) and 2-(2-butoxyethoxy)ethyl acetate. The other compounds listed in Table $\mathrm{C}-17$ were generally present at relatively low abundance. $n$-Butyl ether was detected in the emissions from all of the paints.

The emissions of VOCs from the combinations of LPS2 and FLP3 and LPS2 and SGLP3 (Tables $\mathrm{C}-13$ and $\mathrm{C}-14$ ) were in qualitative agreement with the emissions determined for the individual paints.

Only a few compounds at low abundance were detected in the emissions from the GB substrate (Table C-15). A larger number of compounds, including terpene hydrocarbons and aldehydes, were detected in the emissions from the PW substrate (Table C-16).

Oxidized VOCs were detected in the emissions from the non-VOC paints, but these compounds occurred at considerably lower levels than the compounds emitted by the conventional paints. The qualitative results for FLP4 are presented in Table C-08. By 96-h elapsed time, all but three of the compounds (nonanal, decanal and Texano/(B) were below their lower limits of detection. These same three compounds were also detected in the emissions from the GB substrate and may have been present due to background contamination of the chamber

system. More compounds were detected in the emissions from SGLP4 (Table C-12). However, a 
number of these compounds, including many of the aldehydes, were also detected in the emissions from the PW substrate.

\subsection{CONCENTRATIONS OF THE TARGET COMPOUNDS}

Nine of the most abundant and prevalent VOCs detected in the emissions from the conventional latex paints were selected for quantitative analysis (Table $\mathrm{C}-17$ ). The results for hexylene glycol were highly uncertain because of difficulties with the calibration for this compound. The samples collected during the experiments with the conventional paints and the substrates were analyzed for this list of target VOCs. The samples for the non-VOC paints were analyzed for the generally more abundant compounds. The samples collected at all five time periods were quantitatively analyzed. The results for are presented in Appendix C, Tables C-18-C35. The concentration units for these tables are milligrams per cubic meter $\left(\mathrm{mg} \mathrm{m}^{-3}\right)$.

Generally, the concentrations of the target VOCs in the experiments with the two substrates were at or below the lower limit of quantitation of approximately $0.01 \mathrm{mg} \mathrm{m}^{-3}$. The quantitative results for the duplicate experiments with FLP1 were in excellent agreement. There was more variation between the duplicate experiments with SGLP3. For all of the conventional paints except SGLP1, the compounds with the highest chamber concentrations at the conclusion of the 96-h experimental period were the principal solvent component, either ethylene glycol, propylene glycol, or hexylene glycol, and the coalescing aid, Texanokß. If present, DEGBE frequently had elevated initial concentrations that decayed down to relatively low levels by the conclusions of the experiments. For SGLP1, ethylene glycol and DEGBE were the dominant compounds throughout the experiment.

The concentrations of VOCs emitted by FLP4 and SGLP4 were all substantially lower than the concentrations of VOCs emitted by the conventional paints. By the conclusions of the experiments with these paints, the VOC concentrations were all less than $0.01 \mathrm{mg} \mathrm{m}^{-3}$.

Since the emissions of VOCs from the paints were dominated by a few compounds (i.e., solvents and Texanol@) all of which were quantified, the best measure of concentration for the total emissions was simply the sum of the concentrations of the individual compounds. This measure was termed SigmaVOC (i.e., the sum of the concentrations of the target compounds). The SigmaVOC concentrations for the screening measurements are presented In Appendix C, Table C-36.

The concentrations of SigmaVOC at a given time period vary by a factor of three or less among the conventional flat paints and among the conventional semi-gloss paints. By the conclusions of the experiments, the SigmaVOC concentrations for the non-VOC paints were factors of about 25 to 50 lower than the concentrations for the conventional paints.

The chamber concentrations of formaldehyde and acetaldehyde for the screening measurements with paints are presented in Appendix C, Tables C-37 and C-38, respectively. The concentration units for these two tables are micrograms per cubic meter $\left(\mu \mathrm{g} \mathrm{m}^{-3}\right)$. Many of the paints had somewhat elevated initial concentrations of formaldehyde. The paints with the highest initial formaldehyde concentrations were LPS1, FLP3 and FLP4. Typically, formaldehyde concentrations decreased rapidly with time. With one exception (Paint FLP2), the 96-h concentrations were less than $10 \mu \mathrm{g} \mathrm{m}^{-3}$. Many of the paints also had elevated initial concentrations of acetaldehyde. The paints with the highest initial acetaldehyde concentrations were LPS2, FLP1, FLP2, FLP3, and SGLP1. In general, these concentrations also decreased rapidly with time.

\subsection{EMISSION RATES OF THE TARGET COMPOUNDS}

The quasi-steady state emission rates of the target compounds at the 48- and 96-h time periods were calculated using the mass balance equation (Equation 1). Since the chamber concentrations generally did not change rapidly over this time interval, the steady-state model was expected to produce reliable estimates of the emission rates. These emission rates were calculated as area-specific emission rates $\left(\mathrm{mg} \mathrm{m}^{-2} \mathrm{~h}^{-1}\right)$ based on the painted surface area of $0.0195 \mathrm{~m}^{2}$ and as mass-specific emission rates $\left(\mathrm{mg} \mathrm{kg}^{-1} \mathrm{~h}^{-1}\right)$ based on the masses of paint that 
were applied. The emission rates of the individual compounds emitted by the paints are presented in Appendix C, Tables C-39 - C-54. The SigmaVOC emission rates are presented in Appendix $\mathrm{C}$, Table $\mathrm{C}-55$. The formaldehyde and acetaldehyde emission rates are respectively presented in Appendix C, Tables C-56 and C-57. Figures 2 - 9 individually compare the $96-\mathrm{h}$ area-specific and mass-specific emission rates of ethylene glycol, DEGBE, Texanoß and SigmaVOC.

The paints with the highest area- and mass-specific emission rates of ethylene glycol were FLP1, SGLP1 and SGLP2. Paints FLP2 and SGLP3 did not emit ethylene glycol. Although there was considerable variation among the emission rates of ethylene glycol from the combination of Paints LPS2 and FLP3, there was no apparent direct relationship between the emission rate and humidity over the range of $30-70$ percent relative humidity. The emission rates of ethylene glycol from these combined paints were generally lower than the sum of the emission rates of ethylene glycol from the individual paints. On the other hand, the emission rate of ethylene glycol from the combination of Paints LPS2 and SGLP3 was substantially higher than the emission rate of ethylene glycol from LPS2 (FLP3 was not a source of ethylene glycol).

Paint SGLP1 had the distinctly highest emission rate of DEGBE. Generally, the semigloss paints had higher emission rates of DEGBE than the latex primer sealers and the flat paints. Paints LPS2 and FLP3 and the combination of these paints did not emit DEGBE.

Paints LPS2 and FLP3 had the highest emission rates of TexanoK. On a mass-specific basis, LPS2 clearly had the highest rate. There was an apparent decrease in the area-specific emission rates of Texanok from the combination of Paints LPS2 and FLP3 with increasing relative humidity. However, this trend was less apparent in the mass-specific emission rates. The emission rates of Texanoß from the combination of Paints LPS2 and FLP3 were generally similar to the sum of the emission rates of Texanoß from the individual paints. On the other hand, the emission rate of Texanol from the combination of Paints LPS2 and SGLP3 was similar to the emission rate of Texanol from SGLP3 alone and substantially lower than the sum of the emission rates of Texanoß from LPS2 and SGLP3.

The semi-gloss paints had higher area-specific emission rates of SigmaVOC than the flat paints. The difference was less pronounced among the mass-specific emission rates since semigloss paints generally had higher mass per-unit area coverages. Paint LPS2 had a higher SigmaVOC emission rate than Paint LPS1. The emission rates of SigmaVOC from both of the paint combinations were lower than the respective sums of the emission rates for the individual paints.

The emission rates of formaldehyde and acetaldehyde are reported in units of $\mu \mathrm{g} \mathrm{m}^{-2} \mathrm{~h}^{-1}$ and $\mu \mathrm{g} \mathrm{kg}^{-1} \mathrm{~h}^{-1}$. A number of the paints had quantifiable emission rates of formaldehyde at 48and 96-h elapsed time. The paints with the highest formaldehyde emission rates were LPS1, FLP2 and FLP3. The emission rates of acetaldehyde at 48- and 96-h elapsed time were generally below the lower limits of quantitation.

\subsection{SELECTION OF THE PAINTS FOR THE LARGE-SCALE EXPERIMENTS}

Paint LPS2 was selected for use as the primer in the large-scale experiments with latex paint primarily because the manufacturer specified that the finish coat could be applied after only a one-hour drying period. This simplified the paint application procedure for the large-scale experiments and reduced the number of air samples that needed to be collected and analyzed. Although LPS2 was formulated to be fast drying, it was shown not to be atypical with respect to its qualitative emissions of VOCs.

Paints FLP3 and SGLP3 were selected as the finish coats for the large-scale experiments, in part, because the solvent compositions of the two paints were distinct from each other. FLP3 utilized ethylene glycol, while SGLP3 utilized a combination of propylene glycol and hexylene glycol. In addition, another research group was studying the emissions from one of the national paint brands, and it was thought to be desirable to obtain data for a different paint combination, specifically higher-quality painis produced by a California manufacturer for the California market. 


\subsection{BULK COMPOSITION OF THE MAJOR VOCS IN THE SELECTED PAINTS}

The percent weight compositions of ethylene glycol, propylene glycol and Texanol in Paints LPS2, FLP3 and SGLP3 are presented in Appendix C, Table C-58. The principal solvent component (either ethylene glycol or propylene glycol) was 2.0 to 3.6 percent of the paints by weight. Texanoß was 0.7 to 1.5 percent of the paints by weight.

\subsection{COMPARISON WITH LITERATURE DATA}

Latex paints have generally been shown to contain relatively few VOCs. In addition, studies have shown that there is considerable consistency in the VOC composition of latex paints and other water-based architectural coatings.

Using a direct analysis method, Censullo et al. (1996) obtained VOC emissions profiles for 52 water-based coating samples. These samples included flat, semi-gloss and gloss latex paints and other coating types including varnishes, stains and traffic paints. The predominant VOC components were: Texanoß (37 samples); propylene glycol (31 samples); DEGBE (23 samples); and ethylene glycol (14 samples). A large majority of the samples contained only two or three major VOC components.

Sheldon and Naugle (1994) determined the compositions of VOCs in six interior latex paints (flat, semi-gloss and gloss) produced by two National manufacturers. The measurements were performed by both bulk analysis of paint samples and by a small-scale chamber method for several of the paints. The most prevalent major compounds were ethylene glycol, propylene glycol, diethylene glycol, DEGBE and Texanol@. By bulk analysis, the weight composition of ethylene glycol in the paints was found to be in the range of $6-48 \mathrm{~g} \mathrm{~kg}^{-1}$; the composition of DEGBE was $1.5-13 \mathrm{~g} \mathrm{~kg}^{-1}$; and the composition of Texano® was $3-10 \mathrm{~g} \mathrm{~kg}^{-1}$. These results are in general agreement with the percent weight compositions of VOCs in the three paints that were selected for the large-scale experiments in this investigation. The chamber air samples were analyzed for aldehydes in addition to the other VOCs. The paints were shown to emit elevated concentrations of acetaldehyde in the initial 1 -h samples. These concentrations decreased rapidly with time and were substantially lower in subsequent samples. The paints were also shown to be low sources of formaldehyde. Similar results were found in this study.

Most of the small-scale chamber experiments conducted with latex paints have used an impermeable material for the painting substrate. These experiments have investigated the effects of various parameters on chamber concentrations and emission rates of VOCs. Clausen (1993) investigated the effect of film thickness on the emissions of VOCs from a single latex paint applied to a metal plate. The results indicated that the emissions of VOCs were controlled by evaporation and showed that emission rates decreased with increasing film thickness. Eighteen laboratories in ten countries took part in an interlaboratory comparison study of the emissions of DEGBE and Texanok from a single latex paint applied to metal plates (De Bortolli et al., 1995). Again, differences in paint thickness were found to affect the results. More importantly, differences in the quality of the analyses were found to be the most substantial contributor to the variance in the results. This emphasizes the difficulty of analyzing polar organic compounds by sorbent sampling and thermal desorption GC or CG/MS. Sheldon and Naugle (1994) investigated the effect of surface air velocity on the emissions of VOCs from latex paints applied to glass plates. The test with a fan showed higher air concentrations at the earlier time points and a more rapid decrease in concentrations with time compared to the test without a fan.

It is now known that the type of substrate has a profound effect on the emission characteristics of VOCs from latex paints. Chang et al. (1997) investigated the effect of substrate type on the VOC emissions from a single latex paint. It was found that the amount of VOCs emitted from a painted metal plate was 2 - 10 times more than that emitted from painted gypsum board over a two-week test period in small-scale chambers. The VOC emissions from the metal plate predominantly occurred in the first 100 hours via a fast evaporation process. The VOCs were emitted very slowly from the gypsum board. It was postulated that this process was controlled by diffusion through the substrate. Only nine percent of the ethylene glycol, 14 percent of the DEGBE and 29 percent of the Texanol 8 in the paint applied to the gypsum board were 
emitted over 336 hours. Emissions of these components were still detectable 11 months after paint application. Chang et al. (1997) estimated that if 100 percent of the ethylene glycol and Texanoß in latex paint applied to gypsum board can be re-emitted, it might take as long as 3.5 years for the complete release of these compounds.

\subsection{SUMMARY}

The results of the screening measurements with latex paints are summarized as follows:

1. The emissions of VOCs from all of the conventional paints were qualitatively similar, predominantly consisting of a solvent component (most frequently ethylene glycol or propylene glycol) and the Texanol coalescing aid.

2. There were clear differences both within and among the categories of conventional paints with respect to the emission rates of the individual VOCs and SigmaVOC.

3. The relative humidity of chamber air did not have substantial effects on the emission rates of the VOCs emitted by the combination of the latex primer sealer and the flat latex paint applied to gypsum board.

4. The emission rates of the individual VOCs and SigmaVOC from combinations of the latex primer sealer and the flat and semi-gloss latex paints could not simply be predicted based on the emission rates measured for the individual paints.

5. The compositions of the emissions from the non-VOC paints were generally different than the compositions of the emissions from the conventional paints.

6. The emission rates of SigmaVOC from the non-VOC paints were factors of about 25 to 50 lower than the emission rates of SigmaVOC from the conventional paints.

\subsection{SCREENING MEASUREMENTS OF THE CARPET MATERIALS}

\subsection{EXPERIMENTAL DESIGN}

The carpet materials are listed in Appendix D, Table D-01. There were four carpets, $a$ thermal carpet seaming tape, and four carpet cushions. Materials CP1 and CP3 were intermediate grade residential action back carpets with nylon pile face fiber. Materials CP2 and CP4 were commercial action back carpets with textured loop olefin face fiber. These two carpets were nearly equivalent products from the same manufacturer. Carpets CP1, CP2 and CP4 were from lines certified under the CRI Indoor Air Quality Carpet Testing Program. Materials CC1, CC2 and $\mathrm{CC} 4$ were bonded urethane carpet cushions. Material CC3 was a synthetic fiber cushion.

The screening measurements conducted with the carpet materials are summarized in Appendix D, Table D-02. Blank chamber runs were conducted for both the carpets and the carpet cushions. Duplicate experiments were conducted with materials CP1 and CC2.

The procedures used to test the carpet materials are described in detail in Appendix A. The environmental conditions for the experiments are summarized in Appendix B.

\subsection{QUALITATIVE EMISSIONS OF VOCS}

The 6- and 48-h samples for each material were qualitatively analyzed to identify the VOCs that were emitted by the material. These qualitative results are presented in Appendix $D$, Tables D-03 - D-13. The qualitative emissions of VOCs from the carpets and carpet cushions are summarized in Tables 6 and 7 . Table 6 shows the numbers of compounds detected in the 48-h samples by chemical class. Compounds detected in the chamber background samples wert omitted from the sums. Table 7 lists the most abundant VOCs in the 6-and 48-h samples br:-ed 
on the relative TIC areas of the chromatographic peaks. A maximum of six components was listed for each material. For a number of the materials, there were fewer than six compounds with distinctly elevated TIC areas.

There were qualitative distinctions among the carpets. The two residential nylon pile carpets emitted relatively few compounds after 48 hours of exposure in the chambers. These compounds were predominantly branched alkane hydrocarbons. 4-Phenylcyclohexene (4-PCH) was one of the most abundant VOCs emitted by these carpets. Styrene was not detected in the 48-h samples. Carpets CP2 and CP4 emitted considerably more compounds after 48 hours of exposure. Both carpets, particularly CP2, emitted relatively large numbers of different branched alkane hydrocarbons. Some of these alkanes were among the most abundant compounds emitted by the carpets. Both carpets also emitted some alkene (olefinic) hydrocarbons. 4-PCH was present in the emissions from both carpets while styrene was only detected in the 48-h sample for CP4. Tetrachloroethene was one of the most abundant compounds emitted by CP2. The emissions from Carpet CP4 were distinct in that they contained a relatively large number of glycol ethers, some of which were not identified (Table D-06). After 48 hours, Carpet CP4 emitted two nitrogen containing compounds, N,N-dimethylacetamide and 2-methyleneglutaronitrile.

The use of Seaming Tape ST on Carpet CP3 resulted in the emissions of more VOCs than were detected in the test of the carpet by itself. More branched alkane hydrocarbons were emitted and 2,6-di-tert-butyl-4-methylphenol (butylated hydroxytoluene or BHT) was additionally present.

The three bonded urethane carpet cushions, $\mathrm{CC} 1, \mathrm{CC} 2$ and $\mathrm{CC} 4$, were similar to each other in that they all emitted relatively high amounts of BHT and a complex mixture of unsaturated hydrocarbons. This mixture contained numerous chromatographically unresolved compounds. The mixture eluted near the ends of the chromatographic runs indicating that the compounds had relatively high molecular weights and low volatility. These three cushions also emitted some nitrogen-containing compounds including $\mathrm{N}, \mathrm{N}$-dimethylbenzylamine, $\mathrm{N}, \mathrm{N}$-dimethylacrylamide and 2,2'-azobisisobutryonitrile. Carpet Cushion $\mathrm{CC} 1$ was unique in that it emitted a number of aromatic hydrocarbons including $\mathrm{C}_{3}-\mathrm{C}_{4}$ alkylbenzenes, naphthalene and alkyl substituted naphthalenes. Naphthalene and 2-methylnaphthalene were among the most abundant compounds emitted by this material. Carpet Cushion $\mathrm{CC} 1$ also emitted relatively high amounts of triethylphosphate.

The synthetic fiber carpet cushion, $\mathrm{CC} 3$, predominantly emitted branched alkane hydrocarbons. Small amounts of 4-PCH and caprolactam were detected in the samples. As described above, 4- $\mathrm{PCH}$ is characteristic of emissions from action back carpets. Its presence in the emissions from CC3 possibly suggests that recycled carpet material was used in the manufacture of the cushion.

Small amounts of aldehydes, acetic acid and siloxane compounds were detected in the blank chamber runs (Tables D-08 and D-13). 
Table 6. Summary of the qualitative emissions of VOCs by chemical class for the screening measurements of the carpet and carpet cushion materials. The numbers of compounds identified in each class in the 48-h chamber samples are shown.

\begin{tabular}{|c|c|c|c|c|c|c|c|c|}
\hline \multirow[b]{2}{*}{ Chemical Class } & \multicolumn{4}{|c|}{ Carpet Materials } & \multicolumn{4}{|c|}{ Carpet Cushion Materials } \\
\hline & CP1 & $\mathrm{CP3}$ & $\mathrm{CP2}$ & $\mathrm{CP} 4$ & $\mathrm{CC1}$ & $\mathrm{Cc} 2$ & $\mathrm{CC} 4$ & $\mathrm{CC3}$ \\
\hline Alkane $\mathrm{HCs}$ & 6 & 4 & 38 & 13 & 2 & & & 15 \\
\hline Aromatic $\mathrm{HCs}$ & 1 & 1 & 1 & 2 & 12 & & 1 & 1 \\
\hline Other HCs & & & 5 & 6 & $++^{*}$ & ++ & ++ & 2 \\
\hline Chlorinated Cmpds. & & & 1 & & & & & \\
\hline Carbonyl Cmpds. & & & 1 & 1 & & & 1 & \\
\hline Oxidized Cmpds. & 1 & & 4 & 7 & 5 & 2 & 3 & 1 \\
\hline Nitrogen Cmpds. & & & 1 & 2 & 3 & 1 & 3 & \\
\hline Misc. Cmpds. & & 1 & & & 1 & & & \\
\hline Unident. Cmpds. & & & 4 & 5 & 1 & 3 & & 1 \\
\hline Total No. of Cmpds. & 8 & 6 & 55 & 36 & $>>27$ & $>>8$ & $>>9$ & 20 \\
\hline
\end{tabular}

${ }^{*}++$ Indicates that a complex mixture of numerous unsaturated HCs was emitted.

\subsection{CONCENTRATIONS OF THE TARGET COMPOUNDS}

Forty-four of the most abundant and prevalent VOCs detected in the emissions from the carpet and carpet cushion materials were selected for quantitative analysis (Appendix D, Table $D-14)$. The individual materials were only analyzed for those compounds that they were known to emit based on the qualitative results. The samples collected at all four time periods were analyzed. The results are presented in Appendix C, Tables D-15 - D-27. The concentration units for these tables are micrograms per cubic meter $\left(\mu \mathrm{g} \mathrm{m}^{-3}\right)$. Generally, the lower limits of quantitation for the individual compounds were in the range of $1-5 \mu \mathrm{g} \mathrm{m}^{-3}$.

The chamber concentrations of the target VOCs emitted by the carpets, particularly Carpets CP1 and CP3, were very low. By the 48-h time period, the individual concentrations were frequently less than $10 \mu \mathrm{g} \mathrm{m}^{-3}$, and often less than their lower limits of quantitation. 4-PCH had the highest concentration among the target VOCs by the ends of the experiments with Carpets CP1, CP3 and CP2. Among all of the carpets, the concentrations of the target VOCs were highest for CP4. The compounds emitted at 48 hours by this carpet with chamber concentrations of $10 \mu \mathrm{g} \mathrm{m}^{-3}$ or more were 4-PCH, di(propylene glycol) methyl ether isomers, $\mathrm{N}, \mathrm{N}$-dimethylacetamide and 2-methyleneglutaronitrile.

Carpet Cushion CC1 was analyzed for the most target VOCs. By the end of the experiment with this material, the compounds with the highest concentrations were naphthalene, the two methylnaphthalenes, the combined isomers of Texanoß $B, \mathrm{BHT}, \mathrm{N}, \mathrm{N}$-dimethylbenzylamine, and triethylphoshate. BHT was the predominant and most abundant target VOC emitted by Carpet Cushion CC2. BHT, N,N-dimethylbenzylamine and 2,2'-azobisisobutryonitrile quantitatively dominated the emissions of the target VOCs emitted by Carpet Cushion CC4. Cushion CC3 had relativelv low concentrations of target VOCs. 
Table 7. Most abundant VOCs emitted in the screening measurements of the carpet and carpet cushion materials.

\begin{tabular}{ll}
\hline Material ID / Compound & Material ID / Compound \\
\hline Carpet CP1 & Carpet Cushion CC1 \\
4-PCH & Naphthalene \\
Alkene HCs (mixture) & 2-Methylnaphthalene \\
1-Dodecanol & Unsaturated HCs (complex mixture) \\
& BHT \\
Carpet CP3 & N,N-Dimethylbenzylamine \\
4-PCH & Triethylphosphate \\
& \\
Carpet CP2 & Carpet Cushion CC2 \\
Branched alkane HCs (5) & Unsaturated HCs (complex mixture) \\
Tetrachloroethene & BHT \\
& Unident. compound \\
Carpet CP4 & \\
Branched alkane HCs (2) & Carpet Cushion CC4 \\
4-PCH & Unsaturated HCs (Complex mixture) \\
Triethylene glycol monomethyl ether & BHT \\
1-Dodecanol & \\
Unident. glycol ether & Carpet Cushion CC3 \\
\hline
\end{tabular}

*Numbers of individual compounds in a classification are indicated in parentheses.

The concentrations of TVOC for the screening measurements with the carpet materials are presented in Appendix D, Table D-28. Among the carpets, CP1 and CP3 had the distinctly lowest TVOC concentrations. Among the cushions, CC3 had the distinctly lowest TVOC concentrations. For Cushions $\mathrm{CC} 1, \mathrm{CC} 2$ and $\mathrm{CC} 4$, the TVOC concentrations were predominately a measure of the concentrations of the complex mixture of unsaturated hydrocarbons discussed above. The differences between the TVOC concentrations for the duplicate experiments with CC2 were approximately a factor of 1.5 perhaps due to material heterogeneity.

The concentrations of the individual target VOCs were also summed for each material. This measure was termed SigmaVOC. The SigmaVOC concentrations for the screening measurements are presented in Appendix D, Table D-29. The ratios of the SigmaVOC and the TVOC concentrations for the 24- and 48-h samples are plotted in Figure 10. This comparison shows that relatively small fractions of the total emissions of VOCs from both the carpets and the cushions were quantified as individual compounds. For materials $\mathrm{CP} 4$ and $\mathrm{CC} 1$ the ratios were about 0.25 . For most of the other materials, the ratios were closer to 0.1 and often less. 
The chamber concentrations of formaldehyde and acetaldehyde are presented in Appendix D, Tables D-30 and D-31, respectively. The formaldehyde concentrations were all near the lower limit of quantitation, indicating that these materials were not sources of formaldehyde. The initial, $1-\mathrm{h}$, chamber concentrations of acetaldehyde were elevated for Carpet Cushions CC2 and CC4. By the ends of the experiments, the acetaldehyde concentrations for these two materials were substantially lower, and the values for all materials except CC4 were at or near the lower limit of quantitation.

\subsection{EMISSION RATES OF THE TARGET COMPOUNDS}

The quasi-steady state emission rates of the target compounds at the 24- and 48-h time periods were calculated using the mass balance equation (Equation 1). Since the chamber concentrations did not change rapidly over this time interval, the steady-state model was expected to produce reliable estimates of the emission rates. These emission rates were calculated as area-specific values $\left(\mu \mathrm{g} \mathrm{m}^{-2} \mathrm{~h}^{-1}\right)$ based on the exposed surface areas of $0.0232 \mathrm{~m}^{2}$. The emission rates of the individually quantified VOCs emitted by the carpet materials are presented in Appendix D, Table D-32 - D-42. The TVOC emission rates are presented in Appendix D, Table $\mathrm{D}-43$. The formaldehyde and acetaldehyde emission rates are respectively presented in Appendix D, Tables D-44 and D-45.

Figure 11 shows the specific emission rates of 4-PCH for the carpets. Carpets $\mathrm{CP} 1$ and $\mathrm{CP} 3$ had the lowest emission rates of 4-PCH by the ends of the experiments. These values were in the range of $10-18 \mu \mathrm{g} \mathrm{m}^{-2} \mathrm{~h}^{-1}$. The CRI has established upper limits for the $24-\mathrm{h}$ emission rates of 4-PCH, styrene, formaldehyde and TVOC that must not be exceeded in order for a carpet line to be certified under its Indoor Air Quality Carpet Testing Program (CRI, 1994). The 24-h limit for 4-PCH is $50 \mu \mathrm{g} \mathrm{m}^{-2} \mathrm{~h}^{-1}$. Carpet CP4 did not meet this criterion. However, by 48 hours, the emission rate of 4-PCH from Carpet $\mathrm{CP} 4$ was below $50 \mu \mathrm{g} \mathrm{m}^{-2} \mathrm{~h}^{-1}$.

The methods used in this study and for the CRI Indoor Air Quality Carpet Testing Program were both based on ASTM Standard Guide D-5116 90 (ASTM, 1990). However, when making comparisons with the CRI guidelines, it must be noted that the methods differed with respect to some details such as the loading ratio for the specimen and the chamber ventilation rate (Black et al., 1991). No laboratory inter comparison studies have been conducted for carpets, and it is not known to what extent these methodological differences would be expected to affect the results. Also, there may be considerable heterogeneity in the finished product. Due to practical necessity, the Indoor Air Quality Testing Program is limited to testing only a few specimens from the large amount of material that is produced on a manufacturing line.

The CRI limit for the emission rate of styrene at 24 hours is $400 \mu \mathrm{g} \mathrm{m}^{-2} \mathrm{~h}^{-1}$. All of the carpets tested in this study were low to negligible emitters of styrene. Only Carpets CP2 and CP4 had measurable styrene emissions at 24 hours. These values were $10-12 \mu \mathrm{g} \mathrm{m}^{-2} \mathrm{~h}^{-1}$. Thus, the emissions of styrene from the carpets were approximately a factor of 40 below the CRI limit.

Carpet CP4 generally had the highest emission rates of target VOCs. At 48 hours, the compounds with the highest emission rates, in addition to 4-PCH, were n-undecane, di(propylene glycol)methyl ether isomers, N,N-dimethylacetamide and 2-methyleneglutaronitrile.

The CRI limit for the emission rate of formaldehyde at 24 hours is $50 \mu \mathrm{g} \mathrm{m}^{-2} \mathrm{~h}^{-1}$. The formaldehyde emission rates from all of the study carpets were $5 \mu \mathrm{g} \mathrm{m}^{-2} \mathrm{~h}^{-1}$, or less.

Figure 12 shows the specific emission rates of TVOC for the carpets. Carpets CP1 and CP3 had the lowest TVOC emission rates. These values were in the range of $164-269$ $\mu \mathrm{g} \mathrm{m}^{-2} \mathrm{~h}^{-1}$ at 24 and 48 hours. These values were substantially below the current CRI limit for TVOC emissions at 24 hours of $500 \mu \mathrm{g} \mathrm{m}^{-2} \mathrm{~h}^{-1}$. The application of the seaming tape to Carpet CP3 increased the TVOC emission rate at 48 hours by more than a factor of three. Carpet CP2 had the highest TVOC emission rates. This line was discontinued and replaced by a line of which Carpet CP4 was an example. The TVOC emission rates from this newer material were lower by more than a factor of two; however, the 24-h value of $1,100 \mu \mathrm{g} \mathrm{m}^{-2} \mathrm{~h}^{-1}$ was about twice the CRI limit. In addition to the caveats discussed above regarding comparion with the CRI limits, there is likely to be considerably more uncertainty in the measurement of TVOC than in the calibrated 
measurement of individual VOCs. Also, there may have been differences in analytical methodologies that could have had substantial effects on the results (Hodgson, 1995).

All of the bonded urethane carpet cushions had relatively high emission rates of BHT. These emission rates are plotted in Figure 13. There was generally good agreement between the duplicate experiments with Carpet Cushion CC2. The 24-h values for the three cushions were in the range of 79 to $191 \mu \mathrm{g} \mathrm{m}^{-2} \mathrm{~h}^{-1}$. There was a general increase in the emission rate of BHT from 24 to 48 hours. This increase was most pronounced for Carpet Cushion CC4. The CRI in partnership with the Carpet Cushion Council has also established an Indoor Air Quality Carpet Cushion Testing Program (CRI, 1998). This program sets upper limits for the 24-h emission rates of $\mathrm{BHT}$, 4- $\mathrm{PCH}$, formaldehyde and TVOC from carpet cushions. The 24-h limit for BHT is 300 $\mu \mathrm{g} \mathrm{m}^{-2} \mathrm{~h}^{-1}$. None of the cushions exceeded this limit.

Other compounds with relatively high emission rates from at least one of the bonded urethane materials were $\mathrm{N}, \mathrm{N}$-dimethylbenzylamine, 2,2'-azobisisobutyronitrile and triethylphosphate. The emission rates of the target VOCs from the synthetic fiber cushion, $\mathrm{CC} 3$, were quite low. The 24-h emission rate of 4-PCH from Carpet Cushion CC3 was $5 \mu \mathrm{g} \mathrm{m}^{-2} \mathrm{~h}^{-1}$. This value was substantially lower than the emission rates of 4-PCH from the carpets and well below the CRI limit for 4-PCH from carpet cushions of $50 \mu \mathrm{g} \mathrm{m}^{-2} \mathrm{~h}^{-1}$. The emission rates of formaldehyde from the carpet cushions were generally at or near the lower limit of quantitation of $3 \mu \mathrm{g} \mathrm{m}^{-2} \mathrm{~h}^{-1}$. The CRI limit for formaldehyde from carpet cushions is $50 \mu \mathrm{g} \mathrm{m}^{-2} \mathrm{~h}^{-1}$.

The TVOC emission rates from the bonded urethane cushions shown in Figure 14 were generally higher than the TVOC emission rates from the carpets. At 24 hours, these values were in the range of $1,650-4,050 \mu \mathrm{g} \mathrm{m}^{-2} \mathrm{~h}^{-1}$. The $48-\mathrm{h}$ emission rates were similar. As discussed above, the TVOC values were largely a measure of the emissions of a complex mixture of predominantly unsaturated hydrocarbons. The CRI 24-h limit for the emissions of TVOC from carpet cushions is $1,000 \mu \mathrm{g} \mathrm{m}^{-2} \mathrm{~h}^{-1}$. All of the bonded urethane cushions substantially exceeded the CRI limit. However, it is noted that there was no indication that any of the cushions were certified by the Indoor Air Quality Carpet Cushion Testing Program. The 24-h TVOC emission rate from synthetic fiber Carpet Cushion CC3 was about $250 \mu \mathrm{g} \mathrm{m}^{-2} \mathrm{~h}^{-1}$. This value was substantially lower than the emission rates of TVOC from the bonded urethane cushions and well below the CRI limit.

\subsection{SELECTION OF THE CARPET MATERIALS FOR THE LARGE-SCALE EXPERIMENTS}

The intermediate grade nylon carpets were shown to be relatively low sources of VOCs. In fact, the 48-h emission rates of the target VOCs, including 4-PCH and styrene, and TVOC were so low, that these materials were judged to be marginally useful for evaluating the treatment effects in the large-scale experiments. Consequently, the olefin-fiber carpet, CP2, which had the highest VOC emissions was selected. However, as previously explained, this line was discontinued, and Carpet CP4 from the new line was obtained as a replacement. Carpet CP4 was primarily intended for commercial installations and, therefore, would not typically be installed in residences. It is not known if the emissions of VOCs from this carpet are characteristic of the emissions from base-grade olefin fiber carpets intended for residential use.

Bonded urethane Carpet Cushion CC2 was selected for use over Carpet Cushion CC1 because it was believed to have a relatively high share of the California market. Additionally, it had higher emission rates of BHT and unsaturated hydrocarbons. Carpet Cushion CC4, a nearly equivalent product from the same manufacturer, was purchased for use in the large-scale experiments. It had similar emissions characteristics plus it additionally emitted measurable amounts of several nitrogen-containing compounds.

\subsection{COMPARISON WITH LITERATURE DATA}

A VOC emissions testing program was developed for carpets as part of the Carpet Policy Dialogue conducted by the U.S. EPA with carpet industry representatives (Leukroth, 1991). An initial study was designed to characterize the emissions of TVOC and selected VOCs from SBR carpets representative of the products being manufactured in the U.S. at that time. A test method 
using small-scale chambers was developed based on ASTM Standard Guide D 5116-90 (ASTM, 1990). Nineteen different carpet products were tested using this method (Black et al., 1991). Air samples were collected from the chambers at 1,24 and approximately 140-h elapsed time. Individual compounds in the samples were identified and their concentrations were determined. Specific emission rates were calculated for the major constituents and TVOC. The results of this study were summarized by Hetes et al., (1992) and used as the basis for an exposure and health risk assessment. Sixty-nine compounds were identified, 35 of which were observed for only a single carpet product. The most prevalent compounds included styrene, 4- $\mathrm{PCH}$, 4-ethenylcycohexene, n-undecane, propylbenzene and n-decane. The average and maximum 24-h emission rates of styrene from the 19 carpets were 37 and $173 \mu \mathrm{g} \mathrm{m}^{-2} \mathrm{~h}^{-1}$, respectively. The average and maximum 24-h emission rates of 4-PCH were 64 and $152 \mu \mathrm{g} \mathrm{m}^{-2} \mathrm{~h}^{-1}$, respectively; and the average and maximum 24-h emission rates of TVOC were 151 and $500 \mu \mathrm{g} \mathrm{m}^{-2} \mathrm{~h}^{-1}$, respectively.

Another chamber study was conducted at about the same time to characterize the emission of VOCs from several newly manufactured carpets, including two SBR carpets (Hodgson et al., 1992 and 1993). This study utilized a 20- $\mathrm{m}^{3}$ stainless-steel lined chamber. The individual experiments were conducted over one week with samples of chamber air collected periodically throughout this period. Individual compounds were identified, and concentrations and specific emission rates were determined for selected compounds and TVOC. The 24-h emission rates of styrene from the two carpets were 30 and $260 \mu \mathrm{g} \mathrm{m}^{-2} \mathrm{~h}^{-1}$; the 24-h emission rates of 4-PCH were 64 and $82 \mu \mathrm{g} \mathrm{m}^{-2} \mathrm{~h}^{-1}$; and the 24-h emission rates of TVOC were 196 and 399 $\mu \mathrm{g} \mathrm{m} \mathrm{m}^{-2} \mathrm{~h}^{-1}$.

In the current study of four carpets, the 24-h emission rates of styrene were in the range of $<3-12 \mu \mathrm{g} \mathrm{m}^{-2} \mathrm{~h}^{-1}$; the $24-\mathrm{h}$ emission rates of $4-\mathrm{PCH}$ were in the range of $10-68 \mu \mathrm{g} \mathrm{m}^{-2} \mathrm{~h}^{-1}$; and the 24-h emission rates of TVOC were in the range of $233-3,380 \mu \mathrm{g} \mathrm{m}^{-2} \mathrm{~h}^{-1}$. Thus, the four study carpets had emission rates of styrene that were well below the previously determined rates. One carpet, $\mathrm{CP} 1$, had a distinctly low 4-PCH emission rate compared to the previous data, another carpet, CP2, had a substantially elevated TVOC emission rate.

A study was conducted by the U.S. Consumer Product Safety Commission (CPSC) to identify and quantify VOCs emitted by 17 new carpet cushions covering the five major product types (Hodgson and Phan, 1994; Schaeffer et al., 1996). The survey specimens included four bonded urethane, the most prevalent product type, and three synthetic fiber cushions. The experiments were conducted over 96 hours in small-scale environmental chambers. There were substantial differences in the emissions of VOCs among the cushion types that were presumably due to differences in the manufacturing processes. The synthetic fiber cushions had the lowest TVOC specific emission rates of $95-180 \mu \mathrm{g} \mathrm{m}^{-2} \mathrm{~h}^{-1}$ at 96 hours. Alkane and other hydrocarbons dominated the emissions from these cushions. The bonded urethane cushions generally had the highest TVOC specific emission rates. At 96-h, these rates were in the range of $261-4,350 \mu \mathrm{g}$ $m^{-2} h^{-1}$. All of the bonded urethane cushions emitted BHT. Three of the four specimens emitted a complex mixture of co-eluting higher molecular weight unsaturated compounds. The results of the current study are largely consistent with the CPSC survey study.

A subsequent animal assay study was conducted by the CPSC to measure the sensory irritancy of chemicals emitted by carpets and carpet cushions. One of the target compounds selected for this investigation was BHT, which is used as a heat stabilizer in the production of polyurethane foam. Thirty-five specimens of bonded and prime urethane cushions were collected from manufacturing mills and retail outlets. The emission rates of BHT from these specimens were measured at 24-h exposure in small-scale chambers (Schaeffer, 1998). The specific emission rate of $\mathrm{BHT}$ from the production line, bonded urethane specimens was $210 \pm 120$ $\mu \mathrm{g} \mathrm{m}^{-2} \mathrm{~h}^{-1}$, which is about a factor of three lower than the BHT emission rate measured in the first CPSC survey. The BHT emission rates measured for the three bonded urethane cushions in the current study were in the range of $79-191 \mu \mathrm{g} \mathrm{m}^{-2} \mathrm{~h}^{-1}$, which is consistent with this most recent CPSC survey. 


\subsection{SUMMARY}

follows:

The results of the screening measurements with the carpet materials are summarized as

1. The emissions of VOCs from the carpets were qualitatively dominated by a variety of hydrocarbons. All of the carpets emitted 4-PCH, an unwanted byproduct in the production of SBR latex.

2. Styrene was detected in the emissions from three carpets. The emission rates of styrene were near detection limits demonstrating a significant reduction relative to previously reported results.

3. The two intermediate grade, nylon pile carpets were similar to each other with respect to the composition of their emissions. They emitted fewer VOCs than the base-grade, olefin fiber carpets.

4. The olefin fiber carpets had higher emission rates of TVOC and individual VOCs, including 4-PCH, than the nylon carpets.

5. The two olefin fiber carpets emitted a mixture of glycol ethers not detected in the emissions from the nylon carpets.

6. There were clear qualitative differences between the two types of carpet cushions. All of the bonded urethane carpet cushions emitted $\mathrm{BHT}$ and a mixture of predominantly unsaturated hydrocarbons. The emissions from the synthetic fiber cushion were dominated by alkane hydrocarbons.

7. The emission rates of individual VOCs and TVOC from the synthetic carpet cushion were low.

8. For most of the materials, the quantified compounds only accounted for relatively small fractions of the total emissions. This was largely due to the fact that the emissions were frequently dominated by isomers of various hydrocarbon classes.

\subsection{SCREENING MEASUREMENTS OF THE VINYL FLOORING MATERIALS}

\subsection{EXPERIMENTAL DESIGN}

The vinyl flooring materials are listed in Appendix E, Table E-01. There were five sheet vinyl materials, a rubber cove base, sheet flooring and cove base adhesives, a sheet vinyl seam sealer, aged particleboard underlayment and gypsum board. Sheet Vinyl SV1 was an intermediate grade commercial material designed for full-spread adhesive application. Sheet Vinyl SV2 was an intermediate grade no-wax material designed for perimeter adhesive application. Sheet Vinyls SV3 and SV5 varied with respect to printed pattern and color, but otherwise were the same material from the same manufacturer. This was an intermediate grade no-wax material designed for full-spread adhesive application. Sheet Vinyl SV4 was a base-grade no-wax residential product also designed for full-spread adhesive application. It was produced by the same manufacturer as SV1 and SV2. The adhesives were "solvent free" or "low VOC content" products. The seam sealer was a two-part liquid product. The underlayment and gypsum board respectively served as substrates for the application of the sheet vinyls and the cove base.

The screening measurements conducted with the vinyl flooring materials are summarized

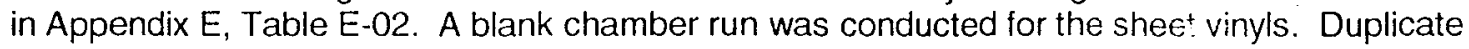
experiments were conducted with material SV3. The adhesives and composite assemblies were also tested. One composite assembly consisted of Sheet VinyI SV5 bonded ro Underlayment UL 
with Sheet Flooring Adhesive SFA. The other assembly consisted of Cove Base CB bonded to Gypsum Board GB with Cove Base Adhesive CBA.

The procedures used to test the sheet vinyls and other materials are described in detail in Appendix A. The environmental conditions for the experiments are summarized in Appendix B.

\subsection{QUALITATIVE EMISSIONS OF VOCS}

The VOCs that were emitted by each material and assembly were identified in the 6- and 48-h samples. The qualitative results for the five sheet vinyl materials are presented in Appendix E, Tables E-03 - E-08. The emissions of VOCs from the sheet vinyls are compared in Appendix $E$, Table E-09. This table is a composite listing of all of the VOCs that were emitted by the five materials at any time period. The data is summarized in Tables 8 and 9. Table 8 shows the numbers of compounds that were detected by chemical class. Compounds that were present in the chamber background were omitted from the sums. Table 9 lists the most abundant VOCs for each material based on the relative TIC areas of the chromatographic peaks. Six compounds were listed for each material.

Table 8. Summary of the qualitative emissions of VOCs by chemical class for the screening measurements of the five sheet vinyl materials. The numbers of compounds identified in each class are shown.

\begin{tabular}{lrrrrr}
\hline Chemical Class & Sv1 & Sv2 & sv3 & sv5 & Sv4 \\
\hline Alkane HCs & 4 & 31 & 29 & 24 & 26 \\
Aromatic HCs & & 13 & 31 & 35 & 20 \\
Other HCs & 2 & 2 & 32 & 23 & 2 \\
Carbonyl Cmpds. & 14 & 8 & 6 & 6 & 8 \\
Other Oxidized Cmpds. & 1 & 1 & & 1 & 1 \\
Nitrogen Cmpds. & 4 & 4 & 7 & 4 & 8 \\
Miscellaneous Cmpds. & & & & & \\
Unidentified Cmpds. & 25 & 60 & 107 & 95 & 66 \\
Total No. of Cmpds. & & & & & \\
\hline
\end{tabular}

There were distinct differences as well as clear similarities among the sheet vinyl materials with respect to their qualitative emissions of VOCs. Commercial Sheet Vinyl SV1 emitted only several alkane hydrocarbons. On the other hand, relatively large numbers of alkane, aromatic and other hydrocarbons numerically dominated the emissions from the residential materials, all of which had a clear vinyl top coat. These compounds included branched $\mathrm{C}_{9}-\mathrm{C}_{12}$ alkane hydrocarbons, normal alkane hydrocarbons, and $\mathrm{C}_{2}-\mathrm{C}_{5}$ alkyl substituted benzenes. The nearly identical Sheet Vinyls SV3 and SV5 additionally emitted numerous unsaturated and cyclic hydrocarbons and a related and distinct series of $\mathrm{C}_{10}-\mathrm{C}_{12}$ alkyl substituted benzenes. Sheet Vinyl SV1 emitted the highest number of oxidized compounds including isomers of di(propylene glycol) methyl ether and several octanol isomers. All of the 
materials emitted the oxidized compounds phenol and 2,2,4-trimethyl-1,3-pentanediol diisobutyrate $(T X \mid B \otimes)$. All but one emitted diethylphthalate. Both $T X \mid B \otimes$ and diethylphthalate are used as plasticizers in vinyl materials.

Sheet Vinyls SV2 and SV4 were different grades and styles of materials produced by the same manufacturer. The listing of compounds in Table E-09 shows that these two materials were very similar with respect to the composition of their VOC emissions.

The most abundant VOCs emitted by the sheet vinyl materials frequently included normal or branched alkane hydrocarbons, phenol and TXIBQ. The emissions from Sheet Vinyls SV3 and SV5 were characterized by the same list of abundant VOCs (i.e., n-decane, n-tridecane, n-tetradecane, benzyl alcohol, phenol and TXIB $®$ ).

Small amounts of aldehydes, decanoic acid, diethylphthalate and siloxane compounds were detected at 48 hours in the blank chamber run for the sheet vinyls (Table E-08).

The two principal solvent components of Seam Sealer SS were tetrahydrofuran and cyclohexanone (Appendix E, Table E-10). They comprised about 60 and $5-10$ percent of the components by volume, respectively.

The VOCs that were emitted by rubber Cove Base CB are listed in Appendix E, Table $\mathrm{E}-11$. A relatively large number of aromatic hydrocarbons were detected. The dominant compounds were toluene, three $\mathrm{C}_{2}$ alkyl substituted tetrahydronaphthalenes, tert-butyl isothiocyanate and benzothiazole.

Two terpene hydrocarbons, aldehydes and acetic acid dominated the emissions from Underlayment UL (Appendix E, Table E-12). The composition of the samples for Gypsum Board GB (Appendix E, Table E-13) was very similar to the composition of the samples for the blank chamber run.

Sheet Flooring Adhesive SFA was shown to emit some VOCs (Appendix E, Table E-14). The most abundant compound was toluene. Other relatively abundant compounds included the sesquiterpene hydrocarbons longifolene and caryophyilene, BHT, and two unidentified compounds. The emissions from Cove Base Adhesive CBA were almost exclusively dominated by hydrocarbons (Appendix E, Table E-15). The compounds included $\mathrm{C}_{8}-\mathrm{C}_{12}$ branched and normal alkane hydrocarbons and numerous alkyl substituted cyclohexanes.

The qualitative results for the composite assemblies are presented in Appendix E, Tables $E-16$ and E-17. The emissions from the assembly with Sheet Vinyl SV5 were very similar to the emissions from SV5 by itself with the exception of a relatively high abundance of toluene, the dominant solvent component of SFA. The assembly with Cove Base CB emitted compounds from both $\mathrm{CB}$ and CBA. Unlike the assembly with the sheet vinyl, the edges of the cove base were not sealed. 
Table 9. Most abundant VOCs emitted in the screening measurements of the five sheet vinyl materials, the cove base and the associated adhesives.

\begin{tabular}{|c|c|}
\hline Material ID / Compound & Material ID / Compound \\
\hline Sheet Vinyl SV1 & Sheet Vinyl SV4 \\
\hline $\mathrm{n}$-Tridecane & $\mathrm{C}_{11}$ Branched alkane HCs (4) \\
\hline 2-Ethyl-1-hexanol & n-Tridecane \\
\hline 4-Methyl-1-heptanol & Phenol \\
\hline \multicolumn{2}{|l|}{ Phenol } \\
\hline $\mathrm{TXIB} \otimes$ & Cove Base CB \\
\hline \multirow[t]{2}{*}{ Diethylphthalate } & Toluene \\
\hline & $\mathrm{C}_{2}$ Tetrahydronaphthalenes (3) \\
\hline Sheet Vinyl SV2 & tert-Butyl isothiocyanate \\
\hline $\mathrm{C}_{10} \& \mathrm{C}_{11}$ Branched alkane $\mathrm{HCs}(5)^{*}$ & Benzothiazole \\
\hline \multicolumn{2}{|l|}{ Phenol } \\
\hline & Sheet Flooring Adhesive SFA \\
\hline Sheet Vinyl SV3 & Toluene \\
\hline n-Decane & Longifolene \\
\hline $\mathrm{n}$-Tridecane & Caryophyllene \\
\hline $\mathrm{n}$-Tetradecane & $\mathrm{BHT}$ \\
\hline Benzyl alcohol & Unidentified compounds (2) \\
\hline \multicolumn{2}{|l|}{ Phenol } \\
\hline \multirow[t]{2}{*}{$\mathrm{TXIB} \otimes$} & Cove Base Adhesive CBA \\
\hline & $\mathrm{C}_{8}$ Branched alkane $\mathrm{HC}$ \\
\hline Sheet Vinyl SV5 & n-Octane \\
\hline n-Decane & n-Decane \\
\hline $\mathrm{n}$-Tridecane & Dimethylcyclohexane isomer \\
\hline $\mathrm{n}$-Tetradecane & Ethylcyclohexane \\
\hline Benzyl alcohol & Trimethylcyclohexane isomer \\
\hline \multicolumn{2}{|l|}{ Phenol } \\
\hline $\mathrm{TXIB} \AA$ & \\
\hline
\end{tabular}

*Numbers of individual compounds in a classification are indicated in parentheses. 


\subsection{CONCENTRATIONS OF THE TARGET COMPOUNDS}

Thirty-seven of the most abundant and prevalent VOCs detected in the emissions from the vinyl flooring materials and assemblies were selected for quantitative analysis (Appendix $E$, Table E-18). The individual materials were only analyzed for those compounds that they were known to emit based on the qualitative results. There were no target compounds for the GB substrate. The samples collected at all four time periods were analyzed. The results for the sheet vinyls are presented in Appendix E, Tables E-19 - E-25. The results for the other materials and assemblies are presented in Appendix E, Tables E-26 - E-31. The lower limit of quantitation was about $1 \mu \mathrm{g} \mathrm{m}^{-3}$ for those individual compounds that approached the limit.

The chamber concentrations of the target VOCs emitted by commercial Sheet Vinyl SV1 were very low. By 48 hours, only the concentration of phenol exceeded $10 \mu \mathrm{g} \mathrm{m}^{-3}$. The concentrations of phenol, normal alkane hydrocarbons, and in some cases TXIB® and benzyl alcohol dominated the emissions of VOCs from the residential sheet vinyl materials. Among these materials, the chamber concentrations of the target VOCs were highest for Sheet Vinyls SV3 and SV5.

For Cove Base $\mathrm{CB}$, the chamber concentrations of toluene, tert-butyl isothiocyanate and benzothiazole were dominant. Toluene dominated the emissions from Sheet Flooring Adhesive SFA. Normal alkane hydrocarbons and ethylcyclohexane dominated the emissions from Cove Base Adhesive CBA.

The concentrations of TVOC for the screening measurements with the vinyl flooring materials are presented in Appendix E, Table E-32. Among the sheet vinyl materials, SV1 had the distinctly lowest TVOC concentrations. At 48 hours, the value was equivalent to the concentration for the blank chamber run. Materials SV3 and SV 5 had the highest TVOC concentrations. Materials SV2 and SV4 had substantially lower TVOC concentrations that were nearly identical. There was good agreement between the duplicate experiments with SV3. The TVOC concentrations for the assembly with SV5 were nearly identical to the TVOC concentrations for SV 5 by itself.

The concentrations of the target VOCs were also summed for each material. These SigmaVOC concentrations are presented in Appendix E, Table E-33. The ratios of the SigmaVOC and the TVOC concentrations for the 24- and 48-h samples of the individual materials and assemblies are plotted in Figure 15. For Sheet Vinyls SV3 and SV5 and the two assemblies, the ratios were approximately 25 percent or better. Smaller fractions of the total emissions of VOCs from the other materials were quantified as individual compounds.

The chamber concentrations of formaldehyde and acetaldehyde are presented in Appendix E, Tables E-34 and E-35, respectively. The formaldehyde concentrations for the sheet vinyl materials were all near or below the lower limit of quantitation, indicating that these materials were not sources of formaldehyde. Only the particleboard underlayment had significant emissions of formaldehyde at 48 hours as shown by the chamber concentrations of formaldehyde for UL by itself and in combination with SFA. The data also showed that CBA was a source of formaldehyde. However, by the end of the experiments with this adhesive, the formaldehyde concentrations were less than $10 \mu \mathrm{g} \mathrm{m}^{-3}$. The underlayment and CBA were initially minor sources of acetaldehyde. By the ends of the experiments, the acetaldehyde concentrations were low for all of the materials.

\subsection{EMISSION RATES OF THE TARGET COMPOUNDS}

The quasi-steady state emission rates of the target compounds at the 24- and 48-h time periods were calculated using the mass balance equation (Equation 1). Since the chamber concentrations did not change rapidly over this time interval, the steady-state model was expected to produce reliable estimates of the emission rates. The emission rates for the sheet vinyl materials were calculated as area-specific values $\left(\mu \mathrm{g} \mathrm{m}^{-2} \mathrm{~h}^{-1}\right)$ based on the exposed surface area of $0.0195 \mathrm{~m}^{2}$. The emission rates for the cove base were calculated as length-specific values $\left(\mu \mathrm{g} \mathrm{m}^{-1} \mathrm{~h}^{-1}\right)$, and the emission rates for the adhesives were calculated as mass-specific values $\left(\mu \mathrm{g} \mathrm{kg}^{-1} \mathrm{~h}^{-1}\right)$. The emission rates of the individually quantified VOCs emitted by the vinyl flooring 
materials are presented in Appendix E, Tables E-36 - E-47. The TVOC emission rates are presented in Appendix E, Table E-48. The formaldehyde and acetaldehyde emission rates are respectively presented in Appendix E, Tables E-49 and E-50. The emission rates of $n$-tridecane, phenol, TXIB@ and TVOC for the sheet vinyl materials are plotted in Figures 16 - 19, respectively.

The commercial Sheet Vinyl SV1 generally had the lowest emission rates both for individual VOCs and TVOC. The same manufacturer also produced Sheet Vinyls SV2 and SV4. Even though SV2 and SV4 differed with respect to grade and the design of the backing (i.e., perimeter or full spread adhesive application), these two materials had similar emissions characteristics. This is apparent from a comparison of the emission rates of the dominant compounds emitted by the materials. Another manufacturer produced the nearly identical Sheet Vinyls SV3 and SV5. In general, the emission rates of VOCs from these two materials were higher than the emission rates for the other sheet vinyl materials.

Figure 16 shows that the specific emission rates of $n$-tridecane were highest for SV3 and SV5 and that there was reasonably good agreement for this compound between the duplicate experiments with SV3 and between the experiments with SV5 by itself and in the assembly. Also the differences in the rates between the 24- and 48-h samples were relatively small. For SV2 and SV 4 , the rates increased from 24 to 48 hours.

Phenol generally had the highest emission rates among the individual compounds. Figure 17 shows that these rates were lowest for SV1 and highest for SV5 by itself and in the assembly. There was good replication in the duplicate experiments, and the differences between the emission rates of phenol at 24 and 48 hours were minor.

The emission rates of TXIBQ from SV1, SV2 and SV4 were all low. The same manufacturer produced all of these materials. The emission rates of TXIB尺 from SV3 and SV5 produced by the other manufacturer were substantially higher as shown in Figure 18. There was a substantial difference in the rates between the duplicate experiments with SV3 that presumably was the result of material heterogeneity. Differences between the emission rates of TXIBB at 24 and 48 hours were minor.

The same general relationships among the sheet vinyl materials were also apparent in their TVOC emission rates. Figure 19 shows that SV1 had TVOC emission rates that were below the lower limit of quantitation of $75 \mu \mathrm{g} \mathrm{m}^{-2} \mathrm{~h}^{-1}$ and that SV3 and SV5 had the highest TVOC emission rates of up to $3,000 \mu \mathrm{g} \mathrm{m}^{-2} \mathrm{~h}^{-1}$. There were small differences between the replicate experiments with SV3 and between the experiments with SV5 by itself and in the assembly.

The emission rates of the target VOCs from Cove Base CB were relatively low. Among the selected compounds, benzothiazole had the highest emission rates. Sheet Flooring Adhesive SFA had relatively mass-specific emission rates of toluene. The toluene emission rate in the experiment with the assembly of SV5, UL and SFA was substantially elevated over the emission rate of toluene from SV5 by itself presumably due to the contribution of SFA. Cove Base Adhesive CBA had mass-specific TVOC emission rates that were five time higher than the rates for SFA.

Underlayment UL had elevated formaldehyde emission rates. The emission rates of formaldehyde from SFA applied to UL were also calculated as area-specific values for direct comparison with the UL results (Table E-49). This showed that the application of SFA to UL resulted in a reduction in the emission rates of formaldehyde from UL. CBA was shown to be a low source of formaldehyde on a mass-specific basis. For the assemblies, the formaldehyde emission rates were near the lower limit of quantitation.

On an area-specific basis, the emission rates of acetaldehyde were low and generally approached the lower limit of quantitation.

\subsection{SELECTION OF THE SHEET VINYL MATERIAL FOR THE LARGE-SCALE EXPERIMENTS}

Sheet Vinyl SV3 was selected for use in the large-scale experiments to evaluate treatment effects. This sheet vinyl was representative of the category of intermediate-grade materials that have the highest share of the residential market. In addition, SV3 had generally 
higher emission rates of VOCs than the other intermediate-grade residential material, SV2. Higher rates were desirable in order to demonstrate the efficacy of the treatments. Sheet Vinyl SV5, which was identical to SV3 except for the printed pattern and color, was purchased for use in the large-scale experiments. The related materials were selected at the outset of the screening measurements based on information that suggested that they were typical of the materials used in the installation of residential vinyl floors.

\subsection{COMPARISON WITH LITERATURE DATA}

The emissions of VOCs from sheet vinyl flooring have received very little attention. All of the reported data is for European products. Bremer et al. (1993) listed the VOCs identified in the emissions from 22 different vinyl flooring materials determined using small-scale chambers.

These compounds included aliphatic and aromatic hydrocarbons, alkylphenols (phenol and alkyl substituted phenols), aliphatic alcohols, aldehydes, ketones, carboxylic acids, and various esters. Generally, the hydrocarbons and aliphatic esters had the highest chamber concentrations. The European Collaborative Action (ECA, 1997) reported the emission rates of VOCs from two vinyl flooring materials. The compounds with the highest emission rates included cyclohexanone, phenol and 2-ethyl-1-hexanol. Others have attempted to model the emissions of VOCs from vinyl flooring materials based on studies conducted in small-scale chambers (Christiansson et al., 1993; Clausen et al., 1993). One of these studies showed that the emission rate of phenol was not affected by the chamber ventilation rate. The model that was developed suggested that the emissions of phenol were controlled by diffusion within the source (Clausen et al., 1993).

\subsection{SUMMARY}

The results of the screening measurements with the vinyl flooring materials are summarized as follows:

1. The residential sheet vinyls with a clear "no-wax" top coat emitted more compounds and had higher emission rates of individual VOCs and TVOC than the single commercial sheet vinyl.

2. There were both qualitative and quantitative differences among the residential sheet vinyls with respect to emissions that may have been related to their manufacturing origin. The emission characteristics for the base and intermediate grade materials from one manufacturer were generally similar to each other and were distinct in some respects from the emission characteristics of the materials from the other manufacturer.

3. All of the sheet vinyl materials were a source of phenol that is an identified TAC.

4. The new-generation "solvent free" or "low VOC content" adhesive products were shown to be sources of a number of VOCs. It is not known to what extent the qualitative and quantitative emissions of VOCs from these products compare to conventional or older-generation products used for the same applications. 


\subsection{EXPOSURE REDUCTION EXPERIMENTS WITH THE LATEX PAINT COMBINATION}

\subsection{EXPERIMENTAL TREATMENTS}

Four large-scale experiments were conducted with the combination of latex paints consisting of materials LPS2, FLP3 and SGLP3. Two of these experiments, $P-1$ and P-2, were conducted at the base-case ventilation rate of $0.5 \mathrm{~h}^{-1}$ over $2-336 \mathrm{~h}$. The results from these experiments were used to assess overall reproducibility of the methods and served as the basis for evaluating the efficacy of the two treatments. One of the treatment experiments, P-3, was conducted using additional ventilation for the first $72 \mathrm{~h}$. The ventilation rate during painting and for the following two hours was $5 h^{-1}$. From $2-72 h$, the ventilation rate was $2 h^{-1}$. The other treatment experiment, P-4, was conducted using the same ventilation conditions as Experiment P-3. In addition, air mixing at the surfaces of the painted gypsum panels was increased by the use of two oscillating room fans operated on their highest speed setting. Increased air velocities should have the effect of decreasing the effective thickness of the air boundary layer at the surface of the paint film that could accelerate the evaporation of the VOCs. This treatment was predicted to be most effective in combination with increased ventilation. The ventilation rate data and the compartment temperatures and relative humidities for the experiments are summarized in Appendix $\mathrm{H}$, Table $\mathrm{H}-01$. The ventilation rates and compartment temperatures were maintained within the established range limits. The relative humidity fell below the lower limit of 30 percent during portions of Experiments P-1 and P-2 (discussed below).

The paint application data for Experiments P-1 - P-4 is presented in Appendix $\mathrm{H}$, Table $H-02$. Generally good uniformity in paint application was achieved as shown by the coverages in grams of wet paint applied per square meter of substrate surface area. The coverages for LPS2 ranged from $101-127 \mathrm{~g} \mathrm{~m}^{-2}$ with the lowest coverage for Experiment $\mathrm{P}-1$. The coverages for the other three experiments were in a range of $118-127 \mathrm{~g} \mathrm{~m}^{-2}$. The coverages for FLP3 in all four experiments were in a narrow range of $105-108 \mathrm{~g} \mathrm{~m}^{-2}$. The coverages for SGLP3 in all four experiments were also in a narrow range of $100-105 \mathrm{~g} \mathrm{~m}^{-2}$.

\subsection{SELECTION OF THE TARGET COMPOUNDS}

A qualitative analysis of the VOCs emitted by the paint combination in base-case Experiment P-2 identified ethylene glycol, propylene glycol and the two isomers of 2,2,4-trimethyl1,3-pentanediol monoisobutyrate (Texanoß) as the most abundant compounds (Appendix $\mathrm{H}$, Table H-03). There were several other oxidized compounds emitted at lower abundance. The six target VOCs selected for quantitative analysis in these experiments were: ethylene glycol; propylene glycol; n-buty ether; 2-ethyl-1-hexanol; 2-(2-butoxyethoxy)ethanol (di(ethylene glycol) butyl ether or DEGBE); and the combined Texanoß isomers (Appendix $\mathrm{H}$, Table $\mathrm{H}-04$ ). Hexylene glycol was not included as a target analyte because the calibration for this compound was unreliable. In addition, the aldehydes, formaldehyde and acetaldehyde, were sampled and analyzed in all four experiments. Formaldehyde, acetaldehyde, ethylene glycol and DEGBE are identified by the ARB as TACs.

\subsection{CONCENTRATIONS OF THE TARGET COMPOUNDS}

The concentrations of the six target VOCs in chamber compartment air and supply air during the four experiments with latex paints are presented in Appendix $\mathrm{H}$, Tables H-05- H-08. The concentrations for the duplicate VOC samples that were collected at several time points are given in these tables. It is noted that Experiment P-4 was terminated early at $240-h$ elapsed time because there was a two-day power outage following this sampling period in which the ventilation and data systems were off. The average concentrations of the six target VOCs in the supply air in all four experiments were below their lower limits of quantitation. The concentrations of these compounds in the background measurement periods were also at or below their lower limits of quantitation with the exception of Texanok in Experiments P-3 and P-4. These were the second experiments to be conducted with paints in the compartments, and high ventilation rate flushing was incapable of reducing the background level of Texanol $\otimes$ to below its lower limit of quantitation. 
The compartment concentrations of the dominant VOCs emitted in Experiments $P-1$ and P-2 were compared in order to assess the reproducibility of the methods. The concentrations of ethylene glycol in the four experiments are plotted in Figures 20a and 20b, and the concentrations of Texanoß are plotted in Figure 21. In these and the subsequent temporal plots, the data points are connected with dashed lines for the sole purpose of improving the readability of the plots. There were concentration differences at the beginning of Experiments P-1 and P-2 that were most notable for ethylene glycol. The cause, which is uncertain, may be related to the exact timing of sample collection with respect to the rapid temporal changes in concentrations immediately following painting. There were also relatively large temporal fluctuations in the concentrations of ethylene glycol in Experiment P-2 from approximately $120 \mathrm{~h}$ through the end of the experiment. Other than these obvious differences, there was generally good agreement between the two base-case experiments.

The environmental data for the experiments, summarized in Table $\mathrm{H}-01$ shows that the relative humidity in Experiments P-1 and P-2 fluctuated over a relatively large range and fell below the lower target limit of 30 percent for some portion of the experiments. The compartment air relative humidity in Experiment $\mathrm{P}-2$ is plotted in Figure 22. Figure $20 \mathrm{~b}$ shows that there was a decrease in the ethylene glycol concentration at the 144- and 168-h sampling periods that corresponded with the period of the lowest relative humidity. This correspondence suggests that the water vapor concentration in air was either affecting the primary emission rate of ethylene glycol from the paints or may have had a secondary effect on the sorption and release of ethylene glycol by surfaces in the compartment. The concentration of propylene glycol may have been similarly affected. In Experiment P-1, the period of lowest relative humidity occurred between 240- and 336-h elapsed time when no air samples were taken. The screening measurements with the combination of paints LPS2 and FLP3 that were conducted at 30,50 and 70 percent relative humidity demonstrated that the effect of water vapor concentration on the primary emission rates of VOCs, including ethylene glycol, from these paints was negligible or relatively minor (Appendix C, Tables C-51 - C-53). Therefore, it is possible that the effect of water vapor on the concentration of ethylene glycol in the large-scale experiments was primarily secondary and related to sorption/desorption phenomena.

The compartment relative humidity was within the target range and generally more stable during Experiment P-3. The supply air humidification system was added prior to initiating Experiment P-4. This system introduced water vapor into the supply air when the relative humidity of the supply air dropped below about 40 percent at room temperature. Thus, it was possible to maintain compartment relative humidity within the target range during the remainder of the experiments.

The compartment concentrations of the dominant VOCs in Experiment P-3 were compared to the corresponding concentrations in base-case Experiments P-1 and P-2 in order to assess whether there were any large-magnitude effects due to additional ventilation. Generally, the concentrations were lower in Experiment P-3 during the period of additional ventilation. However, the concentration differences were less than the four-fold difference in ventilation rate for this period. This suggests that the emission rates of these compounds increased at the higher ventilation rate perhaps due to mass transfer effects at the painted surfaces. Subsequent to $72-h$ elapsed time, the concentrations of both ethylene glycol and Texanok were similar in all three experiments.

Prior to conducting Experiment $P-4$, air velocities were measured $5-\mathrm{cm}$ above the surface at three locations (center of top half, approximate middle, and center of bottom half) for each of the five gypsum board panels with the two oscillating fans operating at their highest speed setting. The average air velocity was $54 \pm 9 \mathrm{~cm} \mathrm{sec}^{-1}$. Velocities previously recorded $5-\mathrm{cm}$ above the wall surfaces without the oscillating fans in the compartment were $20 \pm 4 \mathrm{~cm} \mathrm{sec}^{-1}$. Thus, the added air mixing in Experiment P-4 resulted in about a factor of 2.5 increase in the air velocities near the painted surfaces. The added air mixing, however, did not result in any large-magnitude effect on the compartment air concentrations of the target VOCs as can be seen by comparing the concentrations of ethylene glycol and Texanoß for Experiments P-3 and P-4. 
Since the samples were almost entirely dominated by a few compounds that were individually quantified, the concentrations for the total emissions of VOCs were quantified as SigmaVOC (Appendix H, Table H-09).

The concentrations of formaldehyde and acetaldehyde in compartment and supply air for Experiments P-1 - P-4 are presented in Appendix $\mathrm{H}$, Tables $\mathrm{H}-10$ and $\mathrm{H}-11$, respectively. The background concentrations of these compounds were generally elevated over the supply air concentrations. This was presumably due to emissions from the wood frame and the plywood flooring of the chamber compartments. The chamber concentrations of formaldehyde during the experiments were only clearly elevated over background concentrations during the first day of sampling. The highest concentrations occurred during the first few hours of Experiments P-1 and P-2. The acetaldehyde concentrations substantially exceeded the formaldehyde concentrations. Clearly elevated concentrations of acetaldehyde occurred during the first six hours of sampling, with the highest concentrations occurring for base-case Experiments P-1 and P-2 (Figure 23). This difference may have been affected, in part, by the timing of the sample collection with respect to the occurrence of the peak compartment concentration of acetaldehyde. Nevertheless, the additional ventilation in Experiments P-3 and P-4 was apparently effective at removing the formaldehyde and acetaldehyde released during the painting procedure.

\subsection{EMISSION RATES OF THE TARGET COMPOUNDS}

Quasi steady-state emission rates of the target compounds in the four experiments were calculated with the mass-balance equation (Equation 1). Since the model assumes steady-state conditions and the compound concentrations, in many cases, were changing relatively rapidly during the first hours of the experiments, substantial uncertainty may have been introduced into the estimated emission rates for these beginning periods. The combined painted surface area of $16 \mathrm{~m}^{2}$ was used in the calculations as there was no clear delineation of the sources of the dominant target compounds among the three paints.

The area-specific emission rates in milligrams of compound emitted per square meter of surface per hour $\left(\mathrm{mg} \mathrm{m}^{-2} \mathrm{~h}^{-1}\right)$ are presented Appendix $\mathrm{H}$, Tables $\mathrm{H}-12-\mathrm{H}-15$ for Experiments P-1 - P-4, respectively. The area-specific emission rates of SigmaVOC are presented in Appendix $\mathrm{H}$, Table $\mathrm{H}-16$. An obvious difference among the experiments is that the emission rates of the dominant compounds were generally higher during the period of additional ventilation in Experiments P-3 and P-4 than during the same period of Experiments $\mathrm{P}-1$ and $\mathrm{P}-2$ (Figures $24 a$, $24 b$ and 25). As discussed above, this was probably due to mass transfer effects at the painted surfaces. The estimated emission rates of ethylene glycol and Texanok in the first hours following painting were clearly elevated in Experiments P-3 and P-4. However, as previously noted, there is substantial uncertainty in these estimates. A comparison of the emission rate results for Experiments $\mathrm{P}-3$ and $\mathrm{P}-4$ shows that there were no large magnitude effects due to increased air velocities at the painted surfaces.

The initial formaldehyde emission rates in Experiments P-1, P-3, and P-4 were similar, while the initial formaldehyde emission rate in Experiment P-2 was higher (Appendix $\mathrm{H}$, Table $H-17)$. The initial acetaldehyde emission rates were highest in base-case Experiments $P-1$ and P-2 (Appendix H, Table H-18).

\subsection{CUMULATIVE MASSES AND EXPOSURES FOR THE TARGET COMPOUNDS}

The results were first summarized by calculating the cumulative masses of the target compounds and SigmaVOC that were emitted over the experimental period (Appendix H, Table $H-19$ ). Since Experiment P-4 was terminated early at 240-h elapsed time, cumulative masses were determined for the 0 - to 240-h period for all four experiments and for the 0 - to 336-h period for Experiments P-1, P-2, and P-3. Deletion of the final four-day period did not have any effect on the relative differences among Experiments P-1, P-2, and P-3 for any of the target compounds.

The compounds with the distinctly highest cumulative mass emissions were ethylene glycol, propylene glycol and Texanok. Additional ventilation and additional ventilation with air mixing in Experiments P-3 and P-4 resulted in higher mass emissions of these dominant 
compounds. The data indicates that the mass emissions of acetaldehyde decreased with the two treatments. As previously discussed, this was probably an artifact caused by the timing of sample collection with respect to the rapid removal of acetaldehyde from the compartment. The relative magnitudes of the effects of the treatments on the cumulative mass emissions of the target compounds and SigmaVOC over $-240 \mathrm{~h}$ are illustrated in Figure 26. For this figure, the results for base-case Experiments P-1 and P-2 were averaged and then used to calculate the ratios for the two treatment experiments. For SigmaVOC and all of the compounds, except acetaldehyde, n-butyl ether and 2-ethyl-1-hexanol, the cumulative mass emissions were distinctly higher in the two treatment Experiments P-3 and P-4 than in the base-case experiments. The effects on cumulative mass emissions due to the added air mixing in Experiment P-4 relative to Experiment P-3 were most apparent for ethylene glycol and propylene glycol. The differences between these two experiments for the other compounds were minor.

The fractional compositions of ethylene glycol and Texanol in paints LPS2, FLP3 and SGLP3 were determined by analysis of the bulk paints (Appendix C, Table C-58). The masses of ethylene glycol and Texanoß that were used in Experiments P-1 - P-4 were calculated from the paint application data and the paint compositions. These values are presented in Table 10 where they are compared to the cumulative masses of ethylene glycol and Texanok emitted during the experiments. The average mass of ethylene glycol used in the four experiments was approximately $100 \mathrm{~g}$, and the average mass of Texanoli was approximately $43 \mathrm{~g}$. The cumulative mass emissions of ethylene glycol were $3.7-13.9 \mathrm{~g}$ over $0-240 \mathrm{~h}$ and $4.1-14.2 \mathrm{~g}$ over $0-336 \mathrm{~h}$. Thus, only approximately $4-14$ percent of the ethylene glycol that was applied with the paints was emitted over the 336-h period. The cumulative mass emissions of Texanoß were $6.9-13.2 \mathrm{~g}$ over $0-240 \mathrm{~h}$ and $8.6-15.0 \mathrm{~g}$ over $0-336 \mathrm{~h}$. Only $19-35$ percent of the applied Texanoß was emitted over the 336-h period. The results demonstrate the very large sorption losses and the slow re-emission of the major volatile organic constituents of latex paints applied to gypsum board. These results are excellent agreement with the results obtained in a small-scale chamber with another flat latex paint (Chang et al., 1997). In that study, nine percent of the ethylene glycol and 29 percent of the Texanolß in the paint applied to gypsum board were emitted over the same 336-h time period. This similarity, despite the differences in the paint and the experimental apparatus and conditions, demonstrates that the gypsum board substrate plays a significant role in determining the emissions of VOCs from latex paint. At present, it is not known whether 100 percent of the ethylene glycol and Texanok absorbed by gypsum board are re-emittable (Chang et al., 1998).

The experiments and the treatment effects were next compared by examining the peak exposures to the target compounds that a residential occupant might receive at these conditions. The highest exposure concentrations ( $\mathrm{ppb}$ ) of the target compounds were measured shortly after paint application (Appendix $\mathrm{H}$, Table $\mathrm{H}-20$ ). The maximum concentrations of formaldehyde, acetaldehyde, n-butyl ether and 2-ethyl-1-hexanol were substantially lower in Experiments P-3 and $P-4$ with additional ventilation relative to base-case Experiments $P-1$ and P-2. For Acetaldehyde, there was an approximate ten-fold difference in maximum concentration. The maximum concentrations of ethylene glycol and propylene glycol in Experiment P-4 with additional ventilation combined with air mixing were about 60 percent of the respective maximum concentrations of these compounds in Experiment P-3 with additional ventilation only.

Finally, the experiments and the treatments were compared based on theoretical cumulative exposures in $\mathrm{ppm}-\mathrm{h}$ for a residential occupant that were calculated for two different exposure periods.

The estimated cumulative exposures to the target compounds for the first 48 hours of the experiments are presented in Appendix $\mathrm{H}$, Table $\mathrm{H}-21$. For this calculation, occupancy began at time zero, the conclusion of paint application, and the occupancy factor was assumed to be 0.83 (i.e., an occupancy of 20 hours per day averaged throughout the day). The cumulative exposures to formaldehyde, acetaldehyde, n-butyl ether and 2-ethyl-1-hexanol for this period were clearly lower in Experiments P-3 and P-4 with additional ventilation relative to the base-case experiments. The differences between the cumulative exposures for the two treatment Experiments P-3 and $P-4$ were all less than 25 percent. The relative magnitudes of the effeci: $a^{f}$ the treatments on the 
cumulative exposures to the target compounds over $0-48 \mathrm{~h}$ are illustrated in Figure 27. The results for base-case Experiments $\mathrm{P}-1$ and $\mathrm{P}-2$ were averaged and used to calculate the ratios for the two treatment experiments. This figure shows that the treatments did not result in large fractional reductions in the exposures to the dominant compounds, ethylene glycol, propylene glycol and Texanol( ) for this initial two-day period.

Table 10. Applied masses $(\mathrm{g})$ of ethylene glycol and Texanok compared to the masses of these compounds emitted over $0-336 \mathrm{~h}$ in Experiments P-1 - P-4. The applied masses were calculated from the paint application data (Appendix $\mathrm{H}$, Table $\mathrm{H}-02$ ) and the paint compositions (Appendix C, Table C-58).

\begin{tabular}{lrrrr}
\hline $\begin{array}{l}\text { Compound } / \\
\text { Parameter }\end{array}$ & Exp P-1 & Exp P-2 & Exp P-3 & Exp P-4 \\
\hline Ethylene glycol & & & & \\
Applied mass (g) & 90 & 103 & 100 & 104 \\
Emitted mass (g) & 4.08 & 6.8 & 14.2 & $10.0^{*}$ \\
Fraction emitted (\%) & 4.5 & 6.6 & 14 & 9.6 \\
Texanol@ & & & & \\
Applied mass (g) & 39 & 45 & 43 & 45 \\
Emitted mass (g) & 8.6 & 8.6 & 15.0 & $11.8^{*}$ \\
Fraction emitted (\%) & 22 & 19 & 35 & 26 \\
\hline
\end{tabular}

"Masses were emitted over 0 - $240 \mathrm{~h}$ in Experiment P-4.

The estimated cumulative exposures to the target compounds for the remainders of the experiments are presented in Appendix $\mathrm{H}$, Table $\mathrm{H}-22$. For this calculation, occupancy began exactly $48 \mathrm{~h}$ after painting, thus avoiding the initial period of highest concentration. It was assumed that the daily duration of occupancy was $20 \mathrm{~h}$. The compounds with the distinctly highest cumulative exposures in all of the experiments were ethylene glycol, propylene glycol and Texanol@. The treatment effects were most pronounced for the minor paint constituents. The most obvious difference was the reduction in the exposures to acetaldehyde in the two treatment experiments. Additional ventilation and air mixing decreased the cumulative exposure to n-butyl ether, and both additional ventilation and additional ventilation with air mixing decreased the exposure to 2-ethyl-1-hexanol.

The relative magnitudes of the effects of the treatments on the cumulative exposures to the target VOCs over $48-240 \mathrm{~h}$ are illustrated in Figure 28. The results for base-case Experiments $\mathrm{P}-1$ and $\mathrm{P}-2$ were averaged and used to calculate the ratios for the two treatment experiments. The figure shows that additional ventilation by itself did not result in reduced exposures to the dominant VOCs for this period. There were, however, approximate 25 percent reductions in the exposures to ethylene gls col and Texanol in Experiment P-4 with additional ventilation combined with air mixing relative to the base-case experiments. 
Figure 29 compares the magnitudes of the cumulative exposures estimated for the 0 - to 48-h periods to the total cumulative exposures over $0-240 \mathrm{~h}$ that were calculated as the sums of the exposures for the two periods. For all compounds except Texanok, the cumulative exposures estimated for the 0 - 48-h periods of the experiments accounted for about one-half or

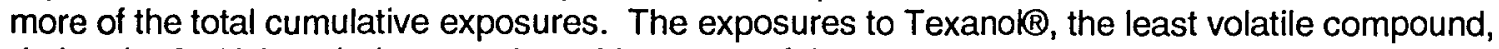
during the $0-48$ - $h$ periods were about 30 percent of the total exposures.

\subsection{COMPARISON WITH THE SCREENING MEASUREMENT RESULTS}

Latex Primer Sealer LPS2 and Flat Latex Paint FLP3 accounted for approximately 97 percent of the wet mass of paint applied in each of the four large-scale experiments. The average combined coverage of these two paints was $224 \mathrm{~g} \mathrm{~m}^{-2}$. Three screening measurements were conducted with a combination of the LPS2 and FLP3 paints over a range of $30-70$ percent relative humidity. The average combined coverage of the two paints in these experiments was $169 \mathrm{~g} \mathrm{~m}^{-2}$ or about 75 percent of the combined coverage of the two paints in the large-scale experiments. Emission rates of the target compounds and SigmaVOC in the small chambers were calculated for elapsed times of 48 and $96 \mathrm{~h}$ (Appendix C, Tables C-51 - C-53).

The 96-h emission rates ( $\mathrm{mg} \mathrm{m}^{-2} \mathrm{~h}^{-1}$ ) of ethylene glycol, propylene glycol, DEGBE, TexanoK, and SigmaVOC in the three screening measurements and in the four large-scale experiments are compared in Table 11. Generally, the results agreed within about a factor of two. The emission rates of ethylene glycol were roughly comparable while the Texanok emission rates were about a factor of two higher in the screening measurements. These discrepancies are relatively small considering that there were large differences between these experiments with respect to chamber ventilation rates, chamber surface-to-volume ratios, and the types and amounts of surface materials.

Table 11. Area-specific emission rates $\left(\mathrm{mg} \mathrm{m}^{-2} \mathrm{~h}^{-1}\right)$ of ethylene glycol, propylene glycol, DEGBE, Texanol and SigmaVOC at 96-h elapsed time in the three screening measurements with the LPS2 and FLP3 paint combination and in large-scale Experiments P-1 - P-4.

\begin{tabular}{|c|c|c|c|c|}
\hline \multirow[b]{3}{*}{ Component } & \multicolumn{4}{|c|}{ Specific Emission Rate, $\mathrm{mg} \mathrm{m}^{-2} \mathrm{~h}^{-1}$} \\
\hline & \multicolumn{2}{|c|}{$\begin{array}{c}\text { Screening Measurements } \\
\text { Combined LPS2 \& FLP3 }\end{array}$} & \multicolumn{2}{|c|}{$\begin{array}{l}\text { Large-Scale } \\
\text { Expts. P-1 - P-4 }\end{array}$} \\
\hline & Avg. & Range & Avg. & Range \\
\hline Ethylene glycol & 0.36 & $0.20-0.57$ & 0.54 & $0.46-0.59$ \\
\hline Propylene glycol & $<0.06$ & --- & 0.09 & $0.07-0.13$ \\
\hline DEGBE & $<0.03$ & -- & 0.01 & $\cdots$ \\
\hline Texanok & 3.24 & $2.68-3.83$ & 1.60 & $1.12-1.92$ \\
\hline SigmaVOC & 3.65 & $3.02-4.09$ & 2.23 & $1.67-2.57$ \\
\hline
\end{tabular}




\subsection{EXPOSURE REDUCTION EXPERIMENTS WITH THE CARPET ASSEMBLY}

\subsection{EXPERIMENTAL TREATMENTS}

Three large-scale experiments were conducted with the carpet assembly consisting of materials CP4, CC4 and ST. The first of these experiments, C-1, was conducted at the basecase ventilation rate of $2 \mathrm{~h}^{-1}$ during carpet installation and for the following two hours. Then the ventilation rate was lowered to $0.5 \mathrm{~h}^{-1}$ and maintained at this condition over $2-336 \mathrm{~h}$. One of the treatment experiments, $\mathrm{C}-2$, was conducted using additional ventilation for the first $72 \mathrm{~h}$. The ventilation rate during carpet installation and for the following two hours was $5 h^{-1}$. From $2-72 \mathrm{~h}$, the ventilation rate was $2 \mathrm{~h}^{-1}$. For the other treatment experiment, $\mathrm{C}-3$, the carpet and cushion were aired out immediately prior to their installation. Two days before the start of the experiment the materials were laid out on the floor of a highly ventilated and well-mixed room as described in Appendix G. At the end of the 48-h airing out period, the materials were taken to the nearby chamber facility. Installation of the materials began within one hour. This experiment was conducted at the base-case ventilation rate conditions described above. The ventilation rate data and the compartment temperatures and relative humidities for the experiments are summarized in Appendix I, Table 1-01. All of these parameters were maintained within the established range limits. The same amounts of materials were used in all three experiments (Appendix I, Table $1-02)$.

\subsection{SELECTION OF THE TARGET COMPOUNDS}

A qualitative analysis of the VOCs emitted by the carpet assembly in base-case Experiment C-1 identified a relatively large number of compounds (Appendix I, Table I-03).

More than half of the individually listed compounds were hydrocarbons including aromatic hydrocarbons and a number of branched alkane and alkene (olefinic) hydrocarbons, most of which were not further identified. 4-Ethenylcyclohexene (4-vinylcyclohexene), styrene and 4-phenylcyclohexene (4-PCH) are characteristic of emissions from the SBR latex adhesive used on the secondary backing of the carpet (Hetes et al., 1992). All of these compounds were detected in the screening measurement of Carpet CP4 (Appendix D, Table D-06). SBR carpets also frequently emit toluene, xylene isomers, isopropylbenzene and propylbenzene (Hetes et al., 1992). However, only the combined $m-, p$-xylene isomers were detected in the screening measurement of the carpet. A relatively large abundance of a complex mixture of unresolved isomers of predominantly unsaturated hydrocarbons eluted near the end of the chromatographic runs. Cushion $\mathrm{CC} 4$ emitted this same mixture in the screening measurement (Appendix D, Table D-11). The emission of a similar mixture was previously reported for a bonded urethane carpet cushion (Schaeffer et al., 1996).

In addition to the hydrocarbons, there were a number of oxidized compounds and nitrogen-containing compounds identified in the emissions from the carpet assembly. All of the aldehydes were present in the chamber background that was probably the dominant source of these compounds. Some of the other oxidized compounds present in the samples were also probable chamber background contaminants. The isomers of di(propylene glycol)methyl ether (DPGME), phenol, phenethyl alcohol and 1-decanol were all emitted by Carpet CP 4 in the screening measurement of this material. The nitrogen-containing compounds, $\mathrm{N}, \mathrm{N}$-dimethylacetamide and 2-methyleneglutaronitrile, were detected in the screening measurement of Carpet $\mathrm{CP} 4$. Butylated hydroxytoluene (BHT) is one of the most abundant compounds emitted by bonded urethane carpet cushions (Schaeffer et al., 1996). It was also among the most abundant compounds emitted by Cushion $\mathrm{CC} 4$ in the screening measurement. Phenol was also detected in the screening measurement of the cushion. The nitrogen-containing compounds, N,N-dimethylbenzylamine and 2,2'-azobisisobutryonitrile, were among the compounds emitted by Cushion CC4 in the screening measurement.

The most abundant VOCs emitted in large-scale experiment $\mathrm{C}-1$ were estimated based on a comparison of the TIC responses of the compounds in the GC/MS analyses. These compounds were two branched alkane hydrocarbons, 4- $\mathrm{PCH}$, the mixture of unsaturated 
hydrocarbons discussed above, DPGME, BHT, N,N-dimethylbenzylamine and 2,2'-azobisisobutryonitrile (Table 1-03).

Fifteen target compounds were selected for analysis in the large-scale experiments. These are listed in Appendix I, Table I-04 along with their dominant sources. The target compounds included formaldehyde and acetaldehyde that had no dominant source and 13 vOCs that were either characteristic of the individual sources and/or were among the most abundant compounds emitted by the assembly. The 13 target VOCs were: styrene; $4-\mathrm{PCH}$;

4-ethenylcyclohexene; 1,2-dichlorobenzene; DPGME; phenol; phenethyl alcohol; 1-decanol; $\mathrm{BHT}$; N,N-dimethylacetamide; N,N-dimethylbenzylamine; 2,2'-azobisisobutryonitrile; and 2-methyleneglutaronitrile. Formaldehyde, acetaldehyde, styrene and phenol are identified by the ARB as TACs.

\subsection{CONCENTRATIONS OF THE TARGET COMPOUNDS}

The concentrations of the 13 target VOCs in the chamber compartment and supply air samples for the three experiments with the carpet assembly are presented in Appendix 1, Tables 1-05 - 1-10. The concentrations for the duplicate VOC samples that were collected at several time points during these experiments are given in the tables. The average concentrations of the target compounds in inlet air were all below their lower limits of quantitation. The compartment background concentrations of these compounds were, with the exception of phenol at $1 \mu \mathrm{g} \mathrm{m}^{-3}$, also below their lower limits of quantitation. The compartment concentrations and average inlet air concentrations of TVOC are presented in Appendix I, Table I-11. The compartment concentrations and average inlet air concentrations of SigmaVOC (i.e., the sum of the concentrations of the 13 target VOCs) are presented in Appendix I, Table |-12. The formaldehyde and acetaldehyde concentrations for Experiment C-1 are presented in Appendix I, Table I-13. The compartment concentrations of formaldehyde and acetaldehyde measured during this basecase experiment were very similar to their respective compartment background concentrations. It was concluded from these results and the results of the screening measurements of the individual materials that the emissions of these compounds from the carpet assembly were negligible. Consequently, the measurement of formaldehyde and acetaldehyde was dropped in the subsequent experiments with the carpet assembly.

The concentrations of styrene, 4-PCH, DPGME, BHT and TVOC in the three experiments are individually compared in Figures 30 - 34, respectively.

The compartment concentrations of the target VOCs emitted by the carpet assembly were low, particularly in comparison with other sources such as the latex paint combination. The four compounds with the highest concentrations in Experiments $\mathrm{C}-1$ and $\mathrm{C}-2$ were 4-PCH, DPGME, BHT, and 2,2'-azobisisobutryonitrile. The concentrations of the more volatile compounds such as styrene (Figure 30) and 4-ethenylcyclohexene decreased rapidly over the initial two to three days of these experiments. The concentrations of the target VOCs were generally lower in Experiment $\mathrm{C}-3$ in which the materials were aired out in comparison to the corresponding concentrations in the other two experiments. Airing out the materials resulted in small and possibly insignificant reductions in the concentrations of 4-PCH and $\mathrm{BHT}$ (Figures 31 and 33); and large reductions in the concentrations of other compounds such as styrene and DPGME (Figures 30 and 32). The reduction in the concentration of 2,2'-azobisisobutryonitrile was dramatic. The general reduction due to airing out is clearly shown by a comparison of the SigmaVOC concentrations presented in Table I-12.

It is notable that the concentrations of $\mathrm{BHT}$ gradually increased with time in all three experiments (Figure 33) while the concentrations of the other target VOCs generally decreased with time. The increase in BHT concentration with time for an assembly of carpet and bonded urethane cushion was previously demonstrated in a small-scale chamber experiment (Schaeffer et al., 1996). This temporal behavior suggests that the carpet was acting as a diffusion barrier for the emission of BHT from the cushion.

The concentrations of $\mathrm{N}, \mathrm{N}$ - dimethylacetamide were all below the lower limit of quantitation except during the first hours of Experiment $\mathrm{C}-1$. The concentrations of both 
$\mathrm{N}, \mathrm{N}$-dimethylbenzylamine and 2-methyleneglutaronitrile were similar in Experiments $\mathrm{C}-1$ and $\mathrm{C}-2$ and near or below the lower limits of quantitation in Experiment $\mathrm{C}-3$.

The combined compartment concentrations of the individually quantified target compounds as measured by SigmaVOC accounted for relatively small portions of the corresponding TVOC concentrations. In Experiments C-1 and C-2, the SigmaVOC concentration at 24-h elapsed time was about 15 percent of the TVOC concentration. By the end of these experiments, the SigmaVOC concentration was less than ten percent of the TVOC concentration. In Experiment C-3, the portion of the TVOC concentration accounted for by the target compounds was even lower.

The TVOC concentrations were, to a large extent, a measure of the complex mixture of unsaturated hydrocarbons emitted by the carpet cushion. The TVOC concentrations in Experiments C-1 and C-3 stayed relatively constant with a slow decrease over the course of the experiments as shown in Figure 34. In Experiment C-2, there was about a factor of two reduction in the TVOC concentration during the 72-h period of additional ventilation in comparison with the corresponding TVOC concentration in Experiment $\mathrm{C}-1$. The concentrations of TVOC during Experiment $\mathrm{C}-3$ with aired-out materials were lower than they were in the other two experiments by roughly a factor of two.

\subsection{EMISSION RATES OF THE TARGET COMPOUNDS}

Quasi steady-state emission rates of the 13 target VOCs and TVOC in the three experiments were calculated with the mass-balance equation (Equation 1). The surface area of the carpet of $10.4 \mathrm{~m}^{2}$ was used in all of the calculations. The concentrations of the target compounds generally did not change as rapidly during the first hours of the experiments as they did in the experiments with the paint combination. Therefore, the amount of uncertainty in the estimated emission rates for the beginning periods with the carpet assembly due to the use of the steady-state model was expected to be lower.

The area-specific emission rates of the target VOCs in micrograms of compound emitted per square meter of surface per hour $\left(\mu \mathrm{g} \mathrm{m}^{-2} \mathrm{~h}^{-1}\right)$ for the three experiments are presented in Appendix I, Tables 1-14-1-19. The area-specific emission rates of TVOC for the experiments are presented in Appendix I, Table 1-20. No calculations were performed for SigmaVOC since emission rates were individually determined for the most abundant compounds. Emission rates could not be calculated for formaldehyde and acetaldehyde since the carpet assembly was not a measurable source of these compounds.

The emission rates of styrene, 4-PCH, DPGME, BHT and TVOC in the three experiments are individually compared in Figures 35 - 39, respectively.

The additional ventilation used during the first 72-h of Experiment $\mathrm{C}-2$ resulted in a clear increase in the emission rates of many of the target VOCs and of TVOC during this period in comparison to the respective emission rates in Experiment $\mathrm{C}-1$. The effect was relatively small for styrene and DPGME that had rapidly decaying concentrations (Figures 35 and 37). The least volatile compounds, such as 4-PCH (Figure 36), BHT (Figure 38), 2,2'-azobisisobutryonitrile and 2-methyleneglutaronitrile, appeared to be the most affected.

It has been hypothesized that the emissions of VOCs originating from the SBR latex on the secondary backing of carpets, such as 4-PCH, are controlled by the physical process of diffusion of the compounds in the backing (Little et al., 1994). If diffusion was the dominant process affecting the compartment air concentrations of 4- $\mathrm{PCH}$, then the difference in the ventilation rate between the experiments should have had very little effect on its emission rate. The results, therefore, suggest that either mass transfer effects at the surfaces of the carpet are more important than previously hypothesized or that other phenomena, such as desorption from fiber and backing surfaces, played a significant role in determining the chamber concentrations of 4-PCH and possibly other compounds.

A comparison of the emission rates of the target VOCs and TVOC between Experiments $\mathrm{C}-1$ and $\mathrm{C}-3$ showed that the 48-h airing out period decreased the emission rates of all of these 
constituents. For some compounds, airing out substantially decreased emission rates. This is illustrated by the temporal profiles of the styrene and DPGME emission rates for these two experiments. The reduction of the emission rate of 2,2'-azobisisobutyronitrile due to airing out was dramatic. On the other hand, the effect was small and possibly insignificant for 4-PCH and $\mathrm{BHT}$. The reduction in the emission rate of TVOC in Experiment $\mathrm{C}-3$ relative to the emission rates of TVOC in the other two experiments was approximately a factor of two (Figure 39). As discussed above, this was primarily due to the reduced emissions of the complex mixture of unsaturated hydrocarbons from the carpet cushion.

\subsection{CUMULATIVE MASSES AND EXPOSURES FOR THE TARGET COMPOUNDS}

The results for the experiments with the carpet assembly were first summarized by calculating the cumulative masses of the target VOCs and TVOC that were emitted over the experimental period (Appendix I, Table I-21). The compounds with the highest mass emissions were 4-PCH, DPGME, BHT and 2,2'-azobisisobutyronitrile. The relative magnitudes of the effects of the treatments on the cumulative mass emissions of the target VOCs are illustrated in Figure 40. For this figure, the results for base-case Experiment C-1 were used to calculate the ratios for the two treatment experiments. These results clearly show that the treatments had substantial effects on the cumulative mass emissions. With the exceptions of 1,2-dichlorobenzene, $\mathrm{N}, \mathrm{N}$-dimethylbenzylamine, and 2,2'-azobisisobutryonitrile, the cumulative emissions were higher in Experiment $\mathrm{C}-2$ with additional ventilation compared to Experiment $\mathrm{C}-1$ conducted at the basecase ventilation rate. As previously discussed, this was the result of higher emission rates during the 72-h period of additional ventilation. For all of the target VOCs and TVOC, the cumulative mass emissions were lower for Experiment $\mathrm{C}-3$ in which the materials were aired out than in base-case Experiment $\mathrm{C}-1$ conducted at the same ventilation rate. This was most likely due to reductions during the airing out period in the masses of the compounds contained in the materials or sorbed onto their surfaces.

Next, the experiments and the treatments were compared based on theoretical cumulative exposures in $\mathrm{ppb}$-h for a residential occupant that were calculated for two different exposure periods.

The estimated cumulative exposures to the target VOCs for the first 48 hours of the three experiments are presented in Appendix I, Table 1-22. For this calculation, occupancy began at time zero, the conclusion of carpet installation, and the occupancy factor was assumed to be 0.83 (i.e., an occupancy of 20 hours per day averaged throughout the day). The exposures were generally low and only exceeded $100 \mathrm{ppb}-\mathrm{h}$ for DPGME, N,N-dimethylacetamide and 2,2'-azobisisobutyronitrile in Experiment $\mathrm{C}-1$.

The relative magnitudes of the effects of the treatments on the cumulative exposures to the target VOCs over $0-48 \mathrm{~h}$ are illustrated in Figure 41. Additional ventilation resulted in reduced exposures relative to the base-case experiment for all compounds except BHT. For most compounds, the fractional reduction was substantially less than the approximate four-fold difference in ventilation rate. The figure also shows that airing out of the materials more effectively reduced exposures for this period than additional ventilation. The exposures for styrene, 4-ethenylcyclohexene, 1,2-dichlorobenzene, DPGME, N,N-dimethylbenzylamine, 2,2'azobisisobutyronitrile and 2-methyleneglutaronitrile in Experiment C-3 were all less than 30 percent of their respective exposures in Experiment C-1.

The estimated cumulative exposures to the target VOCs for the remainders of the experiments are presented in Appendix I, Table I-23. For this calculation, occupancy began exactly $48 \mathrm{~h}$ after installing the materials. It was assumed that the daily duration of occupancy was $20 \mathrm{~h}$. In general, the exposures were low. The compounds with the highest exposures for this period were 4-PCH, DPGME, BHT and 2,2'-azobisisobutyronitrile.

The relative magnitudes of the effects of the treatments on the cumulative exposures to the target VOCs over $48-336 \mathrm{~h}$ are illustrated in Figure 42. The results for base-case Experiment $\mathrm{C}-1$ were used to calculate the ratios for the two treatment experiments. The exposure to BHT was higher in Experiment $\mathrm{C}$-2 while the exposures to 1,2-dichlorobenzen: 
$\mathrm{N}, \mathrm{N}$-dimethylbenzylamine and 2,2'-azobisisobutryonitrile were lower in Experiment $\mathrm{C}$-2 relative to Experiment C-1. For the remaining compounds, the exposure differences between Experiments $\mathrm{C}-1$ and $\mathrm{C}-2$ were minor. With the exceptions of 4-PCH and $\mathrm{BHT}$, the calculated exposures to the target VOCs were lower in Experiment C-3 relative to both Experiments C-1 and C-2. The exposures to 4-PCH were similar for all three experiments while the exposures to BHT were about the same for Experiments $\mathrm{C}-1$ and $\mathrm{C}-3$ and highest in Experiment $\mathrm{C}-2$.

Figure 43 compares the magnitudes of the cumulative exposures estimated for the 0 - to 48-h periods to the total cumulative exposures over $0-336 \mathrm{~h}$ that were calculated as the sums of the exposures for the two periods. For 4-ethenylcyclohexene, one of the most volatile compounds, the exposures during the first $48 \mathrm{~h}$ were near 50 percent of the total exposures. For 4-PCH and $\mathrm{BHT}$, the least volatile compounds, the exposures during the first $48 \mathrm{~h}$ were about 15 percent or less of the total exposures.

\subsection{COMPARISON WITH THE SCREENING MEASUREMENT RESULTS}

Screening measurements were individually conducted for carpet assembly materials CP4 and $\mathrm{CC} 4$. Emission rates of the target VOCs and TVOC in the small chambers were calculated for elapsed times of 24 and $48 \mathrm{~h}$ (Appendix D, Tables D-36, D-41 and D-43). The differences between the 48-h emission rates $\left(\mu \mathrm{g} \mathrm{m}^{-2} \mathrm{~h}^{-1}\right)$ of the target VOCs and TVOC in the screening measurements of $\mathrm{CP} 4$ and $\mathrm{CC} 4$ and in large scale Experiments $\mathrm{C}-1$ and $\mathrm{C}-2$ are compared in Table 12. For styrene, 4-PCH, 4-ethenylcyclohexene, 1,2-dichlorobenzene, and $2,2^{\prime}$-azobisisobutryonitrile, the screening measurement and the large-scale experiment results generally agreed within a factor of two. The 48-h emission rates of the remaining compounds, with the exception of 1-decanol, were higher in the screening measurements. In particular, the emission rate of BHT was roughly a factor of 20 higher in the screening measurement of CC4.

It is expected that covering the cushion with the carpet will retard the emission rates of many of the compounds that originate from the cushion. A substantial reduction in the emission rates of BHT and other VOCs from urethane carpet cushions in the presence of a carpet overlay was previously demonstrated in small-scale chamber experiments (Schaeffer et al., 1996). For $\mathrm{BHT}$, there was a 90 percent reduction over a $96-\mathrm{h}$ period. It is likely that the lower emission rates of BHT in the large-scale experiments relative to the screening measurement of CC4 was due, at least in part, to the carpet overlay.

\subsection{EXPOSURE REDUCTION EXPERIMENTS WITH THE VINYL FLOORING ASSEMBLY}

\subsection{EXPERIMENTAL TREATMENTS}

Four large-scale experiments were conducted with the vinyl flooring assembly consisting of materials SV5, CB, SFA, CBA and UL. Two of these experiments, V-1 and V-4, were conducted at the base-case ventilation rate condition. An operator error occurred during Experiment V-1 at about 168-h elapsed time that invalidated the data from $168 \mathrm{~h}$ through the remainder of the experiment. Therefore, the two base-case experiments can only be compared through 144-h elapsed time. One of the treatment experiments, $\mathrm{V}-2$, was conducted using additional ventilation for the first $72 \mathrm{~h}$. The ventilation rate during flooring installation and for the following two hours was $5 h^{-1}$. From $2-72 \mathrm{~h}$, the ventilation rate was $2 \mathrm{~h}^{-1}$. For the other treatment experiment, $\mathrm{V}-3$, the sheet vinyl flooring material and the rubber cove base were aired out in a highly ventilated room for $48 \mathrm{~h}$ immediately prior to their installation as described in detail in Appendix G. This experiment was conducted at the base-case ventilation rate conditions. The ventilation rate data and the compartment temperatures and relative humdities for the experiments are summarized in Appendix $\mathrm{J}$, Table $\mathrm{J}-01$. The ventilation rates, temperatures and relative humidities were maintained within the established renge limits. 
The material quantities are presented in Appendix J, Table J-02. The same amounts of Sheet Vinyl SV5, Cove Base CB and liquid Seam Sealer SS were used in all four experiments. The masses of the adhesives were determined by weighing the adhesive containers and tools immediately before and after the installations. The average mass of Sheet Flooring Adhesive SFA used in the experiments was approximately $3 \mathrm{~kg}$. The highest mass of $3.4 \mathrm{~kg}$ was used in Experiment V-4, and the lowest mass of $2.7 \mathrm{~kg}$ was used in Experiment $\mathrm{V}-1$. The average mass of Cove Base Adhesive CBA applied in the experiments was approximately $0.5 \mathrm{~kg}$. Variations among the experiments in the use of this product were small.

Table 12. Area-specific emission rates $\left(\mu \mathrm{g} \mathrm{m}^{-2} \mathrm{~h}^{-1}\right)$ of target VOCs and TVOC at 48-h elapsed time in the screening measurements of carpet assembly materials CP4 and CC4 and in large-scale Experiments $\mathrm{C}-1$ and $\mathrm{C}-2$.

\begin{tabular}{|c|c|c|c|c|}
\hline \multirow[b]{3}{*}{ Component } & \multicolumn{4}{|c|}{ Specific Emission Rate, $\mu \mathrm{g} \mathrm{m}^{-2} \mathrm{~h}^{-1}$} \\
\hline & \multicolumn{2}{|c|}{ Screening Measurements } & \multicolumn{2}{|c|}{ Large-Scale Expts. } \\
\hline & $\mathrm{CP} 4$ & $\mathrm{CC} 4$ & $\mathrm{C}-1$ & $\mathrm{C}-2$ \\
\hline Styrene & 4 & --- & 4 & 6 \\
\hline 4-PCH & 33 & --- & 16 & 31 \\
\hline 4-Ethenylcyclohexene & 7 & --- & 7 & 7 \\
\hline 1,2-Dichlorobenzene & --- & 3 & 5 & 4 \\
\hline DPGME & 39 & --- & 14 & 17 \\
\hline Phenol & 6 & 15 & 3 & 4 \\
\hline Phenethyl alcohol & 6 & --- & 1 & 3 \\
\hline 1-Decanol & 2 & $\cdots$ & 3 & 10 \\
\hline $\mathrm{BHT}$ & & 206 & 3 & 13 \\
\hline N,N-Dimethylacetamide & 26 & --- & $<12$ & $<49$ \\
\hline N,N-Dimethylbenzylamine & --- & 43 & 9 & 3 \\
\hline 2,2'-Azobisisobutryonitrile & --- & 94 & 55 & 69 \\
\hline 2-Methyleneglutaronitrile & 40 & --- & 7 & 16 \\
\hline TVOC & 873 & 2,940 & 834 & 1,280 \\
\hline
\end{tabular}

\subsection{SELECTION OF THE TARGET COMPOUNDS}

A qualitative analysis of the VOCs emitted by the vinyl flooring assembly in base-case Experiment $\mathrm{V}-4$ identified a relatively large number of compounds (Appendix J, Table J-03). Approximately 80 percent of the compounds were hydrocarbons. These included normal and branched alkane hydrccarbons, aromatic hydrocarbons, and cyclic or alkene (olefinic)

hydrocarbons. A number of the hydrocarbons were not fully identified. There was a related group 
of $\mathrm{C}_{10}-\mathrm{C}_{12}$ alkyl substituted benzenes that were characteristic of the emissions from SV5 (Appendix E, Table E-06). The most abundant hydrocarbons in the samples based on their TIC area responses were $n$-decane, $n$-tridecane, $n$-tetradecane, and toluene.

Carbonyl compounds, other oxidized compounds and several miscellaneously categorized compounds were additionally identified in the emissions from the vinyl flooring assembly. Some of these were present in the compartment background. Two of the most abundant oxidized compounds, cyclohexanone and tetrahydrofuran, were the principal solvent components of Seam Sealer SS (Appendix E, Table E-10). The other most abundant compounds were phenol, 2,2,4-trimethyl-1,3-pentanediol diisobutyrate (TXIBß) and benzothiazole. Phenol and TXIB尺 were emitted by SV5, and benzothiazole was emitted by Cove Base CB (Appendix E, Table E-11).

Nineteen target compounds were selected for quantitative analysis. These compounds are listed in Appendix $\mathrm{J}$, Table $\mathrm{J}-04$ along with their predominant sources. The target compounds included formaldehyde and acetaldehyde that had no dominant source and 17 VOCs that were either characteristic of the individual sources and/or were among the most abundant compounds emitted by the assembly. The 17 target VOCs were: $n$-decane; $n$-dodecane; $n$-tridecane; $\mathrm{n}$-tetradecane; toluene; combined $\mathrm{m}$-, $\mathrm{p}$-xylene isomers; styrene; 1,2,4-trimethylbenzene; cyclohexanone; benzaldehyde; tetrahydrofuran; 1-octanol; benzyl alcohol; phenol; TXIBQ; tert-butylisothiocyanate; and benzothiazole. Formaldehyde, acetaldehyde, toluene, $m$-, $p$-xylenes, styrene and phenol are identified by the ARB as TACs; 1,2,4-trimethylbenzene is being evaluated for classification as a TAC.

\subsection{CONCENTRATIONS OF THE TARGET COMPOUNDS}

The concentrations of the 17 target VOCs in the chamber compartment and supply air samples for the four experiments with the vinyl flooring assembly are presented in Appendix $\mathrm{J}$, Tables J-05 - J-12. The concentrations for the duplicate VOC samples that were collected at several time points during these experiments are given in the tables. The concentrations of toluene and tetrahydrofuran in inlet air were 1 and $4-12 \mu \mathrm{g} \mathrm{m}^{-3}$, respectively. The inlet air concentrations of the other target VOCs were all below their lower limits of quantitation. Some of the target VOCs were detected in the compartment background. The background concentrations of these compounds were typically at or near their lower limits of quantitation. The compartment concentrations and average inlet air concentrations of TVOC for the four experiments are presented in Appendix $\mathrm{J}$, Table $\mathrm{J}-13$. The compartment concentrations and average inlet air concentrations of SigmaVOC (i.e., the sum of the concentrations of the 17 target VOCs) are presented in Appendix $\mathrm{J}$, Table $\mathrm{J}-14$. The formaldehyde and acetaldehyde concentrations for the four experiments are presented in Appendix $\mathrm{J}$, Tables $\mathrm{J}-15$ and $\mathrm{J}-16$, respectively.

The concentrations of $n$-tridecane, toluene, tetrahydrofuran, phenol, TXIB®, benzothiazole and TVOC in the four experiments are individually compared in Figures $44-50$, respectively.

With the exception of toluene, there was generally good agreement between base-case Experiments V-1 and V-4 with respect to the concentrations of the target VOCs. For toluene, the concentrations were substantially higher in Experiment V-1 than they were in Experiment V-4. This difference was strongly apparent for the first 24-h period (Figure 45a). Toluene was the dominant compound emitted by Sheet Flooring Adhesive SFA (Appendix E, Table E-14). During Experiment $\mathrm{V}-1$, which was the first large-scale use of this product, a small, but unmeasured, amount of adhesive was spilled on the surface of the vinyl. This adhesive could not easily be removed by wiping, and a portion was left on the surface. It is likely that the spilled adhesive was the source of the higher toluene concentrations measured during this experiment. The difference between the toluene concentrations in Experiments V-1 and V-4 is also reflected in the TVOC concentrations for these experiments (Figure 50).

The two compounds with the highest initial concentrations in all four experiments were cyclohexanone and tetrahydrofuran (Figure 46). The initial concentrations of cyclohexanone anci tetrahydrofuran were as high as 3,800 and $7,800 \mu \mathrm{g} \mathrm{m}^{-3}$, respectively. As noted above, Seum 
Sealer SS emitted these compounds. Since $15 \mathrm{~mL}$ of seam sealer were applied to the sheet vinyl in each experiment, it was estimated from the solvent composition of the product that approximately $1.1 \mathrm{~g}$ of cyclohexanone and $8.5 \mathrm{~g}$ of tetrahydrofuran were introduced into the compartment. This would account for the high initial concentrations following the application of the seam sealer. These concentrations decreased rapidly over the first $24 \mathrm{~h}$ of the experiments. By the end of the experimental periods, the concentrations of cyclohexanone and tetrahydrofuran were in the range of $9-17 \mu \mathrm{g} \mathrm{m}^{-3}$.

The temporal behavior of the other target VOCs in the four experiments was substantially different than the behaviors of toluene, cyclohexanone and tetrahydrofuran. The initial concentrations of these other compounds were not has high, and generally their concentrations decreased slowly with time. The concentrations of several of the less volatile compounds, such as n-tridecane and benzothiazole, stayed relatively constant over long time periods. For the least volatile compound, TXIB®, the compartment concentrations slowly increased with time reaching maximum values later in the experiments.

The effects of the treatments varied among the compounds. For $n$-tridecane and toluene (Figures 44 and $45 \mathrm{~b}$ ), the compartment concentrations for Experiment V-3 with airing out were similar to the concentrations for base-case Experiment V-4. The concentrations of these compounds were lower during the period of additional ventilation in Experiment V-2. Additional ventilation also resulted in substantially reduced concentrations of tetrahydrofuran in the first hours of Experiment V-3 (Figure 46). The treatments produced different results for phenol and benzothiazole (Figures 47 and 49). Additional ventilation had no obvious effect on the concentrations of these compounds. However, airing out of the materials appeared to reduce their concentrations over the first several days of Experiment V-3 relative to Experiment $V-4$.

The high concentrations of TVOC during the first $24 \mathrm{~h}$ of the experiments were primarily the result of the high initial concentrations of cyclohexanone and tetrahydrofuran. From $48 \mathrm{~h}$ through the remainder of the experiments, the concentrations of TVOC stayed relatively constant. In fact, the concentrations of TVOC over this period in Experiments V-4, V-2 and V-3 were all near $1,000 \mu \mathrm{g} \mathrm{m}^{-3}$. Thus, the chamber concentrations of TVOC were not substantially affected by the treatments.

Again, the high concentrations of SigmaVOC during the first $24 \mathrm{~h}$ of the experiments were due to high concentrations of cyclohexanone and tetrahydrofuran. At 48-and 72-h elapsed time, the concentrations of SigmaVOC were lowest for Experiment $V-2$ that was run with additional ventilation. However, after the ventilation rate in this experiment was reduced to $0.5 \mathrm{~h}^{-1}$, the SigmaVOC concentrations increased to values that were nearly equivalent to the values for Experiments V-4 and V-3.

A comparison of the TVOC and SigmaVOC concentrations shows that in the initial six hours of the experiments, the SigmaVOC concentrations sometimes exceeded the TVOC concentrations. This anomaly was a consequence of the way in which the TVOC measurements were calibrated. TVOC was calibrated with a mixture of alkane and aromatic hydrocarbons. Oxidized compounds such as cyclohexanone and tetrahydrofuran have lower TIC responses than hydrocarbons. Therefore, the TVOC responses for the initial samples with high concentrations of these two compounds were underestimated. In the latter portions of the experiments, the SigmaVOC concentrations were roughly one third of the TVOC values.

Generally, the concentrations of formaldehyde and acetaldehyde during the experiments reached values that were only about a factor of two above their respective compartment background concentrations at the start of the experiments. Since the wood components of the chamber facility were a known source of formaldehyde and acetaldehyde, it was impossible to determine if the measured concentration increases were attributable to the installation of the vinyl flooring assembly.

\subsection{EMISSION RATES OF THE TARGET COMPOUNDS}

Quasi steady-state emission rates of the 19 target com......nnds and TVOC in the four experiments were calculated with the mass-balance equation (Eyuation 1). The surface area of 
the sheet vinyl of $10.4 \mathrm{~m}^{2}$ was used in all of the calculations. With the exceptions of cyclohexanone and tetrahydrofuran, the concentrations of the target compounds did not change rapidly with time. Therefore, the use of the steady-state model was unlikely to have introduced a large amount of uncertainty into the calculated emission rates of these compounds.

The area-specific emission rates of the target VOCs in micrograms of compound emitted per square meter of surface per hour $\left(\mu \mathrm{g} \mathrm{m}^{-2} \mathrm{~h}^{-1}\right)$ for the four experiments are presented in Appendix J, Tables $\mathrm{J}-17$ - J-24. The area-specific emission rates of TVOC for the experiments are presented in Appendix J, Table J-25. No calculations were performed for SigmaVOC. The area-specific emission rates of formaldehyde and acetaldehyde are presented in Appendix $\mathrm{J}$, Tables J-26 and J-27, respectively.

The specific emission rates of $n$-tridecane, toluene, tetrahydrofuran, phenol, TXIB@, benzothiazole and TVOC in the four experiments are individually compared in Figures 51 - 56; respectively.

With the exception of toluene, the specific emission rates of the target VOCs for basecase Experiments V-1 and V-4 were in generally good agreement. As noted above, the elevated emission rate of toluene at the beginning of Experiment V-1 was probably due to the residue of sheet flooring adhesive left on the surface of the vinyl. The difference between the emission rates of TVOC over the first two days of these experiments largely reflects the elevated emission rate of toluene in Experiment $\mathrm{V}$-1.

The additional ventilation used in the first $72-h$ of Experiment $V-2$ resulted in a general increase in the specific emission rates of most of the target VOCs over this period in comparison to the respective emission rates in base-case Experiment V-4. For compounds such as phenol, $\mathrm{TXIB} \otimes$ and benzothiazole, there was more than a factor of two increase in their apparent emission rates with increased ventilation. This trend, however, was frequently not apparent during the first hour. Most likely, the high ventilation rate of $5 \mathrm{~h}^{-1}$ used during the installation of the vinyl flooring assembly in Experiment V-2 effectively reduced the VOC concentrations resulting from the introduction, unrolling and active handling or use of the materials. After the ventilation rate in Experiment $V$-2 was reduced to $0.5 \mathrm{~h}^{-1}$ at 72 -h elapsed time, the emission rates of the target VOCs decreased to levels that were generally comparable to the levels in Experiment $\mathrm{V}-4$.

The same trend is apparent from a comparison of the specific emission rates of TVOC for Experiments V-4 and V-2 (Figures 56a and 56b). From $24-72 \mathrm{~h}$, the emission rate of TVOC was higher by a factor of two or more in Experiment V-2.

The data presented by Clausen et al. (1993) for small-scale chamber tests of a vinyl flooring material conducted over 600 hours shows that the initial emission rate of phenol was higher by about a factor of two in the experiment conducted at a ventilation rate of $0.25 \mathrm{~h}^{-1}$ relative to the experiment conducted at $0.12 \mathrm{~h}^{-1}$. Later in their experiments, the emission rates of phenol at the two ventilation rates were much more comparable.

The effect of airing out of the sheet vinyl and cove base was evaluated by comparing the emission rates of the target VOCs and TVOC for Experiments V-4 and V-3. Both of these experiments were conducted at the same base-case ventilation rate conditions. The specific emission rates of the target VOCs were generally comparable between the two experiments. In particular, the emission rates of the dominant compounds originating from the sheet vinyl and the cove base, such as benzyl alcohol, phenol, TXIB@ and benzothiazole, were quite similar over the course of these two experiments. The only substantial difference between the experiments was that the emission rates of many of the compounds were lower during the first few hours of Experiment $V-3$. This suggests that the airing out of the materials had only a short-term effect on VOC emission rates. The similarity between these two experiments was also apparent in the emission rates of TVOC. Again, the only notable difference was the lower TVOC emission rates in the first few hours of Experiment $\mathrm{V}-3$. 


\subsection{CUMULATIVE MASSES AND EXPOSURES FOR THE TARGET COMPOUNDS}

The results for the experiments with the vinyl flooring assembly were first summarized by calculating the cumulative masses of the target VOCs and TVOC that were emitted over the experimental period (Appendix J, Table J-28). There were lower cumulative emissions for most of the VOCs in Experiment $V-1$ because the values could only calculated over the first $144 \mathrm{~h}$ of the experiment. $\mathrm{n}$-Tridecane, toluene, cyclohexanone, tetrahydrofuran and phenol generally had the highest mass emissions.

The relative magnitudes of the effects of the treatments on the cumulative mass emissions of the target VOCs are illustrated in Figure 57. The results for the complete base-case Experiment V-4 were used to calculate the ratios for the two treatment experiments. The figure shows that the additional ventilation at the beginning of Experiment $V-2$ resulted in increased emissions for a number of compounds relative to Experiment $V-4$. The effect was most apparent for benzaldehyde, 1-octanol, benzyl alcohol, phenol, TXIB® and benzothiazole. The cumulative emissions of cyclohexanone and tetrahydrofuran were shown to be lower in Experiment V-2 with additional ventilation relative to Experiment $V-4$. It is probable that this was an artifact resulting from the timing of sample collection with respect to the time of the appearance of the maximum compartment concentrations of these two compounds. The cumulative masses of the target compounds in Experiment V-3 were similar to, or somewhat lower than their respective masses in base-case Experiment V-4. The largest decreases in mass emissions due to airing out the materials occurred for phenol emitted by the sheet vinyl and benzothiazole emitted by the cove base. These decreases were approximately 25 percent. Thus, the airing-out treatment had only a limited and relatively minor effect on mass emissions.

Next, the experiments and the treatments were compared based on theoretical cumulative exposures in $\mathrm{ppb}-\mathrm{h}$ for a residential occupant that were calculated for two different exposure periods.

The estimated cumulative exposures to the target compounds for the first 48 hours of the four experiments are presented in Appendix J, Table J-29. For this calculation, occupancy began at time zero, the conclusion of the vinyl floor installation, and the occupancy factor was assumed to be 0.83 (i.e., an occupancy of 20 hours per day averaged throughout the day). In general, cyclohexanone and tetrahydrofuran produced the highest exposures during this period. The exposure to toluene in Experiment $\mathrm{V}$-1 was also high.

The relative magnitudes of the effects of the treatments on the cumulative exposures to the target VOCs over $0-48 \mathrm{~h}$ are illustrated in Figure 58. With the exception of TXIBQ, the exposures for the target VOCs in Experiment V-2 with additional ventilation were lower than the exposures in base-case Experiments V- 1 and V-4. For a number of the compounds, the reductions were 50 percent or more, while the exposures for benzyl alcohol, phenol and benzothiazole were reduced by less than 20 percent. The exposure for TXIB® was unaffected by increased ventilation. Airing out of the materials in Experiment V-3 reduced the exposures during this initial period for all of the VOCs emitted by the materials. These reductions were typically about 25 percent. As expected, the exposure to tetrahydrofuran emitted by the seam sealer was unaffected.

The estimated cumulative exposures to the target compounds for the remainders of the experiments are presented in Appendix $\mathrm{J}$, Table $\mathrm{J}-30$. For this calculation, occupancy began exactly $48 \mathrm{~h}$ after installing the materials. It was assumed that the daily duration of occupancy was $20 \mathrm{~h}$. In general, the treatments had negligible, or only minor effects, on the exposures for this period.

The relative magnitudes of the treatment effects on the cumulative exposures to the target VOCs over $48-336 \mathrm{~h}$ are illustrated in Figure 59. The results for base-case Experiment $\mathrm{V}-4$ were used to calculate the ratios for the two treatment experiments. Additional ventilation in Experiment V-2 decreased the exposures to toluene, tetrahydrofuran and phenol by about 25 percent or less. Airing out the materials decreased the exposures to toluene, styrene, phenol and benzothiazole, again by about 25 percent or less. The exposures for the other compounds over this period were relatively unaffected by either of the two treatments. 
Figure 60 compares the magnitudes of the cumulative exposures estimated for the 0 - to 48-h periods to the total cumulative exposures over $0-336 \mathrm{~h}$ that were calculated as the sums of the exposures for the two periods. For cyclohexanone and tetrahydrofuran emitted by the seam sealer, the exposures during the first $48 \mathrm{~h}$ were about 50 percent or more of the total exposures. For the other target VOCs, the exposures over the first $48 \mathrm{~h}$ were about 20 percent or less of the total exposures.

\subsection{COMPARISON WITH SCREENING MEASUREMENT RESULTS}

Screening measurements were individually conducted for materials Sheet Vinyl SV5 and Cove Base CB. In addition, two composite assemblies were tested in the small-scale chambers. These assemblies were: SV5, Sheet Flooring Adhesive SFA and Underlayment UL; and CB, Cove Base Adhesive CBA and Gypsum Board GB. The emission rates of the target VOCs from these materials and assemblies in the screening measurements were calculated for elapsed times of 24 and $48 \mathrm{~h}$ (Appendix E, Tables E-40, E-42, E-46 and E-47). The differences between the 48-h area-specific emission rates $\left(\mu \mathrm{g} \mathrm{m}^{-2} \mathrm{~h}^{-1}\right)$ of the target VOCs in the screening measurements and in the large-scale experiments are compared in Table 13. The specific emission rates for the cove base and cove base assemblies were reported in Appendix $E$ on a length basis $\left(\mu \mathrm{g} \mathrm{m}^{-1} \mathrm{~h}^{-1}\right)$. For the comparison with the large-scale results, these values were multiplied by a factor of 1.2 since there were $1.2 \mathrm{~m}$ of CB per square meter of SV5 in Experiments $\mathrm{V}-1-\mathrm{V}-4$

For most of the compounds, the 48-h emission rates for the screening measurements were higher than the average values for Experiments $V-1-V-4$. However, for the majority of the compounds (n-decane, n-tridecane, n-tetradecane, m-,p-xylene, styrene, 1,2,4-trimethylbenzene, 1-octanol, benzyl alcohol, phenol, TXIB尺, and tert-butylisothiocyanate) the screening measurement values fell within the range or narrowly above of the range of the values measured in the large-scale experiments. The screening measurement values for benzothiazole emitted by the cove base were lower than the average value but were within the range of values measured for Experiments V-1 - V-4.

\subsection{EXPOSURE REDUCTION AND LONG-TERM EXPERIMENTS WITH THE COMBINED ASSEMBLIES}

\subsection{EXPERIMENTAL DESIGN}

Two large-scale experiments were conducted with a combination of all three source assemblies (paint combination, carpet assembly and vinyl flooring assembly). For these experiments, painting was conducted prior to the installation of the flooring materials. The combination of latex paints LPS2, FLP3 and SGLP3 was applied as previously described. The only difference from Experiments P-1 - P-4 was that the floor of the compartment was left bare (i.e., aluminum sheeting). The ventilation rate during painting and for the following two hours was $5 \mathrm{~h}^{-1}$. Then, the ventilation rate was reduced to $2 \mathrm{~h}^{-1}$ and maintained at this condition for the remainder of the three-day (72-h) drying period. This drying period was designed to allow the initial concentrations of the major compounds emitted by the paints to decay down to lower levels so that a broad range of VOCs could be more practically sampled and analyzed. It also simulated a common situation in both new construction and renovation or remodeling projects in which rooms are painted before the installation of the finish flooring materials. Air samples were collected three hours prior to the end of the 72-h drying period to establish the compartment background and the initial concentrations of target compounds emitted by the paint combination. 
Table 13. Area-specific emission rates $\left(\mu \mathrm{g} \mathrm{m}^{-2} \mathrm{~h}^{-1}\right)$ of target VOCs and TVOC at $48-\mathrm{h}$ elapsed time in the screening measurements of individual and assembled vinyl flooring materials and in large-scale Experiments $V 1-\mathrm{V}-4$.

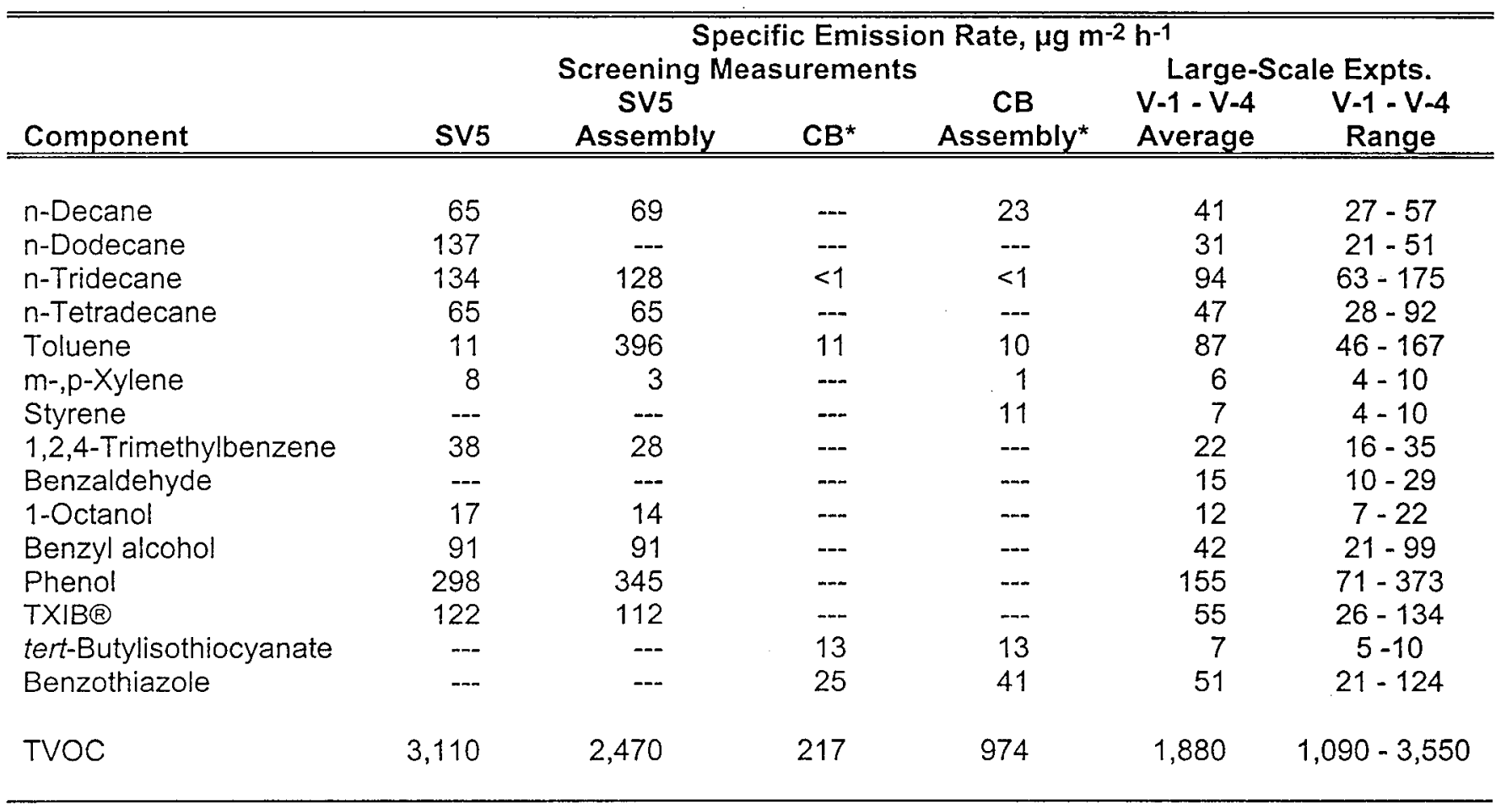

*Values were multiplied by 1.2 since there were $1.2 \mathrm{~m}$ of $\mathrm{CB}$ per $\mathrm{m}^{2}$ of sheet vinyl in Expts. $\mathrm{V}-1-\mathrm{V}-4$. 
The carpet and vinyl flooring assemblies were installed next following the previously established procedures. First, the vinyl flooring assembly consisting of materials SV5, SFA, CB, CBA, SS and UL was installed at one end of the compartment to cover one-third of the floor. Then, the remainder of the floor was carpeted with materials $\mathrm{CP} 4, \mathrm{CC} 4$ and ST. The ventilation rate was maintained at $2 \mathrm{~h}^{-1}$ during flooring installation and for the next 72 hours. Air sampling, following the previously established regime for the exposure reduction experiments, commenced one hour after the flooring installation was completed.

The compartment was actively heated from $6-72$ hours following flooring installation in treatment Experiment $\mathrm{A}-1$. The average temperature during this period was about $33^{\circ} \mathrm{C}$, a $10^{\circ} \mathrm{C}$ increase over the base-case compartment temperature. The compartment was fully insulated, and temperature stratification in the compartment was no more than $\pm 2^{\circ} \mathrm{C}$ from the average temperature measured at the center of the compartment. The ventilation rate was maintained at $2 \mathrm{~h}^{-1}$ during the heating period to provide adequate air flushing as is recommended for building "bake-out" (Alevantis, 1996). The relative humidity of the inlet air was maintained near 40 percent at typical room temperature. This resulted in an average relative humidity of 28 percent in the compartment during heating. At the end of the heating period when the heater was turned off, the ventilation rate was lowered to the base-case rate of $0.5 \mathrm{~h}^{-1}$. The compartment temperatures and relative humidities and the ventilation rate data for Experiment $A-1$ are summarized by period in Appendix K, Table K-01. Additional air samples were collected during this experiment at 12-and 80-h elapsed timie to capture the periods of increasing and decreasing compartment temperature.

The reference experiment, $\mathrm{A}-2$, was conducted identically to the treatment experiment except that there was no heating. This experiment was additionally maintained for a total period of 12 weeks $(2,016$ hours) with samples collected weekly from $2-12$ weeks. The primary purpose for extending this experiment was to provide data on the longer-term changes in the emission rates of the target compounds since this information was largely lacking in the literature. The ventilation rate data and the compartment temperatures and relative humidities for the first phase of Experiment A-2 are summarized in Table K-01. (These parameters are summarized on a weekly basis in Appendix K, Table K-19, for the second phase of the experiment.) The ventilation rates, temperatures and relative humidities were maintained within the established range limits.

The quantities of the materials used in Experiments $A-1$ and $A-2$ are given in Appendix $K$, Table K-02. The amounts of paints LPS2 and FLP3 used in Experiment A-2 were slightly higher than the amounts used in Experiment $A-1$ and in the previous experiments $\mathrm{C}-1-\mathrm{C}-4$. The vinyl flooring assembly was installed to cover $3.48 \mathrm{~m}^{2}$ of floor area. The carpet assembly covered the remaining $6.97 \mathrm{~m}^{2}$ of floor area.

\subsection{SELECTION OF THE TARGET COMPOUNDS}

The results of the qualitative analysis of the VOCs emitted by the combined assemblies in Experiment A-2 are presented in Appendix K, Table K-03. Approximately two-thirds of the identified compounds were hydrocarbons. These included normal and branched alkane hydrocarbons, aromatic hydrocarbons, and cyclic or alkene (olefinic) hydrocarbons. The most abundant individual hydrocarbons based on their TIC area responses were n-decane and $\mathrm{n}$-tridecane emitted by the sheet vinyl flooring.

Carbonyl compounds, other oxidized compounds, nitrogen-containing compounds and a nitrogen and sulfur-containing compound (benzothiazole) were additionally identified in the emissions from the combined assemblies. The aldehydes were present in the compartment background. The most abundant oxidized compounds were ethylene glycol and the isomers of 2,2,4-trimethyl-1,3-pentanediol monoisobutyrate (Texanol( ) emitted by the latex paints. The other abundant oxidized compounds were phenol and 2,2,4-trimethyl-1,3-pentanediol diisobutyrate (TXIB(B) emitted by the sheet vinyl flooring.

Twenty target compounds were selected for quantitative analysis. These compounds are listed in Appendix K, Table K-04 along with their predominant sources. The target compounds $i_{1}$ ivluded formaldehyde and acetaldehyde that had no dominant source and 18 VOCs that were 
either characteristic of the individual sources and/or were among the most abundant compounds emitted by these sources. All of these VOCs had been analyzed in the previous experiments with the individual source assemblies. The 18 target VOCs were: $n$-decane; n-tridecane; toluene; styrene; 1,2,4-trimethylbenzene; 4-phenylcyclohexene (4-PCH); cyclohexanone; tetrahydrofuran; ethylene glycol; propylene glycol; benzyl alcohol; phenol; 2-(2-butoxyethoxy)ethanol (DEGBE); Texanol$($; TXIB®; butylated hydroxytoluene (BHT); 2,2'-azobisisobutryonitrile; and benzothiazole. The source areas used to calculate the emission rates of the individual compounds, which are listed in Table K-04, were selected based on the dominant sources of the compounds.

\subsection{EFFECTS OF HEATING}

\subsubsection{Concentrations of the Target Compounds}

The concentrations of the 18 target VOCs in the chamber compartment and supply air samples for the first two-week period of the two experiments with the combined source assemblies are presented in Appendix K, Tables K-05-K-08. The compounds are grouped in these tables by their dominant source. The vinyl flooring assembly emitted all ten of the Group 1 compounds. The carpet assembly emitted the first four compounds of Group 2. The remaining four compounds were emitted by the paint combination. Approximately half of the samples for Experiment A-1 were collected and analyzed in duplicate, and all of the samples for Experiment A-2 were duplicated. The values presented in the tables are the averages for the duplicate samples. A detailed analysis of the precision for these compounds is presented in Appendix $L$. The compartment concentrations and average inlet air concentrations of SigmaVOCP for the paint-derived compounds (i.e., the sum of the concentrations of the four target VOCs for paint) in Experiments A-1 and A-2 are presented in Appendix K, Table K-09. The corresponding concentrations of TVOCR (i.e., TVOC calculated for the TIC response minus the areas of the dominant compounds emitted by the paints) are also presented in this table. The formaldehyde and acetaldehyde concentrations for the two experiments are presented in Appendix K, Table K-10.

The concentrations of n-tridecane, toluene, phenol, TXIBQ and benzothiazole emitted by the vinyl flooring assembly are presented in Figures 61 - 65, respectively; the concentrations of 4-PCH and BHT emitted by the carpet assembly are presented in Figures 66 and 67, respectively; the concentrations of ethylene glycol and Texanoß emitted by the paint combination are presented in Figures 68 and 69, respectively; and the concentrations of TVOC $C_{R}$ are presented in Figure 70.

The figures show that the compartment air concentrations of $n$-tridecane, phenol and TXIBQ were clearly affected by heating. For all of these compounds, there was a rapid increase in concentration coincident with the onset of heating. The peak measured concentrations occurred at 12- or 24-h elapsed time (i.e., 6 - 12 hours after initiation of heating). Subsequently, the concentrations declined. When the heat was furned off, the concentrations of the three compounds returned to approximately the same concentrations measured in reference Experiment A-2. Toluene behaved differently (Figure 62). There was very little difference between the concentrations measured in the two experiments. Initially, the concentrations of toluene in both experiments were elevated due to the application of Sheet Flooring Adhesive SFA that was its dominant source. During the heating period, toluene should not have been directly exposed to air. That may have accounted for its lack of response. The concentrations of benzothiazole were low throughout both experiments (Figure 65). There appeared to be a small and short-lived increase in concentration due to heating. Subsequent to heating, the concentrations of benzothiazole were lower by several $\mu \mathrm{g} \mathrm{m}^{-3}$ in Experiment $A-1$ than in reference Experiment A-2.

The concentrations of both 4-PCH emitted by CP4 and BHT emitted by CC4 increased with heating. Subsequent to heating and beginning at $96-\mathrm{h}$ elapsed time, the concentrations of both of these compounds in Experiment A-1 returned to levels that were nearly the same as those measured in reference Experiment A-2. As previously observed, the conceittration of BHT slowly increased with time with the compartment running a constant ventilation rate oi $0.5 \mathrm{n}^{-1}$. 
There were several interesting features to the temporal behavior of ethylene glycol emitted by the paint combination (Figure 68). Firstly, there was a substantial and rapid drop in concentration following the installation of the flooring materials. Three hours prior to the installation of these materials, the compartment concentrations in both experiments were about $600 \mu \mathrm{g} \mathrm{m}^{-3}$. This value is in excellent agreement with the concentrations measured at $72-\mathrm{h}$ elapsed time in Experiments P-3 and P-4 (approximately equivalent conditions and time). One hour after installation of the flooring materials, the compartment concentrations were about the same. Then, there was a sudden approximately two-fold decrease in concentration in both experiments. One possible explanation is that the flooring materials, possibly the carpet, acted as a significant sink for ethylene glycol. It is also notable that the concentration of ethylene glycol increased only slightly in response to heating. Finally, there was no apparent increase in the concentration of ethylene glycol in either experiment when the ventilation rate was decreased from $2 h^{-1}$ to $0.5 h^{-1}$.

The concentration of Texanol in Experiment A-1, on the other hand, did increase substantially in response to heating (Figure 69). By the end of the heating period at 72-h elapsed time, the concentrations of Texanoß were approximately equivalent in both experiments. Subsequently to the heating period, the concentrations of Texanok were consistently lower in Experiment A-1 than they were in reference Experiment A-2.

The TVOC $R$ concentration, which was largely a measure of the hydrocarbons emitted by both the carpet and vinyl flooring assemblies, also responded to heating (Figure 70). However, by 96- $h$ elapsed time, the TVOC ${ }_{R}$ concentrations in both experiments were approximately the same and remained equivalent throughout the remainder of the 336-h period.

A comparison of the TVOC $R$ and SigmaVOC $P$ concentrations in Table K-09 illustrates that the latex paint combination was the dominant source in these experiments in terms of total mass concentrations.

The concentrations of formaldehyde and acetaldehyde during these experiments with the combined sources were approximately equivalent to their respective compartment background concentrations at the start of the experiments. This demonstrates that these assemblies were not sources of formaldehyde or acetaldehyde over this time period.

\subsubsection{Emission Rates of the Target Compounds}

Quasi steady-state emission rates of the 18 target VOCs and TVOC $R$ in the two experiments were calculated with the mass-balance equation (Equation 1). The surface areas given in Table K-04 were used in the calculations. The area-specific emission rates $\left(\mu \mathrm{g} \mathrm{m}^{-2} \mathrm{~h}^{-1}\right)$ of the target VOCs are presented in Appendix K, Tables K-11 - K-14. The area-specific emission rates of TVOC $_{R}$ for the experiments are presented in Appendix K, Table K-15. Emission rates were not calculated for formaldehyde and acetaldehyde since the assemblies were not obvious sources of these compounds over the period in which samples were collected.

The specific emission rates of n-tridecane, toluene, phenol, TXIBA, benzothiazole, 4- $\mathrm{PCH}, \mathrm{BHT}$, ethylene glycol, Texanoß and $\mathrm{TVOC}_{\mathrm{R}}$ for the two experiments are presented and compared in Figures $71-80$, respectively.

The emission rate of n-tridecane was increased by heating, but not substantially affected by the change in ventilation rate. Either heating or the change in ventilation rate did not substantially affect the emission rate of toluene. The emission rates of phenol and TXIB尺 changed by a factor of two or more in response to heating. The responses (both the increase and the decrease) were rapid. Both of these compounds had higher apparent emission rates at the higher ventilation rate. The emission rate of benzothiazole was also higher at the higher ventilation rate. The same relationship between the emission rates of these three compounds and ventilation was observed in Experiment $\mathrm{V}-2$.

The emission rate of 4-PCH was substantially increased due to heating and was also higher at the higher ventilation rate. The emission rate of BHT increased by approximately a factor of two in response to heating. 
The emission rate of ethylene glycol was only moderately affected by heating but was higher at the higher ventilation rate. The emission rate of Texanoß was affected both by heating and the ventilation rate. The increase due to heating was substantial. Subsequent to the heating period in Experiment A-1, the Texano@ emission rate was consistently lower than in Experiment A-2.

The emission rate of TVOC $\mathrm{A}$ in Experiment A-1 increased in response to heating. Subsequent to heating, the emission rate rapidly returned to the same value measured in reference Experiment A-2. This, and the results for the individual VOCs, demonstrated that an elevation in temperature of $10^{\circ} \mathrm{C}$ for a relatively short period of 72 hours generally only affected the emission rates during the period that the heat was applied. For most compounds, the emission rates subsequent to heating quickly returned to values that would have been expected to occur in the absence of heating.

\subsubsection{Cumulative Masses and Exposures for the Target Compounds}

The cumulative masses of the target VOCs and TVOC $_{\mathrm{A}}$ emitted by the combined source assemblies over 0 - 336 hours in reference Experiment A-2 and treatment Experiment A-1 are presented in Appendix K, Table K-16. These results show that ethylene glycol and Texanol® from the latex paints and cyclohexanone and tetrahydrofuran from the carpet seam sealer dominated the mass emissions in both experiments.

The relative magnitudes of the effects due to heating are illustrated in Figure 81 . The results for reference Experiment A-2 were used to calculate the ratios. Heating increased the cumulative mass emissions of a number of the target VOCs and of TVOCR by as much as 60 percent. On the other hand, heating did not increase the cumulative mass emissions of toluene, styrene, cyclohexanone, tetrahydrofuran, ethylene glycol, 2,2'-azobisisobutyronitrile and benzothiazole. For styrene and benzothiazole, there were relative decreases in the heating experiment due to small numerical decreases in their cumulative masses.

For reference Experiment $A-2$, cumulative exposures in ppb-h were estimated for both the 0 - to 48-h and the 48- to 336-h periods. These are presented in Appendix K, Table K-17. It was assumed that the daily duration of occupancy was $20 \mathrm{~h}$. The ratios of the cumulative exposures for the 0- to 48-h period to the total cumulative exposures for the entire 336-h period are also presented in Table $\mathrm{K}-17$. In the first $48 \mathrm{~h}$, cyclohexanone, tetrahydrofuran, ethylene glycol and Texanok produced the highest exposures. In fact, 70 to 90 percent of the total exposures for cyclohexanone and tetrahydrofuran occurred in the first $48 \mathrm{~h}$. Over the 48 - to 336 -h period, toluene, ethylene glycol, phenol and Texanoß produced the highest exposures.

The estimated cumulative exposures to the target VOCs for the 96- to 336-h periods of both experiments are presented in Appendix K, Table K-18. For the purpose of this comparison, it was assumed that occupancy began $96 \mathrm{~h}$ after installing the materials. This initial time point was selected since occupancy of a building following a "bake-out" would most likely not occur until after the building returned to its normal operating temperature. As for the previous experiments, it was further assumed that the daily duration of occupancy over this period was $20 \mathrm{~h}$.

The relative magnitudes of the effects of the heating treatment on the cumulative exposures to the target VOCs over $96-336 \mathrm{~h}$ are illustrated in Figure 82. The results for reference Experiment A-2 were used to calculate the ratios. The figure shows that, with a few exceptions, exposures to many of the VOCs over this period were not substantially affected by heating. There was a factor of 2.5 increase in the calculated exposure to tetrahydrofuran in Experiment A-1 that was presumably caused by heating. On the other hand, there were relatively large decreases in the exposures to styrene, Texanoß and benzothiazole that were apparently due to heating. The exposure to Texanol $($ was most beneficially impacted by the heating treatment. The numerical decrease in the exposure to Texanok, the dominant compound in these experiments, was $19 \mathrm{ppm}-\mathrm{h}$, and the relative decrease was more than 50 percent. In general, the results demonstrated that moderate heating produced only short-lived beneficial effects wit? respect to estimated occupant exposures for most VOCs emitted by these sources. The primary exception was Texanol emitted by latex paint. 


\subsubsection{Comparison with Literature Data}

There have been several attempts to evaluate the effects of a bake-out procedure on concentrations of VOCs in buildings. In one building for example, emission rates of TVOC and individual VOCs were measured over a three-week period before and immediately after a four-day bake-out in which the indoor temperatures reached up to $34^{\circ} \mathrm{C}$ (Offermann et al., 1993). Immediately after the bake-out there were substantial reductions in the emission rates of most of the individual VOCs. However, the measurements made the following week showed that the rates had increased to levels that were generally comparable to those measured before the bake-out procedure.

Borrazzo and Girman (1993) reviewed the data from five investigations of bake-outs conducted in California buildings. There were measurable effects for some VOCs. However, the volatility of the compounds did not adequately explain the variability in bake-out effectiveness among the compounds. The authors suggested that compound-dependent properties should play a role in determining the extent to which emission rates increase as a function of temperature. They predicted that more polar compounds, such as alcohols, would be more effected than less polar compounds.

Recently, the influence of chamber temperature on the emissions of TVOC and individual VOCs from latex paint, carpet and vinyl flooring were investigated in small-scale (6-L) chambers (van der Wal et al., 1997). Experiments were conducted over $300-600$ hours at different chamber temperatures in the range of $23-50^{\circ} \mathrm{C}$. In some cases, the chamber was kept at $30^{\circ} \mathrm{C}$ for several weeks and then cooled to $23^{\circ} \mathrm{C}$. For 4-PCH from the carpet and 2-ethyl-1-hexanol from the vinyl flooring, the chamber concentrations dropped upon cooling to the same levels measured for the materials kept at a constant $23^{\circ} \mathrm{C}$. For Texano@ emitted by the latex paint, there was an increased initial emission rate followed by a substantially increased decay rate in the experiment conducted at $30^{\circ} \mathrm{C}$ relative to the experiment at $23^{\circ} \mathrm{C}$. The results from the current study conducted in the large-scale chambers appear to corroborate these results since the emissions of Texanol $\otimes$ were substantially effected by the heating procedure, while most of the other target VOCs were marginally effected by heating.

\subsection{COMPARISON WITH EXPERIMENTS USING THE INDIVIDUAL SOURCE ASSEMBLIES}

The experiments with the combined source assemblies produced area-specific emission rates that were generally consistent with those produced in the large-scale experiments with the individual source assemblies. However, a simple evaluation of the similarities among these experiments is difficult because: 1) the paints were applied earlier in the experiments with the combined sources; 2) the initial ventilation rate scenarios were somewhat different; and 3) the individual areas of the carpet and vinyl flooring assemblies were smaller. Area-specific emission rates measured at the ends of the experiments that employed similar treatments provide the most direct way to evaluate the similarities since temporal changes in emission rates were typically small by this time.

Table 14 compares the emission rates of 17 VOCs at the end of Experiment A-2 with the respective emission rates measured at the ends of experiments with individual source assemblies that used additional ventilation as the treatment. The 336-h emission rate of $n$-decane was a factor of two higher in Experiment A-2 than in Experiment V-2 conducted at nearly the same conditions. For the other compounds emitted by the vinyl flooring assembly, the agreement between the two experiments was within approximately \pm 40 percent. There was also excellent agreement between Experiment A-2 and Experiment C-2 for three of the individual VOCs emitted by the carpet assembly. When the data for approximately equivalent elapsed times was compared, the agreement between Experiment A-2 and Experiment P-3 for the dominant compounds emitted by the latex paints was within \pm 20 percent.

\subsection{LONG-TERM CONCENTRATIONS, EMISSION RATES AND EXPOSURES}

The compartment air concentrations of the individual target VOCs measured weeklv $r_{1}$ Experiment A-2 over 336 - 2,016-h elapsed time are presented in Appendix K, Tables K-20 and 
K-21. The concentrations of SigmaVOC $P$ and $T V O C_{R}$ for this time period are presented in Appendix K, Table K-22. The long-term temporal profiles of the compartment concentrations of nine of the VOCs are shown in Figures 83 - 86. The temporal profiles of the concentrations of SigmaVOCp and TVOC $A$ are shown in Figure 87. The ventilation rate and the environmental conditions were stable over this time period (Appendix K, Table K-19).

For most of the individual VOCs, there were only relatively small (i.e., less than factor of four) changes in concentration over this ten-week time period. Among the compounds emitted by the carpet and vinyl flooring assemblies, the largest decreases generally occurred for the most volatile compounds. For example, the concentration of toluene dropped from 13 to $4 \mathrm{\mu g} \mathrm{m}^{-3}$. The concentration of TXIBQ, one of the least volatile compounds, remained constant while the concentration of BHT increased with time from 8 to approximately $14 \mathrm{\mu g} \mathrm{m}^{-3}$. As discussed above, the increase in the concentration of $\mathrm{BHT}$, which originated from the carpet cushion, was probably due to its slow diffusion through the overlying carpet. Texanol emitted by the latex paints had the highest concentration at 336-h elapsed time. By the end of 12 weeks, the concentration of Texanol$@$ had declined by four fold to $294 \mu \mathrm{g} \mathrm{m}^{-3}$. The relative decrease in the concentration of ethylene glycol, a more volatile compound emitted by the paints, was smaller so that the concentrations of these two compounds were more similar by the end of the experiment. The temporal profile of SigmaVOC $P$ predominantly reflects the changes in the concentrations of Texanol and ethylene glycol. As noted above, $\mathrm{TVOC}_{\mathrm{R}}$ was largely a measure of hydrocarbons emitted by the carpet cushion and, to a lesser extent, the vinyl flooring assembly. The concentration of $T_{V O C}$ remained relatively stable.

The area-specific emission rates of the individual target VOCs for the $336-2,016-h$ period time are presented in Appendix K, Tables K-23 and K-24. The area-specific emission rates of TVOC $C_{R}$ calculated using the floor area of the compartment are presented in Appendix $K$, Table $\mathrm{K}-25$. Since the ventilation rate was unchanged over this time period, the emission rates follow the same temporal profiles as the compartment concentrations.

The cumulative masses of the target VOCs and TVOC $R$ emitted by the combined source assemblies over 336 - 2,016 hours are presented in Appendix K, Table K-26. Ethylene glycol and Texanol $\otimes$ had the highest cumulative mass emissions. The sum of the mass emissions of these two paint compounds was $15.9 \mathrm{~g}$. The sum of the other target VOCs shown in Table K-26 that were predominantly from the carpet and vinyl flooring assemblies was $2.5 \mathrm{~g}$. The sum for TVOC $\mathrm{R}$ that was predominantly hydrocarbons from these other sources was $10.8 \mathrm{~g}$.

Approximately $115 \mathrm{~g}$ of ethylene glycol and $50 \mathrm{~g}$ of Texanok were applied in Experiment A-2 based on the paint application data (Table K-02) and the paint compositions (Appendix C, Table C-58). The cumulative emitted mass of ethylene glycol over $336-2,016$ hours of $2.4 \mathrm{~g}$ was two percent of the applied mass. The cumulative emitted mass of Texanoß over $336-2,016$ hours of $13.5 \mathrm{~g}$ was seven percent of the applied mass. Depending upon the ventilation rate conditions, approximately $4-14$ percent of the applied ethylene glycol and $19-35$ percent of the applied Texanoß would be emitted prior to this period (Table 10). Thus, less than 20 percent of the applied ethylene glycol and less than 50 percent of the applied Texanoß would be expected to be emitted over a period of three months following paint application. This suggests that if ethylene glycol and Texanoß applied to gypsum board were 100 percent re-emittable it would take more than one year for them to be completely released to room air. Chang et al. (1997) found that there were measurable emissions of these VOCs 11 months after paint application to gypsum board and concluded that it might take as long as 3.5 years for the complete release of these VOCs.

The estimated cumulative exposures to the target VOCs that an occupant of a residence would receive over a period of 336 - 2,016 hours are presented in Appendix K, Table K-27. It was assumed that occupancy occurred daily throughout the ten-week period and that the daily duration of occupancy was $20 \mathrm{~h}$. Again, the highest exposures were for ethylene glycol and Texanol at 63 and $100 \mathrm{ppm}-\mathrm{h}$, respectively. The exposure to phenol was estimated to be $8.6 \mathrm{ppm}-\mathrm{h}$, and the exposure to toluene was estimated to be about $3 \mathrm{ppm}-\mathrm{h}$. 
These results demonstrate that many VOCs, particularly, the least volatile compounds, are emitted from common sources over relatively long periods and that the net effective emission rates of these compounds change slowly with time over a period of several months. The most significant changes in emission rates only occur over the first few days following installation of the sources.

Table 14. Area-specific 336-h emission rates $\left(\mu \mathrm{g} \mathrm{m}^{-2} \mathrm{~h}^{-1}\right)$ of target VOCs in large-scale experiments with the individual source assemblies and in Experiment A-2 with the combined source assemblies. All of the compared experiments were conducted with additional ventilation as the treatment.

\begin{tabular}{|c|c|c|c|}
\hline \multirow[b]{2}{*}{ Compound } & \multicolumn{3}{|c|}{ Specific Emission Rate, $\mu \mathrm{g} \mathrm{m}^{-2} \mathrm{~h}^{-1}$} \\
\hline & $\begin{array}{r}\text { Individu } \\
\text { Experiment }\end{array}$ & $\begin{array}{l}\text { urces } \\
\text { Value }\end{array}$ & $\begin{array}{c}\text { Combined } \\
\text { Sources } \\
\text { Exp A-2 } \\
\end{array}$ \\
\hline n-Decane & V-2 & 31 & 66 \\
\hline n-Tridecane & V-2 & 63 & 58 \\
\hline Toluene & V-2 & 37 & 47 \\
\hline 1,2,4-Trimethylbenzene & V-2 & 17 & 16 \\
\hline Cyclohexanone & $V-2$ & 16 & 23 \\
\hline Tetrahydrofuran & V-2 & 7 & 8 \\
\hline Benzyl alcohol & $V-2$ & 23 & 16 \\
\hline Phenol & V-2 & 75 & 100 \\
\hline TXIB@ & V-2 & 39 & 47 \\
\hline Benzothiazole & $V-2$ & 22 & 28 \\
\hline Styrene & $\mathrm{C}-2$ & $<1$ & 2 \\
\hline 4-PCH & $\mathrm{C}-2$ & 8 & 8 \\
\hline $\mathrm{BHT}$ & $\mathrm{C}-2$ & 14 & 15 \\
\hline 2,2'-Azobisisobutryonitrile & $\mathrm{C}-2$ & 11 & 14 \\
\hline Ethylene glycol & P-3 & 159 & $133^{*}$ \\
\hline DEGBE & P-3 & 3 & $3^{*}$ \\
\hline Texanok & $P-3$ & 1,110 & $1,200^{\star}$ \\
\hline
\end{tabular}

*240-h Emission rates; elapsed time from paint application was approximately $312 \mathrm{~h}$. 


\subsection{DATA QUALITY EVALUATION}

\subsection{RELATIVE PRECISION}

The overall measurement precisions for the VOCs were computed from the data generated by the numerous paired samples collected during large-scale Experiments A-1 and A-2 with the combined source assemblies. These precision values take into account both sampling and analytical uncertainties. There were 18 target VOCs selected for these experiments that were among the most abundant compounds and that were characteristic of the individual sources. The VOCs were representative of the major classes of compounds detected in these various experiments and also spanned a broad range of volatility. All 18 of the compounds had been analyzed in the previous large-scale experiments with the individual source assemblies and in the small-scale screening measurements. For all compounds except propylene glycol, there were 23 -30 pairs of co-located samples. There were only five pairs for propylene glycol.

A pooled variance was calculated for each compound from the sets of duplicate measurements according to standard analysis of variance techniques $(\mathrm{Ku}, 1969)$. The pooled standard deviations calculated as the square roots of the variances are presented in Table 15. The average concentrations and the median concentrations for the sets of paired samples are also shown in the table. The average and median concentrations were similar for most compounds, although the median concentrations were often somewhat lower. The relative precisions in percent were calculated by dividing the pooled standard deviations by the median concentrations.

For 12 of the target VOCs, the relative precisions were better than ten percent (Table 15). The compounds with the most overall uncertainty were ethylene glycol and propylene glycol with relative precisions greater than 30 percent and 2-(2-butoxyethoxy)ethanol ether (di(ethylene glycol)butyl ether or DEGBE) with a relative precision of 21 percent. All three of these compounds were emitted by the latex paints. The difficulty of analyzing glycol ethers in the emissions from the latex paints was previously discussed. The other compounds with elevated precision values were toluene (11 percent), tetrahydrofuran (20 percent) and styrene (18 percent). The concentrations for styrene were frequently at the lower limit of quantitation, which probably accounted for the higher uncertainty for this compound.

Several sets of paired samples were collected and analyzed in the other large-scale experiments; but, the numbers of pairs were generally too small to calculate relative uncertainties. Nevertheless, the results for the target VOCs in Experiments A-1 and A-2 can probably be extrapolated to the analysis of other VOCs in the other experiments since the methods were identical. With the exception of the glycol ethers, and possibly those compounds that were near their lower limits of quantitation, the relative precisions for the sampling and analysis of target VOCs would be expected to be around ten percent. The relative precisions for VOCs analyzed in a previous chamber study conducted by this laboratory for the ARB using similar methods were estimated by a propagation of errors method to be in the range $10-15$ percent (Daisey et al., 1994).

\subsection{PROPAGATION OF UNCERTAINTY}

The uncertainties in the specific emission rates of target VOCs can be estimated using a propagation of error method. This method estimates the uncertainty in a dependent variable (e.g., specific emission rate reported as $\mu \mathrm{g} \mathrm{m}^{-2} \mathrm{~h}^{-1}$ ) by propagating the uncertainties for the individual variables that were used to calculate the dependent variable. It is assumed that the errors for the variables are random and independent of each other. The uncertainty in the dependent variable will be dominated by the terms with the greatest uncertainties. For the calculation of a specific emission rate, the terms were the compound concentration, the amount of material installed and the compartment ventilation rate. The material amounts were measured and known with a high degree of certainty, and the ventilation rates were shown to quite stable. Therefore, these terms can be eliminated, and the total uncertainty in the specific emission rate: can be assumed to be very similar to the relative precisions for the sampling and analysis of the VOCs. 
Table 15. Relative precisions for the 18 target VOCs in large-scale Experiments A-1 and A-2. The pooled standard deviations were calculated for $\mathrm{n}$ pairs of side-by-side samples.

\begin{tabular}{lccccc}
\hline \hline & $\begin{array}{c}\text { No. of } \\
\text { Pairs } \\
\text { Compound }\end{array}$ & $\begin{array}{c}\text { Average } \\
\text { Conc. } \\
\left(\mu \mathbf{~ m}^{-3}\right)\end{array}$ & $\begin{array}{c}\text { Median } \\
\text { Conc. } \\
\left(\mu \mathbf{g ~ m}^{-3}\right)\end{array}$ & $\begin{array}{c}\text { Pooled } \\
\text { Std. Dev. } \\
\left(\mu \mathbf{~ m}^{-3}\right)\end{array}$ & $\begin{array}{c}\text { Relative } \\
\text { Precision* } \\
(\%)\end{array}$ \\
\hline n-Decane & 26 & 22.9 & 20.6 & 1.4 & 6.6 \\
n-Tridecane & 26 & 20.9 & 18.4 & 1.4 & 7.6 \\
Toluene & 26 & 16.7 & 13.8 & 1.5 & 10.8 \\
1,2,4-Trimethylbenzene & 26 & 5.0 & 4.5 & 0.38 & 8.4 \\
Cyclohexanone & 25 & 9.5 & 9.8 & 0.81 & 8.3 \\
Tetrahydrofuran & 26 & 6.2 & 4.9 & 1.0 & 19.6 \\
Benzyl alcohol & 26 & 4.4 & 4.5 & 0.38 & 8.4 \\
Phenol & 27 & 27.5 & 28.7 & 1.8 & 6.4 \\
TXIB® & 27 & 14.1 & 13.9 & 1.0 & 7.0 \\
Benzothiazole & 26 & 6.5 & 5.6 & 0.37 & 6.7 \\
Styrene & 26 & 1.9 & 1.0 & 0.17 & 17.7 \\
4-PCH & 26 & 4.0 & 3.9 & 0.20 & 6.3 \\
BHT & 26 & 8.4 & 7.9 & 0.40 & 6.0 \\
2,2'-Azobisisobutyronitrile & 26 & 7.9 & 8.3 & 0.58 & 7.0 \\
Ethylene glycol & 23 & 185 & 146 & 53.2 & 36.4 \\
Propylene glycol & 5 & 15.3 & 5.7 & 1.9 & 33.8 \\
DEGBE & 27 & 5.2 & 3.9 & 0.83 & 21.3 \\
Texanol@ & 30 & 1,120 & 1,040 & 95.1 & 9.1 \\
\hline
\end{tabular}

*The relative precision was calculated as the pooled std. deviation / median concentration $x 100$. 


\subsection{CONCLUSIONS}

\subsection{EFFECTS OF THE EXPOSURE REDUCTION TECHNIQUES}

\subsubsection{Ventilation}

Summary - The results of the study showed that a short period of additional ventilation (i.e., three days) immediately following material installation typically reduced VOC concentrations during this period. This resulted in lower predicted cumulative exposures for most VOCs over the first 48 hours after installation. Therefore, the short-term ventilation treatment would help to protect the installers and any occupants that were present during this initial period. Short-term ventilation, however, was shown to be marginally effective for reducing exposures to many VOCs for full-time occupants that inhabited the building beginning two days after material installation. Other ventilation rate strategies may produce more beneficial results. For example, additional ventilation is likely to be a more effective treatment for reducing cumulative exposures if it can be maintained for longer time periods. However, it is also likely that the improvements with respect to exposure would not be directly proportional to the increase in the ventilation rate. This prediction is based on the observed increase in the net effective emission rates of many compounds with increased ventilation rate. Consequently, the amount of additional ventilation should be maximized whenever possible.

Discussion - The application of additional ventilation during and for several days following the installation of an indoor source of VOCs is probably the most readily accessible exposure reduction technique that consumers can employ in an attempt to reduce the concentrations of indoor airborne contaminants and their exposures to these compounds. The manufacturers of products that are used indoors often recommend the use of additional ventilation during the installation period. The primary purpose of this recommendation is, frequently, to limit the exposures of the installers. Additional ventilation is also considered to be one of the primary mechanisms for reducing the VOC exposures of building occupants following new construction or partial renovation/remodeling (Alevantis, 1996). Intuitively, it is assumed that increased ventilation will beneficially reduce exposures. However, the effectiveness of this strategy has only rarely been quantified. For example, not much is known about the longer-term effectiveness of relatively short-term ventilation strategies.

One of the primary goals of this investigation was to evaluate the effectiveness of a shortterm ventilation scenario that could relatively easily be employed in a residence. The selected scenario called for the use of high ventilation, such as might be induced by the use of a fan in an open exterior window and/or door, during the installation period. This was followed by three days of additional ventilation that might be passively induced by leaving some exterior windows and/or doors open. This strategy was tested for each individual source assembly.

In many cases, the air concentrations of the target VOCs were reduced to some degree by the use of additional ventilation. However, the reductions in concentrations during the period of additional ventilation were generally not directly proportional to the magnitude of the increase in the ventilation rate. Also, the concentrations of the VOCs following the termination of additional ventilation very often quickly returned to values similar to those that would likely have occurred in the absence of additional ventilation.

In the large-scale experiments with paint that produced the highest VOC concentrations, additional ventilation and additional ventilation with air mixing resulted in higher cumulative mass emissions of the distinctly dominant compounds ethylene glycol, propylene glycol and Texanoß. However, additional ventilation did not result in large fractional reductions in the estimated cumulative exposures to these compounds for the first 48 hours after paint.application (i.e., the reductions were 18 percent or less). For the paints, this initial time period was critical because typically more than 50 percent of the total exposures occurred in the first 48 hours. The cumulative exposures to the dominant compounds over the duration of the experimental period $(48-240 \mathrm{~h})$ were unaffected by the initial application of additional ventilation. Additional ventilation only clearly reduced the estimated exposures to acetaldehyde and 2-ethyl-1-hexanol for both time periods. The use of fans to increase air velocities near the painted surfaces in 
combination with additional ventilation reduced the exposures to most compounds in both time periods relative to use of additional ventilation by itself. However, the magnitude of this effect was 25 percent or less.

An explanation for the lack of effectiveness of extra ventilation and extra ventilation with increased air mixing for substantially reducing exposures to the dominant VOCs from latex paint, is that gypsum board is a very large sink for these compounds (Chang et al., 1997 and 1998). It was estimated that only about 14 percent of the ethylene glycol and 35 percent of the Texanoß were emitted over the two-week period in the experiment that utilized additional ventilation. Thus, even though extra ventilation resulted in the emission of more mass of these VOCs compared to the base-case experiments, the effect on the total available reservoir of the compounds in the gypsum board was relatively minor. This reservoir is so large that Chang et al. (1997) estimated that If 100 percent of the ethylene glycol and Texanok in latex paint applied to gypsum board can be re-emitted, it might take as long as 3.5 years for the complete release of these compounds.

The emission rates of the dominant VOCs emitted by latex paint are also high and are likely to be limited by mass-transfer effects at the painted surfaces. Thus, an increase in the ventilation rate would be expected to result in higher emission rates. This would at least partially explain why the concentrations of the dominant compounds did not decrease proportionally with increased ventilation.

Other researchers have observed such mass-transfer effects. In a study employing small-scale chambers, it was demonstrated that emissions of Texanoß from a water-based paint were strongly influenced by ventilation rate (Gunnarsen, 1997). The paint was applied to aluminum plates and was conditioned in the chambers for six days. When the ventilation rate was varied over a range equivalent to about $<0.3-6 \mathrm{~h}^{-1}$ for a building with a 2.5- $\mathrm{m}$ high ceiling, there was more than a ten-fold increase in the emission rate of Texanoß.

Additional ventilation had similar effects in the large-scale experiments with the carpet assembly. The concentrations of many of the target VOCs were lowered during the period of additional ventilation. After the ventilation rate was reduced, the concentrations typically increased to values that were similar to those that were measured in the base-case experiment. One exception was BHT emitted by the underlying carpet cushion. The concentrations of this compound increased with time and reached higher values in the treatment experiment with additional ventilation. For most compounds, the initial improvements in concentration were less than would be predicted based on the amount of additional ventilation. Consequently, the effective specific emission rates were higher during the period of additional ventilation.

The dominant VOCs emitted by carpets are, for the most part, constituents of the SBR latex backing material. It has been hypothesized that the emissions of these compounds are limited by their slow diffusion in the SBR backing (Little et al., 1994). A physically based diffusion model was developed to characterize the emissions process. The basic parameters of this model are: 1) the initial concentration of the VOC in the polymer backing; 2) the diffusion coefficient of the VOC in the polymer; and 3) an equilibrium partitioning coefficient for the VOC between the bulk air and the surface of the source. Resistance to diffusion within the polymer layer was shown to be significantly greater than resistance to diffusion between the surface and the bulk air. Consequently, the model predicts that changes in ventilation rate should have little effect on the emission rates of VOCs from carpet.

The results from the large-scale experiments with the carpet assembly are apparently inconsistent with the predictions of the diffusion model. The emission rate of toluene from a carpet has also been observed in a small-scale chamber study to increase with increasing ventilation rate (Gunnarsen, 1997). These experimental observations illustrate that relatively simple emission models, such as the diffusion model for carpet, are currently inadequate for predicting the impacts of ventilation for complex building materials.

In the large-scale experiments with the carpet assembly, it is possible that desorption of the VOCs from the surfaces of materials, such as the face fiber of the carpet, played a significant role in determining the effective emission rates of the VOCs during the initial portions of the 
experiments. If so, this might help to explain the apparent increase in the emission rates with additional ventilation.

Additional ventilation resulted in increased cumulative mass emissions for most of the target VOCs for the carpet assembly. Additional ventilation also resulted in reduced cumulative exposures for all compounds except BHT over the first 48 hours after installation of the carpet. The magnitude of the effect varied depending upon the compound but was typically a factor of two or less. For the duration of the experiment $(48-336 \mathrm{~h})$, there was no apparent effect of additional ventilation on the predicted cumulative exposures of most compounds. The exceptions were 1,2-dichlorobenzene and two nitrogen-containing compounds emitted by the carpet cushion that had substantially lower exposures for this period apparently in response to additional ventilation.

The initial compartment concentrations of the less volatile compounds emitted by the vinyl flooring assembly, such as phenol and TXIBQ from the sheet vinyl and benzothiazole from the cove base, were very similar during the experiment with additional ventilation to their concentrations in the base-case experiment. Consequently, the effective emission rates of these compounds were considerably elevated as the result of increased ventilation. This result appears to contradict the theoretical prediction that the emissions of these VOCs should be primarily limited by their rates of diffusion within the source materials and, therefore, be insensitive to the ventilation rate (Clausen et al., 1993). The cause of this discrepancy is unknown. However, as was suggested for the carpet materials, desorption phenomena may have played a substantial role in determining the compartment concentrations of these compounds.

Additional ventilation generally resulted in increased cumulative mass emissions of the target VOCs for the vinyl flooring assembly. The exceptions were n-decane, toluene, cyclohexanone and tetrahydrofuran. The latter two compounds were the principal solvent components of the seam sealer applied to the surface of the vinyl. Additional ventilation also resulted in reduced cumulative exposures for all compounds except TXIBQ over the first 48 hours after installation of the vinyl flooring. For a number of the compounds, these reductions were substantial, being 50 percent or more. In general, the effect was most pronounced for the most volatile compounds. For the duration of the experiment $(48-336 \mathrm{~h})$, there was no apparent effect of additional ventilation on the predicted cumulative exposures of most compounds. The exceptions were toluene, tetrahydrofuran and phenol for which the reductions in exposure were 25 percent or less.

\subsubsection{Airing Out of the Materials}

Summary - The experimental results demonstrated that airing out of carpet and carpet cushion for two days prior to their installation effectively reduced the subsequent VOC emissions and predicted occupant exposures to VOCs. The treatment was generally effective over the entire two-week experimental period. Airing out of sheet vinyl and cove base at the same conditions was considerably less effective. The treatment generally only reduced predicted occupant exposures during the first 48 hours after installation of the vinyl flooring materials.

Discussion - For some solid materials, it may be possible to reduce their emissions of VOCs by airing them out prior to their installation in a building. Alevantis (1996) discussed the development of protocols for this material conditioning process. Logically, materials should be aired out in a clean, dry and weil-ventilated space with low levels of background contamination. Due to the lack of experimental data, there is almost no guidance on the time required to adequately condition various materials.

Airing out of materials, such as carpets and sheet vinyl flooring, may actually be more practical for individual residences than for large-scale buildings since the quantities of materials are relatively small. For this study, it was assumed that a consumer might realistically be able to air out carpeting or sheet vinyl on-site in another space such as a garage. Alternately, the supplier or installer might be able to unroll and ventilate the materials at an off-site location. In either case, the site and conditions should be carefully selected since, as examples, unrolling a carpet in a warehouse in which carpets are stored or in a garage with gasoline contamination might be counter productive. 
The effectiveness of airing out materials was investigated for both the carpet and the vinyl flooring assemblies. For each of the experiments, the major materials were aired out in a wellventilated room with very low levels of background contamination. Fans were used to increase the airflow over the surfaces of the materials. Conditioning lasted for two days as this was considered to be a practical time limit for on-site conditioning by consumers.

The airing out treatment was effective for reducing the emissions of VOCs from the carpet assembly materials. There were clear reductions in the initial and subsequent compartment concentrations of the individual VOCs and TVOC in the treatment experiment relative to the base-case experiment conducted at the same environmental conditions. As a result, the cumulative mass emissions of all of the constituents were lower. The treatment also reduced the predicted exposures to the target VOCs over both the initial $(0-48 \mathrm{~h})$ and longerterm (48 - $336 \mathrm{~h}$ ) exposure periods. In general, the treatment had a larger impact on the exposures for the more volatile VOCs. For some compounds the reductions were substantial (i.e., more than a factor of two). On the other hand, the exposures for two of the dominant and less volatile compounds, 4-PCH from the carpet and BHT from the carpet cushion, were only reduced by about 20 percent over the 48- to 336-h period. For all VOCs, the airing out treatment was more effective than additional ventilation for reducing predicted occupant exposures.

The results for the carpet materials are perhaps consistent with the notion that the amounts of the VOCs sorbed onto the surfaces of the materials comprised relatively large fractions of the total available masses of these compounds.

Considerably different results were obtained for the vinyl flooring materials. Airing out of the sheet vinyl and the cove base generally had a short-lived effect on the compartment concentrations of the target VOCs. This was seen as typically small to moderate (i.e., less than 10 to approximately 25 percent) reductions in the cumulative mass emissions of these compounds. Since the initial concentrations of most VOCs were lower in the treatment experiment than in the base-case experiments, the treatment reduced the predicted exposures to these compounds over the first 48 hours after installation of the vinyl flooring. These reductions were in the range of approximately 15 to 50 percent. For most compounds, the airing out treatment was less effective than additional ventilation for reducing exposures during this period. For the duration of the experiment $(48-336 \mathrm{~h})$, the reductions in the predicted cumulative exposures due to the airing out treatment were less than 10 percent for most compounds.

The effect of the airing out treatment on the vinyl flooring materials is perhaps consistent with the notion that diffusion of the VOCs in these materials was limiting the emission rates of these compounds. Since the diffusion process is relatively slow, the concentrations of the VOCs in the surface layers of the materials might have been depleted over the two-day airing out period but only small fractions of the total available masses might have been emitted. This argument in favor of diffusion control of the emissions of VOCs from the sheet vinyl and cove base, however, appears to be inconsistent with the observed dependence of the emission rates of the VOCs on the compartment ventilation rate.

\subsubsection{Moderate In-situ Heating}

Summary - The experimental results demonstrated that moderate heating in combination with additional ventilation did not substantially reduce subsequent occupant exposures to many of the VOCs emitted by the three source assemblies. There was, however, a significant reduction in the exposure to the most abundant compound, Texanok. The use of higher temperatures and/or longer heating periods to produce more substantial effects would probably be impractical in many residential situations. Higher temperatures might also result in material damage (Girman et al., 1990).

Discussion - Building "bake-out" has been used as an in-situ conditioning treatment in new and renovated office buildings. The purpose of the treatment is to artificially reduce the VOC content of materials after they have been installed and before the building is occupied. This procedure is based on the theory that emission rates of VOCs are typically higher at elevated temperatures due to increased surface evaporation or diffusior in materials. Field trials have been attempted and 
reported by several investigators (Girman et al., 1989, 1990; Hicks et al., 1990; Offermann et al., 1993). Generally, the interior temperature was raised to $32-38^{\circ} \mathrm{C}$ while maintaining constant ventilation. Heating was applied for a relatively short period that, in most cases, was limited to several days for practical reasons. The results of the field studies were generally inconclusive, in part, because of the difficulty of conducting controlled experiments in real buildings. Therefore, it was impossible to use these existing studies as the basis for recommendations for controlling occupant exposures to VOCs in office buildings (Alevanis, 1996).

Moderate heating in combination with additional ventilation was one of the exposure reduction techniques investigated in this study. A heating scenario was developed that could be practically employed in a residence by a consumer. The temperature of the space was held at approximately $10^{\circ} \mathrm{C}$ over typical room temperature for a period of three days while maintaining a relatively high ventilation rate. Heat was applied with electric space heaters. The entire procedure would have required approximately four days to implement in a residence.

The experimental heating scenario increased the compartment concentrations of many of the individual VOCs and TVOC emitted by the combined source assemblies. The effects were generally highest at the onset of heating and diminished with time during heating. For many of the VOCs, there was a substantial increase in the cumulative mass emissions for the entire experimental period that was attributable to heating. However, the effect of heating was largely limited to the heating period. The concentrations and emission rates of many of these compounds quickly returned to values that were similar to those measured in the experiment with additional ventilation but no heating. It was realistically assumed that occupancy would not begin until one day after the heating procedure was concluded and the space returned to typical room temperature. Consequently, heating only clearly decreased the predicted cumulative exposures for several compounds. The largest decrease in exposure of approximately 60 percent occurred for Texano@, the most abundant VOC and the dominant compound emitted by the latex paints. Heating did not substantially reduce the exposure to ethylene glycol, the other abundant compound emitted by the paints.

\subsection{OTHER CONTROL OPTIONS}

\subsubsection{Delayed Occupancy}

In general, the compartment concentrations of VOCs emitted by the latex paints decayed rapidly over the first several days following painting. Peak exposures to these VOCs were highest during this period and the predicted cumulative exposures for the first 48 hours after painting accounted for more than 50 percent of the total exposures over the entire $0-240$ hours of the experiments. The experimental data also showed that additional ventilation and additional ventilation with added air mixing had very limited effectiveness for reducing exposures to the dominant VOCs from latex paints over both the initial and longer-term periods. Thus, delayed occupancy for at least several days following painting appears to be an effective control option for reducing exposures to VOCs emitted by latex paints.

The compartment concentrations of VOCs emitted by the carpet and vinyl flooring assemblies decayed slowly in comparison to the concentrations of the paint VOCs.

Consequently, the predicted cumulative exposures to VOCs emitted by the carpet and vinyl flooring assemblies for the first 48 hours after material installation were often 20 percent or less of the total exposures for the two-week experimental period. Additional ventilation was also frequently effective for reducing cumulative exposures to these VOCs over the initial 48-h time period. Delayed occupancy for this initial period would also effectively reduce exposures. However, delayed occupancy for periods considerably longer than several days might be required to substantially reduce cumulative exposures to VOCs emitted by the carpet and vinyl flooring assemblies.

One of the objectives of the long-term experiment with the combined assemblies was to determine if exposures to VOCs emitted by these assemblies could be substantially reduced by delaying occupancy for a period of a week or more. It is often assumed that concentrations of 
VOCs emitted by new building materials decrease rapidly, and perhaps exponentially, over a period of a several weeks. However, few long-term measurements have been made in new or renovated buildings to substantiate or refute this hypothesis. In one case, the emission rate of 4-PCH emitted by a new carpet was shown to remain relatively constant over a period of two to seven weeks (Hodgson et al., 1993).

The results of the long-term experiment demonstrated that under constant environmental conditions the concentrations and emission rates of many of the dominant VOCs and TVOC emitted by the three source assemblies remained relatively constant over a period of two to 12 weeks. The 75 percent decrease in the concentration of Texanoß over this period was the most significant reduction; however, even at 12 weeks this compound still had the highest concentration among the target VOCs. Since VOC concentrations did not decay rapidly or exponentially there was no obviously optimal time for resumption of occupancy demarcated by a substantial reduction in VOC concentrations.

\subsubsection{Product Substitution}

As discussed above, additional ventilation, the most readily accessible control option, had limited effectiveness for reducing occupant exposures to VOCs emitted by latex paint over the initial two-day period and to VOCs emitted by all of the sources over the longer-term period. Airing out of vinyl flooring materials and moderate heating also had limited effectiveness for reducing occupant exposures to VOCs. These findings emphasize the importance of selecting low VOC-emitting or low-VOC impact interior materials for use in new construction and renovation. If smaller amounts of toxic or otherwise objectionable VOCs (i.e., compounds with strong odors) are introduced into a building because lower-emitting materials are used, occupant exposures to these VOCs will be lower and the need for subsequent control strategies should be diminished.

Strategies for screening and selecting building materials and products based on their emissions of VOCs have been presented (Alevantis, 1996; Levin and Hodgson, 1996). However, these strategies, which require information about the emissions characteristics of materials, are infrequently implemented. Levin and Hodgson (1996) discussed some of the major barriers or disincentives to making selections of materials based on their emissions characteristics. These barriers include: 1) the lack of sufficient data; 2) the difficulty of obtaining existing data; 3) the lack of standardized test procedures; 4 ) the difficulty of evaluating potential toxicity and other health effects; 5) the high costs associated with the testing of materials; and 6) the possibility of increased liability for design and building professionals. The situation is further complicated by the fact that there are a number of factors, other than emissions characteristics, which must be considered when selecting a building material. One or more of these factors that include appearance characteristics, durability, initial costs, and maintenance costs is frequently the dominant issue in the final selection. Consequently, the most realistic role for VOC emissions testing and emissions test data in the near term may be to help consumers avoid problematic materials and select lower emitting materials from among those that meet the other selection criteria.

European scientists have proposed a relatively elaborate procedure for the testing and evaluation of VOC emissions from building products and materials (ECA, 1997; Maroni and Lundgren, 1997). This procedure includes: 1) the determination of emission factors for individual VOCs and TVOC using small-scale chamber measurements; 2 ) an exposure assessment based on realistic exposure scenarios and made with an indoor air quality model; 3) a toxicological evaluation that attempts to establish the "lowest concentrations of interest" for the individual VOCs; and 4) a sensory evaluation of the emissions. Because of the complexity and presumably high cost of the scheme, it is unlikely to be widely adopted and used in the near future, at least in the United States.

Some of the data generated in the current study by the screening measurements may be universal enough to assist consumers in their selection of materials. However, it needs to te emphasized that this study was not an exhaustive survey of available materials in any of the product categories. Such a survey would be a major undertaking and would have to be 
continually updated since new materials and reformulated products are constantly being introduced into the market. Also, this study did not attempt to perform a health hazard assessment for any of the materials that were tested. Such an assessment would be another major undertaking. It must further be cautioned that lower overall emissions of VOCs indicated by relatively low TVOC emission rates is not, by itself, an adequate measure of the potential for health impacts since there is a wide range of potency among individual compounds that are emitted by materials.

In the screening measurements, "non-VOC" flat and semi-gloss paints were compared to conventional paints. These new generation paints did emit VOCs, but at levels based on the SigmaVOC values that were 25 to 50 times lower than the conventional paints. Since this study was initiated, more "non-VOC" or "low-VOC" paints have been introduced into the market. The data suggests that exposures to the dominant VOCs in latex paints, such as ethylene glycol and propylene glycol solvents and the Texanol $B$ coalescing aid, may be dramatically reduced by the use of these new generation paints. However, there are other issues, such as color availability, coverage and durability relative to conventional paints, which were not addressed here, but which must also be considered in the selection process.

Two 100 percent nylon fiber, intermediate grade carpets from different manufacturers were compared to two versions of a less-expensive olefin fiber carpet in the screening measurements. The emissions of TVOC and individual VOCs including the odorous compound, 4- $\mathrm{PCH}$, were substantially lower from the nylon carpets than from the olefin carpet. Among these specific materials, the nylon carpets would be the better choices with respect to the emissions of VOCs and potential odor. It is not known if the results for the olefin fiber material are indicative of emissions of VOCs from other olefin fiber carpets.

Two bonded urethane carpet cushions from different manufacturers were compared to a synthetic fiber cushion in the screening measurements. The emission rates of TVOC and individual VOCs were substantially lower from the synthetic fiber material than from the bonded urethane materials. These results are in agreement with a previous study of 17 carpet cushions of five different types that showed that synthetic fiber materials were generally the lowest emitting cushions (Shaeffer et al., 1996). Although synthetic fiber cushions would be expected to result in relatively low exposures to VOCs, other issues, such as the resilient feeling of the carpet installation, must also be considered in the selection process.

A commercial sheet vinyl flooring material was compared to three different residential "no-wax" materials in the screening measurements. The emission rate of TVOC was below the lower limit of quantitation and the emissions rates of individually quantified VOCs were relatively low for the commercial sheet vinyl. From this data alone, it would appear that the commercial sheet vinyl was the best installation choice. However, subsequent maintenance of the commercial product would presumably result in exposures to some VOCs since the product would require periodic waxing and stripping. Additionally, the two product types are not closely comparable, and appearance and maintenance issues would likely play a major role in their selection.

These results illustrate some of the complexities and difficulties involved in selecting building materials. There are many factors to consider and tradeoffs must frequently be made, often in the absence of adequate information regarding emissions of VOCs and potential health effects. VOC emission characteristics may be an important consideration for consumers; however, it is frequently made subordinate to other issues such as appearance, cost or durability.

\subsection{RECOMMENDED PRACTICES TO REDUCE OCCUPANT EXPOSURES}

This study has shown that there are a few general practices that consumers can follow that are likely to reduce their exposures to VOCs emitted by building materials used in new construction and renovation. These recommended practices are outlined below.

1. Materials that emit lower amounts of toxic VOCs shou!r he selected whenever the opportunity exists and there is sufficient data with respect to VOi. Ensicions and possible health effects upon which to base informed decisions. 
2. Renovation/remodeling work should generally be done during periods when a building is unoccupied so occupants are not exposed to the relatively high concentrations of VOCs that can occur during and shortly after the application or installation of materials. If possible, the building should remain unoccupied for several days following painting to reduce occupant exposures to the dominant VOCs in paint.

3. Very high mechanically induced ventilation rates should be used during the application or installation of materials. This will help to protect the installers and any occupants that are present. It may also reduce the subsequent occupant exposures to some compounds from some sources.

4. Subsequent to the installation, additional ventilation should be maintained for as long as practically possible. In many cases, additional ventilation during the period of occupancy will reduce exposures. The amount of additional ventilation should be maximized since the beneficial effects of ventilation with respect to exposures are not, in many cases, in direct proportion to the increase in the ventilation rate.

5. When the opportunity exists, carpets and carpet cushions should be aired out for several days in a well-ventilated clean, dry environment prior to their installation.

6. The in-situ treatment of materials accomplished by applying heat to a room or building in conjunction with additional ventilation after materials are installed is not recommended as a general practice. Heating was shown to be relatively ineffective for reducing the subsequent occupant exposures to most compounds. In addition, it may be difficult to implement an effective heating strategy for an entire residence or building.

\subsection{POTENTIAL COSTS OF RECOMMENDED PRACTICES}

There may be substantial costs associated with implementing the recommended practices for reducing exposures to VOCs emitted by building materials.

There are commercial laboratories that can test small samples of materials for emissions of VOCs following the guidelines of ASTM D 5516-90 (ASTM, 1990). Typical costs for such dynamic, small-scale, chamber tests range between $\$ 1,000-2,000$ per test (Levin and Hodgson, 1996). There would be additional expenditures if it were necessary to have a toxicologist or other specialist interpret the results of the chamber tests with respect to potential health effects. Because of these high costs, it is improbable that individual consumers would test materials to obtain data for their selection process. Consumers would more likely seek information that was freely available from manufacturers or from the scientific literature. Unfortunately, such information is not available for most materials.

The initial purchase price of low-VOC content or low-VOC emitting materials may be higher than the price of conventional or lower-grade materials. This is true for new-generation low-VOC content latex paints for which the current price premium can be substantial. If 20 gallons ( $76 \mathrm{~L}$ ) of paint were required to apply two coats of paint to the interior of a typical house, the additional material cost might be as high as $\$ 200$. If substantial demand for these products develops, the price differential will likely be reduced. Higher quality nylon carpets are also generally more expensive than carpets with olefin fibers. For example, it is estimated that upgrading from a base-grade olefin carpet to an intermediate grade nylon carpet similar to those tested in this study could increase the initial purchase price by a factor of two or roughly $\$ 7.50$ per square yard based on the materials used in this study. If 200 square yards $\left(167 \mathrm{~m}^{2}\right)$ of material were required to fully carpet a typical house, the additional material cost would be $\$ 1,500$.

The costs associated with airing out carpet assembly materials prior to their installation are unknown but are likely to vary considerably depending upon the availability of a suitable space. The costs might be relatively small if the materials could be aired out on site in a garage, for example. In this case, the cost should largely be limited to any additional labor that was required. If the materials had to be aired out off site in a commercial space, there would likely be charges for rental of the space, labor and possibly transportation of the materials. These charges could easily add ten percent $c r$ more to the cost of the material. 
The potential costs of in-situ heat treatment depend upon the duration and extent of the heating and the unit costs of the energy that is consumed. The direct costs for a similar "bakeout" procedure ranged up to $\$ 0.23$ per square foot $\left(\$ 2.50 \mathrm{~m}^{-2}\right)$ for commercial buildings (Girman et al., 1990). In addition, there are potential costs associated with lost or delayed occupancy of commercial buildings during the treatment period.

The costs for additional ventilation are difficult to estimate. The scenario tested in this study assumed that the additional ventilation used after installing the materials could be achieved passively by opening exterior windows and doors in which case the cost would be negligible.

Passive ventilation might not be desirable or practical for security reasons or because of climatic conditions. If mechanical ventilation and/or thermal conditioning of the indoor environment were required, there would be associated energy costs. These costs would depend upon the amount of energy consumed and the unit costs of the energy. If the period of additional ventilation was limited to one month or less, it can reasonably be assumed that the energy costs would typically be small relative to the costs of the materials and labor for painting or flooring installation. 


\section{REFERENCES}

Alevantis, L.E. 1996. Reducing occupant exposure to volatile organic compounds (VOCs) from office building construction materials: Non-binding guidelines. California Department of Health Services Report released July, 1996, 43 p.

ARB-CAPCOA (Air Resources Board and California Air Pollution Control Officers Association). 1989. ARB-CAPCOA Suggested Control Measure for Architectural Coatings. California Air Resources Board Stationary Source Division and California Air Pollution Control Officers Association, Sacramento, CA. July, 1989.

ASTM. 1990. ASTM Standard D 5116-90, Standard Guide for Small-Scale Environmental Chamber Determinations of Organic Emissions From Indoor Materials/Products. American Society for Testing and Materials, Philadelphia, PA.

Black, M., W.J. Pearson and L.M. Work. 1991. A methodology for determining VOC emissions from new SBR latex-backed carpet, adhesives, cushions, and installed systems and predicting their impact on indoor air quality. Proceedings of IAQ '91, Healthy Buildings. American Society of Heating, Refrigerating, and Air-Conditioning Engineers, Inc., Atlanta GA, pp. 39-42.

Bremer, J., E. Witte, and D. Schneider. 1993. Measurement and characterization of emissions form PVC materials for indoor use. Proceedings of the 6th International Conference on Indoor Air Quality and Climate, Helsinki, Finland, July 4 - 8, Vol. 2. pp. 419-424.

Borazzo, J. and J. Girman. 1993. Temperature effects in building bake-out. Proceedings of the 6th International Conference on Indoor Air Quality and Climate, Helsinki, Finland, July 4 - 8 , Vol. 6. pp. 575-580.

Carpet \& Rug Industry Magazine. April, 1995. Top fifty North American carpet \& rug manufacturers by 1994 sales.

CRI (Carpet and Rug Institute). 1994. CRI Indoor Air Quality Testing Program - Fact Sheet. The Carpet and Rug Institute, Dalton, GA.

CRI (Carpet and Rug Institute). 1997. CRI 105, Standard for Installation of Residential Floor Covering Materials. The Carpet and Rug Institute, Dalton, GA.

CRI (Carpet and Rug Institute). 1998. CRI Indoor Air Quality Testing Programs - Fact Sheet. The Carpet and Rug Institute, Dalton, GA.

Censullo, A.C., D.R. Jones and M.T. Wills. 1996. Improvement of speciation profiles for architectural and industrial maintenance coating operations. Final Report to California Air Resources Board under Contract No. 93-319.

Chang, J.C.S., B.A. Tichenor, Z. Guo and K.A. Krebs. 1997. Substrate effects on VOC emissions from a latex paint. Indoor Air 7: 241-247.

Chang, J.C.S., L.E. Sparks, Z. Guo and R. Fortmann. 1998. Evaluation of sink effects from a latex paint. J. Air \& Waste Management Assoc. 48: 953-958.

C\&EN (Chemical \& Engineering News). October 3, 1994. Paints and Coatings, Product Report, pp. 44-66.

Christiansson, J., J.-W. Yu and I. Neretnieks. 1993. Emission of VOCs from PVC-floorings models for predicting the time dependent emission rates and resulting concentrations in the indoor air. Proceedings of the 6th International Conference on Indoor Air Quality and Climate, Helsinki, Finland, July 4 - 8, Vol. 2. pp. 389-394.

Clausen, P.A. 1993. Emission of volatile and semivolatile organic compounds from waterborne paint - The effect of film thickness. Indoor Air 3: 269-275. 


\section{REFERENCES, CONTINUED}

Clausen, P.A., B. Laursen, P. Wolkoff, E. Rasmusen and P.A. Nielsen. 1993. Emission of volatile organic compounds from a vinyl floor covering. Modeling of Indoor Air Quality and Exposure, ASTM STP 1205, N.L. Nagda, Ed., American Society for Testing and Materials, Philadelphia, PA, pp. 3-13.

Daisey, J.M., K.R.R. Mahanama and A.T. Hodgson. 1994. Toxic volatile organic compounds in environmental tobacco smoke: Emission factors for modeling exposures for California populations. Final report to California Air Resources Board under Contract No. A133-186. Report LBL-36379, Lawrence Berkeley Laboratory, Berkeley, CA.

De Bortolli, M., S. Kephalopaoulos and H. Knoppel. 1995. Report No. 16. Determination of VOCs emitted from indoor materials and products. Second interlaboratory comparison of small chamber measurements. Report EUR 16284 EN, European Collaborative Action, Indoor Air Quality \& Its Impact on Man.

ECA (European Collaborative Action). 1997. Report No. 18. Evaluation of VOC emissions from building products. Solid flooring materials. Report EUR 173344 EN, European Collaborative Action, Indoor Air Quality \& its Impact on Man.

Girman, J.R., L.E. Alevantis, G.C. Kulasingam, M.X. Petreas and L.M. Webber. 1989. The bakeout of an office building: A case study. Environ. Int. 15: 449-453.

Girman, J.R., L.E. Alevantis, M.X. Petreas and L.M. Webber. 1990. Building bake-out studies. Proceedings of the 5th International Conference on Indoor Air Quality and Climate, Toronto, Canada, July 29 - August 3, Vol. 3, pp. 349-354.

Gunnarsen, L. 1997. The influence of area-specific ventilation rate on the emissions from construction products. Indoor Air 7: 116-120.

Heller, S.R. and G.W.A. Milne. 1978. EPA/NIH Mass Spectral Database. U.S. Nat. Bur. Stand., Nat Stand. Ref. Data Ser., 63, U.S. Government Printing Office, Washington D.C.

Hetes, R.G., D.S. Womack, T.K. Pierson and D.F. Naugle. 1992. Evaluation of exposures to volatile organics offgassing from new carpets. Final Report to U.S. EPA under Contract No. CR-815509. Report 4479-001/12-F, Research Triangle Institute, Research Triangle Park, NC.

Hicks, J., K. Worl and K. Hall. 1990. Building bake-out during commissioning: Effects on VOC concentrations. Indoor Air '90, Proceedings of the 5th International Conference on Indoor Air Quality and Climate, Toronto, Canada, July 29 - August 3, Vol. 3, pp. 413-418.

Hodgson, A.T. 1995. A review and a limited comparison of methods for measuring total volatile organic compounds in indoor air. Indoor Air 5: 247-257.

Hodgson, A.T. and J.R. Girman. 1989. Application of a multisorbent sampling technique for investigations of volatile organic compounds in buildings. Design and Protocol for Monitoring Indoor Air Quality, ASTM STP 1002, N.L. Nagda and J.P. Harper, Eds., American Society for Testing and Materials, Philadelphia, PA, pp. 244-256.

Hodgson, A.T. and T.A. Phan. 1994. Volatile organic chemical emissions from carpet cushions: Screening measurements. Final Report to U.S. Consumer Product Safety Commission under Interagency Agreement CPSC-IAG-92-1134. Report LBL-35334, Lawrence Berkeley Laboratory, Berkeley, CA.

Hodgson, A.T. and J.D. Wooley. 1991. Assessment of indoor concentrations, indoor sources and source emissions of selected volatile organic compounds. Final Report to California Air Resources Board under Contract No. A933-063. 


\section{REFERENCES, CONTINUED}

Hodgson, A.T., J.D. Wooley and J.M. Daisey. 1992. Volatile organic chemical emissions from carpets. Final report to U.S. Consumer Product Safety Commission under Interagency Agreement CPSC-IAG-90-1256. Report LBL-31916, Lawrence Berkeley Laboratory, Berkeley, CA.

Hodgson, A.T., J.D. Wooley and J.M. Daisey. 1993. Emissions of volatile organic compounds from new carpets measured in a large-scale environmental chamber. J. Air Waste Manage. Assoc. 43: 316-324.

Kovanen, K., O. Seppänen, K. Sirèn and A. Majanen. 1987. Turbulent air flow measurements in ventilated spaces. Indoor Air '87, Proceedings of the 4th International Conference on Indoor Air Quality and Climate, Berlin, West Germany, Aug. 17 - 21, Vol. 3, pp. 329-333.

Ku, H.H. 1969. Statistical concepts in metrology. In: Precision Measurements and Calibration, Statistical Concepts and Procedures. U.S. National Bureau of Standards, NBS Spec. Publ. No. 300 , Vol. 1.

Leukroth, R.W. 1991. Carpet Policy Dialogue. Compendium Report. Report prepared for Office of Toxic Substances, U.S. Environmental Protection Agency, Washington, D.C, September 27, 1991.

Levin, H. and A.T. Hodgson. 1996. Screening and selecting building materials and products based on their emissions of volatile organic compounds (VOCs). Characterizing Sources of Indoor Air Pollution and Related Sink Effects, ASTM STP 1287, B.A. Tichenor, Ed., American Society for Testing and Materials, Philadelphia, PA, pp. 376-391.

Little, J.C., A.T. Hodgson and A.J. Gadgil. 1994. Modeling emissions of volatile organic compounds from new carpets. Atmos. Environ. 28: 227-234.

Maroni, M. and B. Lundrgen. 1998. Assessment of the health and comfort effects of chemical emissions from building materials: the state of the art in the European Union. Indoor Air Suppl. 4: 26-31.

Matson, N.E. and H.E. Feustel. 1998. Residential ventilation systems, Report 98-7. Final report to the New York State Energy Research and Development Authority and the California Institute for Energy Efficiency. Lawrence Berkeley National Laboratory, Berkeley, CA.

Offermann, F.J., S.A. Loiselle, G.D. Ander and H. Lau. 1993. Indoor contaminant emission rates before and after a building bake-out. Proceedings of the 6th International Conference on Indoor Air Quality and Climate, Helsinki, Finland, July 4 - 8, Vol. 6, pp. 687-692.

Riggan, R.M. 1984. Compendium of methods for the determination of toxic organic compounds in ambient air. U.S. EPA Report EPA-600/4-84-041, Environmental Monitoring Systems Laboratory, Research Triangle Park, NC.

Schacter, L. 1990. Carpet related health complaints. U.S. Consumer Product Safety Commission Report released July, 1990.

Schaeffer, V.H. 1998. Hazard assessment of butylated hydroxytoluene from urethane carpet cushions. U.S. Consumer Product Safety Commission Report released March 2, 1998.

Schaeffer, V.H., B. Bhooshan, S.-B. Chen, J.S. Sonenthal and A.T. Hodgson. 1996. Characterization of volatile organic chemical emissions from carpet cushions. Screening measurements with carpet cushions. J. Air \& Waste Manage. Assoc. 46: 813-820.

Sheldon, L.S. and D.F. Naugle. 1994. Determination of test methods for interior architectural coatings. Revised Final Report to ICF Incorporated under U.S. EPA Contract No. 68-D20131, Work Assignment No. 2-1. Report RTI/5522/042-02 FR, Research Triangle Institute, Research Triangle Park, NC. 


\section{REFERENCES, CONTINUED}

Tichenor, B.A. and L.E. Sparks. 1996. Managing exposure to indoor air pollutants in residential and office environments. Indoor Air 6: 259-270.

USEPA (U.S. Environmental Protection Agency). 1993. Targeting indoor air pollution: EPA's approach and progress. U.S. EPA Report EPA 400-R-92-012, Washington, D.C.

van der Wal, J.F., A.K. Hoogeveen and P. Wouda. 1997. The Influence of temperature on the emission of volatile organic compounds from PVC flooring, carpet and paint. Indoor Air 7: 215-221.

Wallace, L., E. Pellizarri and C. Wendel. 1991. Total volatile organic concentrations in 2700 personal, indoor, and outdoor air samples collected in the US EPA Team studies. Indoor Air 1: 465-477.

Winberry, W.T., N.T. Murphy and R.M. Riggan. 1988. Method TO-1. Determination of volatile organic compounds in ambient air using Tenax adsorption and gas chromatography/mass spectrometry (GC/MS). In: Compendium of methods for the determination of toxic organic compounds in ambient air. U.S. EPA Report, Environmental Monitoring Systems Laboratory, Research Triangle Park, NC.

Winberry, W.T., N.T. Murphy and R.M. Riggan. 1988. Method TO-11. Determination of formaldehyde in ambient air using adsorbent cartridge followed by high performance liquid chromatography (HPLC). In: Compendium of methods for the determination of toxic organic compounds in ambient air. U.S. EPA Report, Environmental Monitoring Systems Laboratory, Research Triangle Park, NC. 


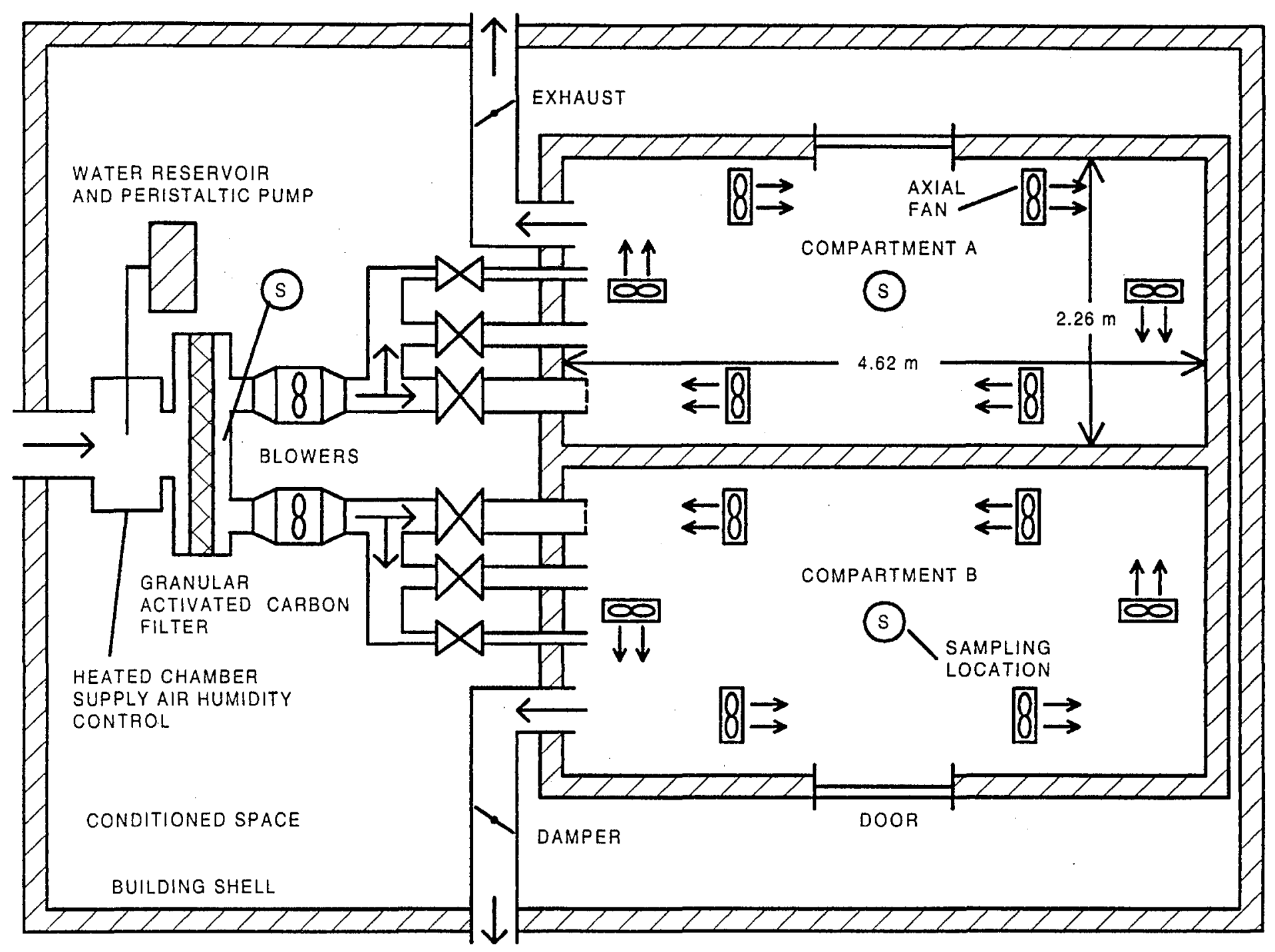

Figure 1. Schematic diagram of dual-compartment, large-scale chamber facility. 


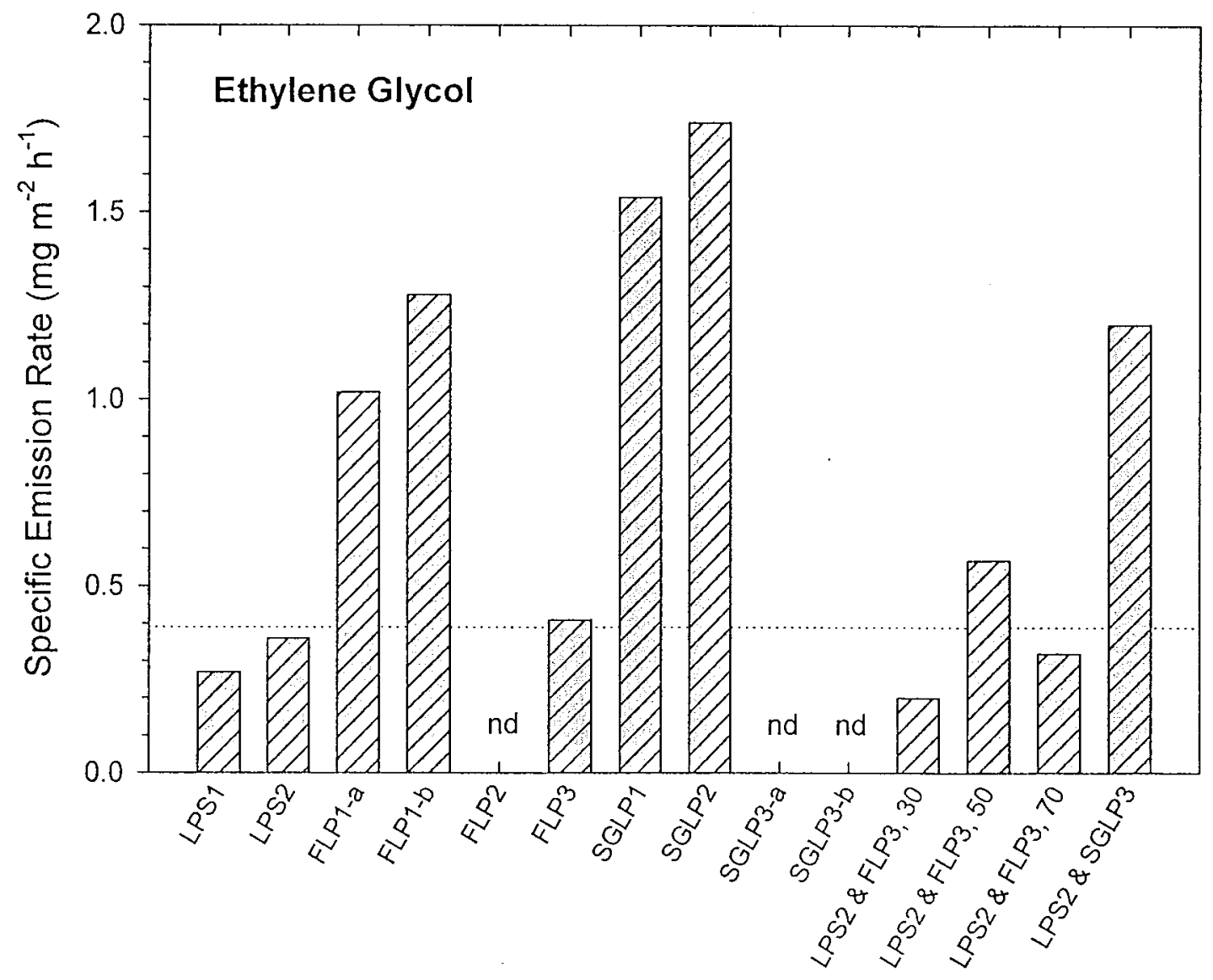

Figure 2. Area-specific emission rates $\left(\mathrm{mg} \mathrm{m}^{-2} \mathrm{~h}^{-1}\right)$ of ethylene glycol emitted at 96 hours by the individual and combined conventional paints in the screening measurements. Median (dotted line) equals $0.39 \mathrm{mg} \mathrm{m}^{-2} \mathrm{~h}^{-1}$. nd $=$ Not detected. Combined paints LPS2 and FLP3 were tested at 30,50 and $70 \% \mathrm{RH}$. Non-VOC paints did not emit Ethylene glycol. 


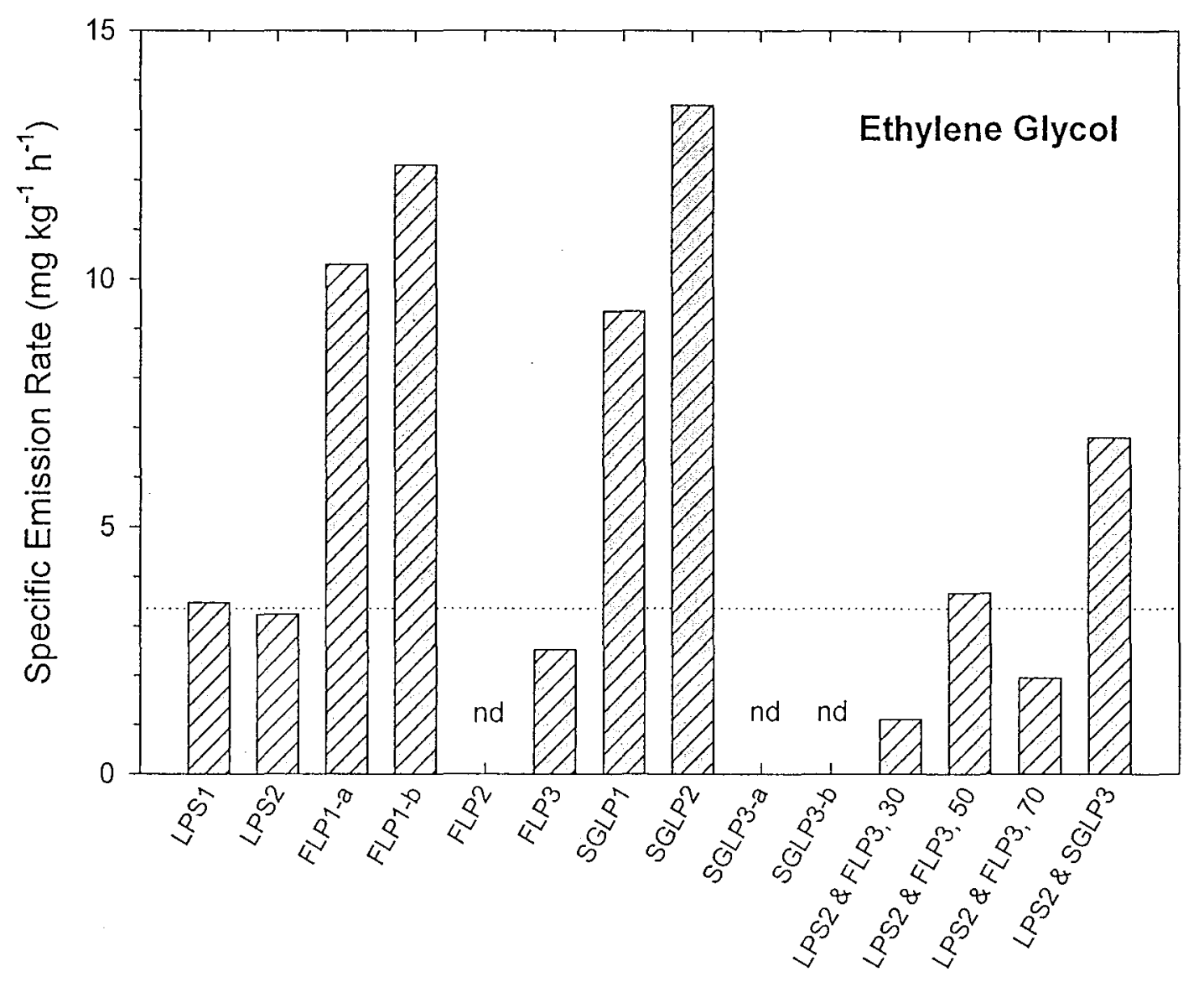

Figure 3. Mass-specific emission rates $\left(\mathrm{mg} \mathrm{kg}^{-1} \mathrm{~h}^{-1}\right)$ of ethylene glycol emitted at 96 hours by the individual and combined conventional paints in the screening measurements. Median (dotted line) equals $3.4 \mathrm{mg} \mathrm{kg}^{-1} \mathrm{~h}^{-1}$. nd $=$ Not detected. Combined paints LPS2 and FLP3 were tested at 30,50 and $70 \% \mathrm{RH}$. Non-VOC paints did not emit ethylene glycol. 


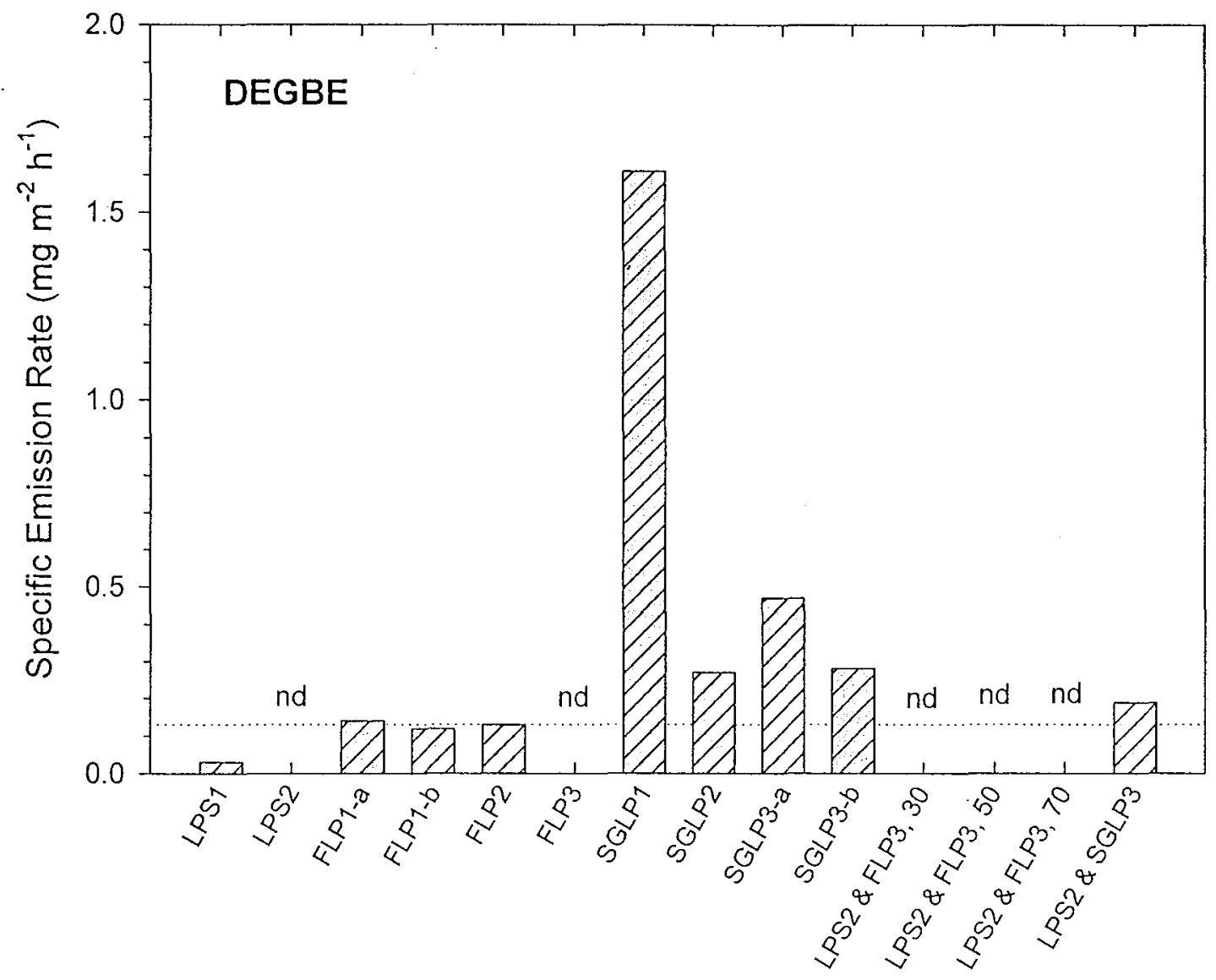

Figure 4. Area-specific emission rates $\left(\mathrm{mg} \mathrm{m}^{-2} \mathrm{~h}^{-1}\right)$ of DEGBE emitted at 96 hours by the individual and combined conventional paints in the screening measurements. Median (dotted line) equals $0.13 \mathrm{mg} \mathrm{m}^{-2} \mathrm{~h}^{-1} . \mathrm{nd}=$ Not detected. Combined paints LPS2 and FLP3 were tested at 30,50 and 70\% RH. Non-VOC paint SGLP4 emitted $0.006 \mathrm{mg} \mathrm{m}^{-2} \mathrm{~h}^{-1}$ of DEGBE at 96 hours. 


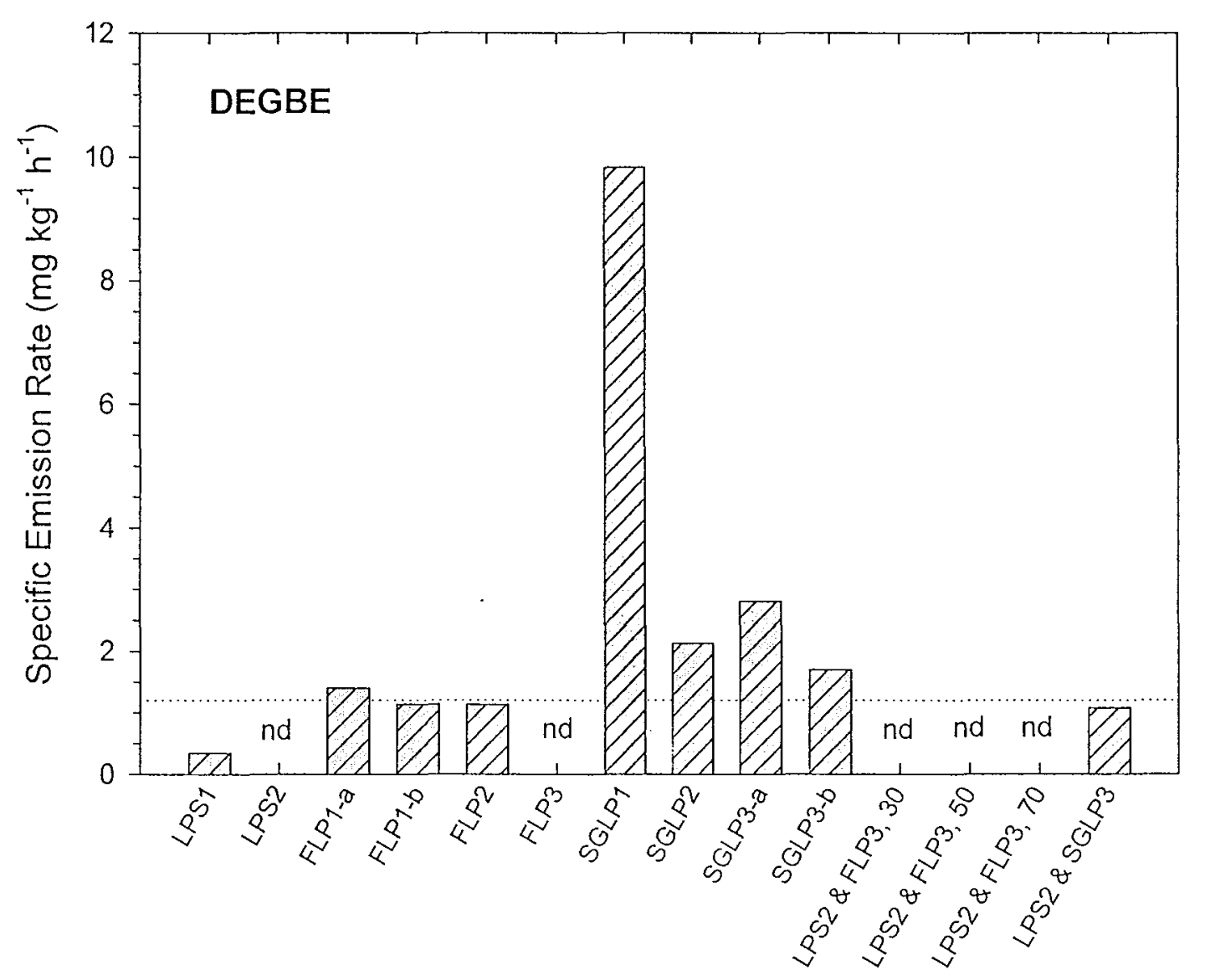

Figure 5. Mass-specific emission rates $\left(\mathrm{mg} \mathrm{kg}^{-1} \mathrm{~h}^{-1}\right)$ of DEGBE emitted at 96 hours by the individual and combined conventional paints in the screening measurements. Median (dotted line) equals $1.2 \mathrm{mg} \mathrm{kg}^{-1} \mathrm{~h}^{-1}$. nd $=$ Not detected. Combined paints LPS2 and FLP3 were tested at 30,50 and $70 \% \mathrm{RH}$. Non-VOC paint SGLP4 emitted $0.042 \mathrm{mg} \mathrm{kg}^{-1} \mathrm{~h}^{-1}$ of DEGBE at 96 hours. 


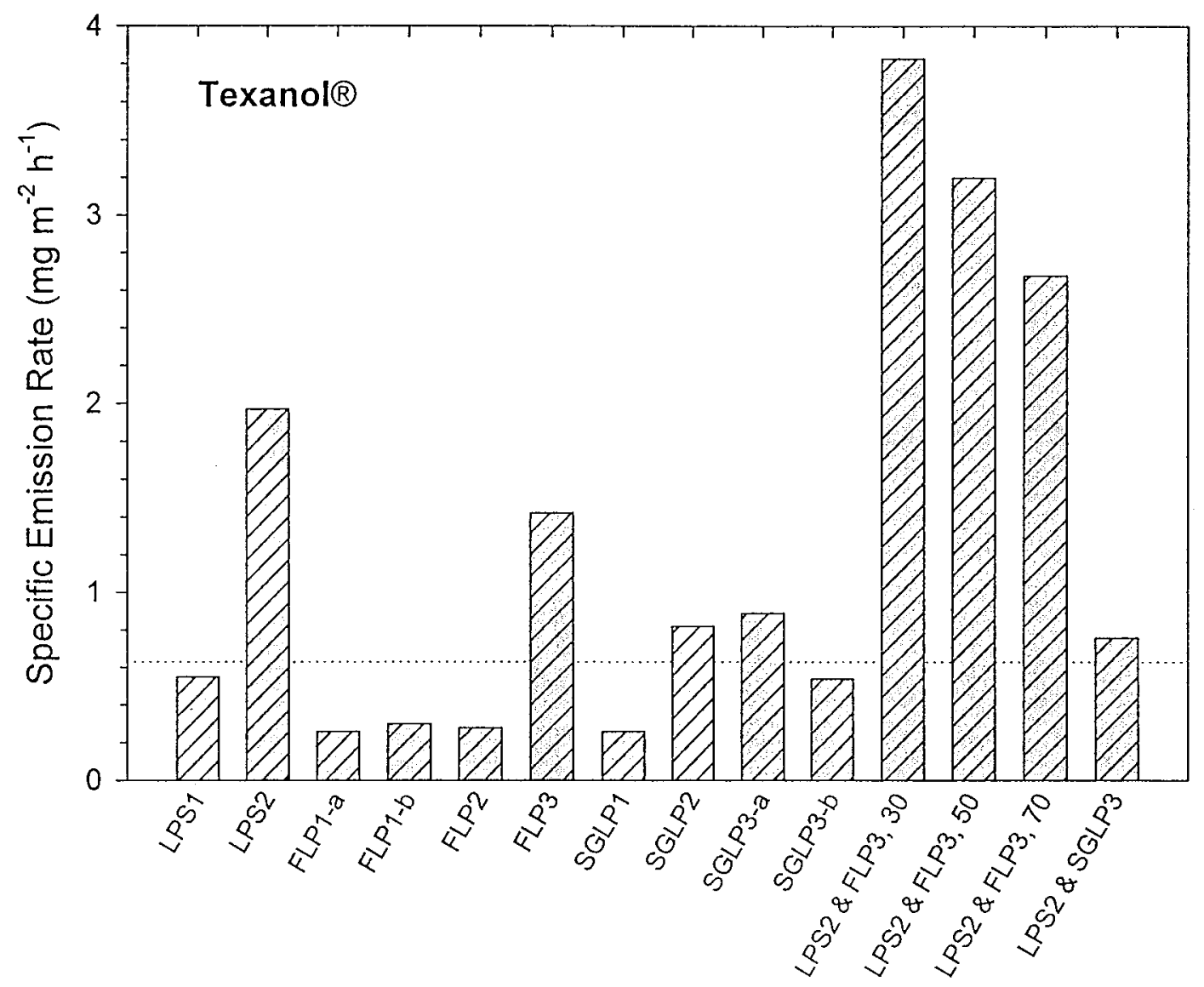

Figure 6. Area-specific emission rates $\left(\mathrm{mg} \mathrm{m}^{-2} \mathrm{~h}^{-1}\right)$ of Texanol® emitted at 96 hours by the individual and combined conventional paints in the screening measurements. Median (dotted line) equals $0.63 \mathrm{mg} \mathrm{m}^{-2} \mathrm{~h}^{-1}$. Combined paints LPS2 and FLP3 were tested at 30,50 and $70 \% \mathrm{RH}$. Non-VOC paint FLP4 emitted $0.22 \mathrm{mg} \mathrm{m}^{-2} \mathrm{~h}^{-1}$ of Texano|@ at 96 hours. 


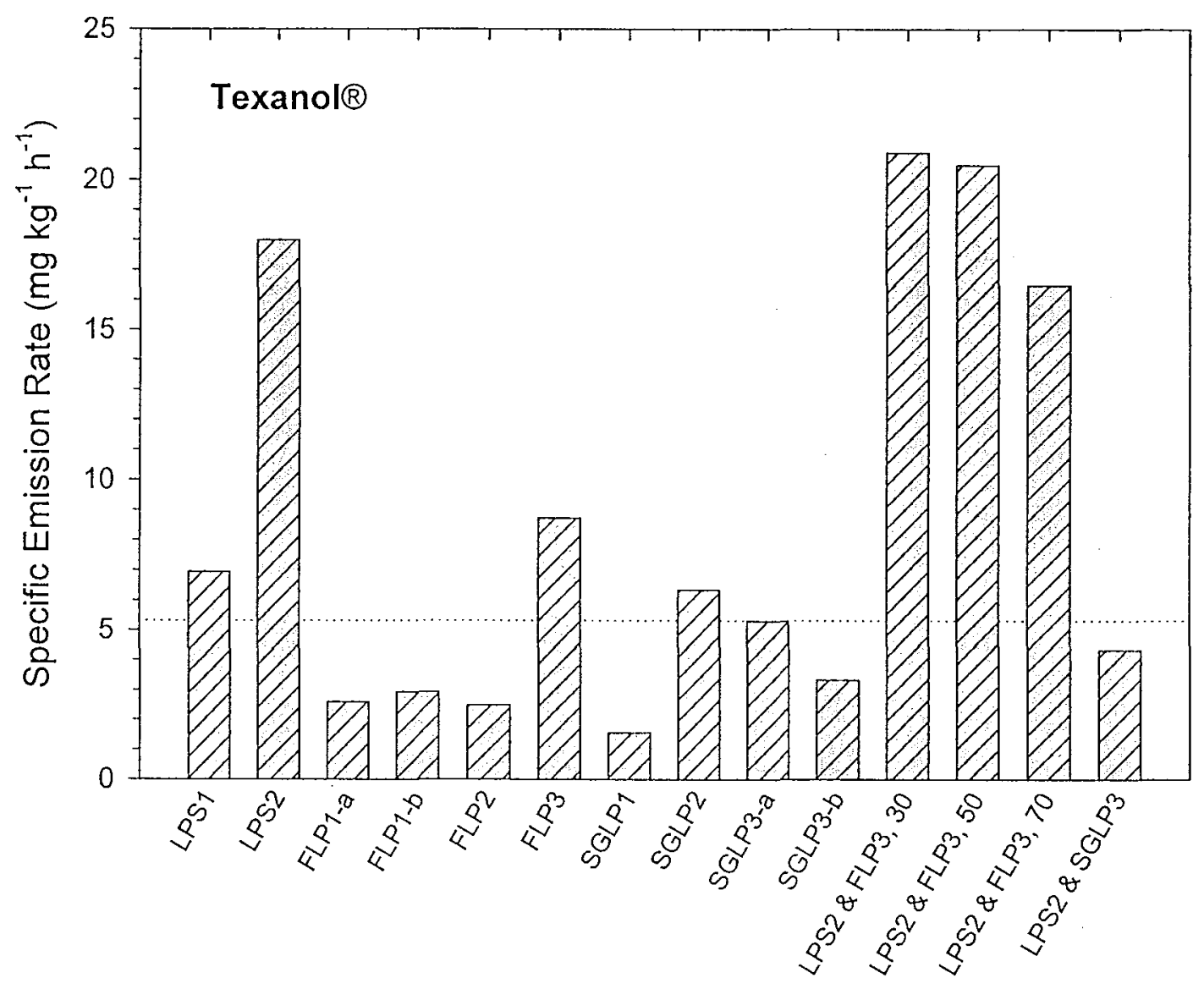

Figure 7. Mass-specific emission rates $\left(\mathrm{mg} \mathrm{kg}^{-1} \mathrm{~h}^{-1}\right)$ of Texano(ه emitted at 96 hours by the individual and combined conventional paints in the screening measurements. Median (dotted line) equals $5.3 \mathrm{mg} \mathrm{kg}^{-1} \mathrm{~h}^{-1}$. Combined paints LPS2 and FLP3 were tested at 30,50 and $70 \% \mathrm{RH}$. Non-VOC paint FLP4 emitted $0.209 \mathrm{mg} \mathrm{kg}-1 \mathrm{~h}-1$ of Texanol@ at 96 hours. 


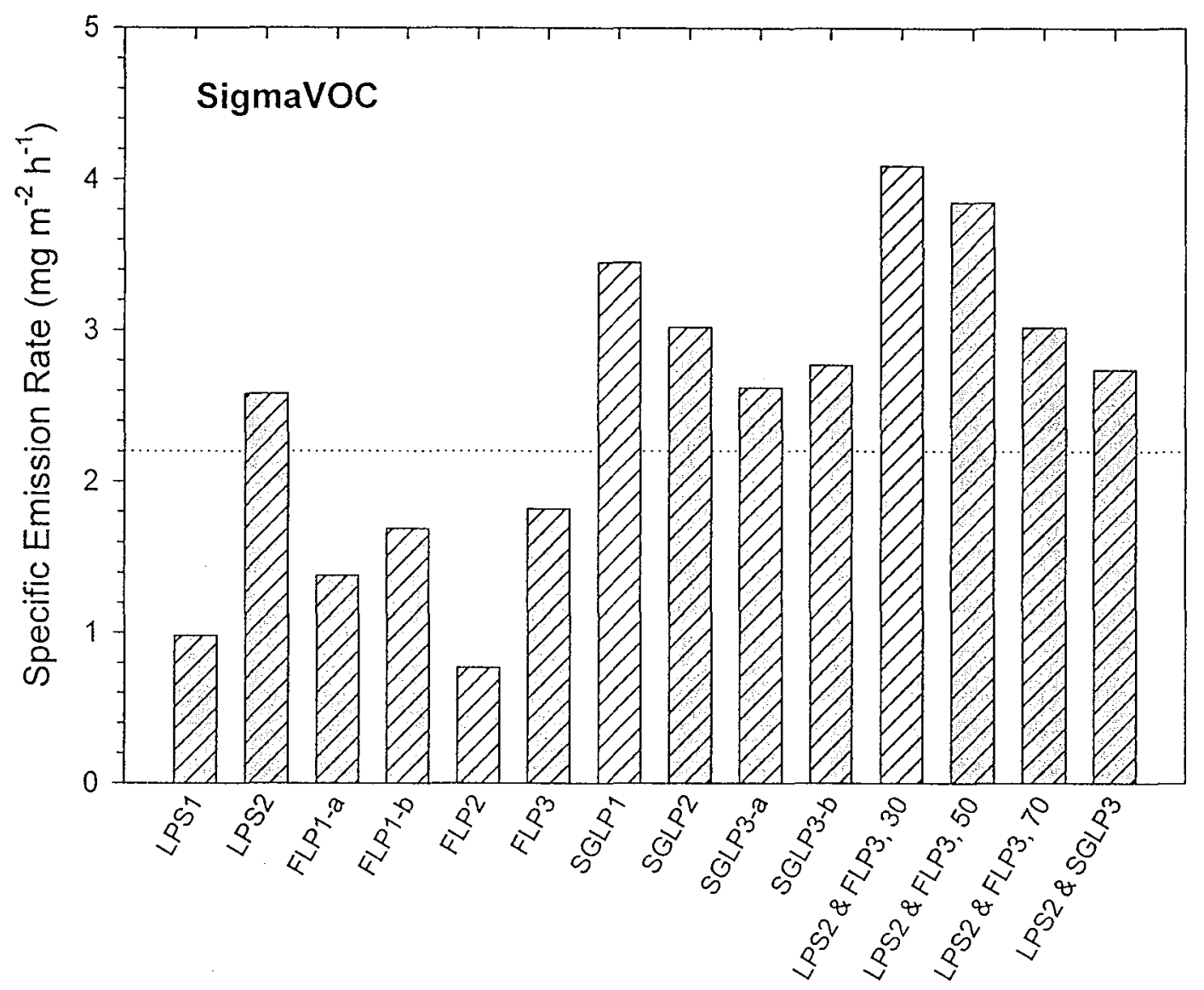

Figure 8. Area-specific emission rates $\left(\mathrm{mg} \mathrm{m}^{-2} \mathrm{~h}^{-1}\right)$ of SigmaVOC emitted at 96 hours by the individual and combined conventional paints in the screening measurements. Median (dotted line) equals $2.2 \mathrm{mg} \mathrm{m}^{-2} \mathrm{~h}^{-1}$. Combined paints LPS2 and FLP3 were tested at 30,50 and $70 \% \mathrm{RH}$. Non-VOC paints FLP4 and SGLP4 respectively emitted 0.03 and $0.09 \mathrm{mg} \mathrm{m}^{-2} \mathrm{~h}^{-1}$ of SigmaVOC at 96 hours. 


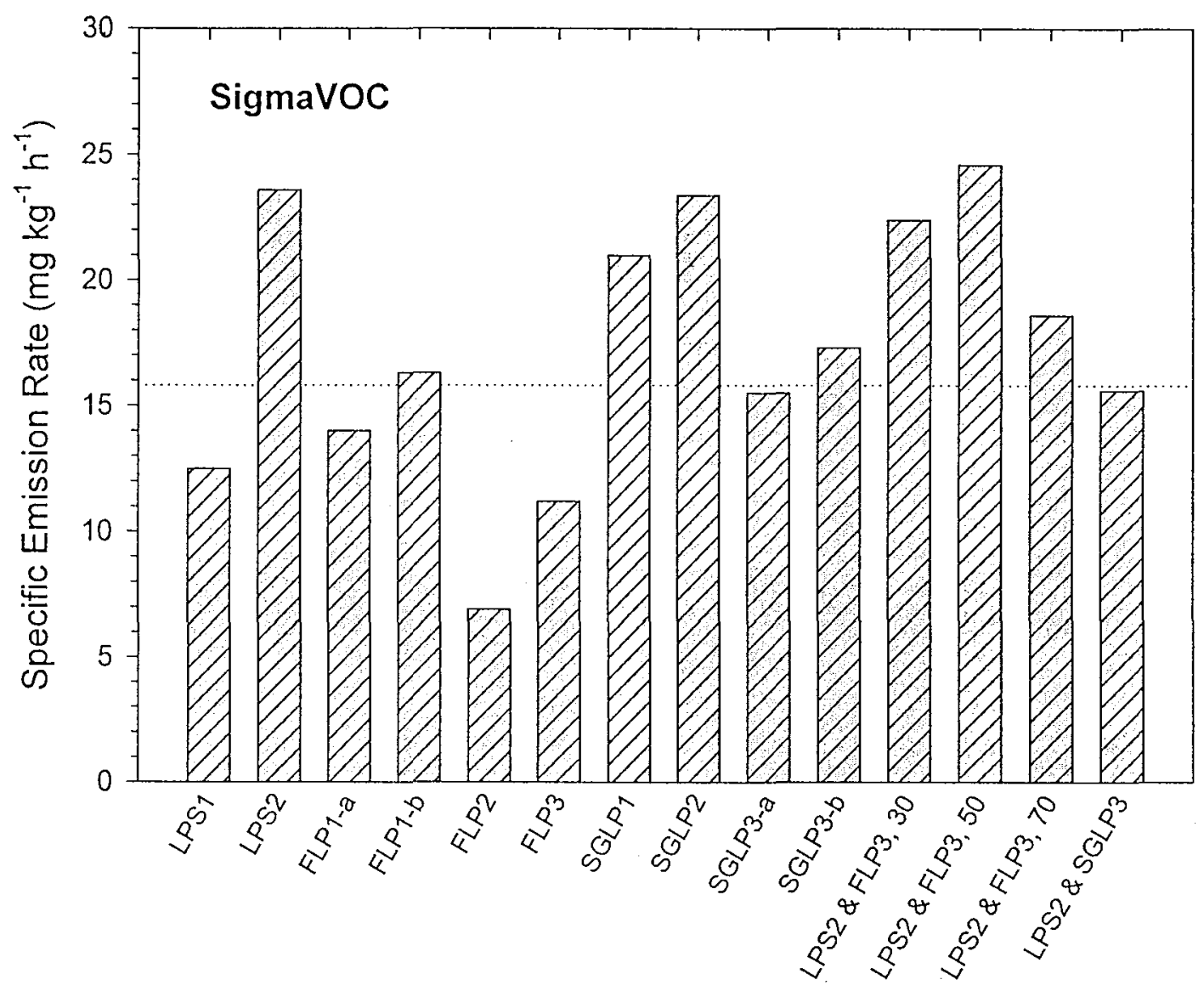

Figure 9. Mass-specific emission rates $\left(\mathrm{mg} \mathrm{kg}^{-1} \mathrm{~h}^{-1}\right)$ of SigmaVOC emitted at 96 hours by the individual and combined conventional paints in the screening measurements. Median (dotted line) equals $15.8 \mathrm{mg} \mathrm{kg}^{-1} \mathrm{~h}^{-1}$. Combined paints LPS2 and FLP3 were tested at 30,50 and $70 \%$ RH. Non-VOC paints FLP4 and SGLP4 respectively emitted 0.30 and $0.63 \mathrm{mg} \mathrm{kg}^{-1} \mathrm{~h}^{-1}$ of SigmaVOC at 96 hours. 


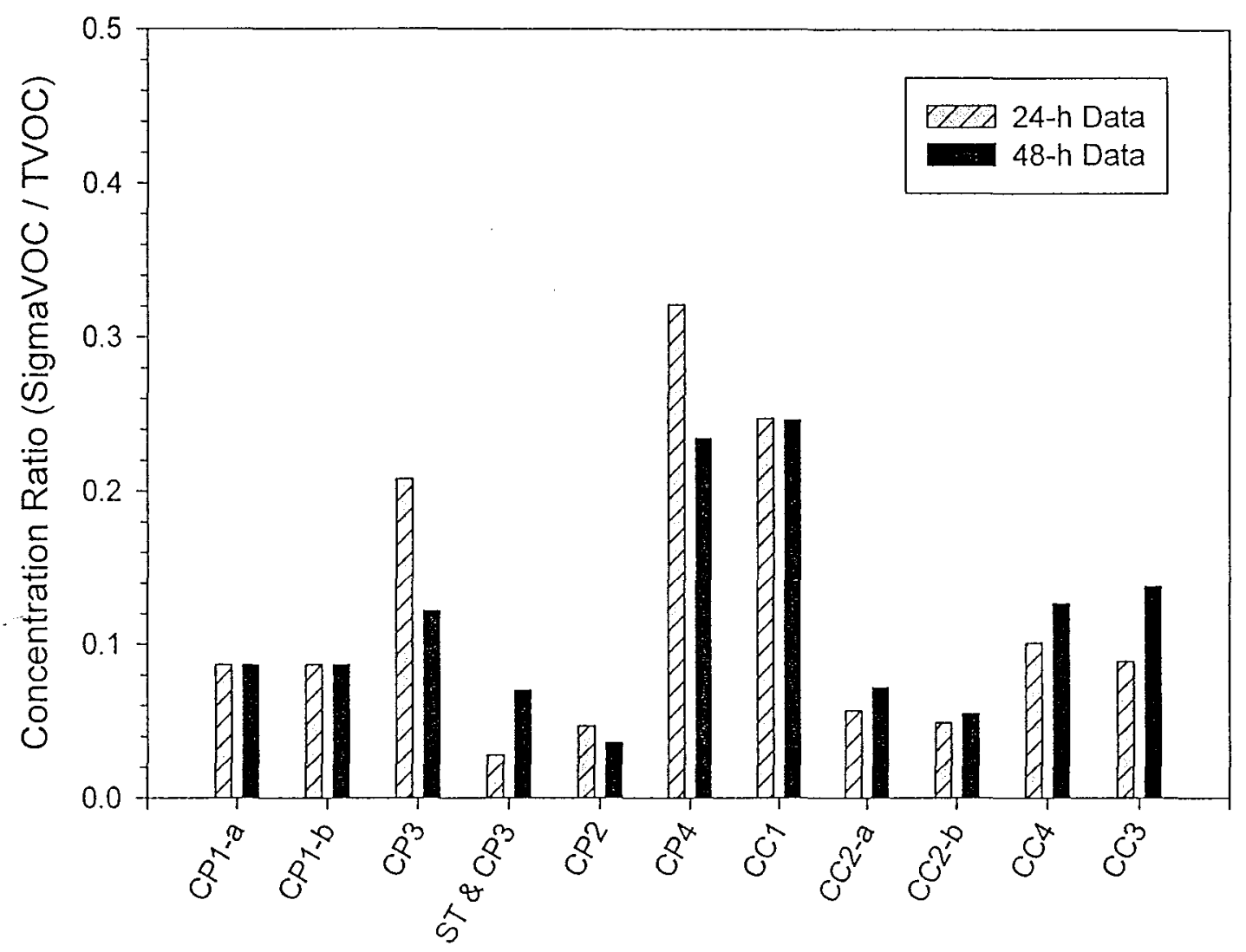

Figure 10. Concentration ratios of SigmaVOC to TVOC at 24 and 48 hours in the screening measurements of the carpet and carpet cushion materials. 


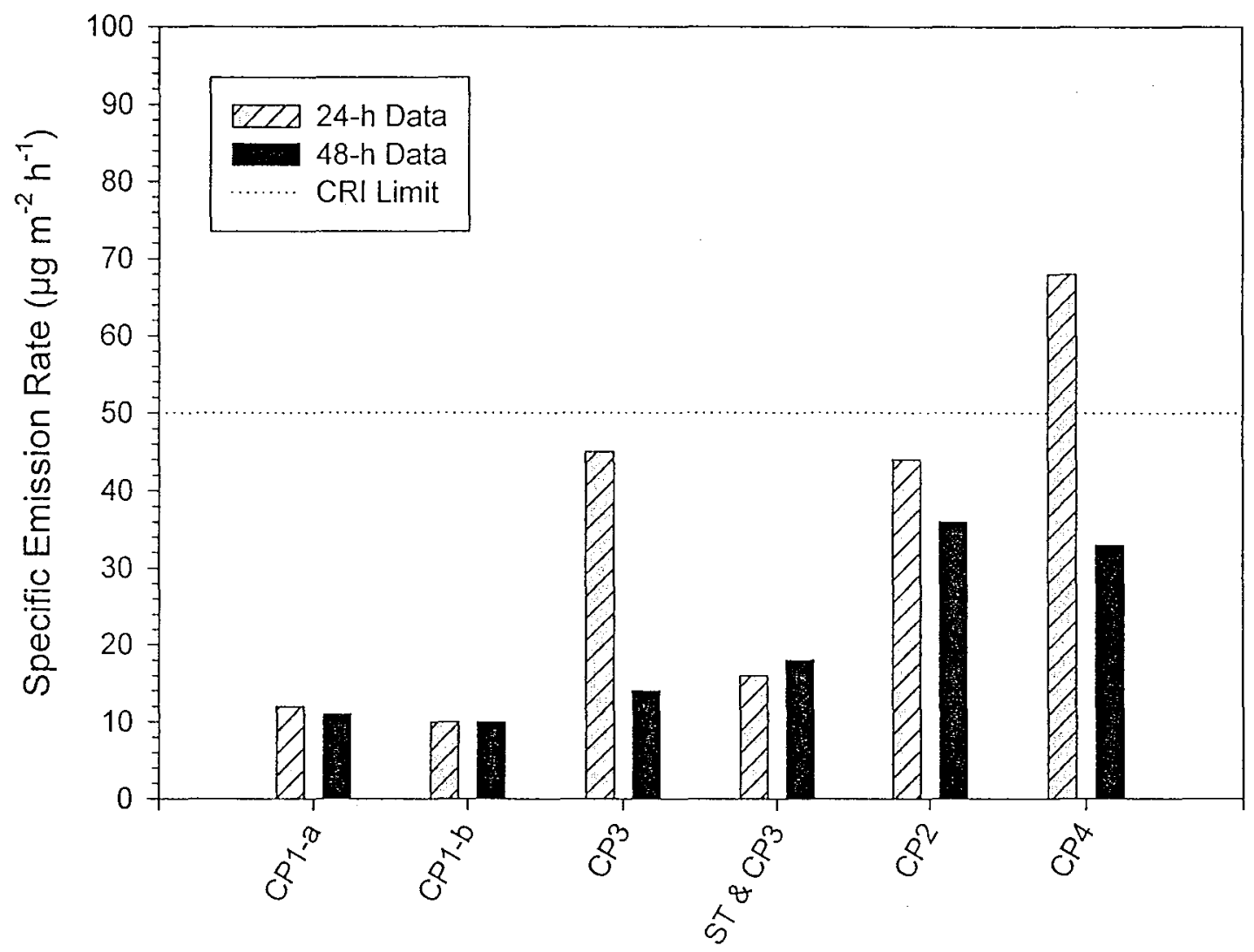

Figure 11. Specific emission rates $\left(\mu \mathrm{g} \mathrm{m}^{-2} \mathrm{~h}^{-1}\right)$ of $4-\mathrm{PCH}$ at 24 and 48 hours in the screening measurements of the carpet materials. Carpet and Rug Institute (CRI) upper limit for the 24-h emission rate of 4-PCH is $50 \mu \mathrm{g} \mathrm{m}^{-2} \mathrm{~h}^{-1}$ (dotted line). 


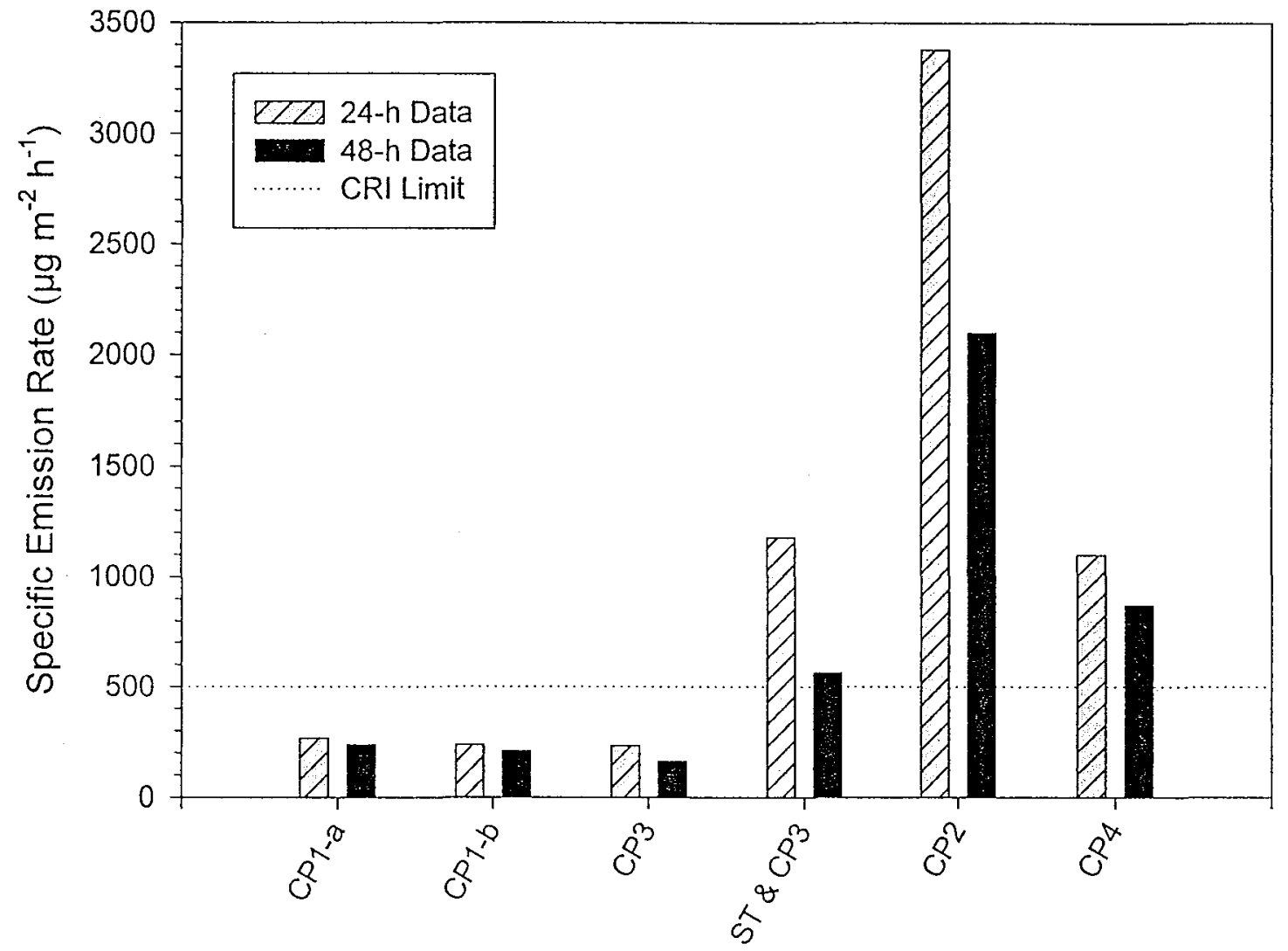

Figure 12. Specific emission rates $\left(\mu \mathrm{g} \mathrm{m}^{-2} \mathrm{~h}^{-1}\right)$ of TVOC at 24 and 48 hours in the screening measurements of the carpet materials. The Carpet and Rug Institute (CRI) upper limit for the 24 -h emission rate of TVOC is $500 \mu \mathrm{g} \mathrm{m}^{-2} \mathrm{~h}^{-1}$ (dotted line). 


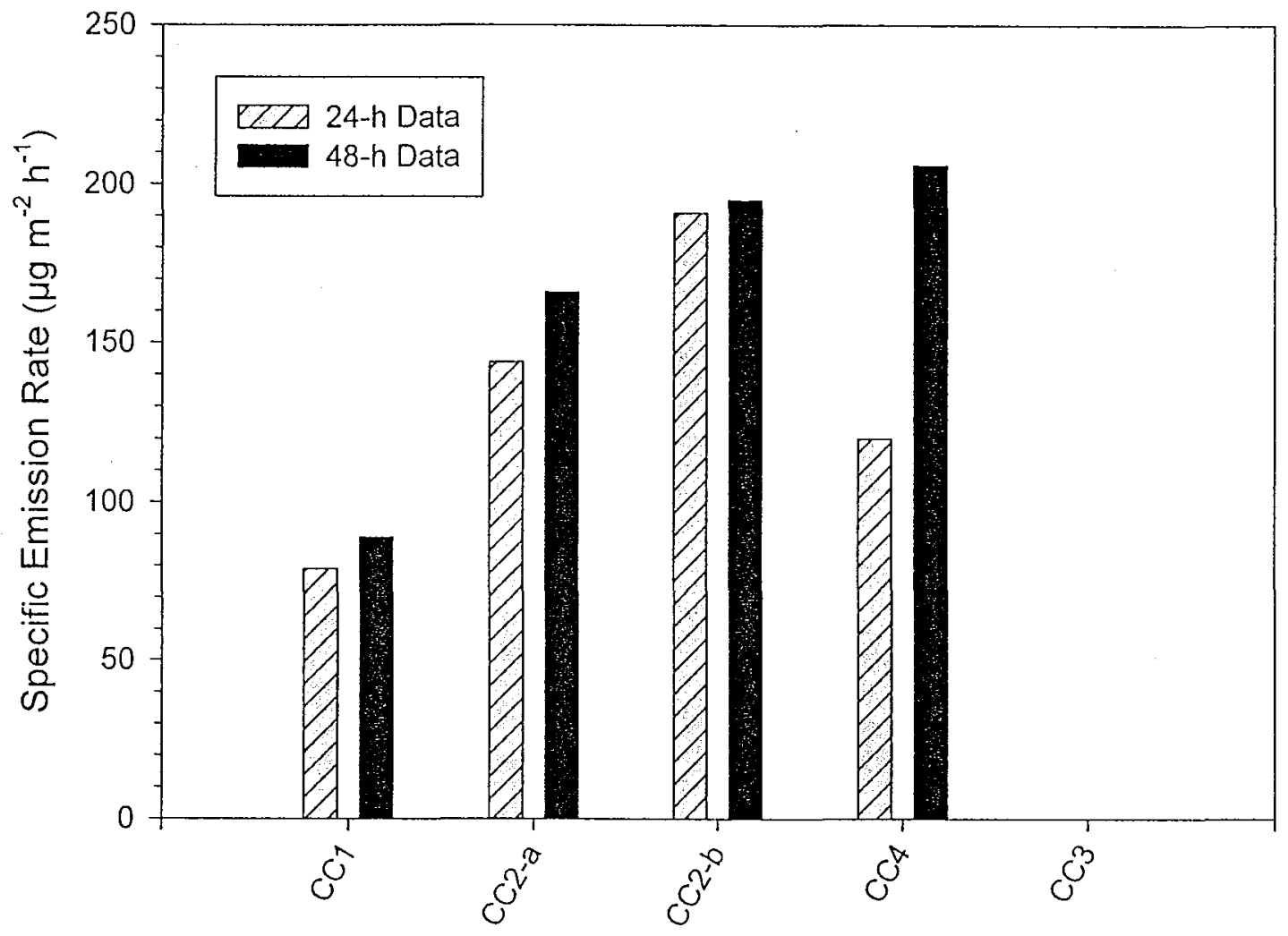

Figure 13. Specific emission rates $\left(\mu \mathrm{g} \mathrm{m}^{-2} \mathrm{~h}^{-1}\right)$ of $\mathrm{BHT}$ at 24 and 48 hours in the screening measurements of the carpet cushion materials. $n d=$ Not detected. Carpet and Rug Institute (CRI) upper limit for the 24-h emission rate of BHT from carpet cushions is $300 \mu \mathrm{g} \mathrm{m}^{-2} \mathrm{~h}^{-1}$. 


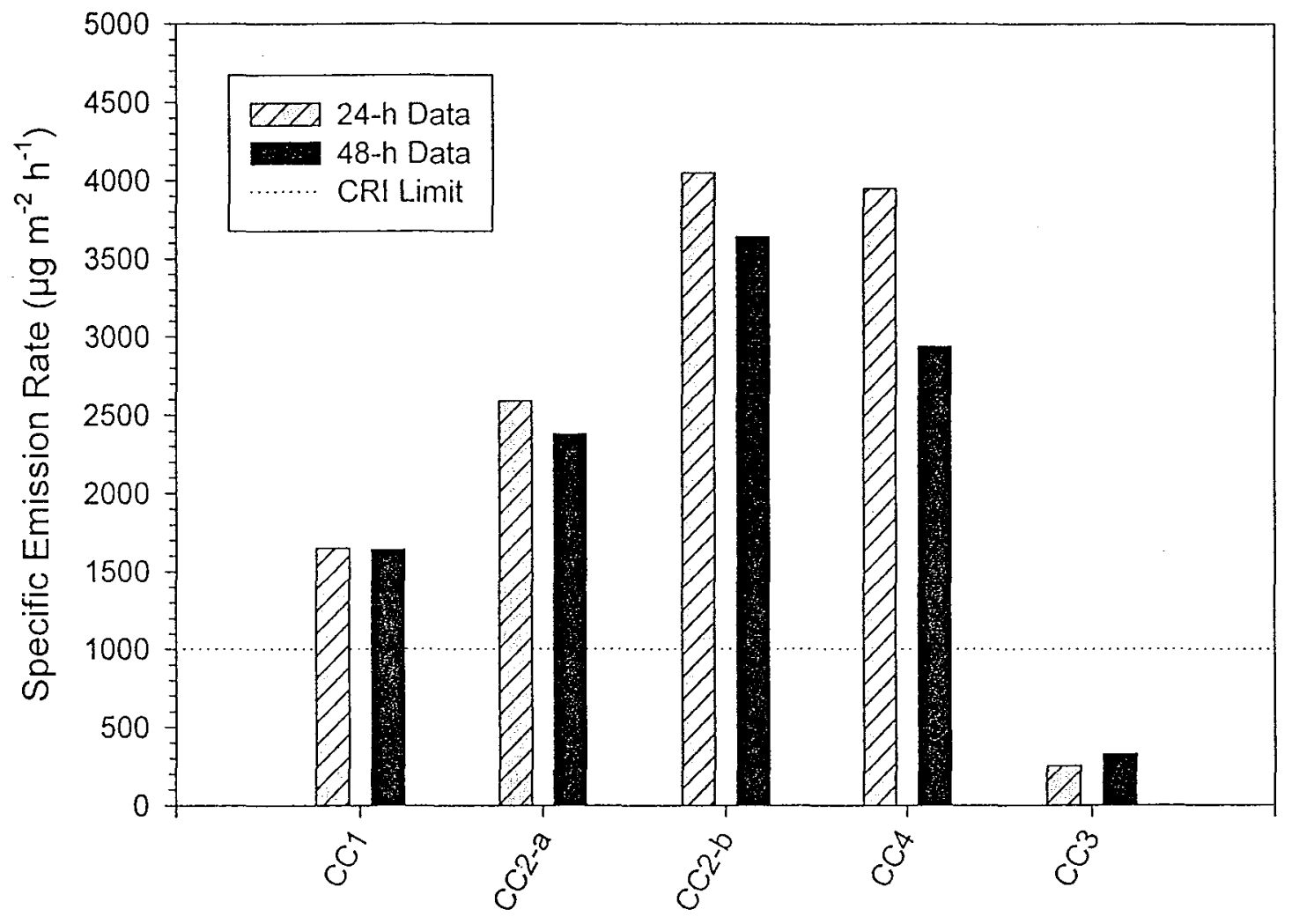

Figure 14. Specific emission rates $\left(\mu \mathrm{g} \mathrm{m}^{-2} \mathrm{~h}^{-1}\right)$ of TVOC at 24 and 48 hours in the screening measurements of the carpet cushion materials. Carpet and Rug Institute (CRI) upper limit for the 24-h emission rate of TVOC from carpet cushions is $1,000 \mu \mathrm{g} \mathrm{m}^{-2} \mathrm{~h}^{-1}$ (dotted line). 


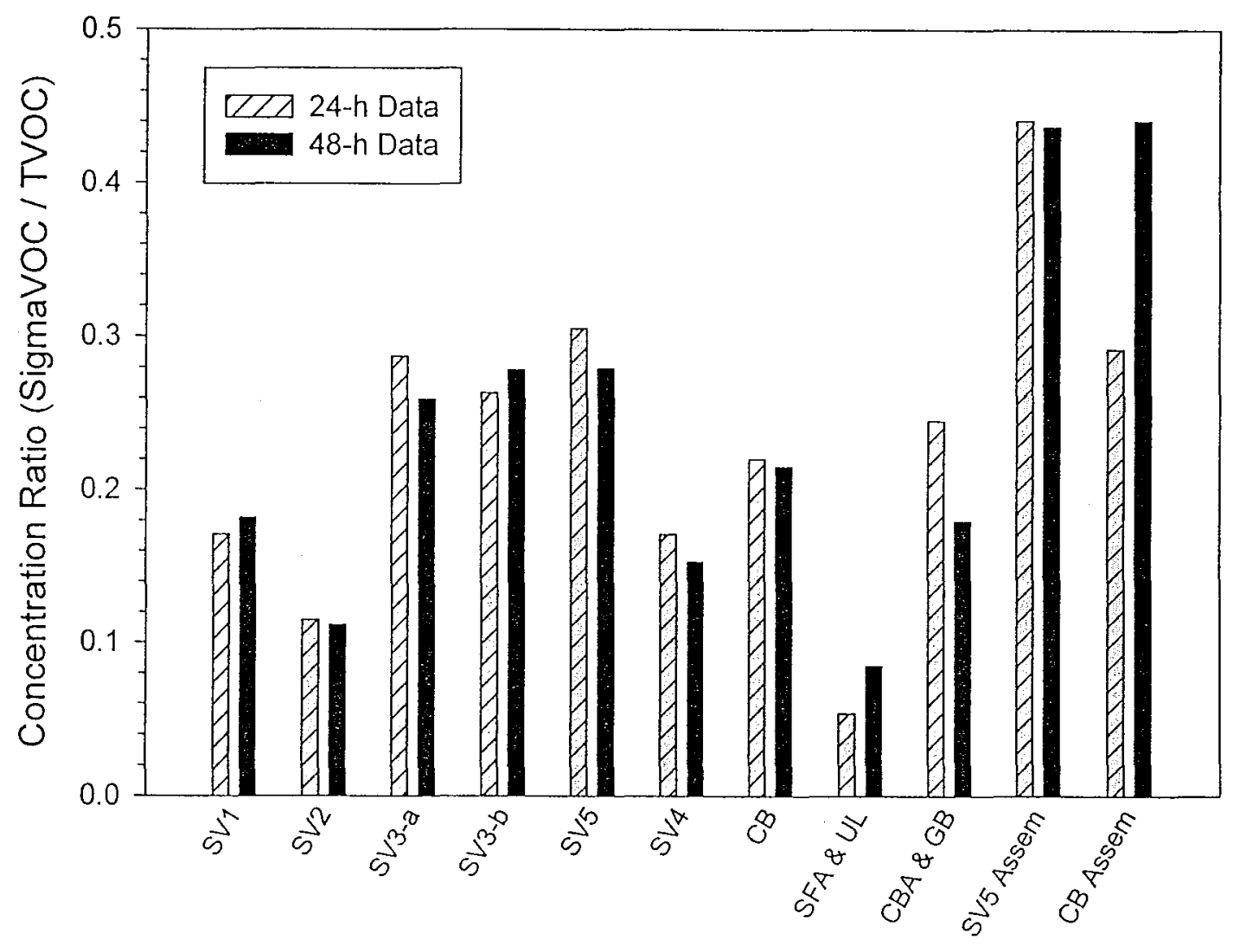

Figure 15. Concentration ratios of SigmaVOC to TVOC at 24 and 48 hours in the screening measurements of the vinyl flooring materials. 


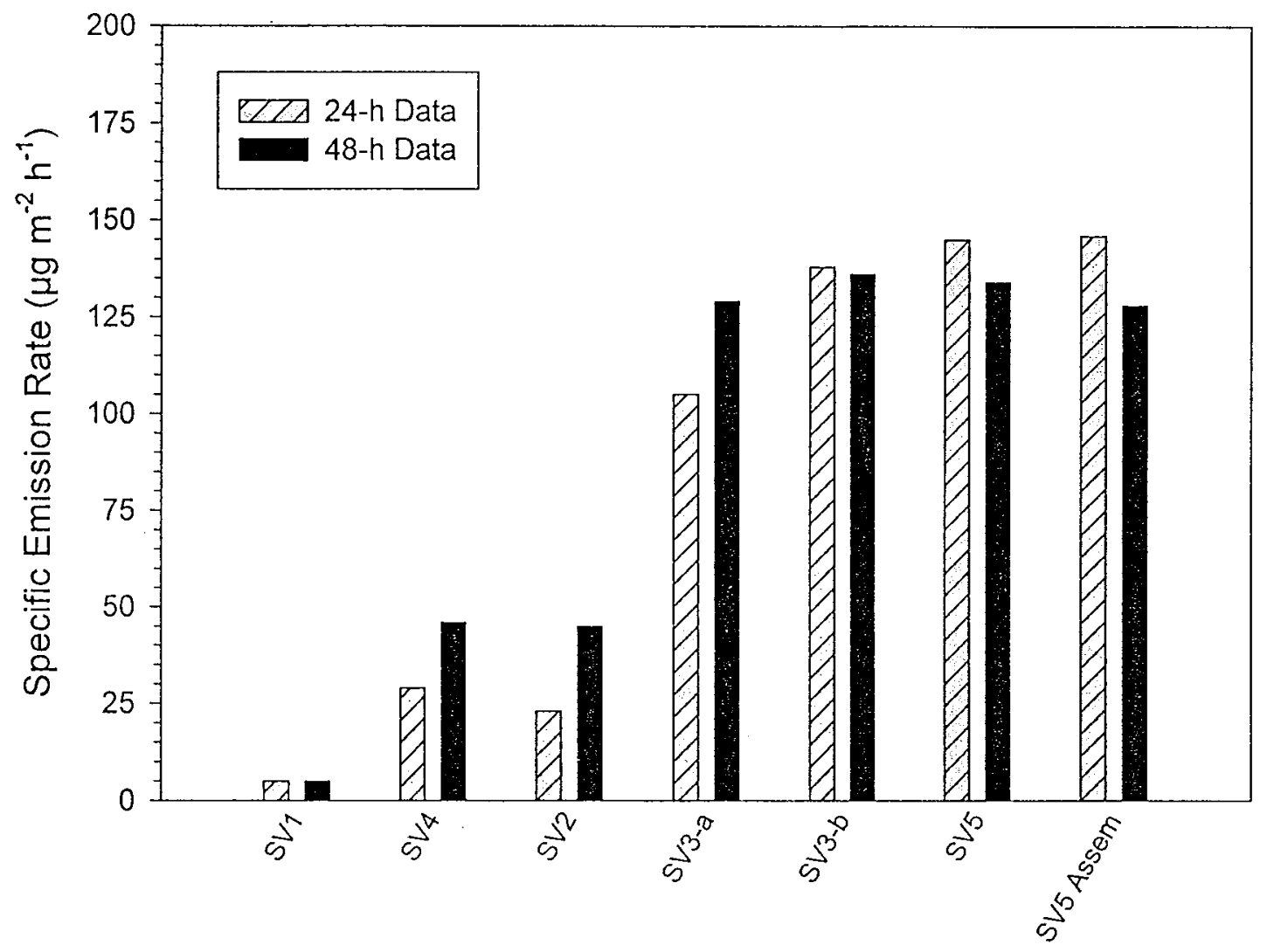

Figure 16. Specific emission rates $\left(\mu \mathrm{g} \mathrm{m}^{-2} \mathrm{~h}^{-1}\right)$ of $n$-tridecane at 24 and 48 hours in the screening measurements of the sheet vinyl materials. 


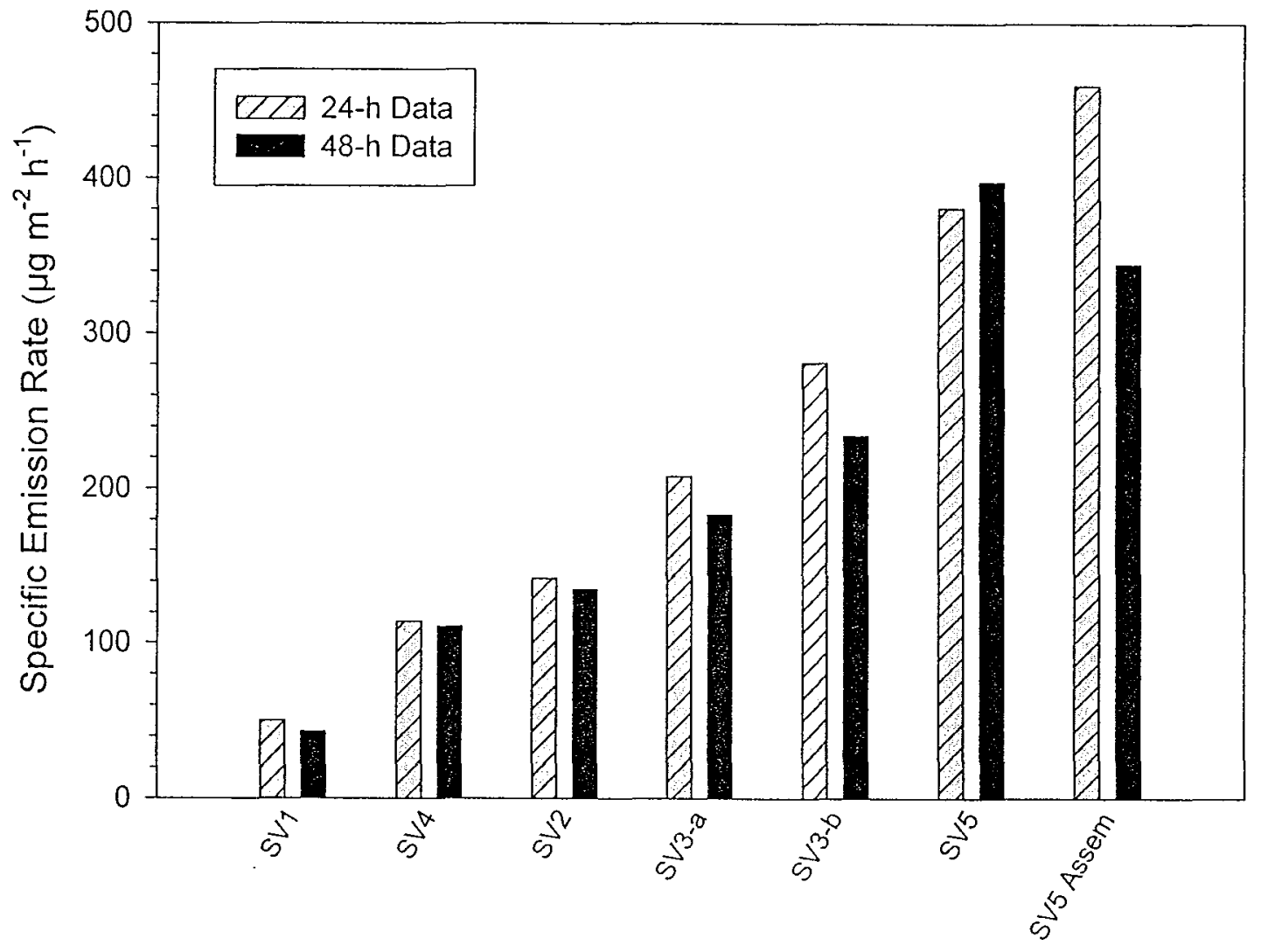

Figure 17. Specific emission rates $\left(\mu \mathrm{g} \mathrm{m}^{-2} \mathrm{~h}^{-1}\right)$ of phenol at 24 and 48 hours in the screening measurements of the sheet vinyl materials. 


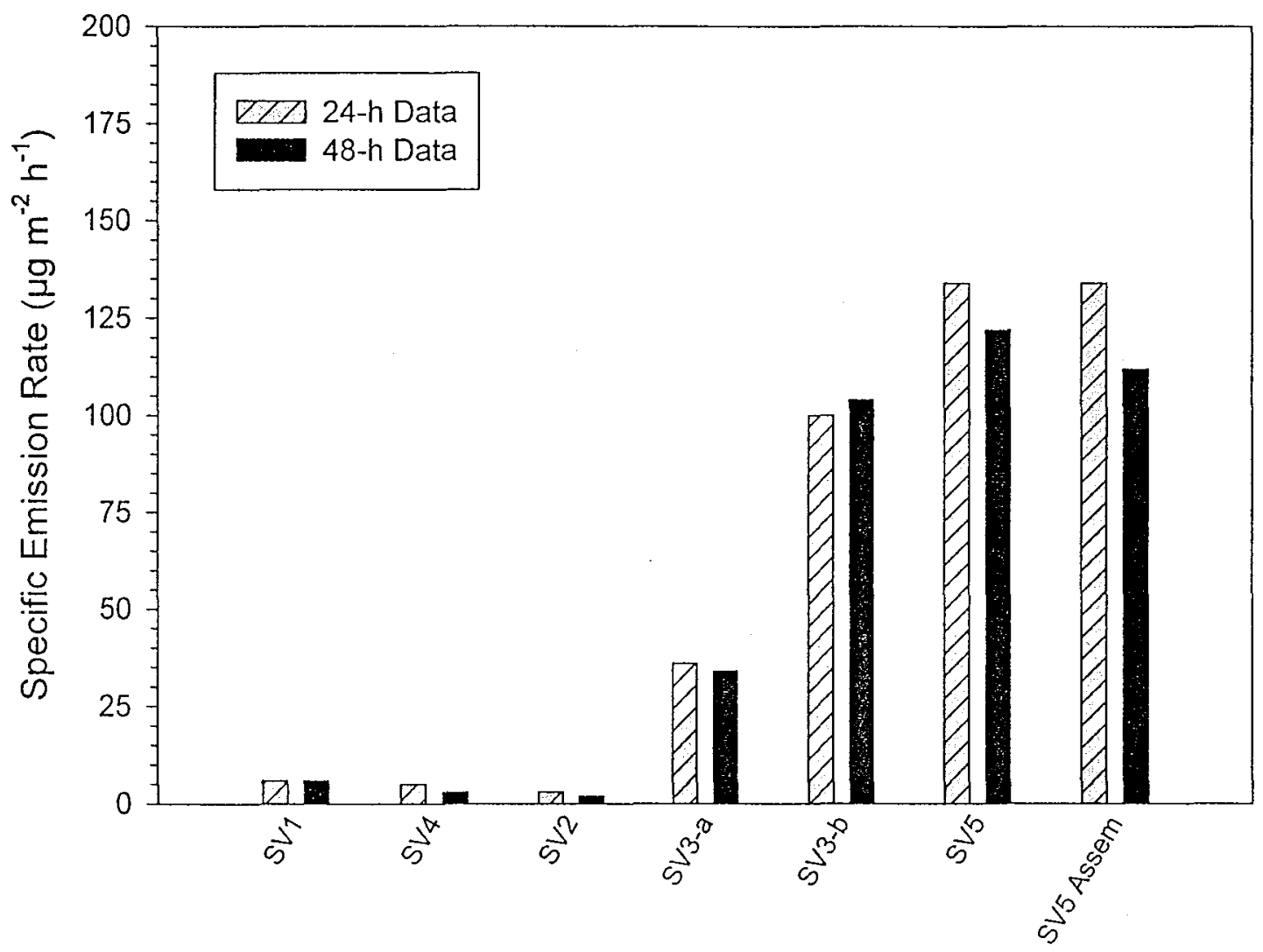

Figure 18. Specific emission rates $\left(\mu \mathrm{g} \mathrm{m}^{-2} \mathrm{~h}^{-1}\right)$ of $T X I B \circledast$ at 24 and 48 hours in the screening measurements of the sheet vinyl materials. 


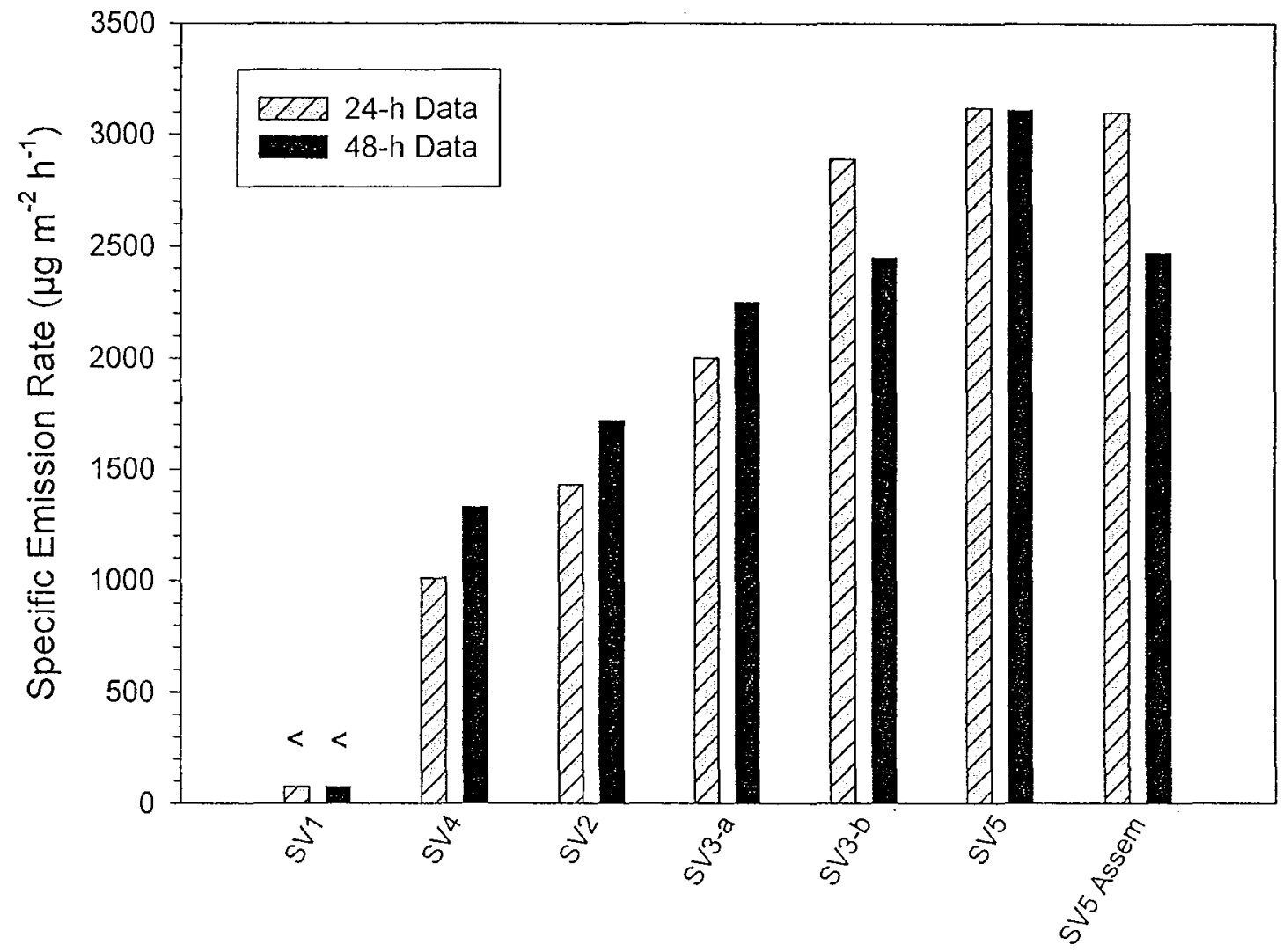

Figure 19. Specific emission rates $\left(\mu \mathrm{g} \mathrm{m}^{-2} \mathrm{~h}^{-1}\right)$ of TVOC at 24 and 48 hours in the screening measurements of the sheet vinyl materials. " $<"=$ Below lower limit of quantitation. 


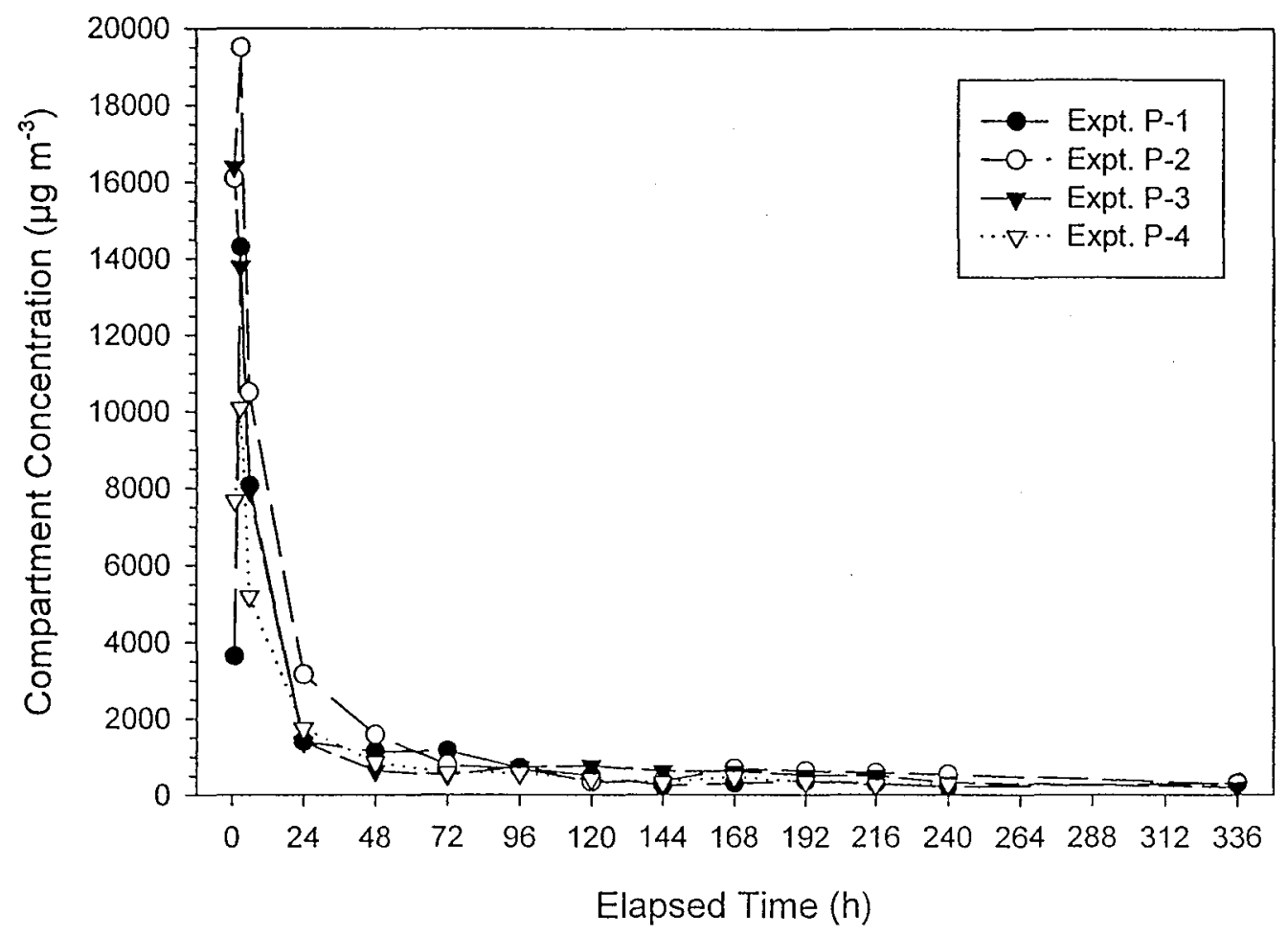

Figure 20a. Compartment concentrations $\left(\mu \mathrm{g} \mathrm{m}^{-3}\right)$ of ethylene glycol in Experiments P-1 - P-4.

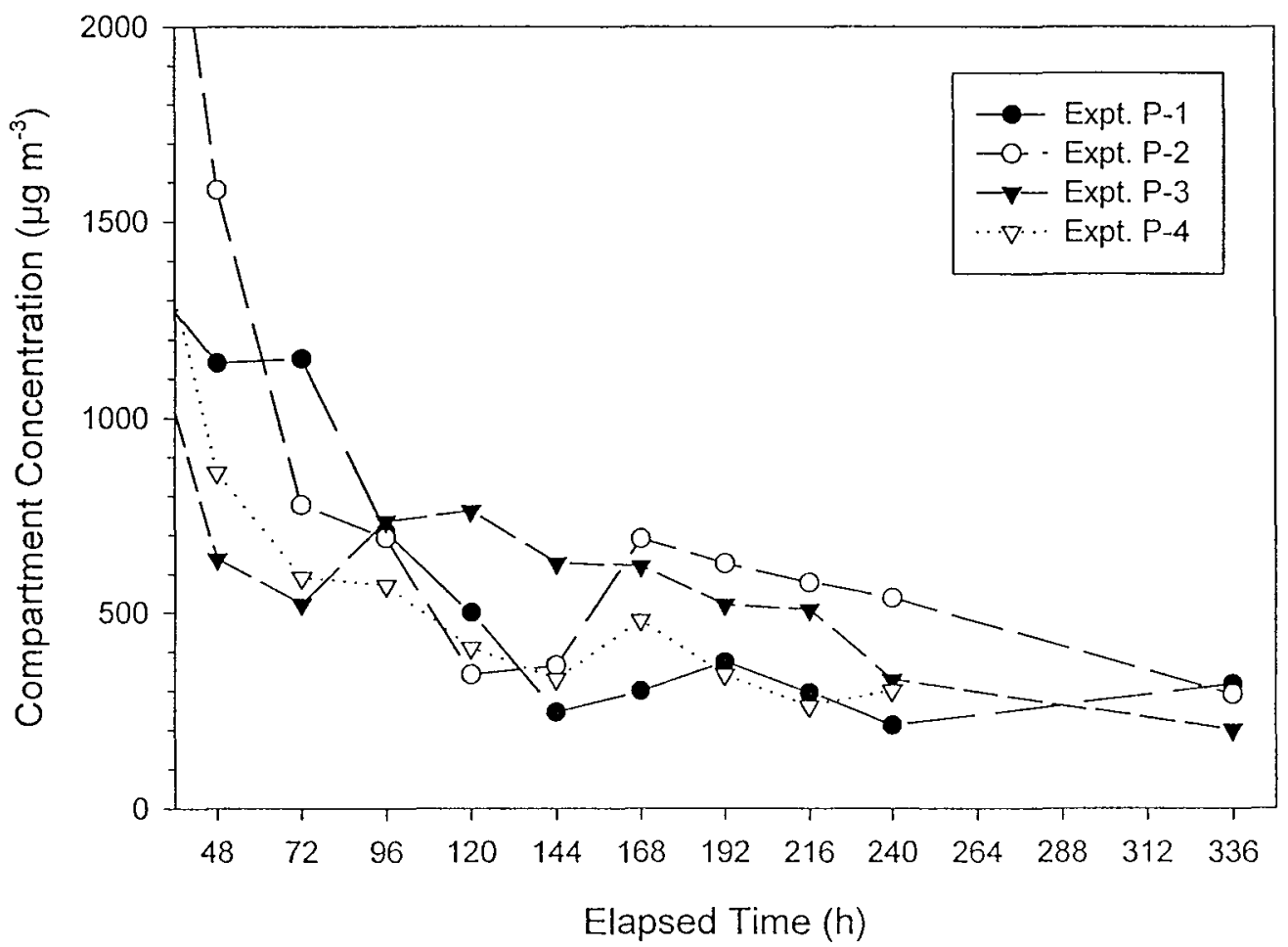

Figure 20b. Compartment concentrations $\left(\mu \mathrm{g} \mathrm{m}^{-3}\right)$ of ethylene glycol over $48-336$ hours in Experiments P-1 - P-4. 


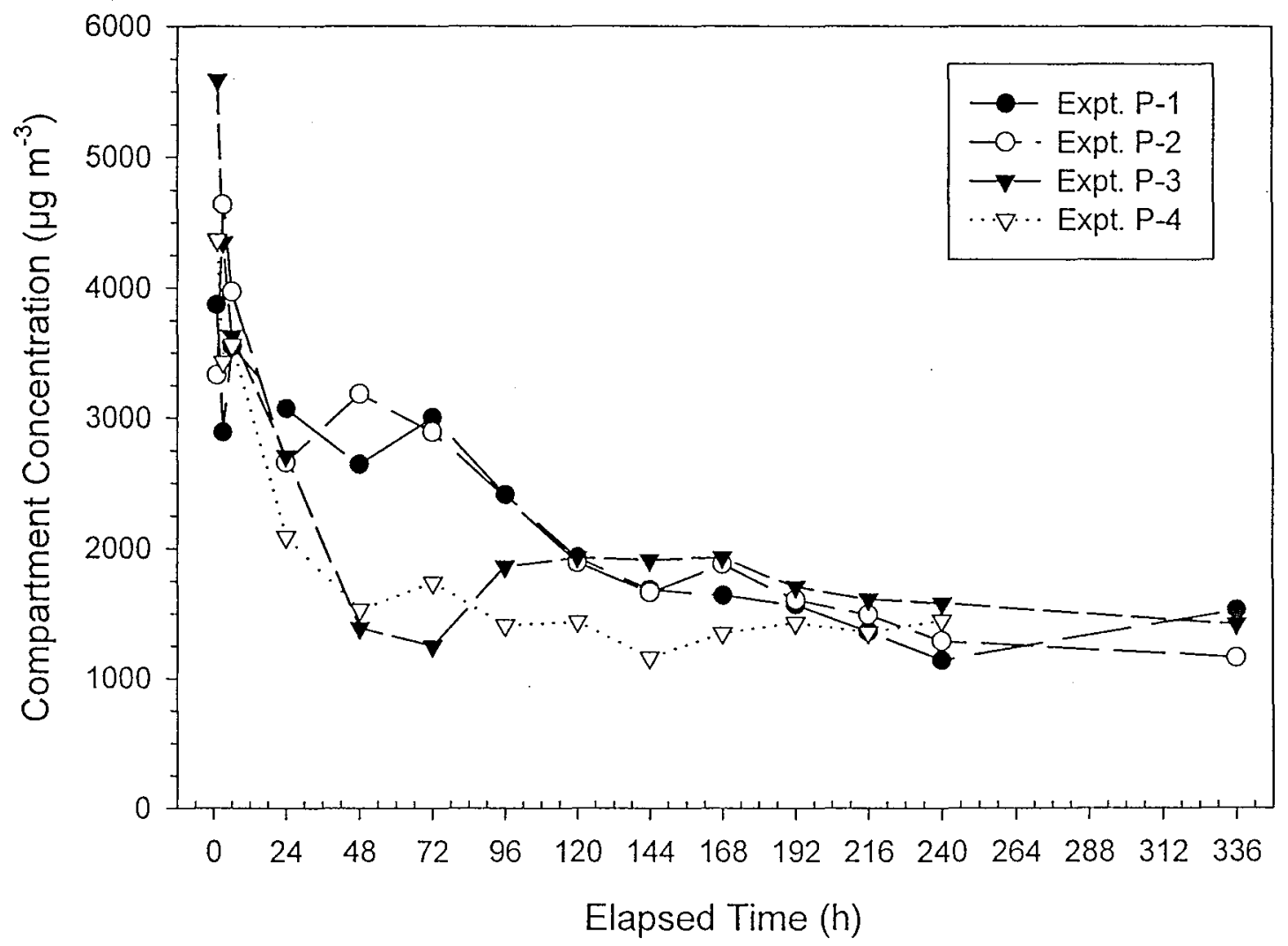

Figure 21. Compartment concentrations $\left(\mu \mathrm{g} \mathrm{m}^{-3}\right)$ of Texanol $($ in Experiments P-1 - P-4. 


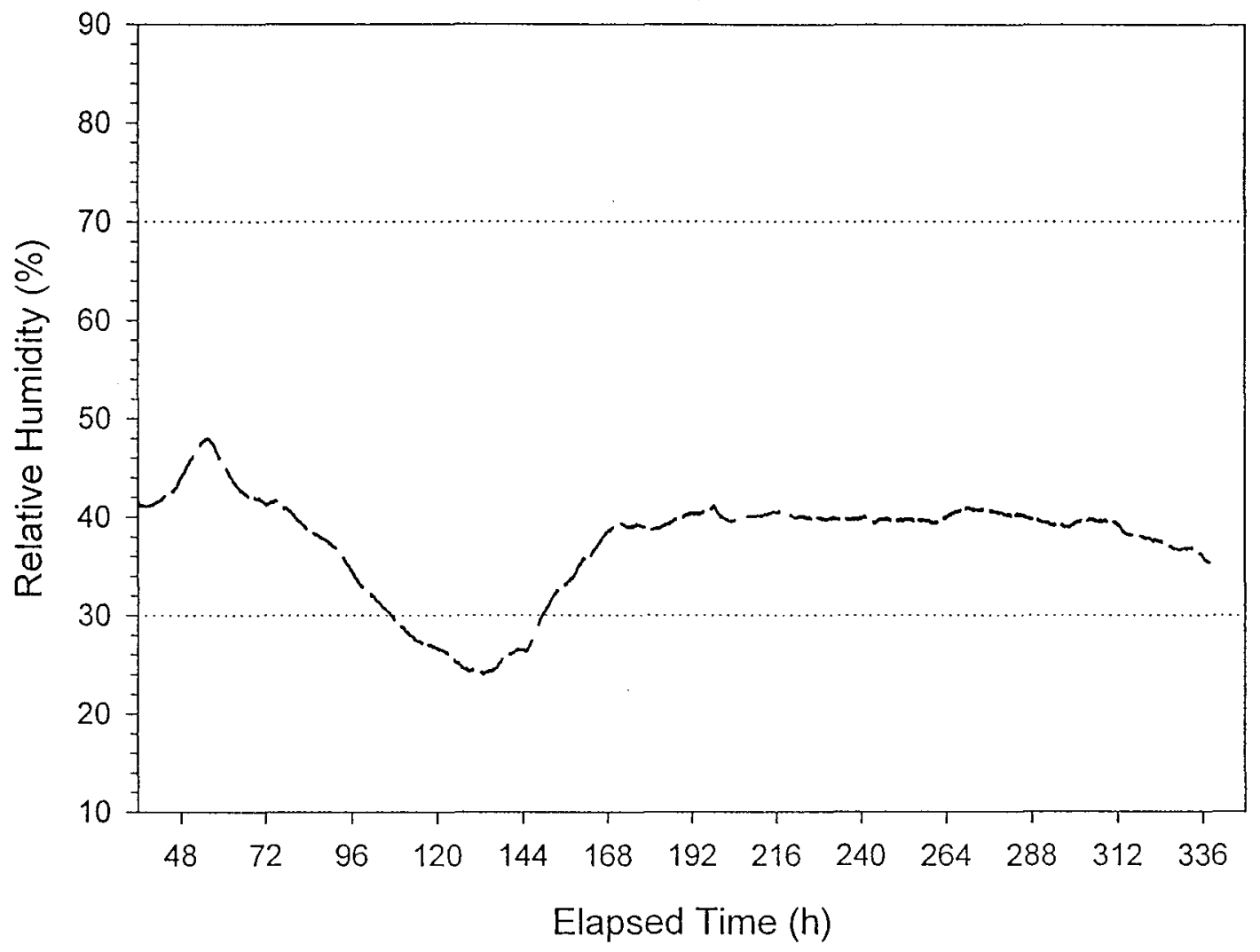

Figure 22. Compartment air relative humidity over 48 - 336 hours in Experiment P-2. 


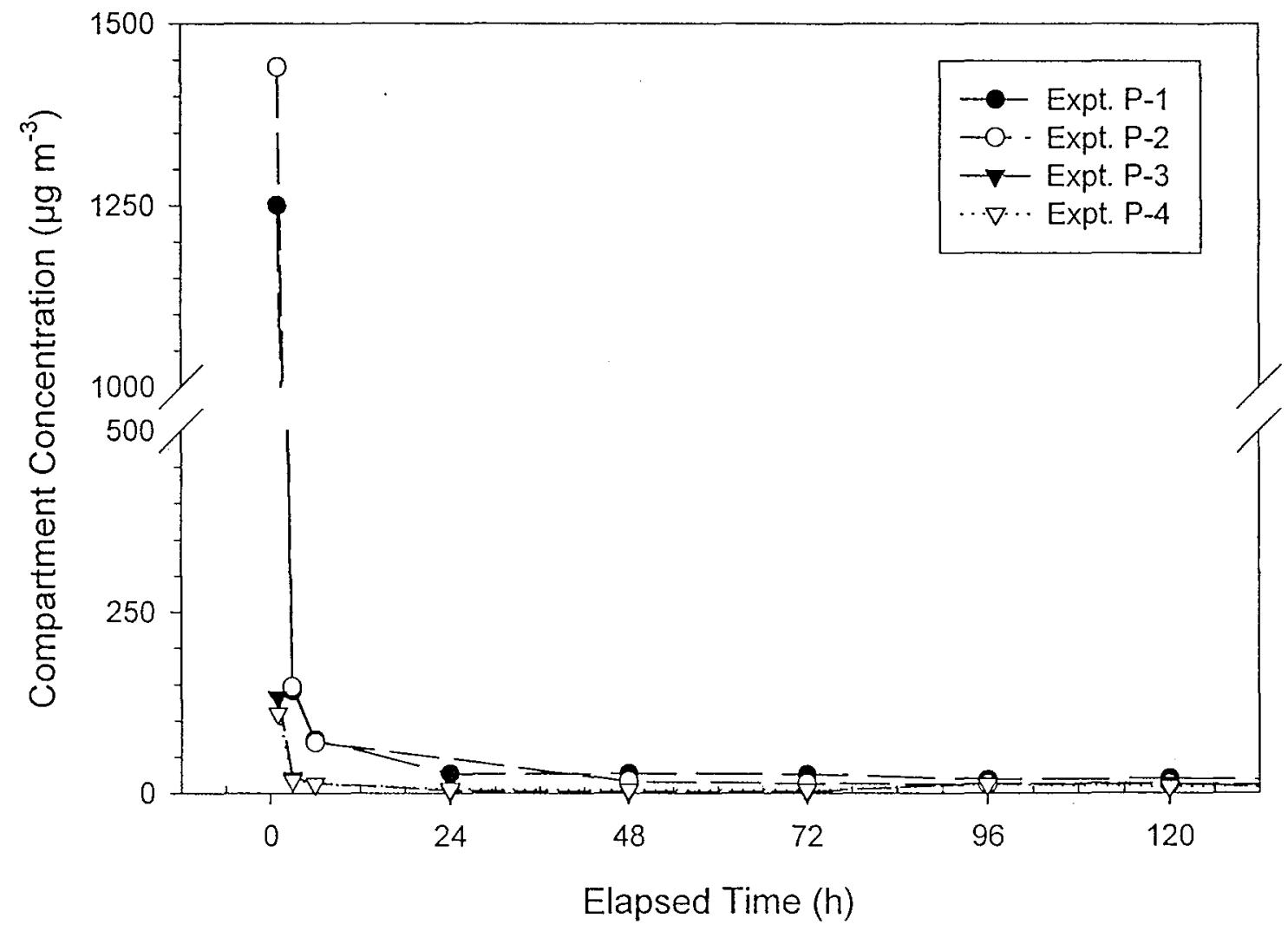

Figure 23. Compartment concentrations $\left(\mu \mathrm{g} \mathrm{m}^{-3}\right)$ of acetaldehyde over $1-120$ hours in Experiments P-1 - P-4. 


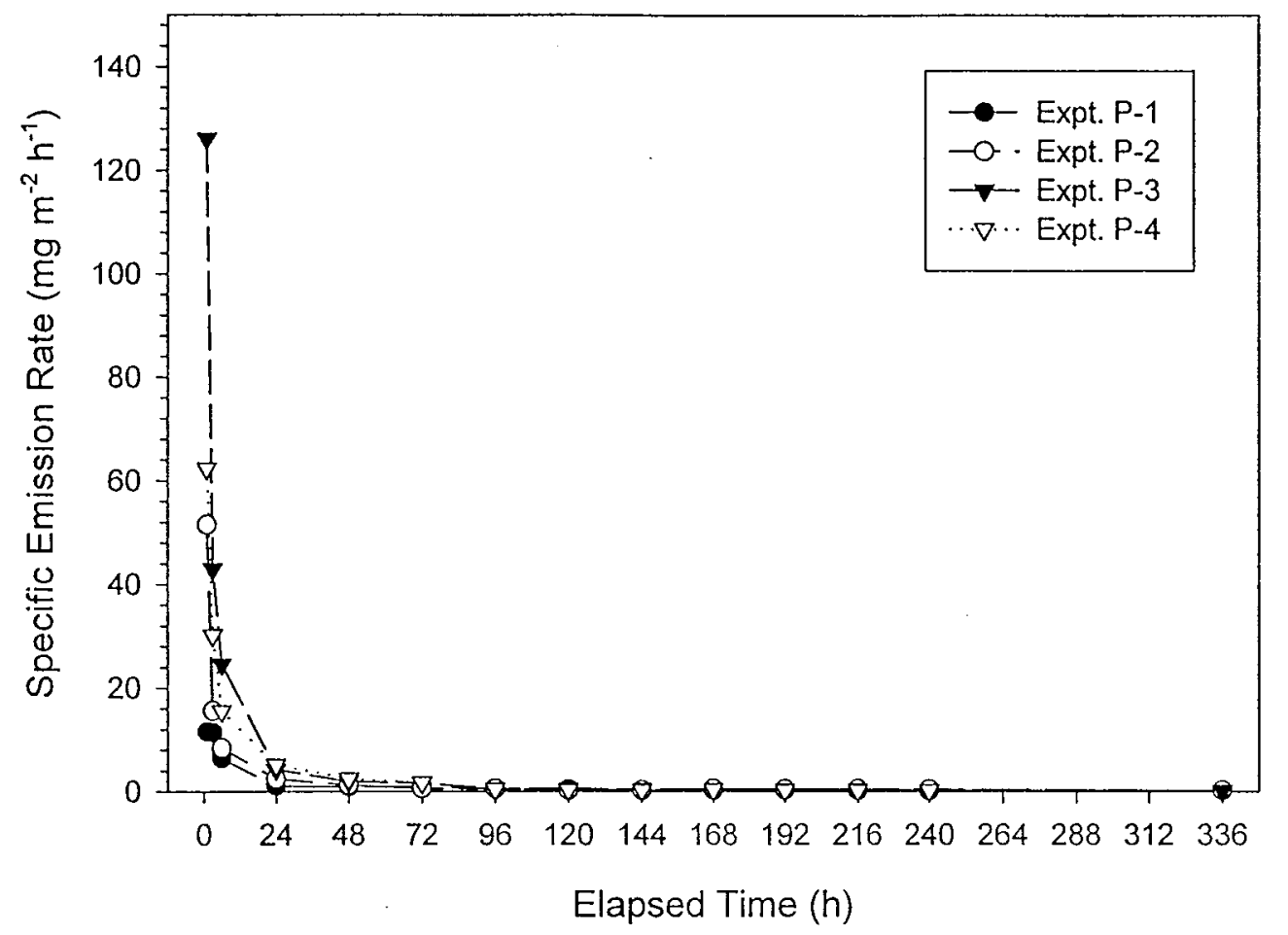

Figure 24a. Specific emission rates $\left(\mathrm{mg} \mathrm{m}^{-2} \mathrm{~h}^{-1}\right)$ of ethylene glycol in Experiments P-1 - P-4.

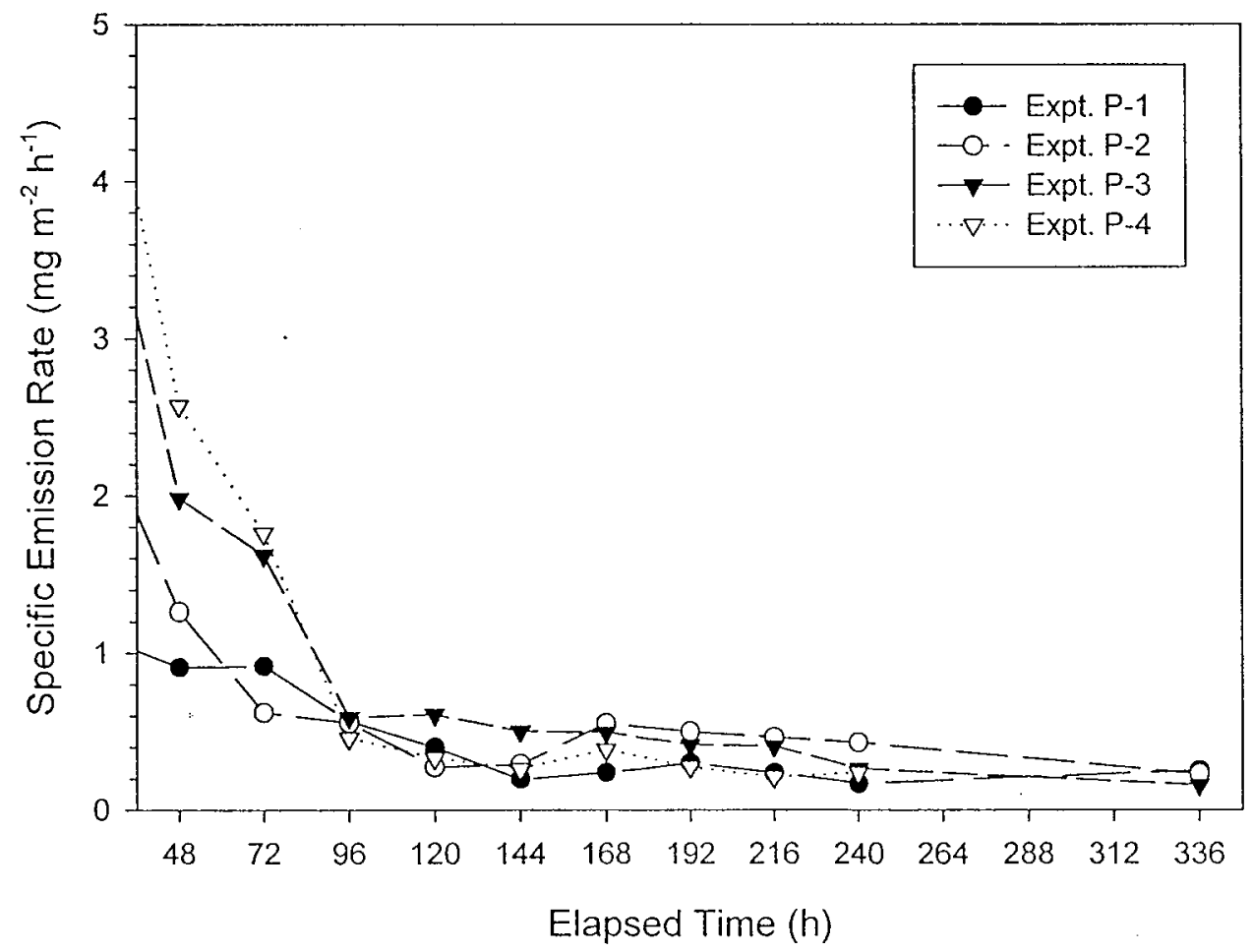

Figure 4 . Specific emission rates $\left(\mathrm{mg} \mathrm{m}^{-2} \mathrm{~h}^{-1}\right)$ of ethylene glycol over $48-336$ hours in Experiments P-1 - P-4. 


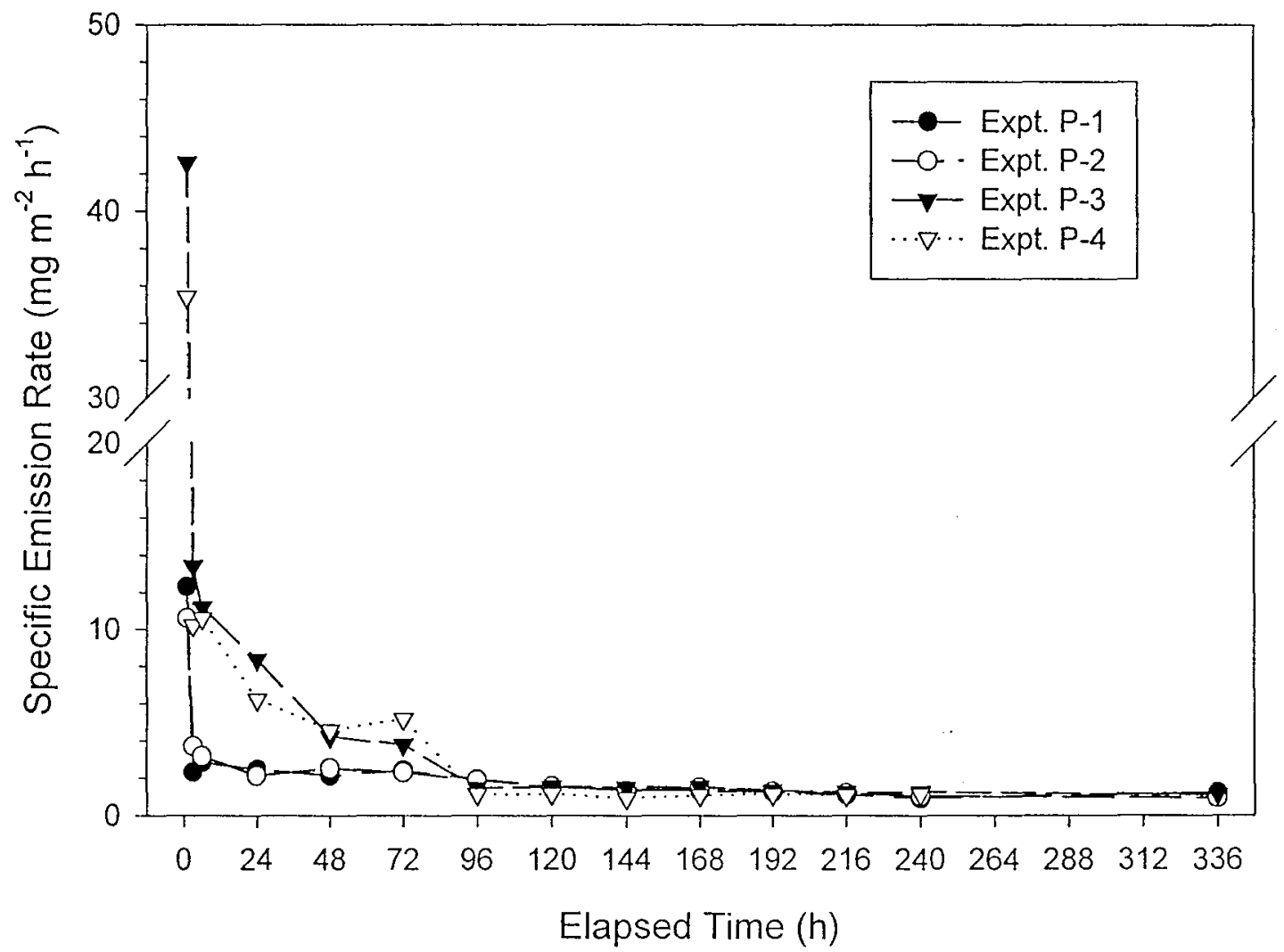

Figure 25. Specific emission rates $\left(\mathrm{mg} \mathrm{m}^{-2} \mathrm{~h}^{-1}\right)$ of Texanol $($ in Experiments $\mathrm{P}-1$ - P-4. 


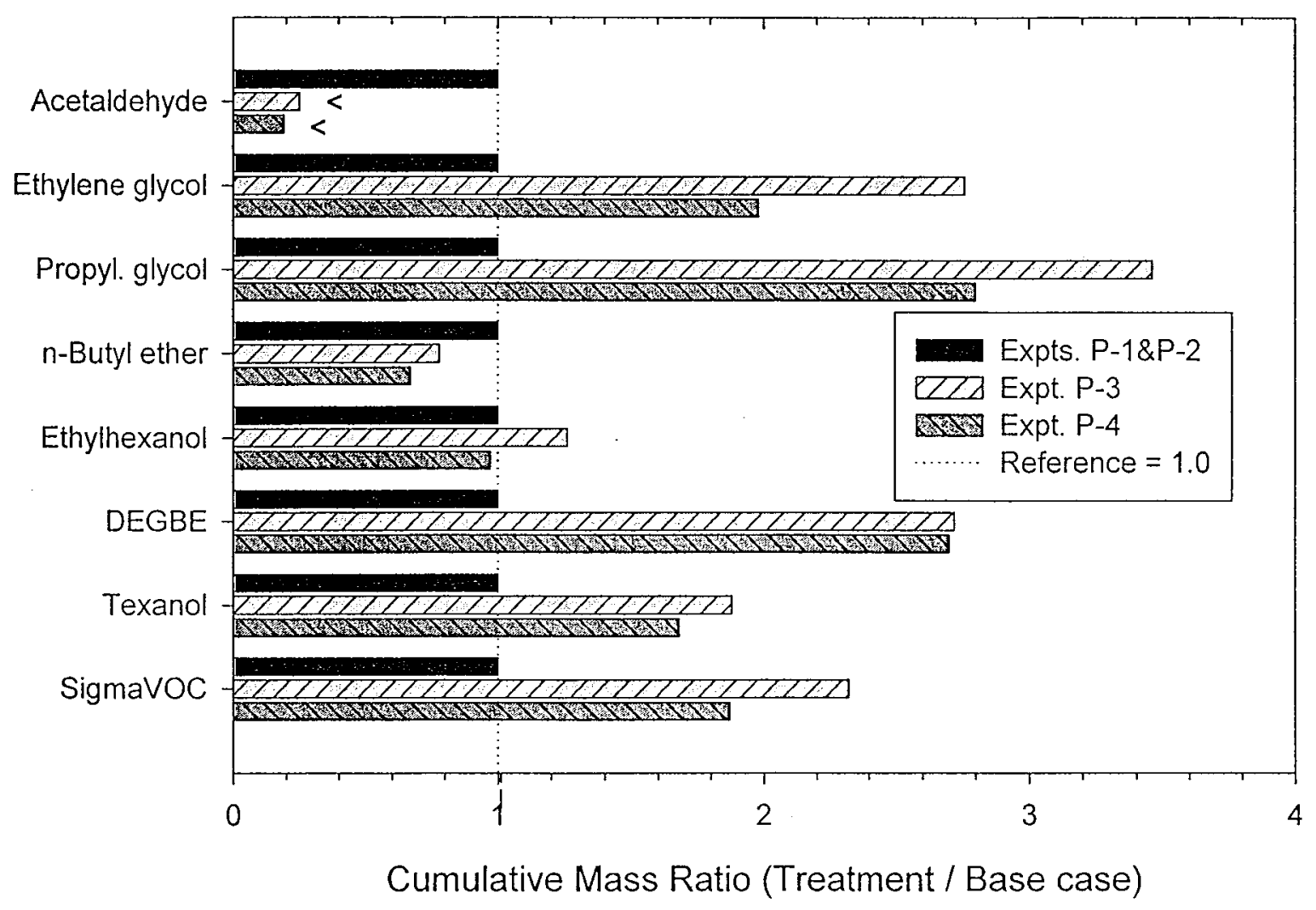

Figure 26. Effects of the treatments on the 0- to 240-h cumulative mass emissions of acetaldehyde, target VOCs and SigmaVOC from paint relative to averaged base-case Experiments P-1 and P-2. " $<$ " Indicates that treatment value was less than lower limit of quantiation. 


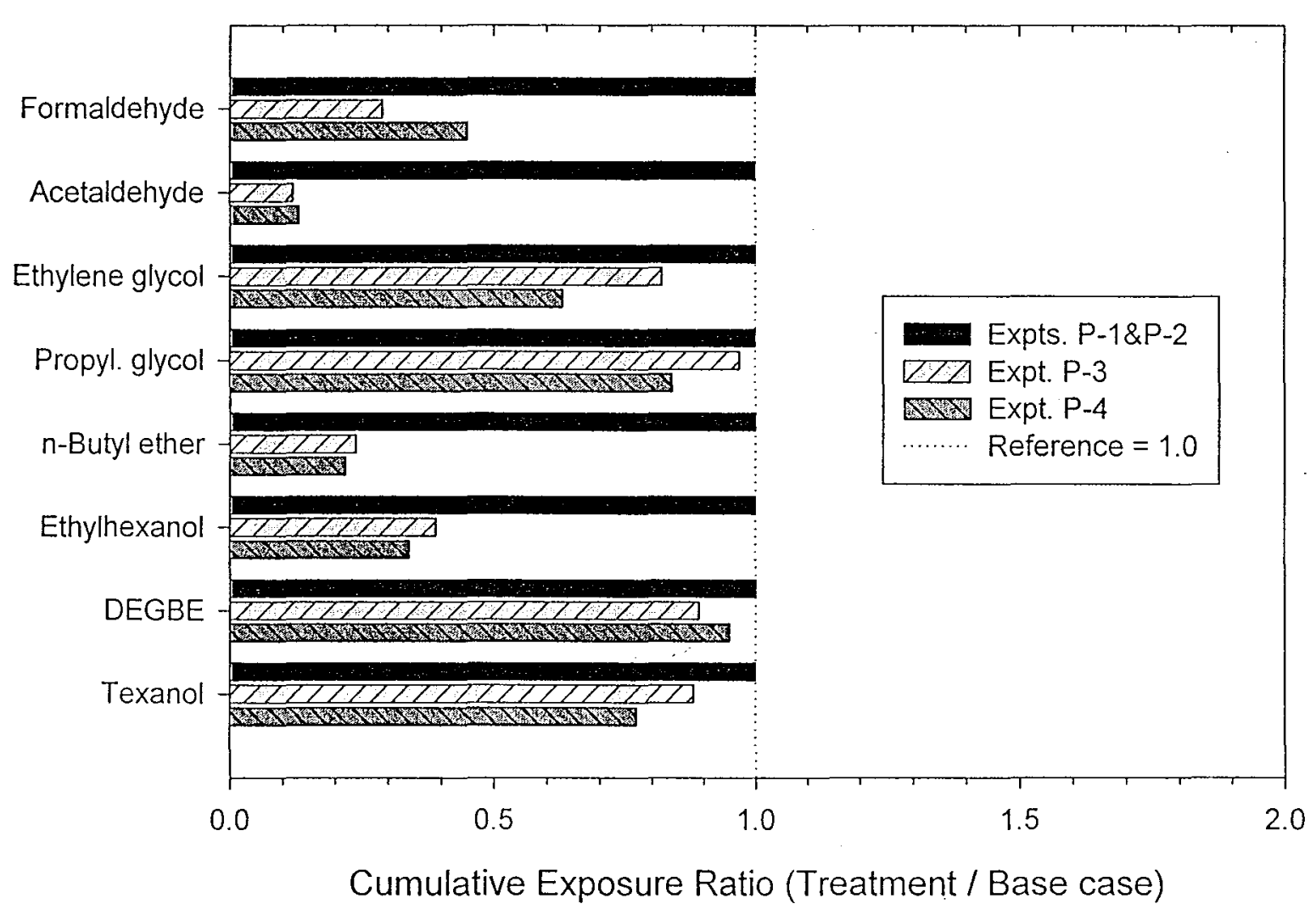

Figure 27. Effects of the treatments on the 0- to 48-h cumulative exposures to formaldehyde, acetaldehyde and target VOCs from paint relative to averaged base-case Experiments $\mathrm{P}-1$ and P-2. The calculation assumes an occupancy of 20 hours per day. 


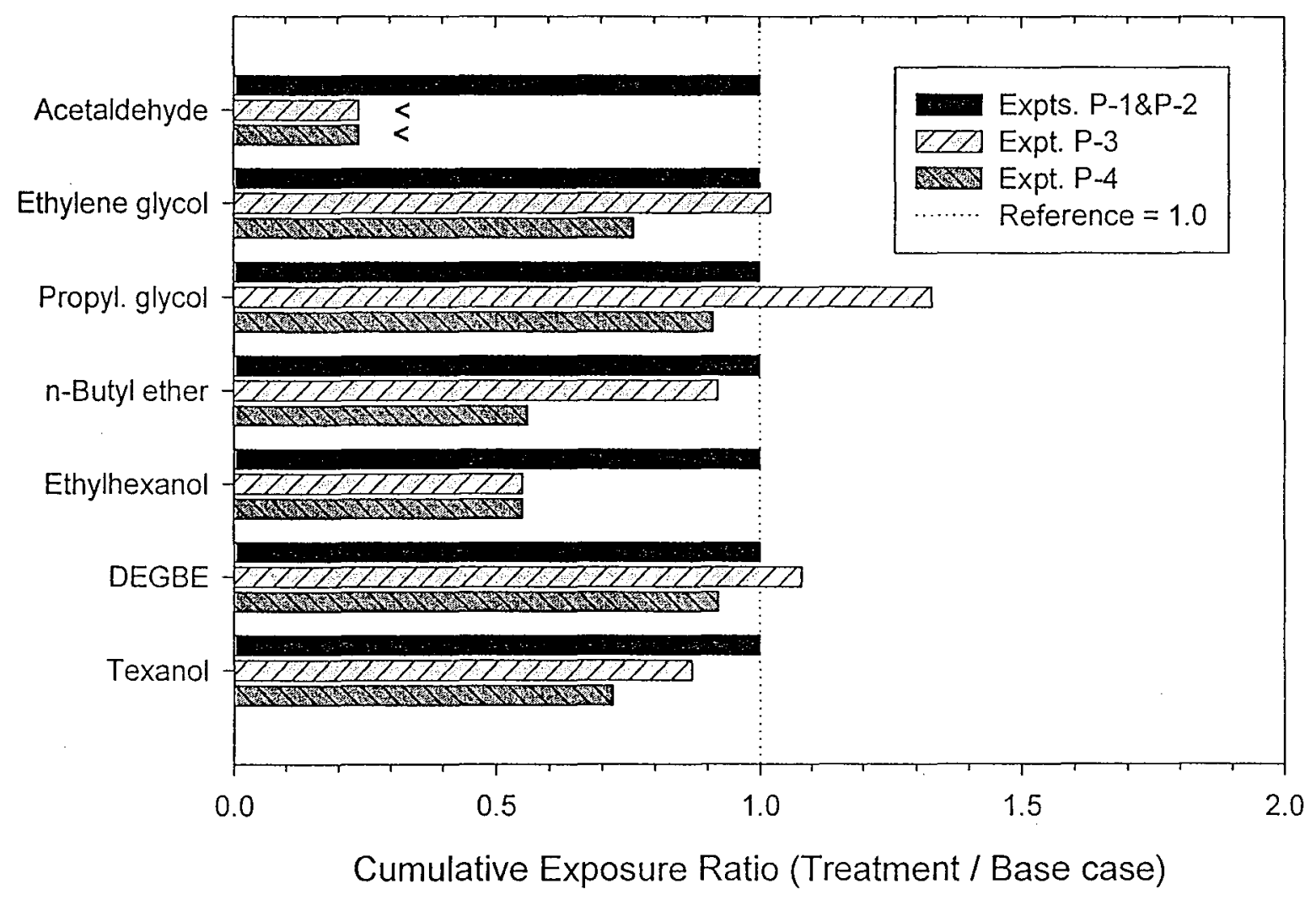

Figure 28. Effects of the treatments on the 48- to 240-h cumulative exposures to acetaldehyde and target VOCs from paint relative to averaged base-case Experiments P-1 and P-2. "<" Inidcates that treatment value was less than lower limit of quantitaion. The calculation assumes occupancy began two days after painting and averaged 20 hours per day. 


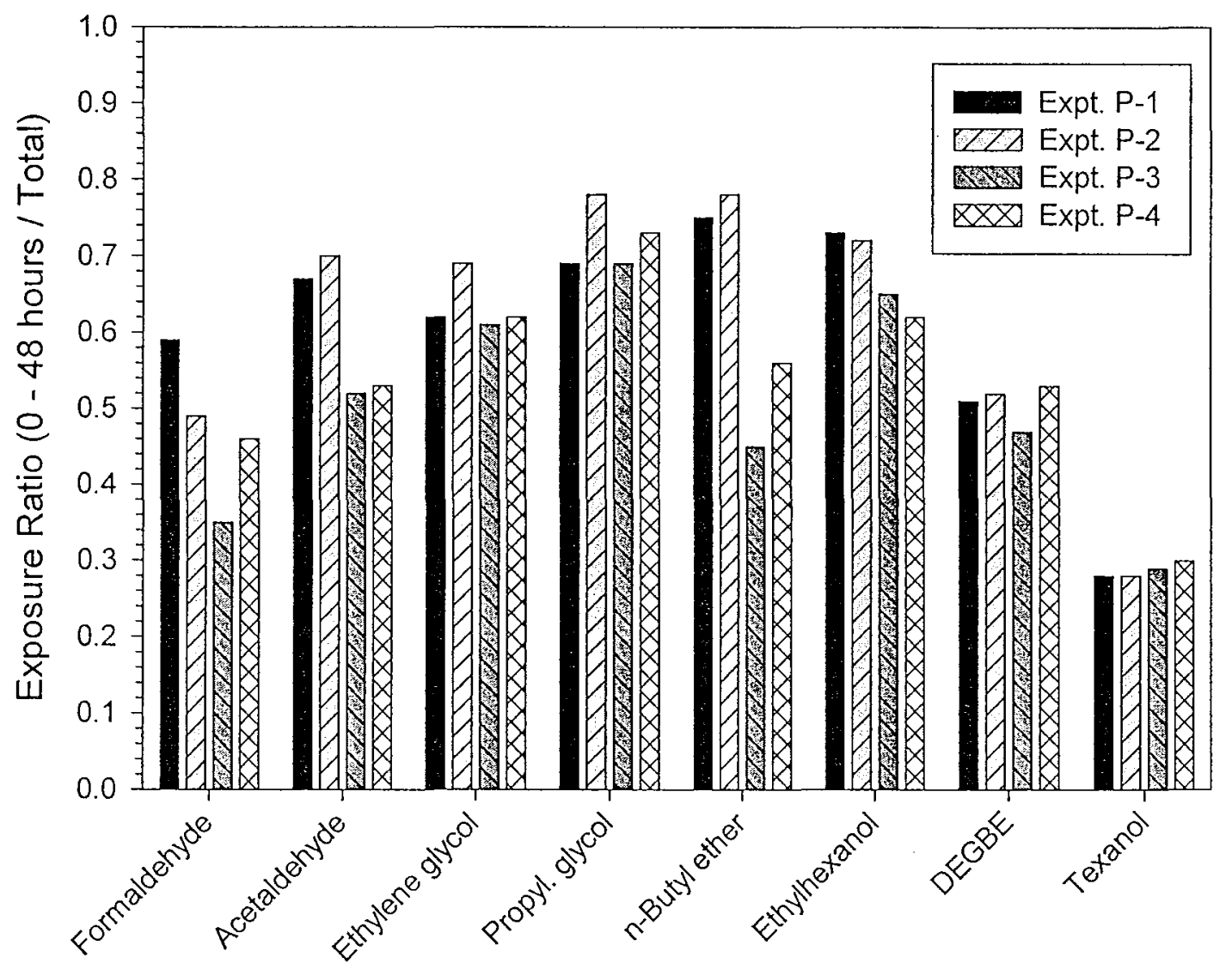

Figure 29. Cumulative exposures to target compounds over $0-48$ hours divided by total cumulative exposures over 0 - 240 hours for Experiments P-1 - P-4. 


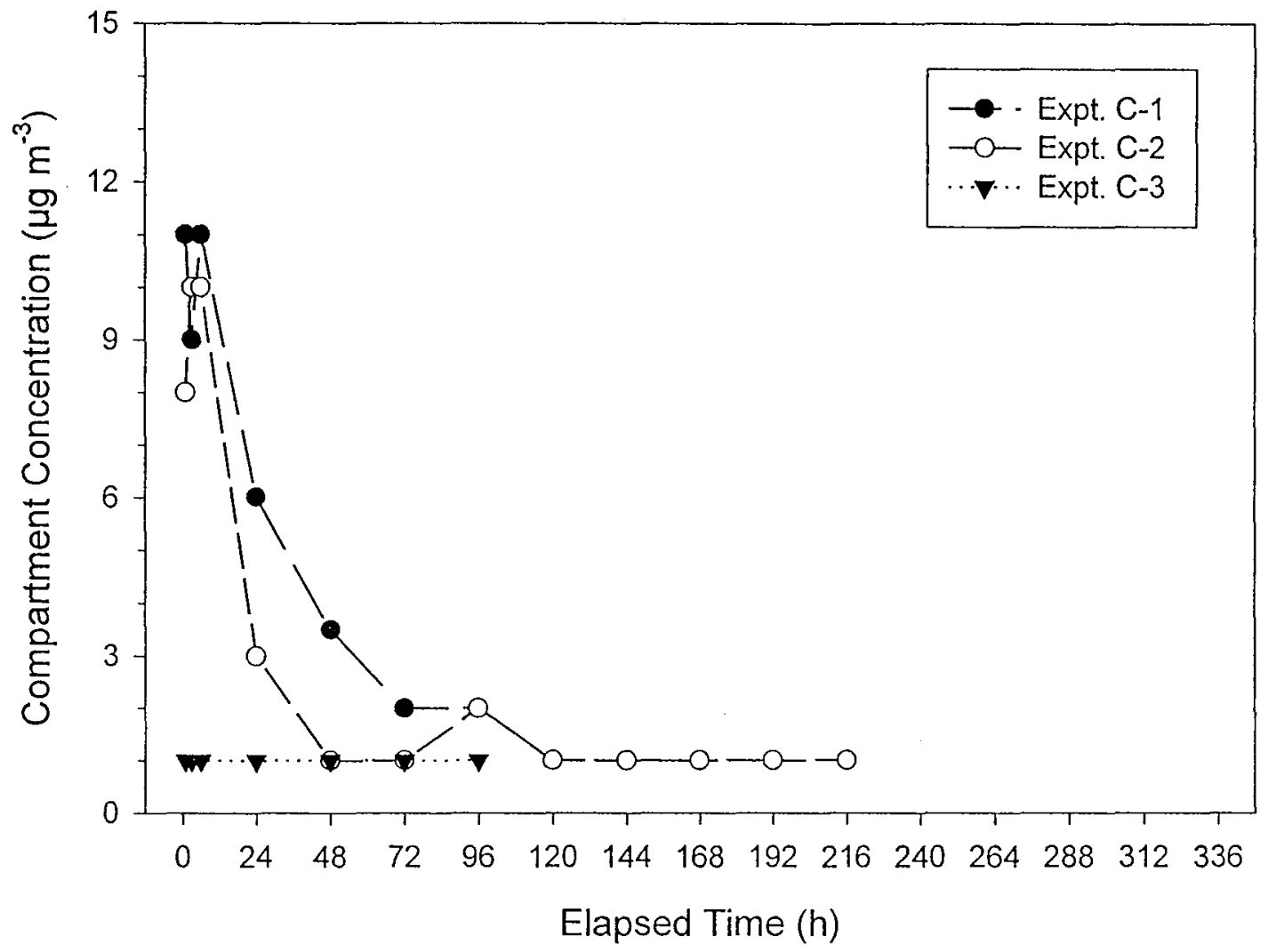

Figure 30. Compartment concentrations $\left(\mu \mathrm{g} \mathrm{m}^{-3}\right)$ of styrene in Experiments $\mathrm{C}-1-\mathrm{C}-3$. 


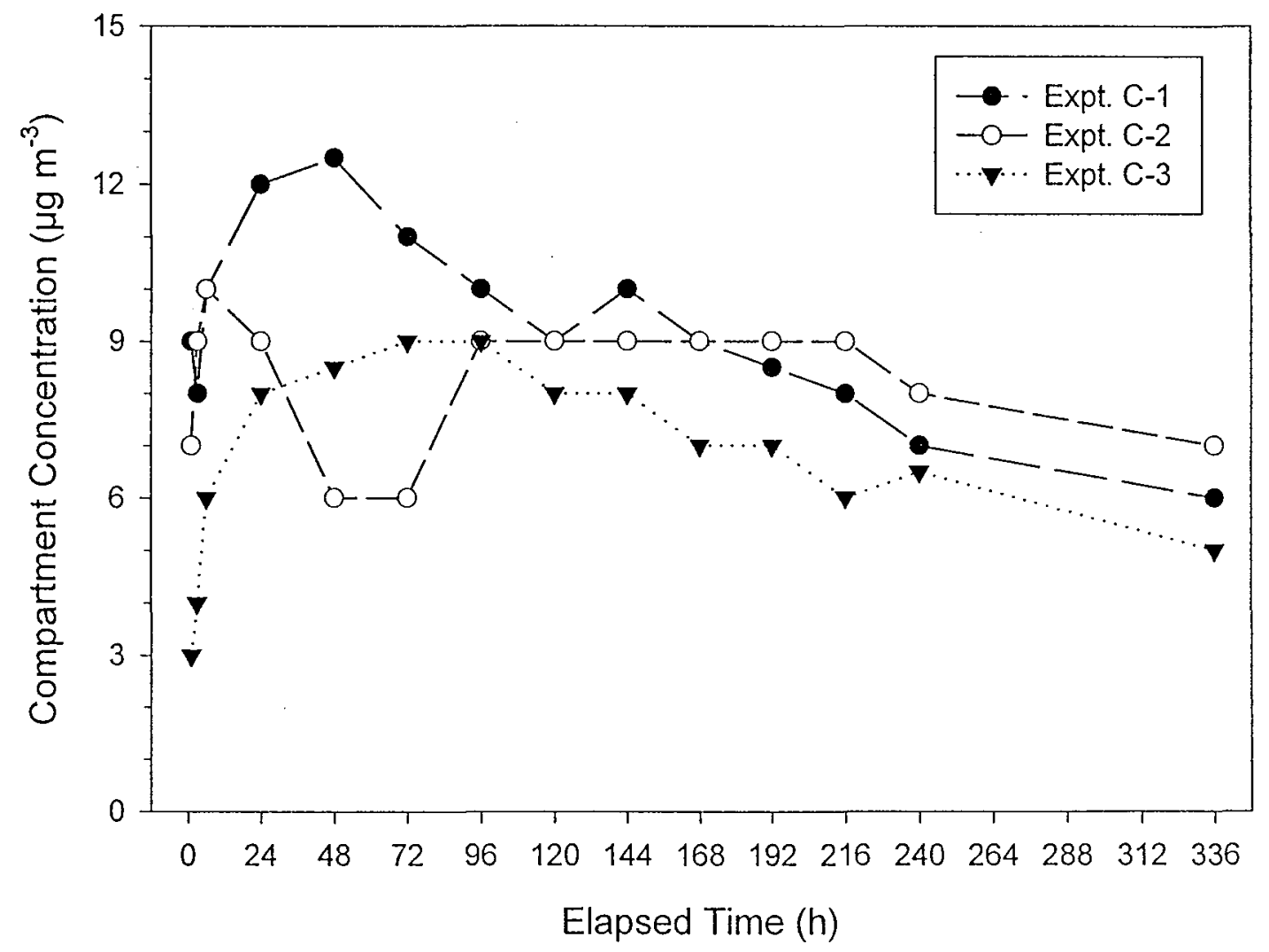

Figure 31. Compartment concentrations $\left(\mu \mathrm{g} \mathrm{m}^{-3}\right)$ of $4-\mathrm{PCH}$ in Experiments $\mathrm{C}-1-\mathrm{C}-3$. 


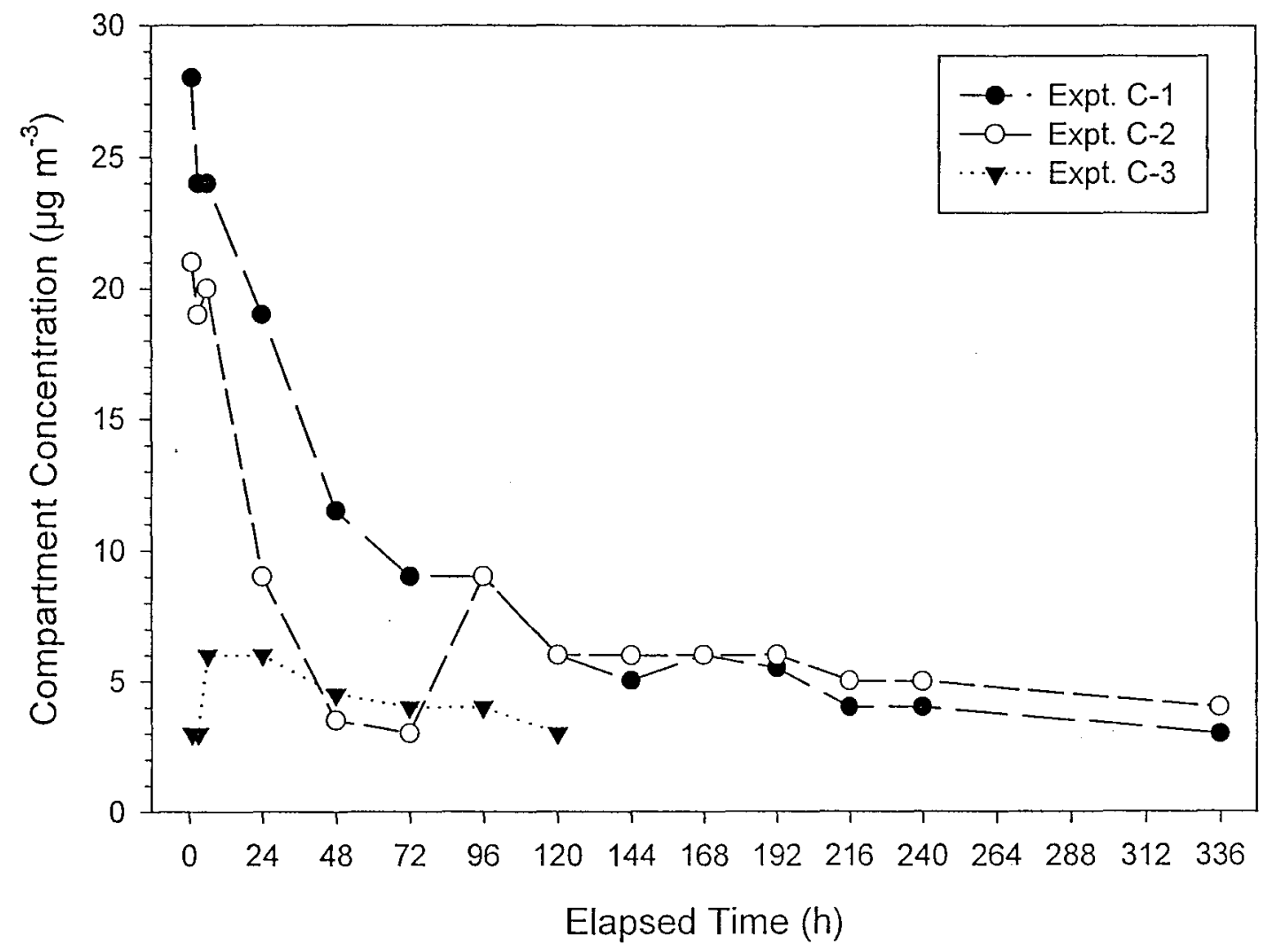

Figure 32. Compartment concentrations $\left(\mu \mathrm{g} \mathrm{m}^{-3}\right)$ of DPGME in Experiments $\mathrm{C}-1$ - C-3. 


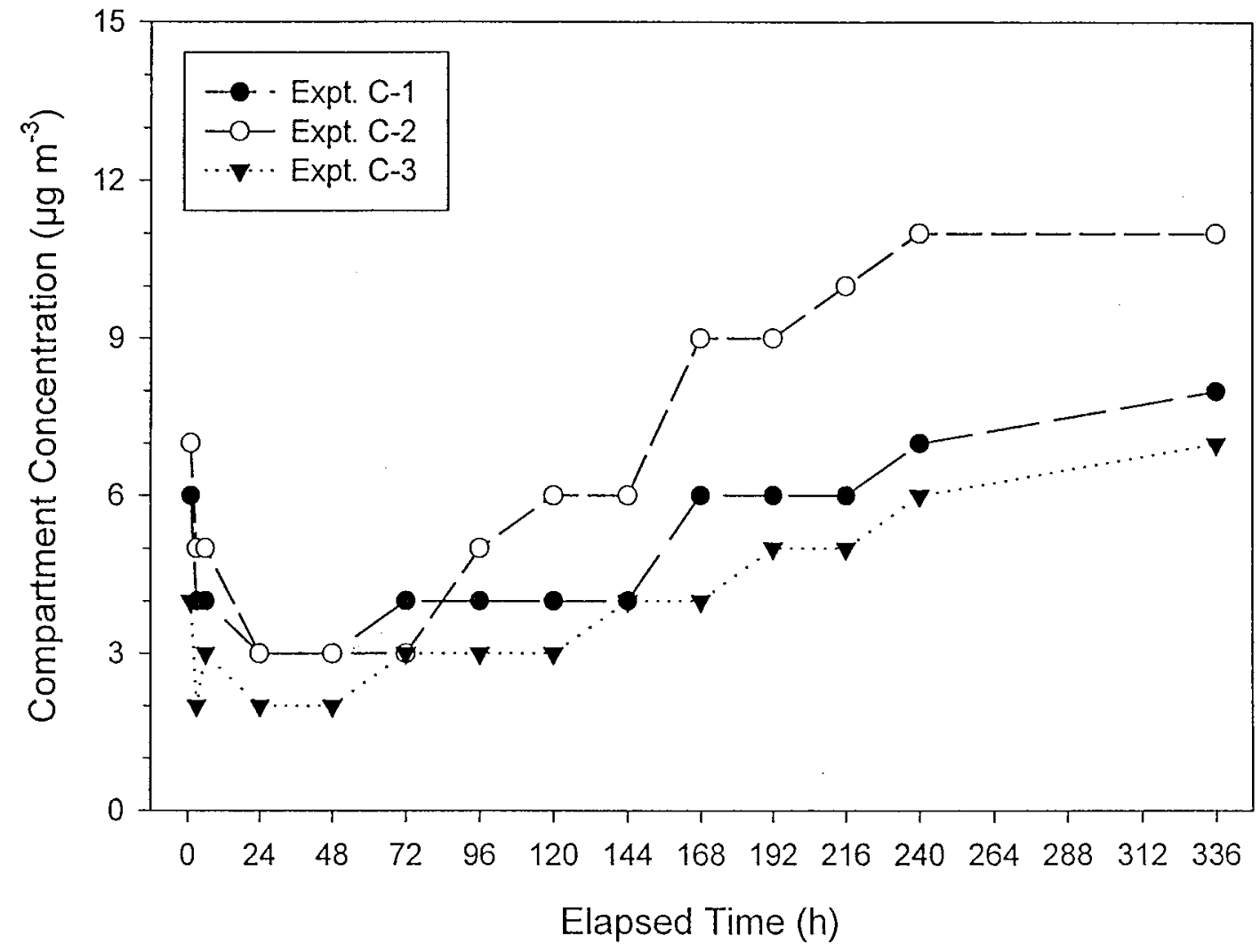

Figure 33. Compartment concentrations $\left(\mu \mathrm{g} \mathrm{m}^{-3}\right)$ of BHT in Experiments C-1 - C-3. 


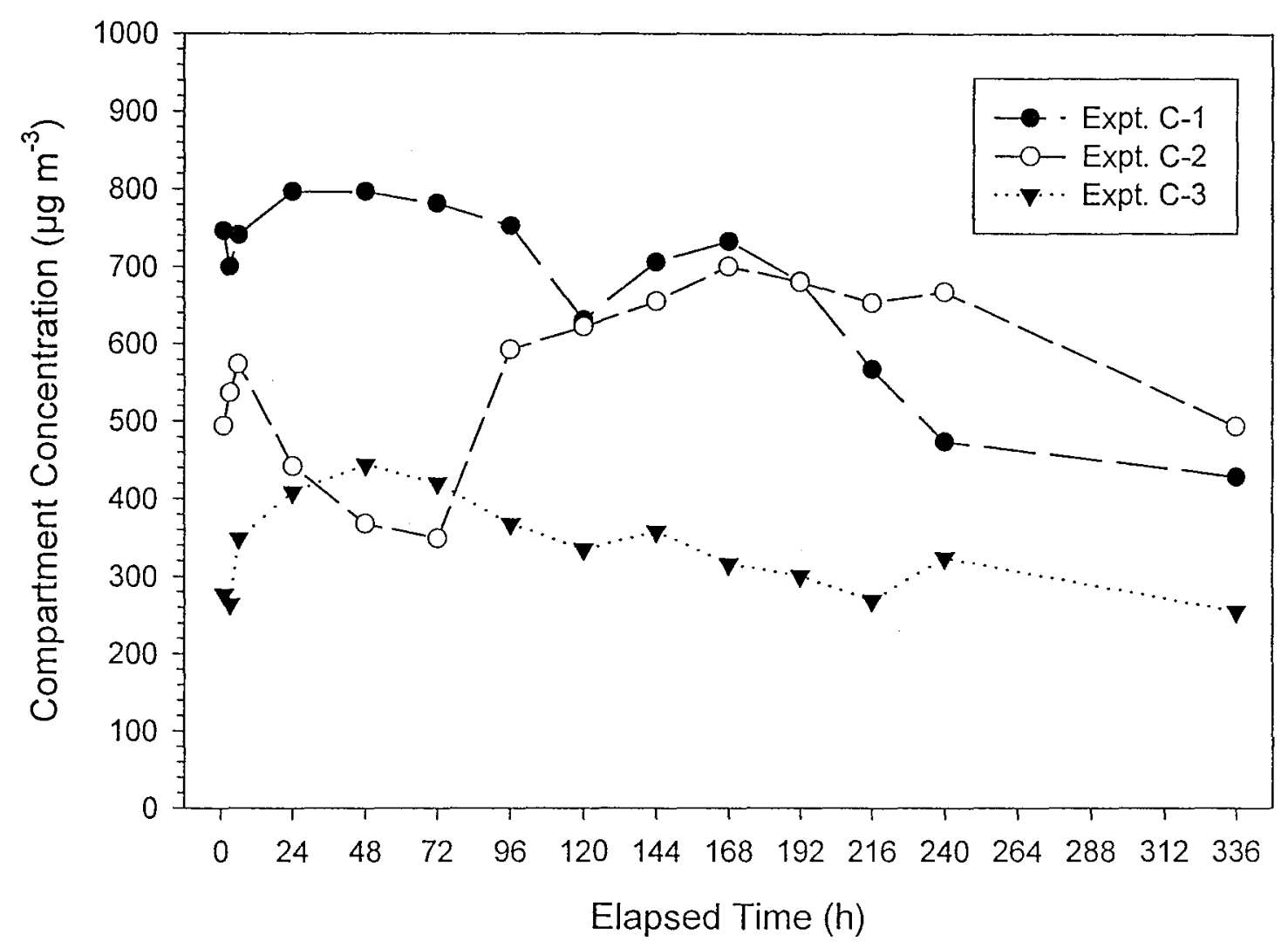

Figure 34. Compartment concentrations $\left(\mu \mathrm{g} \mathrm{m}^{-3}\right.$ ) of TVOC in Experiments $\mathrm{C}-1-\mathrm{C}-3$. 


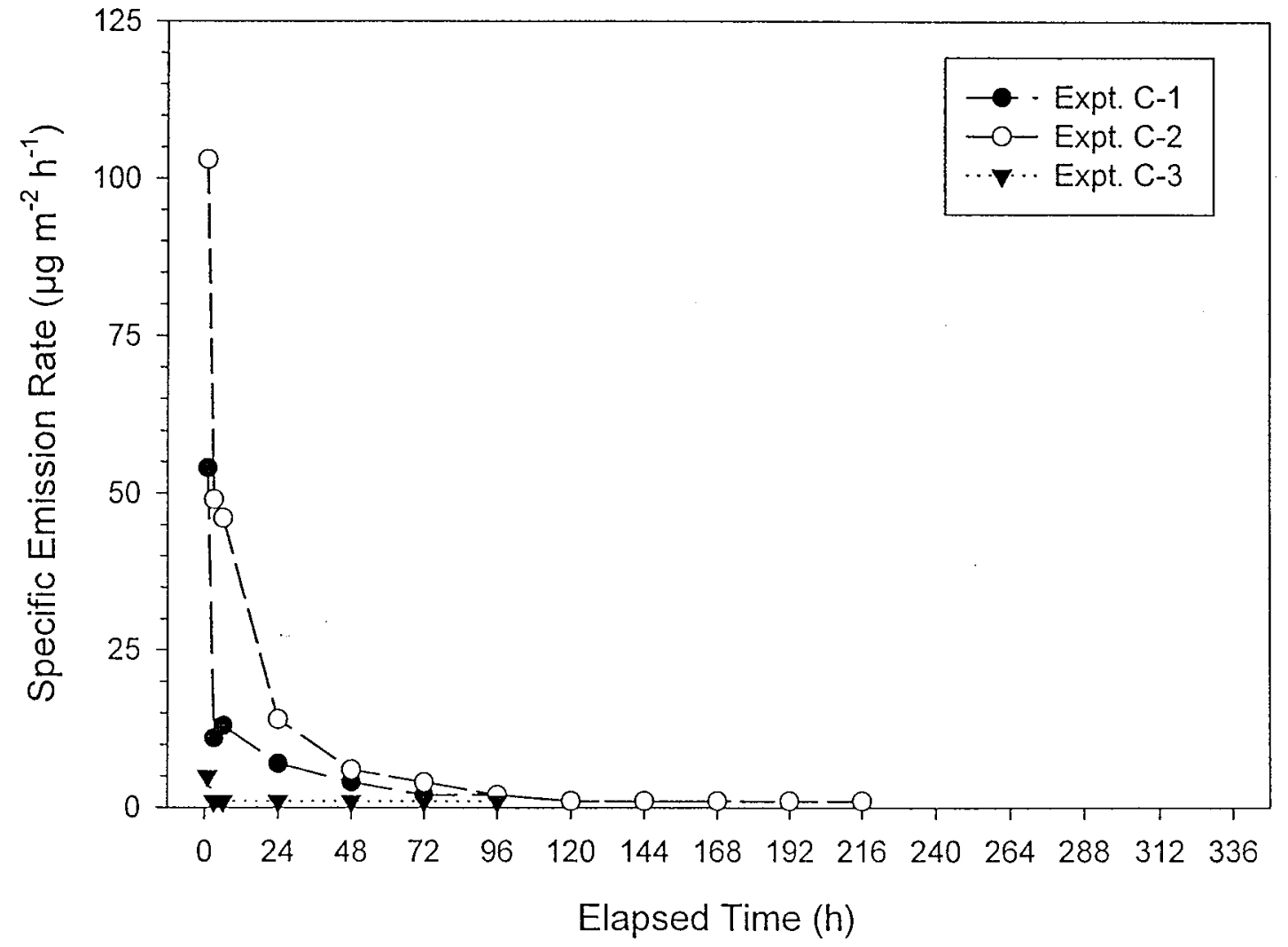

Figure 35. Specific emission rates $\left(\mu \mathrm{g} \mathrm{m}^{-2} \mathrm{~h}^{-1}\right)$ of styrene in Experiments $\mathrm{C}-1$ - C-3. 


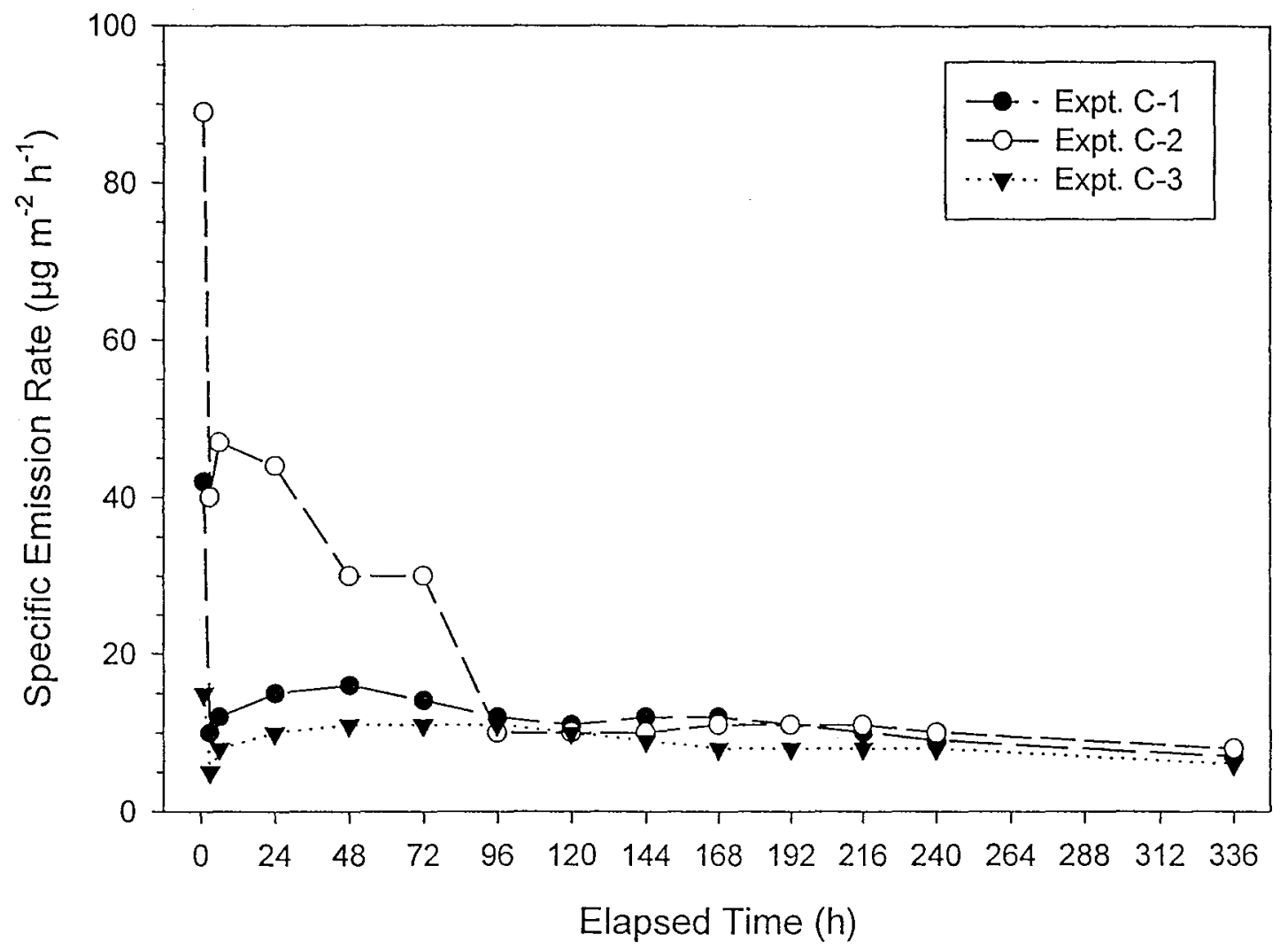

Figure 36. Specific emission rates $\left(\mu \mathrm{m} \mathrm{m}^{-2} \mathrm{~h}^{-1}\right)$ of 4-PCH in Experiments C-1 - C-3. 


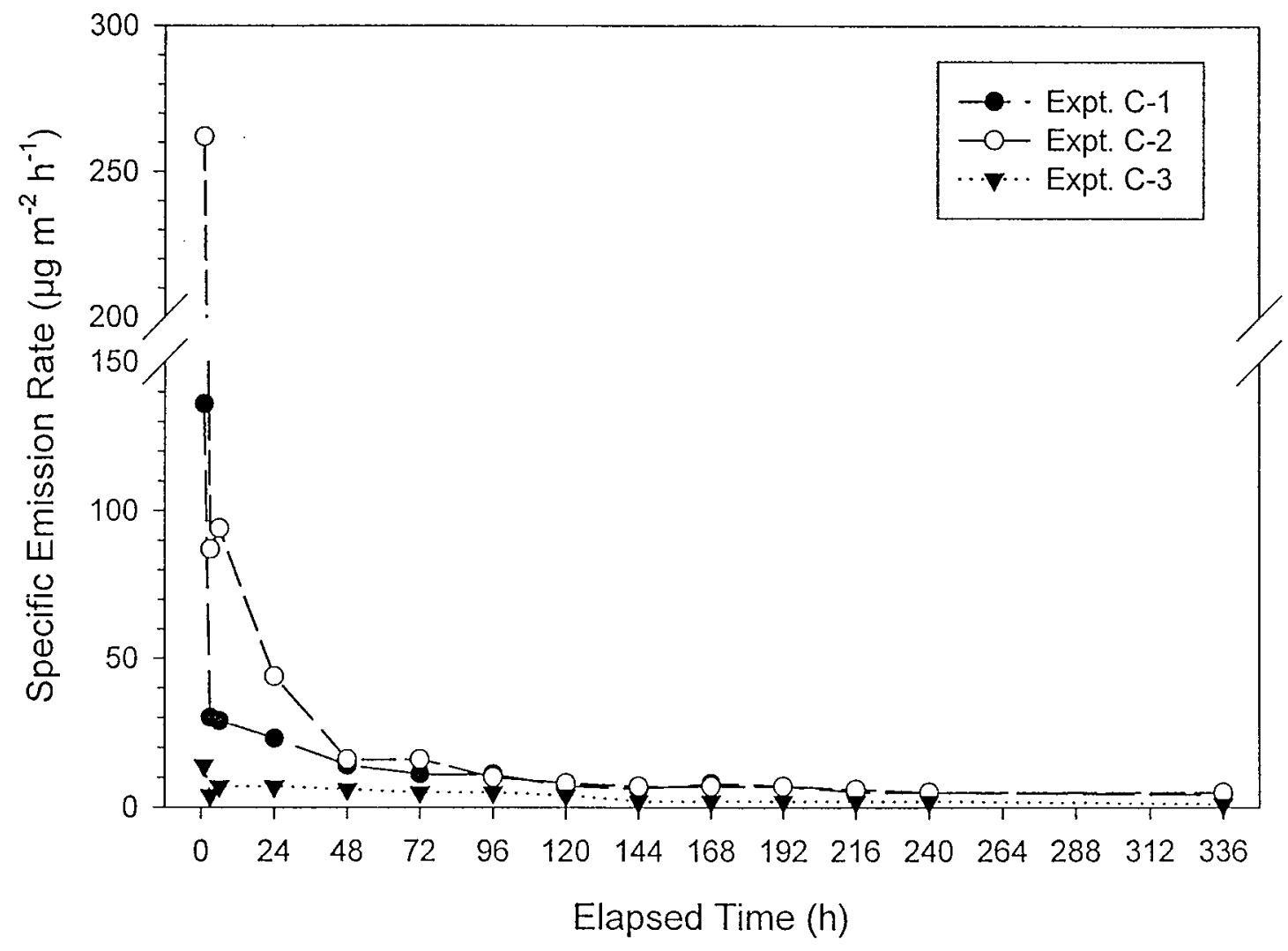

Figure 37. Specific emission rates $\left(\mu \mathrm{g} \mathrm{m}^{-2} \mathrm{~h}^{-1}\right)$ of DPGME in Experiments $\mathrm{C}-1$ - C-3. 


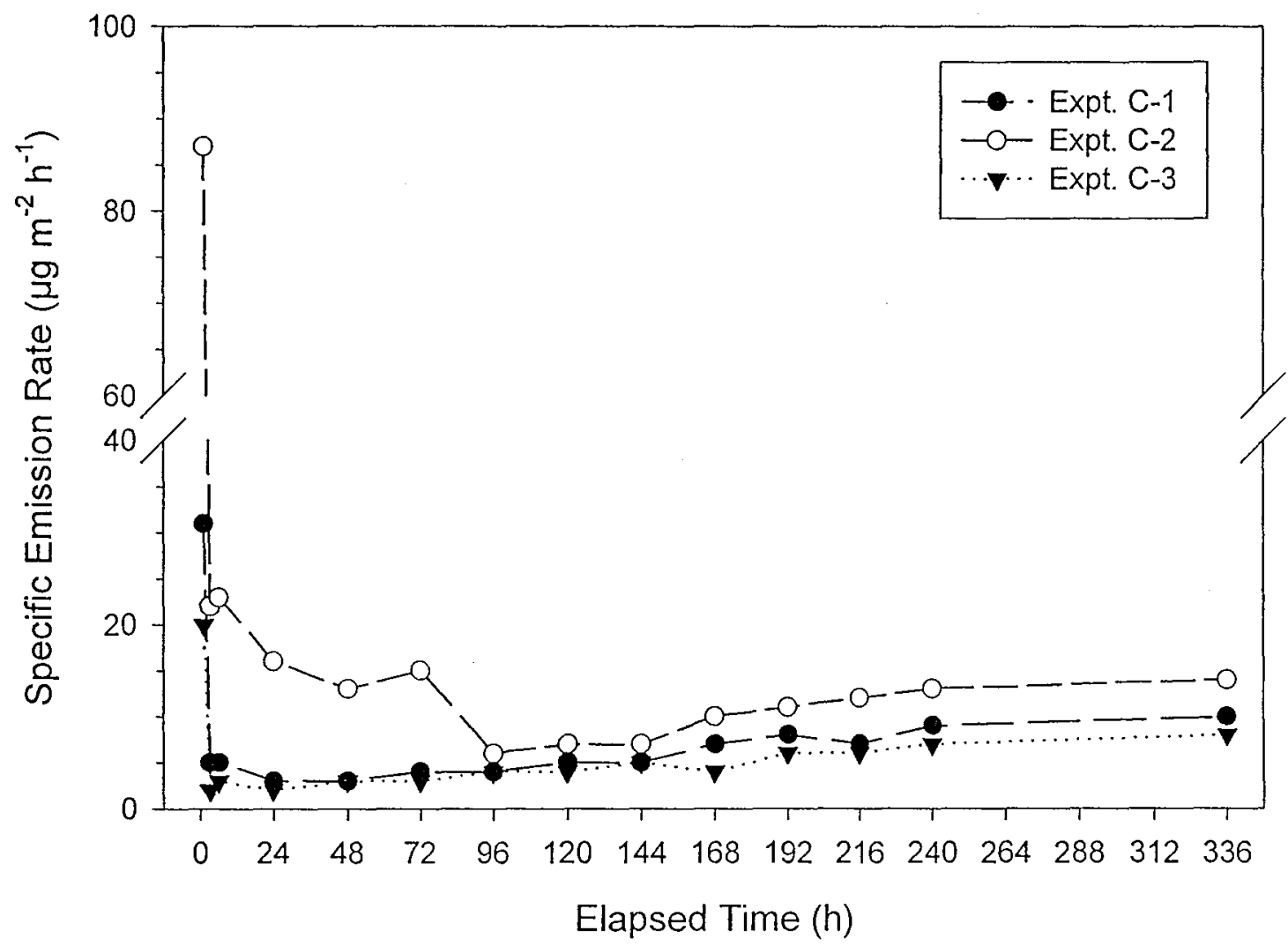

Figure 38. Specific emission rates $\left(\mu \mathrm{g} \mathrm{m}^{-2} \mathrm{~h}^{-1}\right)$ of BHT in Experiments $\mathrm{C}-1-\mathrm{C}-3$. 


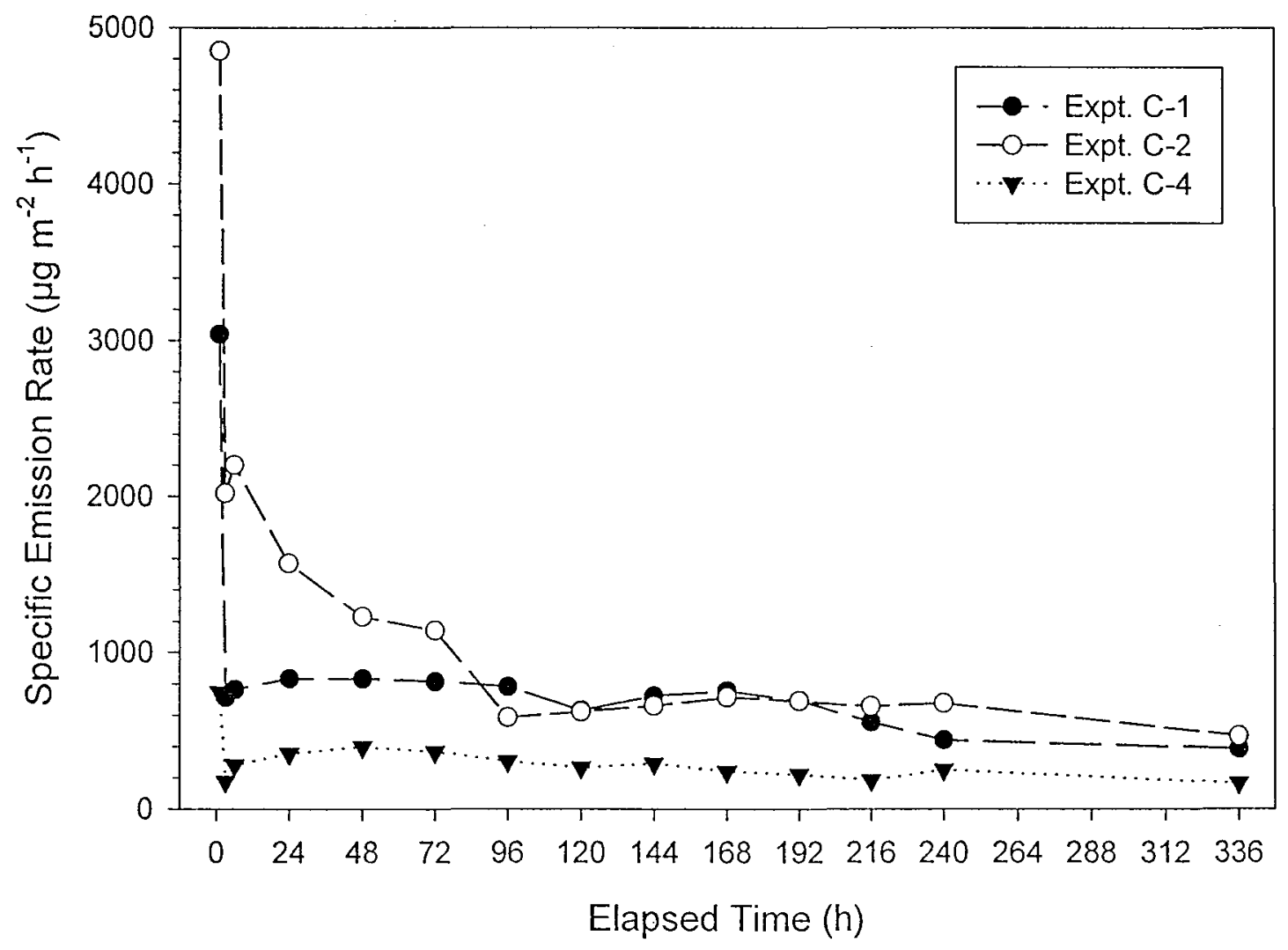

Figure 39. Specific emission rates $\left(\mu \mathrm{g} \mathrm{m}^{-2} \mathrm{~h}^{-1}\right)$ of TVOC in Experiments C-1 - C-3. 


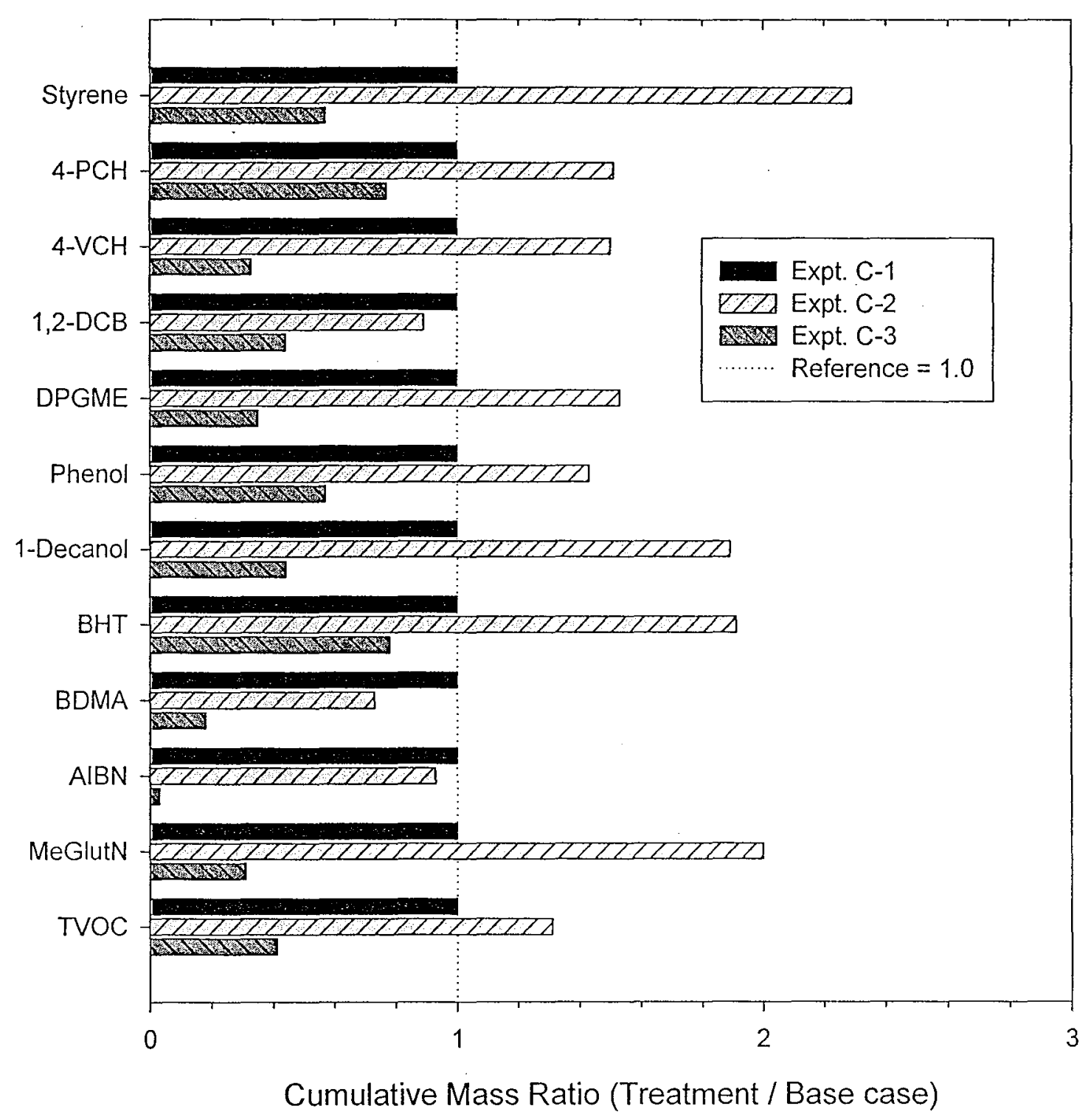

Figure 40. Effects of the treatments on the 0- to 336-h cumulative mass emissions of target VOCs and TVOC from the carpet assembly relative to base-case Experiment $\mathrm{C}-1$. " $<$ " Indicates that treatment value was less than lower limit of quantitation. 


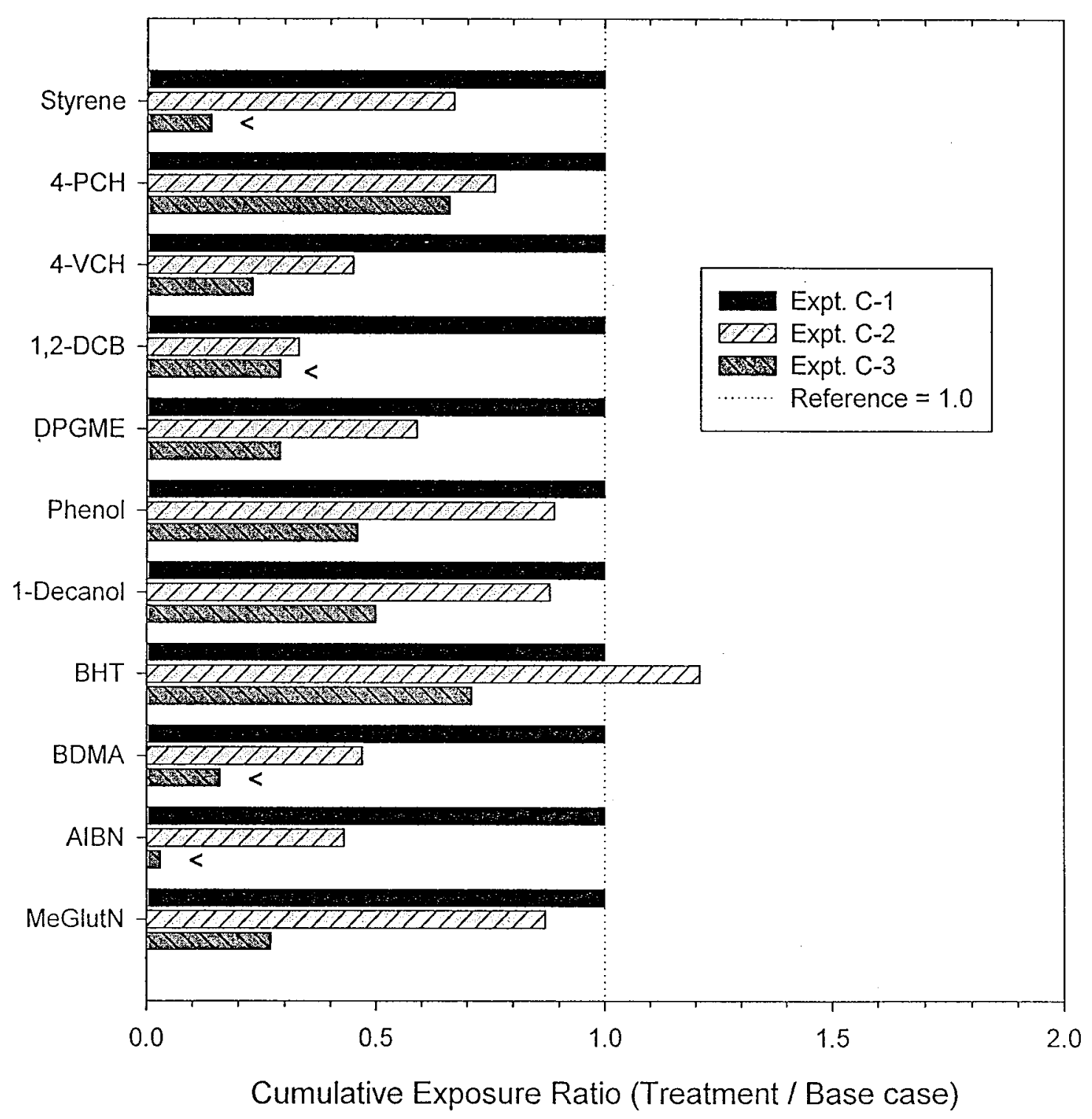

Figure 41. Effects of the treatments on the 0 - to 48-h cumulative exposures to target VOCs from the carpet assembly relative to base-case Experiment $\mathrm{C}-1$.

" $<$ "Indicates that treatment value was less than lower limit of quantitation.

The calculation assumes an occupancy of 20 hours per day. 


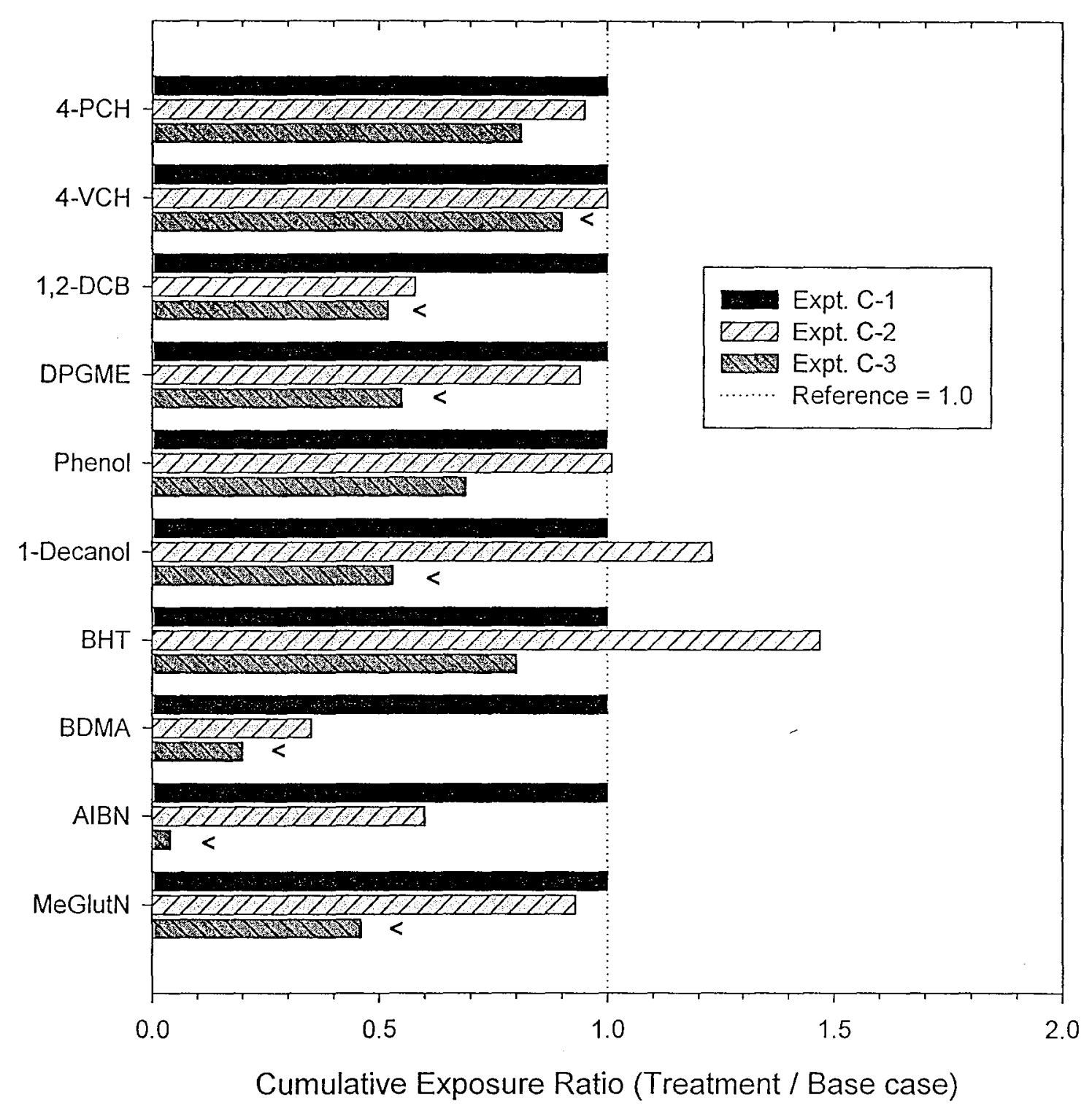

Figure 42. Effects of the treatments on the 48- to 336-h cumulative exposures to target VOCs from the carpet assembly relative to base-case Experiment C-1. " $<$ "Indicates that treatment value was less than lower limit of quantitation. The calculation assumes occupancy began two days after installation and averaged 20 hours per day. 


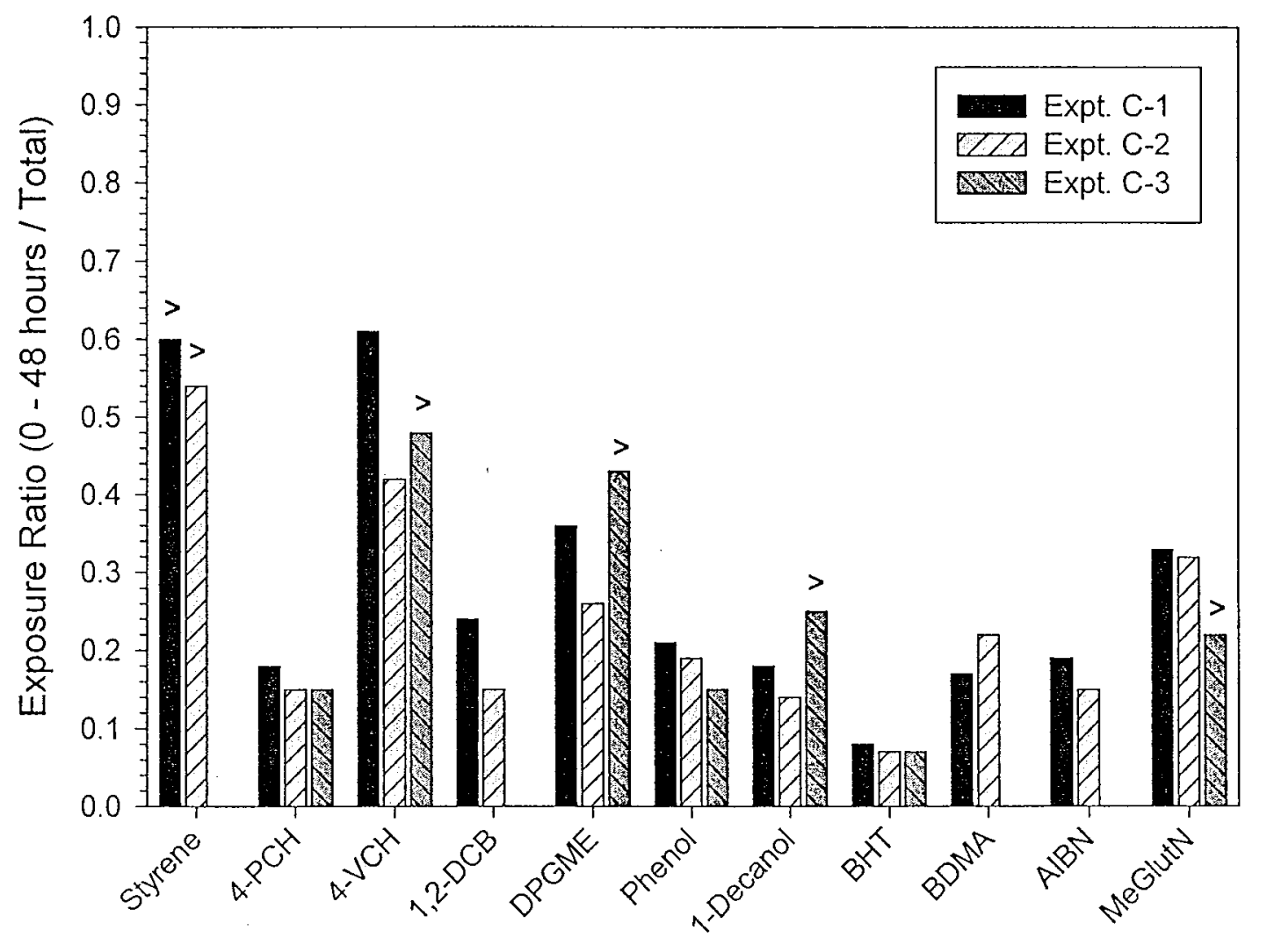

Figure 43. Cumulative exposures to target VOCs over 0 - 48 hours divided by total cumulative exposures over $0-336$ hours for Experiments C-1 - C-3.

" $>$ "Indicates that ratio is greater that plotted value. Ratios could not be calculated for some compounds in Experiment $\mathrm{C}-3$ because exposures were less than the lower limits of quantitation in both periods. 


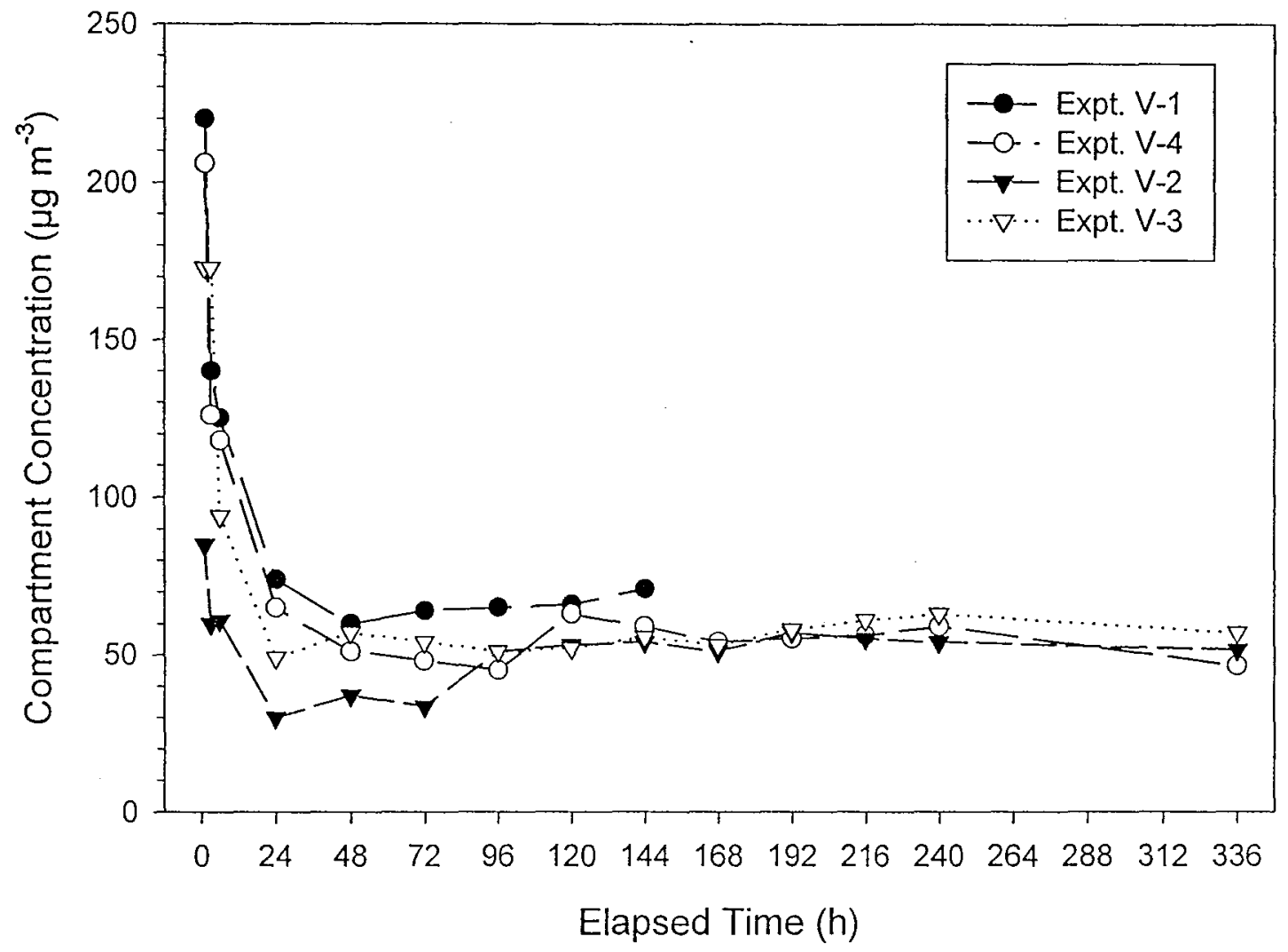

Figure 44. Compartment concentrations $\left(\mu \mathrm{g} \mathrm{m}^{-3}\right)$ of $n$-tridecane in Experiments $V-1-\mathrm{V}-4$. 


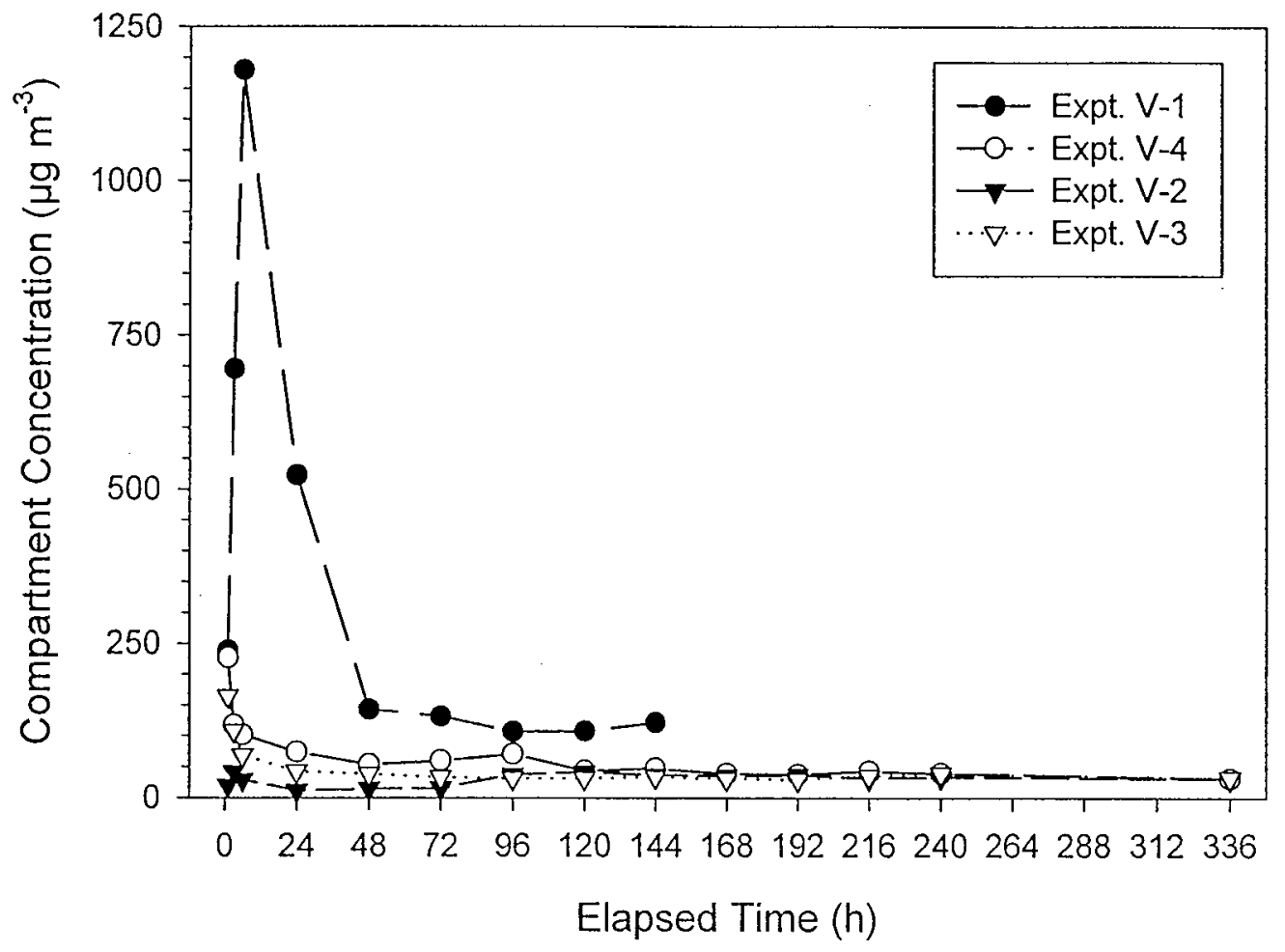

Figure 45a. Compartment concentrations $\left(\mu \mathrm{g} \mathrm{m}^{-3}\right)$ of toluene in Experiments $\mathrm{V}-1-\mathrm{V}-4$.

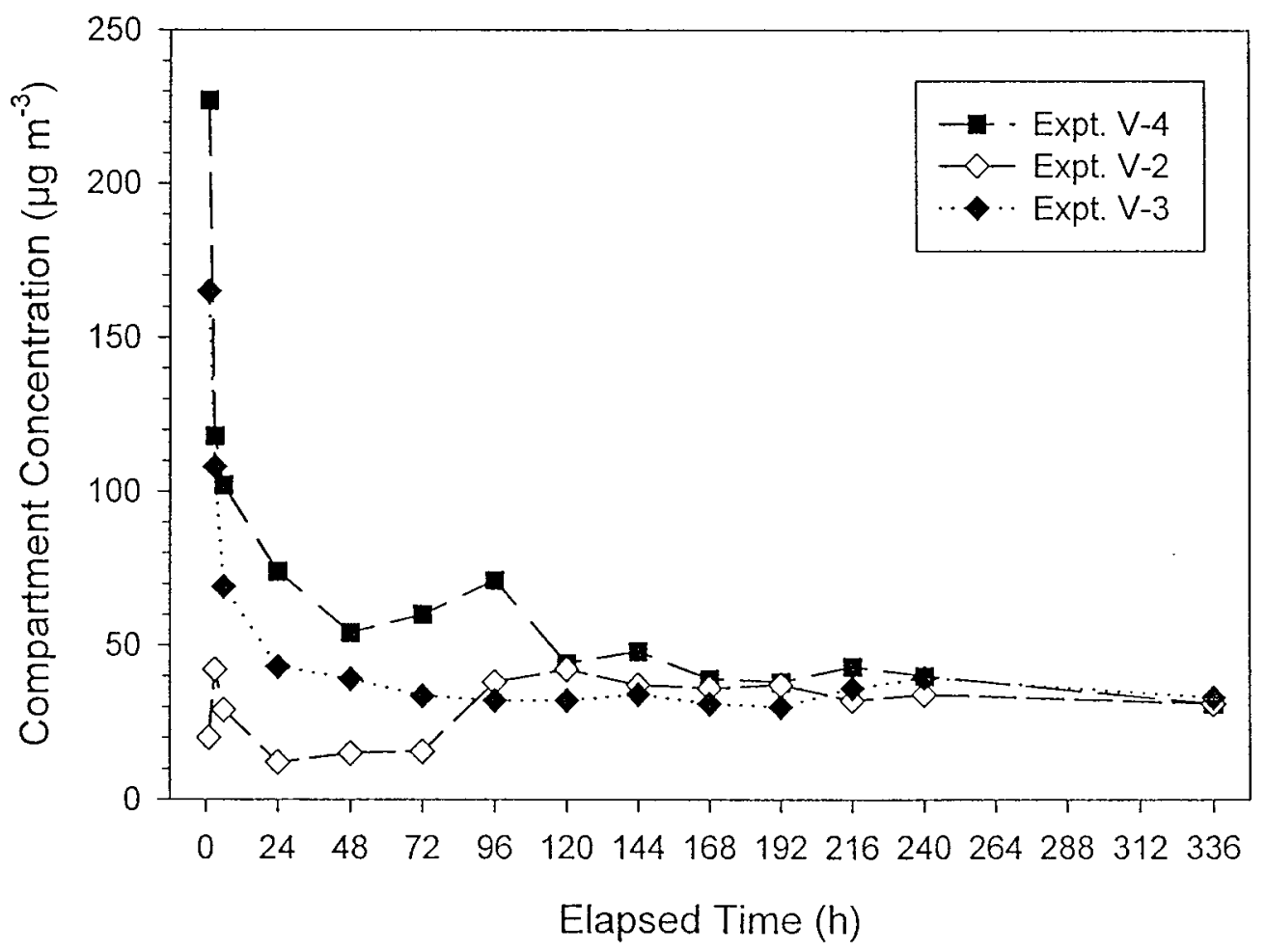

Figure 45b. Compartment concentrations $\left(\mu_{\mathrm{g} \mathrm{m}}^{-3}\right)$ of toluene in Experiments $-2-4$ 


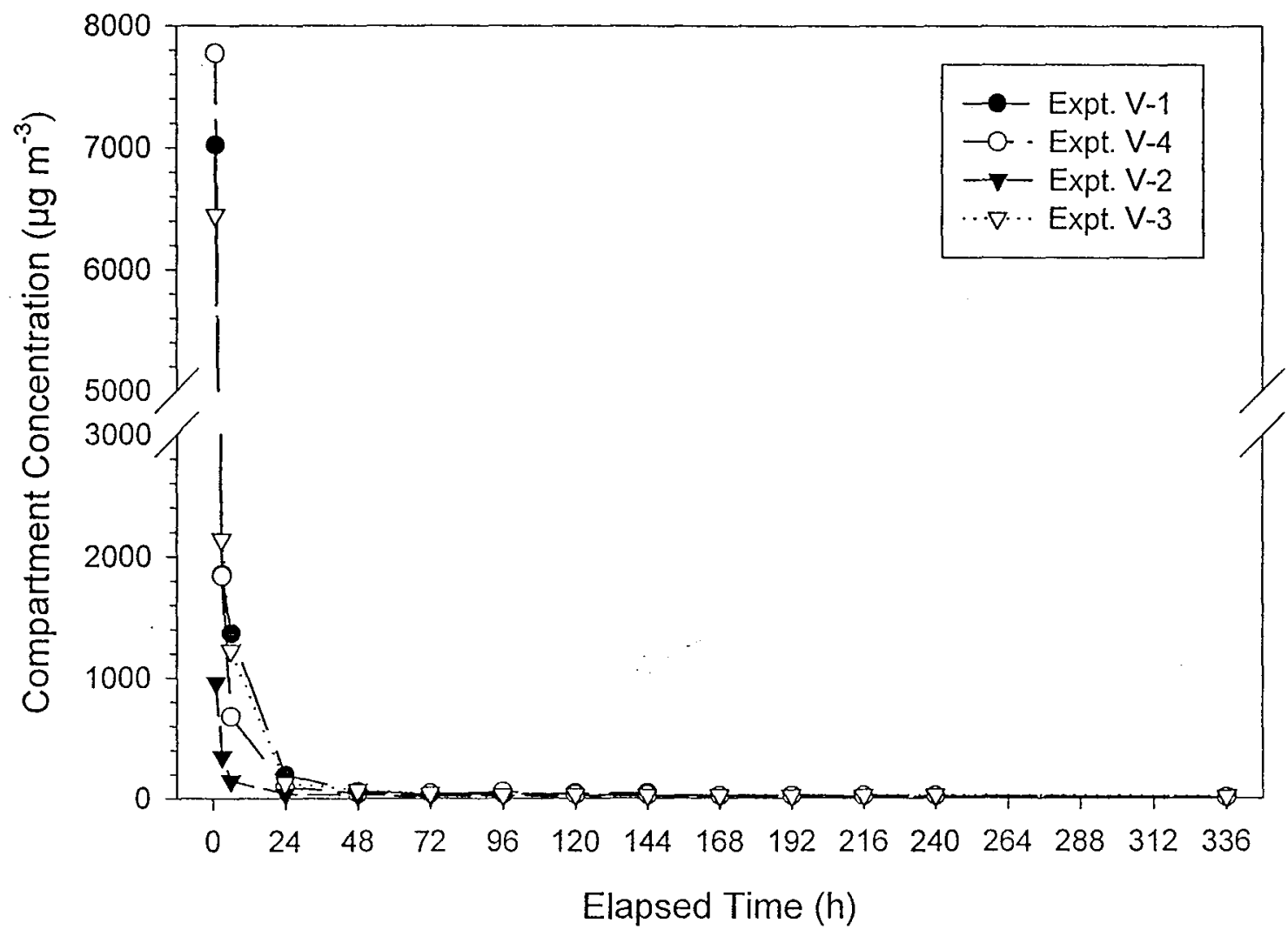

Figure 46. Compartment concentrations $\left(\mu \mathrm{g} \mathrm{m}^{-3}\right.$ ) of tetrahydrofuran in Experiments $\mathrm{V}-1-\mathrm{V}-4$. 


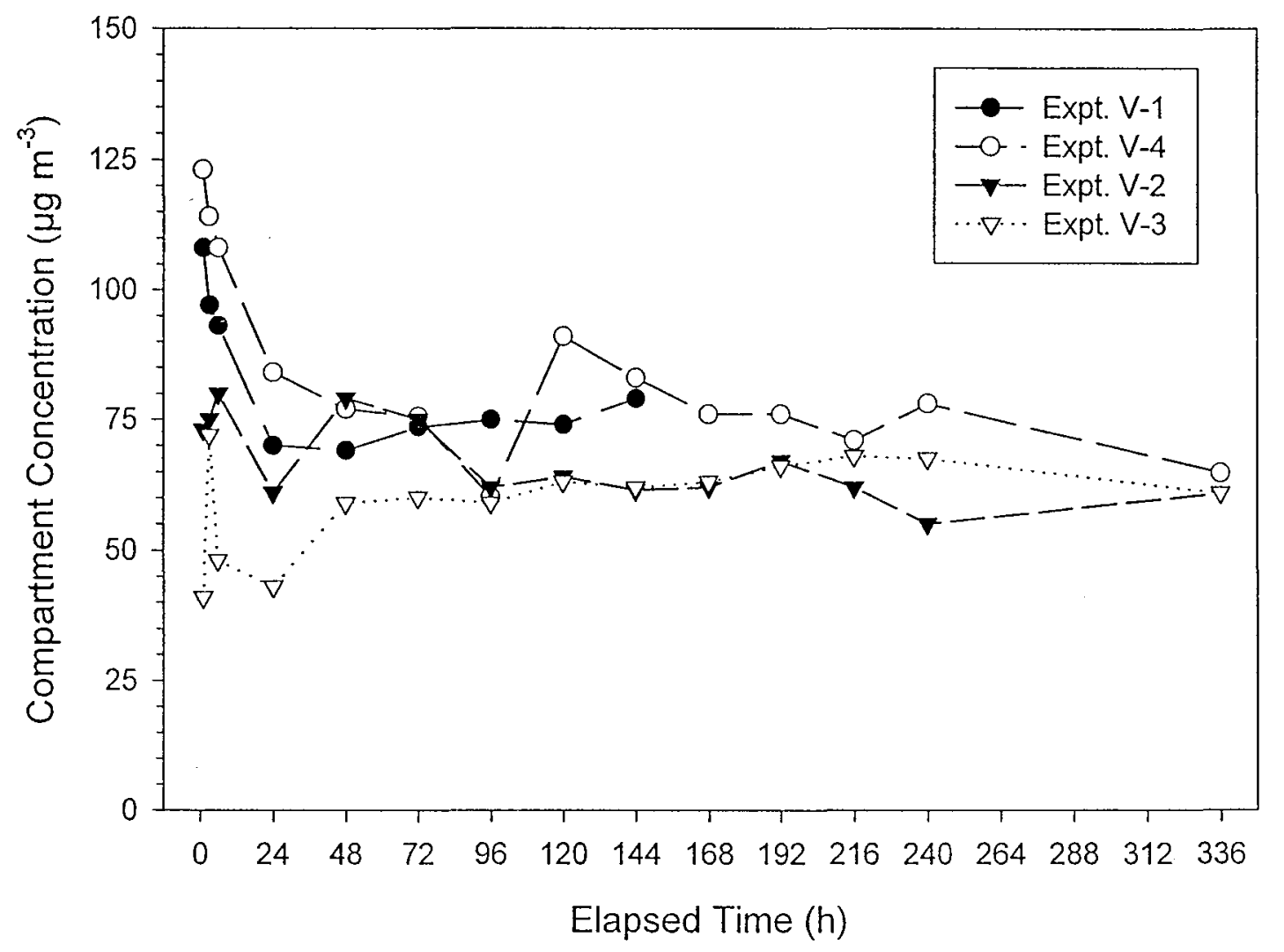

Figure 47. Compartment concentrations $\left(\mu \mathrm{g} \mathrm{m}^{-3}\right)$ of phenol in Experiments $\mathrm{V}-1-\mathrm{V}-4$. 


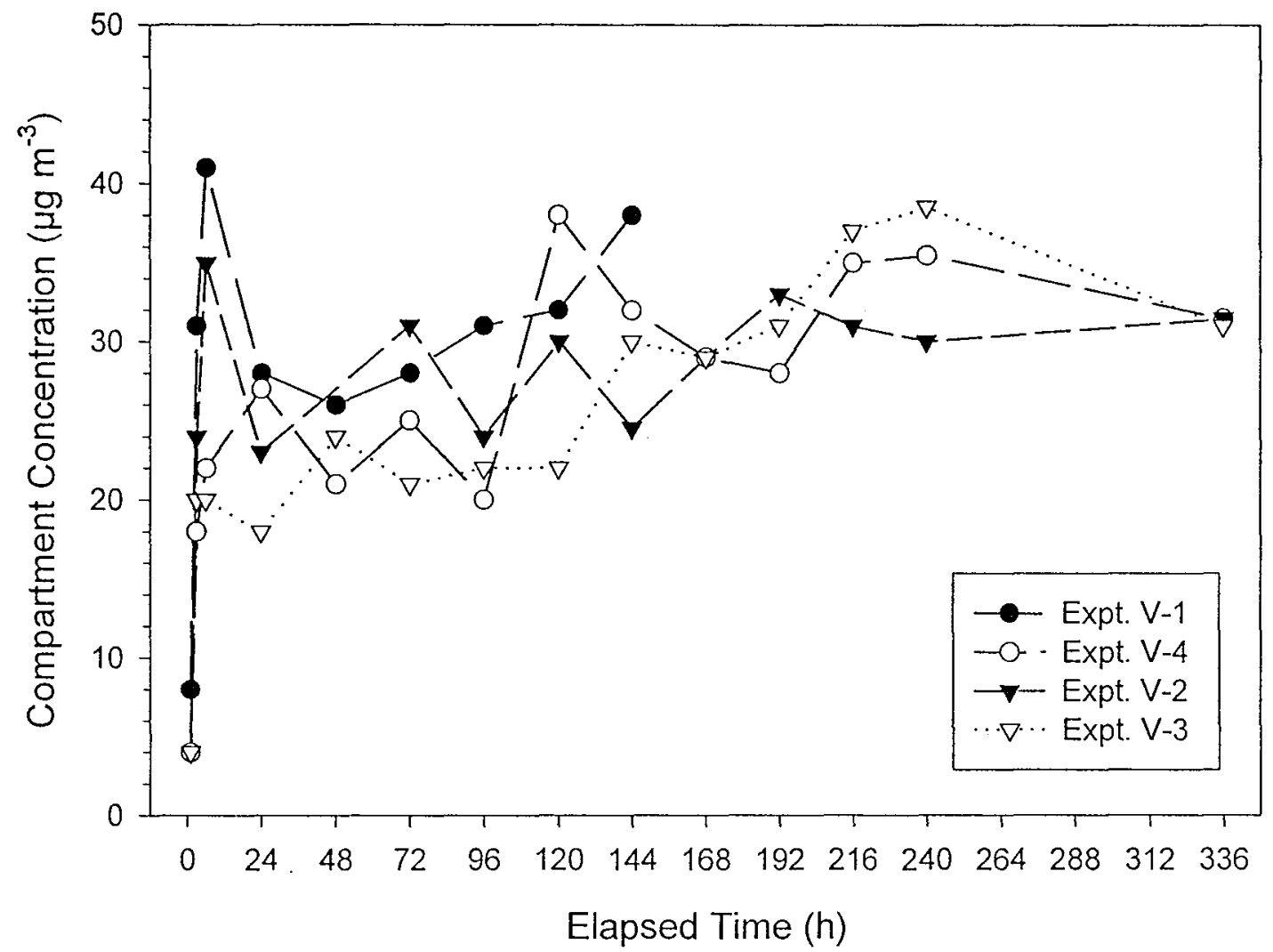

Figure 48. Compartment concentrations $\left(\mu \mathrm{g} \mathrm{m}^{-3}\right)$ of $\mathrm{TXIB} B$ in Experiments $\mathrm{V}-1-\mathrm{V}-4$. 


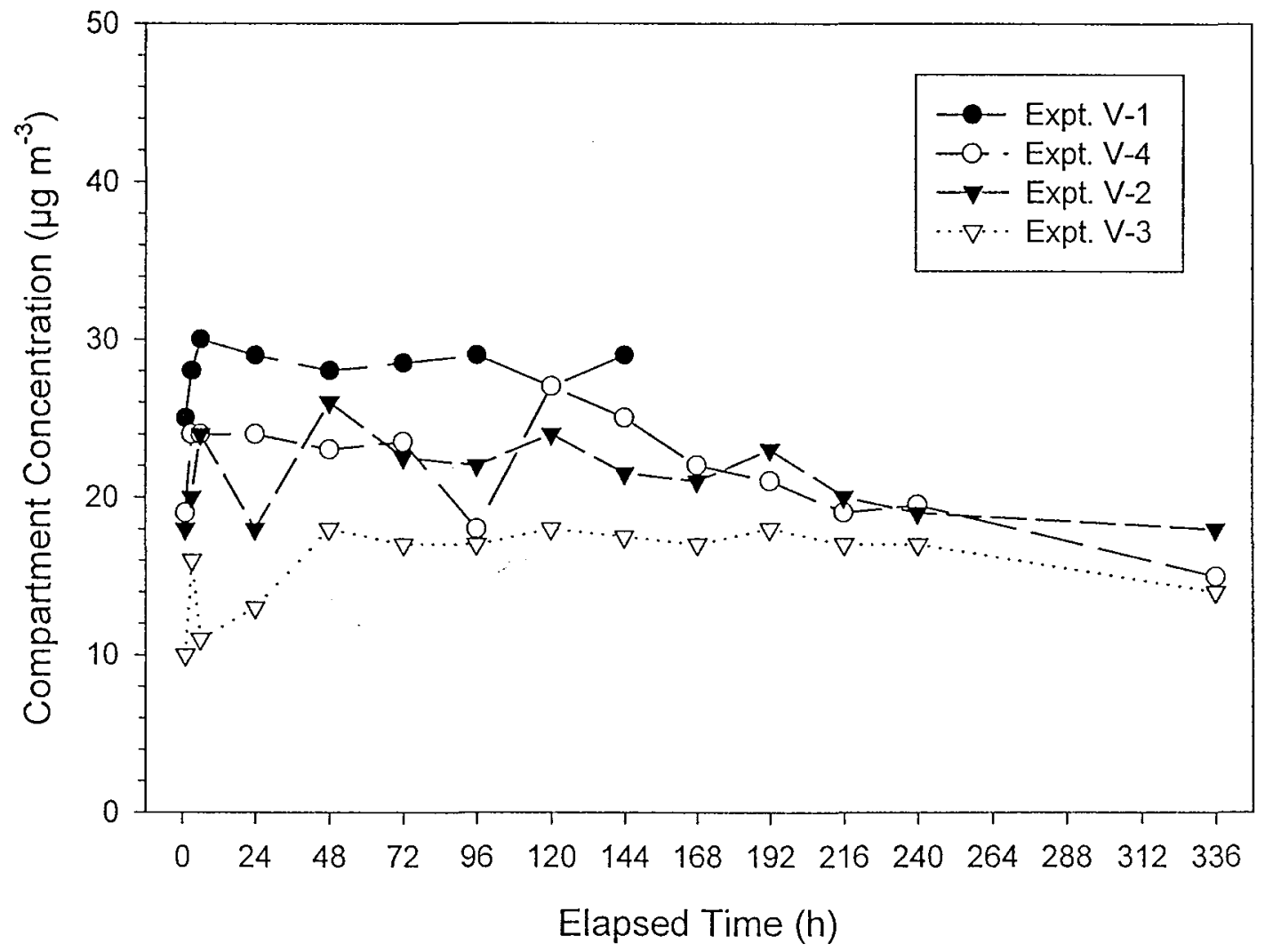

Figure 49. Compartment concentrations $\left(\mu \mathrm{g} \mathrm{m}^{-3}\right)$ of benzothiazole in Experiments $\mathrm{V}-1-\mathrm{V}-4$. 


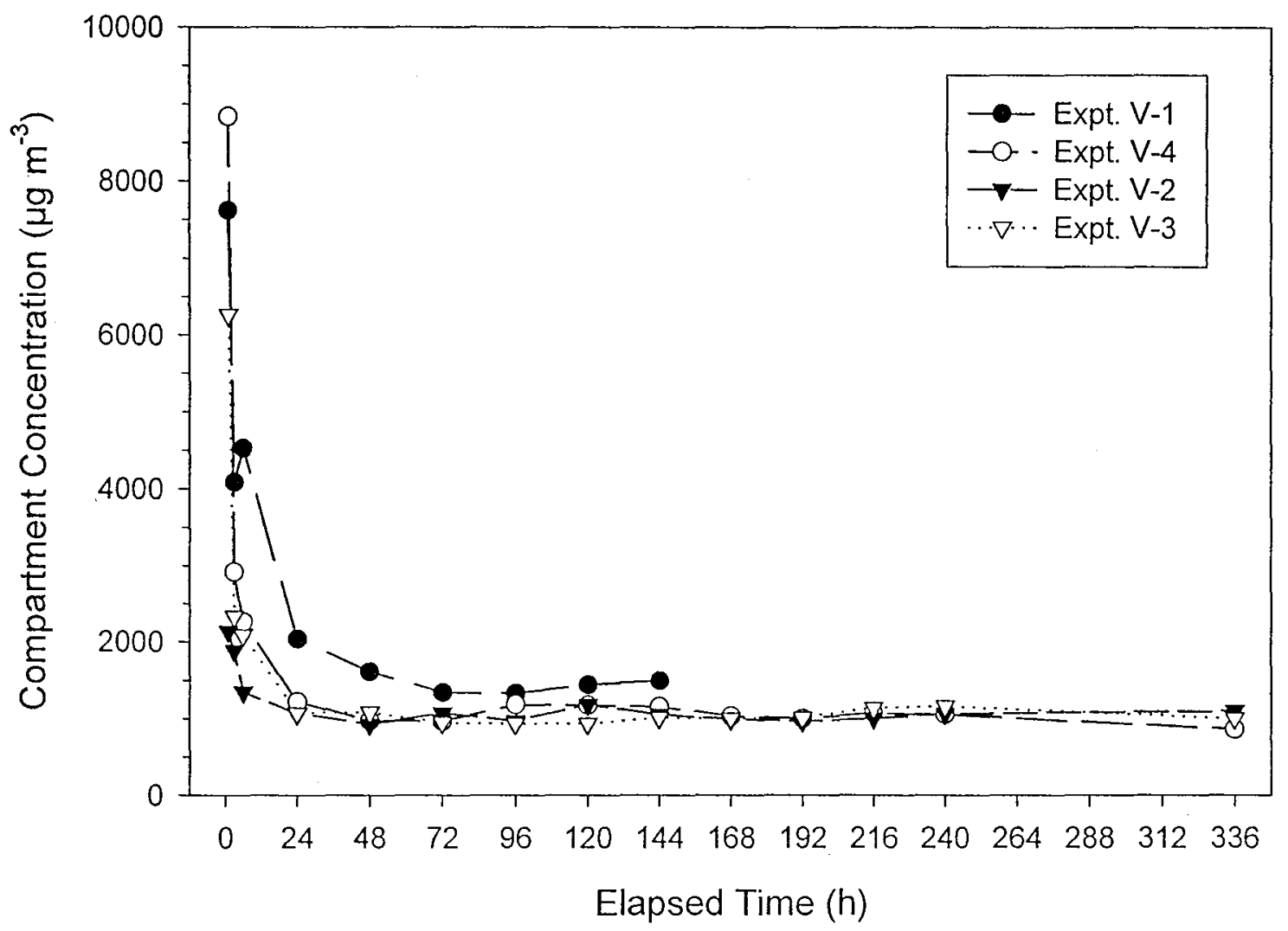

Figure 50. Compartment concentrations $\left(\mu \mathrm{g} \mathrm{m}^{-3}\right)$ of TVOC in Experiments $\mathrm{V}-1-\mathrm{V}-4$. 


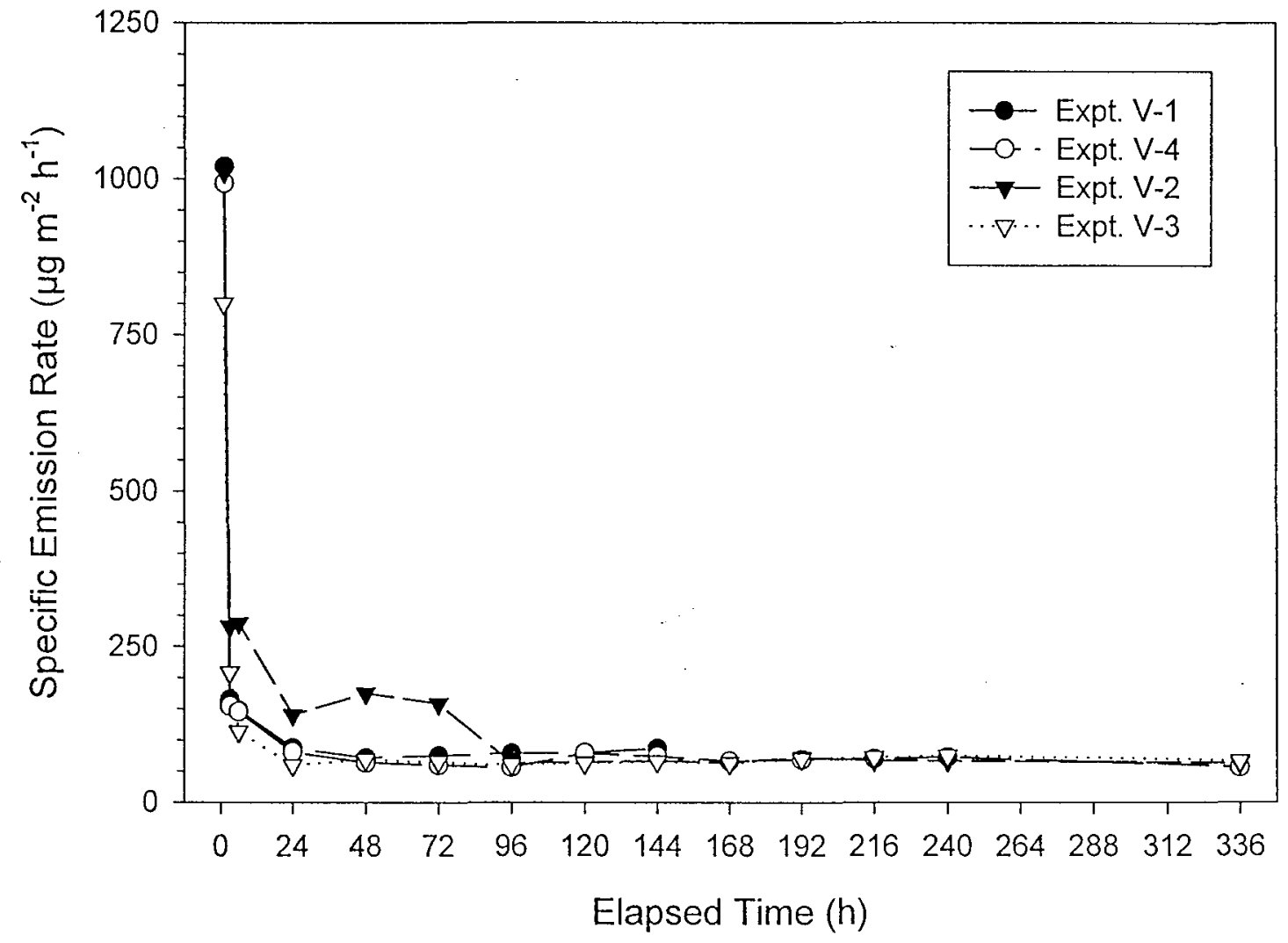

Figure 51. Specific emission rates $\left(\mu g \mathrm{~m}^{-2} \mathrm{~h}^{-1}\right)$ of $\mathrm{n}$-tridecane in Experiments $\mathrm{V}-1-\mathrm{V}-4$. 


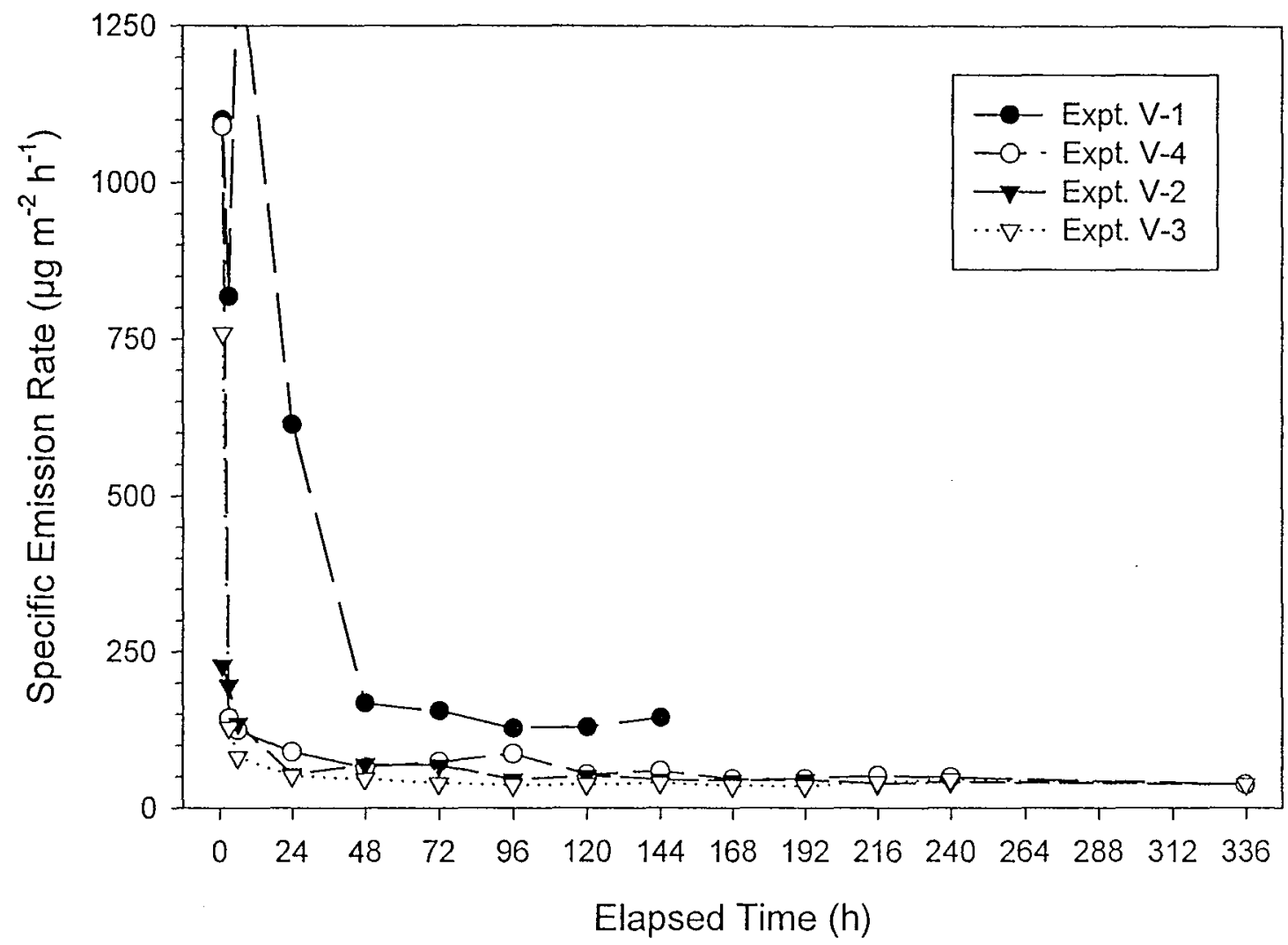

Figure 52. Specific emission rates $\left(\mu \mathrm{g} \mathrm{m}^{-2} h^{-1}\right)$ of toluene in Experiments $\mathrm{V}-1-\mathrm{V}-4$. The 6-h emission rate in Experiment $\mathrm{V}-1$ was $1,390 \mu \mathrm{g} \mathrm{m}^{-2} \mathrm{~h}^{-1}$. 


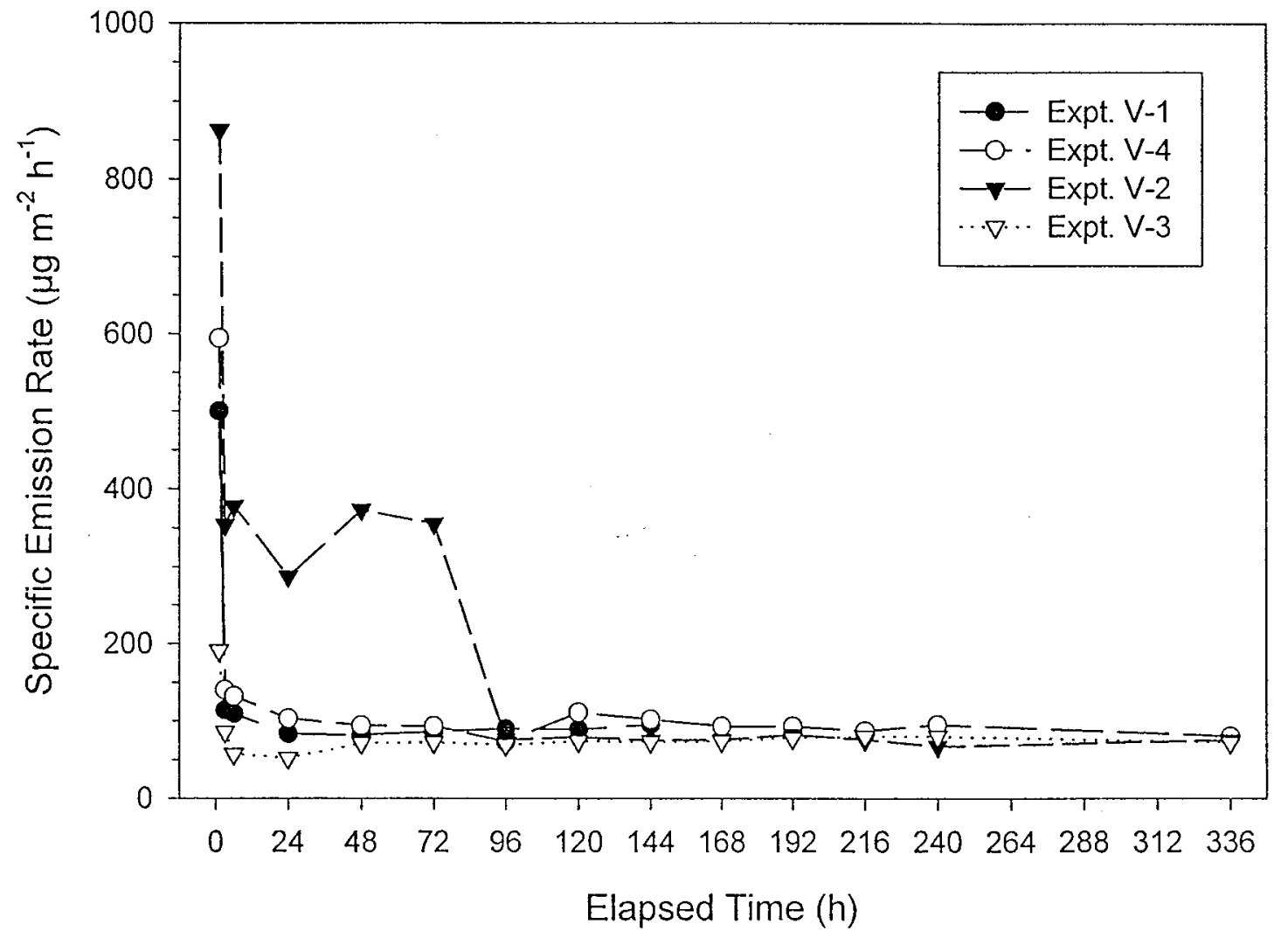

Figure 53. Specific emission rates $\left(\mu \mathrm{g} \mathrm{m}^{-2} \mathrm{~h}^{-1}\right)$ of phenol in Experiments $\mathrm{V}-1-\mathrm{V}-4$. 


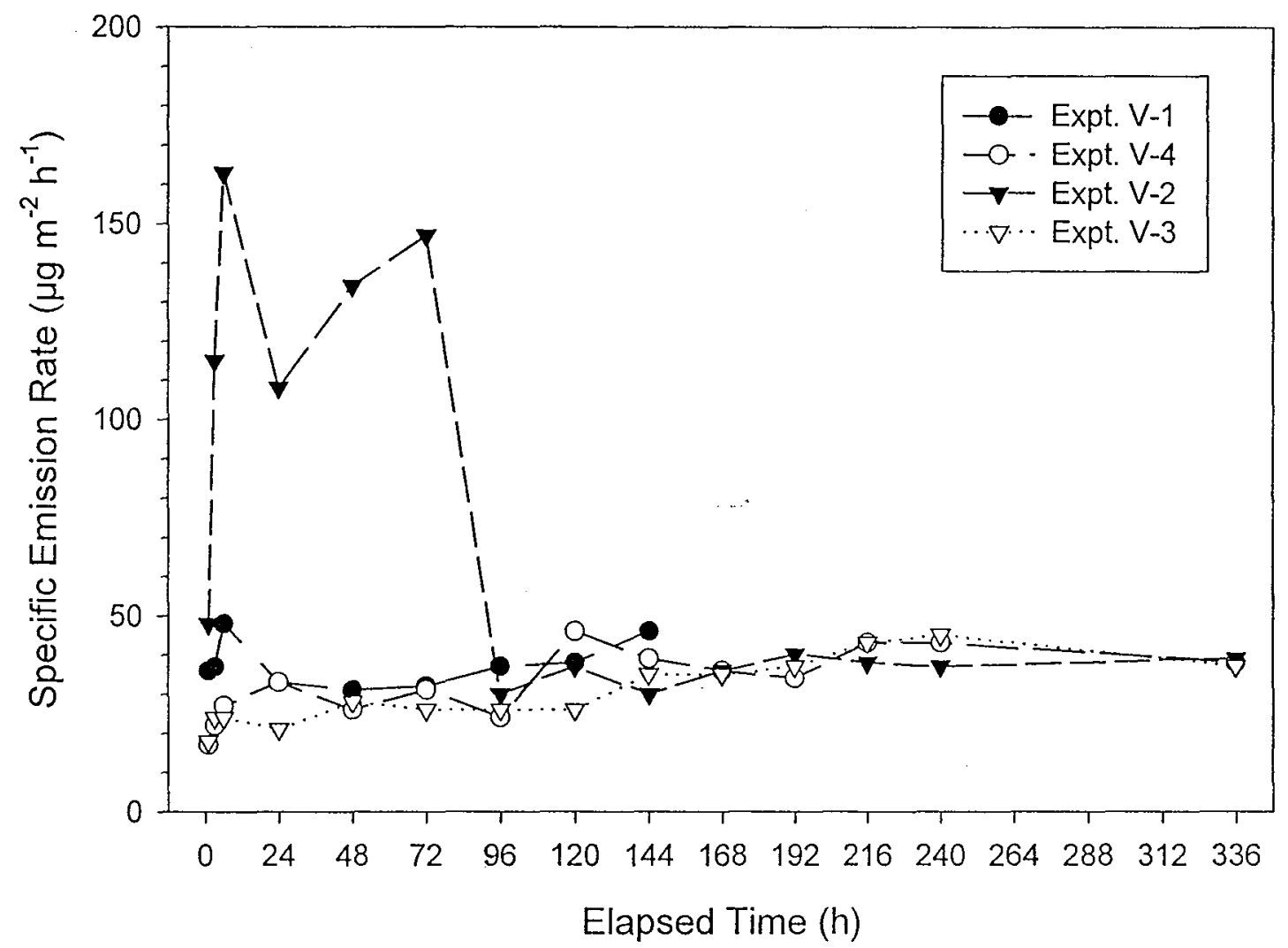

Figure 54. Specific emission rates $\left(\mu \mathrm{g} \mathrm{m}^{-2} \mathrm{~h}^{-1}\right)$ of TXIB® in Experiments $\mathrm{V}-1-\mathrm{V}-4$. 


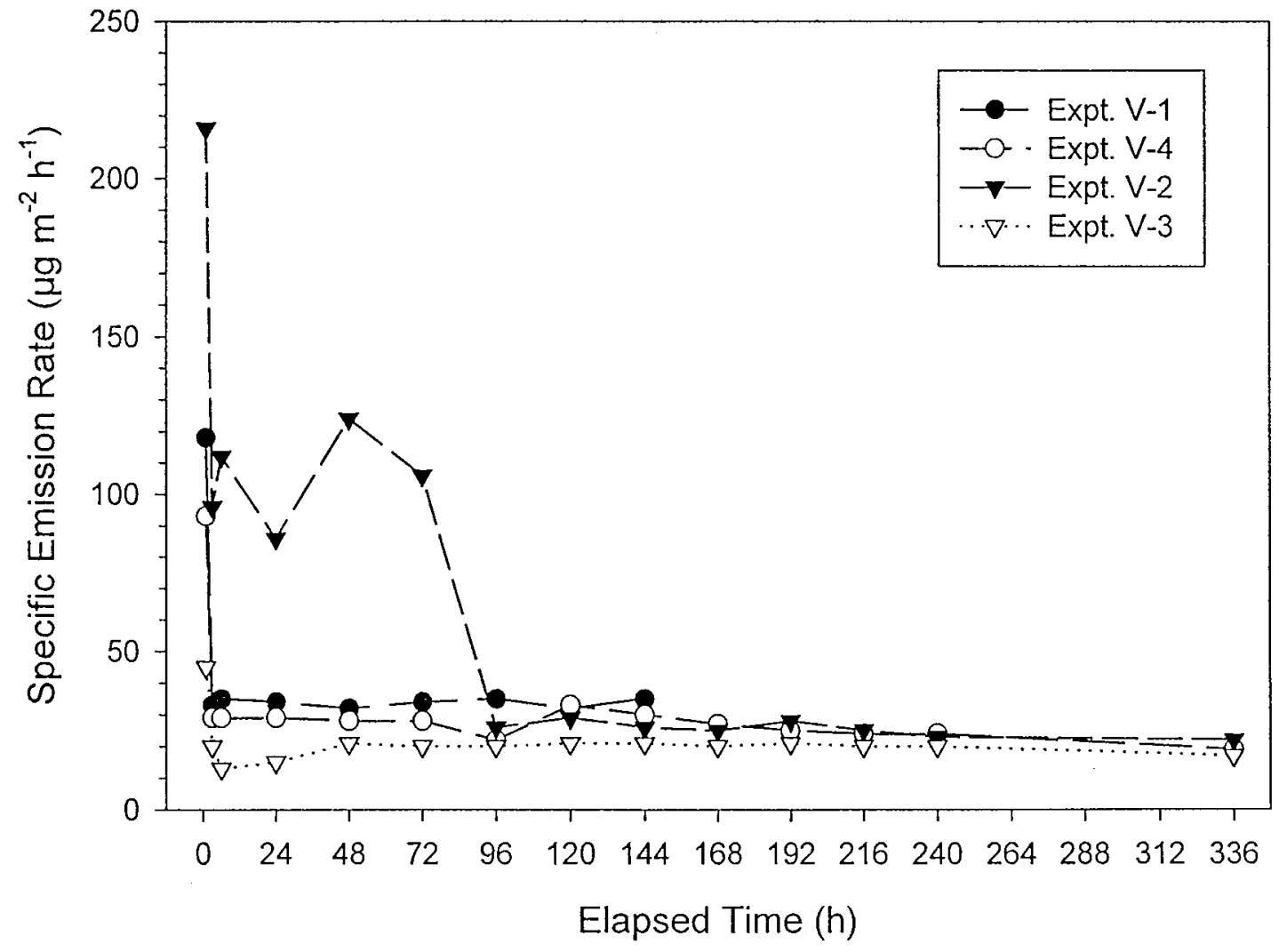

Figure 55. Specific emission rates $\left(\mu \mathrm{g} \mathrm{m}^{-2} \mathrm{~h}^{-1}\right)$ of benzothiazole in Experiments $\mathrm{V}-1-\mathrm{V}-4$. 


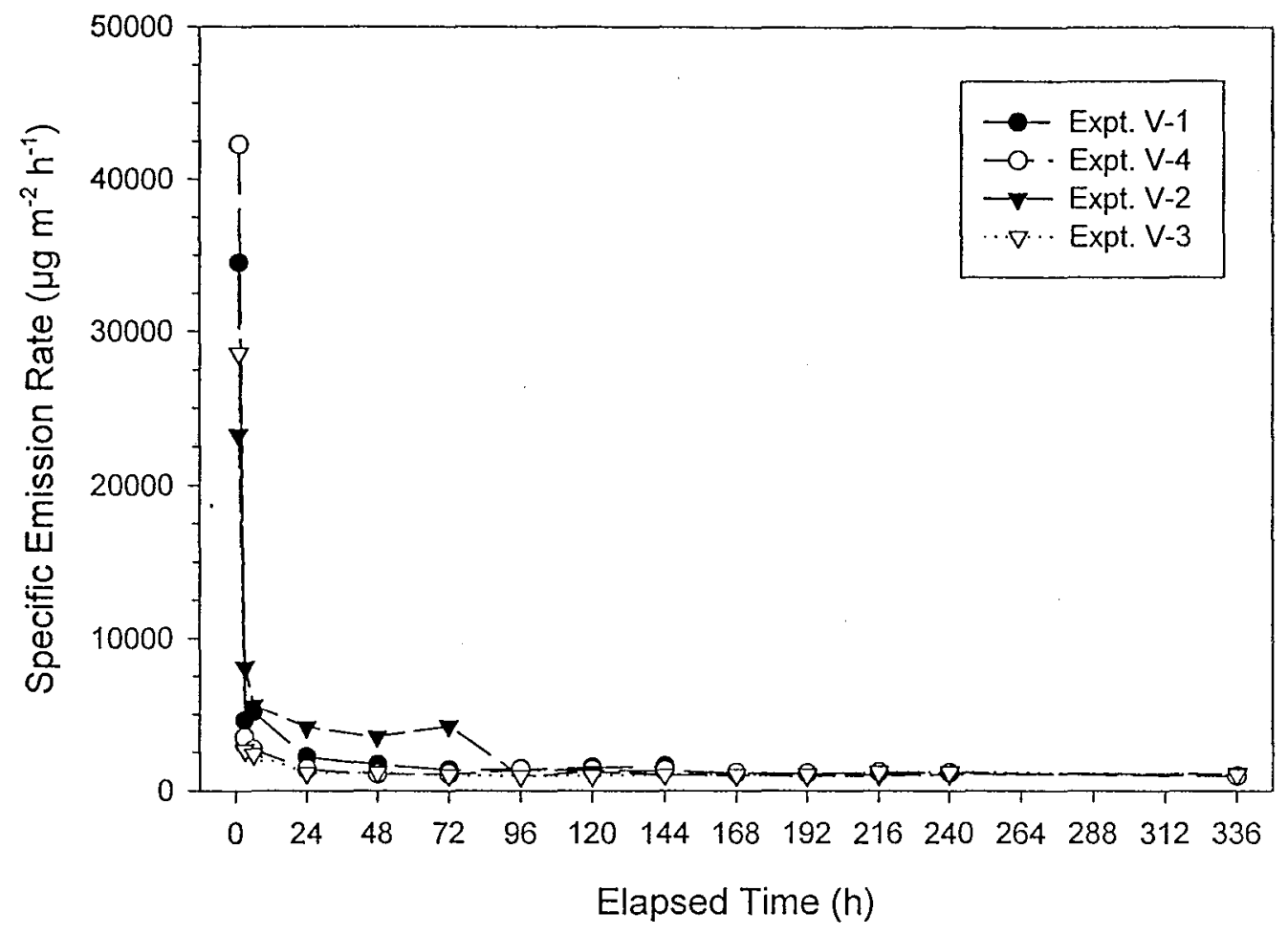

Figure 56a. Specific emission rates $\left(\mu \mathrm{g} \mathrm{m}^{-2} \mathrm{~h}^{-1}\right)$ of $T V O C$ in Experiments $\mathrm{V}-1-\mathrm{V}-4$.

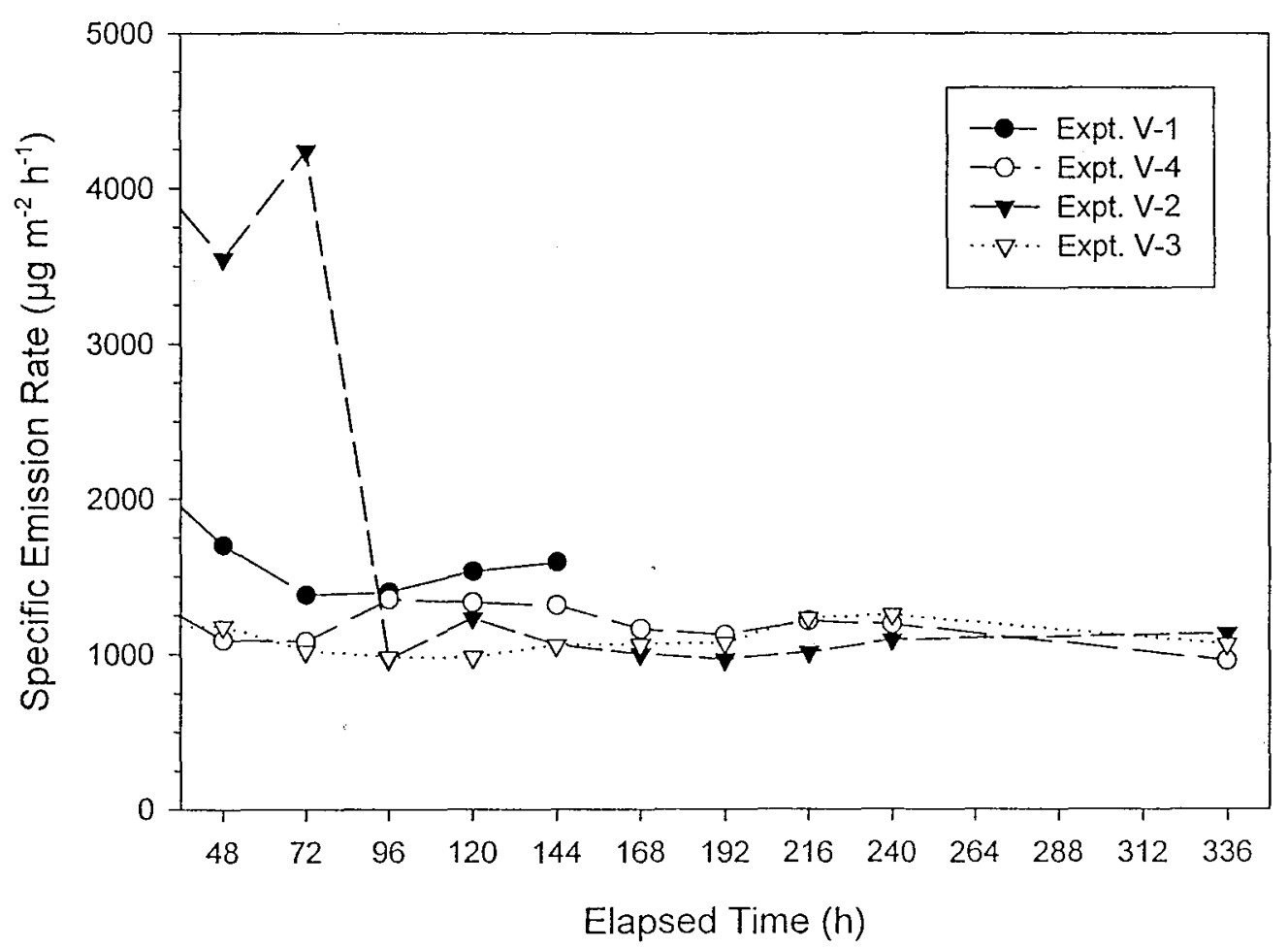

Figure 56b. Specific emission rates $\left(40 \mathrm{~m}^{-1} \mathrm{~h}^{-1}\right)$ of TVOC over $48-336$ hours in Experiments V-1 - V-4. 


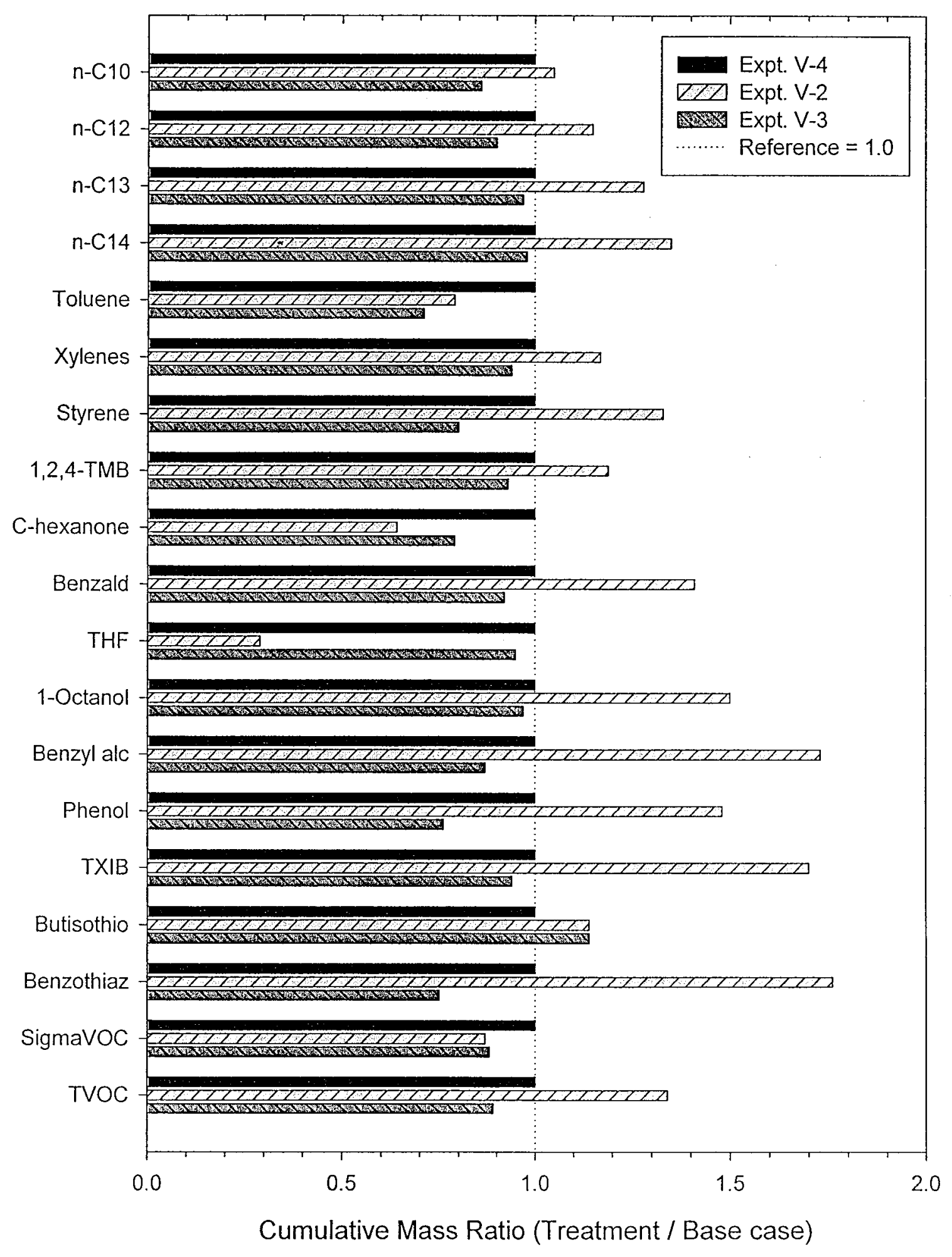

Figure 57. Effects of the treatments on the 0 - to 336-h cumulative mass emissions of target VOCs and TVOC from the vinyl flooring assembly relative to base-case Experiment $V-4$. 


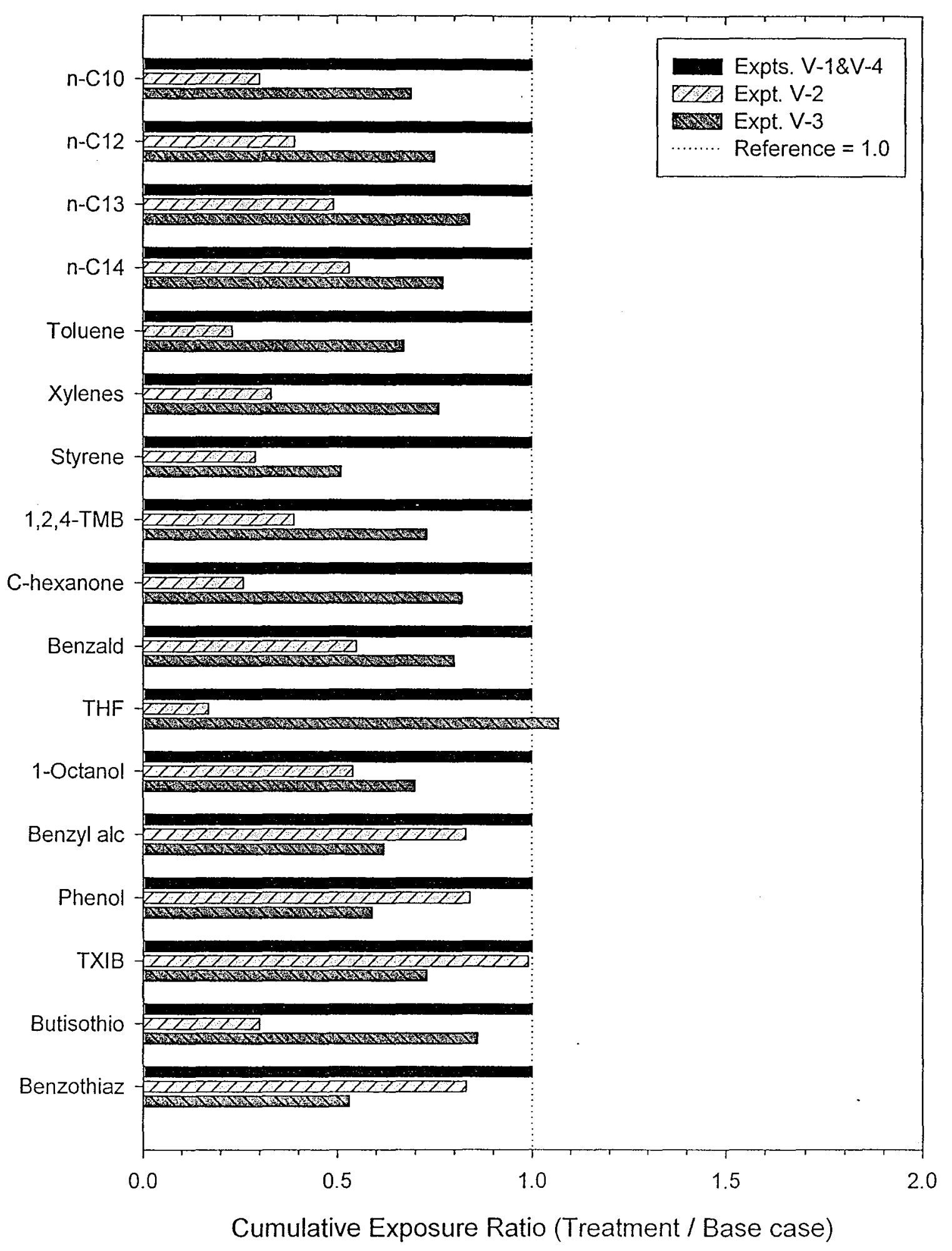

Figure 58. Effects of the treatments on the 0 - to 48-h cumulative exposures to target VOCs from the vinyl flooring assembly relative to averaged base-case Experiments $V-1$ and $V-4$. Toluene ratios were calculated using Experiment $V-4$ as the reference. The calculation assumes an occupancy of 20 hours per day. 


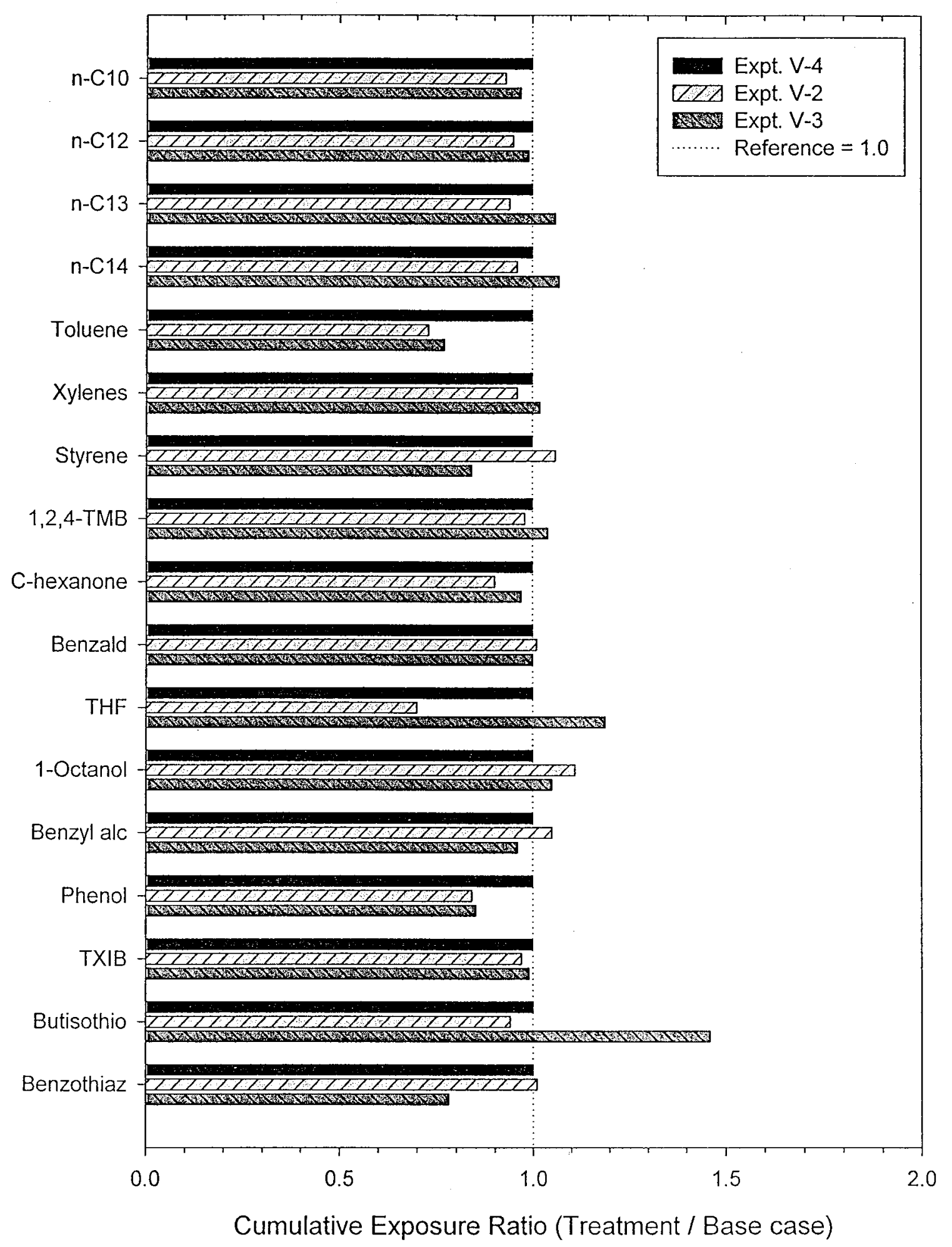

Figure 59. Effects of the treatments on the 48- to 336-h cumulative exposures to target VOCs from the vinyl flooring assembly relative to base-case Experiment $V-4$. The

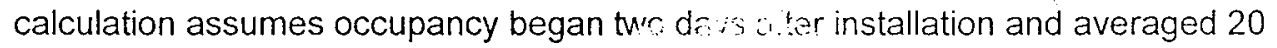
hours per day. 


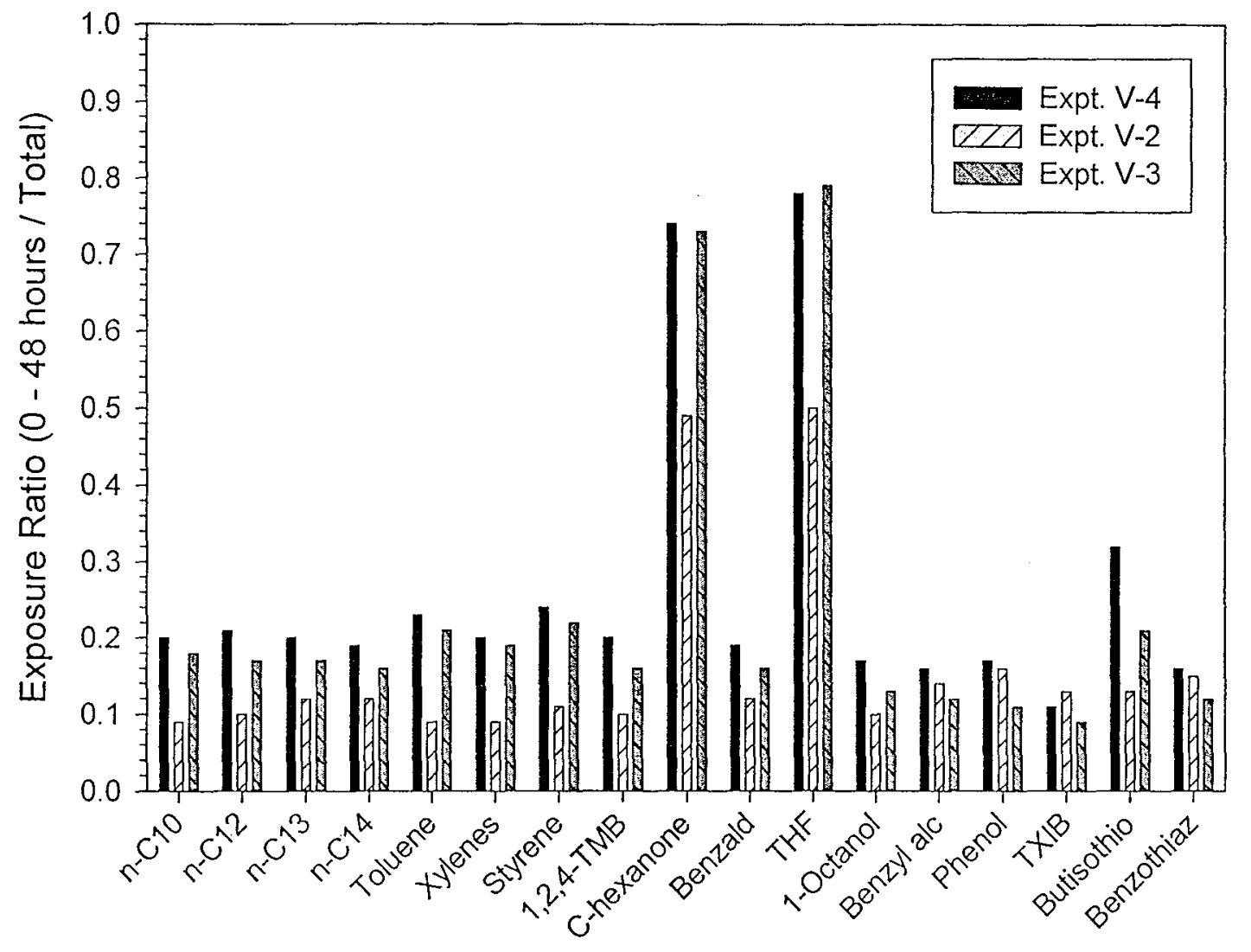

Figure 60. Cumulative exposures to target VOCs over $0-48$ hours divided by total cumulative exposures over $0-336$ hours for Experiments $V-2, V-3$ and $\mathrm{V}-4$. 


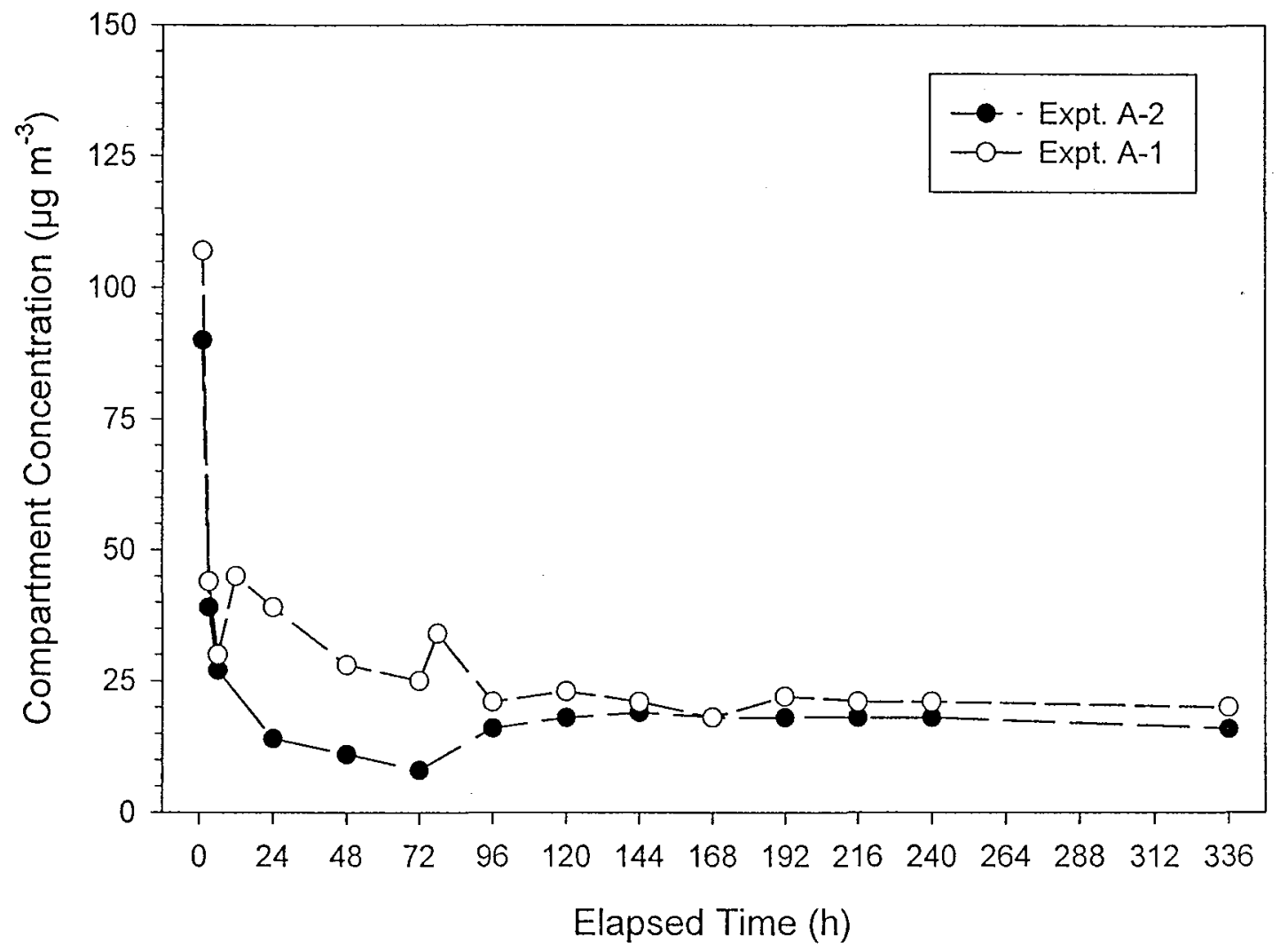

Figure 61. Compartment concentrations $\left(\mu \mathrm{g} \mathrm{m}^{-3}\right)$ of $n$-tridecane in Experiments $\mathrm{A}-1$ and $\mathrm{A}-2$. Compartment was heated to $33^{\circ} \mathrm{C}$ from $6-72$ hours in Experiment $\mathrm{A}-1$. 


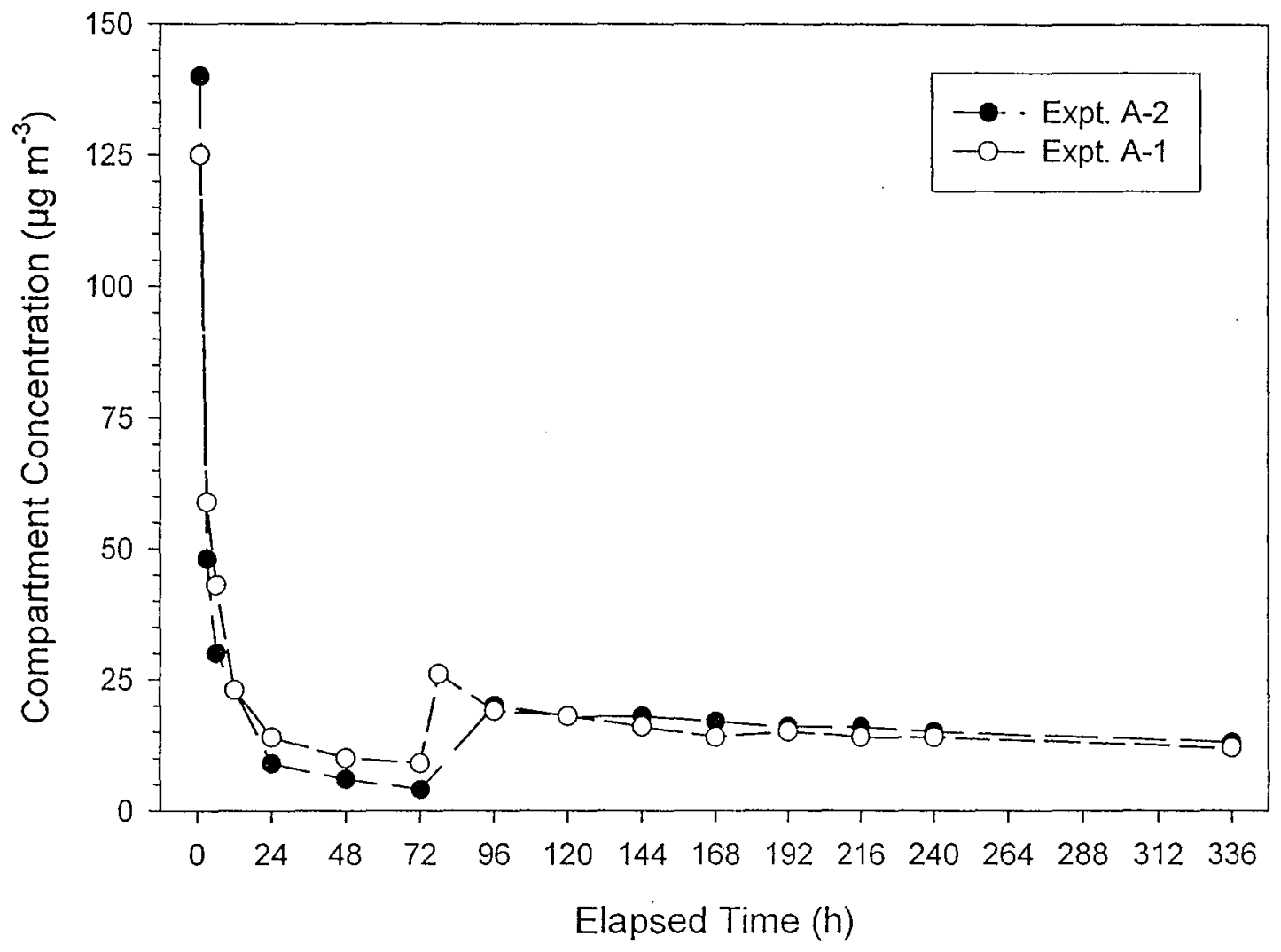

Figure 62. Compartment concentrations $\left(\mu \mathrm{g} \mathrm{m}^{-3}\right)$ of toluene in Experiments $\mathrm{A}-1$ and $\mathrm{A}-2$. Compartment was heated to $33^{\circ} \mathrm{C}$ from $6-72$ hours in Experiment $\mathrm{A}-1$. 


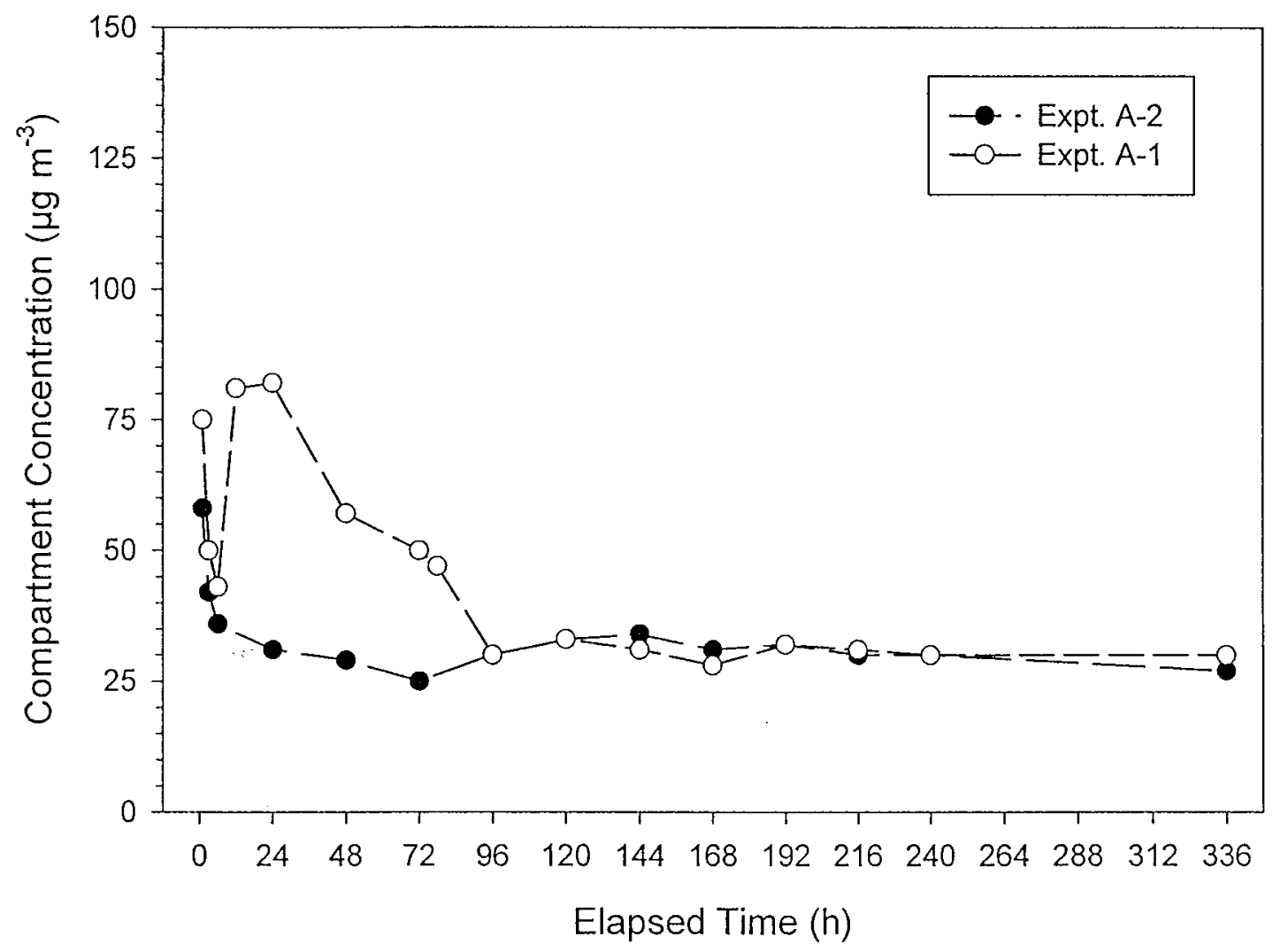

Figure 63. Compartment concentrations $\left(\mu \mathrm{g} \mathrm{m}^{-3}\right.$ ) of phenol in Experiments $\mathrm{A}-1$ and $\mathrm{A}-2$. Compartment was heated to $33^{\circ} \mathrm{C}$ from $6-72$ hours in Experiment A-1. 


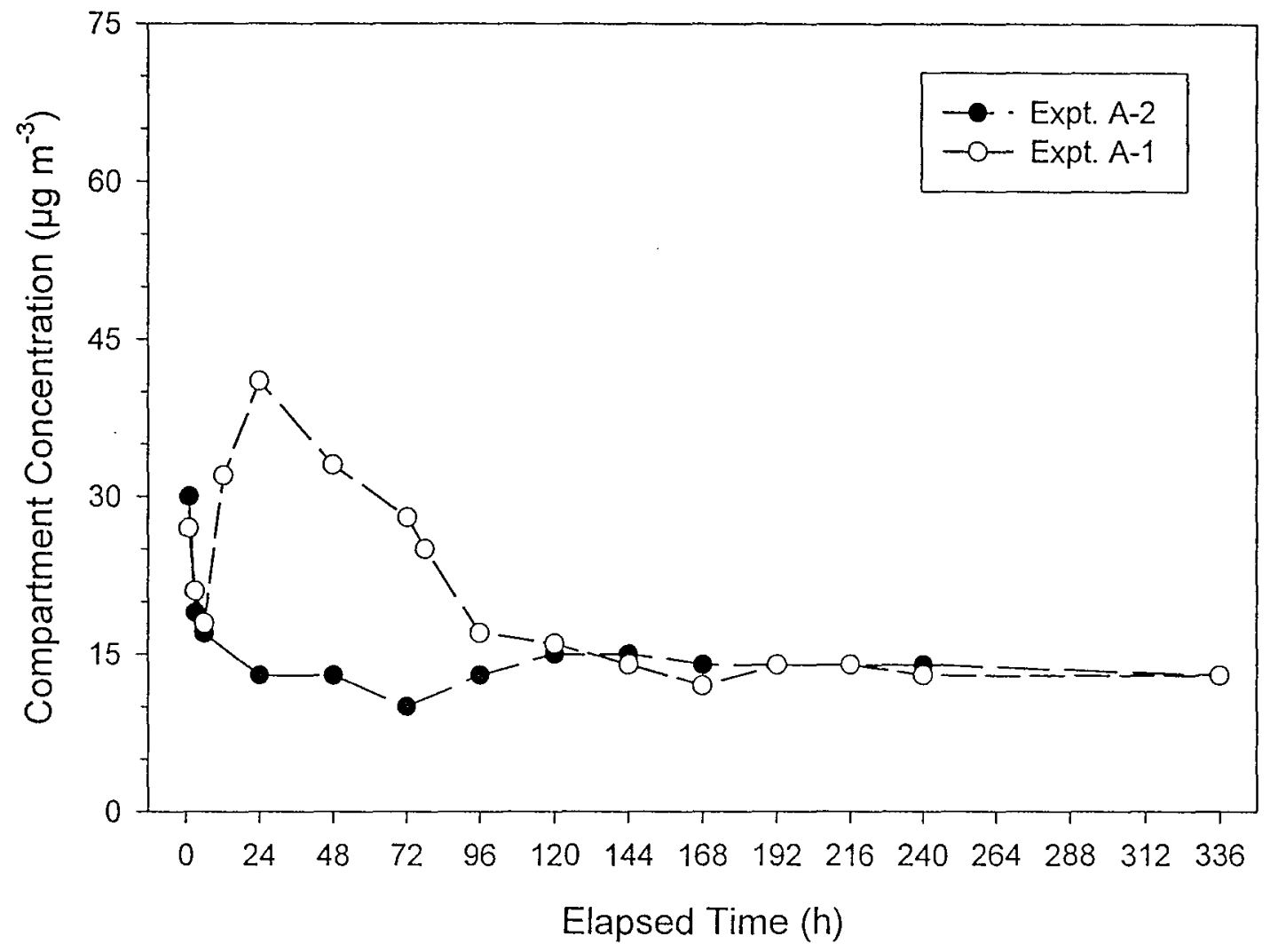

Figure 64. Compartment concentrations $\left(\mu \mathrm{g} \mathrm{m}^{-3}\right)$ of TXIB $\otimes$ in Experiments $\mathrm{A}-1$ and $\mathrm{A}-2$. Compartment was heated to $33^{\circ} \mathrm{C}$ from $6-72$ hours in Experiment $\mathrm{A}-1$. 


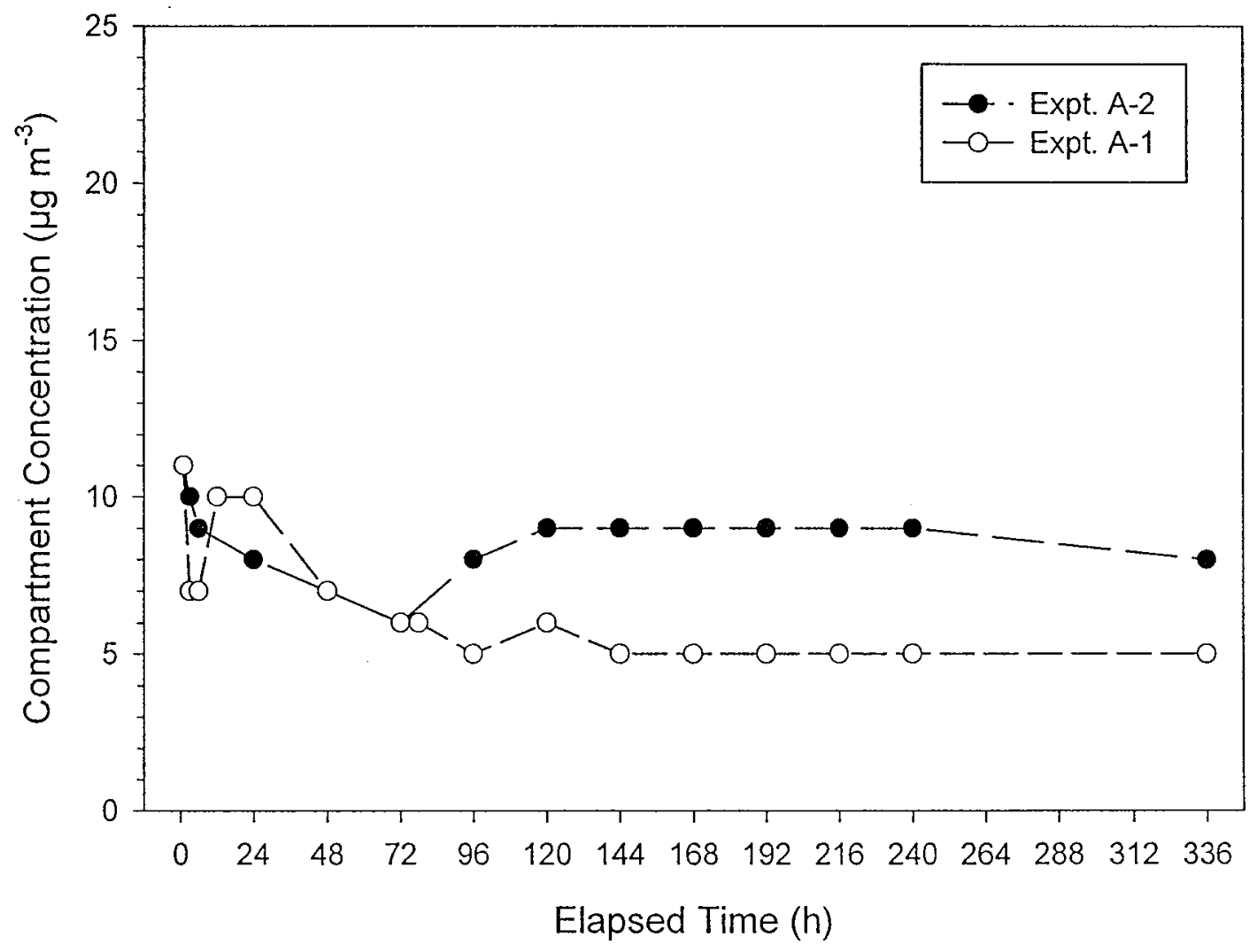

Figure 65. Compartment concentrations $\left(\mu \mathrm{g} \mathrm{m}^{-3}\right)$ of benzothiazole in Experiments $A-1$ and $\mathrm{A}-2$. Compartment was heated to $33^{\circ} \mathrm{C}$ from $6-72$ hours in Experiment $\mathrm{A}-1$. 


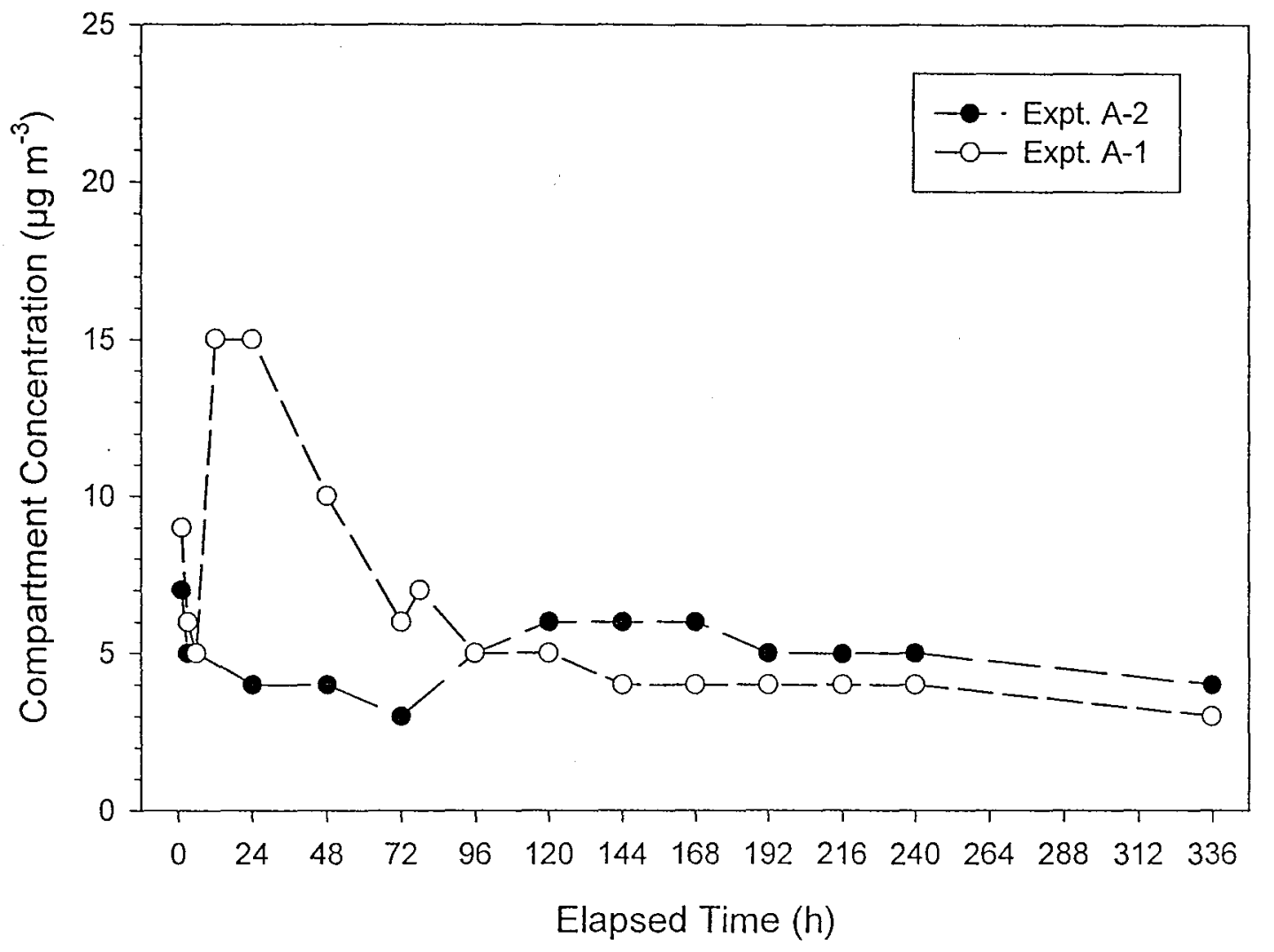

Figure 66. Compartment concentrations $\left(\mu \mathrm{g} \mathrm{m}^{-3}\right.$ ) of 4-PCH in Experiments $\mathrm{A}-1$ and $\mathrm{A}-2$. Compartment was heated to $33^{\circ} \mathrm{C}$ from $6-72$ hours in Experiment $\mathrm{A}-1$. 


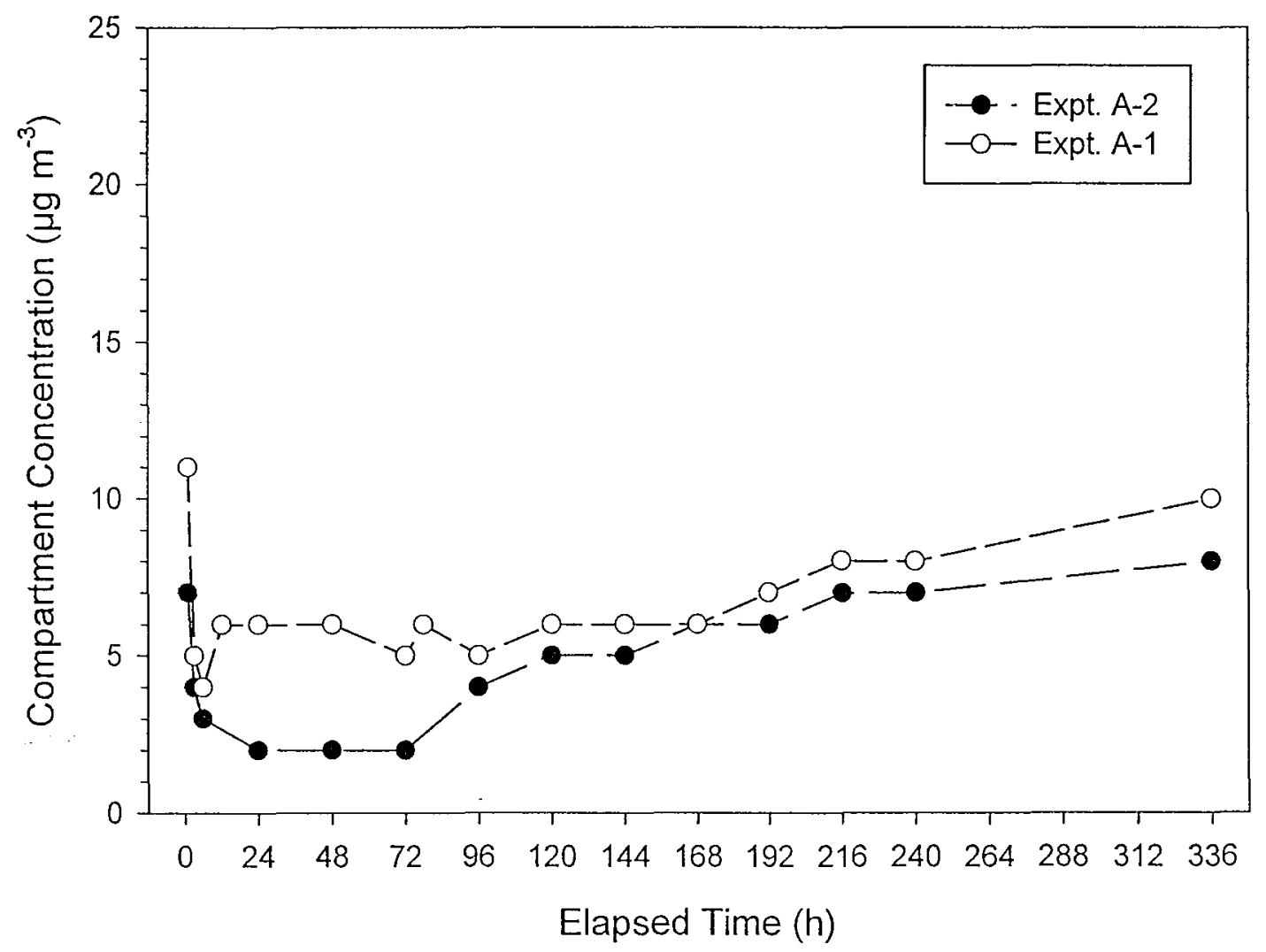

Figure 67. Compartment concentrations $\left(\mu \mathrm{g} \mathrm{m}^{-3}\right)$ of BHT in Experiments $A-1$ and $A-2$. Compartment was heated to $33^{\circ} \mathrm{C}$ from $6-72$ hours in Experiment A-1. 


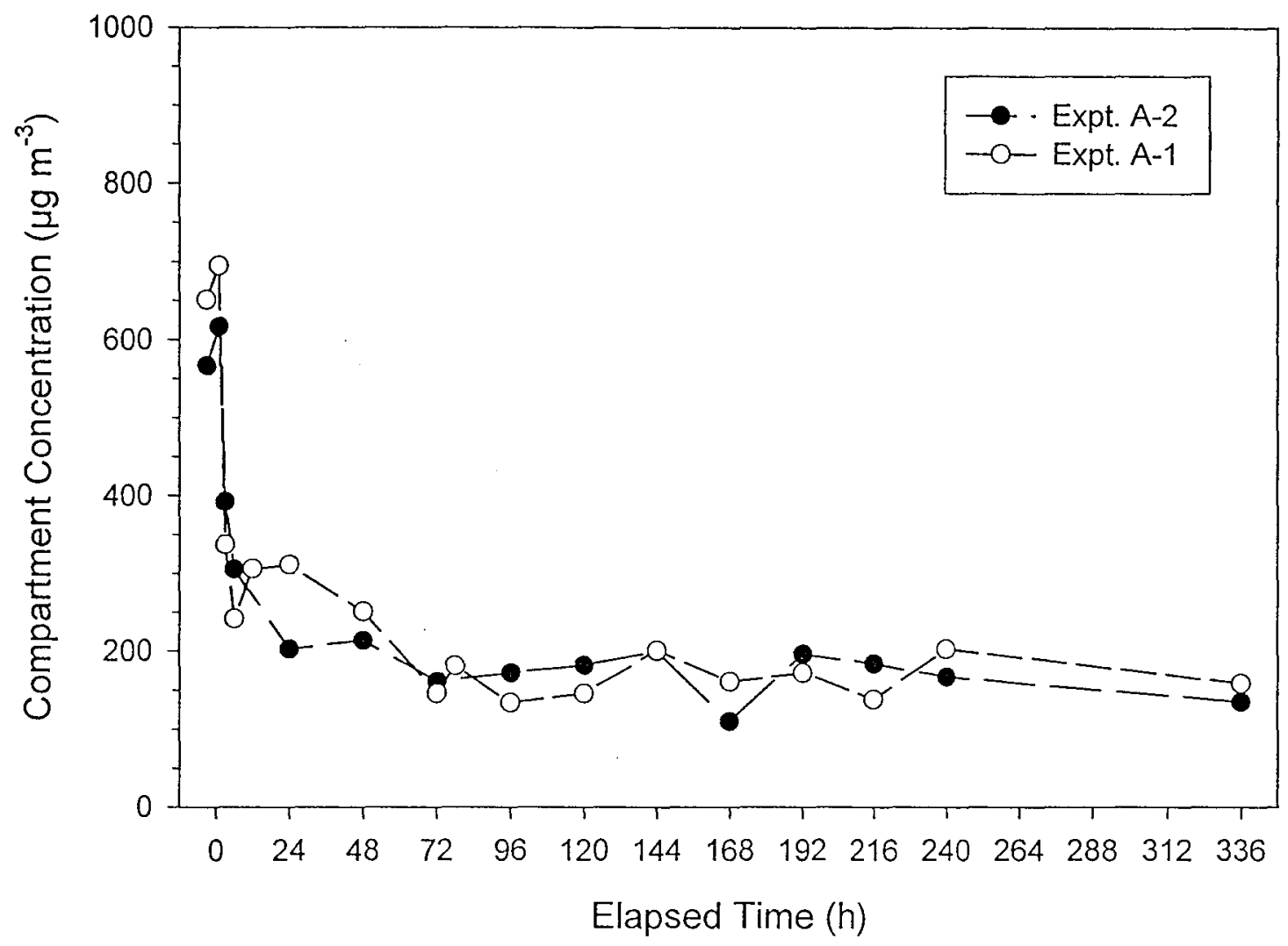

Figure 68. Compartment concentrations $\left(\mu \mathrm{g} \mathrm{m}^{-3}\right.$ ) of ethylene glycol in Experiments A-1 and $\mathrm{A}-2$. Compartment was heated to $33^{\circ} \mathrm{C}$ from $6-72$ hours in Experiment $\mathrm{A}-1$. 


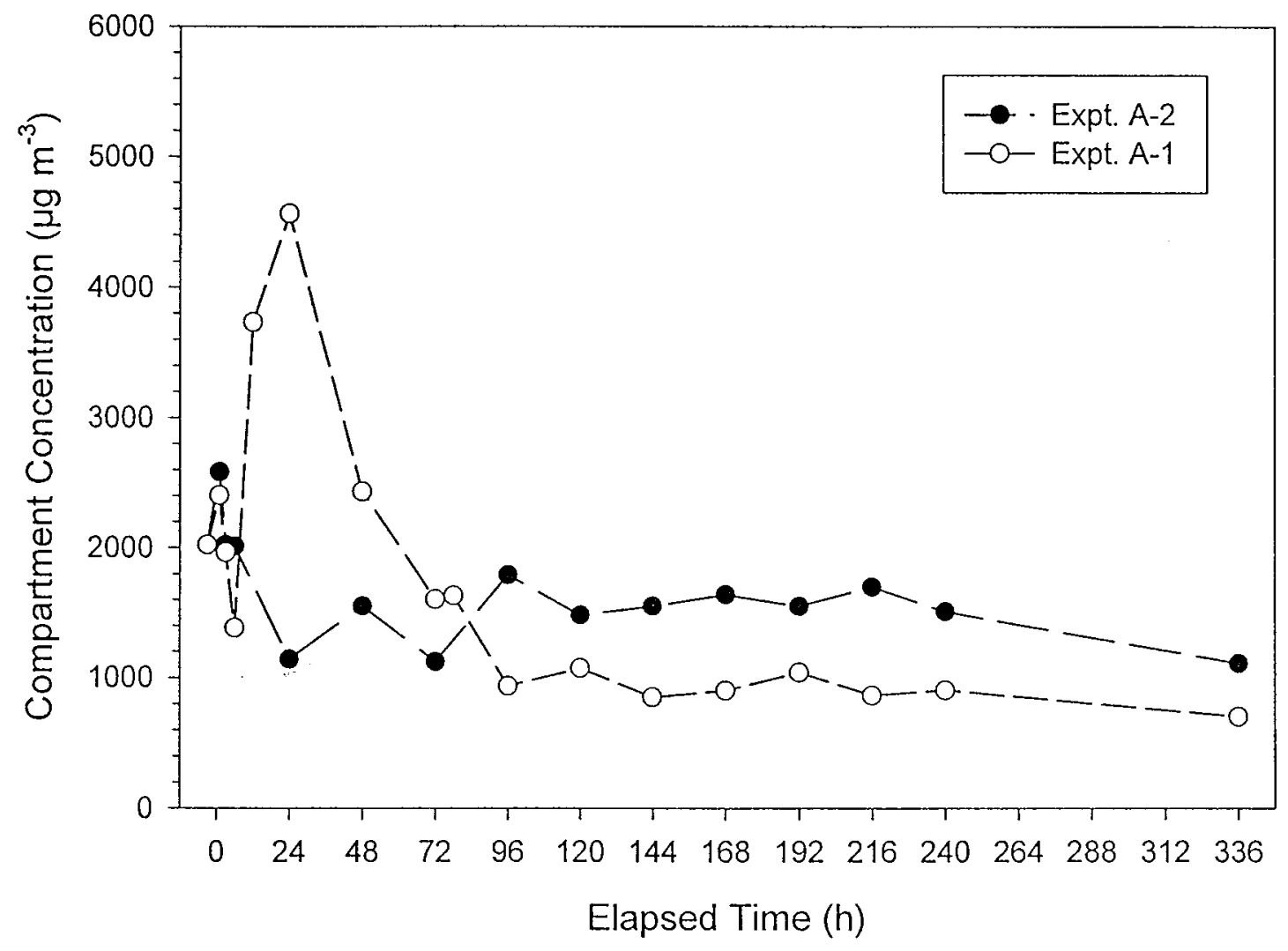

Figure 69. Compartment concentrations $\left(\mu \mathrm{g} \mathrm{m}^{-3}\right)$ of Texanol $\cap$ in Experiments A-1 and $\mathrm{A}-2$. Compartment was heated to $33^{\circ} \mathrm{C}$ from $6-72$ hours in Experiment $\mathrm{A}-1$. 


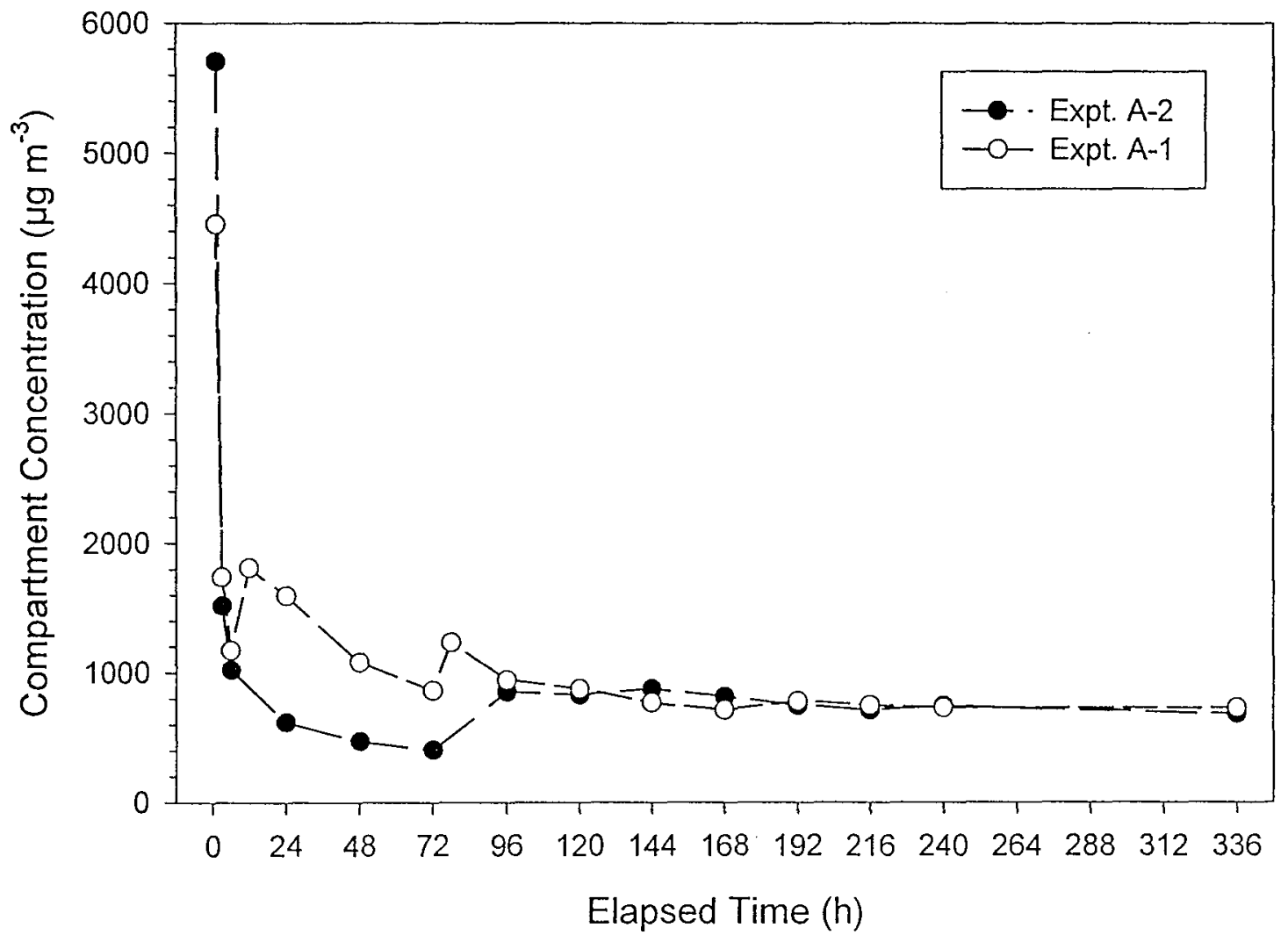

Figure 70. Compartment concentrations $\left(\mu \mathrm{g} \mathrm{m}^{-3}\right)$ of $T_{V O C}$ in Experiments A-1 and $\mathrm{A}-2$. Compartment was heated to $33^{\circ} \mathrm{C}$ from $6-72$ hours in Experiment $\mathrm{A}-1$. 


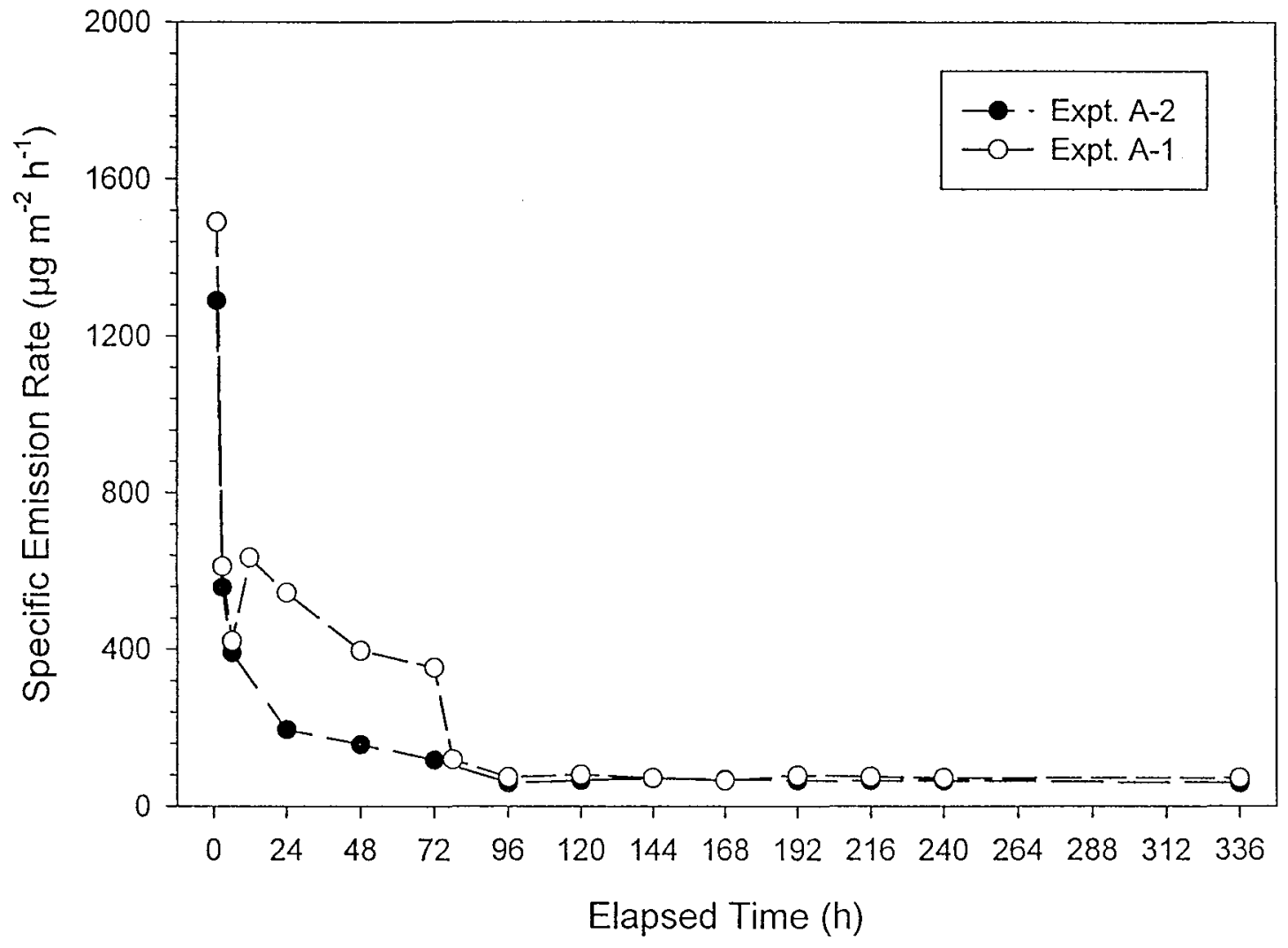

Figure 71. Specific emission rates $\left(\mu \mathrm{g} \mathrm{m}^{-2} \mathrm{~h}^{-1}\right)$ of $\mathrm{n}$-tridecane in Experiments $\mathrm{A}-1$ and A-2. Compartment was heated to $33^{\circ} \mathrm{C}$ from $6-72$ hours in Experiment $\mathrm{A}-1$. 


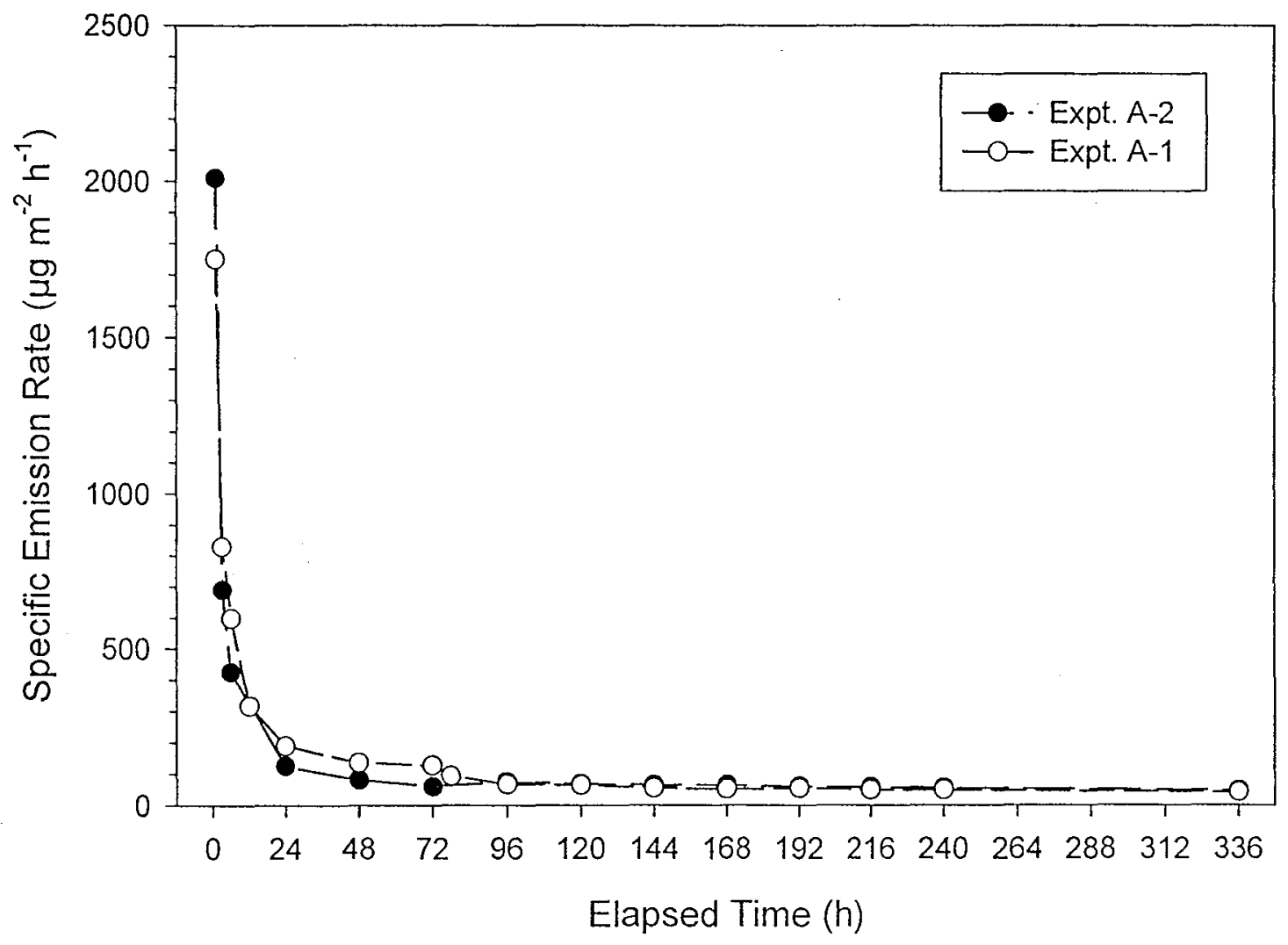

Figure 72. Specific emission rates $\left(\mu \mathrm{g} \mathrm{m}^{-2} \mathrm{~h}^{-1}\right)$ of toluene in Experiments A-1 and A-2. Compartment was heated to $33^{\circ} \mathrm{C}$ from $6-72$ hours in Experiment $\mathrm{A}-1$. 


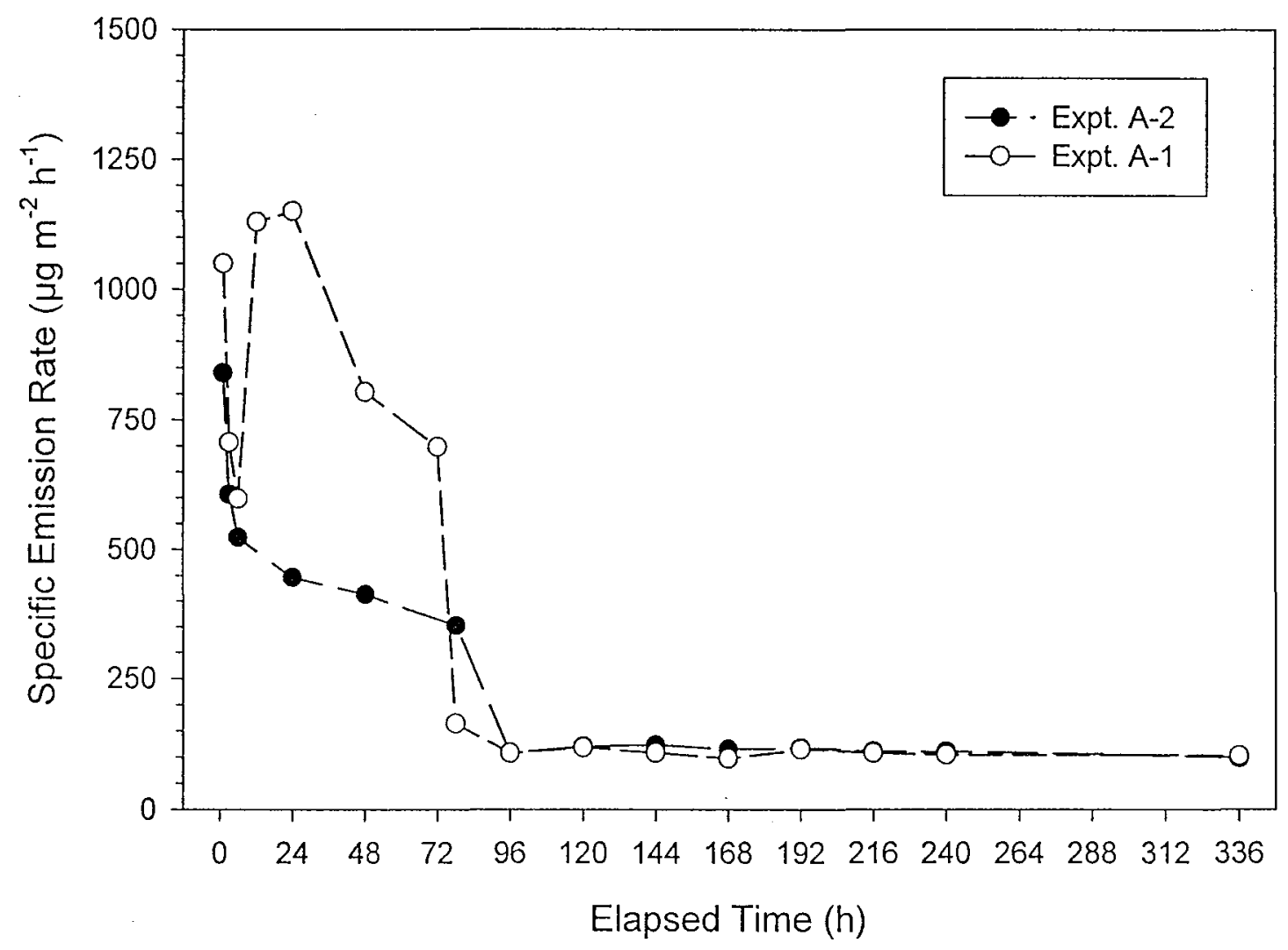

Figure 73. Specific emission rates $\left(\mu \mathrm{g} \mathrm{m}^{-2} \mathrm{~h}^{-1}\right)$ of phenol in Experiments A-1 and A-2. Compartment was heated to $33^{\circ}$ from $6-72$ hours in Experiment $A-1$. 


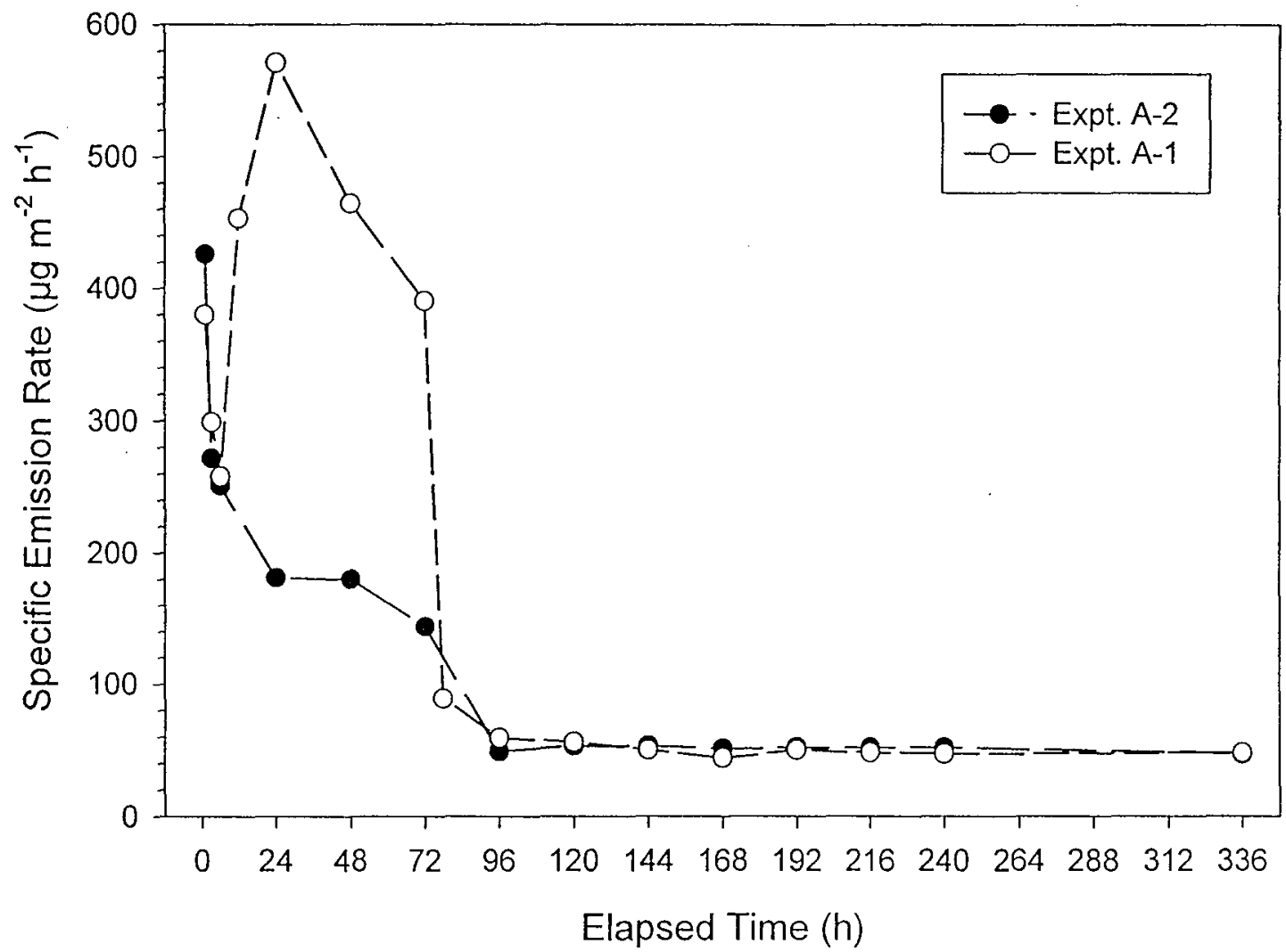

Figure 74. Specific emission rates $\left(\mu \mathrm{g} \mathrm{m}^{-2} \mathrm{~h}^{-1}\right)$ of TXIB@ in Experiments A-1 and A-2. Compartment was heated to $33^{\circ}$ from $6-72$ hours in Experiment A-1. 


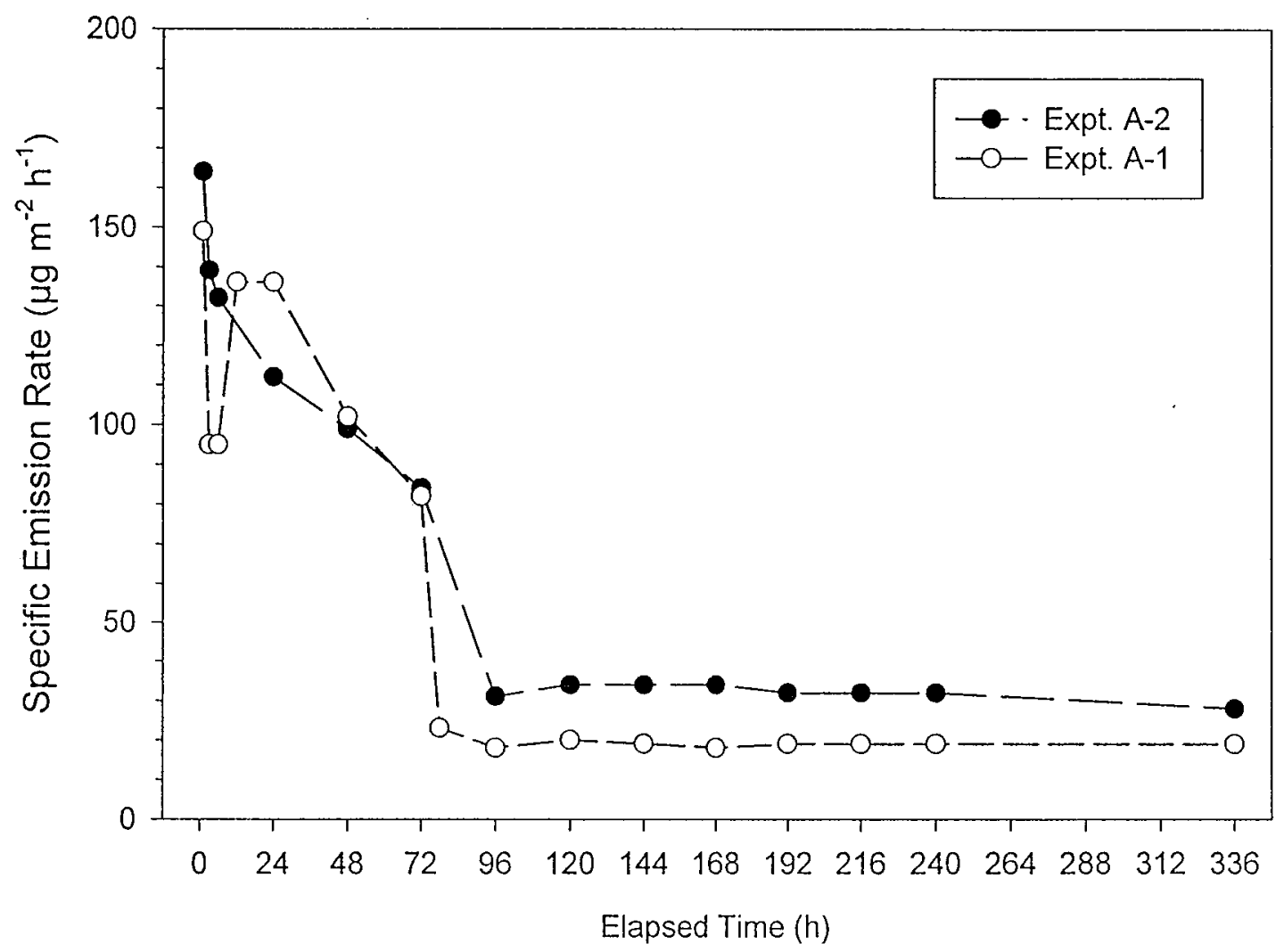

Figure 75. Specific emission rates $\left(\mu \mathrm{g} \mathrm{m}^{-2} \mathrm{~h}^{-1}\right)$ of benzothiazole in Experiments $A-1$ and A-2. Compartment was heated to $33^{\circ} \mathrm{C}$ from $6-72$ hours in Experiment $\mathrm{A}-1$. 


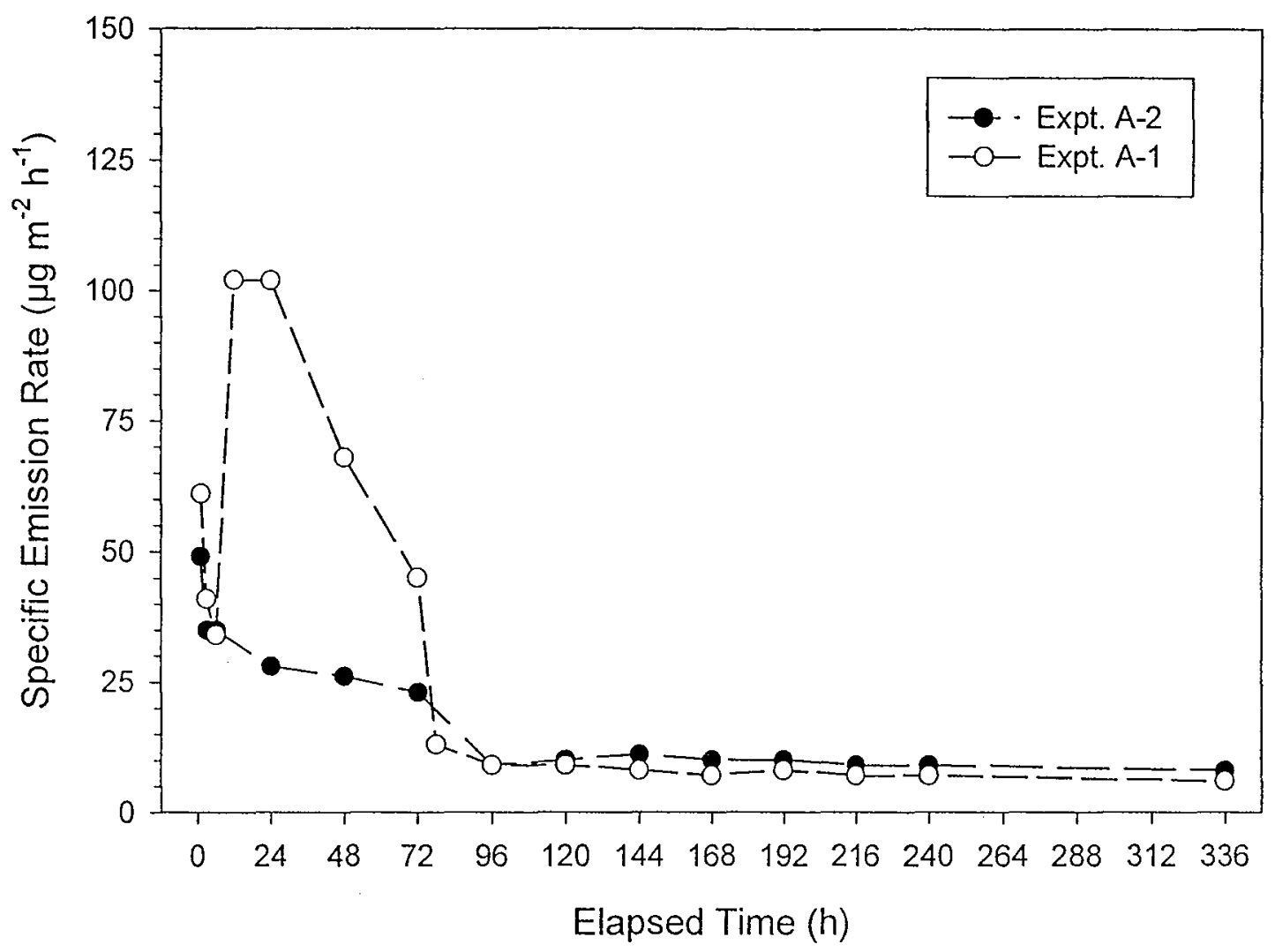

Figure 76. Specific emission rates $\left(\mu \mathrm{g} \mathrm{m}^{-2} \mathrm{~h}^{-1}\right)$ of 4-PCH in Experiments A-1 and A-2. Compartment was heated to $33^{\circ}$ from $6-72$ hours in Experiment A-1. 


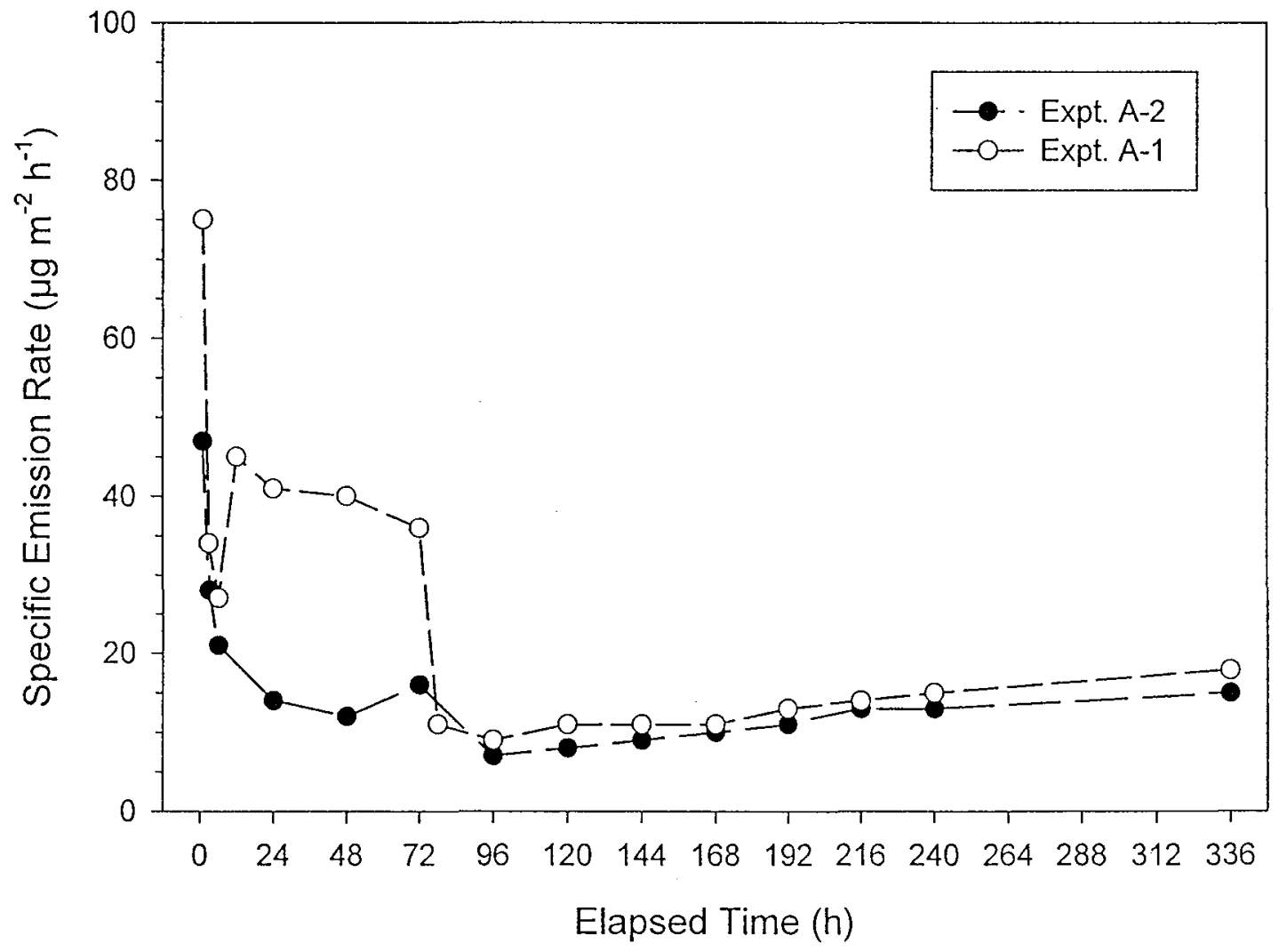

Figure 77. Specific emission rates $\left(\mu \mathrm{g} \mathrm{m}^{-2} \mathrm{~h}^{-1}\right)$ of BHT in Experiments A-1 and A-2. Compartment was heated to $33^{\circ} \mathrm{C}$ from $6-72$ hours in Experiment A-1. 


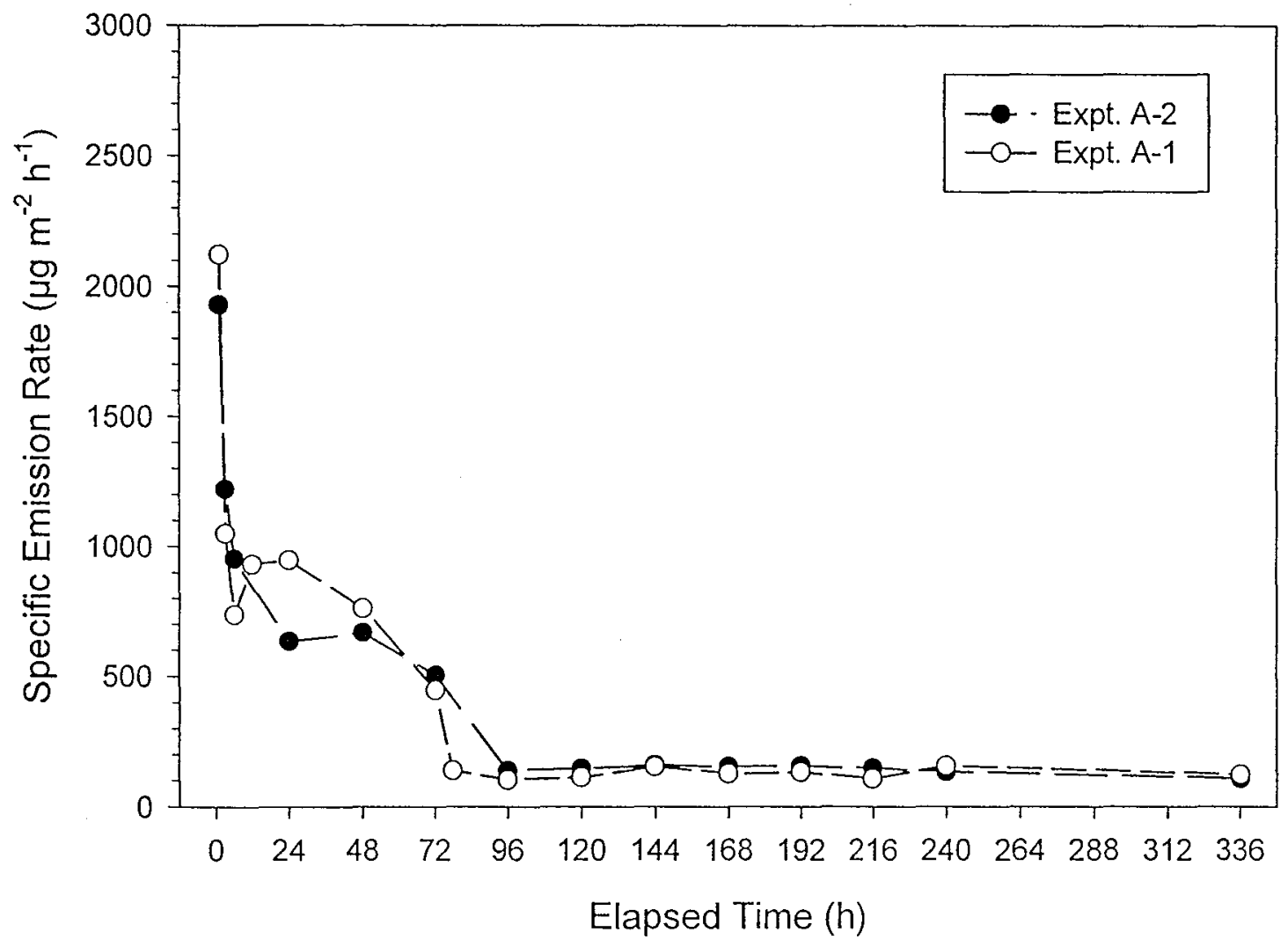

Figure 78. Specific emission rates $\left(\mu \mathrm{g} \mathrm{m}^{-2} \mathrm{~h}^{-1}\right)$ of ethylene glycol in Experiments A-1 and $\mathrm{A}-2$. Compartment was heated to $33^{\circ} \mathrm{C}$ from $6-72$ hours in Experiment $\mathrm{A}-1$. 


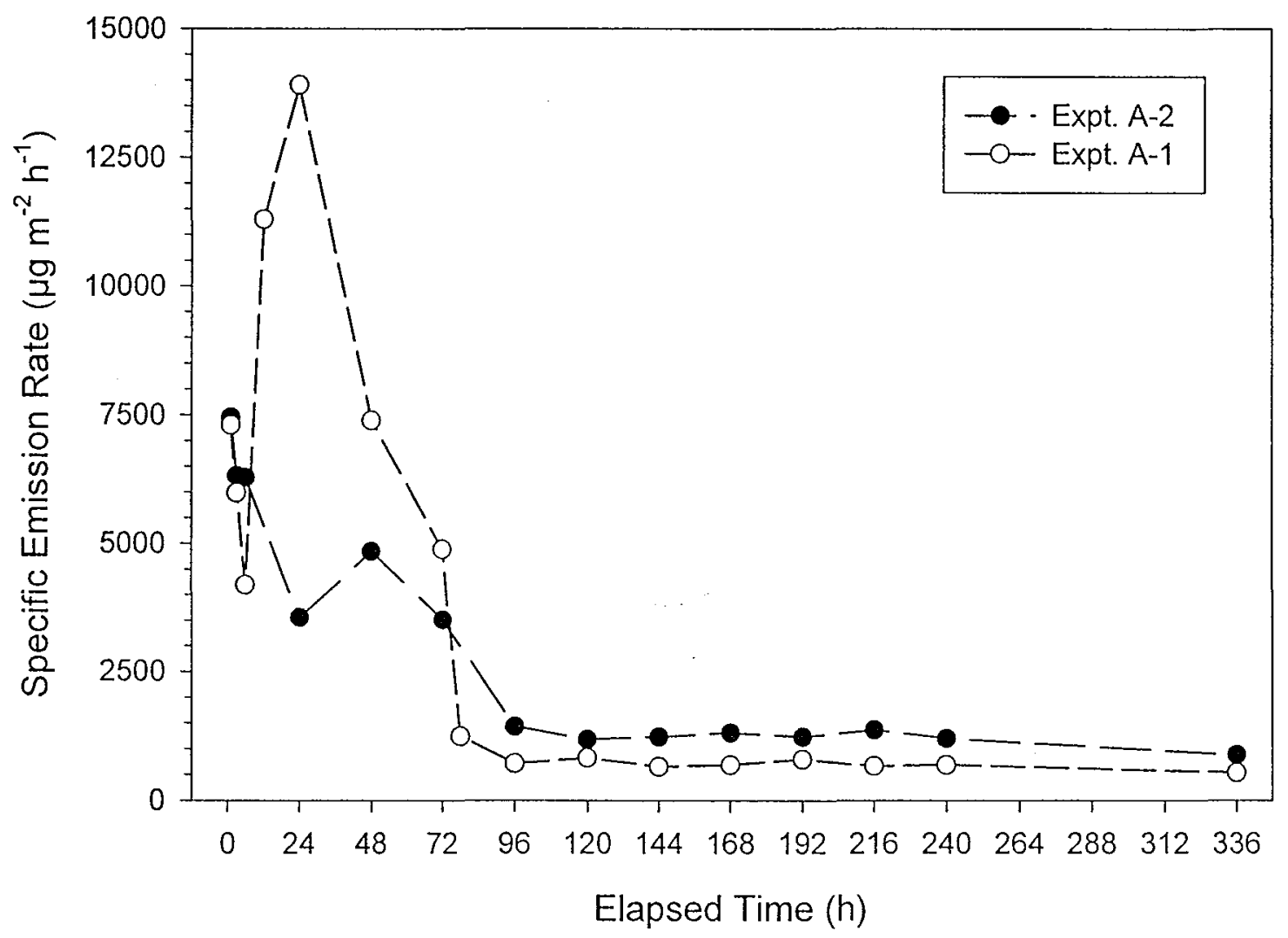

Figure 79. Specific emission rates $\left(\mu \mathrm{g} \mathrm{m}^{-2} \mathrm{~h}^{-1}\right)$ of Texanol $($ in Experiments $A-1$ and $\mathrm{A}-2$. Compartment was heated to $33^{\circ} \mathrm{C}$ from $6-72$ hours in Experiment A-1. 


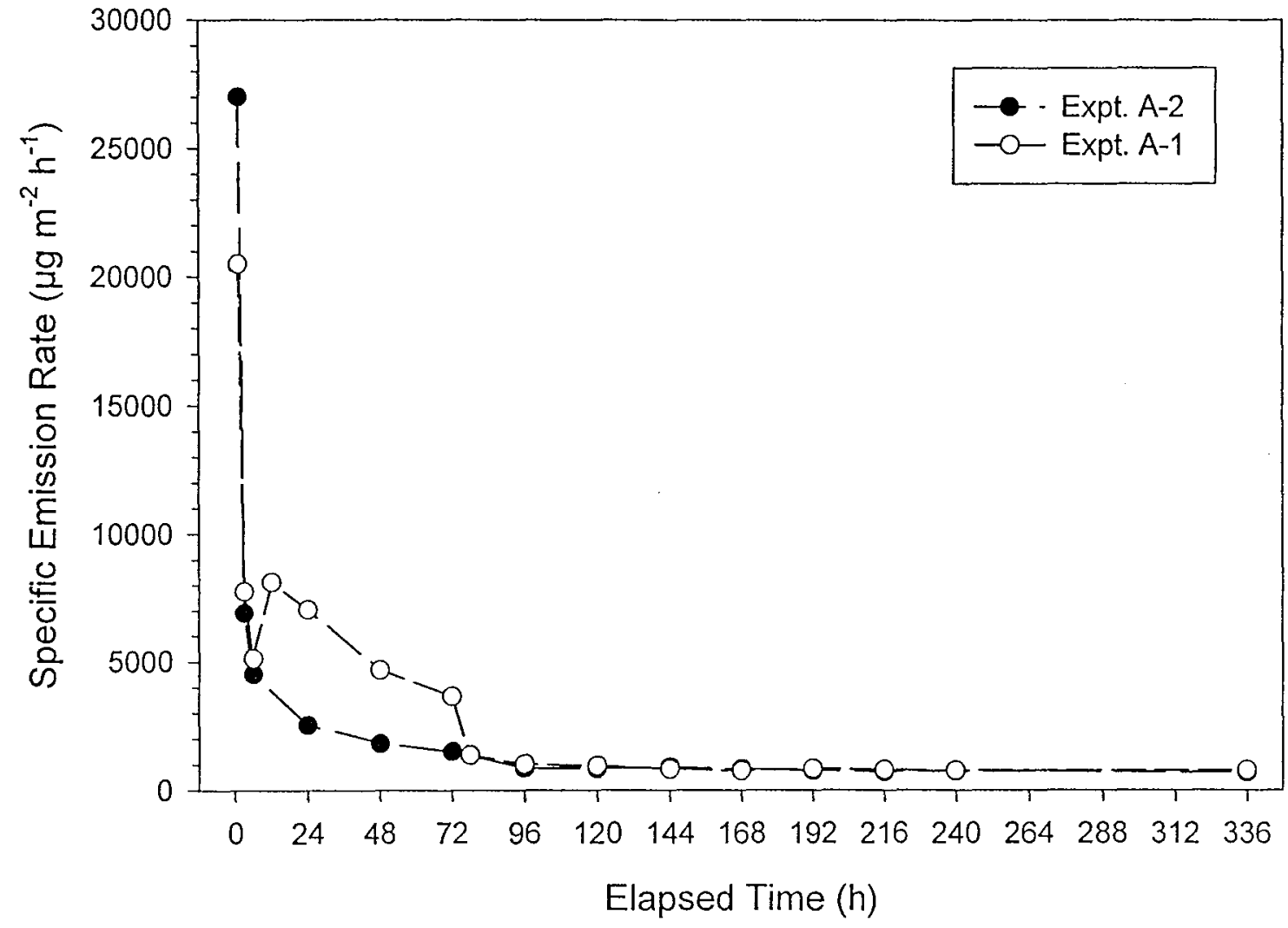

Figure 80. Specific emission rates $\left(\mu \mathrm{g} \mathrm{m}^{-2} \mathrm{~h}^{-1}\right)$ of TVOC $_{R}$ in Experiments $A-1$ and A-2. Compartment was heated to $33^{\circ} \mathrm{C}$ from $6-72$ hours in Experiment $\mathrm{A}-1$. 


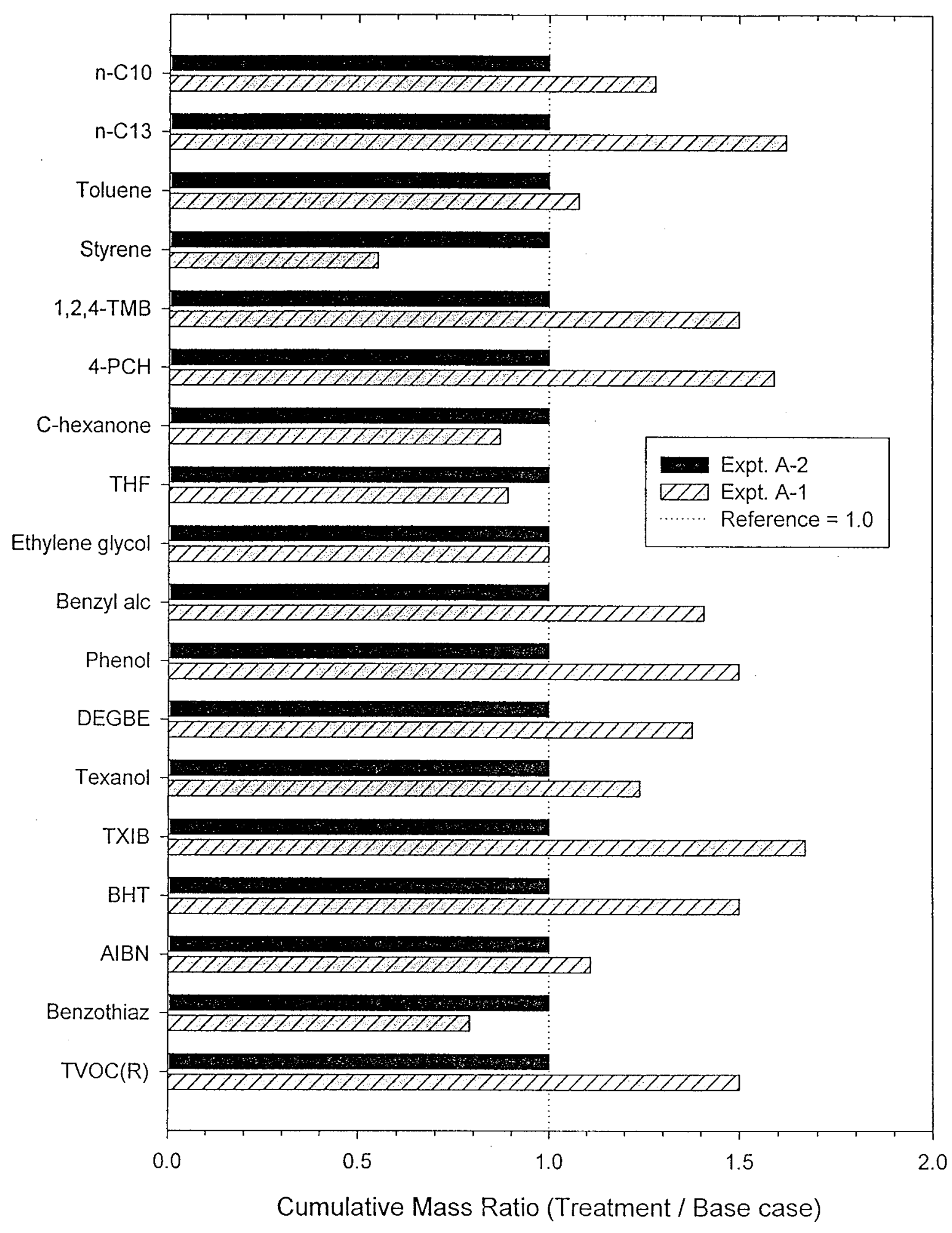

Figure 81. Effects of the heating treatment in Experiment A-1 on the 0- to 336-h cumulative mass emissions of target VOCs and $\mathrm{TVOC}_{R}$ from the combined assembles relative to base-case Experiment A-2. 


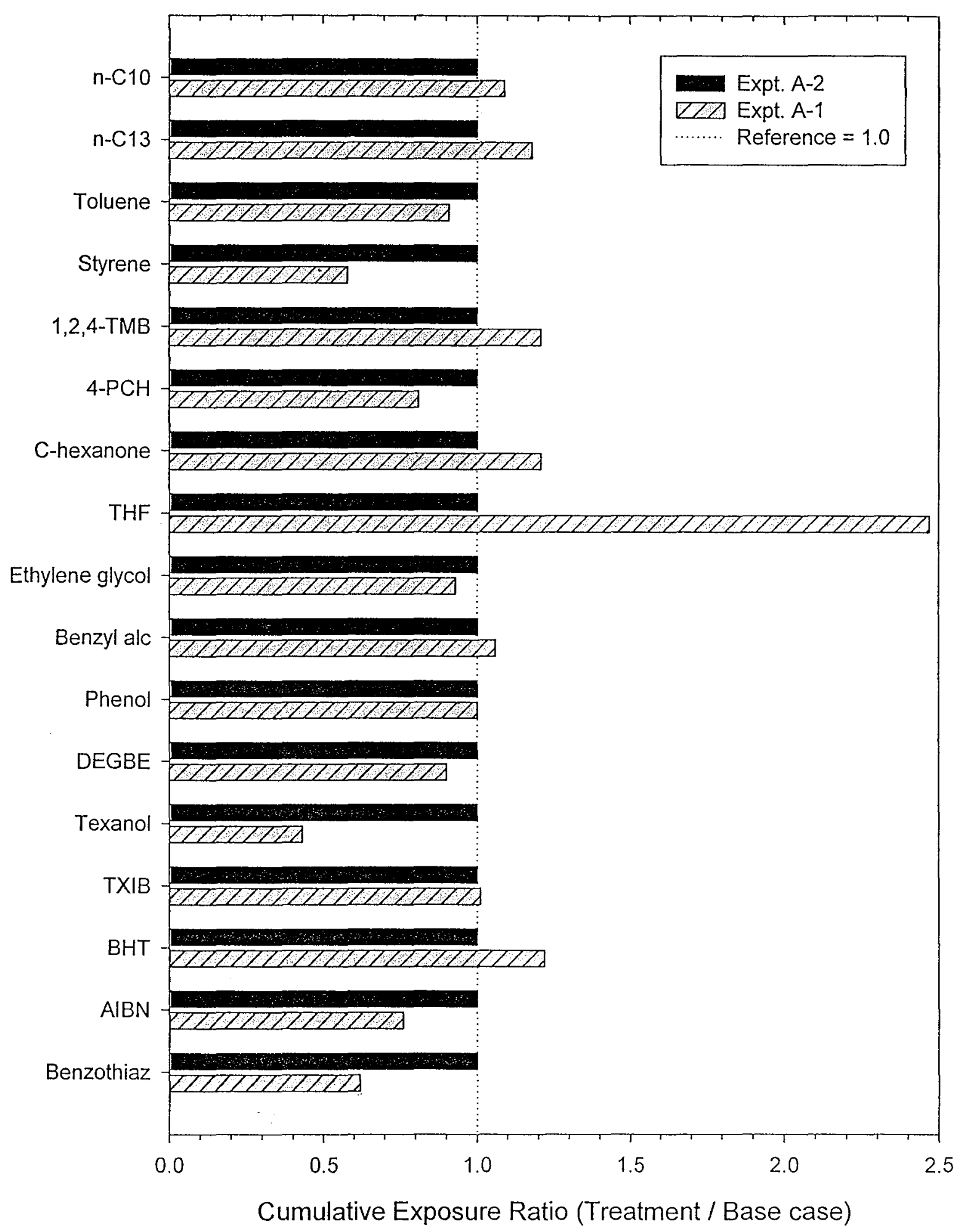

Figure 82. Effects of the heating treatment in Experiment A-1 on the 96- to 336-h cumulative exposures to target VOCs from the combined assemblies relative to base-case Experiment A-2. The calculation assumes an cccupancy of 20 hours per day. 


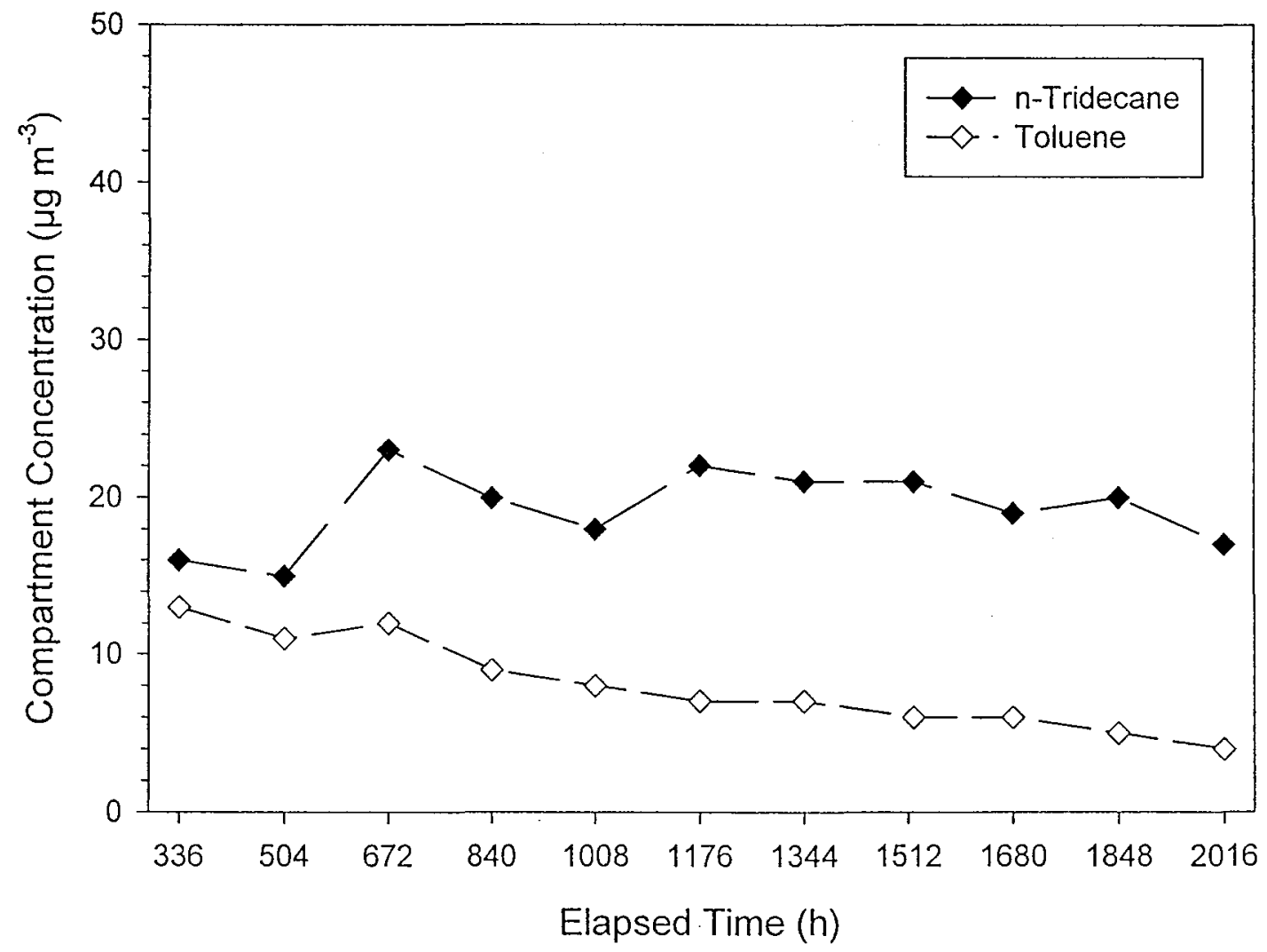

Figure 83. Compartment concentrations $\left(\mu \mathrm{g} \mathrm{m} \mathrm{m}^{-3}\right)$ of $\mathrm{n}$-tridecane and toluene over 336 -2,016 hours in Experiment A-2. 


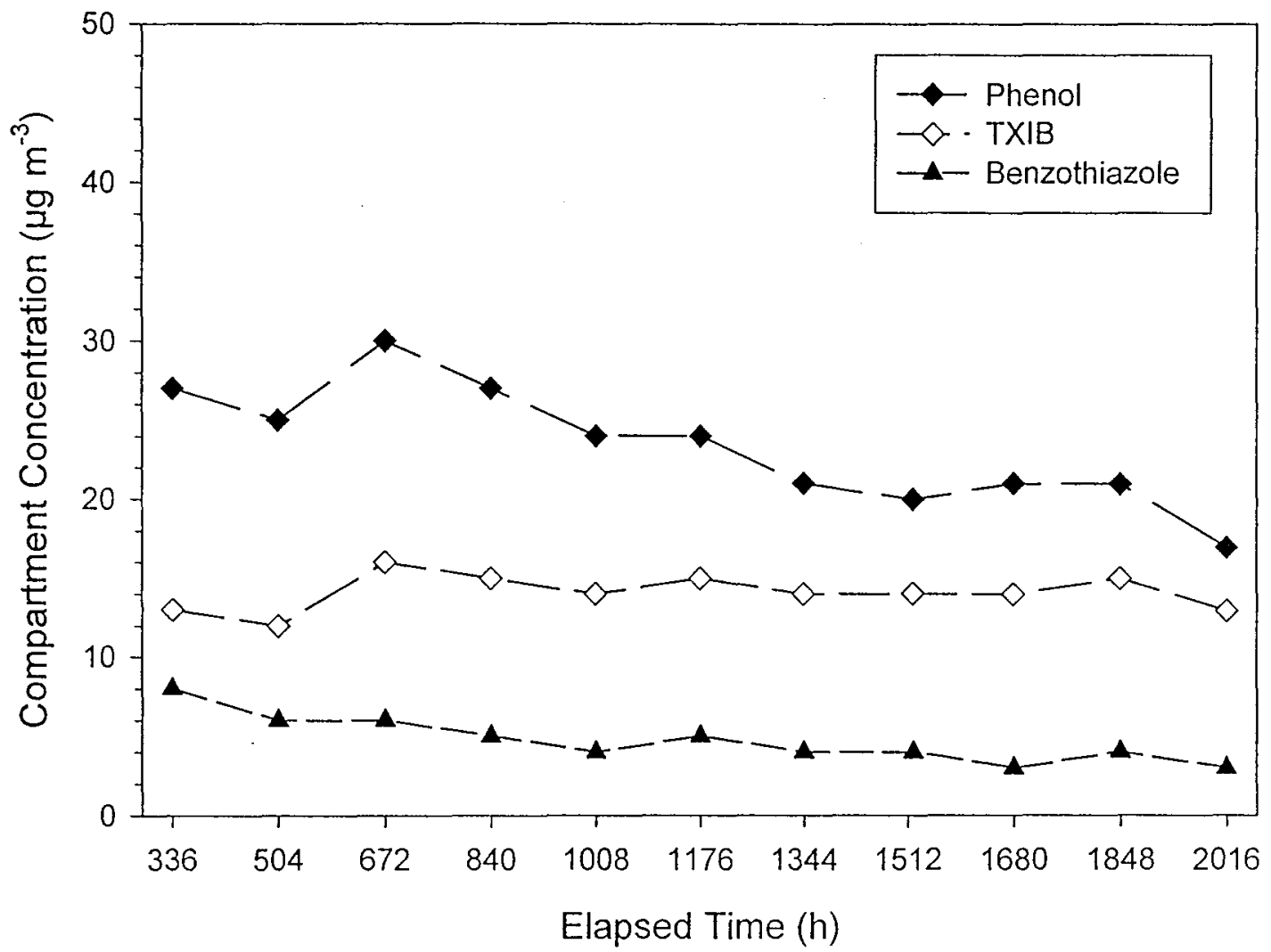

Figure 84. Compartment concentrations $\left(\mu \mathrm{g} \mathrm{m}^{-3}\right)$ of phenol, TXIB® and benzothiazole over $336-2,016$ hours in Experiment A-2. 


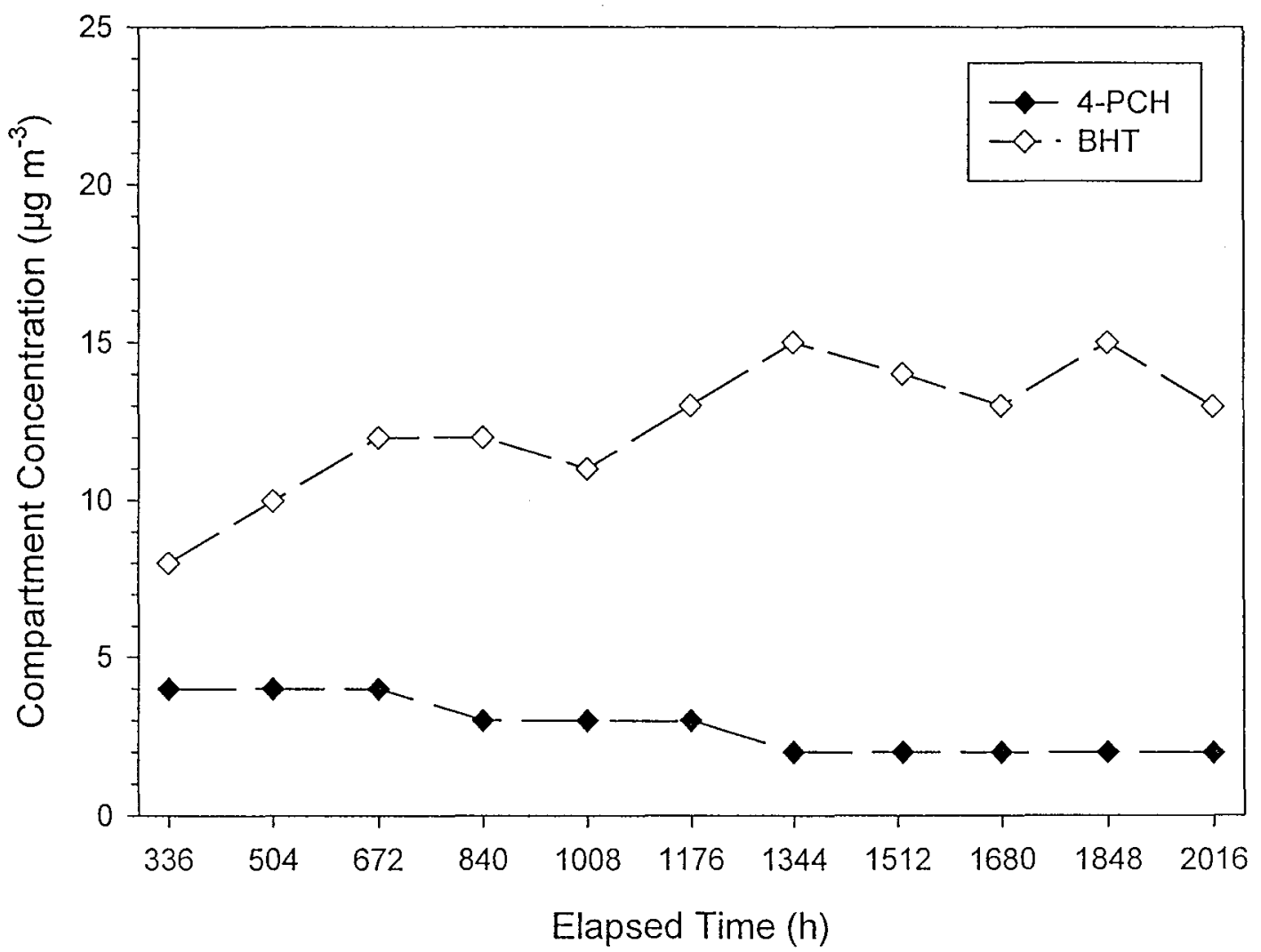

Figure 85. Compartment concentrations $\left(\mu \mathrm{g} \mathrm{m}^{-3}\right)$ of 4-PCH and BHT over 336 - 2,016 hours in Experiment A-2. 


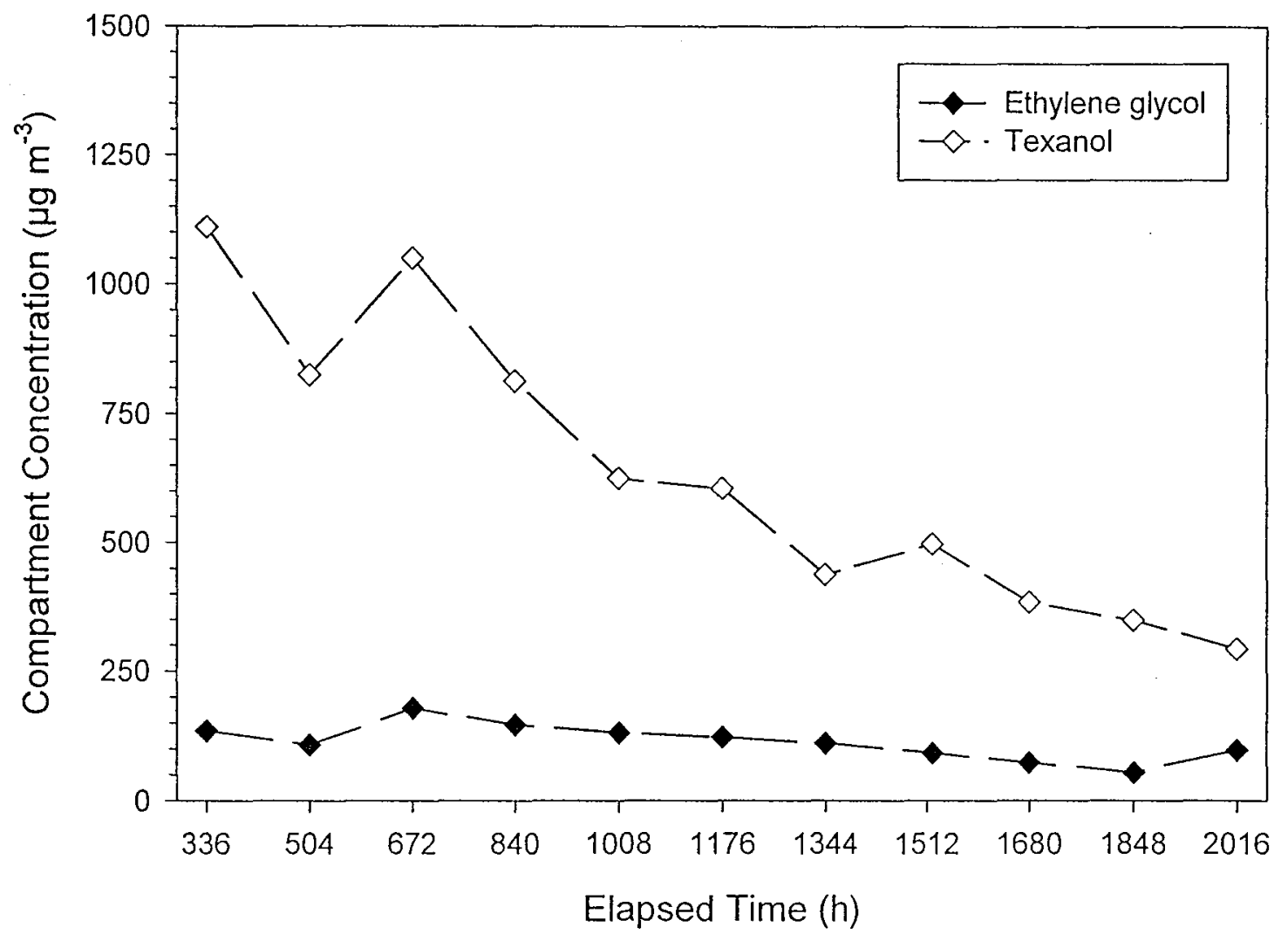

Figure 86. Compartment concentrations $\left(\mu \mathrm{g} \mathrm{m}^{-3}\right)$ of ethylene glycol and Texanol@ over $336-2,016$ hours in Experiment A-2. 


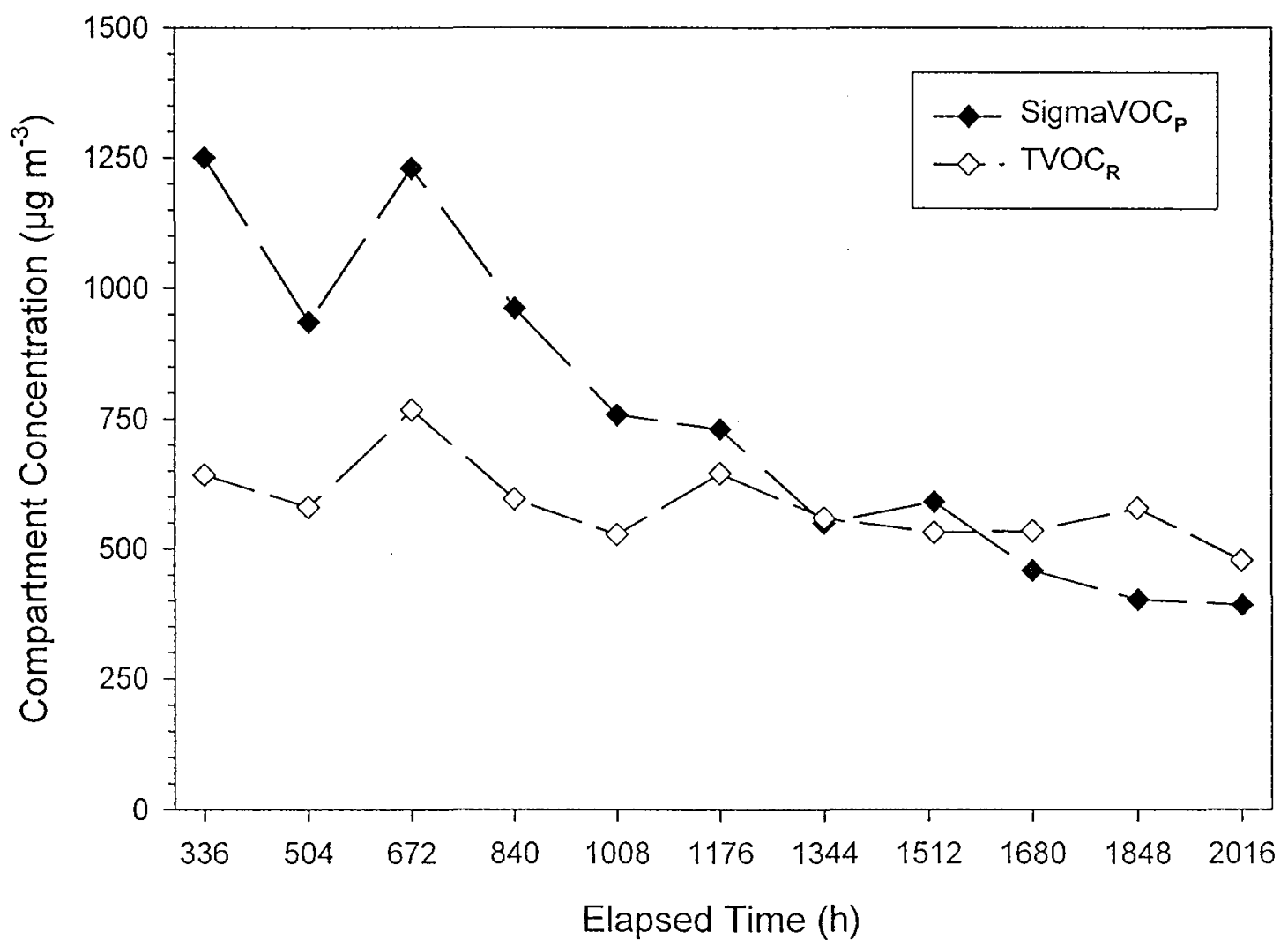

Figure 87. Compartment concentrations $\left(\mu \mathrm{g} \mathrm{m}^{-3}\right)$ of SigmaVOC $\mathrm{P}_{\mathrm{p}}$ (paint) and TVOC $_{R}$ (remainder) over 336 - 2,016 hours in Experiment A-2. 


\section{GLOSSARY OF TERMS, ABBREVIATIONS AND SYMBOLS}

\begin{tabular}{|c|c|}
\hline 1,2-DCB & 1,2-Dichlorobenzene \\
\hline $1,2,4-\mathrm{TMB}$ & 1,2,4-Trimethylbenzene \\
\hline 4-PCH & 4-Phenylcyclohexene \\
\hline 4-VCH & 4-Vinylcyclohexene or 4-ethenylcyclohexene \\
\hline AIBN & 2,2'-Azobisisobutyronitrile \\
\hline ARB & (California) Air Resources Board \\
\hline ASTM & American Society for Testing and Materials \\
\hline BDMA & N,N-Dimethylbenzylamine \\
\hline Benz alc & Benzyl alcohol \\
\hline Benzald & Benzaldehyde \\
\hline Benzothiaz & Benzothiazole \\
\hline BHT & Butylated hydroxytoluene or 2,6-di-tert-butyl-4-methylphenol \\
\hline Butisothio & tert-Butylisothiocyanate \\
\hline $\mathrm{CB}$ & Cove Base \\
\hline CBA & Cove Base Adhesive \\
\hline $\mathrm{CC}(1-4)$ & Carpet Cushion (1 - 4) \\
\hline C-hexone & Cyclohexanone \\
\hline $\mathrm{CP}(1-4)$ & Carpet $(1-4)$ \\
\hline CRI & Carpet and Rug Institute \\
\hline CSTR & Continuously-stirred tank reactor \\
\hline DEGBE & Di(ethylene glycol)butyl ether or 2-(2-butoxyethoxy)ethanol \\
\hline DHS & (California) Department of Health Services \\
\hline DMA & N,N-Dimethylacetamide \\
\hline DPGME & Di(propylene glycol)methyl ether isomers \\
\hline$E G$ & Ethylene glycol \\
\hline EPA & (U.S.) Environmental Protection Agency \\
\hline ER & Specific emission rate \\
\hline ET & Elapsed time \\
\hline FLP(1-4) & Flat Latex Paint (1 - 4) \\
\hline GB & Gypsum Board \\
\hline GC & Gas chromatograph \\
\hline GC/MS & Gas chromatography/mass spectrometry \\
\hline HCs & Hydrocarbons \\
\hline HPLC & High-performance liquid chromatography \\
\hline HVAC & Heating, ventilating and air conditioning \\
\hline ISTD & Internal standard \\
\hline LPS(1\&2) & Latex Primer Sealer (1 \& 2) \\
\hline MeGlutN & 2-Methyleneglutaronitrile \\
\hline$n-C 10$ & n-Decane \\
\hline $\mathrm{n}-\mathrm{C} 12$ & n-Dodecane \\
\hline $\mathrm{n}-\mathrm{C} 13$ & $\mathrm{n}$-Tridecane \\
\hline $\mathrm{n}-\mathrm{C} 14$ & $\mathrm{n}$-Tetradecane \\
\hline nd & Not detected or below lower limit of quantitation \\
\hline PG & Propylene glycol \\
\hline Phen alc & Phenethyl alcohol \\
\hline PW & Plywood \\
\hline SBR & Styrene-butadiene rubber \\
\hline SFA & Sheet Flooring Adhesive \\
\hline $\operatorname{SGLP}(1-4)$ & Semi-Gloss Latex Paint $(1-4)$ \\
\hline SigmaVOC & Sum of individually quantified VOCs \\
\hline SigmaVOC $_{P}$ & Sum of individually quantified VOCs for latex paints \\
\hline SS & Seam Sealer \\
\hline ST & Seaming Tape \\
\hline $\operatorname{SV}(1-5)$ & Sheet Vinyl $(1-5)$ \\
\hline
\end{tabular}




\section{GLOSSARY OF TERMS, ABBREVIATIONS AND SYMBOLS, CONTINUED}

TAC

TACs

Tenax-TA@

Texanol@

THF

TIC

TVOC

TVOC $_{R}$

TXIBQ

UL

UV

VOC

VOCs
Toxic air contaminant

Toxic air contaminants

Trade name for sorbent material based on 2,6-diphenylene oxide

Trade name for 2,2,4-Trimethyl-1,3-pentanediol monoisobutyrate isomers

Tetrahydrofuran

Total-ion current

A measure of total VOCs

Total VOCs minus the concentrations of quantified compounds

Trade name for 2,2,4-Trimethyl-1,3-pentanediol diisobutyrate

Underlayment

Ultra violet

Volatile organic compound

Volatile organic compounds 


\title{
APPENDIX A
}

\author{
SMALL-SCALE SCREENING MEASUREMENT TO CHARACTERIZE EMISSIONS OF VOCS FROM \\ INDOOR MATERIALS/PRODUCTS
}

\section{OBJECTIVES}

This test procedure is applicable to solid materials that can be represented by relatively smallsized specimens and to paints and other liquid finishes that can be uniformly applied to small-sized substrates. The test is used to screen a material or product for emissions of volatile organic compounds (VOCs), total VOCs (TVOC), and low-molecular weight aldehydes at standardized environmental conditions in a small-volume chamber over a relatively short time period (typically one to four days). The chambers are constructed and operated generally following American Society for Testing and Materials Standard Guide D-5116-90 (ASTM, 1990).

\section{TEST SPECIMENS}

The complete identification and history of the material, including the manufacturer, material description, product and lot numbers, manufacturing date, subsequent shipping, storage conditions, and storage duration should be documented. The material specimen is packaged so as to preserve its chemical integrity and to prevent its contamination from other sources. Acceptable packaging materials are sealed Tediar bags and heavy-duty aluminum foil. If aluminum foil is used, the specimen is packaged in at least two layers with the edges carefully sealed by folding. It is generally good practice to collect and store larger or more pieces of a material than are needed for testing as this will help to preserve the chemical integrity of the material. As examples, sheet-flooring materials such as carpets can be stored as tight rolls, and floor tiles can be stored in stacks. For testing, a specimen is cut from near the center of the larger piece or taken from near the center of the stack.

\section{SPECIMEN HANDLING AND HEADSPACE SCREENING}

In the laboratory, packaged materials are stored at room conditions in a clean environment without strong or obvious chemical sources. All handling and preparation of tests specimens are conducted in a clean environment using gloves, if necessary, and clean utensils.

Packaged materials can be pre-screened for VOCs by headspace sampling. A material, or a representative sample of the material, should be transferred to a Tedlar bag if required. The package should contain a relatively small amount of headspace air (e.g., no more than $\sim 1 \mathrm{~L}$ for a small-sized package). The package is stored at room conditions for at least one day. A sample of headspace air $(-100 \mathrm{~mL})$ is withdrawn from the Tedlar bag for qualitative analysis of VOCs.

\section{PREPARATION OF SPECIMENS OF SOLID MATERIALS}

A test specimen of a solid material is cut from the larger piece of stored material. For sheet materials, such as sheet flooring and fabrics, the surface dimensions of the specimens are typically 15 by $15 \mathrm{~cm}$. A relatively thick material that has only one exposed surface when it is installed in a building (e.g., a carpet) is placed in a specially fabricated holder so that only this surface is exposed in the test chamber. The holder is constructed of stainless-steel sheet metal. It has the same interior dimensions as the specimen and adequate depth so that the specimen fits tightly in the holder and the freshly cut edges are covered. A relative thin material with one exposed surface (e.g., sheet vinyl flooring) is attached to a stainless-steel sheet metal plate so that only the finish surface is exposed in the chamber. Thin strips of aluminized tape can be used to attach the material to the plate. This tape has been found to have low emissions of VOCs. The exposed surface area of a specimen is accurately measured and used to calculate area-specific emission rates. Blank specimens are similarly prepared for testing. These specimens consist of either an empty hoider or a plate with attached strips of aluminized tape. 


\section{PREPARATION OF PAINT SPECIMENS}

Paint specimens are prepared by applying the paints to representative substrates. For latex wall paints, the most typical substrate is gypsum wallboard. Another typical substrate is a wood surface, such as plywood or particleboard. The surface dimensions of the substrates are typically 15 by $15 \mathrm{~cm}$. The substrate is accurately weighed prior to painting. Then, the borders of the substrate are masked off with tape leaving a 14 by $14-\mathrm{cm}$ area in the center of the substrate for painting.

Paints are applied to the substrates using standardized procedures that simulate the application of the paints in buildings. Typically wall paints are applied with a roller. Small-dimension rollers and covers can be purchased. A 10-cm wide roller with a cover intended for smooth surfaces is recommended. Paints can also be applied with a brush. A 5-cm wide brush is an appropriate size for small-scale substrates.

The paint is first thoroughly mixed in its container by shaking or stirring. For roller application, approximately $100 \mathrm{~mL}$ of the paint are transferred to a small tray. A disposable tray with approximate dimensions of $15 \times 15 \mathrm{~cm}$ can be simply fabricated from heavy-duty aluminum foil. The roller cover is saturated with paint by running the roller back and forth in the tray. The paint is applied to the substrate using four strokes, two in the vertical direction and two in the horizontal direction, so that the entire area is uniformly covered. For brush application, the paint is applied as uniformly as possible using back-andforth strokes running in only one dimension.

The tape is then removed from the borders of the substrate, and the substrate is re weighed. The difference in the weights before and after painting is used to determine the coverage in grams of wet paint applied per square meter of substrate surface. A painted substrate is placed into a stainless-steel holder that covers the back and edges of the substrate as described above. The substrate is sealed into the holder with thin strips of aluminized tape so that only the painted surface is exposed. Blank substrates without paint are similarly prepared.

If multiple coats of paint are to be tested, the paints should applied following the manufacturer's instructions with respect to drying times between coats. The drying is best performed in a chamber under controlled conditions. Alternatively, the paint may be dried in another clean, controlled environment.

\section{CHAMBER SPECIFICATIONS AND OPERATING CONDITIONS}

The specifications and operating conditions for the small-scale chambers are summarized in Table 1. The chambers consist of polished 316 stainless steel, cylindrical vessels. The chambers are equipped with stainless-steel lined lids that are sealed with Teflon-coated silicone gaskets. The internal volume of the chambers is $10.5 \mathrm{~L}$.

The inlet gas is high-purity nitrogen supplied by gas cylinders. The flow rate of nitrogen is regulated at $1.0 \pm 0.05 \mathrm{~L} \mathrm{~min}^{-1}$ (average \pm one standard deviation) and monitored with an electronic flow controller or sensor. The inlet gas stream is split into two streams. One of these passes through a bubbler containing distilled water held at the same temperature as the chamber. This stream is mixed with the dry gas stream to generate a humidified inlet gas stream. The humidified gas stream is introduced into the chamber through a fitting on the lid with a tubing extension that terminates near the bottom of the chamber. Gas exits the chamber through another fitting on the lid. A sampling manifold constructed of stainless-steel tube fittings is attached to the outlet of this fitting. A combined temperature and humidity probe is inserted into the chamber through a third fitting. Chamber temperature, relative humidity and inlet gas flow rate are monitored and recorded throughout a test using a computer-based data acquisition system. 
Table 1. Specifications and operating conditions for screening measurements conducted in small-scale chambers.

\begin{tabular}{|c|c|}
\hline Parameter & Value \\
\hline Chamber material & 316 Stainless steel \\
\hline Chamber volume, $\mathrm{m}^{3}$ & $10.5 \times 10^{-3}$ \\
\hline Gas & Humidified $\mathrm{N}_{2}$ \\
\hline Inlet gas flow rate, $m^{3} h^{-1}$ & $6.0 \pm 0.3 \times 10-2^{*}$ \\
\hline Ventilation rate, $h^{-1}$ & $5.7 \pm 0.3^{*}$ \\
\hline Temperature, ${ }^{\circ} \mathrm{C}$ & $23 \pm 1^{\star}$ \\
\hline Relative humidity, \% & $50 \pm 5^{\star}$ \\
\hline Air velocity ${ }^{\star \star}, \mathrm{m} \mathrm{s}^{-1}$ & $\sim 0.25$ \\
\hline Sample surface area, $\mathrm{m}^{2}$ & $\sim 2 \times 10^{-2}$ \\
\hline
\end{tabular}

*Average \pm one standard deviation.

${ }^{\star \star}$ For experiments with paints only; other experiments are conducted without the use of a fan for air mixing.

Screening measurements are typically conducted at room conditions of temperature and relative humidity. These conditions are standardized at $23 \pm 1^{\circ} \mathrm{C}$ and $50 \pm 5$ percent relative humidity (averages \pm one standard deviation). Chambers are placed on their sides in a constant temperature enclosure, such as an incubator with heating and refrigeration controls, to achieve and maintain the desired temperature. The flow rates of the wet and dry inlet gas streams are adjusted to achieve the desired relative humidity. For a specimen of a product emitting water, (e.g., latex paint), the inlet gas is periodically adjusted throughout a test to maintain the relative humidity within the specified range.

For tests of paints and other liquid finishes, a small 3.8-cm diameter axial fan (12 VDC) is mounted under the wire screen and operated to increase the air velocity near the painted surface. The output of the fan is controlled by attaching layers of a fine mesh screen to the outlet of the fan to achieve an air velocity near the painted surface of approximately $0.25 \mathrm{~m} \mathrm{sec}^{-1}$. For solid materials, no additional mixing of the air in the chamber is provided.

\section{GENERAL OPERATING PROCEDURES}

Prior to each use, a chamber a washed with laboratory detergent, thoroughly rinsed with water and dried. The chamber is completely assembled without the test specimen and placed in the temperature-controlled enclosure. The inlet gas and the instrumentation are attached. The empty chamber is then operated at the temperature, humidity and ventilation conditions of the test for at least one hour (i.e., approximately six air changes). It is desirable to collect gas samples at the exhaust manifold near the end of this period to determine the concentrations of any background chamber contamination.

The chamber is opened, and the prepared specimen is placed on a metal rack that holds the specimen near the approximate center of the chamber. The chamber lid is quickly re attached and data system is initiated. This establishes the initial time point for the test. 
Samples for VOCs and aldehydes are simultaneously collected from the sampling manifold at predetermined intervals throughout a test. Samples for VOCs are collected on thermally desorbed sorbent samplers. Samples for aldehydes are collected on cartridges coated with acidified 2,4-dinitrophenylhydrazine (DNPH) as a derivatizing agent. Typically, duplicate VOC samples and single aldehyde samples are collected at each sampling interval. The sample flow rates are regulated with electronic mass-flow controllers. The flow rate for the VOC samples is typically $0.1 \mathrm{~L} \mathrm{~min}^{-1}$; however, this rate is varied according the magnitude of the source to achieve optimum mass loading of the sampler. The flow rate for the aldehyde samples is typically $0.5 \mathrm{~L} \mathrm{~min}^{-1}$. The total sample flow rate should always substantially less than the inlet flow rate of gas to the chamber. Solenoid values operated by the data acquisition system can be used to automate the collection of samples.

Typically, screening measurements are conducted over a period of one to four days with samples collected at least daily.

\section{SAMPLE ANALYSES}

Chamber and headspace samples for VOCs are analyzed by thermal desoption gas chromatography/mass spectrometry. The compounds comprising the majority of the mass in a sample are identified. If possible, the identifications are confirmed by the analysis of pure standards. The major compounds of interest in a sample are quantified using calibrations prepared from these standards. Target compounds are primarily selected based on their abundance and toxicological significance. A sample is analyzed for TVOC by the total-ion-current method using an average response factor for common hydrocarbons to calculate the mass. The DNPH cartridges are analyzed for formaldehyde and other aldehydes by high-performance liquid chromatography.

\section{DATA ANALYSIS}

The VOCs identified in the chamber and headspace samples are listed. The concentrations of the selected individual VOCs, TVOC and aldehydes in the chamber samples are determined. Then, the quasi steady-state, area-specific emission rates (ER) of these components $\left(\mu \mathrm{g} \mathrm{h}^{-1}\right)$ are calculated using the following equation:

$$
E R=\frac{Q\left(C-C_{o}\right)}{A}
$$

Where $Q$ is the volumetric flow rate $\left(\mathrm{m}^{3} \mathrm{~h}^{-1}\right)$ through the chamber, $\mathrm{C}$ is the average chamber concentration at the sampling period $\left(\mu \mathrm{g} \mathrm{m}^{-3}\right), \mathrm{C}_{0}$ is the chamber blank concentration $\left(\mu \mathrm{g} \mathrm{m}^{-3}\right)$, and $A$ is the exposed area $\left(\mathrm{m}^{2}\right)$. A mass-specific emission rate can be calculated by dividing by the mass of the specimen rather than the area. The use of the steady-state assumption introduces unknown amounts of error into the estimates of specific emission rates when the chamber concentrations are changing rapidly with time.

\section{REFERENCE}

ASTM. 1990. Standard Guide for Small-Scale Environmental Chamber Determinations of Organic Emissions from Indoor Materials/Products. American Society for Testing and Materials, Philadelphia, PA. 


\section{APPENDIX B}

\section{SUMMARY OF ENVIRONMENTAL DATA FOR THE SCREENING MEASUREMENTS}

Appendix $B$ summarizes the environmental data for the screening measurements of the latex paints, the carpet assembly materials and the vinyl flooring assembly materials. The average values for the inlet nitrogen flow rates and the chamber temperatures and relative humidities are presented along with the coefficients of variation for these values.

\section{List of Tables}

Table B-01. Summary of environmental data for the screening measurements of latex paints in 10-L chambers.

Table B-02. Summary of environmental parameters for the screening measurements of carpet materials in 10-L chambers

Table B-03. Summary of environmental parameters for the screening measurements of vinyl flooring materials in $10-\mathrm{L}$ chambers. 
Table B-01. Summary of environmental data for the screening measurements of latex paints in 10-L chambers.

\begin{tabular}{|c|c|c|c|c|c|c|}
\hline \multirow[b]{2}{*}{ Materials } & \multicolumn{2}{|c|}{$\begin{array}{l}\mathrm{N}_{2} \text { Flow Rate } \\
\left(\mathrm{L} \min ^{-1}\right)\end{array}$} & \multicolumn{2}{|c|}{$\begin{array}{c}\text { Temperature } \\
\left({ }^{\circ} \mathrm{C}\right)\end{array}$} & \multicolumn{2}{|c|}{$\begin{array}{l}\text { Relative Humidity } \\
(\%)\end{array}$} \\
\hline & Avg. & $\mathrm{CV}^{*}, \%$ & Avg. & $\mathrm{CV}, \%$ & Avg. & $\mathrm{CV}, \%$ \\
\hline \multicolumn{7}{|l|}{ Primer sealers } \\
\hline LPS1 & $0.93^{\star *}$ & 25 & 23 & 0.2 & 53 & 2.7 \\
\hline LPS2 & 0.98 & 0.6 & 24 & 0.3 & 47 & 5.6 \\
\hline \multicolumn{7}{|l|}{ Flats } \\
\hline FLP1-a & 0.99 & 1.0 & 23 & 0.2 & 54 & 4.3 \\
\hline FLP1-b & 0.99 & 1.2 & 22 & 0.4 & 51 & 4.6 \\
\hline FLP2 & 0.98 & 1.4 & 23 & 0.4 & 54 & 5.0 \\
\hline FLP3 & 0.98 & 1.7 & 22 & 0.6 & 51 & 6.5 \\
\hline FLP4 & 0.99 & 0.6 & 23 & 0.3 & 54 & 2.6 \\
\hline \multicolumn{7}{|l|}{ Semi-Glosses } \\
\hline SGLP1 & 0.98 & 1.0 & 23 & 0.4 & 53 & 6.3 \\
\hline SGLP2 & 0.98 & 1.2 & 22 & 0.4 & 50 & 7.0 \\
\hline SGLP3-a & $0.93^{\star \star}$ & 25 & 22 & 0.4 & 50 & 7.0 \\
\hline SGLP3-b & 0.99 & 0.6 & 22 & 0.5 & 48 & 7.3 \\
\hline SGLP4 & 0.98 & 0.7 & 23 & 0.6 & 51 & 7.0 \\
\hline \multicolumn{7}{|l|}{ Combinations } \\
\hline LPS2 \& FLP3 @ 50\% RH & 0.99 & 0.5 & 23 & 1.6 & 50 & 3.9 \\
\hline LPS2 \& FLP3 @ $30 \%$ RH & 0.98 & 0.6 & 24 & 0.2 & 31 & 11.8 \\
\hline LPS2 \& FLP3 @ 70\% RH & 0.98 & 0.4 & 23 & 0.3 & 69 & 2.2 \\
\hline LPS2 \& SGLP3 & 0.98 & 0.5 & 23 & 2.4 & 50 & 3.5 \\
\hline \multicolumn{7}{|l|}{ Substrates } \\
\hline GB (Gypsum Board) & 0.99 & 0.5 & 23 & 0.5 & 48 & 3.6 \\
\hline PW (Plywood). & 0.98 & 0.4 & 22 & 0.6 & 48 & 3.2 \\
\hline
\end{tabular}

${ }^{*} \mathrm{CV}=$ Coefficient of variation in percent calculated as the std. deviation / average value $\times 100$. * Inlet $\mathrm{N}_{2}$ flow interrupted from 64 - 69-h elapsed time. 
Table B-02. Summary of environmental parameters for the screening measurements of carpet materials in 10-L chambers.

\begin{tabular}{|c|c|c|c|c|c|c|}
\hline \multirow[b]{2}{*}{ Materials } & \multicolumn{2}{|c|}{$\begin{array}{l}\text { Flow Rate } \\
\left(L \min ^{-1}\right)\end{array}$} & \multicolumn{2}{|c|}{$\begin{array}{c}\text { Temperature } \\
\left({ }^{\circ} \mathrm{C}\right)\end{array}$} & \multicolumn{2}{|c|}{$\begin{array}{l}\text { Relative Humidity } \\
\qquad(\%)\end{array}$} \\
\hline & Avg. & $\mathrm{CV}^{\star}, \%$ & Avg. & CV, $\%$ & Avg. & $\mathrm{CV}, \%$ \\
\hline \multicolumn{7}{|l|}{ Carpets } \\
\hline CP1-a & 1.00 & 0.4 & 23 & 0.3 & 49 & 5.5 \\
\hline$C P 1-b$ & 1.00 & 0.5 & 23 & 0.5 & 48 & 4.7 \\
\hline $\mathrm{CP} 3$ & 0.99 & 0.7 & 25 & 0.8 & 48 & 2.9 \\
\hline $\mathrm{CP} 2$ & 1.00 & 0.6 & 23 & 0.2 & 51 & 5.0 \\
\hline $\mathrm{CP} 4$ & 0.99 & 0.5 & 22 & 0.5 & 48 & 5.5 \\
\hline CP Blank Run & 0.99 & 0.6 & 22 & 0.6 & 49 & 4.5 \\
\hline \multicolumn{7}{|c|}{ Seaming Tape \& Carpet } \\
\hline ST \& CP3 & 0.99 & 0.9 & 25 & 1.1 & 48 & 4.8 \\
\hline \multicolumn{7}{|l|}{ Cushions } \\
\hline $\mathrm{CC} 1$ & 0.99 & 0.6 & 23 & 0.3 & 48 & 4.7 \\
\hline $\mathrm{CC} 2-\mathrm{a}$ & 1.00 & 0.5 & 23 & 0.2 & 50 & 2.6 \\
\hline$c c 2-b$ & 1.00 & 0.8 & 23 & 0.2 & 48 & 5.8 \\
\hline $\mathrm{CC} 4$ & 0.99 & 0.4 & 23 & 0.5 & 49 & 4.4 \\
\hline $\mathrm{CC} 3$ & 1.00 & 0.5 & 23 & 0.2 & 47 & 3.3 \\
\hline CC Blank Run & 0.99 & 0.8 & 22 & 0.2 & 53 & 5.0 \\
\hline
\end{tabular}

${ }^{*} \mathrm{CV}=$ Coefficient of variation in percent calculated as the std. deviation / average value $\times 100$. 
Table B-03. Summary of environmental parameters for the screening measurements of vinyl flooring materials in 10-L chambers.

\begin{tabular}{|c|c|c|c|c|c|c|}
\hline \multirow[b]{2}{*}{ Materials } & \multicolumn{2}{|c|}{$\begin{array}{c}\text { Flow Rate } \\
\left(L_{\text {min }}-1\right)\end{array}$} & \multicolumn{2}{|c|}{$\begin{array}{l}\text { Temperature } \\
\left({ }^{\circ} \mathrm{C}\right)\end{array}$} & \multicolumn{2}{|c|}{$\begin{array}{l}\text { Relative Humidity } \\
(\%)\end{array}$} \\
\hline & Avg. & $\mathrm{CV}^{\star}, \%$ & Avg. & CV,$\%$ & Avg. & $\mathrm{CV}, \%$ \\
\hline \multicolumn{7}{|l|}{ Sheet Vinyls } \\
\hline SV1 & 0.99 & 0.8 & 23 & 0.4 & 48 & 4.0 \\
\hline SV2 & 0.99 & 0.8 & 23 & 0.5 & 49 & 5.9 \\
\hline sv3-a & 0.99 & 0.5 & 23 & 0.5 & 48 & 4.2 \\
\hline SV3-b & 0.99 & 1.1 & 23 & 0.6 & 46 & 2.8 \\
\hline SV5 & 0.99 & 0.9 & 23 & 0.3 & 46 & 2.8 \\
\hline SV4 & 0.99 & 0.6 & 22 & 0.5 & 51 & 5.3 \\
\hline SV Blank Run & 0.99 & 1.0 & 23 & 0.5 & 46 & 2.3 \\
\hline \multicolumn{7}{|l|}{ Cove Base } \\
\hline $\mathrm{CB}$ & 0.99 & 0.7 & 23 & 0.3 & 44 & 1.7 \\
\hline \multicolumn{7}{|l|}{ Substrates } \\
\hline UL (Underlayment) & 0.99 & 0.9 & 23 & 0.4 & 44 & 1.2 \\
\hline GB (Gypsum Bd.) & 0.99 & 0.9 & 23 & 0.2 & 46 & 1.7 \\
\hline \multicolumn{7}{|c|}{ Adhesives on Substrates } \\
\hline SFA \& UL & 0.99 & 1.1 & 23 & 0.6 & 48 & 11.0 \\
\hline $\mathrm{CBA} \& \mathrm{~GB}$ & 0.99 & 0.9 & 24 & 0.6 & 50 & 11.2 \\
\hline \multicolumn{7}{|c|}{ Composite Assemblies } \\
\hline SV5, SFA \& UL & 0.99 & 5.5 & 23 & 0.6 & 48 & 3.4 \\
\hline $\mathrm{CB}, \mathrm{CBA} \& \mathrm{~GB}$ & 0.99 & 5.3 & 23 & 0.5 & 51 & 5.7 \\
\hline
\end{tabular}

${ }^{*} \mathrm{CV}=$ Coefficient of variation in percent calculated as the std. deviation / average value $\mathrm{x} 100$. 


\title{
APPENDIX C
}

\author{
SCREENING MEASUREMENTS OF LATEX PAINTS
}

Appendix $C$ presents the analytical data for the screening measurements of ten latex paints including two primer sealers, four flat paints and four semi-gloss paints. The experiments were conducted in 10-L chambers over a period of 96 hours. Individual VOCs emitted by the paints were identified. The concentrations of selected compounds and SigmaVOC were measured at five time intervals. Specific emission rates of these components were calculated for the 48- and 96-h time intervals.

\section{List of Tables}

Table C-01. Latex paints selected for study.

$\underline{\text { Page }}$

Table C-02. Summary of screening measurements of latex paints and substrates in 10-L chambers.........182

Table C-03. VOCs emitted by Paint LPS1 in 10-L chamber at 6- and 96-hours elapsed times...................183

Table C-04. VOCs emitted by Paint LPS2 in 10-L chamber at 6- and 96-hours elapsed times. ..................183

Table C-05. VOCs emitted by Paint FLP1 in 10-L chamber at 6- and 96-hours elapsed times. ...................184

Table C-06. VOCs emitted by Paint FLP2 in 10-L chamber at 6- and 96-hours elapsed times. ..................184

Table C-07. VOCs emitted by Paint FLP3 in 10-L chamber at 6- and 96-hours elapsed times. ...................184

Table C-08. VOCs emitted by Paint FLP4 in 10-L chamber at 6- and 96-hours elapsed times. ....................185

Table C-09. VOCs emitted by Paint SGLP1 in 10-L chamber at 6- and 96-hours elapsed times. ...............185

Table C-10. VOCs emitted by Paint SGLP2 in 10-L chamber at 6- and 96-hours elapsed times. ................186

Table C-11. VOCs emitted by Paint SGLP3 in 10-L chamber at 6- and 96-hours elapsed times. ................186

Table C-12. VOCs emitted by Paint SGLP4 in 10-L chamber at 6- and 96-hours elapsed times. ................187

Table C-13. VOCs emitted by Paints LPS2 and FLP3 in 10-L chamber at 6- and 96-hours

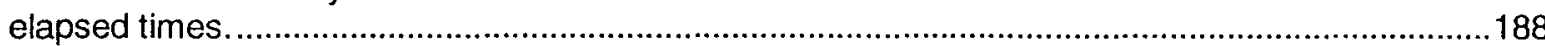

Table C-14. VOCs emitted by Paints LPS2 and SGLP3 in 10-L chamber at 6- and 96-hours elapsed times

Table C-15. VOCs emitted by Gypsum Board GB in 10-L chamber at 6-and 96-hours elapsed times........189

Table C-16. VOCs emitted by Plywood PW in 10-L chamber at 6- and 96-hours elapsed times.................190

Table C-17. Summary of VOCs emitted by conventional latex paints in 10-L chambers...........................191

Table C-18. Chamber concentrations of target VOCs for 96-h screening measurement of Paint LPS1 .......192

Table C-19. Chamber concentrations of target VOCs for 96-h screening measurement of Paint LPS2 .......192

Table C-20. Chamber concentrations of target VOCs for 96-h screening measurement of Paint FLP1-a....192

Table C-21. Chamber concentrations of target VOCs for 96-h screening measurement of Paint FLP1-b....193

Table C-22. Chamber concentrations of target VOCs for 96-h screening measurement of Paint FLP2 .......193

Table C-23. Chamber concentrations of target VOCs for 96-h screening measurement of Paint FLP3.......193

Table C-24. Chamber concentrations of selected VOCs for 96-h screening measurement of Paint FLP4... 194

Table C-25. Chamber concentrations of target VOCs for 96-h screening measurement of Paint SGLP1....194

Table C-26. Chamber concentrations of target VOCs for 96-h screening measurement of Paint SGLP2....195

Table C-27. Chamber concentrations of target VOCs for 96-h screening measurement of

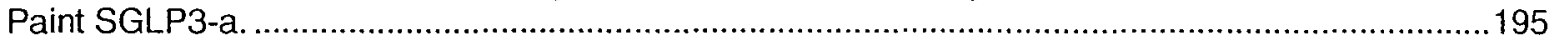

Table C-28. Chamber concentrations of target VOCs for 96-h screening measurement of

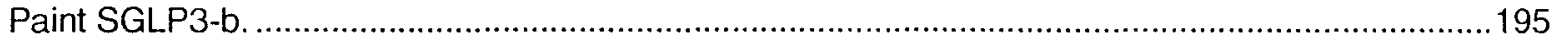

Table C-29. Chamber concentrations of selected VOCs for $96-\mathrm{h}$ screening measurement of Paint SGLP4.

Table C-30. Chamber concentrations of target VOCs for 96-h screening measurement of Paints LPS2 and FLP3 at standard humidity $(50 \% \mathrm{RH})$

Table C-31. Chamber concentrations of target VOCs for 96 -h screening measurement of Paints LPS2 and FLP3 at reduced humidity $(30 \% \mathrm{RH})$.

Table C-32. Chamber concentrations of target VOCs for 96 -h screoning measurement of Paints LPS2 and FLP3 at elevated humidity $(70 \% \mathrm{RH})$. 
Table C-33. Chamber concentrations of target VOCs for 96-h screening measurement of Paints LPS2 and SGLP3.

Table C-34. Chamber concentrations of latex paint target VOCs for $96-\mathrm{h}$ screening measurement of Gypsum Board GB.

Table C-35. Chamber concentrations of latex paint target VOCs for 96-h screening measurement of Plywood PW.

Table C-36. Chamber concentrations of SigmaVOC for $96-\mathrm{h}$ screening measurements of paints and substrates.

Table C-37. Chamber concentrations $\left(\mu \mathrm{g} \mathrm{m}^{-3}\right)$ of formaldehyde for $96-\mathrm{h}$ screening measurements

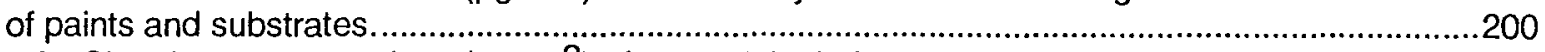

Table C-38. Chamber concentrations $\left(\mu \mathrm{g} \mathrm{m}^{-3}\right)$ of acetaldehyde for 96 -h screening measurements of paints and substrates.

Table C-39. Quasi steady-state emission rates of target VOCs at 48- and 96-h elapsed time for screening measurement of Paint LPS1

Table C-40. Quasi steady-state emission rates of target VOCs at 48- and 96-h elapsed time for screening measurement of Paint LPS2..

Table C-41. Quasi steady-state emission rates of target VOCs at 48- and 96-h elapsed time for screening measurement of Paint FLP1-a.

Table C-42. Quasi steady-state emission rates of target VOCs at 48- and 96-h elapsed time for screening measurement of Paint FLP1-b..

Table C-43. Quasi steady-state emission rates of target VOCs at 48- and 96-h elapsed time for screening measurement of Paint FLP2.

Table C-44. Quasi steady-state emission rates of target VOCs at 48- and 96-h elapsed time for screening measurement of Paint FLP3.

Table C-45. Quasi steady-state emission rates of selected VOCs at 48- and 96-h elapsed time for screening measurement of Paint FLP4.

Table C-46. Quasi steady-state emission rates of target VOCs at 48-and 96-h elapsed time for screening measurement of Paint SGLP1.

Table C-47. Quasi steady-state emission rates of target VOCs at 48- and 96-h elapsed time for screening measurement of Paint SGLP2..

Table C-48. Quasi steady-state emission rates of target VOCs at 48- and 96-h elapsed time for screening measurement of Paint SGLP3-a..

Table C-49. Quasi steady-state emission rates of target VOCs at 48- and 96-h elapsed time for screening measurement of Paint SGLP3-b.

Table C-50. Quasi steady-state emission rates of selected VOCs at 48- and 96-h elapsed time for screening measurement of Paint SGLP4.

Table C-51. Quasi steady-state emission rates of target VOCs at 48-and 96-h elapsed time for screening measurement of Paints LPS2 and FLP3......

Table C-52. Quasi steady-state emission rates of target VOCs at 48- and 96-h elapsed time for

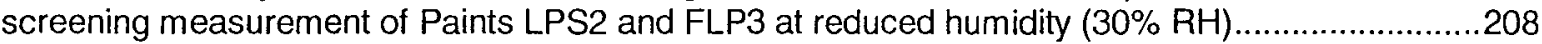

Table C-53. Quasi steady-state emission rates of target VOCs at 48- and 96-h elapsed time for screening measurement of Paints LPS2 and FLP3 at elevated humidity $(70 \% \mathrm{RH}) \ldots \ldots \ldots \ldots \ldots \ldots \ldots . . .209$

Table C-54. Quasi steady-state emission rates of target VOCs at 48- and 96-h elapsed time for screening measurement of Paints LPS2 and SGLP3.......

Table C-55. Quasi steady-state emission rates of SigmaVOC at 48- and 96-h elapsed times for screening measurements of paints.

Table C-56. Quasi steady-state emission rates of formaldehyde at 48- and 96-h elapsed times for

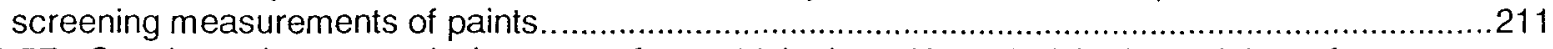

Table C-57. Quasi steady-state emission rates of acetaldehyde at 48- and 96-h elapsed times for screening measurements of paints.

Table C-58. Percent compositions of ethylene glycol, propylene glycol and Texanol in Paints LPS2, FLP3 and SGLP3 selected for use in the large-scale experiments. 
Table C-01. Latex paints selected for study.

\begin{tabular}{lclll}
\hline \hline Material ID & $\begin{array}{c}\text { Manufacturer } \\
\text { Code }\end{array}$ & $\begin{array}{c}\text { Primary } \\
\text { Market }\end{array}$ & Type & Color \\
\hline $\begin{array}{l}\text { Primer Sealer } \\
\text { LPS1 }\end{array}$ & A & National & Conventional & White \\
LPS2* & D & National & Conventional & White \\
Flat & B & National & Conventional & Pure white \\
FLP1 & A & National & Conventional & Pure white \\
FLP2 & C & California & Conventional & Off white \\
FLP3* & A & National & Non-VoC & Pure white \\
FLP4 & & & & \\
Semi-Gloss & B & National & Conventional & Pure white \\
SGLP1 & A & National & Conventional & Pure white \\
SGLP2 & C & California & Conventional & Off white \\
SGLP3* & A & National & Non-VOC & Pure white \\
SGLP4 & & & & \\
\hline
\end{tabular}

*Selected for use in large-scale chamber experiments. 
Table C-02. Summary of screening measurements of latex paints and substrates in 10-L chambers.

\begin{tabular}{|c|c|c|c|c|c|}
\hline Materials & $\begin{array}{c}\text { Duration } \\
\text { (h) }\end{array}$ & Substrate $^{\star}$ & $\begin{array}{l}\text { Area } \\
\left(m^{2}\right)\end{array}$ & $\begin{array}{c}\text { Mass } \\
(\mathrm{g})\end{array}$ & $\begin{array}{c}\text { Coverage } \\
\left(\mathrm{g} \mathrm{m}^{-2}\right)\end{array}$ \\
\hline \multicolumn{6}{|l|}{ Primer sealer } \\
\hline LPSt & 96 & GB & 0.0195 & 1.54 & 79 \\
\hline LPS2 & 96 & GB & 0.0195 & 2.14 & 110 \\
\hline \multicolumn{6}{|l|}{ Flat } \\
\hline FLP1-a & 96 & GB & 0.0195 & 1.93 & 99 \\
\hline FLP1-b & 96 & $\mathrm{~GB}$ & 0.0195 & 2.02 & 104 \\
\hline FLP2 & 96 & GB & 0.0195 & 2.17 & 111 \\
\hline FLP3 & 96 & GB & 0.0195 & 3.17 & 163 \\
\hline FLP4 & 96 & GB & 0.0195 & 2.02 & 104 \\
\hline \multicolumn{6}{|l|}{ Semi-Gloss } \\
\hline SGLP1 & 96 & PW & 0.0195 & 3.20 & 164 \\
\hline SGLP2 & 96 & PW & 0.0195 & 2.51 & 129 \\
\hline SGLP3-a & 96 & PW & 0.0195 & 3.29 & 169 \\
\hline SGLP3-b & 96 & PW & 0.0195 & 3.12 & 160 \\
\hline SGLP4 & 96 & PW & 0.0195 & 2.88 & 148 \\
\hline \multicolumn{6}{|l|}{ Combination } \\
\hline LPS2 \& FLP3 @ $50 \%$ RH & 96 & GB & 0.0195 & 3.05 & 156 \\
\hline LPS2 \& FLP3 @ 30\% RH & 96 & GB & 0.0195 & 3.56 & 183 \\
\hline LPS2 \& FLP3 @ 70\% RH & 96 & GB & 0.0195 & 3.17 & 163 \\
\hline LPS2 \& SGLP3 & 96 & PW & 0.0195 & 3.43 & 176 \\
\hline \multicolumn{6}{|l|}{ Substrate } \\
\hline GB (Gypsum Board) & 96 & $-\cdots$ & 0.0195 & --- & --- \\
\hline PW (Plywood) & 96 & --- & 0.0195 & --- & --- \\
\hline
\end{tabular}

${ }^{\star} \mathrm{GB}=$ Gypsum board; PW = Plywood. 
Table C-03. VOCs emitted by Paint LPS1 in 10-L chamber at 6- and 96-hours elapsed times.

\begin{tabular}{|c|c|c|c|}
\hline COMPOUND & $\begin{array}{l}\text { 6-h } \\
\mathrm{ET} \\
\end{array}$ & $\begin{array}{c}\text { 96-h } \\
\mathrm{ET} \\
\end{array}$ & $\begin{array}{l}\text { Match } \\
\text { Quality }\end{array}$ \\
\hline Ethylene glycol & + & + & Confirmed \\
\hline n-Butyl ether & + & + & Confirmed \\
\hline 2-(2-Butoxyethoxy)ethanol & + & + & Confirmed \\
\hline 2-(2-Butoxyethoxy)ethyl acetate & + & + & Confirmed \\
\hline $\begin{array}{l}\text { 2,2,4-Trimethyl-1,3-pentanediol } \\
\text { monoisobutyrate isomers (Texanol) }\end{array}$ & + & + & Confirmed \\
\hline
\end{tabular}

Table C-04. VOCs emitted by Paint LPS2 in 10-L chamber at 6- and 96-hours elapsed times.

\begin{tabular}{|c|c|c|c|}
\hline COMPOUND & $\begin{array}{l}\text { 6-h } \\
\mathrm{ET} \\
\end{array}$ & $\begin{array}{c}96-h \\
\mathrm{ET}\end{array}$ & $\begin{array}{l}\text { Match } \\
\text { Quality }\end{array}$ \\
\hline Ethylene glycol & + & + & Confirmed \\
\hline n-Butyl ether & & + & Confirmed \\
\hline Propylene glycol & + & & Confirmed \\
\hline Butyl propionate & + & & Confirmed \\
\hline 2-Ethyl-1-hexanol & + & & Confirmed \\
\hline Acetic acid, 2-ethylhexyl ester & + & & Probable \\
\hline 2-(2-Butoxyethoxy)ethanol & & + & Confirmed \\
\hline 1-Decanol & + & & Confirmed \\
\hline 2-(2-Butoxyethoxy)ethyl acetate & & + & Confirmed \\
\hline $\begin{array}{l}\text { 2,2,4-Trimethyl-1,3-pentanediol } \\
\text { monoisobutyrate isomers (Texanol) }\end{array}$ & + & + & Confirmed \\
\hline Unidentified compound & + & + & Unident. \\
\hline
\end{tabular}


Table C-05. VOCs emitted by Paint FLP1 in 10-L chamber at 6- and 96-hours elapsed times.

\begin{tabular}{lccc}
\hline COMPOUND & $\begin{array}{c}6-\mathrm{h} \\
\text { ET }\end{array}$ & $\begin{array}{c}\text { 96-h } \\
\text { ET }\end{array}$ & $\begin{array}{c}\text { Match } \\
\text { Quality }\end{array}$ \\
\hline Ethylene glycol & + & + & Confirmed \\
n-Butyl ether & + & + & Confirmed \\
2-(2-Butoxyethoxy) ethanol & + & + & Confirmed \\
2,2,4-Trimethyl-1,3-pentanediol & & + & Confirmed \\
monoisobutyrate isomers (Texanol) & + & & \\
\end{tabular}

Table C-06. VOCs emitted by Paint FLP2 in 10-L chamber at 6- and 96-hours elapsed times.

\begin{tabular}{lccc}
\hline COMPOUND & $\begin{array}{c}\text { 6-h } \\
\text { ET }\end{array}$ & $\begin{array}{c}\text { 96-h } \\
\text { ET }\end{array}$ & $\begin{array}{c}\text { Match } \\
\text { Quality }\end{array}$ \\
\hline \hline n-Butyl ether & + & & Confirmed \\
Propylene glycol & + & + & Confirmed \\
2-(2-Butoxyethoxy)ethanol & + & + & Confirmed \\
1-Decanol & & + & Confirmed \\
2,2,4-Trimethyl-1,3-pentanediol \\
monoisobutyrate isomers (Texanol)
\end{tabular}

Table C-07. VOCs emitted by Paint FLP3 in 10-L chamber at 6- and 96-hours elapsed times.

\begin{tabular}{|c|c|c|c|}
\hline COMPOUNDD & $\begin{array}{l}\text { 6-h } \\
\text { ET }\end{array}$ & $\begin{array}{c}\text { 96-h } \\
\text { ET }\end{array}$ & $\begin{array}{l}\text { Match } \\
\text { Quality }\end{array}$ \\
\hline Ethylene glycol & + & + & Confirmed \\
\hline n-Butyl ether & + & + & Confirmed \\
\hline Ethylene glycol monoacetate & + & & Probable \\
\hline $\begin{array}{l}\text { 2,2,4-Trimethyl-1,3-pentanediol } \\
\text { monoisobutyrate isomers (Texanol) }\end{array}$ & + & + & Confirmed \\
\hline
\end{tabular}


Table C-08. VOCs emitted by Paint FLP4 in 10-L chamber at 6- and 96-hours elapsed times.

\begin{tabular}{lccc}
\hline COMPOUND & $\begin{array}{c}6-\mathrm{h} \\
\text { ET }\end{array}$ & $\begin{array}{c}96-\mathrm{h} \\
\text { ET }\end{array}$ & $\begin{array}{c}\text { Match } \\
\text { Quality }\end{array}$ \\
\hline \hline Carbonyl Compounds & & & \\
Hexanal & + & & Confirmed \\
Benzaldehyde & + & & Confirmed \\
Nonanal & + & + & Confirmed \\
Decanal & + & + & Confirmed \\
Other Oxidized Compounds & & & \\
1-Butanol & + & & Confirmed \\
Acetic acid & + & & Confirmed \\
n-Butyl ether & + & & Confirmed \\
Butyl propionate & + & & Confirmed \\
Benzyl alcohol & + & & Confirmed \\
2-Phenoxyethanol & + & & Probable \\
2,2,4-Trimethyl-1,3-pentanediol & & & Confirmed \\
monoisobutyrate isomers (Texanol) & & & \\
\hline
\end{tabular}

Table C-09. VOCs emitted by Paint SGLP1 in 10-L chamber at 6- and 96-hours elapsed times.

\begin{tabular}{lccc}
\hline \hline COMPOUND & $\begin{array}{c}6-\mathrm{h} \\
\text { ET }\end{array}$ & $\begin{array}{c}\text { 96-h } \\
\text { ET }\end{array}$ & $\begin{array}{c}\text { Match } \\
\text { Quality }\end{array}$ \\
\hline & + & + & Confirmed \\
Ethylene glycol & + & + & Confirmed \\
n-Butyl ether & + & + & Confirmed \\
2-Butoxyethanol & + & + & Confirmed \\
2-Ethyl-1-hexanol & + & + & Confirmed \\
2-(2-Butoxyethoxy)ethanol & + & + & Confirmed \\
2-(2-Butoxyethoxy)ethyl acetate & + & + & Confirmed \\
2,2,4-Trimethyl-1,3-pentanediol & & & \\
monoisobutyrate isomers (Texanol) & & &
\end{tabular}


Table C-10. VOCs emitted by Paint SGLP2 in 10-L chamber at 6- and 96-hours elapsed times.

\begin{tabular}{lccc}
\hline \hline COMPOUND & $\begin{array}{c}6-\mathrm{h} \\
\text { ET }\end{array}$ & $\begin{array}{c}\text { 96-h } \\
\text { ET }\end{array}$ & $\begin{array}{l}\text { Match } \\
\text { Quality }\end{array}$ \\
\hline $\begin{array}{l}\text { Ethylene glycol } \\
\text { n-Butyl ether }\end{array}$ & + & + & Confirmed \\
2-Butoxyethanol & + & + & Confirmed \\
Ethylene glycol monoacetate & + & & Confirmed \\
2-(2-Butoxyethoxy)ethanol & + & + & Probable \\
2-(2-Butoxyethoxy)ethyl acetate & + & + & Confirmed \\
2,2,4-Trimethyl-1,3-pentanediol & + & + & Confirmed \\
monoisobutyrate isomers (Texanol) & + & + & Confirmed \\
& & & \\
\hline
\end{tabular}

Table C-11. VOCs emitted by Paint SGLP3 in 10-L chamber at 6- and 96-hours elapsed times.

\begin{tabular}{lccc}
\hline \hline COMPOUND & 6-h & 96-h & Match \\
\hline n-Butyl ether & ET & ET & Quality \\
Propylene glycol & & & \\
Hexylene glycol & + & + & Confirmed \\
2-(2-Butoxyethoxy)ethanol & + & + & Confirmed \\
2,2,4-Trimethyl-1,3-pentanediol & + & + & Confirmed \\
monoisobutyrate isomers (Texanol) & + & + & Confirmed \\
& & & \\
\hline
\end{tabular}


Table C-12. VOCs emitted by Paint SGLP4 in 10-L chamber at 6 - and 96-hours elapsed times.

COMPOUND

Pentanal

3-Hydroxy-2-butanone

Hexanal

Benzaldehyde

2-Octenal

Nonanal

Decanal

\section{Other Oxidizied Compounds}

1-Butanol

Acetic acid

1-Pentanol

n-Butyl ether

Butyl propionate

2-Pentylfuran

2-Ethyl-1-hexanol

Benzyl alcohol

Phenol

2-(2-Butoxyethoxy)ethanol

2,2,4-Trimethyl-1,3-pentanediol monoisobutyrate isomers (Texanol)

$\begin{array}{ccl}+ & + & \text { Confirmed } \\ + & + & \text { Probable } \\ + & + & \text { Confirmed } \\ + & + & \text { Confirmed } \\ + & & \text { Confirmed } \\ + & + & \text { Confirmed } \\ + & + & \text { Confirmed }\end{array}$

$\begin{array}{ccc}+ & & \text { Confirmed } \\ + & + & \text { Confirmed } \\ + & + & \text { Confirmed } \\ + & + & \text { Confirmed } \\ + & & \text { Confirmed } \\ + & & \text { Probable } \\ + & + & \text { Confirmed } \\ + & & \text { Confirmed } \\ + & & \text { Confirmed } \\ + & & \text { Confirmed } \\ + & + & \text { Confirmed }\end{array}$


Table C-13. VOCs emitted by Paints LPS2 and FLP3 in 10-L chamber at 6- and 96-hours elapsed times.

\begin{tabular}{|c|c|c|c|}
\hline COMPOUND & $\begin{array}{l}6-\mathrm{h} \\
\text { ET }\end{array}$ & $\begin{array}{c}\text { 96-h } \\
\text { ET }\end{array}$ & $\begin{array}{l}\text { Match } \\
\text { Quality } \\
\end{array}$ \\
\hline Ethylene glycol & + & + & Confirmed \\
\hline Propylene glycol & + & & Confirmed \\
\hline Ethylene glcycol monoacetate & + & & Probable \\
\hline 2-Ethyl-1-hexanol & + & & Confirmed \\
\hline Acetic acid, 2-ethylhexyl ester & + & + & Probable \\
\hline $\begin{array}{l}\text { 2,2,4-Trimethyl-1,3-pentanediol } \\
\text { monoisobutyrate isomers (Texanol) }\end{array}$ & + & + & Confirmed \\
\hline Unidentified compound & & + & Unident. \\
\hline
\end{tabular}

Table C-14. VOCs emitted by Paints LPS2 and SGLP3 in 10-L chamber at 6- and 96-hours elapsed times.

\begin{tabular}{|c|c|c|c|}
\hline COMPOUND & $\begin{array}{l}\text { 6-h } \\
\text { ET }\end{array}$ & $\begin{array}{c}96-h \\
\text { ET }\end{array}$ & $\begin{array}{l}\text { Match } \\
\text { Quality }\end{array}$ \\
\hline Ethylene glycol & + & + & Confirmed \\
\hline n-Butyl ether & + & & Confirmed \\
\hline Propylene glycol & + & + & Confirmed \\
\hline Hexylene glycol & + & + & Confirmed \\
\hline 2-(2-Butoxyethoxy)ethanol & + & + & Confirmed \\
\hline $\begin{array}{l}\text { 2,2,4-Trimethyl-1,3-pentanediol } \\
\text { monoisobutyrate isomers (Texanol) }\end{array}$ & + & + & Confirmed \\
\hline Unidentified compound & & + & Unident. \\
\hline
\end{tabular}


Table C-15. VOCs emitted by Gypsum Board GB in 10-L chamber at 6- and 96-hours elapsed times.

\begin{tabular}{|c|c|c|c|}
\hline COMPOUND & $\begin{array}{l}\text { 6-h } \\
\mathrm{ET}\end{array}$ & $\begin{array}{c}\text { 96-h } \\
\text { ET }\end{array}$ & $\begin{array}{l}\text { Match } \\
\text { Quality } \\
\end{array}$ \\
\hline \multicolumn{4}{|l|}{ Hydrocarbons } \\
\hline p-Cymene & + & & Confirmed \\
\hline \multicolumn{4}{|l|}{ Carbonyl Compounds } \\
\hline Hexanal & + & & Confirmed \\
\hline Cyclohexanone & + & & Confirmed \\
\hline Octanal & + & & \\
\hline Nonanal & + & + & Confirmed \\
\hline Decanal & + & + & Confirmed \\
\hline \multicolumn{4}{|l|}{ Other Oxidizied Compounds } \\
\hline $\begin{array}{l}\text { 2,2,4-Trimethyl-1,3-pentanediol } \\
\text { monoisobutyrate isomers (Texanol) }\end{array}$ & + & + & Confirmed \\
\hline
\end{tabular}


Table C-16. VOCs emitted by Plywood PW in 10-L chamber at 6- and 96-hours elapsed times.

\begin{tabular}{lccc}
\hline \hline & $6-\mathrm{h}$ & $96-\mathrm{h}$ & Match \\
COMPOUND & ET & ET & Quality \\
\hline
\end{tabular}

\section{Hydrocarbons}

alpha-Pinene

beta-Pinene

d-Limonene

p-Cymene

$\begin{array}{ccc}+ & + & \text { Confirmed } \\ + & + & \text { Confirmed } \\ & + & \text { Confirmed } \\ + & & \text { Confirmed }\end{array}$

\section{Carbonyl Compounds}

Pentanal

3-Hydroxy-2-butanone

Hexanal

Octanal

Nonanal

Decanal

\section{Other Oxidizied Compounds}

Acetic acid

2-Ethyl-1-hexanol

Hexanoic acid

2-(2-Butoxyethoxy)ethanol

2-(2-Butoxyethoxy)ethyl acetate

2,2,4-Trimethyl-1,3-pentanediol monoisobutyrate isomers (Texanol)

$+\quad+\quad$ Confirmed

$+\quad$ Probable

$+\quad+\quad$ Confirmed

$+\quad$ Confirmed

$+\quad$ Confirmed

$+\quad+\quad$ Confirmed

$\begin{array}{ccc}+ & & \text { Confirmed } \\ + & & \text { Confirmed } \\ + & + & \text { Confirmed } \\ + & + & \text { Confirmed } \\ + & + & \text { Confirmed } \\ + & + & \text { Confirmed }\end{array}$

$+$ 
Table C-17. Summary of VOCs emitted by conventional latex paints in 10-L chambers.

\begin{tabular}{|c|c|c|c|c|c|c|c|c|c|}
\hline \multirow[b]{2}{*}{ COMPOUND } & \multirow{2}{*}{$\begin{array}{l}\text { CAS } \\
\text { No. }\end{array}$} & \multicolumn{2}{|c|}{ LPS } & \multicolumn{3}{|c|}{ FLP } & \multicolumn{3}{|c|}{ SGLP } \\
\hline & & 1 & 2 & 1 & 2 & 3 & 1 & 2 & 3 \\
\hline Ethylene glycol* & $107-21-1$ & $A^{* *}$ & $A$ & $A$ & & A & A & $A$ & \\
\hline n-Butyl ether & $142-96-1$ & + & + & + & + & + & + & + & + \\
\hline Propylene glycol* & $57-55-6$ & & + & & A & & & & A \\
\hline Butyl propionate* & $590-01-2$ & & + & & & & & & \\
\hline 2-Butoxyethanol* & $111-76-2$ & & & & & & + & + & \\
\hline Ethylene glycol monoacetate & $542-59-6$ & & & & & + & & + & \\
\hline Hexylene glycol* & $107-41-5$ & & & & & & & & A \\
\hline 2-Ethyl-1-hexanol* & $104-76-7$ & & + & & & & + & & \\
\hline Acetic acid, 2-ethylhexyl ester & $103-09-3$ & & + & & & & & & \\
\hline 2-(2-Butoxyethoxy)ethanol* & $112-34-5$ & + & + & A & A & & A & A & A \\
\hline 1-Decanol & $112-30-1$ & & + & & + & & & & \\
\hline 2-(2-Butoxyethoxy)ethyl acetate* & $124-17-4$ & A & + & & & & + & A & \\
\hline $\begin{array}{l}2,2,4-\text { Trimethyl-1,3-pentanediol* }^{*} \\
\text { monoisobutyrate isomers (Texanol) }\end{array}$ & $25265-77-4$ & A & A & A & A & A & A & A & A \\
\hline
\end{tabular}

*Target compound selected for quantitative analysis.

${ }^{* *} A=$ Relatively abundant compound. 
Table C-18. Chamber concentrations of target VOCs for $96-\mathrm{h}$ screening measurement of Paint LPS1.

\begin{tabular}{|c|c|c|c|c|c|}
\hline \multirow[b]{2}{*}{ COMPOUND } & \multicolumn{5}{|c|}{ Chamber Concentration ( $\mathrm{mg} \mathrm{m}^{-3}$ ) } \\
\hline & $1-h$ & $6-h$ & 24-h & 48-h & 96-h \\
\hline Ethylene glycol & 0.95 & 0.46 & 0.23 & 0.19 & 0.09 \\
\hline n-Butyl ether & 0.03 & 0.01 & 0.01 & $<0.01$ & $<0.01$ \\
\hline 2-(2-Butoxyethoxy)ethanol & 0.66 & 0.16 & 0.03 & 0.02 & 0.01 \\
\hline 2-(2-Butoxyethoxy)ethyl acetate & 2.80 & 1.32 & 0.31 & 0.10 & 0.04 \\
\hline $\begin{array}{c}\text { 2,2,4-Trimethyl-1,3-pentanediol } \\
\text { monoisobutyrates (Texanol) }\end{array}$ & 8.30 & 2.10 & 0.67 & 0.31 & 0.18 \\
\hline
\end{tabular}

Table C-19. Chamber concentrations of target VOCs for $96-\mathrm{h}$ screening measurement of Paint LPS2.

\begin{tabular}{lrrrrrr}
\hline \hline & \multicolumn{7}{c}{ Chamber Concentration (mg m-3) } \\
COMPOUND & 1-h & 6-h & 24-h & 48-h & 96-h \\
\hline Ethylene glycol & 10.4 & 2.56 & $\mathrm{md}^{*}$ & 0.17 & 0.12 \\
n-Butyl ether & 0.19 & 0.04 & $\mathrm{md}$ & $<0.01$ & $<0.01$ \\
Propylene glycol & 0.47 & 0.12 & $\mathrm{md}$ & $<0.04$ & $<0.04$ \\
2-Ethyl-1-hexanol & 0.12 & 0.04 & $\mathrm{md}$ & $<0.01$ & $<0.01$ \\
2-(2-Butoxyethoxy)ethanol & 0.01 & $<0.01$ & $\mathrm{md}$ & $<0.01$ & $<0.01$ \\
2,2,4-Trimethyl-1,3-pentanediol & 9.15 & 4.28 & 1.75 & 1.03 & 0.69 \\
monoisobutyrates (Texanol) & & & & & \\
\hline *md = Missing data. & & & & & & \\
\hline
\end{tabular}

Table C-20. Chamber concentrations of target VOCs for 96-h screening measurement of Paint FLP1-a.

\begin{tabular}{|c|c|c|c|c|c|}
\hline \multirow[b]{2}{*}{ COMPOUND } & \multicolumn{5}{|c|}{ Chamber Concentration (mg m-3) } \\
\hline & $1-\mathrm{h}$ & $6-h$ & 24-h & $48-\mathrm{h}$ & 96-h \\
\hline Ethylene glycol & 3.09 & 1.45 & 0.90 & 0.53 & 0.33 \\
\hline n-Butyl ether & 0.08 & 0.02 & 0.01 & $<0.01$ & $<0.01$ \\
\hline 2-(2-Butoxyethoxy)ethanol & $\mathrm{md}^{*}$ & 1.28 & 0.24 & 0.11 & 0.04 \\
\hline $\begin{array}{c}\text { 2,2,4-Trimethyl-1,3-pentanediol } \\
\text { monoisobutyrates (Texanol) }\end{array}$ & md & 9.50 & 2.55 & 0.67 & 0.08 \\
\hline
\end{tabular}


Table C-21. Chamber concentrations of target VOCs for 96-h screening measurement of Paint FLP1-b.

\begin{tabular}{lrrrrrr}
\hline & \multicolumn{5}{c}{ Chamber Concentration (mg m-3) } \\
COMPOUND & 1-h & 6-h & 24-h & 48-h & 96-h \\
\hline Ethylene glycol & 3.01 & 1.18 & 0.72 & 0.54 & 0.41 \\
n-Butyl ether & 0.10 & 0.03 & 0.01 & $<0.01$ & $<0.01$ \\
2-(2-Butoxyethoxy)ethanol & 3.60 & 1.30 & 0.27 & 0.12 & 0.04 \\
2,2,4-Trimethyl-1,3-pentanediol & 14.0 & 9.14 & 2.50 & 0.75 & 0.10 \\
monoisobutyrates (Texanol) & & & & & \\
\end{tabular}

Table C-22. Chamber concentrations of target VOCs for 96-h screening measurement of Paint FLP2.

\begin{tabular}{lrrrrrr}
\hline & \multicolumn{5}{c}{ Chamber Concentration (mg m-3) } \\
COMPOUND & 1-h & 6-h & 24-h & 48-h & 96-h \\
n-Butyl ether & 0.04 & 0.01 & $<0.01$ & $<0.01$ & $<0.01$ \\
Propylene glycol & 1.69 & 0.61 & 0.27 & 0.18 & 0.12 \\
2-(2-Butoxyethoxy)ethanol & 1.95 & 0.47 & 0.12 & 0.10 & 0.04 \\
$\begin{array}{l}\text { 2,2,4-Trimethyl-1,3-pentanediol } \\
\text { monoisobutyrates (Texanol) }\end{array}$ & 27.4 & 12.9 & 2.02 & 0.46 & 0.09 \\
& & & & & \\
\hline
\end{tabular}

Table C-23. Chamber concentrations of target VOCs for 96-h screening measurement of Paint FLP3.

\begin{tabular}{lrrrrrr}
\hline & \multicolumn{5}{c}{ Chamber Concentration (mg m-3) } \\
COMPOUND & 1-h & 6-h & 24-h & 48-h & 96-h \\
\hline Ethylene glycol & 4.77 & 0.89 & 0.31 & 0.27 & 0.13 \\
n-Butyl ether & 0.17 & 0.02 & 0.01 & 0.01 & $<0.01$ \\
$\begin{array}{l}\text { 2,2,4-Trimethyl-1,3-pentanediol } \\
\text { monoisobutyrates (Texanol) }\end{array}$ & 11.8 & 4.97 & 2.94 & 1.26 & 0.46 \\
& & & & & \\
\hline
\end{tabular}


Table C-24. Chamber concentrations of selected VOCs for 96-h screening measurement of Paint FLP4.

\begin{tabular}{|c|c|c|c|c|c|}
\hline \multirow[b]{2}{*}{ COMPOUND } & \multicolumn{5}{|c|}{ Chamber Concentration ( $\mathrm{mg} \mathrm{m}^{-3}$ ) } \\
\hline & 1-h & 6-h & 24-h & 48-h & 96-h \\
\hline \multicolumn{6}{|l|}{ Carbonyl Compounds } \\
\hline Hexanal & 0.006 & 0.005 & 0.001 & 0.001 & $<0.001$ \\
\hline Nonanal & 0.008 & 0.007 & 0.002 & 0.001 & 0.001 \\
\hline \multicolumn{6}{|l|}{ Other Oxidized Compounds } \\
\hline 1-Butanol & 0.019 & 0.007 & 0.003 & 0.001 & $<0.001$ \\
\hline Benzyl alcohol & 0.017 & 0.004 & 0.001 & 0.001 & $<0.001$ \\
\hline $\begin{array}{l}\text { 2,2,4-Trimethyl-1,3-pentanediol } \\
\text { monoisobutyrates (Texanol) }\end{array}$ & 0.052 & 0.024 & 0.013 & 0.010 & 0.007 \\
\hline
\end{tabular}

Table C-25. Chamber concentrations of target VOCs for 96-h screening measurement of Paint SGLP1.

\begin{tabular}{llllllr}
\hline \hline & \multicolumn{5}{c}{ Chamber Concentration (mg m-3) } \\
COMPOUND & 1-h & 6-h & 24-h & 48-h & 96-h \\
\hline Ethylene glycol & 6.15 & 1.93 & 1.65 & 0.87 & 0.50 \\
n-Butyl ether & 0.36 & 0.10 & 0.02 & 0.01 & $<0.01$ \\
2-Butoxyethanol & 0.42 & 0.03 & 0.01 & 0.01 & $<0.01$ \\
2-Ethyl-1-hexanol & 0.27 & 0.04 & 0.01 & 0.01 & 0.01 \\
2-(2-Butoxyethoxy)ethanol & 8.50 & 6.73 & 1.88 & 0.85 & 0.52 \\
2-(2-Butoxyethoxy)ethyl acetate & 0.04 & 0.02 & 0.02 & 0.01 & 0.01 \\
2,2,4-Trimethyl-1,3-pentanediol & 0.90 & 0.51 & 0.24 & 0.12 & 0.08 \\
monoisobutyrates (Texanol) & & & & & \\
\end{tabular}


Table C-26. Chamber concentrations of target VOCs for 96-h screening measurement of Paint SGLP2.

\begin{tabular}{lcrrrrr}
\hline & \multicolumn{5}{c}{ Chamber Concentration (mg m-3) } \\
COMPOUND & 1-h & 6-h & 24-h & 48-h & 96-h \\
\hline \hline Ethylene glycol & 11.5 & 2.14 & 1.09 & 0.71 & 0.56 \\
n-Butyl ether & 0.16 & 0.04 & 0.01 & $<0.01$ & $<0.01$ \\
2-Butoxyethanol & 0.32 & $\mathrm{md}^{*}$ & $<0.01$ & $<0.01$ & $<0.01$ \\
2-(2-Butoxyethoxy)ethanol & 2.00 & 0.74 & 0.31 & 0.12 & 0.07 \\
2-(2-Butoxyethoxy)ethyl acetate & 0.85 & 0.52 & 0.23 & 0.16 & 0.09 \\
2,2,4-Trimethyl-1,3-pentanediol & 5.10 & 1.15 & 0.47 & 0.30 & 0.26 \\
monoisobutyrates (Texanol) & & & & & \\
\hline${ }^{*}$ md = Missing data. & & & & & & \\
\hline
\end{tabular}

Table C-27. Chamber concentrations of target VOCs for 96-h screening measurement of Paint SGLP3-a.

\begin{tabular}{llllll}
\hline \hline & \multicolumn{5}{c}{ Chamber Concentration (mg m-3) } \\
COMPOUND & $\mathbf{1 - h}$ & $\mathbf{6 - h}$ & $\mathbf{2 4 - h}$ & $\mathbf{4 8 - h}$ & 96-h \\
\hline n-Butyl ether & 0.35 & 0.04 & 0.02 & 0.01 & $<0.01$ \\
Propylene glycol & 15.4 & 3.81 & 1.02 & 0.60 & 0.25 \\
Hexylene glycol & 18.0 & 3.21 & 1.16 & 0.54 & 0.17 \\
2-(2-Butoxyethoxy)ethanol & 3.20 & 1.23 & 0.42 & 0.24 & 0.15 \\
$\begin{array}{l}\text { 2,2,4-Trimethyl-1,3-pentanediol } \\
\text { monoisobutyrates (Texanol) }\end{array}$ & 6.90 & 1.20 & 0.42 & 0.31 & 0.29 \\
\hline
\end{tabular}

Table C-28. Chamber concentrations of target VOCs for 96-h screening measurement of Paint SGLP3-b.

\begin{tabular}{lllllll}
\hline & \multicolumn{5}{c}{ Chamber Concentration (mg m-3) } \\
COMPOUND & 1-h & 6-h & 24-h & 48-h & 96-h \\
\hline \hline n-Butyl ether & 0.20 & 0.04 & 0.01 & $<0.01$ & $<0.01$ \\
Propylene glycol & 9.85 & 3.56 & 1.14 & 0.59 & 0.36 \\
Hexylene glycol & 18.3 & 2.36 & 0.96 & 0.59 & 0.28 \\
2-(2-Butoxyethoxy)ethanol & 2.90 & 0.88 & 0.39 & 0.18 & 0.09 \\
$\begin{array}{l}\text { 2,2,4-Trimethyl-1,3-pentanediol } \\
\text { monoisobutyrates (Texanol) }\end{array}$ & 5.00 & 1.00 & 0.42 & 0.24 & 0.18 \\
\hline
\end{tabular}


APPENDIX C - LATEX PAINTS

Table C-29. Chamber concentrations of selected VOCs for 96-h screening measurement of Paint SGLP4.

\begin{tabular}{|c|c|c|c|c|c|}
\hline \multirow[b]{2}{*}{ COMPOUND } & \multicolumn{5}{|c|}{ Chamber Concentration ( $\mathrm{mg} \mathrm{m}^{-3}$ ) } \\
\hline & 1-h & 6-h & 24-h & 48-h & 96-h \\
\hline \multicolumn{6}{|l|}{ Carbonyl Compounds } \\
\hline Pentanal $^{*}$ & 0.050 & 0.017 & 0.005 & 0.005 & 0.003 \\
\hline Hexanal $^{*}$ & 0.252 & 0.079 & 0.029 & 0.023 & 0.011 \\
\hline Benzaldehyde & 0.142 & 0.022 & 0.006 & 0.004 & 0.001 \\
\hline Nonanal $^{*}$ & 0.004 & 0.001 & 0.001 & 0.001 & $<0.001$ \\
\hline \multicolumn{6}{|l|}{ Other Oxidized Compounds } \\
\hline 1-Butanol & 0.124 & 0.032 & 0.005 & 0.005 & 0.003 \\
\hline 1-Pentanol & 0.041 & 0.014 & 0.009 & 0.008 & 0.005 \\
\hline n-Butyl ether & 0.683 & 0.182 & 0.023 & 0.008 & 0.001 \\
\hline 2-Ethyl-1-hexanol* & 0.023 & 0.005 & 0.002 & 0.002 & 0.001 \\
\hline Benzyl alcohol & 0.020 & 0.007 & 0.003 & 0.002 & 0.001 \\
\hline 2-(2-Butoxyethoxy)ethanol ${ }^{\star}$ & 0.032 & 0.010 & 0.006 & 0.003 & 0.002 \\
\hline $\begin{array}{l}\text { 2,2,4-Trimethyl-1,3-pentanediol } \\
\text { monoisobutyrates (Texanol)* }\end{array}$ & 0.064 & 0.014 & 0.010 & 0.011 & 0.009 \\
\hline
\end{tabular}

*Detected in emissions from Plywood PW; see Table C-16.

Table C-30. Chamber concentrations of target VOCs for $96-h$ screening measurement of Paints LPS2 and FLP3 at standard humidity $(50 \% \mathrm{RH})$.

\begin{tabular}{lrrrrrr}
\hline & \multicolumn{5}{c}{ Chamber Concentration (mg m-3) } \\
COMPOUND & 1-h & 6-h & 24-h & 48-h & 96-h \\
\hline \hline & 26.7 & 10.3 & 0.80 & 0.43 & 0.19 \\
Ethylene glycol & 0.20 & 0.03 & $<0.01$ & $<0.01$ & $<0.01$ \\
n-Butyl ether & $<4.00$ & 0.62 & $<0.20$ & $<0.08$ & $<0.03$ \\
Propylene glycol & 0.15 & 0.03 & $<0.01$ & 0.01 & $<0.01$ \\
2-Ethyl-1-hexanol & $\mathrm{md}^{*}$ & 0.01 & 0.01 & $<0.01$ & $<0.01$ \\
2-(2-Butoxyethoxy)ethanol & 13.4 & 8.13 & 3.17 & 2.03 & 1.04 \\
2,2,4-Trimethyl-1,3-pentanediol & & & & & \\
monoisobutyrates (Texanol) & & & & & &
\end{tabular}


Table C-31. Chamber concentrations of target VOCs for $96-h$ screening measurement of Paints LPS2 and FLP3 at reduced humidity $(30 \% \mathrm{RH})$.

\begin{tabular}{lrrrrrr}
\hline \hline & \multicolumn{5}{c}{ Chamber Concentration (mg m-3) } \\
COMPOUND & 1-h & 6-h & 24-h & 48-h & 96-h \\
\hline Ethylene glycol & 56.5 & 7.53 & 1.08 & 0.49 & 0.07 \\
n-Butyl ether & 0.15 & 0.30 & $<0.01$ & $<0.01$ & $<0.01$ \\
Propylene glycol & $<4.00$ & $<0.50$ & $<0.16$ & $<0.05$ & $<0.02$ \\
2-Ethyl-1-hexanol & 0.10 & $<0.03$ & 0.01 & $<0.01$ & $<0.01$ \\
2-(2-Butoxyethoxy)ethanol & 0.05 & $<0.03$ & $<0.01$ & $<0.01$ & $<0.01$ \\
2,2,4-Trimethyl-1,3-pentanediol & 17.1 & md $^{*}$ & 2.67 & 1.82 & 1.24 \\
monoisobutyrates (Texanol) & & & & & \\
\hline
\end{tabular}

${ }^{*} \mathrm{md}=$ Missing data.

Table C-32. Chamber concentrations of target VOCs for 96-h screening measurement of Paints LPS2 and FLP3 at elevated humidity $(70 \% \mathrm{RH})$.

\begin{tabular}{lrrrrr}
\hline \hline & \multicolumn{5}{c}{ Chamber Concentration (mg m-3) } \\
COMPOUND & 1-h & 6-h & 24-h & 48-h & 96-h \\
\hline Ethylene glycol & 12.3 & 8.51 & 0.81 & 0.46 & 0.10 \\
n-Butyl ether & 0.12 & 0.03 & 0.01 & $<0.01$ & $<0.01$ \\
Propylene glycol & $<2.00$ & $<0.25$ & $<0.16$ & $<0.05$ & $<0.02$ \\
2-Ethyl-1-hexanol & 0.12 & 0.04 & $<0.01$ & $<0.01$ & $<0.01$ \\
2-(2-Butoxyethoxy)ethanol & 0.02 & 0.01 & $<0.01$ & $<0.01$ & $<0.01$ \\
2,2,4-Trimethyl-1,3-pentanediol & 12.3 & 7.87 & 3.33 & 1.73 & 0.87 \\
monoisobutyrates (Texanol) & & & & & \\
\hline
\end{tabular}


Table C-33. Chamber concentrations of target VOCs for $96-\mathrm{h}$ screening measurement of Paints LPS2 and SGLP3.

\begin{tabular}{|c|c|c|c|c|c|}
\hline \multirow[b]{2}{*}{ COMPOUND } & \multicolumn{5}{|c|}{ Chamber Concentration ( $\mathrm{mg} \mathrm{m}^{-3}$ ) } \\
\hline & $1-h$ & 6-h & 24-h & 48-h & 96-h \\
\hline Ethylene glycol & 8.75 & 4.63 & 0.60 & 0.75 & 0.39 \\
\hline n-Butyl ether & 0.25 & 0.03 & 0.01 & $<0.01$ & $<0.01$ \\
\hline Propylene glycol & 57.5 & 13.4 & 0.81 & 0.34 & 0.09 \\
\hline Hexylene glycol & 22.1 & 3.05 & 0.76 & 0.20 & 0.10 \\
\hline 2-Ethyl-1-hexanol & 0.15 & 0.02 & $<0.01$ & 0.01 & $<0.01$ \\
\hline 2-(2-Butoxyethoxy)ethanol & 3.75 & 1.78 & 0.34 & 0.19 & 0.06 \\
\hline $\begin{array}{l}\text { 2,2,4-Trimethyl-1,3-pentanediol } \\
\text { monoisobutyrates (Texanol) }\end{array}$ & 7.35 & 1.85 & 0.38 & 0.48 & 0.25 \\
\hline
\end{tabular}

Table C-34. Chamber concentrations of latex paint target VOCs for 96-h screening measurement of Gypsum Board GB.

\begin{tabular}{lcccccc}
\hline \hline & \multicolumn{5}{c}{ Chamber Concentration (mg m-3) } \\
COMPOUND & 1-h & 6-h & 24-h & 48-h & 96-h \\
\hline $\begin{array}{c}\text { 2,2,4-Trimethyl-1,3-pentanediol } \\
\text { monoisobutyrates (Texanol) }\end{array}$ & $<0.01$ & $<0.01$ & $<0.01$ & $<0.01$ & $<0.01$ \\
\hline
\end{tabular}

Table C-35. Chamber concentrations of latex paint target VOCs for 96-h screening measurement of Plywood PW.

\begin{tabular}{lrrrrrr}
\hline \hline & \multicolumn{5}{c}{ Chamber Concentration (mg m-3) } \\
COMPOUND & 1-h & $6-\mathrm{h}$ & $\mathbf{2 4 - h}$ & 48-h & 96-h \\
\hline 2-Ethyl-1-hexanol & $<0.01$ & $<0.01$ & $<0.01$ & $<0.01$ & $<0.01$ \\
2-(2-Butoxyethoxy) ethanol & 0.01 & $<0.01$ & 0.01 & $<0.01$ & $<0.01$ \\
2-(2-Butoxyethoxy) ethyl acetate & $<0.01$ & $<0.01$ & $<0.01$ & $<0.01$ & $<0.01$ \\
$\begin{array}{l}\text { 2,2,4-Trimethyl-1,3-pentanediol } \\
\text { monoisobutyrates (Texanol) }\end{array}$ & 0.02 & 0.02 & 0.01 & 0.01 & $<0.01$ \\
& & & & & \\
\hline
\end{tabular}


Table C-36. Chamber concentrations of SigmaVOC (i.e., sum of target VOCs) for 96-h screening measurements of paints and substrates.

\begin{tabular}{|c|c|c|c|c|c|}
\hline \multirow[b]{2}{*}{ Material ID } & \multicolumn{5}{|c|}{ Chamber Concentration (mg m-3) } \\
\hline & 1-h & 6-h & 24-h & 48-h & 96-h \\
\hline \multicolumn{6}{|l|}{ Primer Sealer } \\
\hline LPS1 & 12.7 & 4.05 & 1.25 & 0.62 & 0.32 \\
\hline LPS2 & 20.3 & 7.04 & $\mathrm{md}^{*}$ & 1.23 & 0.84 \\
\hline \multicolumn{6}{|l|}{ Flat } \\
\hline FLP1-a & md & 12.2 & 3.70 & 1.31 & 0.45 \\
\hline FLP1-b & 20.7 & 11.6 & 3.50 & 1.41 & 0.55 \\
\hline FLP2 & 31.1 & 14.1 & 2.41 & 0.74 & 0.25 \\
\hline FLP3 & 16.7 & 5.88 & 3.26 & 1.54 & 0.59 \\
\hline FLP4 & 0.10 & 0.05 & 0.02 & 0.01 & 0.01 \\
\hline \multicolumn{6}{|l|}{ Semi-Gloss } \\
\hline SGLP1 & 16.6 & 9.36 & 3.83 & 1.88 & 1.13 \\
\hline SGLP2 & 19.9 & 4.59 & 2.11 & 1.30 & 0.99 \\
\hline SGLP3-a & 43.8 & 9.49 & 3.04 & 1.70 & 0.86 \\
\hline SGLP3-b & 36.2 & 7.84 & 2.92 & 1.60 & 0.91 \\
\hline SGLP4 & 1.43 & 0.38 & 0.10 & 0.07 & 0.04 \\
\hline \multicolumn{6}{|l|}{ Combination } \\
\hline LPS2 \& FLP3 @ $50 \%$ RH & 40.4 & 18.5 & 3.99 & 2.48 & 1.25 \\
\hline LPS2 \& FLP3 @ 30\% RH & 73.9 & md & 3.77 & 2.33 & 1.33 \\
\hline LPS2 \& FLP3 @ $70 \%$ RH & 24.9 & 16.5 & 4.16 & 2.20 & 0.98 \\
\hline LPS2 \& SGLP3 & 99.8 & 24.8 & 2.90 & 1.97 & 0.90 \\
\hline \multicolumn{6}{|l|}{ Substrate } \\
\hline $\mathrm{GB}$ & $<0.01$ & $<0.01$ & $<0.01$ & $<0.01$ & $<0.01$ \\
\hline PW & 0.04 & 0.03 & 0.03 & 0.02 & 0.01 \\
\hline
\end{tabular}

$* m d=$ Missing data. 
Table C-37. Chamber concentrations $\left(\mu \mathrm{g} \mathrm{m}^{-3}\right)$ of formaldehyde for $96-\mathrm{h}$ screening measurements of paints and substrates.

\begin{tabular}{|c|c|c|c|c|c|}
\hline \multirow[b]{2}{*}{ Material ID } & \multicolumn{5}{|c|}{ Chamber Concentration ( $\mu \mathrm{g} \mathrm{m}^{-3}$ ) } \\
\hline & $1-\mathrm{h}$ & 6-h & 24-h & 48-h & 96-h \\
\hline \multicolumn{6}{|l|}{ Primer Sealer } \\
\hline LPS1 & 82 & 26 & 13 & 11 & 9 \\
\hline LPS2 & 9 & 7 & 3 & 2 & 1 \\
\hline \multicolumn{6}{|l|}{ Flat } \\
\hline FLP1-a & 4 & 1 & $<1$ & $<1$ & $<1$ \\
\hline FLP1-b & 5 & 1 & $<1$ & $<1$ & $<1$ \\
\hline FLP2 & 17 & 40 & 23 & 17 & 13 \\
\hline FLP3 & 105 & 4 & 4 & 5 & 2 \\
\hline FLP4 & 318 & 83 & 27 & 15 & 8 \\
\hline \multicolumn{6}{|l|}{ Semi-Gloss } \\
\hline SGLP1 & 70 & 7 & 8 & 9 & 6 \\
\hline SGLP2 & 17 & 13 & 9 & 8 & 8 \\
\hline SGLP3-a & 32 & $\mathrm{md}^{*}$ & 6 & 9 & 6 \\
\hline SGLP3-b & 21 & 4 & 3 & 3 & 2 \\
\hline SGLP4 & 27 & 14 & 10 & 7 & 5 \\
\hline \multicolumn{6}{|l|}{ Combination } \\
\hline LPS2 \& FLP3 @ 50\% RH & 20 & 5 & 2 & 3 & $<1$ \\
\hline LPS2 \& FLP3 @ 30\% RH & 16 & 2 & 1 & 2 & $<1$ \\
\hline LPS2 \& FLP3 @ $70 \%$ RH & 29 & 9 & 7 & 3 & 2 \\
\hline LPS2 \& SGLP3 & 23 & 5 & 2 & 4 & 2 \\
\hline \multicolumn{6}{|l|}{ Substrate } \\
\hline GB & 3 & 2 & 1 & 1 & 1 \\
\hline PW & 9 & md & 3 & 3 & 2 \\
\hline
\end{tabular}

*md = Missing data. 
Table C-38. Chamber concentrations $\left(\mu \mathrm{g} \mathrm{m}^{-3}\right)$ of acetaldehyde for $96-\mathrm{h}$ screening measurements of paints and substrates.

\begin{tabular}{|c|c|c|c|c|c|}
\hline \multirow[b]{2}{*}{ Material ID } & \multicolumn{5}{|c|}{ Chamber Concentration ( $\left.\mu \mathrm{g} \mathrm{m}^{-3}\right)$} \\
\hline & $1-h$ & 6-h & 24-h & 48-h & 96-h \\
\hline \multicolumn{6}{|l|}{ Primer Sealer } \\
\hline LPS1 & 73 & 4 & 1 & 1 & $<1$ \\
\hline LPS2 & 15 & 8 & 4 & 3 & 2 \\
\hline \multicolumn{6}{|l|}{ Flat } \\
\hline FLP1-a & 83 & 4 & 2 & 1 & 1 \\
\hline FLP1-b & 104 & 4 & 2 & 1 & 1 \\
\hline FLP2 & 657 & 6 & 4 & 2 & $<1$ \\
\hline FLP3 & 39 & 34 & 3 & 2 & 1 \\
\hline FLP4 & 86 & 9 & 6 & 6 & 3 \\
\hline \multicolumn{6}{|l|}{ Semi-Gloss } \\
\hline SGLP1 & 97 & 21 & 14 & 12 & 10 \\
\hline SGLP2 & 61 & 19 & 10 & 7 & 8 \\
\hline SGLP3-a & 32 & $\mathrm{md}^{*}$ & 12 & 8 & 5 \\
\hline SGLP3-b & 14 & 7 & 6 & 6 & 5 \\
\hline SGLP4 & 40 & 10 & 6 & 8 & 6 \\
\hline \multicolumn{6}{|l|}{ Combination } \\
\hline LPS2 \& FLP3 @ 50\% RH & 29 & 9 & 5 & 5 & 6 \\
\hline LPS2 \& FLP3 @ $30 \%$ RH & 24 & 6 & 2 & 2 & 1 \\
\hline LPS2 \& FLP3 @ 70\% RH & 34 & 11 & 5 & 4 & 4 \\
\hline LPS2 \& SGLP3 & 12 & 8 & 4 & 5 & 4 \\
\hline \multicolumn{6}{|l|}{ Substrate } \\
\hline GB & 3 & 3 & 2 & 2 & 3 \\
\hline PW & 9 & md & 7 & 6 & 6 \\
\hline
\end{tabular}

*md $=$ Missing data. 
Table C-39. Quasi steady-state emission rates of target VOCs at 48- and $96-\mathrm{h}$ elapsed time for screening measurement of Paint LPS1. The coverage of wet paint was $79 \mathrm{~g} \mathrm{~m}^{-2}$.

\begin{tabular}{|c|c|c|c|c|}
\hline \multirow[b]{3}{*}{ COMPOUND } & \multicolumn{4}{|c|}{ Specific Emission Rate } \\
\hline & \multicolumn{2}{|c|}{$\left(\mathrm{mg} \mathrm{m} \mathrm{m}^{-2} \mathrm{~h}^{-1}\right)$} & \multicolumn{2}{|c|}{$\left(\mathrm{mg} \mathrm{kg}^{-1} \mathrm{~h}^{-1}\right)$} \\
\hline & 48-h & 96-h & 48-h & 96-h \\
\hline Ethylene glycol & 0.60 & 0.27 & 7.58 & 3.48 \\
\hline n-Butyl ether & $<0.03$ & $<0.03$ & $<0.39$ & $<0.39$ \\
\hline 2-(2-Butoxyethoxy)ethanol & 0.06 & 0.03 & 0.81 & 0.34 \\
\hline 2-(2-Butoxyethoxy)ethyl acetate & 0.30 & 0.13 & 3.73 & 1.60 \\
\hline $\begin{array}{l}\text { 2,2,4-Trimethyl-1,3-pentanediol } \\
\text { monoisobutyrates (Texanol) }\end{array}$ & 0.94 & 0.55 & 11.9 & 6.94 \\
\hline
\end{tabular}

Table C-40. Quasi steady-state emission rates of target VOCs at 48- and 96-h elapsed time for screening measurement of Paint LPS2. The coverage of wet paint was $110 \mathrm{~g} \mathrm{~m}^{-2}$.

\begin{tabular}{|c|c|c|c|c|}
\hline \multirow[b]{3}{*}{ COMPOUND } & \multicolumn{4}{|c|}{ Specific Emission Rate } \\
\hline & \multicolumn{2}{|c|}{$\left(\mathrm{mg} \mathrm{m}^{-2} \mathrm{~h}^{-1}\right)$} & \multicolumn{2}{|c|}{$\left(\mathrm{mg} \mathrm{kg}^{-1} \mathrm{~h}^{-1}\right)$} \\
\hline & 48-h & 96-h & 48-h & 96-h \\
\hline Ethylene glycol & 0.52 & 0.36 & 4.75 & 3.24 \\
\hline n-Butyl ether & $<0.03$ & $<0.03$ & $<0.28$ & $<0.28$ \\
\hline Propylene glycol & $<0.12$ & $<0.12$ & $<1.12$ & $<1.12$ \\
\hline 2-Ethyl-1-hexanol & $<0.03$ & $<0.03$ & $<0.28$ & $<0.28$ \\
\hline 2-(2-Butoxyethoxy)ethanol & $<0.03$ & $<0.03$ & $<0.28$ & $<0.28$ \\
\hline $\begin{array}{l}\text { 2,2,4-Trimethyl-1,3-pentanediol } \\
\text { monoisobutyrates (Texanol) }\end{array}$ & 2.64 & 1.97 & 24.0 & 18.0 \\
\hline
\end{tabular}


Table C-41. Quasi steady-state emission rates of target VOCs at 48- and 96-h elapsed time for screening measurement of Paint FLP1-a. The coverage of wet paint was $99 \mathrm{~g} \mathrm{~m}^{-2}$.

\begin{tabular}{|c|c|c|c|c|}
\hline \multirow[b]{3}{*}{ COMPOUND } & \multicolumn{4}{|c|}{ Specific Emission Rate } \\
\hline & \multicolumn{2}{|c|}{$\left(m g m^{-2} h^{-1}\right)$} & \multicolumn{2}{|c|}{$\left(\mathrm{mg} \mathrm{kg}^{-1} \mathrm{~h}^{-1}\right)$} \\
\hline & 48-h & 96-h & 48-h & 96-h \\
\hline Ethylene glycol & 1.64 & 1.02 & 16.6 & 10.3 \\
\hline n-Butyl ether & $<0.03$ & $<0.03$ & $<0.31$ & $<0.31$ \\
\hline 2-(2-Butoxyethoxy)ethanol & 0.35 & 0.14 & 3.49 & 1.40 \\
\hline $\begin{array}{l}\text { 2,2,4-Trimethyl-1,3-pentanediol } \\
\text { monoisobutyrates (Texanol) }\end{array}$ & 2.06 & 0.26 & 20.8 & 2.59 \\
\hline
\end{tabular}

Table C-42. Quasi steady-state emission rates of target VOCs at 48-and 96-h elapsed time for screening measurement of Paint FLP1-b. The coverage of wet paint was $104 \mathrm{~g} \mathrm{~m}^{-2}$.

\begin{tabular}{|c|c|c|c|c|}
\hline \multirow[b]{3}{*}{ COMPOUND } & \multicolumn{4}{|c|}{ Specific Emission Rate } \\
\hline & \multicolumn{2}{|c|}{$(\mathrm{mg} \mathrm{m}-2 \mathrm{~h}-1)$} & \multicolumn{2}{|c|}{$\left(\mathrm{mg} \mathrm{kg}^{-1} \mathrm{~h}^{-1}\right)$} \\
\hline & 48-h & 96-h & 48-h & 96-h \\
\hline Ethylene glycol & 1.67 & 1.28 & 16.1 & 12.3 \\
\hline n-Butyl ether & $<0.03$ & $<0.03$ & $<0.30$ & $<0.30$ \\
\hline 2-(2-Butoxyethoxy)ethanol & 0.37 & 0.12 & 3.56 & 1.14 \\
\hline $\begin{array}{l}\text { 2,2,4-Trimethyl-1,3-pentanediol } \\
\text { monoisobutyrates (Texanol) }\end{array}$ & 2.32 & 0.30 & 22.4 & 2.93 \\
\hline
\end{tabular}


Table C-43. Quasi steady-state emission rates of target VOCs at 48- and 96-h elapsed time for screening measurement of Paint FLP2. The coverage of wet paint was $111 \mathrm{~g} \mathrm{~m}-2$.

\begin{tabular}{|c|c|c|c|c|}
\hline \multirow[b]{3}{*}{ COMPOUND } & \multicolumn{4}{|c|}{ Specific Emission Rate } \\
\hline & \multicolumn{2}{|c|}{$\left(\mathrm{mg} \mathrm{m} \mathrm{m}^{-2} \mathrm{~h}-1\right)$} & \multicolumn{2}{|c|}{$\left(\mathrm{mg} \mathrm{kg}^{-1} \mathrm{~h}^{-1}\right)$} \\
\hline & 48-h & 96-h & 48-h & 96-h \\
\hline n-Butyl ether & $<0.03$ & $<0.03$ & $<0.28$ & $<0.28$ \\
\hline Propylene glycol & 0.57 & 0.38 & 5.08 & 3.38 \\
\hline 2-(2-Butoxyethoxy)ethanol & 0.30 & 0.13 & 2.70 & 1.13 \\
\hline $\begin{array}{l}\text { 2,2,4-Trimethyl-1,3-pentanediol } \\
\text { monoisobutyrates (Texanol) }\end{array}$ & 1.43 & 0.28 & 12.9 & 2.48 \\
\hline
\end{tabular}

Table C-44. Quasi steady-state emission rates of target VOCs at 48- and 96-h elapsed time for screening measurement of Paint FLP3. The coverage of wet paint was $163 \mathrm{~g} \mathrm{~m}^{-2}$.

\begin{tabular}{|c|c|c|c|c|}
\hline \multirow[b]{3}{*}{ COMPOUND } & \multicolumn{4}{|c|}{ Specific Emission Rate } \\
\hline & \multicolumn{2}{|c|}{$\left(\mathrm{mg} \mathrm{m}^{-2} \mathrm{~h}^{-1}\right)$} & \multicolumn{2}{|c|}{$\left(\mathrm{mg} \mathrm{kg}^{-1} \mathrm{~h}^{-1}\right)$} \\
\hline & 48-h & 96-h & 48-h & 96-h \\
\hline Ethylene glycol & 0.83 & 0.41 & 5.09 & 2.53 \\
\hline n-Butyl ether & 0.03 & $<0.3$ & 0.19 & $<0.19$ \\
\hline $\begin{array}{l}\text { 2,2,4-Trimethyl-1,3-pentanediol } \\
\text { monoisobutyrates (Texanol) }\end{array}$ & 3.86 & 1.42 & 23.8 & 8.73 \\
\hline
\end{tabular}


Table C-45. Quasi steady-state emission rates of selected VOCs at 48- and 96-h elapsed time for screening measurement of Paint FLP4. The coverage of wet paint was $104 \mathrm{~g} \mathrm{~m}^{-2}$.

\begin{tabular}{|c|c|c|c|c|}
\hline \multirow[b]{3}{*}{ COMPOUND } & \multicolumn{4}{|c|}{ Specific Emission Rate } \\
\hline & \multicolumn{2}{|c|}{$\left(\mathrm{mg} \mathrm{m} \mathrm{m}^{-2} \mathrm{~h}-1\right)$} & \multicolumn{2}{|c|}{$\left(\mathrm{mg} \mathrm{kg}^{-1} \mathrm{~h}-1\right)$} \\
\hline & 48-h & 96-h & 48-h & 96-h \\
\hline \multicolumn{5}{|l|}{ Carbonyl Compounds } \\
\hline Hexanal & 0.003 & $<0.003$ & 0.030 & $<0.030$ \\
\hline Nonanal & 0.003 & 0.003 & 0.030 & $<0.030$ \\
\hline \multicolumn{5}{|l|}{ Other Oxidized Compounds } \\
\hline 1-Butanol & 0.003 & $<0.003$ & 0.030 & $<0.030$ \\
\hline Benzyl alcohol & 0.003 & $<0.003$ & 0.030 & $<0.030$ \\
\hline $\begin{array}{l}\text { 2,2,4-Trimethyl-1,3-pentanediol } \\
\text { monoisobutyrates (Texanol) }\end{array}$ & 0.031 & 0.022 & 0.296 & 0.209 \\
\hline
\end{tabular}

Table C-46. Quasi steady-state emission rates of target VOCs at 48- and 96-h elapsed time for screening measurement of Paint SGLP1. The coverage of wet paint was $164 \mathrm{~g} \mathrm{~m}^{-2}$.

\begin{tabular}{|c|c|c|c|c|}
\hline \multirow[b]{3}{*}{ COMPOUND } & \multicolumn{4}{|c|}{ Specific Emission Rate } \\
\hline & \multicolumn{2}{|c|}{$\left(m g m^{-2} h-1\right)$} & \multicolumn{2}{|c|}{$\left(\mathrm{mg} \mathrm{kg}^{-1} \mathrm{~h}^{-1}\right)$} \\
\hline & 48-h & 96-h & 48-h & 96-h \\
\hline Ethylene glycol & 2.68 & 1.54 & 16.3 & 9.36 \\
\hline n-Butyl ether & 0.03 & $<0.03$ & 0.19 & $<0.19$ \\
\hline 2 Butoxyethanol & 0.03 & $<0.03$ & 0.19 & $<0.19$ \\
\hline 2-Ethyl-1-hexanol & 0.03 & 0.03 & 0.19 & 0.19 \\
\hline 2-(2-Butoxyethoxy)ethanol & 2.61 & 1.61 & 15.9 & 9.83 \\
\hline 2-(2-Butoxyethoxy)ethyl acetate & 0.03 & 0.03 & 0.19 & 0.19 \\
\hline $\begin{array}{l}\text { 2,2,4-Trimethyl-1,3-pentanediol } \\
\text { monoisobutyrates (Texanol) }\end{array}$ & 0.34 & 0.26 & 2.07 & 1.56 \\
\hline
\end{tabular}


Table C-47. Quasi steady-state emission rates of target VOCs at 48-and 96-h elapsed time for screening measurement of Paint SGLP2. The coverage of wet paint was $129 \mathrm{~g} \mathrm{~m}^{-2}$.

\begin{tabular}{|c|c|c|c|c|}
\hline \multirow[b]{3}{*}{ COMPOUND } & \multicolumn{4}{|c|}{ Specific Emission Rate } \\
\hline & \multicolumn{2}{|c|}{$\left(\mathrm{mg} \mathrm{m}^{-2} \mathrm{~h}-1\right)$} & \multicolumn{2}{|c|}{$\left(\mathrm{mg} \mathrm{kg}^{-1} \mathrm{~h}^{-1}\right)$} \\
\hline & 48-h & 96-h & 48-h & 96-h \\
\hline Ethylene glycol & 2.17 & 1.74 & 21.6 & 13.5 \\
\hline n-Butyl ether & $<0.03$ & $<0.03$ & $<0.24$ & $<0.24$ \\
\hline 2 Butoxyethanol & $<0.03$ & $<0.03$ & $<0.24$ & $<0.24$ \\
\hline 2-(2-Butoxyethoxy)ethanol & 0.35 & 0.20 & 2.77 & 1.59 \\
\hline 2-(2-Butoxyethoxy)ethyl acetate & 0.48 & 0.27 & 3.78 & 2.13 \\
\hline $\begin{array}{l}\text { 2,2,4-Trimethyl-1,3-pentanediol } \\
\text { monoisobutyrates (Texanol) }\end{array}$ & 0.88 & 0.82 & 6.88 & 6.33 \\
\hline
\end{tabular}

Table C-48. Quasi steady-state emission rates of target VOCs at 48-and 96-h elapsed time for screening measurement of Paint SGLP3-a. The coverage of wet paint was $169 \mathrm{~g} \mathrm{~m}^{-2}$.

\begin{tabular}{|c|c|c|c|c|}
\hline \multirow[b]{3}{*}{ COMPOUND } & \multicolumn{4}{|c|}{ Specific Emission Rate } \\
\hline & \multicolumn{2}{|c|}{$\left(m g m^{-2} h-1\right)$} & \multicolumn{2}{|c|}{$\left(\mathrm{mg} \mathrm{kg}^{-1} \mathrm{~h}^{-1}\right)$} \\
\hline & 48-h & 96-h & 48-h & 96-h \\
\hline n-Butyl ether & 0.03 & $<0.03$ & 0.18 & $<0.18$ \\
\hline Propylene glycol & 1.86 & 0.78 & 11.0 & 4.66 \\
\hline Hexylene glycol & 1.65 & 0.54 & 9.82 & 3.18 \\
\hline 2-(2-Butoxyethoxy)ethanol & 0.74 & 0.47 & 4.42 & 2.81 \\
\hline $\begin{array}{l}\text { 2,2,4-Trimethyl-1,3-pentanediol } \\
\text { monoisobutyrates (Texanol) }\end{array}$ & 0.93 & 0.89 & 5.55 & 5.30 \\
\hline
\end{tabular}


Table C-49. Quasi steady-state emission rates of target VOCs at 48- and 96-h elapsed time for screening measurement of Paint SGLP3-b. The coverage of wet paint was $160 \mathrm{~g} \mathrm{~m}^{-2}$.

\begin{tabular}{lcccc}
\hline & \multicolumn{4}{c}{$\begin{array}{c}\text { Specific Emission Rate } \\
\left(\mathbf{m g ~ k g}^{-1} \mathbf{~ h}-\mathbf{1}\right)\end{array}$} \\
COMPOUND & $\mathbf{4 8 - \mathbf { h }}$ & $\mathbf{9 6 - \mathbf { h }}$ & $\mathbf{4 8 - \mathbf { h }}$ & $\mathbf{9 6 - \mathbf { h }}$ \\
\hline n-Butyl ether & $<0.03$ & $<0.03$ & $<0.19$ & $<0.19$ \\
Propylene glycol & 1.83 & 1.11 & 11.2 & 6.82 \\
Hexylene glycol & 1.81 & 0.87 & 11.1 & 5.31 \\
2-(2-Butoxyethoxy)ethanol & 0.56 & 0.28 & 3.44 & 1.70 \\
2,2,4-Trimethyl-1,3-pentanediol & 0.72 & 0.54 & 4.39 & 3.33 \\
monoisobutyrates (Texanol) & & & & \\
\hline
\end{tabular}

Table C-50. Quasi steady-state emission rates of selected VOCs at 48- and 96-h elapsed time for screening measurement of Paint SGLP4. The coverage of wet paint was $148 \mathrm{~g} \mathrm{~m}^{-2}$.

\begin{tabular}{|c|c|c|c|c|}
\hline \multirow[b]{3}{*}{ COMPOUND } & \multicolumn{4}{|c|}{ Specific Emission Rate } \\
\hline & \multicolumn{2}{|c|}{$\left(m g m^{-2} h^{-1}\right)$} & \multicolumn{2}{|c|}{$\left(\mathrm{mg} \mathrm{kg}^{-1} \mathrm{~h}^{-1}\right)$} \\
\hline & 48-h & 96-h & 48-h & 96-h \\
\hline \multicolumn{5}{|l|}{ Carbonyl Compounds } \\
\hline Pentanal ${ }^{*}$ & 0.015 & 0.009 & 0.105 & 0.058 \\
\hline Hexanal $^{\star}$ & 0.072 & 0.035 & 0.485 & 0.241 \\
\hline Benzaldehyde & 0.012 & 0.003 & 0.083 & 0.021 \\
\hline Nonanal $^{*}$ & 0.003 & $<0.003$ & 0.021 & $<0.021$ \\
\hline \multicolumn{5}{|l|}{ Other Oxidized Compounds } \\
\hline 1-Butanol & 0.015 & 0.009 & 0.104 & 0.063 \\
\hline 1-Pentanol & 0.024 & 0.014 & 0.164 & 0.098 \\
\hline n-Butyl ether & 0.024 & 0.003 & 0.160 & 0.021 \\
\hline 2-Ethyl-1-hexanol ${ }^{\star}$ & 0.006 & 0.003 & 0.042 & 0.021 \\
\hline Benzyl alcohol & 0.006 & 0.003 & 0.042 & 0.021 \\
\hline 2-(2-Butoxyethoxy)ethanol ${ }^{\star}$ & 0.009 & 0.006 & 0.063 & 0.042 \\
\hline $\begin{array}{l}\text { 2,2,4-Trimethyl-1,3-pentanediol } \\
\text { monoisobuturates (Texanol) }\end{array}$ & $<0.050$ & $<0.040$ & $<0.330$ & $<0.250$ \\
\hline
\end{tabular}

*Detected in e: nissions from Plywood PW; see Table C-16. 
Table C-51. Quasi steady-state emission rates of target VOCs at 48- and 96-h elapsed time for screening measurement of Paints LPS2 and FLP3 at standard humidity $(50 \% \mathrm{RH})$. The coverage of wet paint was $156 \mathrm{~g} \mathrm{~m}^{-2}$.

\begin{tabular}{|c|c|c|c|c|}
\hline \multirow[b]{3}{*}{ COMPOUND } & \multicolumn{4}{|c|}{ Specific Emission Rate } \\
\hline & \multicolumn{2}{|c|}{$\left(\mathrm{mg} \mathrm{m}^{-2} \mathrm{~h}^{-1}\right)$} & \multicolumn{2}{|c|}{$\left(\mathrm{mg} \mathrm{kg}^{-1} \mathrm{~h}^{-1}\right)$} \\
\hline & 48-h & 96-h & 48-h & 96-h \\
\hline Ethylene glycol & 1.34 & 0.57 & 8.54 & 3.67 \\
\hline n-Butyl ether & $<0.03$ & $<0.03$ & $<0.20$ & $<0.20$ \\
\hline Propylene glycol & $<0.25$ & $<0.09$ & $<1.57$ & $<0.59$ \\
\hline 2-Ethyl-1-hexanol & 0.03 & $<0.03$ & 0.20 & $<0.20$ \\
\hline 2-(2-Butoxyethoxy)ethanol & $<0.03$ & $<0.03$ & $<0.20$ & $<0.20$ \\
\hline $\begin{array}{c}\text { 2,2,4-Trimethyl-1,3-pentanediol } \\
\text { monoisobutyrates (Texanol) }\end{array}$ & 6.26 & 3.20 & 40.0 & 20.5 \\
\hline
\end{tabular}

Table C-52. Quasi steady-state emission rates of target VOCs at 48-and 96-h elapsed time for screening measurement of Paints LPS2 and FLP3 at reduced humidity $(30 \% \mathrm{RH})$. The coverage of wet paint was $187 \mathrm{~g} \mathrm{~m}^{-2}$.

\begin{tabular}{|c|c|c|c|c|}
\hline \multirow[b]{3}{*}{ COMPOUND } & \multicolumn{4}{|c|}{ Specific Emission Rate } \\
\hline & \multicolumn{2}{|c|}{$\left(m g m^{-2} h^{-1}\right)$} & \multicolumn{2}{|c|}{$\left(\mathrm{mg} \mathrm{kg}^{-1} \mathrm{~h}^{-1}\right)$} \\
\hline & 48-h & 96-h & 48-h & 96-h \\
\hline Ethylene glycol & 1.52 & 0.20 & 8.30 & 1.11 \\
\hline n-Butyl ether & $<0.03$ & $<0.03$ & $<0.17$ & $<0.17$ \\
\hline Propylene glycol & $<0.15$ & $<0.06$ & $<0.84$ & $<0.34$ \\
\hline 2-Ethyl-1-hexanol & $<0.03$ & $<0.03$ & $<0.17$ & $<0.17$ \\
\hline 2-(2-Butoxyethoxy)ethanol & $<0.03$ & $<0.03$ & $<0.17$ & $<0.17$ \\
\hline $\begin{array}{l}\text { 2,2,4-Trimethyl-1,3-pentanediol } \\
\text { monoisobutyrates (Texanol) }\end{array}$ & 5.61 & 3.83 & 30.7 & 20.9 \\
\hline
\end{tabular}


Table C-53. Quasi steady-state emission rates of target VOCs at 48- and 96-h elapsed time for screening measurement of Paints LPS2 and FLP3 at elevated humidity $(70 \% \mathrm{RH})$. The coverage of wet paint was $163 \mathrm{~g} \mathrm{~m}^{-2}$.

\begin{tabular}{|c|c|c|c|c|}
\hline \multirow[b]{3}{*}{ COMPOUND } & \multicolumn{4}{|c|}{ Specific Emission Rate } \\
\hline & \multicolumn{2}{|c|}{$\left(\mathrm{mg} \mathrm{m}^{-2} \mathrm{~h}^{-1}\right)$} & \multicolumn{2}{|c|}{$\left(\mathrm{mg} \mathrm{kg}^{-1} \mathrm{~h}^{-1}\right)$} \\
\hline & 48-h & 96-h & 48-h & 96-h \\
\hline Ethylene glycol & 1.42 & 0.32 & 8.71 & 1.96 \\
\hline n-Butyl ether & $<0.03$ & $<0.03$ & $<0.19$ & $<0.19$ \\
\hline Propylene glycol & $<0.15$ & $<0.06$ & $<0.95$ & $<0.38$ \\
\hline 2-Ethyl-1-hexanol & $<0.03$ & $<0.03$ & $<0.19$ & $<0.19$ \\
\hline 2-(2-Butoxyethoxy)ethanol & $<0.03$ & $<0.03$ & $<0.19$ & $<0.19$ \\
\hline $\begin{array}{l}\text { 2,2,4-Trimethyl-1,3-pentanediol } \\
\text { monoisobutyrates (Texanol) }\end{array}$ & 5.32 & 2.68 & 32.7 & 16.5 \\
\hline
\end{tabular}

Table C-54. Quasi steady-state emission rates of target VOCs at 48- and 96-h elapsed time for screening measurement of Paints LPS2 and SGLP3. The coverage of wet paint was $176 \mathrm{~g} \mathrm{~m}^{-2}$.

\begin{tabular}{|c|c|c|c|c|}
\hline \multirow[b]{3}{*}{ COMPOUND } & \multicolumn{4}{|c|}{ Specific Emission Rate } \\
\hline & \multicolumn{2}{|c|}{$\left(m g m^{-2} h-1\right)$} & \multicolumn{2}{|c|}{$\left(\mathrm{mg} \mathrm{kg}^{-1} \mathrm{~h}-1\right)$} \\
\hline & 48-h & 96-h & $48-h$ & 96-h \\
\hline Ethylene glycol & 2.30 & 1.20 & 13.1 & 6.81 \\
\hline n-Butyl ether & $<0.03$ & $<0.03$ & $<0.17$ & $<0.17$ \\
\hline Propylene glycol & 1.05 & 0.27 & 5.95 & 1.52 \\
\hline Hexylene glycol & 0.62 & 0.32 & 3.53 & 1.82 \\
\hline 2-Ethyl-1-hexanol & 0.03 & $<0.03$ & 0.17 & $<0.17$ \\
\hline 2-(2-Butoxyethoxy)ethanol & 0.60 & 0.19 & 3.44 & 1.08 \\
\hline $\begin{array}{c}\text { 2,2,4-Trimethyl-1,3-pentanediol } \\
\text { monoisobutyrates (Texanol) }\end{array}$ & 1.32 & 0.76 & 7.49 & 4.33 \\
\hline
\end{tabular}


Table C-55. Quasi steady-state emission rates of SigmaVOC (i.e., sum of target VOCs) at 48- and 96-h elapsed times for screening measurements of paints.

\begin{tabular}{|c|c|c|c|c|}
\hline \multirow[b]{3}{*}{ Material ID } & \multicolumn{4}{|c|}{ Specific Emission Rate } \\
\hline & \multicolumn{2}{|c|}{$\left(\mathrm{mg} \mathrm{m}^{-2} \mathrm{~h}-1\right)$} & \multicolumn{2}{|c|}{$\left(\mathrm{mg} \mathrm{kg}^{-1} \mathrm{~h}^{-1}\right)$} \\
\hline & 48-h & 96-h & 48-h & 96-h \\
\hline \multicolumn{5}{|l|}{ Primer Sealer } \\
\hline LPS1 & 1.91 & 0.98 & 24.2 & 12.5 \\
\hline LPS2 & 3.78 & 2.58 & 34.5 & 23.6 \\
\hline \multicolumn{5}{|l|}{ Flat } \\
\hline FLP1-a & 4.03 & 1.38 & 40.7 & 14.0 \\
\hline FLP1-b & 4.34 & 1.69 & 41.9 & 16.3 \\
\hline FLP2 & 2.28 & 0.77 & 20.5 & 6.91 \\
\hline FLP3 & 4.74 & 1.82 & 29.1 & 11.2 \\
\hline FLP4 & 0.03 & 0.03 & 0.30 & 0.30 \\
\hline \multicolumn{5}{|l|}{ Semi-Gloss } \\
\hline SGLP1 & 5.72 & 3.45 & 34.9 & 21.0 \\
\hline SGLP2 & 3.94 & 3.02 & 30.6 & 23.4 \\
\hline SGLP3-a & 5.17 & 2.62 & 30.6 & 15.5 \\
\hline SGLP3-b & 4.86 & 2.77 & 30.4 & 17.3 \\
\hline SGLP4 & 0.15 & 0.09 & 1.04 & 0.63 \\
\hline \multicolumn{5}{|l|}{ Combination } \\
\hline LPS2 \& FLP3 @ 50\% RH & 7.63 & 3.85 & 48.8 & 24.6 \\
\hline LPS2 \& FLP3 @ 30\% RH & 7.17 & 4.09 & 39.3 & 22.4 \\
\hline LPS2 \& FLP3 @ 70\% RH & 6.77 & 3.02 & 41.6 & 18.6 \\
\hline LPS2 \& SGLP3 & 6.00 & 2.74 & 34.1 & 15.6 \\
\hline
\end{tabular}


Table C-56. Quasi steady-state emission rates of formaldehyde at 48- and 96-h elapsed times for screening measurements of paints.

\begin{tabular}{|c|c|c|c|c|}
\hline \multirow[b]{3}{*}{ Material ID } & \multicolumn{4}{|c|}{ Specific Emission Rate } \\
\hline & \multicolumn{2}{|c|}{$\left(\mu \mathrm{g} \mathrm{m}^{-2} \mathrm{~h}^{-1}\right)$} & \multicolumn{2}{|c|}{$\left(\mu \mathrm{kg}^{-1} \mathrm{~h}^{-1}\right)$} \\
\hline & 48-h & 96-h & 48-h & 96-h \\
\hline \multicolumn{5}{|l|}{ Primer Sealer } \\
\hline LPS1 & 31 & 25 & 390 & 310 \\
\hline LPS2 & 3 & $<3$ & 28 & $<28$ \\
\hline \multicolumn{5}{|l|}{ Flat } \\
\hline FLP1-a & $<3$ & $<3$ & $<31$ & $<31$ \\
\hline FLP1-b & $<3$ & $<3$ & $<30$ & $<30$ \\
\hline FLP2 & 49 & 37 & 440 & 330 \\
\hline FLP3 & 12 & 3 & 76 & 19 \\
\hline FLP4 & 43 & 22 & 420 & 210 \\
\hline \multicolumn{5}{|l|}{ Semi-Gloss } \\
\hline SGLP1 & 18 & 12 & 110 & 75 \\
\hline SGLP2 & 15 & 18 & 120 & 140 \\
\hline SGLP3-a & 18 & 12 & 110 & 73 \\
\hline SGLP3-b & $<9$ & $<6$ & $<58$ & $<38$ \\
\hline SGLP4 & 12 & 9 & 83 & 62 \\
\hline \multicolumn{5}{|l|}{ Combination } \\
\hline LPS2 \& FLP3 \& $50 \%$ RH & 6 & $<3$ & 39 & $<20$ \\
\hline LPS2 \& FLP3 @ $30 \%$ RH & 3 & $<3$ & 17 & $<17$ \\
\hline LPS2 \& FLP3 @ 70\% RH & 6 & 3 & 38 & 19 \\
\hline LPS2 \& SGLP3 & $<9$ & $<6$ & $<52$ & $<35$ \\
\hline
\end{tabular}


Table C-57. Quasi steady-state emission rates of acetaldehyde at 48- and 96-h elapsed times for screening measurements of paints.

\begin{tabular}{|c|c|c|c|c|}
\hline \multirow[b]{3}{*}{ Material ID } & \multicolumn{4}{|c|}{ Specific Emission Rate } \\
\hline & \multicolumn{2}{|c|}{$\left(\mu \mathrm{g} \mathrm{m}^{-2} \mathrm{~h}^{-1}\right)$} & \multicolumn{2}{|c|}{$\left(\mu g \mathrm{~kg}^{-1} \mathrm{~h}^{-1}\right)$} \\
\hline & 48-h & 96-h & 48-h & 96-h \\
\hline \multicolumn{5}{|l|}{ Primer Sealer } \\
\hline LPS1 & $<6$ & $<9$ & $<78$ & $<120$ \\
\hline LPS2 & $<6$ & $<9$ & $<56$ & $<84$ \\
\hline \multicolumn{5}{|l|}{ Flat } \\
\hline FLP1-a & $<6$ & $<9$ & $<62$ & $<93$ \\
\hline FLP1-b & $<6$ & $<9$ & $<59$ & $<89$ \\
\hline FLP2 & $<6$ & $<9$ & $<55$ & $<83$ \\
\hline FLP3 & $<6$ & $<9$ & $<38$ & $<57$ \\
\hline FLP4 & 12 & $<9$ & 120 & $<89$ \\
\hline \multicolumn{5}{|l|}{ Semi-Gloss } \\
\hline SGLP1 & 18 & $<18$ & 110 & $<110$ \\
\hline SGLP2 & $<18$ & $<18$ & $<140$ & $<140$ \\
\hline SGLP3-a & $<18$ & $<18$ & $<110$ & $<110$ \\
\hline SGLP3-b & $<18$ & $<18$ & $<110$ & $<110$ \\
\hline SGLP4 & $<18$ & $<18$ & $<120$ & $<120$ \\
\hline \multicolumn{5}{|l|}{ Combination } \\
\hline LPS2 \& FLP3 @ $50 \% R H$ & 9 & 9 & 59 & 59 \\
\hline LPS2 \& FLP3 @ $30 \%$ RH & $<6$ & $<9$ & $<34$ & $<51$ \\
\hline LPS2 \& FLP3 @ 70\% RH & 6 & $<9$ & 38 & $<57$ \\
\hline LPS2 \& SGLP3 & $<18$ & $<18$ & $<100$ & $<100$ \\
\hline
\end{tabular}


Table C-58. Percent weight compositions of ethylene glycol, propylene glycol and Texanol in Paints LPS2, FLP3 and SGLP3 selected for use in the large-scale experiments.

\begin{tabular}{lrrr}
\hline & \multicolumn{4}{c}{ Percent Weight Composition } \\
COMPOUND & LPS2 & FLP3 & SGLP3 \\
\hline & 3.6 & 2.0 & $<0.7$ \\
Ethylene glycol & $<0.8$ & $<0.8$ & 3.1 \\
Proplyene glycol & 1.5 & 0.9 & 0.7 \\
Texanol & & & \\
\hline
\end{tabular}


(Page is intentionally blank) 


\section{APPENDIX D}

\section{SCREENING MEASUREMENTS OF CARPET MATERIALS}

Appendix $D$ presents the analytical data for the screening measurements of the carpet materials including four carpets and four carpet cushions. The experiments were conducted in 10-L chambers over a period of 48 hours. Individual VOCs emitted by the carpet materials were identified. The concentrations of selected compounds and TVOC were measured at four time intervals. Specific emission rates of these components were calculated for the 24- and 48-h time intervals.

\section{List of Tables}

Table D-01. Carpet materials selected for study.

Table D-02. Summary of screening measurements with carpet materials in $10-\mathrm{L}$ chambers. ...218

Table D-03. VOCs emitted by Carpet CP1 in 10-L chamber at 6- and 48-hours

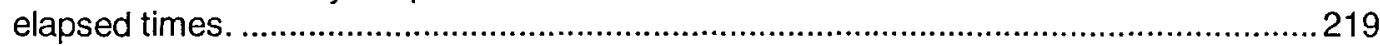

Table D-04. VOCs emitted by Carpet CP3 in 10-L chamber at 6 - and 48-hours elapsed times.

Table D-05. VOCs emitted by Carpet CP2 in 10-L chamber at 6- and 48-hours elapsed times.

Table D-06. VOCs emitted by Carpet CP4 in 10-L chamber at 6-and 48-hours elapsed times

Table D-07. VOCs emitted by Seaming Tape ST and Carpet CP3 in 10-L chamber at 6-

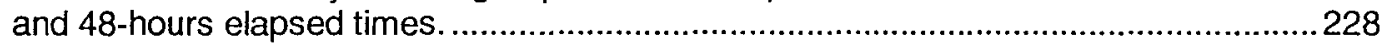

Table D-08. VOCs emitted by CP Blank in 10-L chamber at 6- and 48-hours elapsed times.....230

Table D-09. VOCs emitted by Carpet Cushion CC1 in 10-L chamber at 6 - and 48-hours

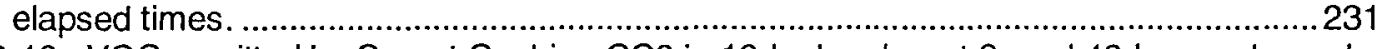

Table D-10. VOCs emitted by Carpet Cushion CC2 in 10-L chamber at 6- and 48-hours elapsed times.

Table D-11. VOCs emitted by Carpet Cushion CC4 in 10-L chamber at 6-and 48-hours elapsed times.

Table D-12. VOCs emitted by Carpet Cushion CC3 in 10-L chamber at 6-and 48-hours

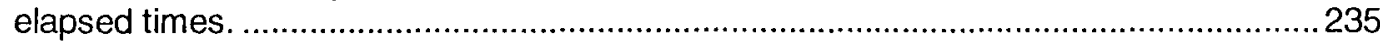

Table D-13. VOCs emitted by CC Blank in 10-L chamber at 6- and 48-hours elapsed times. ...237

Table D-14. Summary of target VOCs for screening measurements with carpet......................238

Table D-15. Chamber concentrations of target VOCs for 48 -h screening measurement of Carpet CP1-a.

Table D-16. Chamber concentrations of target VOCs for 48 -h screening measurement of Carpet CP1-b.

Table D-17. Chamber concentrations of target VOCs for 48-h screening measurement of Carpet CP3.

Table D-18. Chamber concentrations of target VOCs for 48-h screening measurement of Carpet CP2.

Table D-19. Chamber concentrations of target VOCs for 48 -h screening measurement of Carpet CP4.

Table D-20. Chamber concentrations of target VOCs for 48-h screening measurement of Seaming Tape ST and Carpet CP3.

Table D-21. Chamber concentrations of target VOCs for $48-\mathrm{h}$ screening measurement of CP Blank.

Table D-22. Chamber concentrations of target VOCs for 48-h screening measurement of Carpet Cushion CC1 
Table D-23. Chamber concentrations of target VOCs for 48-h screening measurement of Carpet Cushion CC2-a.

Table D-24. Chamber concentrations of target VOCs for 48-h screening measurement

Table D-24. Chamber concentrations
of Carpet Cushion CC2-b.

Table D-25. Chamber concentrations of target VOCs for 48-h screening measurement of Carpet Cushion CC4.

Table D-26. Chamber concentrations of target VOCs for 48 -h screening measurement of Carpet Cushion CC3.

Table D-27. Chamber concentrations of target VOCs for 48-h screening measurement of CC Blank

Table D-28. Chamber concentrations of TVOC for 48-h screening measurements of carpet materials

Table D-29. Chamber concentrations of SigmaVOC for 48-h screening measurements of carpet materials.

Table D-30. Chamber concentrations of formaldehyde for $48-h$ screening measurements of carpet materials

Table D-31. Chamber concentrations of acetaldehyde for 48-h screening measurements of carpet materials.

Table D-32. Quasi steady-state emission rates of target VOCs at 24- and 48-h elapsed time for screening measurement of Carpet CP1-a.

Table D-33. Quasi steady-state emission rates of target VOCs at 24- and 48-h elapsed time for screening measurement of Carpet CP1-b.

Table D-34. Quasi steady-state emission rates of target VOCs at 24- and 48-h elapsed time for screening measurement of Carpet CP3...

Table D-35. Quasi steady-state emission rates of target VOCs at 24- and 48-h elapsed time for screening measurement of Carpet $\mathrm{CP} 2$

Table D-36. Quasi steady-state emission rates of target VOCs at 24- and 48-h elapsed time for screening measurement of Carpet $\mathrm{CP} 4$

Table D-37. Quasi steady-state emission rates of target VOCs at 24- and 48-h elapsed time for screening measurement of Seaming Tape ST and Carpet CP3.

Table D-38. Quasi steady-state emission rates of target VOCs at 24- and 48-h elapsed time for screening measurement of Carpet Cushion CC1.

Table D-39. Quasi steady-state emission rates of target VOCs at 24- and 48-h elapsed time for screening measurement of Carpet Cushion CC2-a.

Table D-40. Quasi steady-state emission rates of target VOCs at 24- and 48-h elapsed time for screening measurement of Carpet Cushion CC2-b.

Table D-41. Quasi steady-state emission rates of target VOCs at 24- and 48-h elapsed time for screening measurement of Carpet Cushion CC4.

Table D-42. Quasi steady-state emission rates of target VOCs at 24- and 48-h elapsed time for screening measurement of Carpet Cushion CC3.

Table D-43. Quasi steady-state emission rates of TVOC at 24- and 48-hours elapsed times for screening measurements of carpet materials.

Table D-44. Quasi steady-state emission rates of formaldehyde at 24- and 48-hours elapsed times for screening measurements of carpet materials.

Table D-45. Quasi steady-state emission rates of acetaldehyde at 24- and 48-hours elapsed times for screening measurements of carpet materials. 
Table D-01. Carpet materials selected for study.

\begin{tabular}{|c|c|c|c|}
\hline Material ID & $\begin{array}{l}\text { Manufacturer } \\
\text { Code }\end{array}$ & $\begin{array}{l}\text { Purchase } \\
\text { Point } \\
\end{array}$ & Material Description \\
\hline \multicolumn{4}{|l|}{ Carpets } \\
\hline $\mathrm{CP} 1$ & A & Dealer & $\begin{array}{l}\text { Residential; intermediate grade; action back } \\
\text { with SBR latex; } 100 \% \text { nylon pile face fiber; } \\
\text { CRI "Green Tag" line }\end{array}$ \\
\hline CP3 & $\mathrm{B}$ & Dealer & $\begin{array}{l}\text { Residential; intermediate grade; action back } \\
\text { with SBR latex; } 100 \% \text { nylon pile face fiber; } \\
\text { CRI "Green Tag" line }\end{array}$ \\
\hline $\mathrm{CP}^{*}$ & $\mathrm{C}$ & Dealer & $\begin{array}{l}\text { Commercial; action back with SBR latex; } \\
28 \text { oz. olefin textured loop face fiber }\end{array}$ \\
\hline $\mathrm{CP}^{\star \star} \dagger$ & $\mathrm{C}$ & Dealer & $\begin{array}{l}\text { Commercial; action back with SBR latex; } \\
28 \text { oz. olefin textured loop face fiber; CRI } \\
\text { "Green Tag" line }\end{array}$ \\
\hline \multicolumn{4}{|c|}{ Seaming Tape } \\
\hline $\mathrm{ST} \mathrm{T}^{\dagger}$ & $\mathrm{D}$ & Retail & $\begin{array}{l}\text { Professional grade thermal adhesive } \\
\text { seaming tape; } 3 \text {-inch wide }\end{array}$ \\
\hline \multicolumn{4}{|l|}{ Cushions } \\
\hline $\mathrm{CC} 1$ & $E$ & Dealer & $\begin{array}{l}\text { Rebonded urethane; } 1 / 2 \text {-inch thick; } 8 \\
\text { pounds per cubic foot }\end{array}$ \\
\hline $\mathrm{CC}^{\star \star *}$ & $\mathrm{~F}$ & Retail & $\begin{array}{l}\text { Rebonded urethane; } 1 / 2 \text {-inch thick; } 4.5 \\
\text { pounds per cubic foot }\end{array}$ \\
\hline $\mathrm{CC} 4^{\star \star \dagger}$ & $F$ & Retail & $\begin{array}{l}\text { Rebonded urethane; } 7 / 16 \text {-inch thick; } 4 \\
\text { pounds per cubic foot }\end{array}$ \\
\hline $\mathrm{CC} 3$ & $E$ & Dealer & Synthetic fiber; $1 / 2$-inch thick \\
\hline
\end{tabular}

${ }^{*} \mathrm{CP} 2$ and $\mathrm{CP} 4$ are equivalent products from the same manufacturer.

${ }^{* *} \mathrm{CC} 2$ and $\mathrm{CC} 4$ are nearly equivalent products from the same manufacturer.

tSelected for use in large-scale experiments. 
Table D-02. Summary of screening measurements with carpet materials in 10-L chambers.

\begin{tabular}{|c|c|c|c|}
\hline Materials & $\begin{array}{c}\text { Duration } \\
\text { (h) }\end{array}$ & $\begin{array}{l}\text { Material } \\
\text { Amount }\end{array}$ & Experimental Procedures \\
\hline \multicolumn{4}{|l|}{ Carpets } \\
\hline CP1-a & 48 & $0.0232 \mathrm{~m}^{2}$ & $\begin{array}{l}\text { Placed into stainless-steel holder; } \\
\text { fiber side exposed }\end{array}$ \\
\hline$C P 1-b$ & 48 & $0.0232 \mathrm{~m}^{2}$ & $\begin{array}{l}\text { Placed into stainless-steel holder; } \\
\text { fiber side exposed }\end{array}$ \\
\hline CP3 & 48 & $0.0232 m^{2}$ & $\begin{array}{l}\text { Placed into stainless-steel holder; } \\
\text { fiber side exposed }\end{array}$ \\
\hline CP2 & 48 & $0.0232 \mathrm{~m}^{2}$ & $\begin{array}{l}\text { Placed into stainless-steel holder; } \\
\text { fiber side exposed }\end{array}$ \\
\hline $\mathrm{CP} 4$ & 48 & $0.0232 \mathrm{~m}^{2}$ & $\begin{array}{l}\text { Placed into stainless-steel holder; } \\
\text { fiber side exposed }\end{array}$ \\
\hline CP Blank Run & 48 & $\cdots$ & Empty stainless-steel holder \\
\hline \multicolumn{4}{|l|}{ Seaming Tape } \\
\hline ST \& CP3 & 48 & $\begin{array}{l}0.152 \mathrm{~m} \& \\
0.0232 \mathrm{~m}^{2}\end{array}$ & $\begin{array}{l}\text { Two pieces of carpet bonded \& } \\
\text { placed into stainless-steel } \\
\text { holder; fiber side exposed }\end{array}$ \\
\hline \multicolumn{4}{|l|}{ Cushions } \\
\hline $\mathrm{CC} 1$ & 48 & $0.0232 \mathrm{~m}^{2}$ & $\begin{array}{l}\text { Placed into stainless-steel holder; } \\
\text { film side exposed }\end{array}$ \\
\hline $\mathrm{CC} 2-\mathrm{a}$ & 48 & $0.0232 \mathrm{~m}^{2}$ & $\begin{array}{l}\text { Placed into stainless-steel holder; } \\
\text { film side exposed }\end{array}$ \\
\hline$c C 2-b$ & 48 & $0.0232 \mathrm{~m}^{2}$ & $\begin{array}{l}\text { Placed into stainless-steel holder; } \\
\text { film side exposed }\end{array}$ \\
\hline $\mathrm{CC} 4$ & 48 & $0.0232 \mathrm{~m}^{2}$ & $\begin{array}{l}\text { Placed into stainless-steel holder; } \\
\text { film side exposed }\end{array}$ \\
\hline CC3 & 48 & $0.0232 \mathrm{~m}^{2}$ & $\begin{array}{l}\text { Placed into stainless-steel holder; } \\
\text { film side exposed }\end{array}$ \\
\hline CC Blank Run & 48 & --- & Empty stainless-steel holder \\
\hline
\end{tabular}


Table D-03. VOCs emitted by Carpet CP1 in 10-L chamber at 6- and 48-hours elapsed times.

COMPOUND

Branched alkane $\mathrm{HC}$

Branched alkane $\mathrm{HC}$

Branched alkane $\mathrm{HC}$

Branched alkane $\mathrm{HC}$

Branched alkane $\mathrm{HC}$

n-Dodecane

Branched alkane $\mathrm{HC}$

Branched alkane $\mathrm{HC}$

Branched alkane $\mathrm{HC}$

Branched alkane $\mathrm{HC}$

Branched alkane $\mathrm{HC}$

\section{Aromatic Hydrocarbons}

Toluene

4-Phenylcyclohexene

\section{Other Hydrocarbons}

Alkene $\mathrm{HC}$

d-Limonene

Alkene $\mathrm{HC}$

Alkene $\mathrm{HCs}$

\section{Carbonyl Compounds}

Benzaldehyde

Nonanal

1-Phenylethanone

Decanal

\section{Other Oxidized Compounds}

Acetic acid

1-Dodecanol

\section{Nitrogen-Containing Cmpds}

2-Methyleneglutaronitrile,

CAS 1572-52-7

\begin{tabular}{ccccc} 
& RT & $6-h$ & $48-h$ & Match \\
Code $^{*}$ & $(\min )$ & ET & ET & Quality \\
\hline
\end{tabular}

$\begin{array}{cccc}26.7 & + & + & \text { Probable } \\ 28.9 & + & + & \text { Probable } \\ 29.2 & + & & \text { Probable } \\ 29.5 & + & + & \text { Probable } \\ 30.2 & + & & \text { Probable } \\ 34.6 & + & + & \text { Confirmed } \\ 36.9 & + & + & \text { Probable } \\ 37.6 & + & & \text { Probable } \\ 38.3 & + & + & \text { Probable } \\ 40.3 & + & & \text { Probable } \\ 42.8 & + & & \text { Probable }\end{array}$

\begin{tabular}{|c|c|c|}
\hline$T, Q$ & 21.1 & + \\
\hline$A, Q$ & 41.1 & + \\
\hline
\end{tabular}

$\begin{array}{cccl} & 30.0 & + & \text { Probable } \\ \text { Q } & 30.2 & + & \text { Confirmed } \\ & 30.3 & + & \text { Probable } \\ \text { A } & 30.5-33.5 & + & \text { Probable }\end{array}$

$\begin{array}{ccccc}\mathrm{B} & 31.0 & + & + & \text { Confirmed } \\ \mathrm{B}, \mathrm{Q} & 34.4 & + & + & \text { Confirmed } \\ \mathrm{T}, \mathrm{Q} & 34.8 & + & & \text { Confirmed } \\ \mathrm{B}, \mathrm{Q} & 37.4 & + & + & \text { Confirmed }\end{array}$

$\begin{array}{lllll}\text { B } & 20.0 & + & + & \text { Probable } \\ \text { A } & 45.1 & + & + & \text { Probable }\end{array}$

39.0

$+\quad$ Probable


Table D-03, Continued. VOCs emitted by Carpet CP1 in 10-L chamber at 6- and 48-hours elapsed times.

\begin{tabular}{|c|c|c|c|c|c|}
\hline COMPOUND & Code $^{\star}$ & $\begin{array}{c}\mathrm{RT} \\
(\mathrm{min})\end{array}$ & $\begin{array}{l}\text { 6-h } \\
\text { ET }\end{array}$ & $\begin{array}{c}\text { 48-h } \\
\text { ET }\end{array}$ & $\begin{array}{l}\text { Match } \\
\text { Quality }\end{array}$ \\
\hline \multicolumn{6}{|l|}{ Miscellaneous Compounds } \\
\hline Hexamethylcyclotrisiloxane & $\mathrm{B}$ & 21.3 & + & + & Confirmed \\
\hline Octamethylcyclotetrasiloxane & $\mathrm{B}$ & 27.4 & + & + & Confirmed \\
\hline Decamethylcyclopentasiloxane & B & 32.5 & & + & Probable \\
\hline Siloxane compound & & 37.6 & + & & Tentative \\
\hline \multicolumn{6}{|l|}{ Unidentified Compounds } \\
\hline Unidentified compound & & 43.0 & + & & Unident. \\
\hline
\end{tabular}

${ }^{\star} \mathrm{T}=$ Toxic air contaminant; $\mathrm{A}=$ Abundant compound; $\mathrm{B}=$ Component of system or chamber background; $Q=$ Quantified target compound. 
Table D-04. VOCs emitted by Carpet CP3 in 10-L chamber at 6 - and 48 -hours elapsed times.

\begin{tabular}{|c|c|c|c|c|c|}
\hline COMPOUND & Code $^{*}$ & $\begin{array}{c}\mathrm{RT} \\
(\mathrm{min})\end{array}$ & $\begin{array}{l}\text { 6-h } \\
\text { ET } \\
\end{array}$ & $\begin{array}{c}\text { 48-h } \\
\text { ET }\end{array}$ & $\begin{array}{l}\text { Match } \\
\text { Quality } \\
\end{array}$ \\
\hline \multicolumn{6}{|l|}{ Alkane Hydrocarbons } \\
\hline Branched alkane $\mathrm{HC}$ & & 26.8 & + & & Probable \\
\hline Branched alkane $\mathrm{HC}$ & & 28.9 & + & & Probable \\
\hline Branched alkane $\mathrm{HC}$ & & 29.2 & + & & Probable \\
\hline Branched alkane $\mathrm{HC}$ & & 29.6 & + & & Probable \\
\hline Branched alkane $\mathrm{HC}$ & & 34.6 & + & & Probable \\
\hline Branched alkane $\mathrm{HC}$ & & 37.0 & + & + & Probable \\
\hline Branched alkane $\mathrm{HC}$ & & 38.3 & + & + & Probable \\
\hline Branched alkane $\mathrm{HC}$ & & 42.8 & + & + & Probable \\
\hline Branched alkane $\mathrm{HC}$ & & 43.9 & + & + & Probable \\
\hline \multicolumn{6}{|l|}{ Aromatic Hydrocarbons } \\
\hline Styrene & $T, Q$ & 26.6 & + & & Confirmed \\
\hline Propylbenzene & & 28.4 & + & & Confirmed \\
\hline 4-Phenylcyclohexene & $A, Q$ & 41.1 & + & + & Confirmed \\
\hline \multicolumn{6}{|l|}{ Other Hydrocarbons } \\
\hline C12 Alkene $\mathrm{HC}$ & & 30.0 & + & & Probable \\
\hline C12 Alkene HC & & 30.2 & + & & Probable \\
\hline C12 Alkene $\mathrm{HC}$ & & 30.9 & + & & Probable \\
\hline C12 Alkene $\mathrm{HC}$ & & 31.1 & + & & Probable \\
\hline C12 Alkene $\mathrm{HC}$ & & 31.2 & + & & Probable \\
\hline $\mathrm{C} 12$ Alkene $\mathrm{HC}$ & & 31.4 & + & & Probable \\
\hline C12 Alkene $\mathrm{HC}$ & & 31.6 & + & & Probable \\
\hline C12 Alkene $\mathrm{HC}$ & & 31.8 & + & & Probable \\
\hline C12 Alkene HC & & 31.9 & + & & Probable \\
\hline C12 Alkene $\mathrm{HC}$ & & 32.0 & + & & Probable \\
\hline C12 Alkene $\mathrm{HC}$ & & 32.2 & + & & Probable \\
\hline C12 Alkene $\mathrm{HC}$ & & 32.3 & + & & Probable \\
\hline Alkene $\mathrm{HC}$ & & 40.6 & + & & Tentative \\
\hline \multicolumn{6}{|l|}{ Carbonyl Compounds } \\
\hline Benzaldehyde & $B$ & 31.1 & + & & Confirmed \\
\hline Nonanal & $\mathrm{B}, \mathrm{Q}$ & 34.4 & + & + & Confirmed \\
\hline Decanal & $B, Q$ & 37.5 & + & & Confirmed \\
\hline \multicolumn{6}{|l|}{ Other Oxidized Compounds } \\
\hline Acetic acid & B & 20.4 & + & + & Probable \\
\hline 2-(2-Butoxyethoxy)ethanol & $\mathrm{T}$ & 38.1 & + & & Confirmed \\
\hline
\end{tabular}


Table D-04, Continued. VOCs emitted by Carpet CP3 in 10-L chamber at 6- and 48-hours elapsed times.

\begin{tabular}{lccccc}
\hline COMPOUND & Code $^{*}$ & $\begin{array}{c}\text { RT } \\
(\mathrm{min})\end{array}$ & $\begin{array}{c}\text { 6-h } \\
\text { ET }\end{array}$ & $\begin{array}{c}\text { 48-h } \\
\text { ET }\end{array}$ & $\begin{array}{c}\text { Match } \\
\text { Quality }\end{array}$ \\
\hline Miscellaneous Compounds & & & & & \\
Hexamethylcyclotrisiloxane & $\mathrm{B}$ & 21.3 & + & + & Confirmed \\
Octamethylcyclotetrasiloxane & $\mathrm{B}$ & 27.4 & + & + & Confirmed \\
Decamethylcyclopentasiloxane & $\mathrm{B}$ & 32.5 & + & + & Probable \\
Siloxane compound & & 37.6 & + & & Tentative \\
Siloxane compound & & 42.2 & + & + & Tentative \\
& & & & & \\
\hline
\end{tabular}

${ }^{\star} \mathrm{T}=$ Toxic air contaminant $\mathrm{A}=$ Abundant compound; $\mathrm{B}=$ Component of system or chamber background; $\mathrm{Q}=$ Quantified target compound. 
Table D-05. VOCs emitted by Carpet CP2 in 10-L chamber at 6- and 48-hours elapsed times.

\begin{tabular}{|c|c|c|c|c|c|}
\hline COMPOUND & Code $^{\star}$ & $\begin{array}{c}\mathrm{RT} \\
(\mathrm{min})\end{array}$ & $\begin{array}{l}\text { 6-h } \\
\text { ET }\end{array}$ & $\begin{array}{c}\text { 48-h } \\
\text { ET }\end{array}$ & $\begin{array}{l}\text { Match } \\
\text { Quality }\end{array}$ \\
\hline \multicolumn{6}{|l|}{ Alkane Hydrocarbons } \\
\hline Branched alkane $\mathrm{HC}$ & & 23.0 & + & & Probable \\
\hline Branched alkane $\mathrm{HC}$ & & 24.4 & + & & Probable \\
\hline Branched alkane $\mathrm{HC}$ & & 26.7 & + & + & Probable \\
\hline Branched alkane $\mathrm{HCs}$ & & $27.0-27.5$ & + & + & Probable \\
\hline Branched alkane $\mathrm{HC}$ & & 27.9 & + & + & Probable \\
\hline Branched alkane $\mathrm{HC}$ & & 28.2 & + & + & Probable \\
\hline Branched alkane $\mathrm{HC}$ & & 28.6 & + & + & Probable \\
\hline Branched alkane $\mathrm{HC}$ & & 28.8 & + & + & Probable \\
\hline Branched alkane $\mathrm{HC}$ & & 29.2 & + & + & Probable \\
\hline Branched alkane $\mathrm{HC}$ & $A$ & 29.5 & + & + & Probable \\
\hline Branched alkane $\mathrm{HC}$ & & 29.7 & + & + & Probable \\
\hline Branched alkane $\mathrm{HC}$ & & 29.9 & + & + & Probable \\
\hline Branched alkane $\mathrm{HC}$ & & 30.0 & + & + & Probable \\
\hline Branched alkane $\mathrm{HC}$ & A & 30.2 & + & + & Probable \\
\hline Branched alkane $\mathrm{HC}$ & & 30.5 & + & + & Probable \\
\hline Branched alkane $\mathrm{HC}$ & & 30.8 & + & + & Probable \\
\hline Branched alkane $\mathrm{HC}$ & & 31.1 & + & + & Probable \\
\hline Branched alkane $\mathrm{HC}$ & & 31.3 & + & + & Probable \\
\hline Branched alkane $\mathrm{HC}$ & A & 31.5 & + & + & Probable \\
\hline Branched alkane $\mathrm{HC}$ & & 31.7 & + & + & Probable \\
\hline Branched alkane $\mathrm{HC}$ & & 31.9 & + & + & Probable \\
\hline Branched alkane $\mathrm{HC}$ & & 32.1 & & + & Probable \\
\hline Branched alkane $\mathrm{HC}$ & & 34.5 & & + & Probable \\
\hline Brarched alkane $\mathrm{HC}$ & $A$ & 36.9 & + & + & Probable \\
\hline Branched alkane $\mathrm{HC}$ & & 37.2 & + & + & Probable \\
\hline Branched alkane $\mathrm{HC}$ & & 37.3 & + & + & Probable \\
\hline Branched alkane $\mathrm{HC}$ & & 37.4 & + & + & Probable \\
\hline Branched alkane $\mathrm{HC}$ & & 37.6 & + & + & Probable \\
\hline Branched alkane $\mathrm{HC}$ & $A$ & 38.2 & + & + & Probable \\
\hline Branched alkane $\mathrm{HC}$ & & 38.4 & + & + & Probable \\
\hline Branched alkane $\mathrm{HC}$ & & 38.5 & + & + & Probable \\
\hline Branched alkane $\mathrm{HC}$ & & 38.6 & & + & Probable \\
\hline Branched alkane $\mathrm{HC}$ & & 38.7 & & + & Probable \\
\hline Branched alkane $\mathrm{HC}$ & & 38.8 & + & + & Probable \\
\hline Branched alkane $\mathrm{HC}$ & & 39.0 & & + & Probable \\
\hline Branched alkane $\mathrm{HC}$ & & 42.8 & + & + & Probable \\
\hline
\end{tabular}


Table D-05, Continued. VOCs emitted by Carpet CP2 in 10-L chamber at 6- and 48-hours elapsed times.

COMPOUND
Alkane Hydrocarbons, Cont.
Branched alkane HC
Branched alkane HC
Branched alkane HC
Branched alkane HC

\section{Aromatic Hydrocarbons}

Toluene
Ethylbenzene
m-,p-Xylene
o-Xylene
Stryene
Propylbenzene
4-Phenylcyclohexene

\section{Other Hydrocarbons}

Alkene $\mathrm{HC}$

Alkene $\mathrm{HC}$

Alkyl substituted cyclohexane

Alkene $\mathrm{HC}$

Alkene $\mathrm{HC}$

Alkene $\mathrm{HC}$

Alkene $\mathrm{HC}$

Alkene $\mathrm{HC}$

Halogenated Compounds

Tetrachloroethene

Carbonyl Compounds

Nonanal
1-Phenylethanone
Decanal

\section{Other Oxidized Compounds}
Acetic acid
Di(propylene glycol)methyl ether, isomer 3
Triethylene glycol monomethyl ether, CAS 112-35-6

$\begin{array}{cc}\mathrm{T}, \mathrm{Q} & 21.0 \\ \mathrm{~T}, \mathrm{Q} & 24.9 \\ \mathrm{~T}, \mathrm{Q} & 25.2 \\ \mathrm{~T}, \mathrm{Q} & 26.3 \\ \mathrm{~T}, \mathrm{Q} & 26.6 \\ \mathrm{Q} & 28.3 \\ \mathrm{Q} & 41.1\end{array}$

32.6

38.0

39.8

43.8

\begin{tabular}{|c|c|}
\hline & Confirmed \\
\hline & Confirmed \\
\hline & Confirmed \\
\hline & Confirmed \\
\hline & Confirmed \\
\hline & Confirmed \\
\hline & Confirmed \\
\hline
\end{tabular}

$$
T, A, Q
$$

22.0

$+$$$
\text { t }
$$$$
+
$$$$
+
$$$$
+
$$$$
+
$$$$
+
$$$$
+
$$

$+$

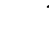

$\begin{array}{cc}+ & \text { Probable } \\ & \text { Probable } \\ + & \text { Tentative } \\ + & \text { Probable } \\ + & \text { Probable } \\ + & \text { Probable } \\ + & \text { Probable } \\ + & \text { Probable }\end{array}$

Probable
Probable
Probable
Probable

Match

Quality

$\begin{array}{cc}\text { 48-h } & \text { Match } \\ \text { ET } & \text { Quality }\end{array}$

$+\quad$ Confirmed

$+\quad$ Confirmed

$+\quad$ Confirmed

$+\quad$ Confirmed

$+\quad$ Confirmed

$\begin{array}{lllll}\mathrm{T}, \mathrm{Q} & 34.8 & + & + & \text { Confirmed } \\ \mathrm{B}, \mathrm{Q} & 37.4 & + & + & \text { Confirmed }\end{array}$

$\begin{array}{ccccc}\text { B } & 20.1 & + & + & \text { Probable } \\ 32.2 & + & & \text { Probable } \\ & 34.7 & + & + & \text { Tentative }\end{array}$


Table D-05, Continued. VOCs emitted by Carpet CP2 in 10-L chamber at 6 - and 48-hours elapsed times.

\begin{tabular}{|c|c|c|c|c|c|}
\hline COMPOUND & Code & $\begin{array}{c}\mathrm{RT} \\
(\mathrm{min})\end{array}$ & $\begin{array}{l}\text { 6-h } \\
\text { ET }\end{array}$ & $\begin{array}{l}\text { 48-h } \\
\text { ET }\end{array}$ & $\begin{array}{l}\text { Match } \\
\text { Quality }\end{array}$ \\
\hline \multicolumn{6}{|l|}{ Other Oxidized Cmpds, Cont. } \\
\hline Benzoic acid & & 40.3 & & + & Probable \\
\hline Decanoic acid & & 43.6 & & + & Probable \\
\hline 1-Dodecanol & & 45.0 & + & + & Tentative \\
\hline \multicolumn{6}{|l|}{ Nitrogen-Containing Cmpds } \\
\hline $\begin{array}{l}\text { 2-Methyleneglutaronitrile, } \\
\text { CAS 1572-52-7 }\end{array}$ & & 39.0 & + & & Probable \\
\hline $\begin{array}{l}\text { N,N-bis(2-Hydroxyethyl)- } \\
\text { dodecanamide, CAS 120-40-1 }\end{array}$ & & 48.3 & & + & Tentative \\
\hline \multicolumn{6}{|l|}{ Miscellaneous Compounds } \\
\hline Hexamethylcyclotrisiloxane & $\mathrm{B}$ & 21.3 & + & + & Probable \\
\hline Octamethylcyclotetrasiloxane & $\mathrm{B}$ & 27.4 & + & + & Probable \\
\hline Decamethylcyclopentasiloxane & $\mathrm{B}$ & 32.5 & + & + & Probable \\
\hline Siloxane compound & & 42.1 & + & & Tentative \\
\hline \multicolumn{6}{|l|}{ Unidentified Compounds } \\
\hline Unidentified glycol ether & & 34.6 & + & + & Unident. \\
\hline $\begin{array}{l}\text { Unidentified glycol ether, } \\
\mathrm{C} 8 \mathrm{H} 18 \mathrm{O} 3\end{array}$ & & 35.7 & + & + & Unident. \\
\hline Unidentified compound & & 39.8 & & + & Unident. \\
\hline Unidentified compound & & 42.1 & & + & Unident. \\
\hline
\end{tabular}

${ }^{*} \mathrm{~T}=$ Toxic air contaminant; $\mathrm{A}=$ Abundant compound; $\mathrm{B}=$ Component of system or chamber background; $\mathrm{Q}=$ Quantified target compound. 
Table D-06. VOCs emitted by Carpet CP4 in 10-L chamber at 6- and 48-hours elapsed times. Samples were analyzed using thermal desorption system two.

\begin{tabular}{llcccc}
\hline & & RT & $6-h$ & $48-h$ & Match \\
COMPOUND & Code $^{*}$ & $(\mathrm{~min})$ & ET & ET & Quality \\
\hline
\end{tabular}

\section{Alkane Hydrocarbons}

Branched alkane $\mathrm{HC}$

Branched alkane $\mathrm{HC}$

Branched alkane $\mathrm{HC}$

n-Undecane

Branched alkane HCs

Branched alkane HC

Branched alkane $\mathrm{HC}$

Branched alkane $\mathrm{HC}$

Branched alkane $\mathrm{HC}$

Branched alkane $\mathrm{HC}$

Branched alkane $\mathrm{HC}$

Branched alkane $\mathrm{HC}$

Branched alkane $\mathrm{HC}$

\section{Aromatic Hydrocarbons}

m-,p-Xylene
Stryene
4-Phenylcyclohexene

\section{Other Hydrocarbons}

4-Ethenylcyclohexene

Alkene $\mathrm{HC}$

Alkene $\mathrm{HC}$

Alkene $\mathrm{HC}$

Alkene $\mathrm{HC}$

Alkene $\mathrm{HC}$

\section{Carbonyl Compounds}

Benzaldehyde

Octanal

Nonanal

Decanal

$\begin{array}{ccccc} & 14.3 & + & + & \text { Probable } \\ & 21.8 & + & + & \text { Probable } \\ & 21.0 & + & + & \text { Probable } \\ \text { Q } & 22.0 & + & + & \text { Confirmed } \\ & 23.4 & + & + & \text { Probable } \\ & 23.6 & + & + & \text { Probable } \\ & 29.1 & + & + & \text { Probable } \\ & 29.4 & + & + & \text { Probable } \\ & 29.6 & + & + & \text { Probable } \\ & 29.8 & + & + & \text { Probable } \\ & 30.6 & + & + & \text { Probable } \\ & 30.8 & + & + & \\ & 31.1 & + & + & \text { Probable } \\ & 35.3 & + & + & \text { Probable }\end{array}$

$\begin{array}{cc}T & 16.6 \\ T, Q & 18.1 \\ \text { A,Q } & 33.5\end{array}$

16.6

18.1

33.5

Q

14.1

23.2

23.8

23.9

32.2

37.7

B

$B, Q$

$B, Q$

B
22.9

26.5

29.8
10.3

22.9

$\begin{array}{lll}+ & \text { Confirmed } \\ + & + & \text { Confirmed } \\ + & + & \text { Confirmed }\end{array}$

Confirmed

Probable

Probable

Probable

Probable

Probable

Confirmed

Confirmed

Confirmed

Confirmed

\section{Other Oxidized Compounds}

Methylheptanol isomer

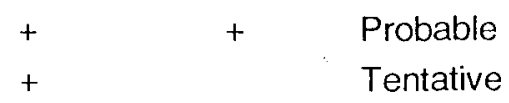


Table D-06, Continued. VOCs emitted by Carpet CP4 in 10-L chamber at 6-and 48-hours elapsed times. Samples were analyzed using thermal desorption system two.

\begin{tabular}{|c|c|c|c|c|c|}
\hline COMPOUND & Code $^{*}$ & $\begin{array}{c}\mathrm{RT} \\
(\mathrm{min}) \\
\end{array}$ & $\begin{array}{l}\text { 6-h } \\
\text { ET }\end{array}$ & $\begin{array}{c}\text { 48-h } \\
\text { ET }\end{array}$ & $\begin{array}{l}\text { Match } \\
\text { Quality } \\
\end{array}$ \\
\hline \multicolumn{6}{|l|}{ Other Oxidized Cmpds, Cont. } \\
\hline $\begin{array}{l}\text { Di(propylene glycol)methyl ether, } \\
\text { isomer } 1\end{array}$ & $Q$ & 23.4 & + & + & Confirmed \\
\hline $\begin{array}{l}\text { Di(propylene glycol)methyl ether, } \\
\text { isomer } 2\end{array}$ & $Q$ & 23.6 & + & & Confirmed \\
\hline $\begin{array}{l}\text { Di(propylene glycol)methyl ether, } \\
\text { isomer } 3\end{array}$ & $Q$ & 24.1 & + & + & Confirmed \\
\hline $\begin{array}{l}\text { Triethylene glycol monomethyl } \\
\text { ether, CAS 112-35-6 }\end{array}$ & A & 26.8 & + & + & Tentative \\
\hline Phenol & $\mathrm{T}, \mathrm{Q}$ & 26.9 & + & + & Confirmed \\
\hline Phenethyl alcohol & $\mathrm{Q}$ & 29.3 & + & + & Confirmed \\
\hline 1-Decanol & $Q$ & 32.2 & + & + & Confirmed \\
\hline 1-Dodecanol & $A$ & 37.7 & + & + & Confirmed \\
\hline \multicolumn{6}{|l|}{ Nitrogen-Containing Cmpds } \\
\hline N,N-Dimethylacetamide & $Q$ & 20.8 & + & + & Confirmed \\
\hline 4-Methyl-1H-imidazole & & 30.3 & + & & Tentative \\
\hline $\begin{array}{l}\text { 2-Methyleneglutaronitrile, } \\
\text { CAS 1572-52-7 }\end{array}$ & $Q$ & 31.6 & + & + & Confirmed \\
\hline \multicolumn{6}{|l|}{ Miscellaneous Compounds } \\
\hline Hexamethylcyclotrisiloxane & $\mathrm{B}$ & 12.3 & + & + & Probable \\
\hline Octamethylcyclotetrasiloxane & $\mathrm{B}$ & 18.9 & + & + & Probable \\
\hline Decamethylcyclopentasiloxane & $\mathrm{B}$ & 30.5 & + & + & Probable \\
\hline \multicolumn{6}{|l|}{ Unidentified Compounds } \\
\hline $\begin{array}{l}\text { Unidentified glycol ether, } \\
\mathrm{C} 8 \mathrm{H} 18 \mathrm{O} 3\end{array}$ & & 27.7 & + & + & Unident. \\
\hline $\begin{array}{l}\text { Unidentified glycol ether, } \\
\mathrm{C} 8 \mathrm{H} 18 \mathrm{O} 3\end{array}$ & $A$ & 27.9 & + & + & Unident. \\
\hline $\begin{array}{l}\text { Unidentified glycol ether, } \\
\mathrm{C} 8 \mathrm{H} 18 \mathrm{O} 3\end{array}$ & & 28.0 & + & + & Unident. \\
\hline Unidentified glycol ether & & 29.3 & + & + & Unident. \\
\hline Unidentified compound & & 37.4 & & + & Unident. \\
\hline Unidentified compound & & 38.7 & + & & Unident. \\
\hline
\end{tabular}

${ }^{*} \mathrm{~T}=$ Toxic air contaminant; $\mathrm{A}=$ Abundant compound; $\mathrm{B}=$ Component of system or chamber background; $\mathrm{Q}=$ Quantified target compound. 
Table D-07. VOCs emitted by Seaming Tape ST and Carpet CP3 in 10-L chamber at 6- and 48hours elapsed times.

\begin{tabular}{lccccc}
\hline & & RT & $6-h$ & $48-h$ & Match \\
COMPOUND & Code $^{*}$ & $(\mathrm{~min})$ & ET & ET & Quality \\
\hline
\end{tabular}

Alkane Hydrocarbons

Branched alkane $\mathrm{HC}$

Branched alkane $\mathrm{HC}$

Branched alkane $\mathrm{HC}$

n-Decane

Branched alkane $\mathrm{HC}$

Branched alkane $\mathrm{HC}$

Branched alkane $\mathrm{HC}$

Branched alkane $\mathrm{HC}$

Branched alkane $\mathrm{HC}$

Branched alkane $\mathrm{HC}$

Branched alkane $\mathrm{HC}$

Branched alkane $\mathrm{HC}$

Branched alkane $\mathrm{HC}$

Branched alkane $\mathrm{HC}$

Branched alkane $\mathrm{HC}$

Branched alkane $\mathrm{HC}$

Branched alkane $\mathrm{HC}$

Branched alkane $\mathrm{HC}$

Branched alkane $\mathrm{HC}$

Branched alkane $\mathrm{HC}$

Branched alkane $\mathrm{HC}$

Branched alkane $\mathrm{HC}$

Branched alkane $\mathrm{HC}$

Branched alkane $\mathrm{HC}$

Branched alkane $\mathrm{HC}$

Branched alkane HC

\begin{tabular}{|c|c|c|c|c|}
\hline & 24.4 & + & & Probable \\
\hline & 26.8 & + & + & Probable \\
\hline & 27.3 & + & + & Probable \\
\hline \multirow[t]{2}{*}{$Q$} & 28.0 & + & & Confirmed \\
\hline & 28.7 & + & + & Probable \\
\hline \multirow[t]{2}{*}{ A } & 28.9 & + & + & Probable \\
\hline & 29.2 & + & + & Probable \\
\hline \multirow[t]{3}{*}{ A } & 29.6 & + & + & Probable \\
\hline & 29.9 & + & + & Probable \\
\hline & 30.1 & + & + & Probable \\
\hline A & 30.3 & + & + & Probable \\
\hline \multirow[t]{2}{*}{$A$} & 30.4 & + & + & Probable \\
\hline & 30.6 & + & & Probable \\
\hline \multirow[t]{13}{*}{ A } & 30.9 & + & + & Probable \\
\hline & 31.1 & + & + & Probable \\
\hline & 31.2 & + & + & Probable \\
\hline & 31.4 & + & + & Probable \\
\hline & 31.6 & + & + & Probable \\
\hline & 31.8 & + & & Probable \\
\hline & 32.1 & + & + & Probable \\
\hline & 32.2 & + & + & Probable \\
\hline & 33.6 & + & & Probable \\
\hline & 37.0 & + & + & Probable \\
\hline & 38.3 & + & + & Probable \\
\hline & 42.8 & + & + & Probable \\
\hline & 43.9 & + & & Probable \\
\hline
\end{tabular}

\section{Aromatic Hydrocarbons}

Styrene
Propylbenzene
4-Phenylcyclohexene

Other Hydrocarbons

Alkene $\mathrm{HC}$

Alkene $\mathrm{HC}$

Alkene $\mathrm{HC}$
27.8

30.3

30.4

$\begin{array}{lll}T, Q & 26.6 & + \\ A, Q & 28.4 & +\end{array}$

Confirmed

Confirmed

Confirmed

Probable

Probable

Probable 
Table D-07, Continued. VOCs emitted by Seaming Tape ST and Carpet CP3 in 10-L chamber at 6- and 48-hours elapsed times.

\begin{tabular}{|c|c|c|c|c|c|}
\hline COMPOUND & Code $^{\star}$ & $\begin{array}{c}\text { RT } \\
(\min ) \\
\end{array}$ & $\begin{array}{l}6-h \\
\text { ET }\end{array}$ & $\begin{array}{c}\text { 48-h } \\
\mathrm{ET}\end{array}$ & $\begin{array}{l}\text { Match } \\
\text { Quality } \\
\end{array}$ \\
\hline \multicolumn{6}{|l|}{ Other Hydrocarbons, Cont. } \\
\hline Alkene $\mathrm{HC}$ & & 30.6 & + & & Probable \\
\hline Alkene $\mathrm{HC}$ & & 30.8 & + & + & Probable \\
\hline Alkene HC & & 31.4 & + & + & Probable \\
\hline Alkene $\mathrm{HC}$ & & 32.0 & + & + & Tentative \\
\hline Alkene $\mathrm{HC}$ & & 32.7 & + & & Probable \\
\hline \multicolumn{6}{|l|}{ Carbonyl Compounds } \\
\hline Nonanal & $\mathrm{B}, \mathrm{Q}$ & 34.4 & + & + & Confirmed \\
\hline Decanal & $\mathrm{B}, \mathrm{Q}$ & 37.5 & + & + & Confirmed \\
\hline \multicolumn{6}{|l|}{ Other Oxidized Compounds } \\
\hline Acetic acid & $\mathrm{B}$ & 20.4 & + & + & Probable \\
\hline $\begin{array}{l}\text { Di(propylene glycol)methyl ether, } \\
\text { isomer } 3\end{array}$ & & 32.3 & + & & Probable \\
\hline 2-(2-Butoxyethoxy)ethanol & & 38.1 & + & + & Confirmed \\
\hline $\begin{array}{l}\text { 2,6-Di-tert-butyl-4-methylphenol } \\
\text { (butylated hydroxytoluene) }\end{array}$ & $Q$ & 45.6 & + & + & Confirmed \\
\hline \multicolumn{6}{|l|}{ Miscellaneous Compounds } \\
\hline Hexamethylcyclotrisiloxane & B & 21.3 & + & + & Confirmed \\
\hline Octamethylcyclotetrasiloxane & $\mathrm{B}$ & 27.4 & + & + & Confirmed \\
\hline Decamethylcyclopentasiloxane & $\mathrm{B}$ & 32.5 & + & + & Probable \\
\hline Siloxane compound & & 42.2 & + & + & Tentative \\
\hline
\end{tabular}

${ }^{*} T=$ Toxic air contaminant; $A=A$ Abundant compound; $B=$ Component of system or chamber background; $\mathrm{Q}=$ Quantified target compound. 
Table D-08. VOCs emitted by CP Blank in 10-L chamber at 6- and 48-hours elapsed times.

\begin{tabular}{|c|c|c|c|c|c|}
\hline COMPOUND & Code $^{\star}$ & $\begin{array}{c}\mathrm{RT} \\
(\mathrm{min})\end{array}$ & $\begin{array}{l}\text { 6-h } \\
\text { ET }\end{array}$ & $\begin{array}{c}\text { 48-h } \\
\text { ET }\end{array}$ & $\begin{array}{l}\text { Match } \\
\text { Quality }\end{array}$ \\
\hline \multicolumn{6}{|l|}{ Carbonyl Compounds } \\
\hline Benzaldehyde & & 31.1 & + & + & Confirmed \\
\hline Nonanal & $\mathrm{Q}$ & 34.4 & + & + & Confirmed \\
\hline 1-Phenylethanone & $\mathrm{T}$ & 34.8 & + & & Confirmed \\
\hline Decanal & $Q$ & 37.5 & + & + & Confirmed \\
\hline \multicolumn{6}{|l|}{ Other Oxidized Compounds } \\
\hline Acetic acid & & 20.2 & + & + & Probable \\
\hline \multicolumn{6}{|l|}{ Miscellaneous Compounds } \\
\hline Hexamethylcyclotrisiloxane & & 21.4 & + & + & Confirmed \\
\hline Octamethylcyclotetrasiloxane & & 27.4 & + & + & Confirmed \\
\hline Decamethylcyclopentasiloxane & & 32.5 & + & + & Probable \\
\hline
\end{tabular}

${ }^{*} \mathrm{~T}=$ Toxic air contaminant; $\mathrm{Q}=$ Quantified target compound. 
Table D-09. VOCs emitted by Carpet Cushion $\mathrm{CC} 1$ in 10-L chamber at 6- and 48-hours elapsed times.

\begin{tabular}{llcccc}
\hline \hline & & RT & $6-h$ & $48-h$ & Match \\
COMPOUND & Code $^{*}$ & $(\mathrm{~min})$ & ET & ET & Quality \\
\hline
\end{tabular}

\section{Alkane Hydrocarbons}

n-Undecane

Branched alkane $\mathrm{HC}$

$n$-Tridecane

$n$-Tetradecane

\section{Aromatic Hydrocarbons}

Toluene

$\mathrm{m}$-, p-Xylene

Propylbenzene

C3 Alkylbenzene

1,3,5-Trimethylbenzene

2-Ethyltoluene

1,2,4-Trimethylbenzene

1,2,3-Trimethylbenzene

1,3-Diethylbenzene

C4 Alkylbenzene

C4 Alkylbenzene

C4 Alkylbenzene

C4 Alkylbenzene

C4 Alkylbenzene

C4 Alkylbenzene

C4 Alkylbenzene

C4 Alkylbenzene

C5 Alkylbenzene

C4 Alkylbenzene

C5 Alkylbenzene

C5 Alkylbenzene

Naphthalene

2-Methylnaphthalene

1-Methylnaphthalene

Dimethylnaphthalene isomer

\section{Other Hydrocarbons}

Dihydromethylindene isomer

Dihydromethylindene isomer Unsaturated hydrocarbons, unresolved isomers

\begin{tabular}{|c|c|c|c|c|}
\hline Q & 31.5 & + & & Confirmed \\
\hline & 34.6 & + & + & Probable \\
\hline$Q$ & 37.5 & & + & Confirmed \\
\hline$Q$ & 40.3 & + & & Con \\
\hline
\end{tabular}

\begin{tabular}{|c|c|c|c|c|}
\hline$T, Q$ & 21.2 & + & & Confirmed \\
\hline$T, Q$ & 25.2 & + & & Confirmed \\
\hline \multirow[t]{2}{*}{$\mathrm{Q}$} & 28.4 & + & & Confirmed \\
\hline & 28.6 & + & + & Probable \\
\hline$Q$ & 28.9 & + & & Confirmed \\
\hline$Q$ & 29.5 & + & & Confirmed \\
\hline$T, Q$ & 29.9 & + & + & Confirmed \\
\hline$Q$ & 31.2 & + & & Confirmed \\
\hline \multirow[t]{13}{*}{$Q$} & 31.6 & + & & Confirmed \\
\hline & 31.9 & + & & Probable \\
\hline & 32.4 & + & & Probable \\
\hline & 32.7 & + & + & Probable \\
\hline & 32.8 & + & & Probable \\
\hline & 33.0 & + & + & Probable \\
\hline & 33.9 & + & + & Probable \\
\hline & 34.1 & + & + & Probable \\
\hline & 34.3 & + & + & Probable \\
\hline & 34.6 & + & & Probable \\
\hline & 35.5 & + & + & Probable \\
\hline & 35.7 & + & & Probable \\
\hline & 36.5 & + & + & Probable \\
\hline$T, A, Q$ & 37.8 & + & + & Confirmed \\
\hline$A, Q$ & 41.0 & + & + & Confirmed \\
\hline \multirow[t]{2}{*}{$\mathrm{Q}$} & 41.5 & + & + & Confirmed \\
\hline & 43.9 & + & & Probable \\
\hline
\end{tabular}

$\begin{array}{ccccc} & 35.0 & + & + & \text { Probable } \\ & 35.6 & + & + & \text { Probable } \\ \text { A } & 35-51 & + & + & \text { Probable }\end{array}$


Table D-09, Continued. VOCs emitted by Carpet Cushion CC1 in 10-L chamber at 6- and 48hours elapsed times.

\begin{tabular}{|c|c|c|c|c|c|}
\hline COMPOUND & Code $^{\star}$ & $\begin{array}{c}\mathrm{RT} \\
(\mathrm{min})\end{array}$ & $\begin{array}{l}\text { 6-h } \\
\text { ET }\end{array}$ & $\begin{array}{l}\text { 48-h } \\
\text { ET }\end{array}$ & $\begin{array}{l}\text { Match } \\
\text { Quality } \\
\end{array}$ \\
\hline \multicolumn{6}{|l|}{ Carbonyl Compounds } \\
\hline Benzaldehyde & $\mathrm{B}$ & 31.1 & + & + & Confirmed \\
\hline 1-Phenylethanone & $\mathrm{T}, \mathrm{Q}$ & 34.8 & + & & Confirmed \\
\hline \multicolumn{6}{|l|}{ Other Oxidized Compounds } \\
\hline 1-Acetoxy-2-propanol & & 29.3 & + & + & Probable \\
\hline 1,3-Dichloro-2-propanol & & 30.9 & + & & Confirmed \\
\hline Phenol & $T$ & 34.7 & + & + & Confirmed \\
\hline $\begin{array}{l}\text { Pentanedioic acid, dimethylester, } \\
\text { CAS } 1119-40-0\end{array}$ & $\mathrm{Q}$ & 36.5 & + & & Confirmed \\
\hline $\begin{array}{l}\text { 2,2,4-Trimethyl-1,3-pentanediol } \\
\text { monoisobutyrate (Texanol } 1 \\
\text { isomer) }\end{array}$ & $Q$ & 43.3 & + & + & Confirmed \\
\hline $\begin{array}{l}\text { 2,2,4-Trimethyl-1,3-pentanediol } \\
\text { monoisobutyrate (Texanol } 3 \\
\text { isomer) }\end{array}$ & $Q$ & 43.6 & + & + & Confirmed \\
\hline $\begin{array}{l}\text { 2,6-Di-tert-butyl-4-methylphenol } \\
\text { (butylated hydroxytoluene) }\end{array}$ & $A, Q$ & 45.6 & + & + & Confirmed \\
\hline \multicolumn{6}{|l|}{ Nitrogen-Containing Cmpds } \\
\hline $\begin{array}{l}\text { 4-Methylmorpholine, } \\
\text { CAS 109-02-4 }\end{array}$ & & 22.9 & + & + & Probable \\
\hline $\begin{array}{l}\text { N,N-Dimethylbenzylamine, } \\
\text { CAS 103-83-3 }\end{array}$ & $A, Q$ & 31.7 & + & + & Confirmed \\
\hline $\begin{array}{l}\text { 2,2'-Azobisisobutyronitrile, } \\
\text { CAS 78-67-1 }\end{array}$ & & 35.4 & + & + & Probable \\
\hline \multicolumn{6}{|l|}{ Miscellaneous Compounds } \\
\hline Decamethylcyclopentasiloxane & $\mathrm{B}$ & 32.5 & + & & Probable \\
\hline Triethylphosphate & $A, Q$ & 37.1 & + & + & Confirmed \\
\hline \multicolumn{6}{|l|}{ Unidentified Compounds } \\
\hline Unidentified compound & & 30.2 & + & & Unident. \\
\hline Unidentified compound & & 30.7 & + & & Unident. \\
\hline Unidentifed compound & & 48.1 & + & + & Unident. \\
\hline
\end{tabular}

${ }^{\star} T=$ Toxic air contaminant; $A=$ Abundant compound; $B=$ Component of system or chamber background; $\mathrm{Q}=$ Quantified target compound. 
Table D-10. VOCs emitted by Carpet Cushion CC2 in 10-L chamber at 6- and 48-hours elapsed times.

\begin{tabular}{|c|c|c|c|c|c|}
\hline COMPOUND & Code & $\begin{array}{c}\mathrm{RT} \\
(\mathrm{min})\end{array}$ & $\begin{array}{l}\text { 6-h } \\
\mathrm{ET} \\
\end{array}$ & $\begin{array}{c}\text { 48-h } \\
\mathrm{ET} \\
\end{array}$ & $\begin{array}{l}\text { Match } \\
\text { Quality } \\
\end{array}$ \\
\hline \multicolumn{6}{|l|}{ Alkane Hydrocarbons } \\
\hline Branched alkane $\mathrm{HC}$ & & 28.8 & + & & Probable \\
\hline Branched alkane $\mathrm{HC}$ & & 29.5 & + & & Probable \\
\hline Branched alkane $\mathrm{HC}$ & & 30.2 & + & & Probable \\
\hline Branched alkane $\mathrm{HC}$ & & 30.3 & + & & Probable \\
\hline Branched alkane $\mathrm{HC}$ & & 30.8 & + & & Probable \\
\hline n-Dodecane & $Q$ & 34.6 & + & & Confirmed \\
\hline \multicolumn{6}{|l|}{ Other Hydrocarbons } \\
\hline d-Limonene & $Q$ & 30.1 & + & + & Confirmed \\
\hline $\begin{array}{l}\text { Unsaturated hydrocarbons, } \\
\text { unresolved isomers }\end{array}$ & $A$ & $35-51$ & + & + & Probable \\
\hline $\begin{array}{l}\text { Unsaturated hydrocarbon, } \\
\mathrm{C} 16 \mathrm{H} 26\end{array}$ & & 44.9 & + & & Probable \\
\hline \multicolumn{6}{|l|}{ Halogenated Compounds } \\
\hline 1,2-Dichlorobenzene & $Q$ & 32.3 & + & & Probable \\
\hline \multicolumn{6}{|l|}{ Carbonyl Compounds } \\
\hline Cyclohexanone & $Q$ & 28.5 & + & & Confirmed \\
\hline Benzaldehyde & $\mathrm{B}$ & 31.0 & + & + & Confirmed \\
\hline Nonanal & $\mathrm{B}, \mathrm{Q}$ & 34.4 & + & + & Confirmed \\
\hline \multicolumn{6}{|l|}{ Other Oxidized Compounds } \\
\hline Benzyl acetate, CAS 140-11-4 & & 37.1 & & + & Probable \\
\hline $\begin{array}{l}\text { 2,6-Di-tert-butyl-4-methylphenol } \\
\text { (butylated hydroxytoluene) }\end{array}$ & $A, Q$ & 45.6 & + & + & Confirmed \\
\hline \multicolumn{6}{|l|}{ Nitrogen-Containing Cmpds } \\
\hline $\begin{array}{l}\text { 2,2'-Azobisisobutyronitrile, } \\
\text { CAS 78-67-1 }\end{array}$ & & 35.4 & + & + & Probable \\
\hline \multicolumn{6}{|l|}{ Miscellaneous Compounds } \\
\hline Decamethylcyclopentasiloxane & $\mathrm{B}$ & 32.5 & + & & Probable \\
\hline \multicolumn{6}{|l|}{ Unidentified Compounds } \\
\hline Unidentified compound & A & 42.3 & + & + & Unident. \\
\hline Unidentified compound & & 44.9 & & + & Unident. \\
\hline Unidentified compound & & 48.0 & + & + & Unident. \\
\hline
\end{tabular}

${ }^{*} \mathrm{~T}=$ Toxic air contaminant; $\mathrm{A}=$ Abundant $\mathrm{c}$ m mpound; $\mathrm{B}=$ Component of system or chamber background; $Q=$ Quantified target compin ir. 
Table D-11. VOCs emitted by Carpet Cushion CC4 in 10-L chamber at 6- and 48-hours elapsed times. Samples were analyzed using thermal desorption system two.

\section{COMPOUND}

Aromatic HC, MW 262

Other Hydrocarbons

Unsaturated hydrocarbons, unresolved isomers

\section{Halogenated Compounds}

1,2-Dichlorobenzene

\section{Carbonyl Compounds}

Benzaldehyde

Octanal

Nonanal

Decanal

\section{Other Oxidized Compounds}

Phenol

2-Ethylhexanoic acid

2,6-Di-tert-butyl-4-methylphenol (butylated hydroxytoluene)

\section{Nitrogen-Containing Cmpds}

$\mathrm{N}, \mathrm{N}$-Dimethylbenzylamine, CAS 103-83-3

N,N-Dimethylacrylamide, CAS 2680-03-7

2,2'-Azobisisobutryonitrile CAS 78-67-1

\section{Miscellaneous Compounds}

Hexamethylcyclotrisiloxane Octamethylcyclotetrasiloxane
A 28-43+

\section{6-h}

Code $^{\star}(\mathrm{min})$

40.8

$+$

$+$

Tentative

Q

24.2

$+$

Confirmed

B $\quad 22.9$

22.9

$B, Q$

26.5

29.7

$+$

$+$

$+$

Confirmed

$+$

Confirmed

$+$

Confirmed

Confirmed

$+$

Confirmed

$+$

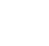

$+\quad$ Confirmed

$+\quad$ Probable

28.7

Confirmed

A,Q $\quad 38.2$

$+$

$+$

$\begin{array}{lllll}\mathrm{Q} & 23.4 & + & + & \text { Confirmed } \\ \mathrm{Q} & 23.6 & + & + & \text { Confirmed } \\ \mathrm{Q} & 27.6 & + & + & \text { Confirmed } \\ & & & & \\ \mathrm{B} & 12.4 & + & + & \text { Confirmed } \\ \mathrm{B} & 18.8 & + & + & \text { Confirmed }\end{array}$

${ }^{\star} \mathrm{T}=$ Toxic air contaminant; $\mathrm{A}=$ Abundant compound; $\mathrm{B}=$ Component of system or chamber background; $\mathrm{Q}=$ Quantified target compound. 
Table D-12. VOCs emitted by Carpet Cushion CC3 in 10-L chamber at 6- and 48-hours elapsed times.

\begin{tabular}{llcccc}
\hline & & RT & $6-\mathrm{h}$ & 48-h & Match \\
COMPOUND & Code $^{*}$ & $(\mathrm{~min})$ & ET & ET & Quality \\
\hline
\end{tabular}

\section{Alkane Hydrocarbons}

Branched alkane $\mathrm{HC}$

Branched alkane $\mathrm{HC}$

Branched alkane $\mathrm{HC}$

Branched alkane $\mathrm{HC}$

Branched alkane $\mathrm{HC}$

Branched alkane $\mathrm{HC}$

Branched alkane $\mathrm{HC}$

Branched alkane $\mathrm{HC}$

Branched alkane $\mathrm{HC}$

Branched alkane $\mathrm{HC}$

Branched alkane $\mathrm{HC}$

Branched alkane $\mathrm{HC}$

Branched alkane $\mathrm{HC}$

Branched alkane $\mathrm{HC}$

Branched alkane $\mathrm{HC}$

Branched alkane $\mathrm{HC}$

Branched alkane $\mathrm{HC}$

Branched alkane $\mathrm{HC}$

Branched alkane $\mathrm{HC}$

Branched alkane $\mathrm{HC}$

Branched alkane $\mathrm{HC}$

Branched alkane $\mathrm{HC}$

Branched alkane $\mathrm{HC}$

Branched alkane $\mathrm{HC}$

\section{Aromatic Hydrocarbons}

C3 Alkylbenzene

C3 Alkylbenzene

1,2,4-Trimethylbenzene

1,2,3-Trimethylbenzene

C4 Alkylbenzene

C4 Alkylbenzene

C4 Alkylbenzene

Naphthalene

2-Methylnaphthalene

4-Phenylcyclohexene

Biphenyl
23.0

26.7

28.8

29.5

30.0

A

30.2

31.4

A

31.5

31.7

31.9

34.6

A

36.9

37.1

37.3

37.6

A

38.2

38.3

38.5

38.7

39.0

40.3

42.7

42.9

43.9

28.6

28.8

Q $\quad 29.9$

Q

31.1

32.9

34.0

34.2

$T, Q$

37.7

Q $\quad 40.9$

Q $\quad 41.1$

$T$
43.1
Probable

Probable

Probable

Probable

$+\quad$ Probable

$+\quad$ Probable

Probable

$+\quad$ Probable

$+\quad$ Probable

Probable

Probable

Probable

Probable

Probable

Probable

Probable

Probable

Probable

Probable

Probable

Probable

Probable

Probable

Probable

Probable

Probable

Confirmed

Confirmed

Probable

Probable

Probable

Confirmed

Confirmed

Confirmed

Confirmed 
Table D-12, Continued. VOCs emitted by Carpet Cushion CC3 in 10-L chamber at 6- and 48hours elapsed times.

\begin{tabular}{|c|c|c|c|c|c|}
\hline COMPOUND & Code $^{*}$ & $\begin{array}{c}\mathrm{RT} \\
(\mathrm{min})\end{array}$ & $\begin{array}{l}\text { 6-h } \\
\text { ET }\end{array}$ & $\begin{array}{l}\text { 48-h } \\
\text { ET }\end{array}$ & $\begin{array}{l}\text { Match } \\
\text { Quality }\end{array}$ \\
\hline \multicolumn{6}{|l|}{ Other Hydrocarbons } \\
\hline Alkene $\mathrm{HC}$ & & 31.1 & + & & Probable \\
\hline Alkene $\mathrm{HC}$ & & 31.2 & + & & Probable \\
\hline C5 Alkyl substituted cyclohexane & & 38.0 & + & + & Tentative \\
\hline Alkene $\mathrm{HC}$ & & 45.0 & + & + & Tentative \\
\hline \multicolumn{6}{|l|}{ Halogenated Compounds } \\
\hline 1,1,1-Trichloroethane & $\mathrm{T}, \mathrm{Q}$ & 15.2 & + & & Confirmed \\
\hline \multicolumn{6}{|l|}{ Carbonyl Compounds } \\
\hline Benzaldehyde & $\mathrm{B}$ & 31.0 & + & & Confirmed \\
\hline Nonanal & $B, Q$ & 34.3 & + & + & Confirmed \\
\hline Decanal & $\mathrm{B}, \mathrm{Q}$ & 37.4 & + & + & Confirmed \\
\hline \multicolumn{6}{|l|}{ Other Oxidized Compounds } \\
\hline 2-Ethyl-1-hexanol & $Q$ & 32.4 & + & + & Confirmed \\
\hline \multicolumn{6}{|l|}{ Nitrogen-Containing Cmpds } \\
\hline Caprolactam & $\mathrm{T}, \mathrm{Q}$ & 43.4 & + & & Probable \\
\hline \multicolumn{6}{|l|}{ Miscellaneous Compounds } \\
\hline Decamethylcyclopentasiloxane & B & 32.5 & & + & Probable \\
\hline \multicolumn{6}{|l|}{ Unidentified Compounds } \\
\hline Unidentified compound & & 31.1 & & + & Unident. \\
\hline
\end{tabular}

${ }^{*} T=$ Toxic air contaminant; $A=$ Abundant compound $B=$ Component of system or chamber background; $\mathrm{Q}=$ Quantified target compound. 
Table D-13. VOCs emitted by CC Blank in 10-L chamber at 6- and 48-hours elapsed times.

\begin{tabular}{|c|c|c|c|c|c|}
\hline COMPOUND & Code $^{\star}$ & $\begin{array}{c}\mathrm{RT} \\
(\mathrm{min})\end{array}$ & $\begin{array}{l}\text { 6-h } \\
\text { ET }\end{array}$ & $\begin{array}{c}\text { 48-h } \\
E T \\
\end{array}$ & $\begin{array}{l}\text { Match } \\
\text { Quality }\end{array}$ \\
\hline \multicolumn{6}{|l|}{ Carbonyl Compounds } \\
\hline Benzaldehyde & & 31.1 & + & & Confirmed \\
\hline Nonanal & $\mathrm{Q}$ & 34.4 & + & + & Confirmed \\
\hline Decanal & $\mathrm{Q}$ & 37.5 & + & + & Confirmed \\
\hline \multicolumn{6}{|l|}{ Other Oxidized Compounds } \\
\hline Acetic acid & & 20.3 & + & + & Probable \\
\hline \multicolumn{6}{|l|}{ Miscellaneous Compounds } \\
\hline Hexamethyicyclotrisiloxane & & 21.4 & + & + & Confirmed \\
\hline Octamethylcyclotetrasiloxane & & 27.4 & + & + & Confirmed \\
\hline Decamethylcyclopentasiloxane & & 32.5 & & + & Probable \\
\hline
\end{tabular}

${ }^{\star} \mathrm{Q}=$ Quantified target compound. 
Table D-14. Summary of target VOCs for screening measurements with carpet materials. See Table D-02 for material codes.

\begin{tabular}{|c|c|c|c|c|c|c|c|c|c|}
\hline COMPOUND & $\mathrm{CP} 1$ & $\mathrm{CP} 3$ & $\mathrm{CP} 2$ & $\mathrm{CP} 4$ & $\begin{array}{l}\text { ST \& } \\
\text { CP3 } \\
\end{array}$ & $\mathrm{CC}_{1}$ & $\mathrm{CC2}$ & $\mathrm{CC} 4$ & $\mathrm{CC} 3$ \\
\hline \multicolumn{10}{|l|}{ Alkane Hydrocarbons } \\
\hline n-Decane & & & & & + & & & & \\
\hline n-Undecane & & & & + & & + & & & \\
\hline n-Dodecane & + & & & & & & + & & \\
\hline n-Tridecane & & & & & & + & & & \\
\hline n-Tetradecane & & & & & & + & & & \\
\hline \multicolumn{10}{|l|}{ Aromatic Hydrocarbons } \\
\hline Toluene & + & & + & & & + & & & \\
\hline Ethylbenzene & & & + & & & & & & \\
\hline m-,p-Xylene & & & + & & & + & & & \\
\hline o-Xylene & & & + & & & & & & \\
\hline Styrene & & + & + & + & + & & & & \\
\hline Propylbenzene & & & + & & & + & & & \\
\hline 1,3,5-Trimethylbenzene & & & & & & + & & & + \\
\hline 2-Ethyltoluene & & & & & & + & & & \\
\hline 1,2,4-Trimethylbenzene & & & & & & + & & & \\
\hline 1,2,3-Trimethylbenzene & & & & & & + & & & + \\
\hline 1,3-Diethylbenzene & & & & & & + & & & + \\
\hline Naphthalene & & & & & & + & & & + \\
\hline 2-Methylnaphthalene & & & & & & + & & & + \\
\hline 1-Methylnaphthalene & & & & & & + & & & \\
\hline 4-Phenylcyclohexene & + & + & + & + & + & & & & + \\
\hline \multicolumn{10}{|l|}{ Other Hydrocarbons } \\
\hline 4-Ethenylcyclohexene & & & & + & & & & & \\
\hline d-Limonene & + & & & & & & + & & \\
\hline
\end{tabular}


Table D-14, Continued. Summary of target VOCs for screening measurements with carpet materials.

\begin{tabular}{|c|c|c|c|c|c|c|c|c|c|}
\hline COMPOUND & $\mathrm{CP} 1$ & $\mathrm{CP} 3$ & $\mathrm{CP} 2$ & $\mathrm{CP} 4$ & $\begin{array}{c}\text { ST \& } \\
\text { CP3 } \\
\end{array}$ & $\mathrm{CC} 1$ & $\mathrm{CC} 2$ & $\mathrm{CC} 4$ & $\mathrm{CC} 3$ \\
\hline $\begin{array}{l}\text { Halogenated Compounds } \\
\text { 1,1,1-Trichloroethane } \\
\text { Tetrachloroethene } \\
\text { 1,2-Dichlorobenzene }\end{array}$ & & & + & & & & + & + & + \\
\hline $\begin{array}{l}\text { Carbonyl Compounds } \\
\text { Cyclohexanone } \\
\text { Nonanal } \\
\text { 1-Phenylethanone } \\
\text { Decanal }\end{array}$ & $\begin{array}{l}+ \\
+ \\
+ \\
\end{array}$ & $\begin{array}{l}+ \\
+ \\
\end{array}$ & $\begin{array}{l}+ \\
+ \\
+\end{array}$ & $\begin{array}{l}+ \\
+ \\
\end{array}$ & $\begin{array}{l}+ \\
+ \\
\end{array}$ & + & + & $\begin{array}{l}+ \\
+ \\
\end{array}$ & $\begin{array}{l}+ \\
+ \\
\end{array}$ \\
\hline $\begin{array}{l}\text { Other Oxidized Compounds } \\
\text { Di(propylene glycol) methyl } \\
\text { ethers } \\
\text { 2-Ethyl-1-hexanol } \\
\text { Phenol } \\
\text { Pentanedioic acid, dimethyl ester } \\
\text { Phenethyl alcohol } \\
\text { 1-Decanol } \\
\text { 2,2,4-Trimethyl-1,3-pentanediol } \\
\text { monoisobutyrates (Texanol) } \\
\text { 2,6-Di-tert-butyl-4-methylphenol } \\
\text { (BHT) }\end{array}$ & & & & $\begin{array}{l}+ \\
+ \\
+\end{array}$ & + & $\begin{array}{l}+ \\
+ \\
+\end{array}$ & + & + & + \\
\hline $\begin{array}{l}\text { Nitrogen-Containing Compounds } \\
\text { N,N-Dimethylacetamide } \\
\text { N,N-Dimethylbenzylamine } \\
\text { N,N-Dimethylacrylamide }\end{array}$ & & & & + & & + & & $\begin{array}{l}+ \\
+\end{array}$ & \\
\hline
\end{tabular}


Table D-14, Continued. Summary of target VOCs for screening measurements with carpet materials.

\begin{tabular}{|c|c|c|c|c|c|c|c|c|c|}
\hline COMPOUND & CP1 & $\mathrm{CP} 3$ & $\mathrm{CP} 2$ & $\mathrm{CP} 4$ & $\begin{array}{l}\text { ST \& } \\
\text { CP3 }\end{array}$ & $\mathrm{CC} 1$ & $\mathrm{CC} 2$ & $\mathrm{CC} 4$ & $\mathrm{CC} 3$ \\
\hline $\begin{array}{l}\text { Nitrogen-Containing Comp } \\
\text { 2-Methylene glutaronitrile } \\
\text { 2,2'-Azobisisobutyronitrile } \\
\text { Caprolacatam } \\
\end{array}$ & & & & + & & & & + & + \\
\hline $\begin{array}{l}\text { Miscellaneous Compounds } \\
\text { Triethylphosphate }\end{array}$ & & & & & & + & & & \\
\hline
\end{tabular}


Table D-15. Chamber concentrations of target VOCs for 48-h screening measurement of Carpet CP1-a.

\begin{tabular}{|c|c|c|c|c|}
\hline \multirow[b]{2}{*}{ Compound } & \multicolumn{4}{|c|}{ Chamber Concentration, $\mu \mathrm{g} \mathrm{m}^{-3}$} \\
\hline & 1-h & $6-h$ & 24-h & 48-h \\
\hline \multicolumn{5}{|l|}{ Alkane Hydrocarbons } \\
\hline n-Dodecane & 2 & 1 & $<1$ & $<1$ \\
\hline \multicolumn{5}{|l|}{ Aromatic Hydrocarbons } \\
\hline Toluene & 11 & 3 & 1 & $<1$ \\
\hline 4-Phenylcyclohexene & 9 & 6 & 5 & 4 \\
\hline \multicolumn{5}{|l|}{ Other Hydrocarbons } \\
\hline d-Limonene & 2 & $<1$ & $<1$ & $<1$ \\
\hline \multicolumn{5}{|l|}{ Carbonyl Compounds } \\
\hline Nonanal & 5 & 2 & $<2$ & $<2$ \\
\hline 1-Phenylethanone & $<1$ & 1 & $<1$ & $<1$ \\
\hline Decanal & 5 & 2 & 2 & 2 \\
\hline
\end{tabular}

Table D-16. Chamber concentrations of target VOCs for 48 -h screening measurement of Carpet CP1-b.

\begin{tabular}{|c|c|c|c|c|}
\hline \multirow[b]{2}{*}{ Compound } & \multicolumn{4}{|c|}{ Chamber Concentration, $\mu \mathrm{g} \mathrm{m}^{-3}$} \\
\hline & 1-h & 6-h & 24-h & 48-h \\
\hline \multicolumn{5}{|l|}{ Alkane Hydrocarbons } \\
\hline n-Dodecane & 2 & 1 & $<1$ & $<1$ \\
\hline \multicolumn{5}{|l|}{ Aromatic Hydrocarbons } \\
\hline Toluene & 9 & 3 & 1 & $<1$ \\
\hline 4-Phenylcyclohexene & 8 & 5 & 4 & 4 \\
\hline \multicolumn{5}{|l|}{ Other Hydrocarbons } \\
\hline d-Limonene & 2 & $<1$ & $<1$ & $<1$ \\
\hline \multicolumn{5}{|l|}{ Carbonyl Compounds } \\
\hline Nonanal & 4 & 2 & $<2$ & $<2$ \\
\hline 1-Phenylethanone & 1 & 1 & $<1$ & $<1$ \\
\hline Decanal & $<2$ & $<2$ & 2 & $<2$ \\
\hline
\end{tabular}


Table D-17. Chamber concentrations of target VOCs for 48-h screening measurement of Carpet CP3.

\begin{tabular}{|c|c|c|c|c|}
\hline \multirow[b]{2}{*}{ Compound } & \multicolumn{4}{|c|}{ Chamber Concentration, $\mu \mathrm{g} \mathrm{m}-3$} \\
\hline & $1-h^{*}$ & 6-h & 24-h & 48-h \\
\hline \multicolumn{5}{|l|}{ Aromatic Hydrocarbons } \\
\hline Styrene & --- & 2 & $<1$ & $<1$ \\
\hline 4-Phenylcyclohexene & -- & 7 & 18 & 6 \\
\hline \multicolumn{5}{|l|}{ Carbonyl Compounds } \\
\hline Nonanal & --- & $<3$ & $<3$ & $<3$ \\
\hline Decanal & --- & $<3$ & $<3$ & $<3$ \\
\hline
\end{tabular}

*Missing data; sample lost during analysis.

Table D-18. Chamber concentrations of target VOCs for 48-h screening measurement of Carpet CP2.

\begin{tabular}{|c|c|c|c|c|}
\hline \multirow[b]{2}{*}{ Compound } & \multicolumn{4}{|c|}{ Chamber Concentration, $\mu \mathrm{g} \mathrm{m} \mathrm{m}^{-3}$} \\
\hline & 1-h & 6-h & 24-h & 48-h \\
\hline \multicolumn{5}{|l|}{ Aromatic Hydrocarbons } \\
\hline Toluene & 78 & 18 & 2 & $<1$ \\
\hline Ethylbenzene & 34 & 11 & 2 & $<1$ \\
\hline m-,p-Xylene & 99 & 39 & 7 & 1 \\
\hline o-Xylene & 32 & 11 & 3 & $<1$ \\
\hline Stryene & 66 & 25 & 4 & $<1$ \\
\hline Propylbenzene & 10 & 4 & 2 & $<1$ \\
\hline 4-Phenylcyclohexene & 27 & 20 & 17 & 14 \\
\hline \multicolumn{5}{|c|}{ Halogenated Compounds } \\
\hline Tetrachloroethene & 62 & 114 & 22 & 5 \\
\hline \multicolumn{5}{|l|}{ Carbonyl Compounds } \\
\hline Nonanal & 4 & $<5$ & $<3$ & $<3$ \\
\hline 1-Phenylethanone & $<1$ & 2 & 1 & 1 \\
\hline Decanal & 5 & $<5$ & 3 & 6 \\
\hline
\end{tabular}


Table D-19. Chamber concentrations of target VOCs for 48-h screening measurement of Carpet CP4.

\begin{tabular}{|c|c|c|c|c|}
\hline \multirow[b]{2}{*}{ Compound } & \multicolumn{4}{|c|}{ Chamber Concentration, $\mu \mathrm{g} \mathrm{m}$} \\
\hline & 1-h & $6-h$ & 24-h & 48-h \\
\hline \multicolumn{5}{|l|}{ Alkane Hydrocarbons } \\
\hline n-Undecane & 39 & 23 & 24 & 9 \\
\hline \multicolumn{5}{|l|}{ Aromatic Hydrocarbons } \\
\hline Stryene & 93 & 18 & 5 & 1 \\
\hline 4-Phenylcyclohexene & 19 & 17 & 26 & 13 \\
\hline \multicolumn{5}{|l|}{ Other Hydrocarbons } \\
\hline 4-Ethenylcyclohexene & 73 & 12 & 5 & 3 \\
\hline \multicolumn{5}{|l|}{ Carbonyl Compounds } \\
\hline Nonanal & 8 & 6 & 3 & 4 \\
\hline Decanal & $<3$ & 15 & 6 & 7 \\
\hline \multicolumn{5}{|l|}{ Other Oxidized Cmpds. } \\
\hline $\begin{array}{l}\text { Di(propylene glycol)methyl } \\
\text { ether isomers }\end{array}$ & 90 & 59 & 27 & 15 \\
\hline Phenol & 6 & 5 & 4 & 2 \\
\hline Phenethyl alcohol & 5 & 4 & 3 & 2 \\
\hline 1-Decanol & 5 & 3 & 4 & 1 \\
\hline \multicolumn{5}{|l|}{$\begin{array}{l}\text { Nitrogen-Containing } \\
\text { Cmpds }\end{array}$} \\
\hline N,N-Dimethylacetamide & 43 & 24 & 11 & 10 \\
\hline 2-Methyleneglutaronitrile & 44 & 37 & 24 & 15 \\
\hline
\end{tabular}


Table D-20. Chamber concentrations of target VOCs for 48-h screening measurement of Seaming Tape ST and Carpet CP3.

\begin{tabular}{|c|c|c|c|c|}
\hline \multirow[b]{2}{*}{ Compound } & \multicolumn{4}{|c|}{ Chamber Concentration, $\mu \mathrm{g} \mathrm{m}^{-3}$} \\
\hline & 1-h & 6-h & 24-h & 48-h \\
\hline \multicolumn{5}{|l|}{ Alkane Hydrocarbons } \\
\hline n-Decane & 4 & 2 & 2 & $<1$ \\
\hline \multicolumn{5}{|l|}{ Aromatic Hydrocarbons } \\
\hline Styrene & 5 & 2 & $<1$ & $<1$ \\
\hline 4-Phenylcyclohexene & 12 & 8 & 6 & 7 \\
\hline \multicolumn{5}{|l|}{ Carbonyl Compounds } \\
\hline Nonanal & 3 & $<3$ & $<3$ & $<3$ \\
\hline Decanal & $<3$ & $<3$ & $<3$ & $<3$ \\
\hline \multicolumn{5}{|l|}{ Other Oxidized Cmpds } \\
\hline $\begin{array}{l}\text { 2,6-Di-tert-butyl-4-methyl- } \\
\text { phenol (BHT) }\end{array}$ & 3 & $<1$ & 2 & 5 \\
\hline
\end{tabular}

Table D-21. Chamber concentrations of target VOCs for 48-h screening measurement of CP Blank.

\begin{tabular}{|c|c|c|c|c|}
\hline \multirow[b]{2}{*}{ Compound } & \multicolumn{4}{|c|}{ Chamber Concentration, $\mu \mathrm{g} \mathrm{m}^{-3}$} \\
\hline & $1-h$ & 6-h & 24-h & 48-h \\
\hline \multicolumn{5}{|c|}{ Carbonyl Compounds } \\
\hline Nonanal & $<3$ & $<3$ & $<3$ & $<3$ \\
\hline Decanal & $<3$ & $<3$ & $<3$ & $<3$ \\
\hline
\end{tabular}


Table D-22. Chamber concentrations of target VOCs for 48-h screening measurement of Carpet Cushion CC1.

\begin{tabular}{|c|c|c|c|c|}
\hline \multirow[b]{2}{*}{ Compound } & \multicolumn{4}{|c|}{ Chamber Concentration, $\mu \mathrm{g} \mathrm{m} \mathrm{m}^{-3}$} \\
\hline & $1-\mathrm{h}$ & 6-h & 24-h & 48-h \\
\hline \multicolumn{5}{|l|}{ Alkane Hydrocarbons } \\
\hline n-Undecane & 2 & 2 & 1 & 1 \\
\hline $\mathrm{n}$-Tridecane & 3 & 2 & 2 & 2 \\
\hline $\mathrm{n}$-Tetradecane & 2 & 2 & 1 & 1 \\
\hline \multicolumn{5}{|l|}{ Aromatic Hydrocarbons } \\
\hline Toluene & 5 & 1 & $<1$ & $<1$ \\
\hline m-,p-Xylene & 3 & 2 & $<1$ & $<1$ \\
\hline Propylbenzene & 4 & 3 & 1 & $<1$ \\
\hline 1,3,5-Trimethylbenzene & 5 & 4 & 1 & $<1$ \\
\hline 2-Ethyltoluene & 4 & 3 & 1 & $<1$ \\
\hline 1,2,4-Trimethylbenzene & 8 & 6 & 3 & 1 \\
\hline 1,2,3-Trimethylbenzene & 5 & 3 & 2 & 2 \\
\hline 1,3-Diethylbenzene & 4 & 3 & 2 & 1 \\
\hline Naphthalene & 15 & 10 & 8 & 9 \\
\hline 2-Methylnaphthalene & 16 & 23 & 10 & 10 \\
\hline 1-Methylnaphthalene & 8 & 8 & 6 & 6 \\
\hline \multicolumn{5}{|l|}{ Carbonyl Compounds } \\
\hline 1-Phenylethanone & 2 & 2 & 1 & $<1$ \\
\hline \multicolumn{5}{|l|}{ Other Oxidized Cmpds } \\
\hline $\begin{array}{l}\text { Pendanedioic acid, dimethyl } \\
\text { ester }\end{array}$ & 4 & 3 & 2 & 3 \\
\hline $\begin{array}{l}\text { 2,2,4-Trimethyl-1,3- } \\
\text { pentanediol } \\
\text { monoisobutyrates }\end{array}$ & 11 & 11 & 11 & 11 \\
\hline $\begin{array}{l}\text { 2,6-Di-tert-butyl-4-methyl- } \\
\text { phenol (BHT) }\end{array}$ & 37 & 62 & 31 & 35 \\
\hline \multicolumn{5}{|l|}{ Nitrogen-Containing Cmpds } \\
\hline N,N-Dimethylbenzylamine & 7 & 60 & 40 & 33 \\
\hline \multicolumn{5}{|l|}{ Miscellaneous Cmpds } \\
\hline Triethylphosphate & 33 & 69 & 48 & 45 \\
\hline
\end{tabular}


Table D-23. Chamber concentrations of target VOCs for 48-h screening measurement of Carpet Cushion CC2-a.

\begin{tabular}{|c|c|c|c|c|}
\hline \multirow[b]{2}{*}{ Compound } & \multicolumn{4}{|c|}{ Chamber Concentration, $\mu \mathrm{g} \mathrm{m}-3$} \\
\hline & $1-h$ & 6-h & 24-h & 48-h \\
\hline \multicolumn{5}{|l|}{ Alkane Hydrocarbons } \\
\hline n-Dodecane & 2 & 1 & $<1$ & $<1$ \\
\hline \multicolumn{5}{|l|}{ Other Hydrocarbons } \\
\hline d-Limonene & 2 & 1 & 1 & $<1$ \\
\hline \multicolumn{5}{|l|}{ Halogenated Compounds } \\
\hline 1,2-Dichlorobenzene & 3 & 2 & 1 & $<1$ \\
\hline \multicolumn{5}{|l|}{ Carbonyl Compounds } \\
\hline Cyclohexanone & 2 & 2 & $<1$ & $<1$ \\
\hline Nonanal & 3 & $<3$ & $<3$ & $<3$ \\
\hline \multicolumn{5}{|l|}{ Other Oxidized Cmpds } \\
\hline $\begin{array}{l}\text { 2,6-Di-tert-butyl-4-methyl- } \\
\text { phenol (BHT) }\end{array}$ & 26 & 62 & 56 & 64 \\
\hline
\end{tabular}

Table D-24. Chamber concentrations of target VOCs for 48-h screening measurement of Carpet Cushion CC2-b.

\begin{tabular}{|c|c|c|c|c|}
\hline \multirow[b]{2}{*}{ Compound } & \multicolumn{4}{|c|}{ Chamber Concentration, $\mu \mathrm{g} \mathrm{m}^{-3}$} \\
\hline & $1-\mathrm{h}$ & 6-h & 24-h & 48-h \\
\hline \multicolumn{5}{|l|}{ Alkane Hydrocarbons } \\
\hline n-Dodecane & 2 & 1 & 1 & $<1$ \\
\hline \multicolumn{5}{|l|}{ Other Hydrocarbons } \\
\hline d-Limonene & 2 & 1 & $<1$ & $<1$ \\
\hline \multicolumn{5}{|l|}{ Halogenated Compounds } \\
\hline 1,2-Dichlorobenzene & 3 & 2 & 1 & 1 \\
\hline \multicolumn{5}{|l|}{ Carbonyl Compounds } \\
\hline Cyclohexanone & 4 & 2 & $<1$ & $<1$ \\
\hline Nonanal & 3 & 4 & $<3$ & $<3$ \\
\hline \multicolumn{5}{|l|}{ Other Oxidized Cmpds } \\
\hline $\begin{array}{l}\text { 2,6-Di-tert-butyl-4-methyl- } \\
\text { phenol (BHT) }\end{array}$ & 36 & 74 & 74 & 76 \\
\hline
\end{tabular}


Table D-25. Chamber concentrations of target VOCs for 48 -h screening measurement of Carpet Cushion CC4.

\begin{tabular}{|c|c|c|c|c|}
\hline \multirow[b]{2}{*}{ Compound } & \multicolumn{4}{|c|}{ Chamber Concentration, $\mu \mathrm{g} \mathrm{m}^{-3}$} \\
\hline & 1-h & 6-h & 24-h & 48-h \\
\hline \multicolumn{5}{|l|}{ Halogenated Compounds } \\
\hline 1,2-Dichlorobenzene & 5 & 4 & 2 & 1 \\
\hline \multicolumn{5}{|l|}{ Carbonyl Compounds } \\
\hline Nonanal & $<6$ & $<6$ & $<6$ & $<6$ \\
\hline Decanal & $<6$ & $<6$ & 8 & $<6$ \\
\hline \multicolumn{5}{|l|}{ Other Oxidized Cmpds } \\
\hline Phenol & 7 & 6 & 7 & 6 \\
\hline $\begin{array}{l}\text { 2,6-Di-tert-butyl-4-methyl- } \\
\text { phenol (BHT) }\end{array}$ & 73 & 43 & 46 & 80 \\
\hline \multicolumn{5}{|c|}{ Nitrogen-Containing Cmpds } \\
\hline N,N-Dimethylbenzylamine & 29 & 39 & 31 & 17 \\
\hline N,N-Dimethylacrylamide & 2 & 2 & 2 & 2 \\
\hline 2,2'-Azobisisobutyronitrile & 95 & 79 & 61 & 36 \\
\hline
\end{tabular}


Table D-26. Chamber concentrations of target VOCs for 48-h screening measurement of Carpet Cushion CC3.

\begin{tabular}{|c|c|c|c|c|}
\hline \multirow[b]{2}{*}{ Compound } & \multicolumn{4}{|c|}{ Chamber Concentration, $\mu \mathrm{g} \mathrm{m}^{-3}$} \\
\hline & 1-h & 6-h & 24-h & 48-h \\
\hline \multicolumn{5}{|l|}{ Aromatic Hydrocarbons } \\
\hline 1,3,5-Trimethylbenzene & 3 & 1 & $<1$ & $<1$ \\
\hline 1,2,4-Trimethylbenzene & 6 & 2 & $<1$ & $<1$ \\
\hline 1,2,3-Trimethylbenzene & 3 & 1 & $<1$ & $<1$ \\
\hline Naphthalene & 2 & $<1$ & $<1$ & $<1$ \\
\hline 2-Methylnaphthalene & 2 & 2 & 1 & $<1$ \\
\hline 4-Phenylcyclohexene & 3 & 3 & 2 & 2 \\
\hline \multicolumn{5}{|l|}{ Halogenated Compounds } \\
\hline 1,1,1-Trichloroethane & 16 & 4 & 1 & $<1$ \\
\hline \multicolumn{5}{|l|}{ Carbonyl Compounds } \\
\hline Nonanal & $<3$ & $<3$ & $<3$ & 3 \\
\hline Decanal & $<3$ & 4 & $<3$ & 8 \\
\hline \multicolumn{5}{|l|}{ Other Oxidized Cmpds } \\
\hline 2-Ethyl-1-hexanol & 9 & 4 & 2 & 2 \\
\hline \multicolumn{5}{|c|}{ Nitrogen-Containing Cmpds } \\
\hline Caprolactam & $<1$ & 2 & 1 & 2 \\
\hline
\end{tabular}

Table D-27. Chamber concentrations of target VOCs for 48-h screening measurement of CC Blank.

\begin{tabular}{lrrrr}
\hline & \multicolumn{5}{c}{ Chamber Concentration $(\mu \mathrm{g} \mathrm{m}-3)$} \\
Compound & $\mathbf{1 h}$ & $\mathbf{6 h}$ & $\mathbf{2 4 h}$ & $\mathbf{4 h ~ h}$ \\
\hline \hline & & & & \\
Carbonyl Compounds & $<3$ & $<3$ & $<3$ & 3 \\
Nonanal & 4 & $<3$ & $<3$ & 7 \\
Decanal & & & & \\
\hline
\end{tabular}


Table D-28. Chamber concentrations of TVOC for $48-\mathrm{h}$ screening measurements of carpet materials.

\begin{tabular}{lrrrr}
\hline & \multicolumn{4}{c}{ Chamber Concentration $\left(\mu \mathrm{g} \mathrm{m}^{-3}\right)$} \\
Material ID & $\mathbf{1 ~ h}$ & $\mathbf{6 h}$ & $\mathbf{2 4} \mathbf{h}$ & $\mathbf{4 8 ~ h}$ \\
\hline Carpets & & & & \\
CP1-a & 440 & 230 & 115 & 103 \\
CP1-b & 390 & 210 & 103 & 92 \\
CP3 & md* & 260 & 101 & 74 \\
CP2 & 5,000 & 2,700 & 1,330 & 830 \\
CP4 & 1,140 & 967 & 439 & 351 \\
CP Blank Run & $<20$ & $<20$ & $<20$ & $<20$ \\
Seaming Tape & & & & \\
ST \& CP3 & 1,830 & 700 & 470 & 230 \\
Cushions & & & & \\
CC1 & 1,150 & 1,150 & 680 & 650 \\
CC2-a & 1,690 & 1,370 & 1,050 & 940 \\
CC2-b & 1,920 & 1,750 & 1,620 & 1,430 \\
CC4 & 1,920 & 1,910 & 1,580 & 1,160 \\
CC3 & 610 & 280 & 135 & 138 \\
CC Blank Run & 27 & 29 & 37 & $<20$ \\
\hline
\end{tabular}

${ }^{*} \mathrm{md}=$ Missing data. 
Table D-29. Chamber concentrations of SigmaVOC (i.e., sum of target VOCs) for 48-h screening measurements of carpet materials.

\begin{tabular}{|c|c|c|c|c|}
\hline \multirow[b]{2}{*}{ Material ID } & \multicolumn{4}{|c|}{ Chamber Concentration $\left(\mu \mathrm{g} \mathrm{m}^{-3}\right)$} \\
\hline & $1 \mathrm{~h}$ & $6 \mathrm{~h}$ & $24 \mathrm{~h}$ & $48 \mathrm{~h}$ \\
\hline \multicolumn{5}{|l|}{ Carpets } \\
\hline CP1-a & 34 & 16 & 10 & 9 \\
\hline CP1-b & 26 & 13 & 9 & 8 \\
\hline CP3 & $\mathrm{md}^{*}$ & 12 & 21 & 9 \\
\hline $\mathrm{CP} 2$ & 416 & 249 & 63 & 30 \\
\hline $\mathrm{CP} 4$ & 426 & 224 & 141 & 82 \\
\hline CP Blank Run & 3 & 3 & 3 & 3 \\
\hline \multicolumn{5}{|l|}{ Seaming Tape } \\
\hline ST \& CP3 & 27 & 15 & 13 & 16 \\
\hline \multicolumn{5}{|l|}{ Cushions } \\
\hline $\mathrm{CC} 1$ & 174 & 275 & 168 & 160 \\
\hline $\mathrm{CC} 2-\mathrm{a}$ & 36 & 69 & 60 & 68 \\
\hline $\mathrm{cC} 2-\mathrm{b}$ & 50 & 84 & 79 & 79 \\
\hline CC4 & 217 & 179 & 160 & 147 \\
\hline $\mathrm{CC} 3$ & 46 & 22 & 12 & 19 \\
\hline CC Blank Run & 6 & 3 & 3 & 10 \\
\hline
\end{tabular}

${ }^{*} \mathrm{md}=$ Missing data. 
Table D-30. Chamber concentrations of formaldehyde for 48-h screening measurements of carpet materials.

\begin{tabular}{|c|c|c|c|c|}
\hline \multirow[b]{2}{*}{ Material ID } & \multicolumn{4}{|c|}{ Chamber Concentration ( $\mu \mathrm{g} \mathrm{m}-3$ ) } \\
\hline & $1 \mathrm{~h}$ & $6 \mathrm{~h}$ & $24 \mathrm{~h}$ & $48 h$ \\
\hline \multicolumn{5}{|l|}{ Carpets } \\
\hline CP1-a & 1 & 2 & 2 & $<1$ \\
\hline$C P 1-b$ & 2 & 3 & 1 & 1 \\
\hline CP3 & 3 & 2 & 1 & 1 \\
\hline $\mathrm{CP} 2$ & 6 & 3 & 1 & 1 \\
\hline $\mathrm{CP} 4$ & 3 & $<1$ & $<1$ & $<1$ \\
\hline CP Blank Run & $<1$ & $<1$ & $<1$ & $<1$ \\
\hline \multicolumn{5}{|l|}{ Seaming Tape } \\
\hline ST \& CP3 & 9 & 7 & 2 & 2 \\
\hline \multicolumn{5}{|l|}{ Cushions } \\
\hline $\mathrm{CC} 1$ & 1 & 2 & 1 & 1 \\
\hline $\mathrm{CC} 2-\mathrm{a}$ & 2 & 2 & $<1$ & 1 \\
\hline$c c 2-b$ & 2 & 3 & $<1$ & 1 \\
\hline $\mathrm{CC} 4$ & 3 & 3 & 3 & 2 \\
\hline $\mathrm{CC} 3$ & 1 & 1 & 1 & $<1$ \\
\hline CC Blank Run & $<1$ & $<1$ & $<1$ & $<1$ \\
\hline
\end{tabular}


Table D-31. Chamber concentrations of acetaldehyde for 48-h screening measurements of carpet materials.

\begin{tabular}{|c|c|c|c|c|}
\hline \multirow[b]{2}{*}{ Material ID } & \multicolumn{4}{|c|}{ Chamber Concentration ( $\mu \mathrm{g} \mathrm{m}^{-3}$ ) } \\
\hline & $1 \mathrm{~h}$ & $6 \mathrm{~h}$ & $24 \mathrm{~h}$ & $48 h$ \\
\hline \multicolumn{5}{|l|}{ Carpets } \\
\hline CP1-a & 2 & 1 & 1 & 1 \\
\hline CP1-b & 1 & 1 & $<1$ & $<1$ \\
\hline CP3 & 2 & 1 & 1 & 1 \\
\hline $\mathrm{CP} 2$ & 6 & 3 & 2 & 2 \\
\hline $\mathrm{CP} 4$ & 5 & 3 & 2 & 1 \\
\hline CP Blank Run & $<1$ & $<1$ & $<1$ & $<1$ \\
\hline \multicolumn{5}{|l|}{ Seaming Tape } \\
\hline $\mathrm{ST} \& \mathrm{CP} 3$ & 3 & 3 & 2 & 2 \\
\hline \multicolumn{5}{|l|}{ Cushions } \\
\hline $\mathrm{CC} 1$ & 4 & 1 & 1 & 1 \\
\hline $\mathrm{CC} 2-\mathrm{a}$ & 33 & 10 & 9 & 1 \\
\hline$C C 2-b$ & 19 & 4 & 1 & 2 \\
\hline $\mathrm{CC} 4$ & 18 & 6 & 5 & 4 \\
\hline $\mathrm{CC} 3$ & 2 & $<1$ & 1 & $<1$ \\
\hline CC Blank Run & $<1$ & $<1$ & $<1$ & $<1$ \\
\hline
\end{tabular}


Table D-32. Quasi steady-state emission rates of target VOCs at 24- and 48-h elapsed time for screening measurement of Carpet CP1-a.

\begin{tabular}{|c|c|c|}
\hline \multirow[b]{2}{*}{ Compound } & \multicolumn{2}{|c|}{$\begin{array}{c}\text { Specific Emission Rate, } \\
\mu \mathrm{g} \mathrm{m} \mathrm{m}^{-2} \mathrm{~h}^{-1}\end{array}$} \\
\hline & 24-h & 48-h \\
\hline \multicolumn{3}{|l|}{ Alkane Hydrocarbons } \\
\hline n-Dodecane & $<3$ & $<3$ \\
\hline \multicolumn{3}{|l|}{ Aromatic Hydrocarbons } \\
\hline Toluene & 3 & $<3$ \\
\hline 4-Phenylcyclohexene & 12 & 11 \\
\hline \multicolumn{3}{|l|}{ Other Hydrocarbons } \\
\hline d-Limonene & $<3$ & $<3$ \\
\hline \multicolumn{3}{|l|}{ Carbonyl Compounds } \\
\hline 1-Phenylethanone & $<3$ & $<3$ \\
\hline
\end{tabular}

Table D-33. Quasi steady-state emission rates of target VOCs at 24- and 48-h elapsed time for screening measurement of Carpet CP1-b.

\begin{tabular}{|c|c|c|}
\hline \multirow[b]{2}{*}{ Compound } & \multicolumn{2}{|c|}{$\begin{array}{c}\text { Specific Emission Rate, } \\
\mu \mathrm{g} \mathrm{m}^{-2} \mathrm{~h}^{-1}\end{array}$} \\
\hline & 24-h & 48-h \\
\hline \multicolumn{3}{|l|}{ Alkane Hydrocarbons } \\
\hline n-Dodecane & $<3$ & $<3$ \\
\hline \multicolumn{3}{|l|}{ Aromatic Hydrocarbons } \\
\hline Toluene & 3 & $<3$ \\
\hline 4-Phenylcyclohexene & 10 & 10 \\
\hline \multicolumn{3}{|l|}{ Other Hydrocarbons } \\
\hline d-Limonene & $<3$ & $<3$ \\
\hline \multicolumn{3}{|l|}{ Carbonyl Compounds } \\
\hline 1-Phenylethanone & $<3$ & $<3$ \\
\hline
\end{tabular}


Table D-34. Quasi steady-state emission rates of target VOCs at 24- and 48-h elapsed time for screening measurement of Carpet CP3.

\begin{tabular}{lcc}
\hline & $\begin{array}{c}\text { Specific Emission Rate, } \\
\mu \mathrm{g} \mathrm{m}^{-2} \mathrm{~h}^{-1}\end{array}$ \\
\hline Compound & $24-\mathrm{h}$ & \multicolumn{2}{c}{$48-\mathrm{h}$} \\
\hline Aromatic Hydrocarbons & $<3$ & $<3$ \\
Styrene & 45 & 14 \\
4-Phenyicyclohexene & & \\
\hline
\end{tabular}

Table D-35. Quasi steady-state emission rates of target VOCs at 24- and 48-h elapsed time for screening measurement of Carpet CP2.

\begin{tabular}{lrr}
\hline & $\begin{array}{c}\text { Specific Emission Rate, } \\
\mu \mathrm{m} \mathrm{m}^{-2} \mathrm{~h}^{-1}\end{array}$ & \\
Compound & $24-\mathrm{h}$ & $<3$ \\
\hline Aromatic Hydrocarbons & 4 & $<3$ \\
$\quad$ Toluene & 5 & 3 \\
Ethylbenzene & 18 & $<3$ \\
m-,p-Xylene & 6 & $<3$ \\
o-Xylene & 10 & $<3$ \\
Styrene & 4 & 36 \\
Propylbenzene & 44 & \\
4-Phenylcyclohexene & & 12 \\
Halogenated Compounds & & \\
$\quad$ Tetrachloroethene & 56 & 3 \\
Carbonyl Compounds & & \\
1-Phenylethanone & 3 & \\
\hline
\end{tabular}


Table D-36. Quasi steady-state emission rates of target VOCs at 24- and 48-h elapsed time for screening measurement of Carpet CP4.

\begin{tabular}{|c|c|c|}
\hline \multirow[b]{2}{*}{ Compound } & \multicolumn{2}{|c|}{$\begin{array}{c}\text { Specific Emission Rate } \\
\mu \mathrm{g} \mathrm{m}^{-2} \mathrm{~h}^{-1}\end{array}$} \\
\hline & 24-h & 48-h \\
\hline \multicolumn{3}{|l|}{ Alkane Hydrocarbons } \\
\hline n-Undecane & 61 & 23 \\
\hline \multicolumn{3}{|l|}{ Aromatic Hydrocarbons } \\
\hline Styrene & 12 & 4 \\
\hline 4-Phenylcyclohexene & 68 & 33 \\
\hline \multicolumn{3}{|l|}{ Other Hydrocarbons } \\
\hline 4-Ethenylcyclohexene & 13 & 7 \\
\hline \multicolumn{3}{|l|}{ Other Oxidized Compounds } \\
\hline Di(propylene glycol)methyl ethers & 70 & 39 \\
\hline Phenol & 9 & 6 \\
\hline Phenethyl alcohol & 8 & 6 \\
\hline 1-Decanol & 11 & 2 \\
\hline \multicolumn{3}{|l|}{ Nitrogen-Containing Compounds } \\
\hline $\mathrm{N}, \mathrm{N}$-Dimethylacetamide & 29 & 26 \\
\hline 2-Methyleneglutaronitrile & 61 & 40 \\
\hline
\end{tabular}


Table D-37. Quasi steady-state emission rates of target VOCs at 24- and 48-h elapsed time for screening measurement of Seaming Tape ST and Carpet CP3.

\begin{tabular}{|c|c|c|}
\hline \multirow[b]{2}{*}{ Compound } & \multicolumn{2}{|c|}{$\begin{array}{c}\text { Specific Emission Rate, } \\
\qquad \mathrm{g} \mathrm{m} \mathrm{m}^{-2} \mathrm{~h}^{-1}\end{array}$} \\
\hline & 24-h & 48-h \\
\hline \multicolumn{3}{|l|}{ Alkane Hydrocarbons } \\
\hline n-Decane & 4 & $<3$ \\
\hline \multicolumn{3}{|l|}{ Aromatic Hydrocarbons } \\
\hline Styrene & $<3$ & $<3$ \\
\hline 4-Phenylcyclohexene & 16 & 18 \\
\hline \multicolumn{3}{|l|}{ Other Oxidized Compounds } \\
\hline 2,6-Di-tert-butyl-4-methyiphenol & 5 & 13 \\
\hline
\end{tabular}


Table D-38. Quasi steady-state emission rates of target VOCs at 24- and 48-h elapsed time for screening measurement of Carpet Cushion CC1.

\begin{tabular}{|c|c|c|}
\hline \multirow[b]{2}{*}{ Compound } & \multicolumn{2}{|c|}{$\begin{array}{l}\text { Specific Emission Rate } \\
\mu \mathrm{g} \mathrm{m} \mathrm{m}^{-2} \mathrm{~h}-1\end{array}$} \\
\hline & 24-h & 48-h \\
\hline \multicolumn{3}{|l|}{ Alkane Hydrocarbons } \\
\hline n-Undecane & 3 & 3 \\
\hline$n$-Tridecane & 4 & 4 \\
\hline $\mathrm{n}$-Tetradecane & 3 & 3 \\
\hline \multicolumn{3}{|l|}{ Aromatic Hydrocarbons } \\
\hline Toluene & $<3$ & $<3$ \\
\hline m-,p-Xylene & $<3$ & $<3$ \\
\hline Propylbenzene & 3 & $<3$ \\
\hline 1,3,5-Trimethylbenzene & 3 & $<3$ \\
\hline 2-Ethyltoluene & 3 & $<3$ \\
\hline 1,2,4-Trimethylbenzene & 7 & 3 \\
\hline 1,2,3-Trimethylbenzene & 4 & 5 \\
\hline 1,3-Diethylbenzene & 4 & 3 \\
\hline Naphthalene & 21 & 22 \\
\hline 2-Methylnaphthalene & 26 & 26 \\
\hline 1-Methylnaphthalene & 14 & 14 \\
\hline \multicolumn{3}{|l|}{ Carbonyl Compounds } \\
\hline 1-Phenylethanone & 3 & $<3$ \\
\hline \multicolumn{3}{|l|}{ Other Oxidized Compounds } \\
\hline Pendanedioic acid, dimethyl ester & 4 & 7 \\
\hline $\begin{array}{l}2,2,4-\text { Trimethyl-1,3-pentanediol } \\
\text { monoisobutyrates }\end{array}$ & 27 & 28 \\
\hline 2,6-Di-tert-butyl-4-methyl-phenol & 79 & 89 \\
\hline \multicolumn{3}{|l|}{ Nitrogen-Containing Compounds } \\
\hline N,N-Dimethylbenzylamine & 103 & 84 \\
\hline \multicolumn{3}{|l|}{ Miscellaneous Compounds } \\
\hline Triethylphosphate & 123 & 115 \\
\hline
\end{tabular}


Table D-39. Quasi steady-state emission rates of target VOCs at 24- and 48-h elapsed time for screening measurement of Carpet Cushion CC2-a.

\begin{tabular}{|c|c|c|}
\hline \multirow[b]{2}{*}{ Compound } & \multicolumn{2}{|c|}{$\begin{array}{c}\text { Specific Emission Rate, } \\
\mu \mathrm{q} \mathrm{m} \mathrm{m}^{-2} \mathrm{~h}^{-1}\end{array}$} \\
\hline & 24-h & 48-h \\
\hline \multicolumn{3}{|l|}{ Alkane Hydrocarbons } \\
\hline n-Dodecane & $<3$ & $<3$ \\
\hline \multicolumn{3}{|l|}{ Other Hydrocarbons } \\
\hline d-Limonene & 3 & $<3$ \\
\hline \multicolumn{3}{|l|}{ Halogenated Compounds } \\
\hline 1,2-Dichlorobenzene & 3 & $<3$ \\
\hline \multicolumn{3}{|l|}{ Carbonyl Compounds } \\
\hline Cyclohexanone & $<3$ & $<3$ \\
\hline \multicolumn{3}{|l|}{ Other Oxidized Compounds } \\
\hline 2,6-Di-tert-butyl-4-methylphenol & 144 & 166 \\
\hline
\end{tabular}

Table D-40. Quasi steady-state emission rates of target VOCs at 24- and 48-h elapsed time for screening measurement of Carpet Cushion CC2-b.

\begin{tabular}{lcc}
\hline \hline & \multicolumn{2}{c}{$\begin{array}{c}\text { Specific Emission Rate, } \\
\text { Comp m-2 h-1 }\end{array}$} \\
\hline $\begin{array}{l}\text { Alkane Hydrocarbons } \\
\text { n-Dodecane }\end{array}$ & $34-\mathrm{h}$ \\
$\begin{array}{l}\text { Other Hydrocarbons } \\
\text { d-Limonene }\end{array}$ & $<3$ \\
Halogenated Compounds & $<3$ & $<3$ \\
$\begin{array}{l}\text { 1,2-Dichlorobenzene } \\
\text { Carbonyl Compounds } \\
\text { Cyclohexanone }\end{array}$ & $<3$ & $<3$ \\
Other Oxidized Compounds \\
2,6-Di-tert-butyl-4-methylphenol
\end{tabular}


Table D-41. Quasi steady-state emission rates of target VOCs at 24- and 48-h elapsed time for screening measurement of Carpet Cushion CC4.

\begin{tabular}{|c|c|c|}
\hline \multirow[b]{2}{*}{ Compound } & \multicolumn{2}{|c|}{$\begin{array}{c}\text { Specific Emission Rate, } \\
\mu \mathrm{g} \mathrm{m} \mathrm{m}^{-2} \mathrm{~h}^{-1}\end{array}$} \\
\hline & 24-h & 48-h \\
\hline \multicolumn{3}{|l|}{ Halogenated Compounds } \\
\hline 1,2-Dichlorobenzene & 5 & 3 \\
\hline \multicolumn{3}{|l|}{ Other Oxidized Compounds } \\
\hline Phenol & 17 & 15 \\
\hline 2,6-Di-tert-butyl-4-methylphenol & 120 & 206 \\
\hline \multicolumn{3}{|l|}{ Nitrogen-Containing Compounds } \\
\hline N,N-Dimethylbenzylamine & 79 & 43 \\
\hline N,N-Dimethylacrylamide & 5 & 4 \\
\hline 2,2'-Azobisisobutyronitrile & 158 & 94 \\
\hline
\end{tabular}

Table D-42. Quasi steady-state emission rates of target VOCs at 24- and 48-h elapsed time for screening measurement of Carpet Cushion CC3.

\begin{tabular}{|c|c|c|}
\hline \multirow[b]{2}{*}{ Compound } & \multicolumn{2}{|c|}{$\begin{array}{l}\text { Specific Emission Rate, } \\
\qquad \mathrm{g} \mathrm{m}^{-2} \mathrm{~h}^{-1}\end{array}$} \\
\hline & 24-h & 48-h \\
\hline \multicolumn{3}{|l|}{ Aromatic Hydrocarbons } \\
\hline 1,3,5-Trimethylbenzene & $<3$ & $<3$ \\
\hline 1,2,4-Trimethylbenzene & $<3$ & $<3$ \\
\hline 1,2,3-Trimethylbenzene & $<3$ & $<3$ \\
\hline Naphthalene & $<3$ & $<3$ \\
\hline 2-Methylnaphthalene & 3 & $<3$ \\
\hline 4-Phenylcyclohexene & 5 & 4 \\
\hline \multicolumn{3}{|l|}{ Halogenated Compounds } \\
\hline 1,1,1-Trichloroethane & 3 & $<3$ \\
\hline \multicolumn{3}{|c|}{ Other Oxidized Compounds } \\
\hline 2-Ethyl-1-hexanol & 4 & 4 \\
\hline \multicolumn{3}{|c|}{ Nitrogen-Containing Compounds } \\
\hline Caprolactam & 3 & 4 \\
\hline
\end{tabular}


Table D-43. Quasi steady-state emission rates of TVOC at 24- and 48-hours elapsed times for screening measurements of carpet materials.

\begin{tabular}{|c|c|c|}
\hline \multirow[b]{2}{*}{ Material ID } & \multicolumn{2}{|c|}{$\begin{array}{c}\text { Specific Emission Rate } \\
\qquad \mu \mathrm{g} \mathrm{m} \mathrm{m}^{-2} \mathrm{~h}^{-1}\end{array}$} \\
\hline & 24-h & 48-h \\
\hline \multicolumn{3}{|l|}{ Carpets } \\
\hline CP1-a & 269 & 238 \\
\hline$C P 1-b$ & 238 & 210 \\
\hline CP3 & 233 & 164 \\
\hline $\mathrm{CP} 2$ & 3,380 & 2,100 \\
\hline $\mathrm{CP} 4$ & 1,100 & 873 \\
\hline \multicolumn{3}{|c|}{ Seaming Tape } \\
\hline ST \& CP3 & 1,180 & 563 \\
\hline \multicolumn{3}{|l|}{ Cushions } \\
\hline $\mathrm{CC1}$ & 1,650 & 1,640 \\
\hline $\mathrm{CC} 2-\mathrm{a}$ & 2,590 & 2,380 \\
\hline$c C 2-b$ & 4,050 & 3,640 \\
\hline $\mathrm{CC} 4$ & 3,950 & 2,940 \\
\hline $\mathrm{CC} 3$ & 251 & 328 \\
\hline
\end{tabular}


Table D-44. Quasi steady-state emission rates of formaldehyde at 24- and 48-hours elapsed times for screening measurements of carpet materials.

\begin{tabular}{|c|c|c|}
\hline \multirow[b]{2}{*}{ Material ID } & \multicolumn{2}{|c|}{$\begin{array}{l}\text { Specific Emission Rate } \\
\mu \mathrm{g} \mathrm{m}^{-2} \mathrm{~h}^{-1}\end{array}$} \\
\hline & 24-h & $48-h$ \\
\hline \multicolumn{3}{|l|}{ Carpets } \\
\hline CP1-a & 5 & $<3$ \\
\hline CP1-b & 3 & 3 \\
\hline CP3 & 3 & 3 \\
\hline $\mathrm{CP} 2$ & $<3$ & $<3$ \\
\hline $\mathrm{CP} 4$ & 3 & 3 \\
\hline \multicolumn{3}{|c|}{ Seaming Tape } \\
\hline ST \& CP3 & 5 & 5 \\
\hline \multicolumn{3}{|l|}{ Cushions } \\
\hline $\mathrm{CC} 1$ & 3 & 3 \\
\hline $\mathrm{cC} 2-\mathrm{a}$ & $<3$ & 3 \\
\hline$c C 2-b$ & $<3$ & 3 \\
\hline $\mathrm{CC} 4$ & 8 & 5 \\
\hline $\mathrm{CC} 3$ & 3 & $<3$ \\
\hline
\end{tabular}


Table D-45. Quasi steady-state emission rates of acetaldehyde at 24- and 48-hours elapsed times for screening measurements of carpet materials.

\begin{tabular}{|c|c|c|}
\hline \multirow[b]{2}{*}{ Material ID } & \multicolumn{2}{|c|}{$\begin{array}{c}\text { Specific Emission Rate } \\
\mu \mathrm{g} \mathrm{m}^{-2} \mathrm{~h}^{-1}\end{array}$} \\
\hline & 24-h & 48-h \\
\hline \multicolumn{3}{|l|}{ Carpets } \\
\hline CP1-a & 3 & 3 \\
\hline CP1-b & $<3$ & $<3$ \\
\hline CP3 & 3 & 3 \\
\hline CP2 & 5 & 5 \\
\hline $\mathrm{CP} 4$ & 5 & 3 \\
\hline \multicolumn{3}{|c|}{ Seaming Tape } \\
\hline ST \& CP3 & 5 & 5 \\
\hline \multicolumn{3}{|l|}{ Cushions } \\
\hline $\mathrm{CC} 1$ & 3 & 3 \\
\hline $\mathrm{CC} 2-\mathrm{a}$ & 23 & 3 \\
\hline$c c 2-b$ & 3 & 5 \\
\hline $\mathrm{CC} 4$ & 13 & 10 \\
\hline CC3 & 3 & $<3$ \\
\hline
\end{tabular}




\section{APPENDIX E}

\section{SCREENING MEASUREMENTS OF SHEET VINYL FLOORING MATERIALS}

Appendix E presents the analytical data for the screening measurements of the sheet vinyl flooring materials including four sheet vinyls, cove base and adhesives. The experiments were conducted in 10-L chambers over a period of 48 hours. Individual VOCs emitted by the sheet vinyl flooring materials were identified. The concentrations of selected compounds and TVOC were measured at four time intervals. Specific emission rates of these components were calculated for the 24 - and $48-\mathrm{h}$ time intervals.

\section{List of Tables}

Table E-01. Sheet vinyl flooring materials selected for study..........................................................265

Table E-02. Summary of screening measurements with sheet vinyl flooring materials .............................266

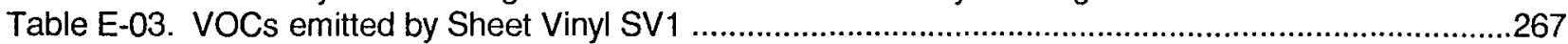

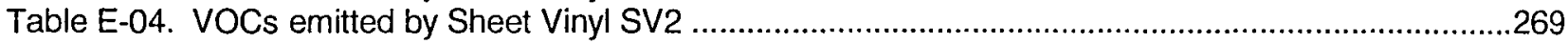

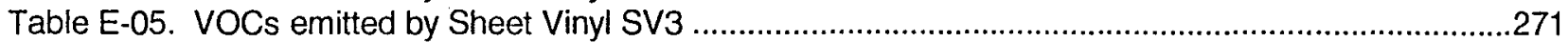

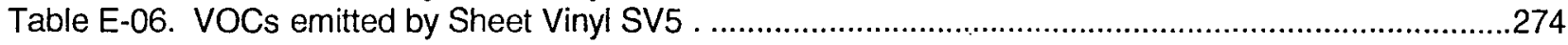

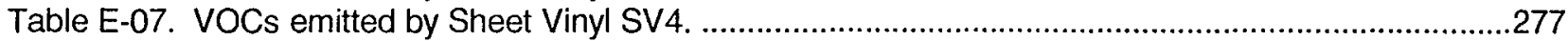

Table E-08. VOCs detected in sheet vinyl blank chamber run. ...........................................................280

Table E-09. Composite listing of VOCs emitted by Sheet Vinyls SV1 through SV5 ...............................281

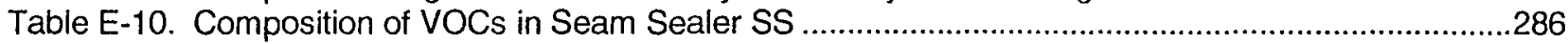

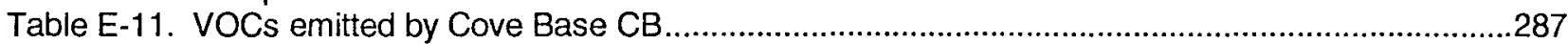

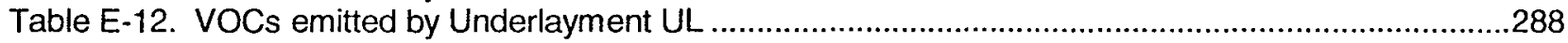

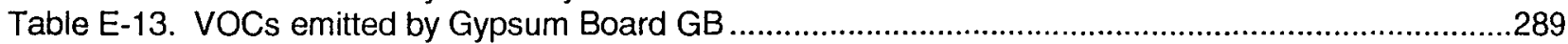

Table E-14. VOCs emitted in by Sheet Flooring Adhesive SFA …........................................................290

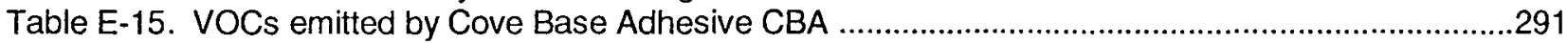

Table E-16. VOCs emitted by Sheet Vinyl SV5 and Sheet Flooring Adhesive SFA ..............................293

Table E-17. VOCs emitted by Cove Base CB and Cove Base Adhesive CBA ......................................295

Table E-18. Summary of target VOCs for sheet vinyl flooring materials .............................................297

Table E-19. Chamber concentrations of target VOCs for Sheet Vinyl SV1 .........................................299

Table E-20. Chamber concentrations of target VOCs for Sheet Vinyl SV2 .............................................300

Table E-21. Chamber concentrations of target VOCs for Sheet Vinyl SV3-a........................................301

Table E-22. Chamber concentrations of target VOCs for Sheet Vinyl SV3-b.......................................302

Table E-23. Chamber concentrations of target VOCs for Sheet Vinyl SV5 ..............................................303

Table E-24. Chamber concentrations of target VOCs for Sheet Vinyl SV4 ..........................................304

Table E-25. Chamber concentrations of target VOCs for sheet vinyl blank run. ........................................305

Table E-26. Chamber concentrations of target VOCs for Cove Base CB. ..............................................306

Table E-27. Chamber concentrations of target VOCs for Underlayment UL..........................................307

Table E-28. Chamber concentrations of target VOCs for Sheet Flooring Adhesive SFA ...........................307

Table E-29. Chamber concentrations of target VOCs for Cove Base Adhesive CBA ...............................308

Table E-30. Chamber concentrations of target VOCs for Sheet Vinyl SV5 and Sheet Flooring

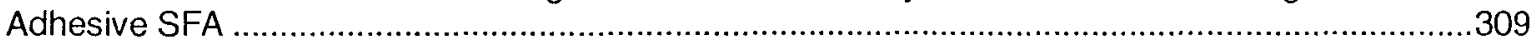

Table E-31. Chamber concentrations of target VOCs for Cove Base CB and Cove Base

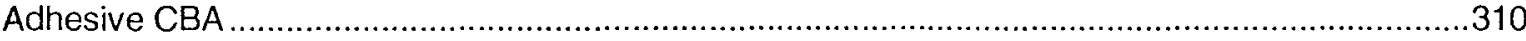

Table E-32. Chamber concentrations of TVOC for sheet vinyl flooring materials ..................................311

Table E-33. Chamber concentrations of SigmaVOC sheet vinyl flooring materials.................................312

Table E-34. Chamber concentrations of formaldehyde for sheet vinyl flooring materials............................313

Table E-35. Chamber concentrations of acetaldehyde for sheet vinyl flooring materials ..........................314

Table E-36. Quasi steady-state emission rates of target VOCs for Sheet Vinyl SV1 ...............................315

Table E-37. Quasi steady-state emission rates of target VOCs for Sheet Vinyl SV2 ...............................316

Table E-38. Quasi stead!--state emission rates of target VOCs for Sheet Vinyl SV3-a............................317 


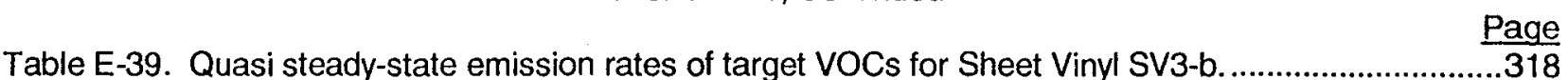

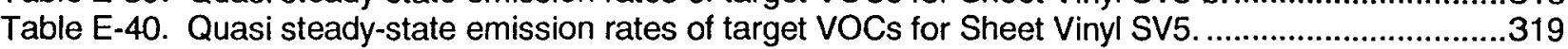

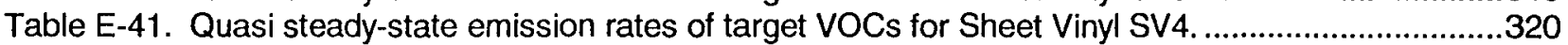

Table E-42. Quasi steady-state emission rates of target VOCs for Cove Base CB. .................................321

Table E-43. Quasi steady-state emission rates of target VOCs for Underlayment UL............................322

Table E-44. Quasi steady-state emission rates of target VOCs for Sheet Flooring Adhesive SFA...........322

Table E-45. Quasi steady-state emission rates of target VOCs for Cove Base Adhesive CBA .................323

Table E-46. Quasi steady-state emission rates of target VOCs for Sheet Vinyl SV5 and Sheet Flooring Adhesive SFA

Table E-47. Quasi steady-state emission rates of target VOCs at 24-and 48-h elapsed time for screening measurement of Cove Base CB and Cove Base Adhesive CBA. 325

Table E-48. Quasi steady-state emission rates of TVOC for sheet vinyl flooring materials .....................326

Table E-49. Quasi steady-state emission rates of formaldehyde for sheet vinyl flooring materials............327

Table E-50. Quasi steady-state emission rates of acetaldehyde for sheet vinyl flooring materials ..............328 
Table E-01. Sheet vinyl flooring materials selected for study.

\begin{tabular}{|c|c|c|c|}
\hline Material ID & $\begin{array}{c}\text { Manufacturer } \\
\text { Code } \\
\end{array}$ & $\begin{array}{c}\text { Purchase } \\
\text { Point } \\
\end{array}$ & Material Description \\
\hline \multicolumn{4}{|c|}{ Sheet Vinyls } \\
\hline SV1 & A & Dealer & $\begin{array}{l}\text { Commercial; intermediate grade; full-spread } \\
\text { adhesive application }\end{array}$ \\
\hline SV2 & A & Dealer & $\begin{array}{l}\text { Residential no wax; intermediate grade; } \\
\text { perimeter adhesive application }\end{array}$ \\
\hline SV3 & B & Dealer & $\begin{array}{l}\text { Residential no wax; intermediate grade; } \\
\text { full-spread adhesive application }\end{array}$ \\
\hline SV5*† & B & Dealer & $\begin{array}{l}\text { Residential no wax; intermediate grade; } \\
\text { full-spread adhesive application }\end{array}$ \\
\hline SV4 & A & Retail & $\begin{array}{l}\text { Residential no wax; base grade; full-spread } \\
\text { adhesive application }\end{array}$ \\
\hline \multicolumn{4}{|l|}{ Cove Base } \\
\hline $\mathrm{CB}^{\dagger}$ & $\mathrm{C}$ & Dealer & Rubber; 4-inch wide, 44-inch long strips \\
\hline \multicolumn{4}{|l|}{ Adhesives } \\
\hline SFAt & $\mathrm{D}$ & Dealer & $\begin{array}{l}\text { Multi-purpose sheet flooring adhesive; } \\
\text { "solvent free" }\end{array}$ \\
\hline CBA $^{\dagger}$ & $\mathrm{D}$ & Dealer & $\begin{array}{l}\text { White acrylic cove base adhesive; "low } \\
\text { VOC" content }\end{array}$ \\
\hline \multicolumn{4}{|l|}{ Seam sealer } \\
\hline SSt & B & Dealer & High-gloss seam sealer; 2 parts \\
\hline \multicolumn{4}{|c|}{ Underlayment } \\
\hline $\mathrm{UL}^{\dagger}$ & $E$ & Retail & $\begin{array}{l}\text { Particle board underlayment; } 3 / 8 \text {-inch thick; } \\
\text { aired out }\end{array}$ \\
\hline
\end{tabular}

*SV3 and SV5 vary with respect to color, pattern and production date, but otherwise are the same material.

tSelected for use in large-scale experiments. 
Table E-02. Summary of screening measurements with sheet vinyl flooring materials in $10-\mathrm{L}$ chambers.

\begin{tabular}{|c|c|c|c|}
\hline Materials & $\begin{array}{c}\text { Duration } \\
\text { (h) }\end{array}$ & $\begin{array}{l}\text { Material } \\
\text { Amount } \\
\end{array}$ & Experimental Procedures \\
\hline \multicolumn{4}{|l|}{ Sheet Vinyls } \\
\hline SV1 & 48 & $0.0195 \mathrm{~m}^{2}$ & $\begin{array}{l}\text { Taped onto metal plate; top } \\
\text { surface exposed }\end{array}$ \\
\hline SV2 & 48 & $0.0195 \mathrm{~m}^{2}$ & $\begin{array}{l}\text { Taped onto metal plate; top } \\
\text { surface exposed }\end{array}$ \\
\hline SV3-a & 48 & $0.0195 \mathrm{~m}^{2}$ & $\begin{array}{l}\text { Taped onto metal plate; top } \\
\text { surface exposed }\end{array}$ \\
\hline SV3-b & 48 & $0.0195 \mathrm{~m}^{2}$ & $\begin{array}{l}\text { Duplicate run; taped onto metal } \\
\text { plate; top surface exposed }\end{array}$ \\
\hline SV5 & 48 & $0.0195 \mathrm{~m}^{2}$ & $\begin{array}{l}\text { Taped onto metal plate; top } \\
\text { surface exposed }\end{array}$ \\
\hline SV4 & 48 & $0.0195 \mathrm{~m}^{2}$ & $\begin{array}{l}\text { Taped onto metal plate; top } \\
\text { surface exposed }\end{array}$ \\
\hline SV Blank Run & 48 & -- & Metal plate with aluminum tape \\
\hline \multicolumn{4}{|l|}{ Cove Base } \\
\hline $\mathrm{CB}$ & 48 & $0.229 \mathrm{~m}$ & Both sides exposed \\
\hline \multicolumn{4}{|l|}{ Substrates } \\
\hline UL & 48 & $0.0195 \mathrm{~m}^{2}$ & $\begin{array}{l}\text { Taped into metal holder; one } \\
\text { surface exposed }\end{array}$ \\
\hline $\begin{array}{l}\text { GB (Gypsum } \\
\text { Board) }\end{array}$ & 48 & $0.0195 \mathrm{~m}^{2}$ & $\begin{array}{l}\text { Taped into metal holder; top } \\
\text { surface exposed }\end{array}$ \\
\hline \multicolumn{4}{|c|}{ Adhesives on Substrates } \\
\hline SFA \& UL & 48 & $0.0070 \mathrm{~kg}$ & $\begin{array}{l}\text { SFA applied with 1/16"-notched } \\
\text { spreader, } 0.020 \mathrm{~m}^{2} \text { surface area }\end{array}$ \\
\hline CBA \& GB & 48 & $0.0083 \mathrm{~kg}$ & $\begin{array}{l}\text { CBA applied with trowel; } \\
\sim 0.012 \mathrm{~m}^{2} \text { surface area }\end{array}$ \\
\hline \multicolumn{4}{|c|}{ Composite Assemblies } \\
\hline SV5, SFA \& UL & 48 & $0.0195 \mathrm{~m}^{2}$ & $\begin{array}{l}\text { Taped into metal holder; SV } 5 \text { top } \\
\text { surface exposed; } 5.7 \mathrm{~g} \text { adhesive }\end{array}$ \\
\hline $\mathrm{CB}, \mathrm{CBA} \& \mathrm{~GB}$ & 48 & $0.114 \mathrm{~m}$ & $\begin{array}{l}\text { GB taped into metal holder; CB } \\
\text { applied with } 2.2 \mathrm{~g} \text { adhesive }\end{array}$ \\
\hline
\end{tabular}


Table E-03. VOCs emitted by Sheet Vinyl SV1 in 10-L chamber at 6- and 48-hours elapsed times.

\begin{tabular}{|c|c|c|c|c|c|}
\hline COMPOUND & Code $^{*}$ & $\begin{array}{c}\mathbf{R T} \\
(\mathrm{min})\end{array}$ & $\begin{array}{l}6-\mathrm{h} \\
\mathrm{ET} \\
\end{array}$ & $\begin{array}{l}\text { 48-h } \\
\mathrm{ET}\end{array}$ & $\begin{array}{l}\text { Match } \\
\text { Quality } \\
\end{array}$ \\
\hline \multicolumn{6}{|l|}{ Alkane Hydrocarbons } \\
\hline $\mathrm{n}$-Tridecane & $A, Q$ & 29.3 & + & + & Confirmed \\
\hline n-Tetradecane & & 32.2 & + & + & Confirmed \\
\hline n-Pentadecane & & 35.0 & + & + & Confirmed \\
\hline n-Hexadecane & & 37.6 & + & + & Confirmed \\
\hline \multicolumn{6}{|l|}{ Carbonyl Compounds } \\
\hline 6-Methyl-5-hepten-2-one & & 22.3 & + & & Confirmed \\
\hline Benzaldehyde & & 22.5 & + & + & Confirmed \\
\hline Octanal & & 22.6 & + & + & Confirmed \\
\hline Nonanal & & 26.1 & + & + & Confirmed \\
\hline 1-Phenylethanone & $\mathrm{T}, \mathrm{Q}$ & 26.4 & + & + & Confirmed \\
\hline Decanal & & 29.3 & + & + & Confirmed \\
\hline \multicolumn{6}{|l|}{ Other Oxidized Compounds } \\
\hline Acetic acid & & 11.1 & + & & Confirmed \\
\hline Isooctanol & & 22.7 & + & + & Probable \\
\hline Di(propylene glycol) methyl ether 1 & $Q$ & 23.1 & + & + & Confirmed \\
\hline Di(propylene glycol) methyl ether 2 & Q & 23.2 & + & + & Confirmed \\
\hline Di(propylene glycol) methyl ether 3 & $\mathrm{Q}$ & 23.8 & + & + & Confirmed \\
\hline 2-Ethyl-1-hexanol & $A, Q$ & 23.9 & + & + & Confirmed \\
\hline 4-Methyl-1-heptanol & $A$ & 24.2 & + & + & Probable \\
\hline 6-Methyl-1-heptanol & & 24.3 & + & + & Probable \\
\hline 5-Methyl-1-heptanol & & 24.5 & + & + & Probable \\
\hline Phenol & $T, A, Q$ & 26.6 & + & + & Confirmed \\
\hline 2-Phenyl-2-propanol & & 27.2 & + & + & Confirmed \\
\hline 2-(2-Butoxyethoxy)ethanol & $T, Q$ & 29.9 & + & + & Confirmed \\
\hline $\begin{array}{c}\text { 2,2,4-Trimethyl-1,3-pentanediol } \\
\text { monoisobutyrate (Texanol } 1 \text { ) }\end{array}$ & & 35.2 & + & + & Confirmed \\
\hline $\begin{array}{l}\text { 2,2,4-Trimethyl-1,3-pentanediol } \\
\text { monoisobutyrate (Texanol 3) }\end{array}$ & & 35.7 & + & + & Confirmed \\
\hline Decanoic acid & & 35.9 & + & + & Probable \\
\hline $\begin{array}{l}\text { 2,6-Di-tert-butyl-4-methylphenol } \\
\text { (BHT) }\end{array}$ & $Q$ & 37.7 & + & + & Confirmed \\
\hline $\begin{array}{l}\text { 2,2,4-Trimethyl-1,3-pentanediol } \\
\text { diisobutyrate (TXIB) }\end{array}$ & $A, Q$ & 40.3 & + & + & Confirmed \\
\hline Diethylphthalate & $A, Q$ & 42.3 & + & + & Confirmed \\
\hline \multicolumn{6}{|l|}{ Nitrogen-Containing Compounds } \\
\hline 1-Methyl-2-pyrrolidinone & $\mathrm{Q}$ & 27.5 & + & + & Confirmed \\
\hline \multicolumn{6}{|l|}{ Miscellaneous Compounds } \\
\hline Hexamethylcyclotrisiloxane & & 11.8 & + & + & Confirmed \\
\hline Octamethylcyclotrisiloxane & & 18.2 & + & + & Confirmed \\
\hline
\end{tabular}


Table E-03, Continued. VOCs emitted by Sheet Vinyl SV1 in 10-L chamber at 6- and 48-hours elapsed times.

\begin{tabular}{|c|c|c|c|c|c|}
\hline COMPOUND & Code $^{\star}$ & $\begin{array}{c}\mathrm{RT} \\
(\mathrm{min})\end{array}$ & $\begin{array}{l}\text { 6-h } \\
\text { ET }\end{array}$ & $\begin{array}{c}\text { 48-h } \\
\text { ET }\end{array}$ & $\begin{array}{l}\text { Match } \\
\text { Quality }\end{array}$ \\
\hline \multicolumn{6}{|l|}{ Unidentified Compounds } \\
\hline Unidentified alcohol & & 29.2 & + & & Unident. \\
\hline Unidentified glycol ether 1 & & 30.5 & + & + & Unident. \\
\hline Unidentified glycol ether 2 & & 30.7 & + & + & Unident. \\
\hline Unidentified oxidized compound & & 35.4 & + & + & Unident. \\
\hline
\end{tabular}

${ }^{*} \mathrm{~T}=$ Toxic air contaminant; $\mathrm{A}=$ Abundant compound; $\mathrm{Q}=$ Quantified target compound. 
Table E-04. VOCs emitted by Sheet Vinyl SV2 in 10-L chamber at 6- and 48-hours elapsed times.

\begin{tabular}{|c|c|c|c|c|c|}
\hline COMPOUND & Code & $\begin{array}{c}\mathrm{RT} \\
(\mathrm{min}) \\
\end{array}$ & $\begin{array}{l}\text { 6-h } \\
\text { ET }\end{array}$ & $\begin{array}{c}\text { 48-h } \\
\text { ET }\end{array}$ & $\begin{array}{l}\text { Match } \\
\text { Quality } \\
\end{array}$ \\
\hline \multicolumn{6}{|l|}{ Alkane Hydrocarbons } \\
\hline C10 Branched alkane $\mathrm{HC}$ & & 15.9 & + & + & Probable \\
\hline C10 Branched alkane $\mathrm{HC}$ & & 16.3 & + & + & Probable \\
\hline C10 Branched alkane $\mathrm{HC}$ & A & 17.7 & + & + & Probable \\
\hline C10 Branched alkane HC & & 18.1 & + & + & Probable \\
\hline C10 Branched alkane HC & & 18.2 & + & + & Probable \\
\hline C10 Branched alkane HC & & 18.3 & + & + & Probable \\
\hline C10 Branched alkane $\mathrm{HC}$ & & 18.4 & + & + & Probable \\
\hline C10 Branched alkane $\mathrm{HC}$ & & 18.5 & + & + & Probable \\
\hline C10 Branched alkane $\mathrm{HC}$ & & 19.0 & + & + & Probable \\
\hline C10 Branched alkane $\mathrm{HC}$ & & 19.1 & + & + & Probable \\
\hline n-Decane & & 19.2 & + & + & Confirmed \\
\hline C11 Branched alkane $\mathrm{HC}$ & & 19.7 & + & + & Probable \\
\hline C11 Branched alkane $\mathrm{HC}$ & $A$ & 20.0 & + & + & Probable \\
\hline C11 Branched alkane $\mathrm{HC}$ & & 20.3 & + & + & Probable \\
\hline C11 Branched alkane $\mathrm{HC}$ & A & 20.6 & + & + & Probable \\
\hline C11 Branched alkane $\mathrm{HC}$ & & 20.8 & + & + & Probable \\
\hline C11 Branched alkane $\mathrm{HC}$ & & 21.0 & + & + & Probable \\
\hline C11 Branched alkane $\mathrm{HC}$ & & 21.2 & + & + & Probable \\
\hline C11 Branched alkane $\mathrm{HC}$ & $A$ & 21.4 & + & + & Probable \\
\hline C11 Branched alkane $\mathrm{HC}$ & & 21.5 & + & + & Probable \\
\hline C11 Branched alkane $\mathrm{HC}$ & $A$ & 21.6 & + & + & Probable \\
\hline C11 Branched alkane $\mathrm{HC}$ & & 21.7 & + & + & Probable \\
\hline C11 Branched alkane $\mathrm{HC}$ & & 22.1 & + & + & Probable \\
\hline C11 Branched alkane $\mathrm{HC}$ & & 22.4 & + & + & Probable \\
\hline C11 Branched alkane $\mathrm{HC}$ & & 22.5 & + & + & Probable \\
\hline C11 Branched alkane $\mathrm{HC}$ & & 22.6 & + & + & Probable \\
\hline C11 Branched alkane $\mathrm{HC}$ & & 22.8 & + & + & Probable \\
\hline C12 Branched alkane $\mathrm{HC}$ & & 23.6 & & + & Probable \\
\hline n-Dodecane & $Q$ & 26.2 & + & + & Confirmed \\
\hline n-Tridecane & $Q$ & 29.3 & + & + & Confirmed \\
\hline $\mathrm{n}$-Tetradecane & & 32.2 & + & + & Confirmed \\
\hline \multicolumn{6}{|l|}{ Aromatic Hydrocarbons } \\
\hline Ethyltoluene isomer & & 19.7 & + & + & Probable \\
\hline 4-Ethyltoluene & & 19.8 & + & + & Confirmed \\
\hline 1,2,4-Trimethylbenzene & $\mathrm{T}, \mathrm{Q}$ & 21.1 & + & + & Confirmed \\
\hline C4 Alkylbenzene & & 23.0 & + & + & Probable \\
\hline C4 Alkylbenzene & & 23.1 & + & + & Probable \\
\hline C4 Alkylbenzene & & 23.2 & + & + & Probable \\
\hline C4 Alkylbenzene & & 24.1 & & + & Probable \\
\hline C4 Alkylbenzene & & 24.2 & + & + & Probable \\
\hline C4 Alkyllbenzene & & 24.4 & + & + & Probable \\
\hline C4 Alkylbenzene & & 25.6 & & + & Probable \\
\hline
\end{tabular}


Table E-04, Continued. VOCs emitted by Sheet Vinyl SV2 in 10-L chamber at 6- and 48-hours elapsed times.

\begin{tabular}{|c|c|c|c|c|c|}
\hline COMPOUND & Code $^{\star}$ & $\begin{array}{c}\mathrm{RT} \\
(\mathrm{min})\end{array}$ & $\begin{array}{l}\text { 6-h } \\
\text { ET }\end{array}$ & $\begin{array}{c}\text { 48-h } \\
\text { ET }\end{array}$ & $\begin{array}{l}\text { Match } \\
\text { Quality } \\
\end{array}$ \\
\hline \multicolumn{6}{|l|}{ Aromatic Hydrocarbons, Cont. } \\
\hline 1,2,3,5-Tetramethylbenzene & & 25.8 & + & + & Confirmed \\
\hline C4 Alkylbenzene & & 27.1 & & + & Probable \\
\hline Naphthalene & $\mathrm{T}, \mathrm{Q}$ & 29.5 & + & + & Confirmed \\
\hline \multicolumn{6}{|l|}{ Other Hydrocarbons } \\
\hline C9 Alkene HC & & 15.2 & + & + & Probable \\
\hline Pentamethylcyclohexane isomer & & 19.6 & + & + & Probable \\
\hline \multicolumn{6}{|l|}{ Carbonyl Compounds } \\
\hline Nonanal & & 26.1 & + & + & Confirmed \\
\hline 1-Phenylethanone & $T$ & 26.5 & + & + & Confirmed \\
\hline \multicolumn{6}{|l|}{ Other Oxidized Compounds } \\
\hline n-Propyl acetate & & 10.1 & + & + & Confirmed \\
\hline Acetic acid & & 10.8 & + & + & Confirmed \\
\hline 2-Propoxyethanol & Q & 15.5 & + & + & Confirmed \\
\hline 1-Octanol & & 25.4 & + & + & Confirmed \\
\hline Phenol & $T, A, Q$ & 26.6 & + & + & Confirmed \\
\hline 2-(2-Butoxyethoxy)ethanol & $\mathrm{T}, \mathrm{Q}$ & 29.9 & + & + & Confirmed \\
\hline $\begin{array}{l}\text { 2,2,4-Trimethyl-1,3-pentanediol } \\
\text { monoisobutyrate (Texanol 1) }\end{array}$ & & 35.2 & + & & Confirmed \\
\hline $\begin{array}{l}\text { 2,2,4-Trimethyl-1,3-pentanediol } \\
\text { monoisobutyrate (Texanol 3) }\end{array}$ & & 35.7 & + & + & Confirmed \\
\hline Decanoic acid & & 35.9 & + & + & Probable \\
\hline 1-Dodecanol & & 37.1 & + & + & Confirmed \\
\hline $\begin{array}{l}\text { 2,2,4-Trimethyl-1,3-pentanediol } \\
\text { diisobutyrate (TXIB) }\end{array}$ & $Q$ & 40.3 & + & + & Confirmed \\
\hline Diethylphthalate & $\mathrm{Q}$ & 42.3 & + & + & Confirmed \\
\hline \multicolumn{6}{|l|}{ Nitrogen-Containing Compounds } \\
\hline 1-Methyl-2-pyrrolidinone & Q & 27.5 & + & + & Confirmed \\
\hline \multicolumn{6}{|l|}{ Miscellaneous Compounds } \\
\hline Hexamethycyclotrisiloxane & & 11.9 & + & + & Confirmed \\
\hline \multicolumn{6}{|l|}{ Unidentified Compounds } \\
\hline Unidentified compound & & 17.2 & + & & Unident. \\
\hline Unidentified compound & & 17.3 & + & + & Unident. \\
\hline Unidentified compound & & 22.0 & + & + & Unident. \\
\hline Unidentified compound & & 22.9 & + & + & Unident. \\
\hline
\end{tabular}

* $T$ = Toxic air contaminant $\mathrm{A}=$ Abundant compound; $\mathrm{Q}=$ Quantified target compound 
Table E-05. VOCs emitted by Sheet Vinyl SV3 in 10-L chamber at 6-and 48-hours elapsed times.

\begin{tabular}{|c|c|c|c|c|c|}
\hline COMPOUND & Code $^{*}$ & $\begin{array}{c}\mathrm{RT} \\
(\mathrm{min})\end{array}$ & $\begin{array}{l}6-h \\
\text { ET } \\
\end{array}$ & $\begin{array}{c}\text { 48-h } \\
\mathrm{ET} \\
\end{array}$ & $\begin{array}{l}\text { Match } \\
\text { Quality } \\
\end{array}$ \\
\hline $\begin{array}{l}\text { Alkane Hydrocarbons } \\
\text { C9 Branched alkane HC } \\
\text { C9 Branched alkane HC } \\
\text { n-Nonane } \\
\text { C10 Branched alkane HC } \\
\text { C10 Branched alkane HC } \\
\text { C10 Branched alkane HC } \\
\text { C10 Branched alkane HC } \\
\text { C10 Branched alkane HC } \\
\text { C10 Branched alkane HC } \\
\text { C10 Branched alkane HC } \\
\text { C10 Branched alkane HC } \\
\text { C10 Branched alkane HC } \\
\text { C10 Branched alkane HC } \\
\text { n-Decane } \\
\text { C11 Branched alkane HC } \\
\text { C11 Branched alkane HC } \\
\text { C11 Branched alkane HC } \\
\text { C11 Branched alkane HC } \\
\text { C11 Branched alkane HC } \\
\text { C11 Branched alkane HC } \\
\text { C11 Branched alkane HC } \\
\text { C11 Branched alkane HC } \\
\text { C11 Branched alkane HC } \\
\text { n-Undecane } \\
\text { C12 Branched alkane HC } \\
\text { n-Dodecane } \\
\text { n-Tridecane } \\
\text { n-Tetradecane } \\
\text { n-Pentadecane }\end{array}$ & $\begin{array}{l}A, Q \\
A, Q\end{array}$ & $\begin{array}{l}13.7 \\
14.1 \\
15.3 \\
15.9 \\
16.2 \\
16.4 \\
16.6 \\
16.8 \\
17.5 \\
17.6 \\
17.7 \\
17.8 \\
18.1 \\
19.2 \\
19.7 \\
20.0 \\
20.2 \\
20.5 \\
20.7 \\
21.3 \\
21.4 \\
21.5 \\
21.8 \\
22.8 \\
23.4 \\
26.2 \\
29.3 \\
32.2 \\
35.0\end{array}$ & $\begin{array}{l}+ \\
+ \\
+ \\
+ \\
+ \\
+ \\
+ \\
+ \\
+ \\
+ \\
+ \\
+ \\
+ \\
+ \\
+ \\
+ \\
+ \\
+ \\
+ \\
+ \\
+ \\
+ \\
+ \\
+ \\
+ \\
+ \\
+\end{array}$ & $\begin{array}{l}+ \\
+ \\
+ \\
+ \\
+ \\
+ \\
+ \\
+ \\
+ \\
+ \\
+ \\
+ \\
+ \\
+ \\
+ \\
+ \\
+ \\
+ \\
+ \\
+ \\
+ \\
+ \\
+ \\
+ \\
+ \\
+ \\
+ \\
+ \\
+\end{array}$ & $\begin{array}{l}\text { Probable } \\
\text { Probable } \\
\text { Confirmed } \\
\text { Probable } \\
\text { Probable } \\
\text { Probable } \\
\text { Probable } \\
\text { Probable } \\
\text { Probable } \\
\text { Probable } \\
\text { Probable } \\
\text { Probable } \\
\text { Probable } \\
\text { Confirmed } \\
\text { Probable } \\
\text { Probable } \\
\text { Probable } \\
\text { Probable } \\
\text { Probable } \\
\text { Probable } \\
\text { Probable } \\
\text { Probable } \\
\text { Probable } \\
\text { Confirmed } \\
\text { Probable } \\
\text { Confirmed } \\
\text { Confirmed } \\
\text { Confirmed } \\
\text { Confirmed }\end{array}$ \\
\hline $\begin{array}{l}\text { Aromatic Hydrocarbons } \\
\text { Toluene } \\
\text { m-,p-Xylene } \\
\text { o-Xylene } \\
\text { Propylbenzene } \\
\text { Ethyltoluene isomer } \\
\text { 4-Ethyltoluene } \\
\text { C3 Alkylbenzene } \\
\text { 2-Ethyltoluene } \\
\text { 1,2,4-Trimethylbenzene } \\
\text { C4 Alkylbenzene } \\
\text { C4 Alkylbenzene } \\
\text { 1,2,3-Trimethylbenzene } \\
\text { 1,3-Diethylbenzene } \\
\text { C4 Alkylbenzene }\end{array}$ & $\begin{array}{l}\mathrm{T}, \mathrm{Q} \\
\mathrm{T}, \mathrm{Q} \\
\mathrm{T}, \mathrm{Q}\end{array}$ & $\begin{array}{l}11.8 \\
16.2 \\
17.3 \\
19.5 \\
19.8 \\
19.9 \\
20.1 \\
20.7 \\
21.2 \\
21.9 \\
22.0 \\
22.5 \\
22.9 \\
23.0\end{array}$ & $\begin{array}{l}+ \\
+ \\
+ \\
+ \\
+ \\
+ \\
+ \\
+ \\
+ \\
+ \\
+ \\
+ \\
+ \\
+\end{array}$ & $\begin{array}{l}+ \\
+ \\
+ \\
+ \\
+ \\
+ \\
+ \\
+ \\
+ \\
+ \\
+ \\
+ \\
+ \\
+\end{array}$ & $\begin{array}{l}\text { Confirmed } \\
\text { Confirmed } \\
\text { Confirmed } \\
\text { Confirmed } \\
\text { Probable } \\
\text { Confirmed } \\
\text { Probable } \\
\text { Confirmed } \\
\text { Confirmed } \\
\text { Probable } \\
\text { Probable } \\
\text { Confirmed } \\
\text { Confirmed } \\
\text { Probable }\end{array}$ \\
\hline
\end{tabular}


Table E-05, Continued. VOCs emitted by Sheet Vinyl SV3 in 10-L chamber at 6- and 48-hours elapsed times.

\begin{tabular}{|c|c|c|c|c|c|}
\hline COMPOUND & Code $^{*}$ & $\begin{array}{c}\mathrm{RT} \\
(\mathrm{min})\end{array}$ & $\begin{array}{l}\text { 6-h } \\
\text { ET }\end{array}$ & $\begin{array}{c}\text { 48-h } \\
\text { ET }\end{array}$ & $\begin{array}{l}\text { Match } \\
\text { Quality }\end{array}$ \\
\hline \multicolumn{6}{|l|}{ Aromatic Hydrocarbons, Cont. } \\
\hline C4 Alkylbenzene & & 23.3 & + & + & Probable \\
\hline C4 Alkylbenzene & & 23.8 & + & + & Probable \\
\hline C4 Alkylbenzene & & 24.2 & + & + & Probable \\
\hline C4 Alkylbenzene & & 24.4 & + & + & Probable \\
\hline C5 Alkylbenzene & & 24.7 & + & + & Probable \\
\hline C4 Alkylbenzene & & 27.1 & & + & Probable \\
\hline Naphthalene & $\mathrm{T}, \mathrm{Q}$ & 29.6 & + & + & Confirmed \\
\hline (1-Butylhexyl)benzene & & 37.2 & + & + & Probable \\
\hline (1-Propylheptyl)benzene & & 37.5 & + & + & Probable \\
\hline (1-Ethyloctyl)benzene & & 38.0 & + & + & Probable \\
\hline ( $\uparrow$-Methylnonyl)benzene & & 39.1 & + & + & Probable \\
\hline (1-Penylhexyl)benzene & & 39.6 & + & + & Probable \\
\hline (1-Butylheptyl)benzene & & 39.7 & + & + & Probable \\
\hline (1-Propyloctyl)benzene & & 39.9 & + & + & Probable \\
\hline (1-Ethylnonyl)benzene & & 40.6 & + & + & Probable \\
\hline (1,1-Dimethylnonyl)benzene & & 40.7 & + & + & Probable \\
\hline (1-Methyldecyl)benzene & & 41.5 & + & + & Probable \\
\hline (1-Penylheptyl)benzene & & 41.8 & + & + & Probable \\
\hline (1-Butyloctyl)benzene & & 42.0 & + & + & Probable \\
\hline (1-Propylnonyl)benzene & & 42.3 & + & + & Probable \\
\hline (1-Ethyldecyl)benzene & & 42.9 & + & + & Probable \\
\hline \multicolumn{6}{|l|}{ Other Hydrocarbons } \\
\hline Ethylcyclohexane & & 12.9 & + & + & Confirmed \\
\hline Trimethylcyclohexane isomer & & 13.0 & + & + & Probable \\
\hline Trimethylcyclohexane isomer & & 13.6 & + & + & Probable \\
\hline Trimethylcyclohexane isomer & & 14.7 & + & + & Probable \\
\hline C9 Alkene or cyclic HC & & 14.9 & + & + & Tentative \\
\hline C9 Alkene or cyclic HC & & 15.0 & + & + & Probable \\
\hline C3 Alkyl substituted cyclohexane & & 15.1 & + & + & Probable \\
\hline C10 Alkene HC & & 15.4 & & + & Probable \\
\hline C3 Alkyl substituted cyclohexane & & 16.1 & + & + & Probable \\
\hline $\mathrm{C} 9 \mathrm{H} 16 \mathrm{HC}$ & & 16.7 & + & + & Tentative \\
\hline Propylcyclohexane & & 17.0 & + & + & Confirmed \\
\hline Alkene or cyclic $\mathrm{HC}$ & & 17.0 & + & + & Tentative \\
\hline C10 Alkene $\mathrm{HC}$ & & 17.1 & + & + & Probable \\
\hline C10 Alkene $\mathrm{HC}$ & & 17.2 & + & + & Probable \\
\hline C4 Alkyl substituted cyclohexane & & 17.4 & & + & Probable \\
\hline C10 Alkene HC & & 17.6 & + & + & Probable \\
\hline C4 Alkyl substituted cyclohexane & & 18.2 & + & + & Probable \\
\hline C10 Alkene $\mathrm{HC}$ & & 18.6 & + & + & Probable \\
\hline C10 Alkene $\mathrm{HC}$ & & 18.8 & + & + & Probable \\
\hline C10 Alkene or cyclic HC & & 18.8 & & + & Probable \\
\hline C4 Alkyl substituted cyclohexane & & 19.0 & + & + & Probable \\
\hline C4 Alkyl substituted cyclohexane & & 19.6 & + & + & Probable \\
\hline
\end{tabular}


Table E-05, Continued. VOCs emitted by Sheet Vinyl SV3 in 10-L chamber at 6- and 48-hours elapsed times.

\begin{tabular}{|c|c|c|c|c|c|}
\hline COMPOUND & Code $^{\star}$ & $\begin{array}{c}\mathrm{RT} \\
(\mathrm{min})\end{array}$ & $\begin{array}{l}\text { 6-h } \\
\text { ET }\end{array}$ & $\begin{array}{c}\text { 48-h } \\
\mathrm{ET}\end{array}$ & $\begin{array}{l}\text { Match } \\
\text { Quality } \\
\end{array}$ \\
\hline \multicolumn{6}{|l|}{ Other Hydrocarbons, Cont. } \\
\hline C11 Alkene HC & & 20.3 & + & + & Tentative \\
\hline $\mathrm{C} 1 \mathrm{OH} 18$ Hydrocarbon & & 20.3 & & + & Tentative \\
\hline C11 Alkene $\mathrm{HC}$ & & 20.6 & + & + & Probable \\
\hline Butylcyclohexane & & 20.9 & + & + & Confirmed \\
\hline C11 Alkene HC & & 21.0 & + & + & Probable \\
\hline Decahydronaphthalene isomer & & 22.3 & + & + & Tentative \\
\hline C11 Alkene $\mathrm{HC}$ & & 22.7 & + & + & Probable \\
\hline Alkene $\mathrm{HC}$ & & 29.9 & & + & Tentative \\
\hline Alkene $\mathrm{HC}$ & & 30.4 & & + & Tentative \\
\hline Alkene $\mathrm{HC}$ & & 37.1 & + & + & Tentative \\
\hline \multicolumn{6}{|l|}{ Carbonyl Compounds } \\
\hline Heptanal & & 18.0 & + & + & Confirmed \\
\hline Benzaldehyde & & 22.5 & + & + & Confirmed \\
\hline Nonanal & & 26.1 & + & & Confirmed \\
\hline 1-Phenylethanone & $\mathrm{T}$ & 26.5 & + & + & Confirmed \\
\hline \multicolumn{6}{|l|}{ Other Oxidized Compounds } \\
\hline 1-Butanol & & 10.0 & + & + & Confirmed \\
\hline Acetic acid & & 11.2 & + & & Confirmed \\
\hline Benzyl alcohol & $A, Q$ & 26.5 & + & + & Confirmed \\
\hline Phenol & $T, A, Q$ & 26.6 & + & + & Confirmed \\
\hline $\begin{array}{l}\text { 2,2,4-Trimethyl-1,3-pentanediol } \\
\text { monoisobutyrate (Texanol 1) }\end{array}$ & & 35.2 & + & + & Confirmed \\
\hline $\begin{array}{l}\text { 2,2,4-Trimethyl-1,3-pentanediol } \\
\text { monoisobutyrate (Texanol 3) }\end{array}$ & & 35.7 & + & + & Confirmed \\
\hline Decanoic acid & & 35.9 & + & + & Probable \\
\hline $\begin{array}{l}\text { 2,2,4-Trimethyl-1,3-pentanediol } \\
\text { diisobutyrate (TXIB) }\end{array}$ & $A, Q$ & 40.3 & + & + & Confirmed \\
\hline Diethylphthalate & $\mathrm{Q}$ & 42.3 & + & + & Confirmed \\
\hline \multicolumn{6}{|l|}{ Miscellaneous Compounds } \\
\hline Hexamethylcyclotrisiloxane & & 11.9 & + & + & Confirmed \\
\hline Octamethylcyclotetrasiloxane & & 18.3 & + & + & Confirmed \\
\hline \multicolumn{6}{|l|}{ Unidentified Compounds } \\
\hline Unidentified compound & & 17.4 & + & & Unident. \\
\hline Unidentified compound & & 29.1 & + & + & Unident. \\
\hline Unidentified compound & & 29.5 & + & + & Unident. \\
\hline Unidentified compound & & 29.6 & + & & Unident. \\
\hline Unidentified compound & & 29.9 & + & & Unident. \\
\hline Unidentified oxidized compound & & 30.1 & + & + & Unident. \\
\hline Unidentified compound & & 30.4 & + & + & Unident. \\
\hline
\end{tabular}

${ }^{*} T=$ Toxic air contaminant; $A=$ Abundant compound; $Q=$ Quantified target compound. 
Table E-06. VOCs emitted by sheet vinyl SV5 in 10-L chamber at 6- and 48-hours elapsed times.

\begin{tabular}{|c|c|c|c|c|c|}
\hline COMPOUND & Code $^{\star}$ & $\begin{array}{c}\mathrm{RT} \\
(\mathrm{min})\end{array}$ & $\begin{array}{l}\text { 6-h } \\
\text { ET }\end{array}$ & $\begin{array}{c}\text { 48-h } \\
\text { ET }\end{array}$ & $\begin{array}{l}\text { Match } \\
\text { Quality }\end{array}$ \\
\hline \multicolumn{6}{|l|}{ Alkane Hydrocarbons } \\
\hline C9 Branched alkane $\mathrm{HC}$ & & 13.7 & + & + & Probable \\
\hline C9 Branched alkane $\mathrm{HC}$ & & 14.0 & + & + & Probable \\
\hline C9 Branched alkane $\mathrm{HC}$ & & 14.1 & + & + & Probable \\
\hline n-Nonane & $Q$ & 15.2 & + & + & Confirmed \\
\hline C10 Branched alkane $\mathrm{HC}$ & & 15.9 & + & + & Probable \\
\hline C10 Branched alkane $\mathrm{HC}$ & & 16.2 & + & + & Probable \\
\hline C10 Branched alkane $\mathrm{HC}$ & & 16.6 & + & + & Probable \\
\hline C10 Branched alkane $\mathrm{HC}$ & & 17.5 & + & + & Probable \\
\hline C10 Branched alkane HC & & 17.6 & + & + & Probable \\
\hline C10 Branched alkane $\mathrm{HC}$ & & 17.7 & + & + & Probable \\
\hline C10 Branched alkane HC & & 17.8 & + & + & Probable \\
\hline C10 Branched alkane $\mathrm{HC}$ & & 18.1 & + & + & Probable \\
\hline $\mathrm{n}$-Decane & $A, Q$ & 19.2 & + & + & Confirmed \\
\hline $\mathrm{C} 11$ Branched alkane $\mathrm{HC}$ & & 20.0 & + & + & Probable \\
\hline C11 Branched alkane $\mathrm{HC}$ & & 21.3 & + & + & Probable \\
\hline $\mathrm{C} 11$ Branched alkane $\mathrm{HC}$ & & 21.4 & + & + & Probable \\
\hline C11 Branched alkane $\mathrm{HC}$ & & 21.5 & + & + & Probable \\
\hline C11 Branched alkane HC & & 21.8 & + & + & Probable \\
\hline n-Undecane & & 22.8 & + & + & Confirmed \\
\hline n-Dodecane & & 26.2 & + & + & Confirmed \\
\hline $\mathrm{n}$-Tridecane & $A, Q$ & 29.3 & + & + & Confirmed \\
\hline n-Tetradecane & $A, Q$ & 32.2 & + & + & Confirmed \\
\hline n-Pentadecane & & 35.0 & + & + & Confirmed \\
\hline \multicolumn{6}{|l|}{ Aromatic Hydrocarbons } \\
\hline Toluene & $T, Q$ & 11.7 & + & + & Confirmed \\
\hline$m-, p$-Xylene & $\mathrm{T}, \mathrm{Q}$ & 16.1 & + & + & Confirmed \\
\hline o-Xylene & $\mathrm{T}, \mathrm{Q}$ & 17.3 & + & + & Confirmed \\
\hline Propylbenzene & & 19.5 & + & + & Confirmed \\
\hline Ethyltoluene isomer & & 19.7 & + & + & Probable \\
\hline 4-Ethyltoluene & & 19.8 & + & + & Confirmed \\
\hline C3 Alkylbenzene & & 20.0 & + & + & Probable \\
\hline 2-Ethyltoluene & & 20.6 & + & + & Confirmed \\
\hline $1,2,4$-Trimethylbenzene & $\mathrm{T}, \mathrm{Q}$ & 21.2 & + & + & Confirmed \\
\hline C4 Alkylbenzene & & 21.8 & + & + & Probable \\
\hline C4 Alkylbenzene & & 22.0 & + & + & Probable \\
\hline 1,2,3-Trimethylbenzene & & 22.4 & + & + & Confirmed \\
\hline 1,3-Diethylbenzene & & 22.9 & + & + & Confirmed \\
\hline C4 Alkylbenzene & & 23.0 & + & + & Probable \\
\hline C4 Alkylbenzene & & 23.2 & + & + & Probable \\
\hline C4 Alkylbenzene & & 23.2 & + & + & Probable \\
\hline C4 Alkylbenzene & & 23.8 & + & + & Probable \\
\hline C4 Alkylbenzene & & 24.1 & + & + & Probable \\
\hline C4 Alkylbenzene & & 24.2 & + & + & Probable \\
\hline
\end{tabular}


Table E-06, Continued. VOCs emitted by sheet vinyl SV5 in 10-L chamber at 6- and 48-hours elapsed times.

\begin{tabular}{|c|c|c|c|c|c|}
\hline COMPOUND & Code* & $\begin{array}{c}\mathbf{R T} \\
(\min )\end{array}$ & $\begin{array}{l}\text { 6-h } \\
\text { ET } \\
\end{array}$ & $\begin{array}{c}48-h \\
E T\end{array}$ & $\begin{array}{l}\text { Match } \\
\text { Quality } \\
\end{array}$ \\
\hline \multicolumn{6}{|l|}{ Aromatic Hydrocarbons, Cont. } \\
\hline C4 Alkylbenzene & & 24.4 & + & + & Probable \\
\hline C5 Alkylbenzene & & 24.6 & + & + & Probable \\
\hline Butenylbenzene isomer & & 24.7 & + & & Probable \\
\hline 1,2,3,5-Tetramethylbenzene & & 25.8 & + & + & Confirmed \\
\hline C4 Alkylbenzene & & 27.1 & + & + & Probable \\
\hline (1-Butoxyhexyl)benzene & & 37.2 & + & + & Probable \\
\hline (1-Propylheptyl)benzene & & 37.4 & + & + & Probable \\
\hline (1-Ethyloctyl)benzene & & 38.0 & + & + & Probable \\
\hline (1-Methylnonyl)benzene & & 39.1 & + & + & Probable \\
\hline (1-Phenylhexyl)benzene & & 39.5 & + & + & Probable \\
\hline (1-Butylheptyl)benzene & & 39.6 & + & + & Probable \\
\hline (1-Propyloctyl)benzene & & 39.9 & + & + & Probable \\
\hline (1-Ethylnonyl)benzene & & 40.5 & + & + & Probable \\
\hline (1,1-Dimethylnonyl)benzene & & 40.7 & + & + & Probable \\
\hline (1-Methyldecyl)benzene & & 41.5 & + & + & Probable \\
\hline (1-Penylheptyl)benzene & & 41.8 & + & + & Probable \\
\hline (1-Butyloctyl)benzene & & 42.0 & + & + & Probable \\
\hline (1-Propylnonyl)benzene & & 42.3 & + & + & Probable \\
\hline (1-Ethyldecyl)benzene & & 42.9 & + & + & Probable \\
\hline \multicolumn{6}{|l|}{ Other Hydrocarbons } \\
\hline C3 Alkyl substituted cyclohexane & & 13.0 & + & + & Probable \\
\hline Trimethylcyclohexane isomer & & 13.6 & + & + & Probable \\
\hline C3 Alkyl substituted cyclohexane & & 15.1 & + & + & Probable \\
\hline C3 Alkyl substituted cyclohexane & & 16.0 & + & + & Probable \\
\hline $\mathrm{C} 9 \mathrm{H} 16 \mathrm{HC}$ & & 16.7 & + & + & Tentative \\
\hline Propylcyclohexane & & 16.9 & + & + & Confirmed \\
\hline C10 Alkene or cyclic $\mathrm{HC}$ & & 17.0 & + & + & Probable \\
\hline C10 Alkene $\mathrm{HC}$ & & 17.2 & + & + & Probable \\
\hline C4 Alkyl substituted cyclohexane & & 17.4 & + & + & Probable \\
\hline C4 Alkyl substituted cyclohexane & & 18.2 & + & + & Probable \\
\hline C10 Alkene $\mathrm{HC}$ & & 18.6 & + & + & Probable \\
\hline C10 Alkene or cyclic HC & & 18.8 & + & + & Probable \\
\hline C4 Alkyl substituted cyclohexane & & 19.0 & + & + & Probable \\
\hline Butylcyclohexane & & 20.9 & + & + & Confirmed \\
\hline C11 Alkene HC & & 21.0 & + & + & Probable \\
\hline Decahydronaphthalene isomer & & 22.3 & + & + & Probable \\
\hline C11 Alkene HC & & 22.6 & + & + & Probable \\
\hline C11 Alkene HC & & 22.7 & + & + & Probable \\
\hline Alkene $\mathrm{HC}$ & & 29.2 & + & + & Tentative \\
\hline Alkene $\mathrm{HC}$ & & 29.5 & + & + & Tentative \\
\hline Alkene $\mathrm{HC}$ & & 29.9 & + & + & Tentative \\
\hline Alkene $\mathrm{HC}$ & & 30.4 & + & + & Tentative \\
\hline Alkene $\mathrm{HC}$ & & 37.1 & + & + & Tentative \\
\hline
\end{tabular}


Table E-06, Continued. VOCs emitted by sheet vinyl SV5 in 10-L chamber at 6- and 48-hours elapsed times.

\begin{tabular}{|c|c|c|c|c|c|}
\hline COMPOUND & Code $^{*}$ & $\begin{array}{c}\mathrm{RT} \\
(\mathrm{min}) \\
\end{array}$ & $\begin{array}{l}\text { 6-h } \\
\mathrm{ET} \\
\end{array}$ & $\begin{array}{c}\text { 48-h } \\
\mathrm{ET}\end{array}$ & $\begin{array}{l}\text { Match } \\
\text { Quality } \\
\end{array}$ \\
\hline \multicolumn{6}{|l|}{ Carbonyl Compounds } \\
\hline Benzaldehyde & & 22.5 & + & + & Confirmed \\
\hline Nonanal & & 26.0 & + & + & Confirmed \\
\hline 1-Phenylethanone & $\mathrm{T}$ & 26.5 & + & + & Confirmed \\
\hline \multicolumn{6}{|l|}{ Other Oxidized Compounds } \\
\hline 1-Butanol & & 9.9 & + & & Confirmed \\
\hline 1-Octanol & $Q$ & 25.4 & + & + & Confirmed \\
\hline Benzyl alcohol & $A, Q$ & 26.5 & + & + & Confirmed \\
\hline Phenol & $T, A, Q$ & 26.6 & + & + & Confirmed \\
\hline 2-Ethylhexanoic acid & & 28.4 & + & + & Probable \\
\hline $\begin{array}{l}\text { 2,2,4-Trimethyl-1,3-pentanediol } \\
\text { diisobutyrate (TXIB) }\end{array}$ & $A, Q$ & 40.3 & + & + & Confirmed \\
\hline \multicolumn{6}{|l|}{ Nitrogen-Containing Compounds } \\
\hline N-Propylbenzamide & & 40.7 & + & + & Tentative \\
\hline \multicolumn{6}{|l|}{ Miscellaneous Compounds } \\
\hline Hexamethylcyclotrisiloxane & & 11.9 & + & + & Confirmed \\
\hline Octamethylcyclotetrasiloxane & & 18.2 & + & + & Confirmed \\
\hline Siloxane compound & & 33.7 & + & + & Probable \\
\hline \multicolumn{6}{|l|}{ Unidentified Compounds } \\
\hline Unidentified compound & & 17.2 & + & & Unident. \\
\hline Unidentified compound & & 28.9 & + & + & Unident. \\
\hline Unidentified compound & & 29.0 & + & + & Unident. \\
\hline Unidentified oxidized compound & & 30.1 & + & + & Unident. \\
\hline
\end{tabular}

${ }^{\star} \mathrm{T}=$ Toxic air contaminant; $\mathrm{A}=\mathrm{Abundant}$ compound; $\mathrm{Q}=$ Quantified target compound. 
Table E-07. VOCs emitted by Sheet Vinyl SV4 in 10-L chamber at 6- and 48-hours elapsed times.

\begin{tabular}{lccccc}
\hline & & RT & $6-h$ & $48-h$ & Match \\
COMPOUND & Code $^{\star}$ & $(\mathrm{min})$ & ET & ET & Quality \\
\hline
\end{tabular}

\section{Alkane Hydrocarbons}

C10 Branched alkane $\mathrm{HC}$ C10 Branched alkane $\mathrm{HC}$ C10 Branched alkane HC C10 Branched alkane $\mathrm{HC}$ C10 Branched alkane $\mathrm{HC}$ C10 Branched alkane HC C10 Branched alkane $\mathrm{HC}$ C11 Branched alkane HC C11 Branched alkane HC C11 Branched alkane HC C11 Branched alkane HC C11 Branched alkane HC C11 Branched alkane HC C11 Branched alkane HC C11 Branched alkane HC C11 Branched alkane HC C11 Branched alkane HC C11 Branched alkane HC C11 Branched alkane HC C11 Branched alkane HC C11 Branched alkane HC C11 Branched alkane HC C12 Branched alkane HC n-Dodecane

$n$-Tridecane

$\mathrm{n}$-Tetradecane

\section{Aromatic Hydrocarbons}

Ethyltoluene isomer

4-Ethyltoluene

1,2,4-Trimethylbenzene

1,2,3-Trimethylbenzene

1,3-Diethylbenzene

C4 Alkylbenzene

C4 Alkylbenzene

C4 Alkylbenzene

C4 Alkylbenzene

C4 Alkylbenzene

C4 Alkylbenzene

C4 Alkylbenzene

C5 Alkylbenzene

C4 Alkylbenzene

C4 Alkylbenzene

1,2,3,5-Tetramethylbenzene

C5 Alkylbenzene

\begin{tabular}{|c|c|c|c|c|}
\hline & 17.7 & + & + & Probable \\
\hline & 18.1 & + & + & Probable \\
\hline & 18.3 & + & + & Probable \\
\hline & 18.4 & + & + & Probable \\
\hline & 18.5 & + & + & Probable \\
\hline & 19.0 & + & + & Probable \\
\hline & 19.1 & + & + & Probable \\
\hline & 19.3 & + & & Probable \\
\hline & 19.7 & + & + & Probable \\
\hline A & 20.0 & + & + & Probable \\
\hline & 20.3 & + & + & Probable \\
\hline A & 20.6 & + & + & Probable \\
\hline & 20.8 & + & + & Probable \\
\hline & 21.1 & + & + & Probable \\
\hline & 21.2 & + & + & Probable \\
\hline A & 21.4 & + & + & Probable \\
\hline & 21.5 & + & + & Probable \\
\hline A & 21.6 & + & + & Probable \\
\hline & 21.7 & + & + & Probable \\
\hline & 22.1 & + & + & Probable \\
\hline & 22.3 & + & + & Probable \\
\hline & 22.6 & + & + & Probable \\
\hline & 22.9 & + & + & Probable \\
\hline & 26.2 & + & + & Confirmed \\
\hline$A, Q$ & 29.3 & + & + & Confirmed \\
\hline & 32.2 & + & + & Confirmed \\
\hline & 19.8 & + & + & Probable \\
\hline & 19.9 & + & + & Confirmed \\
\hline$T, Q$ & 21.2 & + & + & Confirmed \\
\hline & 22.5 & + & + & Confirmed \\
\hline & 22.9 & + & + & Confirmed \\
\hline & 23.0 & + & + & Probable \\
\hline & 23.2 & + & + & Probable \\
\hline & 23.3 & + & + & Probable \\
\hline & 23.8 & + & + & Probable \\
\hline & 24.1 & + & + & Probable \\
\hline & 24.2 & + & + & Probable \\
\hline & 24.4 & + & + & Probable \\
\hline & 24.7 & & + & Probable \\
\hline & 25.3 & + & + & Probable \\
\hline & $\begin{array}{l}25.6 \\
258\end{array}$ & + & + & $\begin{array}{l}\text { Probable } \\
\text { Confirmed }\end{array}$ \\
\hline & 26.1 & $t$ & + & Probable \\
\hline
\end{tabular}


Table E-07, Continued. VOCs emitted by Sheet Vinyl SV4 in 10-L chamber at 6- and 48-hours elapsed times.

\begin{tabular}{|c|c|c|c|c|c|}
\hline COMPOUND & Code $^{\star}$ & $\begin{array}{c}\mathrm{RT} \\
(\mathrm{min})\end{array}$ & $\begin{array}{l}\text { 6-h } \\
\text { ET }\end{array}$ & $\begin{array}{c}\text { 48-h } \\
\text { ET }\end{array}$ & $\begin{array}{l}\text { Match } \\
\text { Quality }\end{array}$ \\
\hline \multicolumn{6}{|l|}{ Aromatic Hydrocarbons, Cont. } \\
\hline C4 Alkylbenzene & & 27.1 & + & + & Probable \\
\hline C5 Alkylbenzene & & 27.3 & & + & Probable \\
\hline C5 Alkylbenzene & & 28.1 & & + & Probable \\
\hline Naphthalene & $\mathrm{T}, \mathrm{Q}$ & 29.6 & + & + & Confirmed \\
\hline \multicolumn{6}{|l|}{ Other Hydrocarbons } \\
\hline C5 Alkyl substituted cyclohexane & & 22.0 & + & + & Tentative \\
\hline Alkene $\mathrm{HC}$ & & 37.1 & + & + & Tentative \\
\hline \multicolumn{6}{|l|}{ Carbonyl Compounds } \\
\hline Nonanal & & 26.1 & + & + & Confirmed \\
\hline 1-Phenylethanone & $T, Q$ & 26.5 & + & + & Confirmed \\
\hline Decanal & & 29.4 & + & & Confirmed \\
\hline \multicolumn{6}{|l|}{ Other Oxidized Compounds } \\
\hline Isopropyl acetate & & 7.7 & + & + & Confirmed \\
\hline n-Propyl acetate & $\mathbf{Q}$ & 10.1 & + & + & Confirmed \\
\hline Acetic acid & & 11.1 & + & + & Probable \\
\hline 2-Propoxyethanol & $Q$ & 15.5 & + & + & Confirmed \\
\hline Phenol & $T, A, Q$ & 26.6 & + & + & Confirmed \\
\hline 2-(2-Butoxyethoxy)ethanol & $\mathrm{T}, \mathrm{Q}$ & 29.9 & + & + & Confirmed \\
\hline Nonanoic acid & & 33.3 & + & & Probable \\
\hline $\begin{array}{c}\text { 2,2,4-Trimethyl-1,3-pentanediol } \\
\text { monoisobutyrate (Texanol 1) }\end{array}$ & & 35.2 & + & + & Confirmed \\
\hline $\begin{array}{c}\text { 2,2,4-Trimethyl-1,3-pentanediol } \\
\text { monoisobutyrate (Texanol 3) }\end{array}$ & & 35.7 & + & + & Confirmed \\
\hline Decanoic acid & & 35.9 & + & + & Probable \\
\hline $\begin{array}{l}\text { 2,2,4-Trimethyl-1,3-pentanediol } \\
\text { diisobutyrate (TXIB) }\end{array}$ & $Q$ & 40.3 & + & + & Confirmed \\
\hline Diethylphthalate & $\mathrm{Q}$ & 42.3 & + & + & Confirmed \\
\hline \multicolumn{6}{|l|}{ Nitrogen-Containing Compounds } \\
\hline 1-Methyl-2-pyrrolidinone & $\mathrm{Q}$ & 27.5 & + & + & Confirmed \\
\hline \multicolumn{6}{|l|}{ Miscellaneous Compounds } \\
\hline Hexamethylcyclotrisiloxane & & 11.8 & + & + & Confirmed \\
\hline Octamethylcyclotetrasiloxane & & 18.3 & + & + & Confirmed \\
\hline \multicolumn{6}{|l|}{ Unidentified Compounds } \\
\hline Unidentified compound & & 17.3 & + & & Unident. \\
\hline Unidentified compound & & 17.4 & + & + & Unident. \\
\hline Unidentified compound & & 21.8 & + & + & Unident. \\
\hline Unidentified compound & & 22.9 & & + & Unident. \\
\hline
\end{tabular}


Table E-07, Continued. VOCs emitted by Sheet Vinyl SV4 in 10-L chamber at 6- and 48-hours elapsed times.

\begin{tabular}{lccccc}
\hline COMPOUND & Code & $\begin{array}{c}\text { RT } \\
(\min )\end{array}$ & $\begin{array}{c}\text { 6-h } \\
\text { ET }\end{array}$ & $\begin{array}{c}\text { 48-h } \\
\text { ET }\end{array}$ & $\begin{array}{c}\text { Match } \\
\text { Quality }\end{array}$ \\
\hline & & & & & \\
Unidentified Compounds, Cont. & & & + & Unident. \\
Undientified compound & 25.4 & + & + & Unident. \\
Unidentified compound & 27.8 & & + & \\
& & & & & \\
\hline
\end{tabular}

${ }^{*} T=$ Toxic air contaminant; $A=$ Abundant compound; $\mathrm{Q}=$ Quantified target compound. 
Table E-08. VOCs detected in sheet vinyl blank chamber run at 6 - and 48-hours elapsed times.

\begin{tabular}{|c|c|c|c|c|c|}
\hline COMPOUND & Code $^{\star}$ & $\begin{array}{c}\mathrm{RT} \\
(\mathrm{min}) \\
\end{array}$ & $\begin{array}{l}6-h \\
\text { ET }\end{array}$ & $\begin{array}{c}\text { 48-h } \\
\text { ET }\end{array}$ & $\begin{array}{l}\text { Match } \\
\text { Quality }\end{array}$ \\
\hline \multicolumn{6}{|l|}{ Aromatic Hydrocarbons } \\
\hline m-,p-Xylene & $T, Q$ & 16.1 & + & & Confirmed \\
\hline o-Xylene & $T, Q$ & 17.3 & + & & Confirmed \\
\hline 1,2,4-Trimethylbenzene & $T, Q$ & 21.1 & + & & Confirmed \\
\hline \multicolumn{6}{|l|}{ Carbonyl Compounds } \\
\hline Benzaldehyde & & 22.5 & + & & Confirmed \\
\hline Octanal & & 22.6 & + & & Confirmed \\
\hline Nonanal & $Q$ & 26.1 & + & + & Confirmed \\
\hline Decanal & $Q$ & 29.3 & + & + & Confirmed \\
\hline \multicolumn{6}{|l|}{ Other Oxidized Compounds } \\
\hline 2-(2-Butoxyethoxy)ethanol & $T, Q$ & 29.9 & + & & Confirmed \\
\hline Decanoic acid & & 35.9 & + & + & Probable \\
\hline Diethylphthalate & $Q$ & 42.3 & + & + & Confirmed \\
\hline \multicolumn{6}{|l|}{ Miscellaneous Compounds } \\
\hline Hexamethylcyclotrisiloxane & & 11.8 & & + & Confirmed \\
\hline Octamethylcyclotrisiloxane & & 18.2 & & + & Confirmed \\
\hline
\end{tabular}

${ }^{*} T$ = Toxic air contaminant; $Q=$ Quantified target compound. 
Table E-09. Composite listing of VOCs emitted by Sheet Vinyls SV1 through SV5 in 10-L chambers.

\begin{tabular}{|c|c|c|c|c|c|c|c|}
\hline COMPOUND & $\begin{array}{c}\mathrm{RT} \\
(\mathrm{min}) \\
\end{array}$ & SV1 & SV2 & SV3 & Sv5 & SV4 & $\begin{array}{c}\text { Blank } \\
\text { Run }\end{array}$ \\
\hline \multicolumn{8}{|l|}{ Alkane Hydrocarbons } \\
\hline C9 Branched alkane HC & 13.7 & & & + & + & & \\
\hline C9 Branched alkane $\mathrm{HC}$ & 14.0 & & & & + & & \\
\hline C9 Branched alkane $\mathrm{HC}$ & 14.1 & & & + & + & & \\
\hline n-Nonane & 15.2 & & & + & + & & \\
\hline C10 Branched alkane $\mathrm{HC}$ & 15.9 & & + & + & + & & \\
\hline C10 Branched alkane $\mathrm{HC}$ & 16.2 & & + & + & + & & \\
\hline C10 Branched alkane $\mathrm{HC}$ & 16.4 & & & + & & & \\
\hline C10 Branched alkane HC & 16.6 & & & + & + & & \\
\hline C10 Branched alkane $\mathrm{HC}$ & 16.8 & & & + & & & \\
\hline C10 Branched alkane HC & 17.5 & & & + & + & & \\
\hline C10 Branched alkane $\mathrm{HC}$ & 17.6 & & A & + & + & & \\
\hline C10 Branched alkane $\mathrm{HC}$ & 17.7 & & & + & + & + & \\
\hline C10 Branched alkane HC & 17.8 & & & + & + & & \\
\hline C10 Branched alkane $\mathrm{HC}$ & 18.1 & & + & + & + & + & \\
\hline C10 Branched alkane $\mathrm{HC}$ & 18.2 & & + & & & & \\
\hline C10 Branched alkane $\mathrm{HC}$ & 18.3 & & + & & & + & \\
\hline C10 Branched alkane $\mathrm{HC}$ & 18.4 & & + & & & + & \\
\hline C10 Branched alkane $\mathrm{HC}$ & 18.5 & & + & & & + & \\
\hline C10 Branched alkane $\mathrm{HC}$ & 19.0 & & + & & & + & \\
\hline C10 Branched alkane $\mathrm{HC}$ & 19.1 & & + & & & + & \\
\hline n-Decane & 19.2 & & + & A & A & & \\
\hline C11 Branched alkane $\mathrm{HC}$ & 19.3 & & & & & + & \\
\hline C11 Branched alkane $\mathrm{HC}$ & 19.7 & & + & + & & + & \\
\hline C11 Branched alkane $\mathrm{HC}$ & 20.0 & & $A$ & + & + & $A$ & \\
\hline C11 Branched alkane $\mathrm{HC}$ & 20.2 & & + & + & & + & \\
\hline C11 Branched alkane $\mathrm{HC}$ & 20.5 & & A & + & + & A & \\
\hline C11 Branched alkane $\mathrm{HC}$ & 20.7 & & + & + & & + & \\
\hline C11 Branched alkane $\mathrm{HC}$ & 21.0 & & + & & & + & \\
\hline C11 Branched alkane $\mathrm{HC}$ & 21.2 & & + & + & + & + & \\
\hline C11 Branched alkane $\mathrm{HC}$ & 21.4 & & $A$ & + & + & $A$ & \\
\hline C11 Branched alkane $\mathrm{HC}$ & 21.5 & & + & + & + & + & \\
\hline C11 Branched alkane HC & 21.6 & & $A$ & & & $A$ & \\
\hline C11 Branched alkane $\mathrm{HC}$ & 21.7 & & + & + & + & + & \\
\hline C11 Branched alkane $\mathrm{HC}$ & 22.1 & & + & & & + & \\
\hline C11 Branched alkane HC & 22.3 & & + & & & + & \\
\hline C11 Branched alkane $\mathrm{HC}$ & 22.5 & & + & & & & \\
\hline C11 Branched alkane HC & 22.6 & & + & & & + & \\
\hline C11 Branched alkane $\mathrm{HC}$ & 22.8 & & + & & & & \\
\hline n-Undecane & 22.8 & & & + & + & & \\
\hline $\mathrm{C} 12$ Branched alkane $\mathrm{HC}$ & 22.9 & & & & & + & \\
\hline C12 Branched alkane $\mathrm{HC}$ & 23.4 & & & + & & & \\
\hline
\end{tabular}


Table E-09, Continued. Composite listing of VOCs emitted by Sheet Vinyls SV1 through SV5 in 10-L chambers.

\begin{tabular}{|c|c|c|c|c|c|c|c|}
\hline COMPOUND & $\begin{array}{c}\mathrm{RT} \\
\text { (min) }\end{array}$ & SV1 & sv2 & SV3 & Sv5 & SV4 & $\begin{array}{l}\text { Blank } \\
\text { Run }\end{array}$ \\
\hline \multicolumn{8}{|l|}{ Alkane Hydrocarbons, Cont. } \\
\hline C12 Branched alkane HC & 23.6 & & + & & & & \\
\hline n-Dodecane & 26.2 & & + & + & + & + & \\
\hline n-Tridecane & 29.3 & $A$ & + & $A$ & $A$ & $A$ & \\
\hline n-Tetradecane & 32.2 & + & + & A & $A$ & + & \\
\hline n-Pentadecane & 35.0 & + & & + & + & & \\
\hline n-Hexadecane & 37.6 & + & & & & & \\
\hline \multicolumn{8}{|l|}{ Aromatic Hydrocarbons } \\
\hline Toluene & 11.7 & & & + & + & & \\
\hline m-,p-Xylene & 16.1 & & & + & + & & + \\
\hline o-Xylene & 17.3 & & & + & + & & + \\
\hline Propylbenzene & 19.5 & & & + & + & & \\
\hline Ethyltoluene isomer & 19.7 & & + & + & + & + & \\
\hline 4-Ethyltoluene & 19.8 & & + & + & + & + & \\
\hline C3 Alkylbenzene & 20.0 & & & + & + & & \\
\hline 2-Ethyltoluene & 20.6 & & & + & + & & \\
\hline $1,2,4-$ Trimethylbenzene & 21.1 & & + & + & + & + & + \\
\hline C4 Alkylbenzene & 21.8 & & & + & + & & \\
\hline C4 Alkylbenzene & 22.0 & & + & & + & & \\
\hline 1,2,3-Trimethylbenzene & 22.4 & & & + & + & + & \\
\hline 1,3-Diethylbenzene & 22.9 & & & + & + & + & \\
\hline C4 Alkylbenzene & 23.0 & & + & + & + & + & \\
\hline C4 Alkylbenzene & 23.1 & & + & & & & \\
\hline C4 Alkylbenzene & 23.2 & & + & & + & + & \\
\hline C4 Alkylbenzene & 23.2 & & & + & + & + & \\
\hline C4 Alkylbenzene & 23.8 & & & + & + & + & \\
\hline C4 Alkylbenzene & 24.1 & & + & & + & + & \\
\hline C4 Alkylbenzene & 24.2 & & + & + & + & + & \\
\hline C4 Alkyllbenzene & 24.4 & & + & + & + & + & \\
\hline C5 Alkylbenzene & 24.6 & & & + & + & + & \\
\hline Butenylbenzene isomer & 24.7 & & & & + & & \\
\hline C4 Alkylbenzene & 25.3 & & & & & + & \\
\hline C4 Alkylbenzene & 25.6 & & + & & & + & \\
\hline $1,2,3,5$-Tetramethylbenzene & 25.8 & & + & & + & + & \\
\hline C5 Alkylbenzene & 26.1 & & & & & + & \\
\hline C4 Alkylbenzene & 27.1 & & + & + & + & + & \\
\hline C5 Alkylbenzene & 27.3 & & & & & + & \\
\hline C5 Alkylbenzene & 28.1 & & & & & + & \\
\hline Naphthalene & 29.5 & & + & + & & + & \\
\hline (1-Butylhexyl)benzene & 37.2 & & & + & + & & \\
\hline (1-Propylheptyl)benzene & 37.4 & & & + & + & & \\
\hline
\end{tabular}


Table E-09, Continued. Composite listing of VOCs emitted by Sheet Vinyls SV1 through SV5 in 10-L chambers.

\begin{tabular}{llllllll}
\hline COMPOUND & RT & & & & Blank \\
(min) & sv1 & sv2 & sv3 & sv5 & sv4 & Run \\
\hline
\end{tabular}

Aromatic Hydrocarbons, Cont.

(1-Ethyloctyl)benzene

(1-Methylnonyl)benzene

(1-Penylhexyl)benzene

(1-Butylheptyl)benzene

(1-Propyloctyl)benzene

(1-Ethylnonyl)benzene

(1,1-Dimethylnonyl)benzene

(1-Methyldecyl)benzene

(1-Pentylheptyl)benzene

(1-Butyloctyl)benzene

(1-Propylnonyl)benzene

(1-Ethyldecyl)benzene

\section{Other Hydrocarbons}

Ethylcyclohexane

Trimethylcyclohexane isomer

Trimethylcyclohexane isomer

Trimethylcyclohexane isomer

$\mathrm{C} 9$ Alkene $\mathrm{HC}$ or cyclic $\mathrm{HC}$

C9 Alkene $\mathrm{HC}$ or cyclic $\mathrm{HC}$

C3 Alkyl substituted cyclohexane

C9 Alkene $\mathrm{HC}$

C10 Alkene $\mathrm{HC}$

C3 Alkyl substituted cyclohexane

$\mathrm{C} 9 \mathrm{H} 16 \mathrm{HC}$

Propylcyclohexane

C10 Alkene or cyclic HC

C10 Alkene $\mathrm{HC}$

C10 Alkene $\mathrm{HC}$

C4 Alkyl substituted cyclohexane

C10 Alkene HC

C4 Alkyl substituted cyclohexane

C10 Alkene $\mathrm{HC}$

C10 Alkene $\mathrm{HC}$

C10 alkene $\mathrm{HC}$ or cyclic $\mathrm{HC}$

C4 Alkyl substituted cyclohexane

Pentamethylcyclohexane isomer

C4 Alkyl substituted cyclohexane

C11 Alkene $\mathrm{HC}$

C1OH18 Hydrocarbon

C11 Alkene $\mathrm{HC}$
38.0

39.1

39.5

39.6

39.9

40.5

40.7

41.5

41.8

42.0

42.3

42.9

12.9

13.0

13.6

14.7

14.9

15.0

15.1

15.2

15.4

16.0

16.7

16.9

17.0

17.1

17.2

17.4

17.6

18.2

18.6

18.8

18.8

19.0

19.6

19.6

20.3

20.3

20.6

$\begin{array}{ll}+ & + \\ + & + \\ + & + \\ + & + \\ + & + \\ + & + \\ + & + \\ + & + \\ + & + \\ + & + \\ + & + \\ + & +\end{array}$

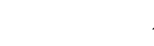

\section{$+$}

$+\quad+$

$+\quad+$

$+$

$+$

$+$

$+$

$+$

$+\quad+$

$+\quad+$

$+\quad+$

$+\quad+$

$+$

$+\quad+$

$+\quad+$

$+$

$+\quad+$

$+\quad+$

$+$

$+\quad+$

$+\quad+$

$+$

$+$

$+$

$+$ 
Table E-09, Continued. Composite listing of VOCs emitted by Sheet Vinyls SV1 through SV5 in 10-L chambers.

\begin{tabular}{|c|c|c|c|c|c|c|c|}
\hline COMPOUND & $\begin{array}{c}\text { RT } \\
\text { (min) }\end{array}$ & SV1 & SV2 & SV3 & SV5 & SV4 & $\begin{array}{c}\text { Blank } \\
\text { Run }\end{array}$ \\
\hline \multicolumn{8}{|l|}{ Other Hydrocarbons, Cont. } \\
\hline Butylcyclohexane & 20.9 & & & + & + & & \\
\hline C11 Alkene $\mathrm{HC}$ & 21.0 & & & + & + & & \\
\hline C5 Alkyl substituted cyclohexane & 22.0 & & & & & + & \\
\hline Decahydronaphthalene isomer & 22.3 & & & + & + & & \\
\hline C11 Alkene HC & 22.6 & & & & + & & \\
\hline C11 Alkene $\mathrm{HC}$ & 22.7 & & & + & + & & \\
\hline Alkene $\mathrm{HC}$ & 29.2 & & & & + & & \\
\hline Alkene $\mathrm{HC}$ & 29.5 & & & & + & & \\
\hline Alkene $\mathrm{HC}$ & 29.9 & & & + & + & & \\
\hline C14 Alkene HC & 30.4 & & & + & + & & \\
\hline Alkene $\mathrm{HC}$ & 37.1 & & & + & + & + & \\
\hline \multicolumn{8}{|l|}{ Carbonyl Compounds } \\
\hline Heptanal & 18.0 & & & + & & & \\
\hline 6-Methyl-5-hepten-2-one & 22.3 & + & & & & & \\
\hline Benzaldehyde & 22.5 & + & & + & + & & + \\
\hline Octanal & 22.6 & + & & & & & + \\
\hline Nonanal & 26.0 & + & + & + & + & + & + \\
\hline 1-Phenylethanone & 26.4 & + & + & + & + & + & \\
\hline Decanal & 29.3 & + & & & & + & + \\
\hline \multicolumn{8}{|l|}{ Other Oxidizded Compounds } \\
\hline Isopropyl acetate & 7.7 & & & & & + & \\
\hline 1-Butanol & 9.9 & & & + & + & & \\
\hline n-Propyl acetate & 10.1 & & + & & & + & \\
\hline Acetic acid & 10.8 & + & + & + & & + & \\
\hline 2-Propoxyethanol & 15.5 & & + & & & + & \\
\hline Isooctanol & 22.7 & + & & & & & \\
\hline Di(propylene glycol) methyl ether 1 & 23.1 & + & & & & & \\
\hline Di(propylene glycol) methyl ether 2 & 23.2 & + & & & & & \\
\hline Di(propylene glycol) methyl ether 3 & 23.8 & + & & & & & \\
\hline 2-Ethyl-1-hexanol & 23.9 & $A$ & & & & & \\
\hline 4-Methyl-1-heptanol & 24.2 & A & & & & & \\
\hline 6-Methyl-1-heptanol & 24.3 & + & & & & & \\
\hline 5-Methyl-1-heptanol & 24.5 & + & & & & & \\
\hline 1-Octanol & 25.4 & & + & & + & & \\
\hline Benzyl alcohol & 26.5 & & & A & A & & \\
\hline Phenol & 26.6 & $A$ & $A$ & A & A & A & \\
\hline 2-Phenyl-2-propanol & 27.2 & + & & & & & \\
\hline 2-Ethylhexanoic acid & 28.4 & & & & + & & \\
\hline 2-(2-Butoxyethoxy)ethanol & 29.9 & + & + & & & + & + \\
\hline
\end{tabular}


Table E-09, Continued. Composite listing of VOCs emitted by Sheet Vinyls SV1 through SV5 in 10-L chambers.

\begin{tabular}{|c|c|c|c|c|c|c|c|}
\hline COMPOUND & $\begin{array}{c}\mathrm{RT} \\
(\mathrm{min})\end{array}$ & SV1 & SV2 & SV3 & SV5 & SV4 & $\begin{array}{c}\text { Blank } \\
\text { Run }\end{array}$ \\
\hline \multicolumn{8}{|l|}{ Other Oxidized Compounds, Cont. } \\
\hline Nonanoic acid & 33.3 & & & & & + & \\
\hline $\begin{array}{c}\text { 2,2,4-Trimethyl-1,3-pentanediol } \\
\text { monisobutyrate (Texanol 1) }\end{array}$ & 35.2 & + & + & + & & + & + \\
\hline $\begin{array}{c}\text { 2,2,4-Trimethyl-1,3-pentanediol } \\
\text { monisobutyrate (Texanol 3) }\end{array}$ & 35.7 & + & + & + & & + & + \\
\hline Decanoic acid & 35.9 & + & + & + & & + & + \\
\hline 1-Dodecanol & 37.1 & & + & & & & \\
\hline $\begin{array}{l}\text { 2,6-Di-tert-butyl-4-methylphenol } \\
(\mathrm{BHT})\end{array}$ & 37.7 & + & & & & & \\
\hline $\begin{array}{l}\text { 2,2,4-Trimethyl-1,3-pentanediol } \\
\text { diisobutyrate (TXIB) }\end{array}$ & 40.3 & A & + & $A$ & A & + & \\
\hline Diethylphthalate & 42.3 & A & + & + & & + & + \\
\hline \multicolumn{8}{|l|}{ Nitrogen-Containing Compounds } \\
\hline 1-Methyl-2-pyrrolidinone & 27.5 & + & + & & & + & \\
\hline N-Propylbenzamide & 40.7 & & & & + & & \\
\hline \multicolumn{8}{|l|}{ Miscellaneous Compounds } \\
\hline Hexamethylcyclotrisiloxane & 11.8 & + & + & + & + & + & + \\
\hline Octamethylcyclotrisiloxane & 18.2 & + & & + & + & + & + \\
\hline Siloxane compound & 33.7 & & 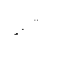 & & + & & \\
\hline \multicolumn{8}{|l|}{ Unidentified Compounds } \\
\hline Unidentified compound & 17.2 & & + & & + & & \\
\hline Unidentified compound & 17.3 & & + & & & + & \\
\hline Unidentified compound & 17.4 & & & + & & + & \\
\hline Unidentified compound & 21.8 & & & & & + & \\
\hline Unidentified compound & 22.0 & & + & & & & \\
\hline Unidentified compound & 22.9 & & + & & & + & \\
\hline Undientified compound & 25.4 & & & & & + & \\
\hline Unidentified compound & 27.8 & & & & & + & \\
\hline Unidentified compound & 28.9 & & & & + & & \\
\hline Unidentified compound & 29.0 & & & + & + & & \\
\hline Unidentified alcohol & 29.2 & + & & & & & \\
\hline Unidentified compound & 29.5 & & & + & & & \\
\hline Unidentified compound & 29.6 & & & + & & & \\
\hline Unidentified compound & 29.9 & & & + & & & \\
\hline Unidentified oxidized compound & 30.1 & & & + & + & & \\
\hline Unidentified compound & 30.4 & & & + & & & \\
\hline Unidentified glycol ether 1 & 30.5 & + & & & & & \\
\hline Unidentified glycol ether 2 & 30.7 & + & & & & & \\
\hline Unidentified oxidized compound & 35.4 & + & & & & & \\
\hline
\end{tabular}

$A=$ Abundant compound. 
Table E-10. Composition of VOCs in Seam Sealer SS, Parts A and B, as determined by analysis of the bulk products.

\begin{tabular}{lcc}
\hline COMPOUND & $\begin{array}{c}\text { Fraction of } \\
\text { Product by } \\
\text { Volume }\end{array}$ & $\begin{array}{c}\text { Mass per Vol. of } \\
\text { Product } \\
(\mathrm{mg} \mathrm{mL}-1)\end{array}$ \\
\hline $\begin{array}{l}\text { Part A } \\
\text { Tetrahydrofuran }\end{array}$ & 0.66 & 590 \\
Cyclohexanone & 0.10 & 91 \\
Part B & & \\
Tetrahydrofuran & 0.61 & 540 \\
Cyclohexanone & 0.06 & 57 \\
\hline
\end{tabular}


Table E-11. VOCs emitted by Cove Base CB in 10-L chamber at 6- and 48-hours elapsed times.

\begin{tabular}{|c|c|c|c|c|c|}
\hline COMPOUND & Code $^{\star}$ & $\begin{array}{c}\mathrm{RT} \\
(\mathrm{min})\end{array}$ & $\begin{array}{l}\text { 6-h } \\
\text { ET }\end{array}$ & $\begin{array}{c}\text { 48-h } \\
\text { ET }\end{array}$ & $\begin{array}{l}\text { Match } \\
\text { Quality } \\
\end{array}$ \\
\hline \multicolumn{6}{|l|}{ Alkane Hydrocarbons } \\
\hline n-Tridecane & $Q$ & 29.3 & + & + & Confirmed \\
\hline \multicolumn{6}{|l|}{ Aromatic Hydrocarbons } \\
\hline Toluene & $T, A, Q$ & 11.7 & + & + & Confirmed \\
\hline Styrene & $\mathrm{T}, \mathrm{Q}$ & 17.6 & + & + & Confirmed \\
\hline 4-tert-Butyltoluene & $\mathrm{Q}$ & 24.3 & + & + & Confirmed \\
\hline bis(1-Methylethyl)benzene & & 26.9 & + & + & Probable \\
\hline 1-(1-Ethylpropyl)-4-methylbenzene & & 27.2 & + & + & Tentative \\
\hline C6 Alkylbenzene & & 27.4 & + & + & Probable \\
\hline $\begin{array}{l}\text { 1,4-Dimethyl-2-(2-methylpropyl)- } \\
\text { benzene }\end{array}$ & & 27.5 & + & + & Tentative \\
\hline (1,1-Dimethylbutyl)benzene & & 27.8 & + & + & Probable \\
\hline C6 Alkylbenzene & & 28.2 & + & + & Probable \\
\hline C2 Tetrahydronaphthalene & & 30.1 & + & + & Probable \\
\hline C2 Tetrahydronaphthalene & & 30.2 & + & + & Probable \\
\hline C2 Tetrahydronaphthalene & $A$ & 32.4 & + & + & Probable \\
\hline C2 Tetrahydronaphthalene & $A$ & 32.6 & + & + & Probable \\
\hline C2 Tetrahydronaphthalene & A & 33.0 & + & + & Probable \\
\hline \multicolumn{6}{|l|}{ Other Hydrocarbons } \\
\hline C3 Alkyl substituted cyclohexane & & 15.1 & + & + & Probable \\
\hline $\begin{array}{l}2,6,6-\text { Trimethylbicyclo[3.1.1]- } \\
\text { heptane }\end{array}$ & & 18.7 & + & + & Probable \\
\hline C4 Alkyl substituted cyclohexane & & 20.1 & + & + & Probable \\
\hline \multicolumn{6}{|l|}{ Carbonyl Compounds } \\
\hline Cyclohexanone & & 19.8 & + & + & Confirmed \\
\hline Benzaldehyde & & 22.5 & + & + & Confirmed \\
\hline 1-Phenylethanone & $T$ & 26.5 & & + & Confirmed \\
\hline \multicolumn{6}{|l|}{ Other Oxidized Compounds } \\
\hline alpha-Terpineol & $Q$ & 29.5 & + & + & Confirmed \\
\hline 2-tert-Butylphenol & $\mathrm{Q}$ & 34.0 & + & + & Confirmed \\
\hline \multicolumn{6}{|l|}{ Nitrogen-Containing Compounds } \\
\hline 4-Methylmorpholine & & 13.2 & + & + & Confirmed \\
\hline Morpholine & & 15.2 & & + & Confirmed \\
\hline \multicolumn{6}{|l|}{ Miscellaneous compounds } \\
\hline tert-Butyl isothiocyanate & $A, Q$ & 16.6 & + & + & Confirmed \\
\hline Benzothiazole & $A, Q$ & 31.7 & + & + & Confirmed \\
\hline \multicolumn{6}{|l|}{ Unidentified Compounds } \\
\hline Unidentified oxidized compound & & 26.6 & + & + & Unident. \\
\hline
\end{tabular}

${ }^{*}-{ }_{1}=$ Toxic air contaminant; $\mathrm{A}=$ Abundant compound; $\mathrm{Q}=$ Quantified target compound 
Table E-12. VOCs emitted by Underlayment UL in 10-L chamber at 6- and 48-hours elapsed times.

\begin{tabular}{|c|c|c|c|c|c|}
\hline COMPOUND & Code $^{\star}$ & $\begin{array}{c}\mathrm{RT} \\
(\mathrm{min}) \\
\end{array}$ & $\begin{array}{l}\text { 6-h } \\
\mathrm{ET} \\
\end{array}$ & $\begin{array}{c}\text { 48-h } \\
\mathrm{ET}\end{array}$ & $\begin{array}{l}\text { Match } \\
\text { Quality } \\
\end{array}$ \\
\hline $\begin{array}{l}\text { Aromatic Hydrocarbons } \\
\text { p-Cymene }\end{array}$ & & 22.0 & + & & Confirmed \\
\hline $\begin{array}{l}\text { Other Hydrocarbons } \\
\text { alpha-Pinene } \\
\text { beta-Pinene } \\
\text { 3-Carene } \\
\text { d-Limonene } \\
\text { Terpene HC }\end{array}$ & & $\begin{array}{l}17.3 \\
19.4 \\
20.4 \\
21.4 \\
21.7\end{array}$ & $\begin{array}{l}+ \\
+ \\
+ \\
+ \\
+\end{array}$ & $\begin{array}{l}+ \\
+\end{array}$ & $\begin{array}{l}\text { Confirmed } \\
\text { Confirmed } \\
\text { Confirmed } \\
\text { Confirmed } \\
\text { Probable }\end{array}$ \\
\hline $\begin{array}{l}\text { Carbonyl Compounds } \\
\text { Pentanal } \\
\text { Hexanal } \\
\text { 2-Furancarboxaldehyde } \\
\text { Heptanal } \\
\text { 6-Methyl-5-hepten-2-one } \\
\text { Benzaldehyde } \\
\text { Octanal } \\
\text { Nonanal } \\
\text { Decanal }\end{array}$ & $\begin{array}{l}Q \\
Q\end{array}$ & $\begin{array}{l}10.0 \\
14.5 \\
18.0 \\
18.7 \\
22.2 \\
22.4 \\
22.5 \\
26.0 \\
29.3\end{array}$ & $\begin{array}{l}+ \\
+ \\
+ \\
+ \\
+ \\
+ \\
+ \\
+ \\
+\end{array}$ & $\begin{array}{l}+ \\
+ \\
+ \\
+\end{array}$ & $\begin{array}{l}\text { Confirmed } \\
\text { Confirmed } \\
\text { Confirmed } \\
\text { Confirmed } \\
\text { Confirmed } \\
\text { Confirmed } \\
\text { Confirmed } \\
\text { Confirmed } \\
\text { Confirmed }\end{array}$ \\
\hline $\begin{array}{l}\text { Other Oxidized Compounds } \\
\text { Acetic acid } \\
\text { Isooctanol } \\
\text { Hexanoic acid } \\
\text { alpha-Terpineol }\end{array}$ & $Q$ & $\begin{array}{l}10.8 \\
22.6 \\
24.4 \\
29.5\end{array}$ & $\begin{array}{l}+ \\
+ \\
+ \\
+\end{array}$ & $\begin{array}{l}+ \\
+\end{array}$ & $\begin{array}{l}\text { Confirmed } \\
\text { Tentative } \\
\text { Confirmed } \\
\text { Confirmed }\end{array}$ \\
\hline $\begin{array}{l}\text { Miscellaneous compounds } \\
\text { Hexamethylcyclotrisiloxane } \\
\text { Octamethylcyclotetrasiloxane } \\
\text { Siloxane compound } \\
\text { Siloxane compound } \\
\text { Siloxane compound }\end{array}$ & & $\begin{array}{l}11.8 \\
18.2 \\
27.8 \\
28.9 \\
33.7\end{array}$ & $\begin{array}{l}+ \\
+ \\
+ \\
+ \\
+\end{array}$ & $\begin{array}{l}+ \\
+ \\
+ \\
+ \\
+\end{array}$ & $\begin{array}{l}\text { Confirmed } \\
\text { Confirmed } \\
\text { Probable } \\
\text { Probable } \\
\text { Probable }\end{array}$ \\
\hline $\begin{array}{l}\text { Unidentified Compounds } \\
\text { Unidentified compound } \\
\text { Unidentified compound }\end{array}$ & & $\begin{array}{l}21.8 \\
22.9\end{array}$ & $\begin{array}{l}+ \\
+\end{array}$ & + & $\begin{array}{l}\text { Unident. } \\
\text { Unident. }\end{array}$ \\
\hline
\end{tabular}

*A = Abundant compound; $\mathrm{Q}=$ Quantified target compound. 
Table E-13. VOCs emitted by Gypsum Board GB in 10-L chamber at 6- and 48-hours elapsed times.

\begin{tabular}{|c|c|c|c|c|c|}
\hline COMPOUND & Code $^{\star}$ & $\begin{array}{c}\mathrm{RT} \\
(\mathrm{min}) \\
\end{array}$ & $\begin{array}{l}6-\mathrm{h} \\
\text { ET }\end{array}$ & $\begin{array}{c}48-h \\
E T \\
\end{array}$ & $\begin{array}{l}\text { Match } \\
\text { Quality } \\
\end{array}$ \\
\hline \multicolumn{6}{|l|}{ Aromatic Hydrocarbons } \\
\hline Toluene & $\mathrm{T}$ & 11.7 & & + & Confirmed \\
\hline \multicolumn{6}{|l|}{ Carbonyl Compounds } \\
\hline Benzaldehyde & & 22.4 & + & + & Confirmed \\
\hline Octanal & & 22.5 & + & + & Confirmed \\
\hline Nonanal & & 26.1 & + & + & Confirmed \\
\hline Decanal & & 29.3 & + & + & Confirmed \\
\hline \multicolumn{6}{|l|}{ Other Oxidized Compounds } \\
\hline Acetic acid & & 10.8 & + & + & Confirmed \\
\hline \multicolumn{6}{|l|}{ Miscellaneous compounds } \\
\hline Hexamethylcyclotrisiloxane & & 11.8 & & + & Confirmed \\
\hline Siloxane compound & & 23.6 & + & + & Probable \\
\hline Siloxane compound & & 33.7 & + & + & Probable \\
\hline
\end{tabular}

${ }^{*} \mathrm{~T}=$ Toxic air contaminant. 
Table E-14. VOCs emitted in 10-L chamber at 6- and 48-hours elapsed times by Sheet Flooring Adhesive SFA applied to particle board Underlayment UL.

\begin{tabular}{|c|c|c|c|c|c|}
\hline COMPOUND & Code $^{*}$ & $\begin{array}{c}\mathbf{R T} \\
(\min )\end{array}$ & $\begin{array}{l}\text { 6-h } \\
\text { ET } \\
\end{array}$ & $\begin{array}{c}\text { 48-h } \\
\mathrm{ET} \\
\end{array}$ & $\begin{array}{l}\text { Match } \\
\text { Quality } \\
\end{array}$ \\
\hline \multicolumn{6}{|l|}{ Aromatic Hydrocarbons } \\
\hline Toluene & $T, A, Q$ & 11.7 & + & + & Confirmed \\
\hline Indene & & 24.0 & + & + & Confirmed \\
\hline 4-Phenylcyclohexene & & 33.0 & + & + & Confirmed \\
\hline \multicolumn{6}{|l|}{ Other Hydrocarbons } \\
\hline C4 Alkyl substituted cyclohexane & & 18.8 & + & + & Probable \\
\hline C4 Alkyl substituted cyclohexane & & 19.4 & + & + & Probable \\
\hline 5-Vinyl-2-norbornene & & 19.6 & + & & Tentative \\
\hline $\mathrm{C} 10 \mathrm{H} 22$ Compound & & 21.3 & + & + & Probable \\
\hline Dicyclopentadiene & & 21.7 & + & + & Probable \\
\hline $\mathrm{C} 1 \mathrm{OH} 14$ Compound & & 22.3 & + & + & Probable \\
\hline $\mathrm{C} 15 \mathrm{H} 24$ Compound & & 32.2 & & + & Probable \\
\hline $\mathrm{C} 15 \mathrm{H} 24$ Compound & & 33.0 & & + & Probable \\
\hline C15H24 Compound & & 33.3 & & + & Probable \\
\hline Longifolene & $A, Q$ & 34.2 & + & + & Confirmed \\
\hline Caryophyllene & A & 34.7 & + & + & Probable \\
\hline \multicolumn{6}{|l|}{ Carbonyl Compounds } \\
\hline Hexanal & & 14.5 & & + & Confirmed \\
\hline Benzaldehyde & & 22.5 & & + & Confirmed \\
\hline Decanal & & 29.3 & + & & Confirmed \\
\hline \multicolumn{6}{|l|}{ Other Oxidized Compounds } \\
\hline Acetic acid & & 11.0 & + & + & Confirmed \\
\hline $\begin{array}{l}\text { Trimethylcyclohexanemethanol } \\
\text { isomer }\end{array}$ & & 27.8 & + & + & Tentative \\
\hline alpha-Terpineol & $\mathrm{Q}$ & 29.5 & + & + & Confirmed \\
\hline $\begin{array}{l}\text { 2,6-Di-tert-butyl-4-methylphenol } \\
\text { (BHT) }\end{array}$ & $A, Q$ & 37.7 & & + & Confirmed \\
\hline \multicolumn{6}{|l|}{ Miscellaneous compounds } \\
\hline Hexamethylcyclotrisiloxane & & 11.8 & + & & Confirmed \\
\hline Octamethylcyclotetrasiloxane & & 18.2 & + & + & Confirmed \\
\hline Siloxane compound & & 23.7 & & + & Probable \\
\hline Siloxane compound & & 29.0 & + & + & Probable \\
\hline Siloxane compound & & 33.7 & + & + & Probable \\
\hline \multicolumn{6}{|l|}{ Unidentified Compounds } \\
\hline Unidentified mixture & & 22.9 & + & & Unident. \\
\hline Unidentified compound & & 32.2 & & + & Unident. \\
\hline Unidentified compound & A & 36.7 & & + & Unident. \\
\hline Unidentified compound & A & 41.5 & & + & Unident. \\
\hline
\end{tabular}

${ }^{*} \mathrm{~T}=$ Toxic air contaminant; $\mathrm{A}=$ Abundant compound; $\mathrm{Q}=$ Quantified target compound . 
Table E-15. VOCs emitted in 10-L chamber at 6- and 48-hours elapsed times by Cove Base Adhesive CBA applied to Gypsum Board GB.

\begin{tabular}{|c|c|c|c|c|c|}
\hline COMPOUND & Code ${ }^{*}$ & $\begin{array}{c}\mathbf{R T} \\
(\mathrm{min})\end{array}$ & $\begin{array}{l}\text { 6-h } \\
\text { ET }\end{array}$ & $\begin{array}{c}\text { 48-h } \\
\text { ET }\end{array}$ & $\begin{array}{l}\text { Match } \\
\text { Quality }\end{array}$ \\
\hline \multicolumn{6}{|l|}{ Alkane Hydrocarbons } \\
\hline C8 Branched alkane $\mathrm{HC}$ & & 7.9 & + & + & Probable \\
\hline C8 Branched alkane HC & & 8.0 & + & + & Probable \\
\hline C8 Branched alkane $\mathrm{HC}$ & & 9.3 & + & + & Probable \\
\hline C8 Branched alkane $\mathrm{HC}$ & $A$ & 9.4 & + & + & Probable \\
\hline C8 Branched alkane $\mathrm{HC}$ & & 9.5 & + & + & Probable \\
\hline C8 Branched alkane HC & & 9.8 & + & + & Probable \\
\hline C8 Branched alkane $\mathrm{HC}$ & & 9.9 & + & + & Probable \\
\hline n-Octane & $A, Q$ & 11.0 & + & + & Confirmed \\
\hline C9 Branched alkane $\mathrm{HC}$ & & 11.9 & + & + & Probable \\
\hline C9 Branched alkane $\mathrm{HC}$ & & 12.2 & + & + & Probable \\
\hline C9 Branched alkane $\mathrm{HC}$ & & 12.5 & + & + & Probable \\
\hline $\mathrm{C} 9$ Branched alkane $\mathrm{HC}$ & & 13.4 & + & + & Probable \\
\hline C9 Branched alkane $\mathrm{HC}$ & & 13.7 & + & + & Probable \\
\hline C9 Branched alkane $\mathrm{HC}$ & & 14.0 & + & + & Probable \\
\hline n-Nonane & $Q$ & 15.2 & + & + & Confirmed \\
\hline C10 Branched alkane $\mathrm{HC}$ & & 16.6 & + & + & Probable \\
\hline C10 Branched alkane $\mathrm{HC}$ & & 17.5 & + & + & Probable \\
\hline C10 Branched alkane HC & & 17.7 & + & + & Probable \\
\hline C10 Branched alkane $\mathrm{HC}$ & & 17.8 & + & + & Probable \\
\hline $\mathrm{C} 10$ Branched alkane $\mathrm{HC}$ & & 18.1 & + & + & Probable \\
\hline n-Decane & $A, Q$ & 19.2 & + & + & Confirmed \\
\hline C11 Branched alkane $\mathrm{HC}$ & & 20.0 & + & + & Probable \\
\hline C11 Branched alkane $\mathrm{HC}$ & & 20.6 & & + & Probable \\
\hline C11 Branched alkane $\mathrm{HC}$ & & 21.3 & + & + & Probable \\
\hline C11 Branched alkane $\mathrm{HC}$ & & 21.4 & + & + & Probable \\
\hline C11 Branched alkane $\mathrm{HC}$ & & 21.5 & + & + & Probable \\
\hline C11 Branched alkane $\mathrm{HC}$ & & 21.8 & + & + & Probable \\
\hline n-Undecane & $\mathrm{Q}$ & 22.8 & + & + & Confirmed \\
\hline C12 Branched alkane HC & & 23.8 & & + & Probable \\
\hline n-Dodecane & & 26.1 & + & + & Confirmed \\
\hline \multicolumn{6}{|l|}{ Aromatic Hydrocarbons } \\
\hline Toluene & $T, Q$ & 11.7 & + & + & Confirmed \\
\hline m-,p-Xylene & $\mathrm{T}, \mathrm{Q}$ & 16.1 & + & + & Confirmed \\
\hline Styrene & $\mathrm{T}, \mathrm{Q}$ & 17.6 & + & + & Confirmed \\
\hline 4-Phenylcyclohexene & $\mathrm{Q}$ & 33.0 & + & + & Confirmed \\
\hline \multicolumn{6}{|l|}{ Other Hydrocarbons } \\
\hline Methylcyclohexane & & 8.1 & + & + & Confirmed \\
\hline C3 Cyclopentane & & 8.6 & + & + & Probable \\
\hline C8 Alkene HC & & 8.9 & + & + & Probable \\
\hline Dimethylcyclohexane isomer & A & 10.4 & + & + & Probable \\
\hline Dimethylcyclohexane isomer & & 10.5 & + & + & Probable \\
\hline Dimethylcyclohexane isomer & & 10.7 & + & + & Probable \\
\hline Dimethylcyclohexane isomer & & 10.9 & + & + & Probable \\
\hline
\end{tabular}


Table E-15, Continued. VOCs emitted in 10-L chamber at 6- and 48-hours elapsed times by Cove Base Adhesive CBA applied to Gypsum Board GB.

\begin{tabular}{|c|c|c|c|c|c|}
\hline COMPOUND & Code $^{\star}$ & $\begin{array}{c}\mathrm{RT} \\
(\mathrm{min})\end{array}$ & $\begin{array}{l}\text { 6-h } \\
\text { ET }\end{array}$ & $\begin{array}{c}\text { 48-h } \\
\text { ET }\end{array}$ & $\begin{array}{l}\text { Match } \\
\text { Quality }\end{array}$ \\
\hline \multicolumn{6}{|l|}{ Other Hydrocarbons, Cont. } \\
\hline Dimethylcyclohexane isomer & & 11.3 & + & + & Probable \\
\hline Dimethylcyclohexane isomer & & 11.6 & + & + & Probable \\
\hline Trimethylcyclohexane isomer & & 12.6 & + & + & Probable \\
\hline Propylcyclopentane & & 12.7 & + & + & Probable \\
\hline Ethylcyclohexane & $A, Q$ & 12.9 & + & + & Confirmed \\
\hline Trimethylcyclohexane isomer & & 13.0 & + & + & Probable \\
\hline Trimethylcyclohexane isomer & $A$ & 13.6 & + & + & Probable \\
\hline C9 Alkene $\mathrm{HC}$ & & 13.7 & + & + & Probable \\
\hline C3 Alkyl substituted cyclohexane & & 14.6 & + & + & Probable \\
\hline C3 Alkyl substituted cyclohexane & & 14.7 & + & + & Probable \\
\hline C3 Alkyl substituted cyclohexane & & 14.9 & + & + & Probable \\
\hline C3 Alkyl substituted cyclohexane & & 15.0 & + & + & Probable \\
\hline C3 Alkyl substituted cyclohexane & & 15.1 & + & + & Probable \\
\hline C3 Alkyl substituted cyclohexane & & 16.0 & + & + & Probable \\
\hline C3 Alkyl substituted cyclohexane & & 16.1 & + & + & Probable \\
\hline $\mathrm{C} 9 \mathrm{H} 16$ Compound & & 16.7 & + & + & Tentative \\
\hline Propylcyclohexane & & 17.0 & + & + & Confirmed \\
\hline C4 Alkyl substituted cyclohexane & & 17.2 & + & + & Probable \\
\hline C10 Alkene $\mathrm{HC}$ & & 18.1 & + & + & Probable \\
\hline C4 Alkyl substituted cyclohexane & & 18.2 & + & + & Probable \\
\hline C4 Alkyl substituted cyclohexane & & 18.6 & + & + & Probable \\
\hline C4 Alkyl substituted cyclohexane & & 18.8 & + & + & Probable \\
\hline C4 Alkyl substituted cyclohexane & & 19.0 & + & + & Probable \\
\hline C10 Alkene $\mathrm{HC}$ & & 19.1 & + & + & Probable \\
\hline C4 Alkyl substituted cyclohexane & & 19.4 & + & + & Tentative \\
\hline C11 Alkene HC & & 20.2 & + & + & Probable \\
\hline Butylcyclohexane & & 20.9 & + & + & Confirmed \\
\hline C11 Alkene HC & & 20.9 & + & + & Probable \\
\hline Decahydronaphthalene isomer & & 22.3 & + & + & Probable \\
\hline C5 Alkyl substituted cyclohexane & & 22.7 & + & + & Probable \\
\hline C12 Alkene HC & & 23.4 & + & + & Probable \\
\hline \multicolumn{6}{|l|}{ Carbonyl Compounds } \\
\hline Decanal & & 29.3 & + & + & Confirmed \\
\hline \multicolumn{6}{|l|}{ Other Oxidized Compounds } \\
\hline n-Butyl ether & & 15.4 & + & + & Confirmed \\
\hline $\begin{array}{l}\text { 2,6-Di-tert-butyl-4-methylphenol } \\
\text { (BHT) }\end{array}$ & $Q$ & 37.7 & & + & Confirmed \\
\hline \multicolumn{6}{|l|}{ Miscellaneous compounds } \\
\hline Octamethylcyclotetrasiloxane & & 18.2 & + & & Confirmed \\
\hline Siloxane compound & & 33.7 & + & + & Probable \\
\hline
\end{tabular}

${ }^{\star} T$ = Toxic air contaminant; $A$ = Abundant compound; $\mathrm{Q}=$ Quantified target compound. 
Table E-16. VOCs emitted in 10-L chamber at 6- and 48-hours elapsed times by Sheet Vinyl SV5 and Sheet Flooring Adhesive SFA applied to particle board Underlayment UL.

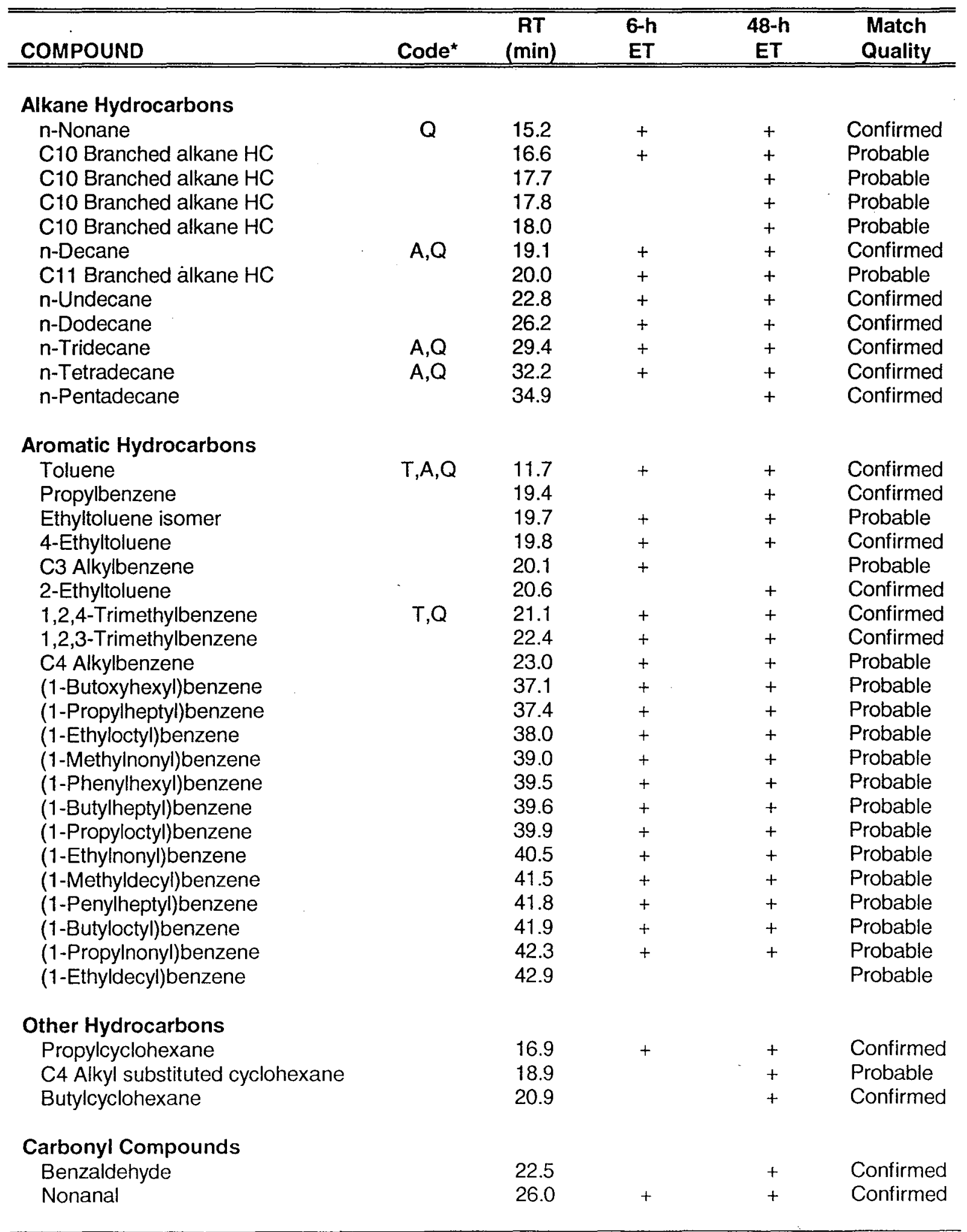


Table E-16, Continued. VOCs emitted in 10-L chamber at 6- and 48-hours elapsed times by Sheet Vinyl SV5 and Sheet Flooring Adhesive SFA applied to particle board Underlayment UL.

\begin{tabular}{|c|c|c|c|c|c|}
\hline COMPOUND & Code ${ }^{*}$ & $\begin{array}{c}\mathrm{RT} \\
(\mathrm{min})\end{array}$ & $\begin{array}{l}\text { 6-h } \\
\text { ET }\end{array}$ & $\begin{array}{l}\text { 48-h } \\
\text { ET }\end{array}$ & $\begin{array}{l}\text { Match } \\
\text { Quality }\end{array}$ \\
\hline \multicolumn{6}{|l|}{ Other Oxidized Compounds } \\
\hline 1-Octanol & $\mathrm{Q}$ & 25.4 & + & + & Confirmed \\
\hline Benzyl alcohol & $\hat{Q}$ & 26.4 & + & + & Confirmed \\
\hline Phenol & $T, A, Q$ & 26.6 & + & + & Confirmed \\
\hline 2-Ethylhexanoic acid & & 28.4 & & + & Probable \\
\hline 2-Ethyl-1-hexanol & & 30.4 & + & + & Confirmed \\
\hline 1-Dodecanol & & 37.0 & + & + & Confirmed \\
\hline $\begin{array}{l}\text { 2,2,4-Trimethyl-1,3-pentanediol } \\
\text { diisobutyrate (TXIB) }\end{array}$ & $A, Q$ & 40.3 & + & + & Confirmed \\
\hline \multicolumn{6}{|l|}{ Nitrogen-Containing Compounds } \\
\hline N-Propylbenzamide & & 40.7 & & + & Tentative \\
\hline \multicolumn{6}{|l|}{ Unidentified Compounds } \\
\hline Unidentified compound & & 29.0 & + & + & Unident. \\
\hline Unidentified compound & & 29.2 & + & + & Unident. \\
\hline Unidentified oxidized compound & & 29.5 & + & + & Unident. \\
\hline Unidentified oxidized compound & & 30.0 & + & + & Unident. \\
\hline
\end{tabular}

${ }^{\star} \mathrm{T}=$ Toxic air contaminant; $\mathrm{A}=$ Abundant compound; $\mathrm{Q}=$ Quantified target compound. 
Table E-17. VOCs emitted in 10-L chamber at 6- and 48-hours elapsed times by Cove Base CB and Cove Base Adhesive CBA applied to Gypsum Board GB.

\begin{tabular}{lccccc}
\hline & & RT & $6-h$ & $48-h$ & Match \\
COMPOUND & Code $^{*}$ & $(\mathrm{~min})$ & ET & ET & Quality \\
\hline \hline
\end{tabular}

Alkane Hydrocarbons

C8 Branched alkane $\mathrm{HC}$

C8 Branched alkane $\mathrm{HC}$

C8 Branched alkane $\mathrm{HC}$

C8 Branched alkane $\mathrm{HC}$

C8 Branched alkane $\mathrm{HC}$ n-Octane

C9 Branched alkane $\mathrm{HC}$

C9 Branched alkane $\mathrm{HC}$

C9 Branched alkane $\mathrm{HC}$

C9 Branched alkane $\mathrm{HC}$

C9 Branched alkane $\mathrm{HC}$

C9 Branched alkane $\mathrm{HC}$

n-Nonane

C10 Branched alkane $\mathrm{HC}$

C10 Branched alkane $\mathrm{HC}$

C10 Branched alkane $\mathrm{HC}$

C10 Branched alkane HC

n-Decane

C11 Branched alkane $\mathrm{HC}$

C11 Branched alkane $\mathrm{HC}$

C11 Branched alkane $\mathrm{HC}$

C11 Branched alkane $\mathrm{HC}$

C11 Branched alkane $\mathrm{HC}$

C11 Branched alkane $\mathrm{HC}$

$\mathrm{n}$-Undecane

n-Dodecane

\section{Aromatic Hydrocarbons}

Toluene

Ethylbenzene

$\mathrm{m}$-, p-Xylene

Styrene

4-tert-Butyltoluene

bis(1-Methylethyl)benzene

(1,1-Dimethylbutyl)benzene

C6 Alkylbenzene

4-Phenylcyclohexene

C2 Tetrahydronaphthalene

C2 Tetrahydronaphthalene

C2 Tetrahydronaphthalene

\section{Other Hydrocarbons}

Methylcyclohexane

Dimethylcyclohexane isomer

Dimethylcyclohexane isomer

\begin{tabular}{|c|c|c|c|c|}
\hline & 9.3 & + & + & Probable \\
\hline \multirow[t]{2}{*}{ A } & 9.5 & + & + & Probable \\
\hline & 9.6 & + & + & Probable \\
\hline \multirow[t]{2}{*}{$A$} & 9.8 & + & + & Probable \\
\hline & 9.9 & + & + & Probable \\
\hline \multirow[t]{7}{*}{$A, Q$} & 11.0 & + & + & Confirmed \\
\hline & 11.9 & + & + & Probable \\
\hline & 12.2 & + & + & Probable \\
\hline & 12.5 & + & + & Probable \\
\hline & 13.4 & + & + & Probable \\
\hline & 13.7 & + & + & Probable \\
\hline & 14.0 & + & + & Probable \\
\hline \multirow[t]{5}{*}{$Q$} & 15.2 & + & + & Confirmed \\
\hline & 16.6 & + & + & Probable \\
\hline & 17.7 & + & + & Probable \\
\hline & 17.8 & & + & Probable \\
\hline & 18.0 & + & + & Probable \\
\hline \multirow[t]{7}{*}{$A, Q$} & 19.2 & + & + & Confirmed \\
\hline & 20.0 & + & + & Probable \\
\hline & 20.6 & & + & Probable \\
\hline & 21.2 & & + & Probable \\
\hline & 21.4 & & + & Probable \\
\hline & 21.5 & & + & Probable \\
\hline & 21.8 & & + & Probable \\
\hline \multirow[t]{2}{*}{$Q$} & 22.8 & + & + & Confirmed \\
\hline & 26.1 & & + & Confirmed \\
\hline
\end{tabular}

\begin{tabular}{|c|c|c|c|c|}
\hline $\mathrm{T}, \mathrm{Q}$ & 11.7 & + & + & Confirmed \\
\hline $\mathrm{T}$ & 15.8 & + & & Confirmed \\
\hline $\mathrm{T}, \mathrm{Q}$ & 16.1 & + & & Confirmed \\
\hline $\mathrm{T}, \mathrm{Q}$ & 17.6 & + & + & Confirmed \\
\hline \multirow[t]{4}{*}{$Q$} & 24.3 & + & + & Confirmed \\
\hline & 26.9 & & + & Probable \\
\hline & 27.8 & + & + & Probable \\
\hline & 28.1 & + & + & Probable \\
\hline \multirow[t]{4}{*}{$\mathrm{Q}$} & 33.0 & & + & Confirmed \\
\hline & 32.3 & + & + & Probable \\
\hline & 32.5 & + & + & Probable \\
\hline & 32.9 & + & + & Probab \\
\hline
\end{tabular}

$\begin{array}{llll}8.1 & + & + & \text { Confirmed } \\ 10.4 & + & + & \text { Probable } \\ 10.5 & + & + & \text { Probable }\end{array}$


Table E-17, Continued. VOCs emitted in 10-L chamber at 6- and 48-hours elapsed times by Cove Base CB and Cove Base Adhesive CBA applied to Gypsum Board GB.

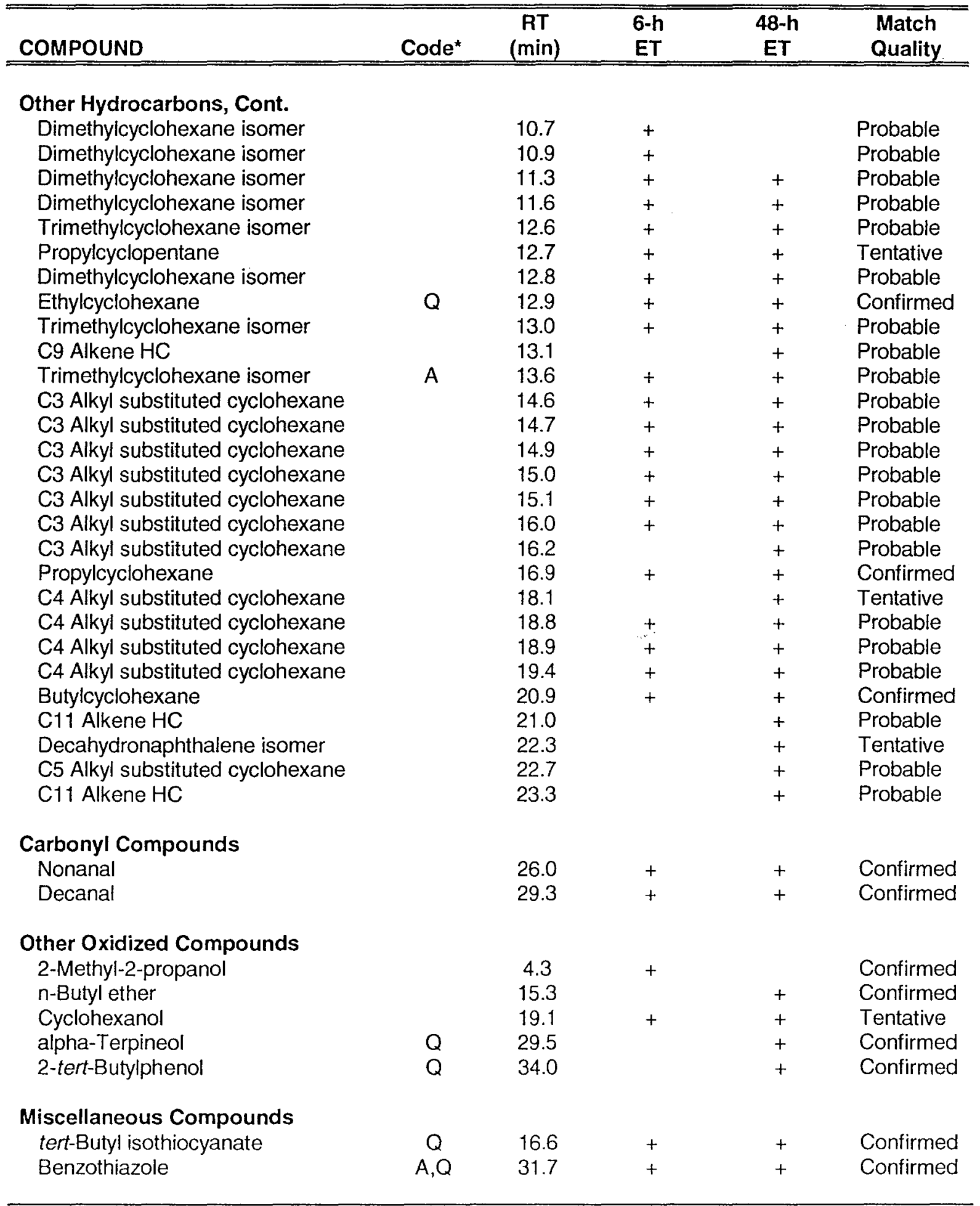

${ }^{*} \mathrm{~T}=$ Toxic air contaminant; $\mathrm{A}=$ Abundant compound; $\mathrm{Q}=$ Quantified target compound . 
Table E-18. Summary of target VOCs for screening measurements with sheet vinyl flooring materials.

\begin{tabular}{|c|c|c|c|c|c|c|c|c|c|c|c|}
\hline COMPOUND & SV1 & SV2 & SV3 & SV5 & SV4 & $\mathrm{CB}$ & UL & SFA & $\mathrm{CBA}$ & $\begin{array}{c}\text { SV5 \& } \\
\text { SFA }\end{array}$ & $\begin{array}{l}\mathrm{CB} \mathrm{\&} \\
\mathrm{CBA} \\
\end{array}$ \\
\hline \multicolumn{12}{|l|}{ Alkane Hydrocarbons } \\
\hline n-Octane & & & & & & & & & + & & + \\
\hline n-Nonane & & & + & + & & & & & + & + & + \\
\hline n-Decane & & & + & + & & & & & + & + & + \\
\hline n-Undecane & & & & & & & & & + & & + \\
\hline n-Dodecane & & + & & & & & & & & & \\
\hline $\mathrm{n}$-Tridecane & + & + & + & + & + & + & & & & + & + \\
\hline n-Tetradecane & & & + & + & & & & & & + & \\
\hline \multicolumn{12}{|l|}{ Aromatic Hydrocarbons } \\
\hline Toluene & & & + & + & & + & & + & + & + & + \\
\hline m-,p-Xylene & & & + & + & & & & & + & + & + \\
\hline o-Xylene & & & + & + & & & & & & + & \\
\hline Styrene & & & & & & + & & & + & & + \\
\hline 1,2,4-Trimethylbenzene & & + & + & + & + & & & & & + & \\
\hline 4-tert-Butyltoluene & & & & & & + & & & & & + \\
\hline Naphthalene & & + & + & + & + & & & & & + & \\
\hline 4-Phenylcyclohexene & & & & & & & & & + & & + \\
\hline \multicolumn{12}{|l|}{ Other Hydrocarbons } \\
\hline Ethylcyclohexane & & & & & & & & & + & & + \\
\hline Longifolene & & & & & & & & + & & + & \\
\hline \multicolumn{12}{|l|}{ Carbonyl Compounds } \\
\hline Hexanal & & & & & & & + & & & & \\
\hline Nonanal & & & & & & & + & & & & \\
\hline 1-Phenylethanone & + & & & & + & & & & & & \\
\hline Decanal & & & & & & & + & & & & \\
\hline
\end{tabular}


Table E-18, Continued. Summary of target VOCs for screening measurements with sheet vinyl flooring materials.

\begin{tabular}{|c|c|c|c|c|c|c|c|c|c|c|c|}
\hline COMPOUND & SV1 & sv2 & SV3 & SV5 & SV4 & $\mathrm{CB}$ & UL & SFA & $\mathrm{CBA}$ & $\begin{array}{c}\text { SV5 \& } \\
\text { SFA } \\
\end{array}$ & $\begin{array}{l}\mathrm{CB} \& \\
\mathrm{CBA} \\
\end{array}$ \\
\hline $\begin{array}{l}\text { Other Oxidized Compounds } \\
\text { n-Propyl acetate } \\
\text { 2-Propoxyethanol } \\
\text { Di(propylene glycol) methyl ethers } \\
\text { 2-Ethyl-1-hexanol } \\
\text { 1-Octanol } \\
\text { Benzyl alcohol } \\
\text { Phenol } \\
\text { alpha-Terpineol } \\
\text { 2-(2-Butoxyethoxy)ethanol } \\
\text { 2-tert-Butylphenol } \\
\text { 2,6-Di-tert-butyl-4-methylphenol } \\
\text { 2,2,4-Trimethyl-1,3-pentanediol } \\
\text { diisobutyrate } \\
\text { Diethylphthalate }\end{array}$ & $\begin{array}{l}+ \\
+ \\
+ \\
+ \\
+ \\
\end{array}$ & $\begin{array}{l}+ \\
+ \\
+\end{array}$ & $\begin{array}{l}+ \\
+ \\
+ \\
+ \\
+\end{array}$ & $\begin{array}{r}+ \\
+ \\
+ \\
\end{array}$ & $\begin{array}{l}+ \\
+ \\
+ \\
+\end{array}$ & $\begin{array}{l}+ \\
+\end{array}$ & + & + & + & $\begin{array}{l}+ \\
+ \\
+\end{array}$ & $\begin{array}{l}+ \\
+ \\
+\end{array}$ \\
\hline $\begin{array}{l}\text { Nitrogen Containing Compounds } \\
\text { 1-Methyl-2-pyrrolidinone }\end{array}$ & + & + & & & + & & & & & & \\
\hline $\begin{array}{l}\text { Mscellaneous Compounds } \\
\text { tert-Butyl isothiocyanate } \\
\text { Eenzothiazole }\end{array}$ & & & & & & $\begin{array}{l}+ \\
+\end{array}$ & & & & & + \\
\hline
\end{tabular}


Table E-19. Chamber concentrations of target VOCs for 48-h screening measurement of Sheet Vinyl SV1.

\begin{tabular}{|c|c|c|c|c|}
\hline \multirow[b]{2}{*}{ Compound } & \multicolumn{4}{|c|}{ Chamber Concentration, $\mu \mathrm{g} \mathrm{m}^{-3}$} \\
\hline & 1-h & 6-h & 24-h & 48-h \\
\hline \multicolumn{5}{|l|}{ Alkane Hydrocarbons } \\
\hline n-Tridecane & 5 & 3 & 2 & 2 \\
\hline \multicolumn{5}{|l|}{ Carbonyl Compounds } \\
\hline 1-Phenylethanone & 2 & 1 & 1 & 1 \\
\hline \multicolumn{5}{|l|}{ Other Oxidized Cmpds. } \\
\hline $\begin{array}{l}\text { Di(propylene glycol) methyl } \\
\text { ethers }\end{array}$ & 7 & 4 & 3 & 3 \\
\hline 2-Ethyl-1-hexanol & 8 & 5 & 3 & 4 \\
\hline Phenol & 41 & 26 & 16 & 14 \\
\hline 2-(2-Butoxyethoxy)ethanol & 2 & 2 & 1 & $<1$ \\
\hline $\begin{array}{l}\text { 2,6-Di-tert-butyl-4- } \\
\text { methylphenol }\end{array}$ & 1 & 1 & $<1$ & $<1$ \\
\hline $\begin{array}{l}\text { 2,2,4-Trimethyl-1,3- } \\
\text { pentanediol diisobutyrate }\end{array}$ & 3 & 3 & 2 & 2 \\
\hline Diethylphthalate & 3 & 3 & 2 & 2 \\
\hline
\end{tabular}


Table E-20. Chamber concentrations of target VOCs for 48 -h screening measurement of Sheet Vinyl SV2.

\begin{tabular}{|c|c|c|c|c|}
\hline \multirow[b]{2}{*}{ Compound } & \multicolumn{4}{|c|}{ Chamber Concentration, $\mu \mathrm{g} \mathrm{m}-3$} \\
\hline & $1-h$ & 6-h & 24-h & $48-h$ \\
\hline \multicolumn{5}{|l|}{ Alkane Hydrocarbons } \\
\hline n-Dodecane & 2 & 2 & 6 & 9 \\
\hline n-Tridecane & 5 & 3 & 8 & 15 \\
\hline \multicolumn{5}{|l|}{ Aromatic Hydrocarbons } \\
\hline 1,2,4-Trimethylbenzene & 1 & 1 & 3 & 3 \\
\hline Naphthalene & 1 & 1 & 1 & 1 \\
\hline \multicolumn{5}{|l|}{ Other Oxidized Cmpds. } \\
\hline 2-Propoxyethanol & 3 & 2 & 4 & 4 \\
\hline Phenol & 51 & 50 & 47 & 44 \\
\hline 2-(2-Butoxyethoxy)ethanol & 3 & 3 & 1 & 2 \\
\hline $\begin{array}{l}\text { 2,2,4-Trimethyl-1,3- } \\
\text { pentanediol diisobutyrate }\end{array}$ & 7 & 2 & 1 & 1 \\
\hline \multicolumn{5}{|l|}{ Nitrogen-Containing Cmpds. } \\
\hline 1-Methyl-2-pyrrolidinone & 3 & 3 & 4 & 3 \\
\hline
\end{tabular}


Table E-21. Chamber concentrations of target VOCs for 48-h screening measurement of Sheet Vinyl SV3-a.

\begin{tabular}{|c|c|c|c|c|}
\hline \multirow[b]{2}{*}{ Compound } & \multicolumn{4}{|c|}{ Chamber Concentration, $\mu \mathrm{g} \mathrm{m}^{-3}$} \\
\hline & 1-h & 6-h & 24-h & 48-h \\
\hline \multicolumn{5}{|l|}{ Alkane Hydrocarbons } \\
\hline n-Nonane & 25 & 24 & 19 & 18 \\
\hline n-Decane & 49 & 60 & 51 & 50 \\
\hline n-Tridecane & 52 & 39 & 34 & 42 \\
\hline n-Tetradecane & 23 & 17 & 13 & 16 \\
\hline \multicolumn{5}{|l|}{ Aromatic Hydrocarbons } \\
\hline Toluene & 3 & 2 & 1 & 2 \\
\hline m-,p-Xylene & 3 & 3 & 2 & 2 \\
\hline o-Xylene & 3 & 3 & 2 & 2 \\
\hline 1,2,4-Trimethylbenzene & 14 & 13 & 12 & 12 \\
\hline Naphthalene & $<1$ & $<1$ & $<1$ & $<1$ \\
\hline \multicolumn{5}{|l|}{ Other Oxidized Cmpds. } \\
\hline Benzyl alcohol & 45 & 32 & 19 & 16 \\
\hline Phenol & 137 & 103 & 68 & 60 \\
\hline $\begin{array}{l}\text { 2,2,4-Trimethyl-1,3- } \\
\text { pentanediol disobutyrate }\end{array}$ & 30 & 21 & 12 & 11 \\
\hline Diethylphthalate & 5 & 5 & 3 & 3 \\
\hline
\end{tabular}


Table E-22. Chamber concentrations of target VOCs for 48-h screening measurement of Sheet Vinyl SV3-b.

\begin{tabular}{|c|c|c|c|c|}
\hline \multirow[b]{2}{*}{ Compound } & \multicolumn{4}{|c|}{ Chamber Concentration, $\mu \mathrm{g} \mathrm{m}-3$} \\
\hline & $1-\mathrm{h}$ & $6-\mathrm{h}$ & 24-h & 48-h \\
\hline \multicolumn{5}{|l|}{ Alkane Hydrocarbons } \\
\hline n-Nonane & 20 & 17 & 18 & 21 \\
\hline n-Decane & 42 & 38 & 36 & 40 \\
\hline $\mathrm{n}$-Tridecane & 79 & 59 & 45 & 44 \\
\hline $\mathrm{n}$-Tetradecane & 43 & 30 & 23 & 21 \\
\hline \multicolumn{5}{|l|}{ Aromatic Hydrocarbons } \\
\hline Toluene & 3 & 2 & 1 & 1 \\
\hline $\mathrm{m}-\mathrm{p}$-Xylene & 10 & 5 & 3 & 2 \\
\hline o-Xylene & 5 & 2 & 2 & 2 \\
\hline 1,2,4-Trimethylbenzene & 20 & 13 & 9 & 9 \\
\hline Naphthalene & 1 & $<1$ & $<1$ & $<1$ \\
\hline \multicolumn{5}{|l|}{ Other Oxidized Cmpds. } \\
\hline Benzyl alcohol & 59 & 40 & 27 & 21 \\
\hline Phenol & 140 & 125 & 92 & 77 \\
\hline $\begin{array}{l}\text { 2,2,4-Trimethyl-1,3- } \\
\text { pentanediol diisobutyrate }\end{array}$ & 67 & 52 & 33 & 34 \\
\hline Diethylphthalate & 1 & 1 & 1 & 1 \\
\hline
\end{tabular}


Table E-23. Chamber concentrations of target VOCs for 48-h screening measurement of Sheet Vinyl SV5.

\begin{tabular}{|c|c|c|c|c|}
\hline \multirow[b]{2}{*}{ Compound } & \multicolumn{4}{|c|}{ Chamber Concentration, $\mu \mathrm{g} \mathrm{m}-3$} \\
\hline & 1-h & $6-h$ & 24-h & 48-h \\
\hline \multicolumn{5}{|l|}{ Alkane Hydrocarbons } \\
\hline n-Nonane & 19 & 21 & 19 & 21 \\
\hline n-Decane & 49 & 44 & 36 & 45 \\
\hline n-Tridecane & 110 & 73 & 48 & 44 \\
\hline n-Tetradecane & 63 & 41 & 26 & 21 \\
\hline \multicolumn{5}{|l|}{ Aromatic Hydrocarbons } \\
\hline Toluene & 5 & 4 & 4 & 3 \\
\hline m-,p-Xylene & 6 & 5 & 3 & 3 \\
\hline o-Xylene & 5 & 3 & 2 & 2 \\
\hline 1,2,4-Trimethylbenzene & 28 & 19 & 12 & 12 \\
\hline Naphthalene & 2 & 1 & $<1$ & $<1$ \\
\hline \multicolumn{5}{|l|}{ Other Oxidized Cmpds. } \\
\hline 1-Octanol & 18 & 11 & 6 & 5 \\
\hline Benzyl alcohol & 87 & 63 & 36 & 30 \\
\hline Phenol & 217 & 162 & 125 & 98 \\
\hline $\begin{array}{l}\text { 2,2,4-Trimethyl-1,3- } \\
\text { pentanediol diisobutyrate }\end{array}$ & 93 & 66 & 44 & 40 \\
\hline Diethylphthalate & 1 & 1 & 1 & 1 \\
\hline
\end{tabular}


Table E-24. Chamber concentrations of target VOCs for 48-h screening measurement of Sheet Vinyl SV4.

\begin{tabular}{|c|c|c|c|c|}
\hline \multirow[b]{2}{*}{ Compound } & \multicolumn{4}{|c|}{ Chamber Concentration, $\mu \mathrm{g} \mathrm{m}^{-3}$} \\
\hline & $1-h$ & 6-h & 24-h & 48-h \\
\hline \multicolumn{5}{|l|}{ Alkane Hydrocarbons } \\
\hline $\mathrm{n}$-Tridecane & 8 & 6 & 9 & 15 \\
\hline \multicolumn{5}{|l|}{ Aromatic Hydrocarbons } \\
\hline 1,2,4-Trimethyibenzene & 2 & 3 & 3 & 3 \\
\hline Naphthalene & 4 & 3 & 4 & 5 \\
\hline \multicolumn{5}{|l|}{ Carbonyl Compounds } \\
\hline 1-Phenylethanone & 2 & 2 & 1 & 2 \\
\hline \multicolumn{5}{|l|}{ Other Oxidized Cmpds. } \\
\hline n-Propyl acetate & 7 & 8 & 8 & 8 \\
\hline 2-Propoxyethanol & 3 & 10 & 9 & 9 \\
\hline Phenol & 47 & 43 & 37 & 36 \\
\hline 2-(2-Butoxyethoxy)ethanol & 8 & 5 & 1 & 1 \\
\hline $\begin{array}{l}\text { 2,2,4-Trimethyl-1,3- } \\
\text { pentanediol diisobutyrate }\end{array}$ & 13 & 5 & 3 & 4 \\
\hline Diethylphthalate & 3 & 2 & 2 & 2 \\
\hline \multicolumn{5}{|l|}{ Nitrogen-Containing Cmpds. } \\
\hline 1-Methyl-2-pyrrolidinone & 6 & 6 & 5 & 6 \\
\hline
\end{tabular}


Table E-25. Chamber concentrations of target VOCs for 48-h sheet vinyl blank run.

\begin{tabular}{|c|c|c|c|c|}
\hline \multirow[b]{2}{*}{ Compound } & \multicolumn{4}{|c|}{ Chamber Concentration, $\mu \mathrm{g} \mathrm{m}^{-3}$} \\
\hline & 1-h & 6-h & 24-h & 48-h \\
\hline \multicolumn{5}{|l|}{ Aromatic Hydrocarbons } \\
\hline $\mathrm{m}-\mathrm{p}$-Xylene & 2 & 5 & 1 & $<1$ \\
\hline o-Xylene & 1 & 2 & $<1$ & $<1$ \\
\hline 1,2,4-Trimethylbenzene & $<1$ & 1 & $<1$ & $<1$ \\
\hline \multicolumn{5}{|l|}{ Carbonyl Compounds } \\
\hline Nonanal & 3 & 2 & 2 & 2 \\
\hline Decanal & 5 & 4 & 4 & 3 \\
\hline \multicolumn{5}{|l|}{ Other Oxidized Cmpds. } \\
\hline 2-(2-Butoxyethoxy)ethanol & 2 & 1 & $<1$ & $<1$ \\
\hline Diethylphthalate & 1 & 2 & 1 & 1 \\
\hline
\end{tabular}


Table E-26. Chamber concentrations of target VOCs for 48-h screening measurement of Cove Base CB.

\begin{tabular}{|c|c|c|c|c|}
\hline \multirow[b]{2}{*}{ Compound } & \multicolumn{4}{|c|}{ Chamber Concentration, $\mu \mathrm{g} \mathrm{m-3}$} \\
\hline & 1-h & 6-h & 24-h & 48-h \\
\hline \multicolumn{5}{|l|}{ Alkane Hydrocarbons } \\
\hline $\mathrm{n}$-Tridecane & 2 & 1 & 1 & 1 \\
\hline \multicolumn{5}{|l|}{ Aromatic Hydrocarbons } \\
\hline Toluene & 95 & 65 & 42 & 34 \\
\hline Styrene & 37 & 23 & 14 & 10 \\
\hline 4-tert-Butyltoluene & 11 & 8 & 5 & 4 \\
\hline \multicolumn{5}{|l|}{ Other Oxidized Cmpds. } \\
\hline alpha-Terpineol & 15 & 13 & 9 & 7 \\
\hline 2-tert-Butylphenol & 4 & 5 & 5 & 4 \\
\hline \multicolumn{5}{|l|}{ Miscellaneous Compounds } \\
\hline tert-Butyl isothiocyanate & 116 & 77 & 48 & 43 \\
\hline Benzothiazole & 136 & 113 & 91 & 80 \\
\hline
\end{tabular}


Table E-27. Chamber concentrations of target VOCs for 48-h screening measurement of Underlayment UL.

\begin{tabular}{|c|c|c|c|c|}
\hline \multirow[b]{2}{*}{ Compound } & \multicolumn{4}{|c|}{ Chamber Concentration, $\mu \mathrm{g} \mathrm{m}^{-3}$} \\
\hline & 1-h & 6-h & 24-h & 48-h \\
\hline \multicolumn{5}{|c|}{ Carbonyl Compounds } \\
\hline Hexanal & 35 & 19 & 8 & 6 \\
\hline Nonanal & 9 & 5 & 3 & 2 \\
\hline Decanal & 5 & 3 & 2 & 1 \\
\hline \multicolumn{5}{|c|}{ Other Oxidized Cmpds. } \\
\hline alpha-Terpineol & 3 & 1 & 1 & 1 \\
\hline
\end{tabular}

Table E-28. Chamber concentrations of target VOCs for 48-h screening measurement of Sheet Flooring Adhesive SFA applied to Underlayment UL.

\begin{tabular}{|c|c|c|c|c|}
\hline \multirow[b]{2}{*}{ Compound } & \multicolumn{4}{|c|}{ Chamber Concentration, $\mu \mathrm{g} \mathrm{m}^{-3}$} \\
\hline & 3-h & 6-h & 24-h & 48-h \\
\hline \multicolumn{5}{|c|}{ Aromatic Hydrocarbons } \\
\hline Toluene & 4,000 & 4,020 & 62 & 57 \\
\hline \multicolumn{5}{|l|}{ Other Hydrocarbons } \\
\hline Longifolene & 190 & 200 & 13 & 10 \\
\hline \multicolumn{5}{|c|}{ Other Oxidized Cmpds. } \\
\hline $\begin{array}{l}\text { 2,6-Di-tert-butyl-4- } \\
\text { methylphenol }\end{array}$ & 10 & 20 & 9 & 13 \\
\hline
\end{tabular}


Table E-29. Chamber concentrations of target VOCs for 48-h screening measurement of Cove Base Adhesive CBA applied to Gypsum Board GB.

\begin{tabular}{|c|c|c|c|c|}
\hline \multirow[b]{2}{*}{ Compound } & \multicolumn{4}{|c|}{ Chamber Concentration, $\mu \mathrm{g} \mathrm{m-3}$} \\
\hline & 1-h & 6-h & 24-h & 48-h \\
\hline \multicolumn{5}{|l|}{ Alkane Hydrocarbons } \\
\hline n-Octane & 3,810 & 4,240 & 887 & 280 \\
\hline n-Nonane & 490 & 380 & 100 & 34 \\
\hline n-Decane & 570 & 460 & 153 & 62 \\
\hline n-Undecane & 260 & 240 & 93 & 48 \\
\hline \multicolumn{5}{|l|}{ Aromatic Hydrocarbons } \\
\hline Toluene & 390 & 260 & 40 & 12 \\
\hline m-,p-Xylene & 100 & 60 & 13 & 6 \\
\hline Styrene & 360 & 260 & 53 & 18 \\
\hline 4-Phenylcyclohexene & 40 & 40 & 20 & 16 \\
\hline \multicolumn{5}{|l|}{ Other Hydrocarbons } \\
\hline Ethylcyclohexane & 920 & 820 & 253 & 110 \\
\hline \multicolumn{5}{|l|}{ Other Oxidized Cmpds. } \\
\hline $\begin{array}{l}\text { 2,6-Di-tert-butyl-4- } \\
\text { methylphenol }\end{array}$ & $<1$ & $<1$ & $<1$ & 4 \\
\hline
\end{tabular}


Table E-30. Chamber concentrations of target VOCs for 48-h screening measurement of Sheet Vinyl SV5 and Sheet Flooring Adhesive SFA applied to Underlayment UL.

\begin{tabular}{|c|c|c|c|c|}
\hline \multirow[b]{2}{*}{ Compound } & \multicolumn{4}{|c|}{ Chamber Concentration, $\mu \mathrm{g} \mathrm{m}-3$} \\
\hline & $1-\mathrm{h}$ & 6-h & 24-h & 48-h \\
\hline \multicolumn{5}{|l|}{ Alkane Hydrocarbons } \\
\hline n-Nonane & 16 & 14 & 10 & 10 \\
\hline n-Decane & 42 & 30 & 23 & 23 \\
\hline $\mathrm{n}$-Tridecane & 106 & 72 & 48 & 42 \\
\hline n-Tetradecane & 52 & 36 & 24 & 21 \\
\hline \multicolumn{5}{|l|}{ Aromatic Hydrocarbons } \\
\hline Toluene & 104 & 208 & 160 & 130 \\
\hline$m-, p-X y l e n e$ & 6 & 4 & 3 & 2 \\
\hline o-Xylene & 4 & 2 & 2 & 1 \\
\hline 1,2,4-Trimethylbenzene & 26 & 14 & 11 & 9 \\
\hline Naphthalene & 2 & $<1$ & $<1$ & $<1$ \\
\hline \multicolumn{5}{|l|}{ Other Hydrocarbons } \\
\hline Longifolene & $<1$ & $<1$ & $<1$ & $<1$ \\
\hline \multicolumn{5}{|l|}{ Other Oxidized Cmpds. } \\
\hline 1-Octanol & 16 & 8 & 6 & 5 \\
\hline Benzyl alcohol & 88 & 54 & 37 & 30 \\
\hline Phenol & 298 & 224 & 151 & 113 \\
\hline $\begin{array}{l}\text { 2,6-Di-tert-butyl-4- } \\
\text { methylphenol }\end{array}$ & $<1$ & $<1$ & $<1$ & $<1$ \\
\hline $\begin{array}{l}\text { 2,2,4-Trimethyl-1,3- } \\
\text { pentanediol diisobutyrate }\end{array}$ & 84 & 70 & 44 & 37 \\
\hline Diethylphthalate & 2 & $<1$ & 1 & $<1$ \\
\hline
\end{tabular}


Table E-31. Chamber concentrations of targèt VOCs for 48-h screening measurement of Cove Base CB and Cove Bàse Adhesive CBA applied to Gypsum Board GB.

\begin{tabular}{|c|c|c|c|c|}
\hline \multirow[b]{2}{*}{ Compound } & \multicolumn{4}{|c|}{ Chamber Concentration, $\mu \mathrm{g} \mathrm{m}^{-3}$} \\
\hline & 1-h & 6-h & 24-h & 48-h \\
\hline \multicolumn{5}{|l|}{ Alkane Hydrocarbons } \\
\hline n-Octane & 712 & 770 & 418 & 193 \\
\hline n-Nonane & 54 & 70 & 46 & 23 \\
\hline n-Decane & 47 & 60 & 56 & 36 \\
\hline n-Undecane & 7 & 13 & 20 & 18 \\
\hline $\mathrm{n}$-Tridecane & 6 & 7 & 2 & 1 \\
\hline \multicolumn{5}{|l|}{ Aromatic Hydrocarbons } \\
\hline Toluene & 258 & 127 & 30 & 16 \\
\hline m-,p-Xylene & 32 & 23 & 8 & 4 \\
\hline Styrene & 130 & 107 & 34 & 17 \\
\hline 4-tert-Butyltoluene & 11 & 7 & 2 & 2 \\
\hline 4-Phenylcyclohexene & 2 & 2 & 2 & 2 \\
\hline \multicolumn{5}{|l|}{ Other Hydrocarbons } \\
\hline Ethylcyclohexane & 233 & 187 & 98 & 54 \\
\hline \multicolumn{5}{|l|}{ Other Oxidized Cmpds. } \\
\hline alpha-Terpineol & 11 & 7 & 4 & 4 \\
\hline 2-tert-Butylphenol & 4 & 3 & 2 & 2 \\
\hline $\begin{array}{l}\text { 2,6-Di-tert-butyl-4- } \\
\text { methylphenol }\end{array}$ & $<1$ & $<1$ & $<1$ & $<1$ \\
\hline \multicolumn{5}{|l|}{ Miscellaneous Compounds } \\
\hline tert-Butyl isothiocyanate & 79 & 43 & 30 & 21 \\
\hline Benzothiazole & 145 & 123 & 84 & 65 \\
\hline
\end{tabular}


Table E-32. Chamber concentrations of TVOC for 48-h screening measurements of sheet vinyl flooring materials.

\begin{tabular}{|c|c|c|c|c|}
\hline \multirow[b]{2}{*}{ Material ID } & \multicolumn{4}{|c|}{ Chamber Concentration $\left(\mu \mathrm{g} \mathrm{m}^{-3}\right)$} \\
\hline & $1-h$ & 6-h & $24-h$ & 48-h \\
\hline \multicolumn{5}{|l|}{ Sheet Vinyls } \\
\hline SV1 & 344 & 265 & 175 & 154 \\
\hline SV2 & 425 & 701 & 633 & 729 \\
\hline SV3-a & 1,320 & 1,010 & 821 & 904 \\
\hline SV3-b & 1,450 & 1,340 & 1,110 & 983 \\
\hline SV5 & 2,220 & 1,730 & 1,190 & 1,180 \\
\hline SV4 & 508 & 450 & 496 & 600 \\
\hline SV Blank Run & 144 & 160 & 190 & 160 \\
\hline \multicolumn{5}{|l|}{ Cove Base } \\
\hline $\mathrm{CB}$ & 1,390 & 1,210 & 980 & 860 \\
\hline \multicolumn{5}{|l|}{ Substrates } \\
\hline UL & 420 & 326 & 213 & 219 \\
\hline GB & 136 & 208 & 193 & 181 \\
\hline \multicolumn{5}{|c|}{ Adhesives on Substrates } \\
\hline SFA \& UL & $8,090^{\star}$ & 6,660 & 1,150 & 674 \\
\hline CBA \& GB & 23,900 & 23,600 & 6,570 & 3,280 \\
\hline \multicolumn{5}{|c|}{ Composite Assemblies } \\
\hline SV5, SFA \& UL & 2,000 & 1,620 & 1,180 & 973 \\
\hline $\mathrm{CB}, \mathrm{CBA} \& \mathrm{~GB}$ & 5,320 & 4,870 & 2,860 & 1,720 \\
\hline
\end{tabular}

*3-h Sample. 
Table E-33. Chamber concentrations of SigmaVOC (i.e., sum of target VOCs) for 48-h screening measurements of sheet vinyl flooring materials.

\begin{tabular}{|c|c|c|c|c|}
\hline \multirow[b]{2}{*}{ Material ID } & \multicolumn{4}{|c|}{ Chamber Concentration ( $\left.\mu \mathrm{g} \mathrm{m}^{-3}\right)$} \\
\hline & $1-h$ & 6-h & 24-h & 48-h \\
\hline \multicolumn{5}{|l|}{ Sheet Vinyls } \\
\hline SV1 & 75 & 51 & 30 & 28 \\
\hline SV2 & 76 & 66 & 73 & 82 \\
\hline SV3-a & 389 & 321 & 236 & 234 \\
\hline SV3-b & 492 & 385 & 292 & 273 \\
\hline SV5 & 704 & 513 & 363 & 329 \\
\hline SV4 & 103 & 95 & 85 & 92 \\
\hline SV Blank Run & 15 & 18 & 10 & 6 \\
\hline \multicolumn{5}{|l|}{ Cove Base } \\
\hline $\mathrm{CB}$ & 416 & 306 & 216 & 185 \\
\hline \multicolumn{5}{|l|}{ Substrates } \\
\hline UL & 52 & 29 & 15 & 11 \\
\hline GB & $\cdots{ }^{\star}$ & -- & --- & $\cdots$ \\
\hline \multicolumn{5}{|c|}{ Adhesives on Substrates } \\
\hline SFA \& UL & $4,200^{\star \star}$ & 4,020 & 62 & 57 \\
\hline $\mathrm{CBA} \& \mathrm{~GB}$ & 6,940 & 6,760 & 1,610 & 590 \\
\hline \multicolumn{5}{|c|}{ Composite Assemblies } \\
\hline SV5, SFA \& UL & 846 & 736 & 520 & 425 \\
\hline $\mathrm{CB}, \mathrm{CBA} \& \mathrm{~GB}$ & 1,730 & 1,550 & 836 & 758 \\
\hline
\end{tabular}

${ }^{\star}$ No individual VOCs were quantified.

**3-h Sample. 
Table E-34. Chamber concentrations of formaldehyde for 48-h screening measurements of sheet vinyl flooring materials.

\begin{tabular}{|c|c|c|c|c|}
\hline \multirow[b]{2}{*}{ Material ID } & \multicolumn{4}{|c|}{ Chamber Concentration ( $\mu \mathrm{g} \mathrm{m}^{-3}$ ) } \\
\hline & 1-h & 6-h & 24-h. & 48-h \\
\hline \multicolumn{5}{|l|}{ Sheet Vinyls } \\
\hline SV1 & $<1$ & $<1$ & $<1$ & $<1$ \\
\hline SV2 & 3 & 1 & 1 & 1 \\
\hline SV3-a & 3 & 1 & 1 & $<1$ \\
\hline SV3-b & $<1$ & $<1$ & $<1$ & $<1$ \\
\hline SV5 & $<1$ & $<1$ & $<1$ & $<1$ \\
\hline SV4 & 3 & 2 & 1 & 1 \\
\hline SV Blank Run & $<1$ & $<1$ & $<1$ & $<1$ \\
\hline \multicolumn{5}{|l|}{ Cove Base } \\
\hline $\mathrm{CB}$ & $<1$ & $<1$ & $<1$ & $<1$ \\
\hline \multicolumn{5}{|l|}{ Substrates } \\
\hline UL & 77 & 69 & 59 & 53 \\
\hline GB & $<1$ & $<1$ & $<1$ & $<1$ \\
\hline \multicolumn{5}{|c|}{ Adhesives on Substrates } \\
\hline SFA \& UL & 39 & 26 & 30 & 31 \\
\hline CBA \& GB & 63 & 15 & 10 & 9 \\
\hline \multicolumn{5}{|c|}{ Composite Assemblies } \\
\hline SV5, SFA \& UL & 1 & 1 & 2 & 2 \\
\hline $\mathrm{CB}, \mathrm{CBA} \& \mathrm{~GB}$ & 6 & 6 & 7 & 7 \\
\hline
\end{tabular}


Table E-35. Chamber concentrations of acetaldehyde for 48-h screening measurements of sheet vinyl flooring materials.

\begin{tabular}{|c|c|c|c|c|}
\hline \multirow[b]{2}{*}{ Material ID } & \multicolumn{4}{|c|}{ Chamber Concentration ( $\mu \mathrm{g} \mathrm{m}^{-3}$ ) } \\
\hline & 1-h & $6-\mathrm{h}$ & 24-h & 48-h \\
\hline \multicolumn{5}{|l|}{ Sheet Vinyls } \\
\hline SV1 & 2 & 1 & 1 & 1 \\
\hline SV2 & 2 & 1 & 1 & 2 \\
\hline SV3-a & 5 & 4 & 2 & 3 \\
\hline SV3-b & 3 & 3 & 5 & 3 \\
\hline SV5 & 3 & 5 & 4 & 6 \\
\hline SV4 & 5 & 4 & 3 & 2 \\
\hline SV Blank Run & $<1$ & $<1$ & 1 & 1 \\
\hline \multicolumn{5}{|l|}{ Cove Base } \\
\hline $\mathrm{CB}$ & $<1$ & $<1$ & $<1$ & $<1$ \\
\hline \multicolumn{5}{|l|}{ Substrates } \\
\hline UL & 12 & 8 & 4 & 2 \\
\hline GB & 2 & $<1$ & 1 & $<1$ \\
\hline \multicolumn{5}{|c|}{ Adhesives on Substrates } \\
\hline SFA \& UL & 19 & 7 & 5 & 4 \\
\hline $\mathrm{CBA} \& \mathrm{~GB}$ & 21 & 18 & 5 & 4 \\
\hline \multicolumn{5}{|c|}{ Composite Assemblies } \\
\hline SV5, SFA \& UL & 3 & 3 & 2 & 2 \\
\hline $\mathrm{CB}, \mathrm{CBA} \& \mathrm{~GB}$ & 18 & 8 & 4 & 2 \\
\hline
\end{tabular}


Table E-36. Quasi steady-state emission rates of target VOCs at 24- and 48-h elapsed time for screening measurement of Sheet Vinyl SV1.

\begin{tabular}{|c|c|c|}
\hline \multirow[b]{2}{*}{ Compound } & \multicolumn{2}{|c|}{$\begin{array}{l}\text { Specific Emission Rate, } \\
\qquad \mu \mathrm{g} \mathrm{m}^{-2} \mathrm{~h}^{-1}\end{array}$} \\
\hline & 24-h & 48-h \\
\hline \multicolumn{3}{|l|}{ Alkane Hydrocarbons } \\
\hline $\mathrm{n}$-Tridecane & 5 & 5 \\
\hline \multicolumn{3}{|l|}{ Carbonyl Compounds } \\
\hline 1-Phenylethanone & 3 & 3 \\
\hline \multicolumn{3}{|l|}{ Other Oxidized Cmpds. } \\
\hline Di(propylene glycol) methyl ethers & 8 & 8 \\
\hline 2-Ethyl-1-hexanol & 10 & 11 \\
\hline Phenol & 50 & 43 \\
\hline 2-(2-Butoxyethoxy)ethanol & 3 & $<3$ \\
\hline 2,6-Di-tert-butyl-4-methylphenol & $<3$ & $<3$ \\
\hline $\begin{array}{l}\text { 2,2,4-Trimethyl-1,3-pentanediol } \\
\text { diisobutyrate }\end{array}$ & 6 & 6 \\
\hline Diethylphthalate & 3 & 3 \\
\hline
\end{tabular}


Table E-37. Quasi steady-state emission rates of target VOCs at 24- and 48-h elapsed time for screening measurement of Sheet Vinyl SV2.

\begin{tabular}{|c|c|c|}
\hline \multirow[b]{2}{*}{ Compound } & \multicolumn{2}{|c|}{$\begin{array}{l}\text { Specific Emission Rate, } \\
\qquad \mathrm{g} \mathrm{m} \mathrm{m}^{-2} \mathrm{~h}^{-1}\end{array}$} \\
\hline & 24-h & 48-h \\
\hline \multicolumn{3}{|l|}{ Alkane Hydrocarbons } \\
\hline n-Dodecane & 18 & 29 \\
\hline $\mathrm{n}$-Tridecane & 23 & 45 \\
\hline \multicolumn{3}{|l|}{ Aromatic Hydrocarbons } \\
\hline 1,2,4-Trimethylbenzene & 8 & 9 \\
\hline Naphthalene & 4 & 4 \\
\hline \multicolumn{3}{|l|}{ Other Oxidized Cmpds. } \\
\hline 2-Propoxyethanol & 11 & 11 \\
\hline Phenol & 142 & 135 \\
\hline 2-(2-Butoxyethoxy)ethanol & 4 & 5 \\
\hline $\begin{array}{l}\text { 2,2,4-Trimethyl-1,3-pentanediol } \\
\text { diisobutyrate }\end{array}$ & 3 & 2 \\
\hline \multicolumn{3}{|l|}{ Nitrogen-Containing Cmpds. } \\
\hline 1-Methyl-2-pyrrolidinone & 11 & 9 \\
\hline
\end{tabular}


Table E-38. Quasi steady-state emission rates of target VOCs at 24- and 48-h elapsed time for screening measurement of Sheet Vinyl SV3-a.

\begin{tabular}{|c|c|c|}
\hline \multirow[b]{2}{*}{ Compound } & \multicolumn{2}{|c|}{$\begin{array}{l}\text { Specific Emission Rate, } \\
\qquad \mu \mathrm{g} \mathrm{m} \mathrm{m}^{-2} \mathrm{~h}^{-1}\end{array}$} \\
\hline & 24-h & 48-h \\
\hline \multicolumn{3}{|l|}{ Alkane Hydrocarbons } \\
\hline n-Nonane & 57 & 54 \\
\hline n-Decane & 157 & 154 \\
\hline n-Tridecane & 105 & 129 \\
\hline n-Tetradecane & 39 & 49 \\
\hline \multicolumn{3}{|l|}{ Aromatic Hydrocarbons } \\
\hline Toluene & 4 & 5 \\
\hline m-,p-Xylene & 3 & 3 \\
\hline o-Xylene & 6 & 6 \\
\hline 1,2,4-Trimethylbenzene & 36 & 35 \\
\hline Naphthalene & $<3$ & $<3$ \\
\hline \multicolumn{3}{|l|}{ Other Oxidized Cmpds. } \\
\hline Benzyl alcohol & 57 & 49 \\
\hline Phenol & 208 & 183 \\
\hline $\begin{array}{l}\text { 2,2,4-Trimethyl-1,3-pentanediol } \\
\text { diisobutyrate }\end{array}$ & 36 & 34 \\
\hline Diethylphthalate & 3 & 5 \\
\hline
\end{tabular}


Table E-39. Quasi steady-state emission rates of target VOCs at 24- and 48-h elapsed time for screening measurement of Sheet Vinyl SV3-b.

\begin{tabular}{|c|c|c|}
\hline \multirow[b]{2}{*}{ Compound } & \multicolumn{2}{|c|}{$\begin{array}{c}\text { Specific Emission Rate, } \\
\mu \mathrm{g} \mathrm{m}^{-2} \mathrm{~h}^{-1}\end{array}$} \\
\hline & 24-h & 48-h \\
\hline \multicolumn{3}{|l|}{ Alkane Hydrocarbons } \\
\hline n-Nonane & 54 & 63 \\
\hline n-Decane & 111 & 121 \\
\hline n-Tridecane & 138 & 136 \\
\hline n-Tetradecane & 69 & 63 \\
\hline \multicolumn{3}{|l|}{ Aromatic Hydrocarbons } \\
\hline Toluene & 4 & 4 \\
\hline m-,p-Xylene & 6 & 5 \\
\hline o-Xylene & 5 & 5 \\
\hline 1,2,4-Trimethylbenzene & 28 & 27 \\
\hline Naphthalene & $<3$ & $<3$ \\
\hline \multicolumn{3}{|l|}{ Other Oxidized Cmpds. } \\
\hline Benzyl alcohol & 83 & 63 \\
\hline Phenol & 281 & 234 \\
\hline $\begin{array}{l}\text { 2,2,4-Trimethyl-1,3-pentanediol } \\
\text { diisobutyrate }\end{array}$ & 100 & 104 \\
\hline Diethylphthalate & $<3$ & $<3$ \\
\hline
\end{tabular}


Table E-40. Quasi steady-state emission rates of target VOCs at 24- and 48-h elapsed time for screening measurement of Sheet Vinyl SV5.

\begin{tabular}{|c|c|c|}
\hline \multirow[b]{2}{*}{ Compound } & \multicolumn{2}{|c|}{$\begin{array}{l}\text { Specific Emission Rate, } \\
\mu \mathrm{g} \mathrm{m}^{-2} \mathrm{~h}^{-1}\end{array}$} \\
\hline & 24-h & 48-h \\
\hline \multicolumn{3}{|l|}{ Alkane Hydrocarbons } \\
\hline n-Nonane & 58 & 65 \\
\hline n-Decane & 109 & 137 \\
\hline n-Tridecane & 145 & 134 \\
\hline $\mathrm{n}$-Tetradecane & 80 & 65 \\
\hline \multicolumn{3}{|l|}{ Aromatic Hydrocarbons } \\
\hline Toluene & 11 & 11 \\
\hline m-,p-Xylene & 5 & 8 \\
\hline o-Xylene & 7 & 8 \\
\hline 1,2,4-Trimethylbenzene & 37 & 38 \\
\hline Naphthalene & $<3$ & $<3$ \\
\hline \multicolumn{3}{|l|}{ Other Oxidized Cmpds. } \\
\hline 1-Octanol & 18 & 17 \\
\hline Benzyl alcohol & 110 & 91 \\
\hline Phenol & 381 & 298 \\
\hline $\begin{array}{l}\text { 2,2,4-Trimethyl-1,3-pentanediol } \\
\text { diisobutyrate }\end{array}$ & 134 & 122 \\
\hline Diethylphthalate & $<3$ & $<3$ \\
\hline
\end{tabular}


Table E-41. Quasi steady-state emission rates of target VOCs at 24- and 48-h elapsed time for screening measurement of Sheet Vinyl SV4.

\begin{tabular}{|c|c|}
\hline & $\begin{array}{c}\text { Specific Emission Rate, } \\
\mu \mathrm{g} \mathrm{m}^{-2} \mathrm{~h}^{-1}\end{array}$ \\
\hline Compound & $24-h$ \\
\hline
\end{tabular}

Alkane Hydrocarbons

n-Tridecane

29

46

Aromatic Hydrocarbons

1,2,4-Trimethylbenzene

$11 \quad 11$

Naphthalene

12

15

Carbonyl Compounds

1-Phenylethanone

3

6

Other Oxidized Cmpds.

n-Propyl acetate

2-Propoxyethanol

Phenol

2-(2-Butoxyethoxy)ethanol

2,2,4-Trimethyl-1,3-pentanediol

diisobutyrate

Diethylphthalate
$24 \quad 24$

$27 \quad 27$

$114 \quad 111$

$5 \quad 5$

$11 \quad 12$

53

Nitrogen-Containing Cmpds.

1-Methyl-2-pyrrolidinone 
Table E-42. Quasi steady-state emission rates of target VOCs at 24- and 48-h elapsed time for screening measurement of Cove Base CB.

\begin{tabular}{lcc}
\hline \hline & $\begin{array}{c}\text { Specific Emission Rate, } \\
\text { Comp m-1 h-1 }\end{array}$ & 24-h \\
\hline \hline Alkane Hydrocarbons & $<1$ & $<1$ \\
n-Tridecane & & \\
Aromatic Hydrocarbons & 11 & 9 \\
Toluene & 4 & 3 \\
Styrene & 1 & 1 \\
4-tert-Butyltoluene & & \\
Other Oxidized Cmpds. & 2 & 2 \\
alpha-Terpineol & 1 & 1 \\
2-tert-Butylphenol & & \\
Miscellaneous Compounds & 12 & 11 \\
tert-Buyl isothiocyanate & 24 & 21 \\
Benzothiazole & & \\
\hline
\end{tabular}


Table E-43. Quasi steady-state emission rates of target VOCs at 24- and 48-h elapsed time for screening measurement of Underlayment UL.

\begin{tabular}{lcc}
\hline & $\begin{array}{c}\text { Specific Emission Rate, } \\
\mu \mathrm{m} \mathrm{m}^{-2} \mathrm{~h}^{-1}\end{array}$ & \\
Compound & $24-\mathrm{h}$ & \multicolumn{2}{c}{$\mathbf{4 8}$} \\
\hline Carbonyl Compounds & 26 & 20 \\
Hexanal & 5 & $<3$ \\
Nonanal & $<3$ & $<3$ \\
Decanal & & \\
Other Oxidized Cmpds. & 3 & 3 \\
alpha-Terpineol & 3 & \\
\hline
\end{tabular}

Table E-44. Quasi steady-state emission rates of target VOCs at 24- and 48-h elapsed time for screening measurement of Sheet Flooring Adhesive SFA applied to Underlayment UL.

\begin{tabular}{|c|c|c|}
\hline \multirow[b]{2}{*}{ Compound } & \multicolumn{2}{|c|}{$\begin{array}{c}\text { Specific Emission Rate } \\
\mu \mathrm{g} \mathrm{kq} \mathrm{h}^{-1}\end{array}$} \\
\hline & 24-h & $48-h$ \\
\hline \multicolumn{3}{|l|}{ Aromatic Hydrocarbons } \\
\hline Toluene & 528 & 481 \\
\hline \multicolumn{3}{|l|}{ Other Hydrocarbons } \\
\hline Longifolene & 113 & 85 \\
\hline \multicolumn{3}{|l|}{ Other Oxidized Cmpds. } \\
\hline 2,6-Di-tert-butyl-4-methylphenol & 75 & 113 \\
\hline
\end{tabular}


Table E-45. Quasi steady-state emission rates of target VOCs at 24- and 48-h elapsed time for screening measurement of Cove Base Adhesive CBA applied to Gypsum Board GB.

\begin{tabular}{|c|c|c|}
\hline \multirow[b]{2}{*}{ Compound } & \multicolumn{2}{|c|}{$\begin{array}{l}\text { Specific Emission Rate, } \\
\qquad \mathrm{g} \mathrm{kg} \mathrm{k}^{-1} \mathrm{~h}^{-1}\end{array}$} \\
\hline & 24-h & 48-h \\
\hline \multicolumn{3}{|l|}{ Alkane Hydrocarbons } \\
\hline n-Octane & 6,350 & 2,000 \\
\hline n-Nonane & 716 & 243 \\
\hline n-Decane & 1,100 & 444 \\
\hline n-Undecane & 668 & 343 \\
\hline \multicolumn{3}{|l|}{ Aromatic Hydrocarbons } \\
\hline Toluene & 286 & 86 \\
\hline m-,p-Xylene & 88 & 36 \\
\hline Styrene & 382 & 129 \\
\hline 4-Phenylcyclohexene & 143 & 114 \\
\hline \multicolumn{3}{|l|}{ Other Hydrocarbons } \\
\hline Ethylcyclohexane & 1,810 & 787 \\
\hline \multicolumn{3}{|l|}{ Other Oxidized Cmpds. } \\
\hline 2,6-Di-tert-butyl-4-methylphenol & $<7$ & 29 \\
\hline
\end{tabular}


Table E-46. Quasi steady-state emission rates of target VOCs at 24- and 48-h elapsed time for screening measurement of Sheet Vinyl SV5 and Sheet Flooring Adhesive SFA applied to Underlayment UL.

\begin{tabular}{|c|c|c|}
\hline \multirow[b]{2}{*}{ Compound } & \multicolumn{2}{|c|}{$\begin{array}{l}\text { Specific Emission Rate, } \\
\mu \mathrm{g} \mathrm{m}^{-2} \mathrm{~h}^{-1}\end{array}$} \\
\hline & 24-h & 48-h \\
\hline \multicolumn{3}{|l|}{ Alkane Hydrocarbons } \\
\hline n-Nonane & 30 & 30 \\
\hline n-Decane & 70 & 69 \\
\hline n-Tridecane & 146 & 128 \\
\hline $\mathrm{n}$-Tetradecane & 73 & 65 \\
\hline \multicolumn{3}{|l|}{ Aromatic Hydrocarbons } \\
\hline Toluene & 487 & 396 \\
\hline m-,p-Xylene & 6 & 3 \\
\hline o-Xylene & 6 & 4 \\
\hline 1,2,4-Trimethylbenzene & 33 & 28 \\
\hline Naphthalene & $<3$ & $<3$ \\
\hline \multicolumn{3}{|l|}{ Other Hydrocarbons } \\
\hline Longifolene & $<3$ & $<3$ \\
\hline \multicolumn{3}{|l|}{ Other Oxidized Cmpds. } \\
\hline 1-Octanol & 18 & 14 \\
\hline Benzyl alcohol & 113 & 91 \\
\hline Phenol & 460 & 345 \\
\hline 2,6-Di-tert-butyl-4-methylphenol & $<3$ & $<3$ \\
\hline $\begin{array}{l}\text { 2,2,4-Trimethyl-1,3-pentanediol } \\
\text { diisobutyrate }\end{array}$ & 134 & 112 \\
\hline Diethylphthalate & $<3$ & $<3$ \\
\hline
\end{tabular}


Table E-47. Quasi steady-state emission rates of target VOCs at 24- and 48-h elapsed time for screening measurement of Cove Base CB and Cove Base Adhesive CBA applied to Gypsum Board GB.

\begin{tabular}{|c|c|c|}
\hline \multirow[b]{2}{*}{ Compound } & \multicolumn{2}{|c|}{$\begin{array}{l}\text { Specific Emission Rate, } \\
\qquad \mu \mathrm{g} \mathrm{m}^{-1} \mathrm{~h}^{-1}\end{array}$} \\
\hline & 24-h & 48-h \\
\hline \multicolumn{3}{|l|}{ Alkane Hydrocarbons } \\
\hline n-Octane & 218 & 101 \\
\hline n-Nonane & 24 & 12 \\
\hline n-Decane & 29 & 19 \\
\hline n-Undecane & 10 & 9 \\
\hline n-Tridecane & 1 & $<1$ \\
\hline \multicolumn{3}{|l|}{ Aromatic Hydrocarbons } \\
\hline Toluene & 16 & 8 \\
\hline m-,p-Xylene & 4 & 1 \\
\hline Styrene & 18 & 9 \\
\hline 4-tert-Butyltoluene & 1 . & 1 \\
\hline 4-Phenylcyclohexene & 1 & 1 \\
\hline \multicolumn{3}{|l|}{ Other Hydrocarbons } \\
\hline Ethylcyclohexane & 51 & 28 \\
\hline \multicolumn{3}{|l|}{ Other Oxidized Cmpds. } \\
\hline alpha-Terpineol & 2 & 2 \\
\hline 2-tert-Butylphenol & 1 & 1 \\
\hline 2,6-Di-tert-butyl-4-methylphenol & $<1$ & $<1$ \\
\hline \multicolumn{3}{|l|}{ Miscellaneous Compounds } \\
\hline tert-Butyl isothiocyanate & 16 & 11 \\
\hline Benzothiazole & 44 & 34 \\
\hline
\end{tabular}


Table E-48. Quasi steady-state emission rates of TVOC at 24- and 48-hours elapsed times for screening measurements of sheet vinyl flooring materials.

\begin{tabular}{|c|c|c|c|c|}
\hline \multirow[b]{2}{*}{ Material ID } & \multirow{2}{*}{$\begin{array}{l}\text { Material } \\
\text { Amount }\end{array}$} & \multicolumn{2}{|c|}{ Specif. Emission Rate } & \multirow[b]{2}{*}{ Units } \\
\hline & & 24-h & 48-h & \\
\hline \multicolumn{5}{|l|}{ Sheet Vinyls } \\
\hline SV1 & $0.0195 \mathrm{~m}^{-2}$ & $<75$ & $<75$ & $\mu g m^{-2} h^{-1}$ \\
\hline SV2 & $0.0195 \mathrm{~m}^{-2}$ & 1,430 & 1,720 & $\mu g \mathrm{~m}^{-2} \mathrm{~h}-1$ \\
\hline SV3-a & $0.0195 \mathrm{~m}^{-2}$ & 2,000 & 2,250 & $\mu \mathrm{g} \mathrm{m}^{-2} \mathrm{~h}^{-1}$ \\
\hline SV3-b & $0.0195 \mathrm{~m}^{-2}$ & 2,890 & 2,450 & $\mu \mathrm{g} \mathrm{m}-2 \mathrm{~h}^{-1}$ \\
\hline SV5 & $0.0195 \mathrm{~m}^{-2}$ & 3,120 & 3,110 & $\mu g m^{-2} h^{-1}$ \\
\hline SV4 & $0.0195 \mathrm{~m}^{-2}$ & 1,010 & 1,330 & $\mu g m^{-2} h^{-1}$ \\
\hline \multicolumn{5}{|l|}{ Cove Base } \\
\hline $\mathrm{CB}$ & $0.229 \mathrm{~m}$ & 212 & 181 & $\mu \mathrm{g} \mathrm{m}^{-1} \mathrm{~h}^{-1}$ \\
\hline \multicolumn{5}{|l|}{ Substrates } \\
\hline UL & $0.0195 \mathrm{~m}^{-2}$ & 151 & 168 & $\mu g m^{-2} h^{-1}$ \\
\hline GB & $0.0195 \mathrm{~m}^{-2}$ & $<75$ & $<75$ & $\mu g m^{-2} h^{-1}$ \\
\hline \multicolumn{5}{|c|}{ Adhesives on Substrates } \\
\hline SFA \& UL & $0.0070 \mathrm{~kg}$ & 8,340 & 4,330 & $\mu \mathrm{g} \mathrm{kg}^{-1} \mathrm{~h}^{-1}$ \\
\hline $\mathrm{CBA} \& \mathrm{~GB}$ & $0.0083 \mathrm{~kg}$ & 45,800 & 22,300 & $\mu g \mathrm{~kg}^{-1} \mathrm{~h}^{-1}$ \\
\hline \multicolumn{5}{|c|}{ Composite Assemblies } \\
\hline SV5, SFA \& UL & $0.0195 \mathrm{~m}^{-2}$ & 3,100 & 2,470 & $\mu g m^{-2} n^{-1}$ \\
\hline $\mathrm{CB}, \mathrm{CBA} \& \mathrm{~GB}$ & $0.114 \mathrm{~m}$ & 1,400 & 812 & $\mu \mathrm{g} \mathrm{m}^{-1} \mathrm{~h}^{-1}$ \\
\hline
\end{tabular}


Table E-49. Quasi steady-state emission rates of formaldehyde at 24- and 48-hours elapsed times for screening measurements of sheet vinyl flooring materials.

\begin{tabular}{|c|c|c|c|c|}
\hline Material ID & $\begin{array}{l}\text { Material } \\
\text { Amount }\end{array}$ & \multicolumn{2}{|c|}{ Specif. Emission Rate } & Units \\
\hline \multicolumn{5}{|l|}{ Sheet Vinyls } \\
\hline SV1 & $0.0195 \mathrm{~m}^{-2}$ & $<3$ & $<3$ & $\mu \mathrm{g} \mathrm{m}^{-2} \mathrm{~h}^{-1}$ \\
\hline SV2 & $0.0195 \mathrm{~m}^{-2}$ & 4 & 3 & $\mu g m^{-2} h^{-1}$ \\
\hline SV3-a & $0.0195 \mathrm{~m}^{-2}$ & 3 & $<3$ & $\mu \mathrm{g} \mathrm{m}^{-2} \mathrm{~h}^{-1}$ \\
\hline SV3-b & $0.0195 \mathrm{~m}^{-2}$ & $<3$ & $<3$ & $\mu \mathrm{g} \mathrm{m}-2 \mathrm{~h}^{-1}$ \\
\hline SV5 & $0.0195 \mathrm{~m}^{-2}$ & $<3$ & $<3$ & $\mu g m^{-2} h^{-1}$ \\
\hline SV4 & $0.0195 \mathrm{~m}^{-2}$ & 4 & 4 & $\mu \mathrm{g} \mathrm{m}-2 \mathrm{~h}^{-1}$ \\
\hline \multicolumn{5}{|l|}{ Cove Base } \\
\hline CB & $0.229 \mathrm{~m}$ & $<1$ & $<1$ & $\mu \mathrm{g} \mathrm{m}^{-1} \mathrm{~h}^{-1}$ \\
\hline \multicolumn{5}{|l|}{ Substrates } \\
\hline UL & $0.0195 \mathrm{~m}^{-2}$ & 180 & 160 & $\mu g m^{-2} h^{-1}$ \\
\hline GB & $0.0195 \mathrm{~m}^{-2}$ & $<3$ & $<3$ & $\mu \mathrm{g} \mathrm{m}^{-2} \mathrm{~h}^{-1}$ \\
\hline \multicolumn{5}{|c|}{ Adhesives on Substrates } \\
\hline SFA \& UL & $0.0070 \mathrm{~kg}$ & 258 & 263 & $\mu \mathrm{g} \mathrm{kg}^{-1} \mathrm{~h}^{-1}$ \\
\hline SFA \& UL & $0.0195 \mathrm{~m}^{-2}$ & 94 & 94 & $\mu g m^{-2} h^{-1}$ \\
\hline CBA \& GB & $0.0083 \mathrm{~kg}$ & 72 & 68 & $\mu g \mathrm{~kg}^{-1} \mathrm{~h}^{-1}$ \\
\hline \multicolumn{5}{|c|}{ Composite Assemblies } \\
\hline SV5, SFA \& UL & $0.0195 \mathrm{~m}^{-2}$ & 5 & 7 & $\mu g m^{-2} h^{-1}$ \\
\hline $\mathrm{CB}, \mathrm{CBA} \& \mathrm{~GB}$ & $0.114 \mathrm{~m}$ & 3 & 3 & $\mu \mathrm{g} \mathrm{m}^{-1} \mathrm{~h}^{-1}$ \\
\hline
\end{tabular}


Table E-50. Quasi steady-state emission rates of acetaldehyde at 24- and 48-hours elapsed times for screening measurements of sheet vinyl flooring materials.

\begin{tabular}{|c|c|c|c|c|}
\hline Material ID & $\begin{array}{l}\text { Material } \\
\text { Amount }\end{array}$ & $\begin{array}{r}\text { Specif. } \\
24-h\end{array}$ & $\begin{array}{l}\text { on Rate } \\
48-h\end{array}$ & Units \\
\hline \multicolumn{5}{|l|}{ Sheet Vinyls } \\
\hline SV1 & $0.0195 \mathrm{~m}^{-2}$ & $<3$ & $<3$ & $\mu \mathrm{g} \mathrm{m}^{-2} \mathrm{~h}^{-1}$ \\
\hline SV2 & $0.0195 \mathrm{~m}^{-2}$ & $<3$ & 3 & $\mu \mathrm{g} \mathrm{m}^{-2} \mathrm{~h}^{-1}$ \\
\hline SV3-a & $0.0195 \mathrm{~m}^{-2}$ & 4 & 5 & $\mu g m^{-2} h^{-1}$ \\
\hline SV3-b & $0.0195 \mathrm{~m}^{-2}$ & 13 & 7 & $\mu g m^{-2} h^{-1}$ \\
\hline SV5 & $0.0195 \mathrm{~m}^{-2}$ & 8 & 14 & $\mu g m^{-2} n^{-1}$ \\
\hline SV4 & $0.0195 \mathrm{~m}^{-2}$ & 6 & 3 & $\mu g m^{-2} h^{-1}$ \\
\hline \multicolumn{5}{|l|}{ Cove Base } \\
\hline $\mathrm{CB}$ & $0.229 \mathrm{~m}$ & $<1$ & $<1$ & $\mu g m^{-1} h^{-1}$ \\
\hline \multicolumn{5}{|l|}{ Substrates } \\
\hline UL & $0.0195 \mathrm{~m}^{-2}$ & 8 & 4 & $\mu g m^{-2} h^{-1}$ \\
\hline GB & $0.0195 \mathrm{~m}^{-2}$ & $<3$ & $<3$ & $\mu g m^{-2} h^{-1}$ \\
\hline \multicolumn{5}{|c|}{ Adhesives on Substrates } \\
\hline SFA \& UL & $0.0070 \mathrm{~kg}$ & 36 & 23 & $\mu \mathrm{g} \mathrm{kg}^{-1} \mathrm{~h}^{-1}$ \\
\hline SFA \& UL & $0.0195 \mathrm{~m}^{-2}$ & 13 & 8 & $\mu \mathrm{g} \mathrm{m}^{-2} \mathrm{~h}^{-1}$ \\
\hline $\mathrm{CBA} \& \mathrm{~GB}$ & $0.0083 \mathrm{~kg}$ & 28 & 19 & $\mu \mathrm{g} \mathrm{kg}^{-1} \mathrm{~h}^{-1}$ \\
\hline \multicolumn{5}{|c|}{ Composite Assemblies } \\
\hline SV5, SFA \& UL & $0.0195 \mathrm{~m}^{-2}$ & 4 & 4 & $\mu g \mathrm{~m}^{-2} \mathrm{~h}^{-1}$ \\
\hline $\mathrm{CB}, \mathrm{CBA} \& \mathrm{~GB}$ & $0.114 \mathrm{~m}$ & 1 & $<1$ & $\mu \mathrm{g} \mathrm{m}^{-1} \mathrm{~h}^{-1}$ \\
\hline
\end{tabular}


APPENDIX F - VENTILATION STUDIES

\title{
APPENDIX F
}

\author{
VENTILATION RATE AND AIR MIXING STUDIES OF THE LARGE-SCALE CHAMBER \\ FACILITY
}

\section{OBJECTIVES}

The objectives of these experiments were to: 1) establish and measure the three fixed air change rates for each of the two large-scale chamber compartments; 2) establish and measure the air velocities in the compartments using small fans; 3 ) measure the quality of air mixing within each compartment at the two lower fixed air change rates; and 4) measure the air leakage between each compartment and the building and between the two compartments.

\section{DESCRIPTION OF CHAMBER FACILITY}

The chamber facility is housed in a small building. This building is equipped with thermostatic controls and a heat-pump system for heating and cooling. This system is capable of maintaining the building temperature within approximately $\pm 3^{\circ} \mathrm{C}$ of the set point under most climatic conditions.

Chamber compartments $A$ and $B$ were designed and constructed to simulate conditions for a small room in a typical residence. The interior dimensions of each compartment are $2.26 \mathrm{~m}$ wide by $4.62 \mathrm{~m}$ long with a $2.44-\mathrm{m}$ high ceiling, yielding a volume of $25.5 \mathrm{~m}^{3}$. The walls and ceiling are finished with gypsum board. The seams between boards were taped and topped with joint compound. The finished gypsum board was painted with a "no VOC" primer and flat latex paint combination. The plywood floor was covered with thin aluminum plates with overlapping joints. The plates were screwed down to the floor and the joints were sealed with aluminized tape. Each compartment has a single, unfinished, composite-wood slab door $0.91 \mathrm{~m}$ wide by $2.13 \mathrm{~m}$ high. The door is weather-stripped on all edges to reduce air leakage. During an experiment, the gaps around the door are sealed with duct tape. There are no windows.

Ventilation air for the chamber compartments is supplied from the exterior of the building through a $15-\mathrm{cm}$ diameter aluminum duct. This air is filtered for gaseous contaminants by passing through a sheet metal box containing a $5-\mathrm{cm}$ thick horizontal bed of activated charcoal with surface dimensions of 0.61 by $0.61 \mathrm{~m}$. The filter box has two exits, each leading to one compartment. The components for each of these systems are identical. An in-line centrifugal blower is attached to the filter box. Downstream of the blower is a manifold leading to three different sized ducts. The internal duct diameters are $3.0 \mathrm{~cm}, 5.1 \mathrm{~cm}$ and $7.6 \mathrm{~cm}$. They are used to provide chamber compartment air change rates of $0.5,2$ and $5 \mathrm{~h}^{-1}$, respectively. Manual valves on the manifold are used to select the desired duct. The ducts are $1.83 \mathrm{~m}$ long. A pitot tube (Dwyer Instruments, Inc.) is installed $0.46 \mathrm{~m}$ from the outlet end of each duct to monitor the air velocity pressure in the pipe. A manual gate valve is installed downstream of the pitot tube for the $3.0-\mathrm{cm}$ and $5.1-\mathrm{cm}$ diameter ducts. This valve is used to set the desired air flow rate. The air then directly enters the compartment $20 \mathrm{~cm}$ above the floor at a long end of the compartment. A cap is placed on the outlet of the 7.6-cm duct in the compartment and drilled with holes which sets the desired air flow rate. All the components of the duct system downstream of the blower are ABS and PVC plastic. They were washed with methanol prior to assembly. All joints are pressed tight and held with screws. No adhesives or sealants were used to assemble the system.

The chamber facility is equipped with a system to add water vapor to the ventilation supply air upstream of the filter box. This system is used to increase the humidity of the supply air when it falls below a minimum value of $40 \%$ relative humidity at room temperature. The components consist of: a humidity probe positioned in the filter box; a water reservoir; a variable speed peristaltic metering pump with electronic controls ${ }^{*}:$ : specially-fabricated aluminum duct 
section heated with a laboratory hot plate; and a water overflow drain. The data system measures the humidity in the filter box and uses a proportional-control algorithm to set the speed of the water-metering pump.

Air exits each compartment on the same wall that air enters the compartment. The center of the outlet is located $38 \mathrm{~cm}$ from the ceiling, about $2.2 \mathrm{~m}$ diagonally across from the air inlet. The dimensions of the outlet are $15 \mathrm{~cm}$ by $25 \mathrm{~cm}$. Exterior to the compartment, the exhaust is fitted with a damper, which is manually adjusted according to the set air change rate. The exhaust duct is a $6-\mathrm{m}$ section of $15-\mathrm{cm}$ diameter flexible tubing leading to a vent on the roof of the building.

Each compartment is fitted with six 7.6-cm diameter axial fans to provide adequate air mixing. These fans are attached to vertically aligned removable metal poles. There are two fans spaced equally along each long wall and one fan located in the middle of each short wall. The fan heights alternate between 0.81 and $1.62 \mathrm{~m}$ from the floor. The fans are positioned $30-38 \mathrm{~cm}$ from a wall and are aligned so that they move air parallel to the wall. They all move air in the same direction. The fan speeds are controlled with a variable transformer to achieve the desired air velocity near the floor of the compartment.

Air is sampled from the mid-point of each compartment. The inlet of a 0.4-cm I.D. stainless-steel tube is positioned $1.5 \mathrm{~m}$ above the floor at the mid point. This tube runs directly to the exterior of the chamber and is connected to a stainless-steel sampling manifold with three ports to allow for the simultaneous collection of duplicate samples for volatile organic compounds and a single sample for aldehydes. Air is continuously pulled through the sampling tube at a rate of $1 \mathrm{~L} \mathrm{~min}^{-1}$ throughout an entire experiment. Sampling flow rates are regulated with electronic mass flow controllers (Model FC 280, Tylan General) connected to vacuum pumps. These devices operate continuously at the established flow rates. The inlets of the sampling lines leading to the mass flow controllers are connected to electronically actuated three-way solenoid valves. The sampling devices are connected to the sampling manifold and to the valves. For sampling, each valve is switched by the data system from the open position to the position connected to the sampling device. At the end of a set time period, the data system switches the valve back to the open position and sampling is terminated. In some cases, the samples are manually collected during the period in which the valves are held in the in-line position.

The instrumentation for each compartment consists of: 1 ) a pressure transducer (Model 239 , Setra Systems, Inc.) to measure the velocity pressure for the selected pitot tube; 2) a combined RTD temperature and relative humidity probe (Model HMD30YB, Vaisala) positioned at the midpoint of the compartment $1.5 \mathrm{~m}$ above the floor; 3 ) four Type $T$ thermocouples to measure floor temperature and air temperature stratification; and 4) an air velocity transducer (Model 84705AM-V-STD-NC, TSI, Inc.) with a range of 0 to $50 \mathrm{~cm} \mathrm{sec}^{-1}$ to monitor air velocity $5 \mathrm{~cm}$ above the floor. The voltage outputs from these devices are measured at one-minute intervals and averaged and recorded at five-minute intervals with a PC-based data system (Series 500, Keithly/Metrabyte) and data acquisition software (Labtech Notebook, Version 7; Laboratory Technologies, Corp.).

\section{METHODS}

The tracer gas employed for these experiments was sulfur hexafluoride $\left(\mathrm{SF}_{6}\right)$. A gas cylinder containing a one-percent mixture of $\mathrm{SF}_{6}$ in air was used as the supply. The flow rate of the $\mathrm{SF}_{6}$ mixture into the chamber system was regulated with a calibrated electronic mass flow controller. Four peristaltic pumps were used to draw air for the analysis of $\mathrm{SF}_{6}$ from the chamber system and the building into a computer-controlled multi-port valve. This allowed four separate locations to be sequentially analyzed with a one-minute interval between successive samples. A gas chromatograph equipped with an automated gas sample valve and an electron capture detector was used for the analysis. This system was calibrated on each day of analysis using dilutions of certified gas mixtures of $\mathrm{SF}_{6}$ and air. 
To set and measure the flow rate of air entering a compartment through a supply duct, the one-percent $\mathrm{SF}_{6}$ gas mixture was metered at a constant rate into the inlet of the selected duct just downstream of the compartment supply manifold. The $\mathrm{SF}_{6}$ concentration was measured in the air in the duct just as it entered the compartment. For the $3.0-\mathrm{cm}$ and $5.1-\mathrm{cm}$ diameter ducts, the gate valve was adjusted until the supply $\mathrm{SF}_{6}$ concentration matched the concentration for the desired air flow rate found using,

$$
C_{A}^{o}=\frac{C_{S F 6} \cdot Q_{S F 6}}{\lambda_{A} V_{A}}
$$

Where $C_{A}{ }^{0}$ is the expected concentration in the supply of compartment $A$ with a volume, $V_{A}$ and an air exchange rate of $\lambda_{A}$. The $S_{F}$ from the tank has a concentration, $\mathrm{C}_{\mathrm{SF}}$, and is supplied with a mass flow controller at a volumetric flowrate $Q_{\mathrm{SF} 6}$. For the 7.6-cm diameter duct, holes were drilled in the cap on the duct outlet until the calculated concentration was reached. When the desired flow rate was established, the velocity pressure drop for the corresponding pitot tube was recorded in volts with the system pressure transducer and in inches of water with a portable electronic pressure meter.

A residence time distribution experiment was performed for each compartment at each fixed air change rate as described in ASTM D 5116-90 (1990). The objectives of this procedure were to identify any bypassing of air between the air inlet and exhaust or any significant air leakage and to quantify the quality of air mixing in the compartment. First, the compartment was ventilated at the maximum rate until the $\mathrm{SF}_{6}$ concentration in the compartment was near the limit of detection. Then, an experiment was initiated starting with the lowest air change rate. The tracer gas was introduced as described above. The time that the $\mathrm{SF}_{6}$ was first introduced into the supply duct established the initial time for the experiment. The $\mathrm{SF}_{6}$ concentration was measured at four locations: 1) clean supply air at the filter box; 2) the inlet air in the duct just as it entered the compartment; 3) the mid point of the compartment; and 4) the air exhaust. Since there was a one-minute interval between successive samples, the $\mathrm{SF}_{6}$ concentration at each location was measured every four minutes. The experiment was continued until steady state $\mathrm{SF}_{6}$ concentrations were achieved in the compartment and the exhaust. This experiment was repeated for the other two fixed air change rates. For each experiment, the effective volume,

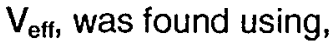

$$
V_{\text {eff }}=\frac{V_{A} \cdot \lambda_{A}}{\lambda_{A}^{R T D}}
$$

Where, the air exchange rate based on the residence time distribution, $\lambda_{A}{ }^{\mathrm{RTD}}$, was found by fitting the tracer concentration data to,

$$
\frac{C_{A}}{C_{A}^{o}}=1-\exp \left(-\lambda_{A}^{R T D} \cdot t\right)
$$

Where, $C_{A}$ is the tracer concentration in compartment $A, C_{A}{ }^{0}$ is the tracer concentration in the supply, and the initial concentration of the tracer is zero. The effective volume was then compared to the actual volume.

In order to measure the rate of air leakage from a compartment to the building, the pressure drop between the compartment and the building was first measured at the 0.5 and $2 \mathrm{~h}^{-1}$ air change rates with the compartment operating normally. Then, the exhaust was sealed by taping a flat plate over the opening in the compartment. Inlet air flow was established in the smallest diameter duct, and the control valve was adjusted to achieve a pressure drop between the compartment and the building that matched the measured pressure drop for normal operation 
at either the low or intermediate air change rates. This inlet air flowrate was, thus, a measure of the leakage rate since the exhaust was sealed. A $0.5-\mathrm{L}$ volume of the one-percent air mixture of $\mathrm{SF}_{6}$ was quickly introduced into the compartment at the mid point, and the concentration of $\mathrm{SF}_{6}$ in the compartment over time was fit to the following equation to quantify the decay or leakage rate.

$$
\frac{C_{A}}{C_{A}^{i}}=\exp \left(-\lambda_{L}^{A} \cdot t\right)
$$

Where, $C_{A}{ }^{i}$ is the initial tracer concentration, $\lambda_{L}{ }^{A}$ is the air leakage rate from compartment $A$ to the building, and $t$ is time.

A separate experiment was conducted to determine the air leakage rate between the two compartments. One compartment was operated at the $2 \mathrm{~h}^{-1}$ air change rate while the second compartment was operated at $0.5 \mathrm{~h}^{-1}$. Thus, there was a pressure differential established between the two compartments. Tracer gas was supplied to the first compartment and measured in both compartments over time. The experiment was run until near steady-state $\mathrm{SF}_{6}$ concentrations were achieved in the second compartment. The data were applied to a simple two-chamber mass-balance model. The first chamber was modeled as having one air supply and two exhausts, the standard exhaust outlet and the leakage to the second compartment operating at lower pressure. The leakage rate was found using the following equation.

$$
\lambda_{L}^{A \rightarrow B}=\frac{\lambda_{B}\left(C_{B}-C_{B}{ }^{0}\right)}{\left(C_{A}{ }^{0}-C_{B}\right)}
$$

Where, $C_{B}$ is the tracer concentration in chamber $B, C_{A}{ }^{0}$ and $C_{B}{ }^{0}$ are the supply $S_{6}$ concentrations for compartments $\mathrm{A}$ and $\mathrm{B}$, and $\lambda_{B}$ is the air exchange rate for compartment $\mathrm{B}$. $\lambda_{L}{ }^{A} \rightarrow^{B}$ is the inter-compartment leakage rate defined as $Q_{L} N_{A}$, where $Q_{L}$ is the inter-compartment volumetric flowrate. Equation (5) is valid at steady state when $C_{A} \cong C_{A}{ }^{0}$.

Air velocities near the floor of each compartment were mapped with the compartment operating at $0.5 \mathrm{~h}^{-1}$ and with the mixing fans operating. The floor of each compartment was marked off into a three by five equal-area grid. The sensor tip of the air velocity transducer was successively placed $5 \mathrm{~cm}$ above the floor at each of the eight grid intersections. The air velocity at each point was recorded with the data system. Air velocities were also measured at the center point of each of the four compartment walls $(5 \mathrm{~cm}$ away from the wall) under the same conditions.

\section{RESULTS AND DISCUSSION}

The results of the measurements of the three fixed air change rates for the two chamber compartments are presented in Table F-1. The established air change rates were within plus or minus four percent of the desired values.

The results of the residence time distribution experiments are presented in Table F-2. For the low and intermediate air change rates in each compartment, the deviation of the effective compartment volume from the actual volume was close to ten percent. At the $5.0 \mathrm{~h}^{-1}$ air change rate, the deviation was close to 20 percent. ASTM D 5116-90 (1990) suggests that a deviation of about ten percent or less is acceptable; however, precise quantitative guidance on how complete the mixing must be is not yet available. In all cases but one, the effective volume was greater than the actual volume. This implies that some of the supply air was escaping through a leak before it became well mixed with the air in the compartment. For one experiment (Compartment $\mathrm{A}$ at the $2.0 \mathrm{~h}^{-1}$ air change rate), the effective volume was smaller indicating that a small amount of supply air was bypassing to the exhaust.

The results of the leakage rate experiments are presented in Table F-3. Six to 13 percent of the air in a compartment leaked out of the compartment to the building at the 0.5 and $2 \mathrm{~h}^{-1}$ air change rates. The inter-compartment leakage experiment indicated that the air leakage between compartments operating at 0.5 and $2 h^{-1}$ rates was less than one ferv nt: of $0.5 h^{-1}$. 
The air velocities measured in the two compartments operating at $0.5 \mathrm{~h}^{-1}$ are presented in Table F-4. The average air velocity ( \pm 1 standard deviation) $5 \mathrm{~cm}$ above the floor in compartment $A$ was $11 \pm 4 \mathrm{~cm} \mathrm{sec}^{-1}$. The average air velocity $5 \mathrm{~cm}$ above the floor in compartment $B$ was $9 \pm$ $4 \mathrm{~cm} \mathrm{sec}^{-1}$. The air velocities measured at the walls averaged $20 \mathrm{~cm} \mathrm{sec}^{-1}$ for each compartment.

\section{REFERENCE}

ASTM. 1990. ASTM Standard D 5116-90, Standard Guide for Small-Scale Environmental Chamber Determinations of Organic Emissions From Indoor Materials/Products, American Society for Testing and Materials, Philadelphia, PA.

\section{List of Tables}

Table F-1. Measured fixed air change rates for chamber compartments $A$ and $B$.

Table F-2. Air residence time distributions for chamber compartments $A$ and $B$.

Table F-3. Air leakage from chamber compartments $A$ and $B$ to the building at the 0.5 and $2.0 \mathrm{~h}^{-1}$ air change rates.

Table F-4. Summary of the floor and wall air velocities measured in chamber compartments $A$ and $B$ at the $0.5 h^{-1}$ air change rate. 
Tajle F-1. Measured fixed air change rates for chamber compartments A and B. The volume of each compartment is $25.5 \mathrm{~m}^{3}$.

\begin{tabular}{|c|c|c|c|c|c|c|}
\hline \multirow{2}{*}{ Parameter } & \multicolumn{3}{|c|}{ Values, Compartment A } & \multicolumn{3}{|c|}{ Values, Compartment B } \\
\hline & 0.5 & 2.0 & 5.0 & 0.5 & 2.0 & 5.0 \\
\hline Expected inlet air flow rate $(\mathrm{L} \mathrm{min}-1)$ & 212 & 850 & 2,120 & 212 & 850 & 2,120 \\
\hline $1 \% \mathrm{SF}_{6}$ flow rate $\left(\mathrm{mL} \mathrm{min}^{-1}\right)$ & 3.35 & 13.4 & 33.5 & 3.35 & 13.4 & 33.5 \\
\hline Velocity pressure (Setra Volts) & 0.72 & 1.06 & 1.36 & 0.80 & 1.23 & 1.42 \\
\hline $\begin{array}{l}\text { Average } S_{6} \text { compartment conc. } \\
\qquad(\mathrm{ppb}) \pm 1 \text { std. dev. }\end{array}$ & $157 \pm 2$ & $160 \pm 2$ & $163 \pm 2$ & $161 \pm 2$ & $167 \pm 3$ & $155 \pm 1$ \\
\hline Measured air change rate $\left(\mathrm{h}^{-1}\right)$ & $0.50 \pm 0.01$ & $2.0 \pm 0.1$ & $4.8 \pm 0.1$ & $0.49 \pm 0.01$ & $1.9 \pm 0.1$ & $5.1 \pm 0.1$ \\
\hline
\end{tabular}

Table F-2. Air residence time distributions for chamber compartments A and B. The volume of each compartment is $25.5 \mathrm{~m}^{3}$.

\begin{tabular}{l|ccc|ccc}
\hline \hline Parameter & \multicolumn{3}{|c|}{ Values, Compartment A } & \multicolumn{3}{c}{ Values, Compartment B } \\
\hline \hline Expected air change rate $\left(\mathrm{h}^{-1}\right)$ & 0.5 & 2.0 & 5.0 & 0.5 & 2.0 & 5.0 \\
Average SF6 conc. $(\mathrm{ppb})$ & 157 & 177 & 167 & 173 & 161 & 149 \\
Measured air change rate $\left(\mathrm{h}^{-1}\right)$ & 0.46 & 2.04 & 3.95 & 0.43 & 1.80 & 4.50 \\
Effective volume $\left(\mathrm{m}^{3}\right)$ & 28.1 & 22.6 & 31.0 & 27.3 & 28.1 & 30.5 \\
Deviation from actual volume (\%) & +10 & -11 & +21 & +7 & +10 & +20 \\
& & & & & &
\end{tabular}


Table F-3. Air leakage from chamber compartments $A$ and $B$ to the building at the 0.5 and $2.0 \mathrm{~h}^{-1}$ air change rates.

\begin{tabular}{l|cc|cc}
\hline \hline Parameter & \multicolumn{2}{|c|}{ Values, Compartment A } & Values, Compartment B \\
\hline \hline Air change rate $\left(\mathrm{h}^{-1}\right)$ & 0.5 & 2.0 & 0.5 & 2.0 \\
Pressure differential $(\mathrm{Pa})$ & 1.0 & 3.0 & 1.0 & 3.0 \\
Leakage rate $\left(\mathrm{h}^{-1}\right)$ & 0.05 & 0.11 & 0.07 & 0.16 \\
Leakage rate $(\%)$ & 10 & 6 & 13 & 9 \\
\hline
\end{tabular}

Table F-4. Summary of the floor and wall air velocities measured in chamber compartments $A$ and $B$ at the $0.5 \mathrm{~h}^{-1}$ air change rate. Velocities were measured $5 \mathrm{~cm}$ from surfaces.

\begin{tabular}{|c|c|c|}
\hline \multirow[b]{2}{*}{ Parameter } & \multicolumn{2}{|c|}{ Air Velocity, cm sec-1 } \\
\hline & Compartment A & Compartment B \\
\hline \multicolumn{3}{|l|}{ Floor } \\
\hline Number of points & 8 & 8 \\
\hline Range & $6-18$ & $2-13$ \\
\hline Average ( \pm 1 std. dev.) & $11 \pm 4$ & $9 \pm 4$ \\
\hline \multicolumn{3}{|l|}{ Walls (mid point) } \\
\hline Number of points & 4 & 4 \\
\hline Range & $11-32$ & $14-23$ \\
\hline Average ( \pm 1 std. dev.) & $20 \pm 11$ & $20 \pm 4$ \\
\hline
\end{tabular}


(Page is intentionally blank) 


\section{APPENDIX G}

\section{PROCEDURES USED FOR EXPOSURE REDUCTION EXPERIMENTS CONDUCTED IN THE LARGE-SCALE CHAMBER FACILITY}

\section{GENERAL PROCEDURES}

These experiments were designed to measure the concentrations and emission rates of the target compounds under simulated residential indoor environmental conditions. The chamber facility is described in detail in Appendix $F$. The walls and ceilings of the two chamber compartments were gypsum board painted with flat latex paint, which is the most typical residential wall treatment. The floors were covered with carpet or sheet vinyl flooring. Furnishings were also included as these may act as additional sinks for the deposition and re-emission for compounds that are emitted by the source assemblies. A set of eight identical chairs was purchased for this purpose. These chairs were fully upholstered, medium-sized, arm chairs. They had previously been used in a hotel. The fabric was a fleecy synthetic material. Drapery from the same source was also purchased. The fabric appeared to be cotton or a cotton blend. The material was cut down into panels with dimensions of 1.5 by $2.1 \mathrm{~m}$. Two chairs and one drapery panel were used for each experiment. The chairs and drapery panels were alternated among the experiments and were aired out between uses.

The chamber compartments were thoroughly ventilated between experiments in order to reduce the background concentrations of volatile organic compounds (VOCs) and aldehydes. Typically, this was done for more than two weeks. Background concentrations of VOCs and aldehydes were monitored occasionally during the airing-out periods between experiments. Just prior to initiating an experiment, the compartment was operated at a ventilation rate of $0.5 \mathrm{~h}^{-1}$ for two or more days. For this background measurement period, the compartment was furnished with two chairs and a single drapery panel hung on a wire at the short wall of the compartment opposite the supply air inlet and the exhaust. Each compartment was fitted with six, speedcontrolled, $7.6-\mathrm{cm}$ diameter axial fans to provide adequate air mixing within the compartment. The placement of these fans is described in Appendix F. For experiments with the latex paint combination, painting substrates consisting of textured gypsum wall board and plywood panels and wall-to-wall carpeting were installed prior to the background measurement period. At the end of this period, air samples for VOCs and aldehydes were collected from the compartment and from the supply air downstream of the charcoal filter.

In preparation for the installation of the source assembly for each experiment, the supply air flow rate was set to provide a ventilation rate of 2.0 or $5.0 \mathrm{~h}^{-1}$ as required. The compartment exhaust damper was adjusted accordingly, and the tubing connections for the pressure transducer were manually moved to the selected pitot tube. The technicians then entered the compartment and removed the chairs, the drapery panel, and the sampling line. For experiments with floor coverings, they also removed the air mixing fans. The technicians installed the source assemblies following the protocols described below. They re-install the fans, the temperature and humidity probe, the thermocouples, and the sampling line and exited the compartment. The data acquisition and sample valve control program was then terminated and re-started. This established the initial time for the experiment.

The first set of air samples was collected from the compartment and the supply air at an average elapsed time of one hour. At 2-h elapsed time, the technicians re-entered the compartment and installed the two chairs, the drapery panel and an air velocity transducer. They exited the compartment, and the door was taped closed. The door was not opened and the compartment was not re-entered after this time. The compartment ventilation rate was reduced to either 0.5 or $2.0 \mathrm{~h}^{-1}$; the exhaust damper was adjusted; and the tubing connections for the pressure trans.ducer were moved. 
Additional air samples were collected from the compartment and the supply air at average elapsed times of three and six hours on the first day of the experiment. Subsequently, samples were collected daily for the next nine days at 24-h intervals from the time the experiment was initiated. The final set of air samples was collected on day fourteen of the experiment at 336-h elapsed time. Replicate samples for VOCs were collected on at least two sampling events during an experiment.

For experiments with elevated ventilation, the compartment was operated at a ventilation rate of $2.0 \mathrm{~h}^{-1}$ for the first 72 hours following the installation of the source assembly. Then, the ventilation rate was reduced to $0.5 \mathrm{~h}^{-1}$; the exhaust damper was adjusted; and the tubing connections for the pressure transducer were moved.

Prior to each experiment, the calibrations of the mass-flow controllers used to regulate the air sampling rates were checked with a flow calibrator.

The environmental and other data were recorded throughout an experimental period and the sampling values were controlled using a computer-based data acquisition and control system. The sensors and the data acquisition and control system are described in more detail in Appendix F. The experimental data consisted of: probe RTD temperature $1.5 \mathrm{~m}$ from floor at the midpoint of the compartment; probe relative humidity from the same location; four thermocouple temperatures in the compartment (one on the floor, one $30 \mathrm{~cm}$ from the ceiling at the end of the compartment nearest the supply air inlet, one $30 \mathrm{~cm}$ from floor at the opposite end of the compartment, and one $1.2 \mathrm{~m}$ above the floor near the midpoint of interior wall); air velocity $5-\mathrm{cm}$ above the floor; flow velocity pressure; supply air relative humidity; several thermocouple temperatures in the building; mass-flow controller flow rates; sampling valve positions; elapsed time and clock time. The data were recorded at 5-minute intervals.

\section{LATEX PAINT COMBINATION}

The substrate used for the application of the flat latex paint consisted of five panels of $1-\mathrm{cm}$ thick, unfinished, gypsum wall board each with surface dimensions of 1.22 by $2.44 \mathrm{~m}$ yielding a total painting surface area of $14.9 \mathrm{~m}^{2}$. More than one month prior to the experiments with paint, the exposed surfaces of all of the panels were textured using a professional texturing gun and commercial texturing compound. The textured surface that was applied was typical of many residential wall board installations.

The substrate used for the application of the semi-gloss latex paint consisted of two $1.3-\mathrm{cm}$ thick panels of smooth-surface plywood each with surface dimensions of 0.61 by $0.91 \mathrm{~m}$ yielding a total painting surface area of $1.1 \mathrm{~m}^{2}$.

The gypsum board and plywood panels were installed in the compartment two or more days prior to initiating an experiment. The gypsum board panels were installed over the painted walls of the compartment and held in place with screws. The panels extended from the floor to the ceiling. Three panels were installed along the long interior wall of the compartment. The edges of these panels were butted against each other. The remaining panels were installed on each side of the door on the opposite long wall of the compartment. The plywood panels were hung on the short walls with one panel at each end of the compartment. The floor of the chamber was swept clean after installing the panels.

Next, the floor of the compartment was completely carpeted. The carpet used for all of these experiments with paints was an action back, tufted level loop, 100 percent olefin fiber material. The carpet was obtained from a local retailer and had been in the retailer's warehouse stock for an extended period prior to the purchase. The carpet was installed directly over the aluminum-clad floor without a carpet cushion. It was held in place with tack strips positioned around the inside perimeter of the compartment. No seam tape or adhesives were used in the installation. 
Following the installation of substrate panels and the carpet, the two chairs, the drapery panel, the air mixing fans, the temperature and humidity probe, and the sampling line were installed. Then the $48-\mathrm{h}$ background measurement period was initiated.

The paints used in the experiments were purchased from a local retailer. They were all obtained in 3.78-L containers. The semi-gloss latex paint was mixed and split into new $0.95-\mathrm{L}$ paint containers following purchase since a relatively small amount of this paint was used in each experiment. Immediately prior to use, each paint container was thoroughly mixed by shaking and stirring.

The paints were applied using typical painting tools. The latex primer sealer was applied to the gypsum board panels by roller using a new $23-\mathrm{cm}$ wide roller cover with a $0.9-\mathrm{cm}$ nap. A brush was used to apply the primer to the upper and lower borders of the gypsum panels and to the two plywood panels. The flat latex paint was applied to the primered gypsum panels using a new roller cover of the same type. Another brush was used to apply the flat latex paint to the upper and lower borders of the gypsum panels. A third brush was used to apply the semi-gloss paint to the primered plywood panels. New plastic liners were used to contain the paints in the roller trays. Plastic drop cloths were used to collect any paint spilled during the painting process. All paint containers, tools and drop cloths were weighed immediately prior to and following an experiment. These weights were used to calculate the masses of the three paints that were applied to the panels.

The latex primer sealer and the flat latex paint were applied to the gypsum board panels by roller using a standardized procedure that followed recommended industry practice. The roller was loaded with paint, and the paint was applied to an approximately 0.6 by $0.1 .2 \mathrm{~m}$ area following an " $N$ " pattern. The pattern was filled in by running the roller horizontally over the area and was then smoothed out with vertical strokes. This procedure was repeated until the entire surface area was painted.

For each of these experiments, the latex primer sealer was applied first. Two technicians entered the compartment with the primer, painting tools and a work light. The door was closed and remained closed during the painting operation. It took approximately 25 minutes to apply the primer. At the end of this period, the technicians exited the compartment with the remaining paint and the painting tools. The primer was allowed to dry for one hour as recommended by the manufacturer. Exactly one hour after initially entering the compartment, the technicians reentered with the flat and semi-gloss latex paints and painting materials. They applied these paints following the same sequence as used for the primer. It took approximately another $20-25$ minutes to apply the finish paints. The remaining paint and painting tools were passed out of the compartment. The temperature and humidity probe, the thermocouples, and the sampling line were installed and positioned. The technicians then exited the chamber with the work light. This established the beginning of the 336-h experimental period. At 2-h elapsed time, the technicians re-entered the compartment and installed the two chairs, the drapery panel, and the air velocity transducer.

For the paint experiment with added air mixing and additional ventilation, two oscillating room fans, $30 \mathrm{~cm}$ in diameter, were installed in the compartment at 2-h elapsed time when the two chairs and the drapery panel were also installed. The fans were placed on a metal hospital cart in the approximate middle of the compartment. The axial centers of the fans were $1.1 \mathrm{~m}$ above the floor. The fans were positioned and operated so that they oscillated along the long walls of the compartment and moved air approximately perpendicular to these walls. The fans were continuously operated at their maximum speed setting until 72-h elapsed time when they were switched off externally to the compartment. The fans remained in the compartment for the duration of the experiment. 
At the end of each experiment, the painted gypsum board, the plywood panels and the carpet were removed from the compartment, and the compartment was ventilated at a high air change rate.

\section{CARPET ASSEMBLY}

The carpet used for these experiments was an action back, tufted textured loop, 100 percent olefin fiber material. This carpet was purchased through a contract flooring dealer. The dealer was able to obtain material that had been manufactured approximately one week prior to its delivery to the dealer. Upon delivery, the large roll was cut into the approximate sizes required for the experiments. For each experiment, there were three sections of carpet. Two of these had dimensions of 1.2 by $2.4 \mathrm{~m}$ and the third had dimensions of 2.4 by $2.4 \mathrm{~m}$. The three sections were tightly rolled together and stored in a sealed Tedlar bag until they were used in an experiment.

A $1.1-\mathrm{cm}$ thick bonded urethane carpet cushion was used for the experiments. This material was manufactured approximately one week prior to the time it was purchased from a local retailer. The $1.8 \mathrm{~m}$ wide roll was cut into approximately $2.4 \mathrm{~m}$ long sections. The material for each experiment, which consisted of three sections, was rolled together and stored in a sealed Tedlar bag until it was used.

Following the 48-h background measurement period, the two chairs, the drapery panel, the air mixing fans, and the sampling line were removed from the compartment. The carpet and carpet cushion materials for the experiment were unpacked from their storage bags outside of the compartment. The rolled materials, the installation tools and a work light were taken into the compartment by two technicians. The compartment door was closed and remained closed during most the installation period. Previously, a carpet tack strip had been attached to the floor around the inside perimeter of the compartment. The sections of carpet cushion were laid out on the floor with the smooth webbing side facing up. The sections were trimmed with a knife so that they fit inside of the tack strip. Next, the sections of carpet were laid out over the cushion. These were aligned so that the two seams ran across the short length of the compartment and were not directly over the seams in the cushion. The two seams were bonded with thermal seam tape applied with a professional seaming iron. The edges of the carpet were then trimmed with a knife so that the carpet fit closely against the walls of the compartment. The carpet was attached to the tack strip by pounding the edges down with a metal block and hammer. The carpet was not stretched, as is typical practice. The air mixing fans, the temperature and humidity probe, the thermocouples, and the sampling line were installed. The technicians exited the compartment with the work light. This established the beginning of the 336-h experimental period. The entire installation and setup procedure required approximately 50 minutes to complete. At 2-h elapsed time, the technicians re-entered the compartment and installed the two chairs, the drapery panel, and the air velocity transducer.

For one experiment, the carpet and carpet cushion materials were aired out prior to their installation in the compartment. Two days before the start of the experiment, the materials were laid out on the floor of a room that was ventilated at over ten air changes per hour. The carpet was positioned with the backing facing up and the cushion was positioned with the webbing side facing up. Four oscillating room fans were used to increase the air velocity over the surfaces of the materials. The fans were placed on the floor at the edges of the materials and were operated in the oscillating mode on their highest speed setting. At the end of the 48-h airing out period, the materials were rolled up and taken to the nearby chamber facility. Installation of the materials, as described above, began within one hour.

At the end of each experiment, the carpet and carpet cushion were removed from the compartment, and the compartment was ventilated at a high air change rate. 


\section{VINYL FLOORING ASSEMBLY}

The primary materials used for the experiments with the vinyl flooring assembly were particle board underlayment, residential sheet vinyl flooring, rubber cove base and associated adhesives.

The particle board underlayment was purchased from a local building supply retailer several months prior to the initiation of these experiments. The 1.22 by $2.44 \mathrm{~m}, 1-\mathrm{cm}$ thick, panels were stored in the chamber facility building. They were leaned up against several walls of the building to maximize the exposure of their surfaces to air. This was intended to reduce the emissions of VOCs from the panels; however, the reduction was not quantified. Prior to an experiment, four panels of underlayment were cut to completely cover the floor of the compartment. They were carefully cut so that the seams fit closely together and no nailing or filling would be required. The panels were then removed from the compartment, and the compartment was set up for the background measurement period as described above.

The sheet vinyl flooring was purchased through the contract flooring dealer. The roll of material was received within one week of its production by the manufacturer. Upon receipt, the roll was cut into the approximate sizes required for the experiments. There were three pieces for each experiment. Two pieces were 1.8 by $2.4 \mathrm{~m}$, and the other piece was 0.9 by $2.4 \mathrm{~m}$. Pieces for two experiments were tightly rolled together and stored in a sealed Tediar bag.

The sheet flooring adhesive was packaged by the manufacturer in a 19-L container. Sufficient adhesive needed to complete the installation was transferred into a separate container for each experiment. The cove base adhesive was packaged in $0.32-\mathrm{L}$ tubes with applicator spouts designed for use in a caulking gun. The seam sealer consisted of two separate liquids that are designed to be mixed together in equal portions just prior to application. The secondary container of sheet vinyl adhesive, two containers of cove base adhesive, and the installation tools consisting of two notched trowel applicators and a spatula were weighed immediately prior to and following an experiment. These weights were used to calculate the applied masses of the two adhesives.

Following the background measurement period, the two chairs, the drapery panel, the air mixing fans, and the sampling line were removed from the compartment. The technicians then installed the four panels of underlayment on top of the aluminum-clad floor. The compartment door was left open during this operation. Next, the sheet vinyl flooring material for the experiment was removed from its storage bag exterior to the compartment. The vinyl flooring, sections of cove base, adhesives, tools and a work light were taken into the compartment by the two technicians. The door was closed and remained closed during most of the installation. The vinyl flooring was laid out on the floor so that the two seams ran across the short length of the compartment. The material was trimmed to approximately the correct size with a knife. The sheet flooring adhesive was applied to the surface of the underlayment with the notched trowels. These trowels had $1.5-$ by $1.5-\mathrm{mm}$ square teeth as recommended by the adhesive manufacturer. The adhesive was applied in stages with two stages for each piece of vinyl flooring. After the vinyl flooring was glued down, the edges were trimmed as required and any large air bubbles were worked out by rubbing the surface and applying pressure with a large towel. The cove base, which was manufactured in 1.1-m sections, was applied next. Three equally-spaced beads of adhesive running the length of a section of cove base were applied with a caulking gun. The beads were approximately $0.6 \mathrm{~cm}$ wide. The cove base with adhesive was then pressed against the base of the wall and down against the vinyl flooring. Finally, the seam sealer was applied to the two seams in the vinyl flooring. The sealer was prepared outside of the compartment by mixing together $7.5 \mathrm{~mL}$ of each of the two components in a glass vial. The mixture was applied using a 10-mL plastic disposable syringe.

The tools and the remaining and scrap materials were passed out of the compartment. The air mixing fans, the temperature and humidity probe, the the nucc:uples, and the sampling line were installed. The technicians exited the compartment wit..... work light. This established 
the beginning of the 336- $h$ experimental period. The entire installation and setup procedure required approximately 90 minutes to complete. At 2-h elapsed time, the technicians re-entered the compartment and installed the two chairs, the drapery panel, and the air velocity transducer.

For one experiment, the sheet vinyl flooring and cove base materials were aired out prior to their installation in the compartment. Two days before the start of the experiment, the materials were laid out on the floor of a room that was ventilated at over ten air changes per hour. The materials were positioned with their finished surfaces facing up. Two oscillating room fans were used to increase the air velocity over the surfaces of the materials. The fans were placed on the floor at the edges of the materials and were operated in the oscillating mode on their highest speed setting. At the end of the 48-h airing out period, the vinyl flooring was rolled up, and both materials were transported to the chamber facility. Installation of the materials began within one hour.

At the end of each experiment, the entire vinyl flooring assembly and the cove base were removed from the compartment, and the compartment was ventilated at a high air change rate.

\section{COMBINED SOURCES}

Two experiments were conducted in which all of the sources were installed in the compartment. The materials used for these installations were the same as those described above for the individual source experiments. The substrates used for the paint combination were also identical in kind and size to those described above. Two-thirds of the floor area of the compartment was covered with the carpet assembly, and the remaining one-third was covered with the vinyl flooring assembly.

The gypsum board and plywood panels, the two chairs, and the drapery panel were installed in the compartment. The aluminum-clad floor was left bare. Following the $48-h$ background measurement period, the furnishings were removed and the paints were applied following procedures that were identical to those described above. The ventilation rate during painting was $5.0 \mathrm{~h}^{-1}$. Immediately after painting, the air mixing fans, the temperature and humidity probe, the thermocouples, and the sampling line were installed. There were no carpet and furnishings in the compartment. The technicians then exited the compartment with the work light. This established the initial time for the paint-drying period. Two hours later, the ventilation rate was reduced to $2.0 \mathrm{~h}^{-1}$.

Since the magnitude of the VOC emissions from the latex paints was known to be significantly greater than the magnitude of the VOC emissions from the other two source assemblies, the flooring materials were installed three days (72 hours) after painting. This initial drying period allowed the VOC emissions from the paints to decay down to somewhat lower levels and made it more practical to collect and analyze VOC samples for compounds that were representative of all of the sources. Additionally, it is common in new construction and remodeling projects for painting to be completed prior to installation of the finish flooring materials.

Approximately 68 hours after painting, VOC and aldehyde samples were collected from the compartment and the supply air. Seventy-two hours after painting and with the chamber still operating at $2.0 \mathrm{~h}^{-1}$, the installation of the carpet and vinyl flooring assemblies was initiated. The vinyl flooring assembly was installed first followed by the carpet assembly. The procedures were the same as those described above for the individual assemblies. The air mixing fans, the temperature and humidity probe, the thermocouples, the sampling line and the air velocity transducer were then installed, and the two technicians exited the compartment with the work light. This established the beginning of the experimental period. The entire installation and setup procedure required approximately 70 minutes to complete. At 2-h elapsed time, the technicians re-entered the compartment and installed the two chairs, the drapery panel, and the air velocity transducer. 
One of these experiments was conducted using mild heating over approximately three days in combination with additional ventilation as a source treatment procedure. Prior to this experiment, the exterior walls, the ceiling and the floor of the compartment were insulated with fiberglass insulation in an attempt to improve the uniformity of the air and surface temperatures in the compartment. Fiberglass bats with R-11 insulation value were installed on the three exterior walls; R-19 fiberglass bats were installed on the roof deck and under the floor. Two hours after the beginning of the experimental period, two portable heaters were placed in the compartment concurrently with the installation of the furnishings. These were identical 1,500 watt, 120 VAC, fan-forced, radiant heaters. The fans were placed on the carpet on aluminum plates and positioned on either side of the door. The operation of one of the heaters was controlled by a digital, laboratory temperature controller with $0.1^{\circ} \mathrm{C}$ resolution that was mounted on the exterior of the compartment. The temperature probe for the controller was positioned in the compartment about $60 \mathrm{~cm}$ above the floor. The other heater was manually controlled from the exterior of the compartment with an on/off switch.

Immediately following the collection of the 6-h air samples, the two heaters were turned on. After several hours when the air and surface temperatures had approached the $33^{\circ} \mathrm{C}$ setpoint temperature, the manually controlled heater was switched off. The single temperaturecontrolled heater was used for the duration of the heating period. The compartment was continuously heated until the collection of the $72-\mathrm{h}$ air samples was completed. At this time, the heater was switched off, and the ventilation rate was reduced to $0.5 \mathrm{~h}^{-1}$. Additional sets of air samples were collected during this experiment at 12- and 80-h elapsed time.

The other experiment with combined source assemblies was conducted with additional ventilation of $2.0 \mathrm{~h}^{-1}$, but without heating, throughout the first 72 hours following installation of the flooring assemblies. After collection of the 72-h air samples, the ventilation rate was reduced to $0.5 \mathrm{~h}^{-1}$. This experiment was conducted over a period of 12 weeks $(2,016$ hours) in order to measure the longer-term emissions of the target VOCs. Air samples were collected weekly following the initial two-week period. 
(Page is intentionally blank) 


\section{APPENDIX H}

\section{EXPOSURE REDUCTION EXPERIMENTS WITH THE LATEX PAINT COMBINATION}

Appendix $\mathrm{H}$ presents the analytical data for the four large-scale exposure reduction experiments with the latex paint combination. The experiments were conducted in $25.5 \mathrm{~m}^{3}$ chamber compartments over a period of 336 hours. Paints LPS2, FLP3 and SGLP3 were applied to gypsum board and plywood panels with a total surface area of $16 \mathrm{~m}^{2}$. The concentrations of selected compounds and SigmaVOC were measured throughout the experiments. Specific emission rates of these components were calculated. Cumulative mass emissions and cumulative exposures were estimated.

List of Tables

Table H-01. Summary of environmental parameters for large-scale Experiments $P-1$ through P-4 with latex paints.

Table H-02. Paint application data for large-scale Experiments P-1 through P-4. 346

Table H-03. VOCs emitted by Paints LPS2, FLP3 and SGLP3 in large-scale Experiment P-2 at 24- and 240-hours elapsed times.

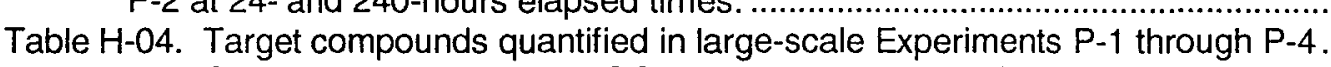
348

Table H-05. Concentrations of target VOCs for large-scale Experiment P-1 .............................350

Table H-06. Concentrations of target VOCs for large-scale Experiment P-2 ..........................351

Table H-07. Concentrations of target VOCs for large-scale Experiment P-3............................352

Table H-08. Concentrations of target VOCs for large-scale Experiment P-4 .............................353

Table $\mathrm{H}$-09. Concentrations of SigmaVOC for large-scale Experiments $\mathrm{P}-1$ through $\mathrm{P}-4 \ldots \ldots \ldots . . .354$

Table $\mathrm{H}-10$. Concentrations of formaldehyde for large-scale Experiments $\mathrm{P}-1$ through $\mathrm{P}-4 \ldots . . .355$

Table $\mathrm{H}-11$. Concentrations of acetaldehyde for large-scale Experiments P-1 through P-4.......356

Table H-12. Quasi steady-state specific emission rates of target VOCs for large-scale Experiment $P-1$

Table H-13. Quasi steady-state specific emission rates of target VOCs for large-scale Experiment P-2.... 358

Table H-14. Quasi steady-state specific emission rates of target VOCs for large-scale Experiment P-3

Table H-15. Quasi steady-state specific emission rates of target VOCs for large-scale Experiment P-4.

Table H-16. Quasi steady-state specific emission rates of SigmaVOC for large-scale Experiments P-1 through P-4 361

Table H-17. Quasi steady-state specific emission rates of formaldehyde for large-scale Experiments P-1 through P-4.

Table H-18. Quasi steady-state specific emission rates of acetaldehyde for large-scale Experiments P-1 through P-4.

Table H-19. Cumulative masses of target compounds and SigmaVOC emitted over $0-240$ and 0 - 336 hours in large-scale Experiments P-1 through P-4. 364

Table $\mathrm{H}-20$. Maximum exposure concentrations of target compounds during the first 48 hours of large-scale Experiments P-1 through P-4 ….............................................................365

Table $\mathrm{H}-21$. Cumulative exposures to target compounds during the first 48 hours of large-scale Experiments $\mathrm{P}-1$ through $\mathrm{P}-4$.. 365

Table H-22. Cumulative exposures to target compounds over $48-240$ and 48 - 336 hours in large-scale Experiments $\mathrm{P}-1$ through $\mathrm{P}-4$. 
Table H-01. Summary of environmental parameters for large-scale Experiments P-1 through P-4 with latex paints.

\begin{tabular}{|c|c|c|c|c|}
\hline \multirow[b]{2}{*}{ Parameter } & \multicolumn{4}{|c|}{ Experiment } \\
\hline & P-1 & P-2 & P-3 & $\mathrm{P}-4^{*}$ \\
\hline Starting date & $10 / 10 / 96$ & $10 / 22 / 96$ & $11 / 13 / 96$ & $12 / 09 / 96$ \\
\hline Treatment & Base case & Base case & Add ventilation & $\begin{array}{l}\text { Add ventilation } \\
\& \text { mixing }\end{array}$ \\
\hline \multicolumn{5}{|l|}{$\begin{array}{l}\text { Ventilation rate, } \\
2-72 \mathrm{~h}\left(\mathrm{~h}^{-1}\right)\end{array}$} \\
\hline Average \pm 1 std. dev & $0.50 \pm 0.01 \dagger$ & $0.50 \pm 0.011^{\dagger}$ & $1.95 \pm 0.01^{\ddagger}$ & $1.88 \pm 0.01^{\ddagger}$ \\
\hline Range & $0.50-0.51$ & $0.49-0.50$ & $1.91-1.98$ & $1.85-1.91$ \\
\hline \multicolumn{5}{|l|}{$\begin{array}{l}\text { Ventilation rate, } \\
72-336 \mathrm{~h}\left(\mathrm{~h}^{-1}\right)\end{array}$} \\
\hline Average \pm 1 std. dev & $0.50 \pm 0.01$ & $0.50 \pm 0.01$ & $0.50 \pm 0.01$ & $0.50 \pm 0.01$ \\
\hline Range & $0.49-0.51$ & $0.49-0.50$ & $0.49-0.51$ & $0.49-0.52$ \\
\hline \multicolumn{5}{|l|}{$\begin{array}{l}\text { Temperature, } \\
2-336 \mathrm{~h}\left({ }^{\circ} \mathrm{C}\right)\end{array}$} \\
\hline Average \pm 1 std. dev. & $23.1 \pm 1.1$ & $23.1 \pm 0.6$ & $23.8 \pm 0.3$ & $23.1 \pm 0.6$ \\
\hline Range & $20.7-25.7$ & $21.7-25.4$ & $23.0-25.2$ & $22.1-24.5$ \\
\hline \multicolumn{5}{|l|}{$\begin{array}{l}\text { Relative Humidity, } \\
2-336 \mathrm{~h}(\%)\end{array}$} \\
\hline Average \pm 1 std. dev. & $43 \pm 10$ & $38 \pm 5$ & $44 \pm 6$ & $48 \pm 5$ \\
\hline Range & $24-61$ & $24-48$ & $33-53$ & $37-56$ \\
\hline
\end{tabular}

*Experiment P-4 was terminated at 240-h elapsed time.

tVentilation rate for $0-2$ hours was $2 h^{-1}$.

掅entilation rate for $0-2$ hours was $5 \mathrm{~h}^{-1}$. 
Table H-02. Paint application data for large-scale Experiments P-1 through P-4. See Appendix C, Table C-01 for paint descriptions.

\begin{tabular}{|c|c|c|c|}
\hline $\begin{array}{c}\text { Experiment/ } \\
\text { Paint }\end{array}$ & $\begin{array}{l}\text { Area } \\
\left(\mathrm{m}^{2}\right) \\
\end{array}$ & $\begin{array}{c}\text { Mass } \\
(\mathrm{g}) \\
\end{array}$ & $\begin{array}{c}\text { Coverage } \\
\left(\mathrm{g} \mathrm{m}^{-2}\right)\end{array}$ \\
\hline \multicolumn{4}{|l|}{ P-1 } \\
\hline LPS2 & 16.0 & 1,610 & 101 \\
\hline FLP3 & 14.9 & 1,590 & 107 \\
\hline SGLP3 & 1.1 & 110 & 100 \\
\hline Total $^{\star}$ & 16.0 & 3,310 & 207 \\
\hline \multicolumn{4}{|l|}{ P-2 } \\
\hline LPS2 & 16.0 & 2,000 & 125 \\
\hline FLP3 & 14.9 & 1,570 & 105 \\
\hline SGLP3 & 1.1 & 114 & 104 \\
\hline Total $^{*}$ & 16.0 & 3,680 & 230 \\
\hline \multicolumn{4}{|l|}{ P-3 } \\
\hline LPS2 & 16.0 & 1,880 & 118 \\
\hline FLP3 & 14.9 & 1,610 & 108 \\
\hline SGLP3 & 1.1 & 115 & 105 \\
\hline Total $^{\star}$ & 16.0 & 3,600 & 225 \\
\hline \multicolumn{4}{|l|}{ P-4 } \\
\hline LPS2 & 16.0 & 2,030 & 127 \\
\hline FLP3 & 14.9 & 1,570 & 106 \\
\hline SGLP3 & 1.1 & 115 & 103 \\
\hline Total $^{\star}$ & 16.0 & 3,720 & 232 \\
\hline
\end{tabular}

*Total area was used in specific emission rate calculations. 
Table H-03. VOCs emitted by Paints LPS2, FLP3 and SGLP3 in large-scale Experiment P-2 at 24- and 240-hours elapsed times.

\begin{tabular}{|c|c|c|c|c|}
\hline COMPOUND & Code $^{*}$ & $\begin{array}{c}\text { 24-h } \\
\text { ET }\end{array}$ & $\begin{array}{c}240-h \\
\text { ET }\end{array}$ & $\begin{array}{l}\text { Match } \\
\text { Quality } \\
\end{array}$ \\
\hline \multicolumn{5}{|l|}{ Aromatic Hydrocarbons } \\
\hline $\mathrm{m}-\mathrm{p}$-Xylene & $\mathrm{T}, \mathrm{B}$ & + & & Confirmed \\
\hline 4-Phenylcyclohexene & $\mathrm{B}$ & & + & Confirmed \\
\hline \multicolumn{5}{|l|}{ Other Hydrocarbons } \\
\hline alpha-Pinene & $B$ & + & + & Confirmed \\
\hline 3-Carene & B & & + & Confirmed \\
\hline \multicolumn{5}{|l|}{ Carbonyl Compounds } \\
\hline Hexanal & B & + & + & Confirmed \\
\hline Octanal & $\mathrm{B}$ & + & & Confirmed \\
\hline Nonanal & $\mathrm{B}$ & + & + & Confirmed \\
\hline Decanal & $\mathrm{B}$ & + & + & Confirmed \\
\hline \multicolumn{5}{|l|}{ Other Oxidized Compounds } \\
\hline Acetic acid & B & + & + & Confirmed \\
\hline Ethylene glycol & $T, A, Q$ & + & + & Confirmed \\
\hline n-Butyl ether & $\mathrm{Q}$ & + & & Confirmed \\
\hline Propylene glycol & $A, Q$ & + & + & Confirmed \\
\hline Ethylene glycol monoacetate & & + & & Probable \\
\hline Hexylene glycol & & + & & Confirmed \\
\hline 2-Ethyl-1-hexanol & $\mathrm{Q}$ & + & & Confirmed \\
\hline 2-(2-Butoxyethoxy)ethanol & $T, Q$ & + & + & Confirmed \\
\hline Unidentified oxidized compound & & + & + & Unident. \\
\hline $\begin{array}{l}\text { 2,2,4-Trimethyl-1,3-pentanediol } \\
\text { monisobutyrate isomers (Texanol) }\end{array}$ & $A, Q$ & + & + & Confirmed \\
\hline
\end{tabular}

${ }^{\star} \mathrm{T}=$ Toxic air contaminant; $\mathrm{A}=$ Abundant compound; $\mathrm{B}=$ Component of chamber background; $\mathrm{Q}=$ Quantified target compound. 
Table H-04. Target compounds quantified in large-scale Experiments P-1 through P-4. Toxic Air Contaminant (TAC) Category for June 1996 is indicated where applicable.

\begin{tabular}{|c|c|c|c|}
\hline Compound & TAC Cat. & $\begin{array}{c}\text { Table } \\
\text { Abbrev. }\end{array}$ & Sources \\
\hline \multicolumn{4}{|l|}{ Carbonyl Compounds } \\
\hline Formaldehyde & 1 & & FLP3,SGLP3 \\
\hline Acetaldehyde & 1 & & FLP3,SGLP3 \\
\hline \multicolumn{4}{|l|}{ Other Oxidized Cmpds. } \\
\hline Ethylene glycol & 1 & $E G$ & LPS2,FLP3 \\
\hline Propylene glycol & & $P G$ & LPS2,SGLP3 \\
\hline n-Butyl ether & & & LPS2,FLP3,SGLP3 \\
\hline 2-Ethyl-1-hexanol & & & LPS2 \\
\hline 2-(2-Butoxyethoxy)ethanol & 1 & DEGBE & LPS2,SGLP3 \\
\hline $\begin{array}{l}\text { 2,2,4-Trimethyl-1,3-pentanediol } \\
\text { monoisbutyrates (combined } \\
\text { isomers) }\end{array}$ & & Texanol & LPS2,FLP3,SGLP3 \\
\hline
\end{tabular}


Table H-05. Concentrations of target VOCs for large-scale Experiment P-1.

\begin{tabular}{|c|c|c|c|c|c|c|}
\hline Sample ID & EG & $\mathbf{P G}$ & $\begin{array}{l}\text { ber Cor } \\
\text { Butyl } \\
\text { ether } \\
\end{array}$ & $\begin{array}{c}\text { tration, } \mu \\
\text { Ethyl- } \\
\text { hexanol }\end{array}$ & DEGBE & Texanol \\
\hline Avg. Inlet & $<33$ & $<12$ & $<1$ & $<1$ & $<1$ & $<2$ \\
\hline Background & $<33$ & $<12$ & $<1$ & $<1$ & $<1$ & $<2$ \\
\hline $1-h$ & 3,640 & 840 & 530 & 160 & 60 & 3,870 \\
\hline 3-h & 14,300 & 2,340 & 276 & 116 & 92 & 2,890 \\
\hline $6-h$ & 8,060 & 1,070 & 214 & 99 & 63 & 3,550 \\
\hline 24-h & 1,400 & 309 & 50 & 30 & 32 & 3,070 \\
\hline $48-h$ & 1,140 & 190 & 27 & 15 & 28 & 2,640 \\
\hline $72-h$ & 1,150 & 148 & 17 & 8 & 15 & 3,000 \\
\hline 96-h & 707 & 96 & 11 & 6 & 15 & 2,410 \\
\hline $120-\mathrm{h}$ & 500 & 36 & 8 & 5 & 10 & 1,930 \\
\hline $144-h$ & 245 & 40 & 7 & 3 & 7 & 1,680 \\
\hline $168-h$ & 299 & 27 & 5 & 3 & 6 & 1,640 \\
\hline $192-h$ & 373 & 27 & 4 & 2 & 5 & 1,560 \\
\hline 216-h & 294 & 31 & 4 & 3 & 5 & 1,360 \\
\hline $240 a-h$ & 220 & 27 & 3 & 2 & 4 & 980 \\
\hline 240b-h & 202 & 26 & 4 & 2 & 4 & 1,310 \\
\hline $336-h$ & 316 & 42 & 3 & 1 & 5 & 1,530 \\
\hline
\end{tabular}


Table H-06. Concentrations of target VOCs for large-scale Experiment P-2.

\begin{tabular}{|c|c|c|c|c|c|c|}
\hline Sample ID & EG & $\mathbf{P G}$ & $\begin{array}{l}\text { ber Cor } \\
\text { Butyl } \\
\text { ether } \\
\end{array}$ & $\begin{array}{c}\text { tration, } \mu \\
\text { Ethyl- } \\
\text { hexanol }\end{array}$ & DEGBE & Texanol \\
\hline Avg. Inlet & $<33$ & $<12$ & $<1$ & $<1$ & $<1$ & $<2$ \\
\hline Background & $<33$ & $<12$ & $<1$ & $<1$ & $<1$ & $<2$ \\
\hline $1-h$ & 16,100 & 4,020 & 554 & 169 & 84 & 3,330 \\
\hline 3-h & 19,500 & 3,740 & 265 & 108 & 108 & 4,640 \\
\hline $6-\mathrm{h}$ & 10,500 & 2,260 & 227 & 91 & 82 & 3,970 \\
\hline 24-h & 3,160 & 756 & 49 & 38 & 60 & 2,660 \\
\hline $48-h$ & 1,580 & 364 & 23 & 18 & 42 & 3,180 \\
\hline $72-h$ & 776 & 114 & 13 & 8 & 20 & 2,890 \\
\hline $96-h$ & 691 & 89 & 8 & 5 & 16 & $\mathrm{md}^{*}$ \\
\hline $120 a-h$ & 396 & 44 & 5 & 4 & 12 & 1,840 \\
\hline $120 b-h$ & 287 & 38 & 5 & 3 & 10 & 1,950 \\
\hline 144-h & 364 & 49 & 4 & 4 & 10 & 1,660 \\
\hline $168 a-h$ & 715 & 89 & 5 & 2 & 14 & 1,920 \\
\hline $168 b-h$ & 664 & 77 & 5 & 3 & 12 & 1,850 \\
\hline $192-h$ & 625 & 58 & 5 & 3 & 11 & 1,600 \\
\hline 216-h & 576 & 53 & 6 & 4 & 10 & 1,490 \\
\hline $240-h$ & 537 & 45 & 5 & 3 & 8 & 1,290 \\
\hline $336 a-h$ & 274 & md & 3 & 3 & 4 & 1,020 \\
\hline $336 b-h$ & 304 & 27 & 3 & 2 & 5 & 1,310 \\
\hline
\end{tabular}

${ }^{*} \mathrm{md}=$ missing or invalidated data. 
Table H-07. Concentrations of target VOCs for large-scale Experiment P-3.

\begin{tabular}{|c|c|c|c|c|c|c|}
\hline Sample ID & EG & PG & $\begin{array}{c}\text { ber Con } \\
\text { Butyl } \\
\text { ether }\end{array}$ & $\begin{array}{c}\text { tration, } \mu \\
\text { Ethyl- } \\
\text { hexanol }\end{array}$ & DEGBE & Texanol \\
\hline Avg. Inlet & $<33$ & $<12$ & $<1$ & $<1$ & $<1$ & $<2$ \\
\hline Background & $<33$ & $<12$ & $<1$ & $<1$ & $<1$ & 27 \\
\hline $1-h$ & 16,400 & 4,640 & 110 & 76 & 119 & 5,590 \\
\hline $3 a-h$ & 13,800 & 2,920 & 96 & 53 & 96 & 4,600 \\
\hline $3 b-h$ & 13,800 & 2,960 & 85 & 51 & 96 & 4,100 \\
\hline $6-h$ & 7,920 & 1,910 & 59 & 38 & 85 & 3,630 \\
\hline 24-h & 1,390 & 284 & 8 & 13 & 34 & 2,710 \\
\hline $48-h$ & 638 & 123 & 3 & 4 & 17 & 1,390 \\
\hline $72-h$ & 522 & 83 & 2 & 2 & 12 & 1,250 \\
\hline $96-h$ & 735 & 166 & 9 & 4 & 16 & 1,860 \\
\hline $120 a-h$ & 758 & 133 & 10 & 3 & 17 & 1,820 \\
\hline $120 b-h$ & 765 & 131 & 11 & 5 & 17 & 2,050 \\
\hline 144-h & 627 & 102 & 9 & 3 & 14 & 1,910 \\
\hline $168 a-h$ & 641 & 91 & 9 & 3 & 14 & 1,930 \\
\hline $168 b-h$ & 597 & 88 & 8 & 3 & 12 & $\mathrm{md}^{*}$ \\
\hline 192-h & 520 & 71 & 8 & 3 & 9 & 1,700 \\
\hline $216-h$ & 507 & 73 & 8 & $<1$ & 10 & 1,610 \\
\hline $240-\mathrm{h}$ & 328 & 42 & 5 & $<1$ & 8 & 1,580 \\
\hline $336-h$ & 199 & $<10$ & 3 & $<1$ & 4 & 1,420 \\
\hline
\end{tabular}

${ }^{*} \mathrm{md}=$ Missing or invalidated data. 
Table H-08. Concentrations of target VOCs for large-scale Experiment P-4. Experiment was terminated at 240-h elapsed time (see Report).

\begin{tabular}{|c|c|c|c|c|c|c|}
\hline Sample ID & EG & PG & $\begin{array}{c}\text { ber Con } \\
\text { Butyl } \\
\text { ether }\end{array}$ & $\begin{array}{c}\text { ntration, } \mu \\
\text { Ethyl- } \\
\text { hexanol }\end{array}$ & $\begin{array}{l}n-3 \\
\text { DEGBE }\end{array}$ & Texanol \\
\hline Avg. Inlet & $<33$ & $<12$ & $<1$ & $<1$ & $<1$ & $<2$ \\
\hline Background & $<33$ & $<12$ & $<1$ & 1 & $<1$ & 10 \\
\hline $1-h$ & 7,670 & 2,970 & 87 & 58 & 87 & 4,370 \\
\hline 3-h & 10,100 & 2,640 & 82 & 43 & 106 & 3,430 \\
\hline $6-h$ & 5,190 & 1,490 & 53 & 34 & 77 & 3,560 \\
\hline 24-h & 1,750 & 370 & 11 & 11 & 45 & 2,090 \\
\hline 48-h & 860 & 160 & 5 & 5 & 23 & 1,530 \\
\hline 72-h & 590 & 94 & 3 & 2 & 14 & 1,740 \\
\hline 96-h & 570 & 90 & 8 & 4 & 15 & 1,410 \\
\hline $120-h$ & 410 & 63 & 6 & 3 & 11 & 1,440 \\
\hline 144-h & 330 & 41 & 4 & 2 & 8 & 1,160 \\
\hline $168-h$ & 480 & 75 & 5 & 3 & 10 & 1,350 \\
\hline $192-h$ & 340 & 54 & 4 & 3 & 9 & 1,430 \\
\hline $216 a-h$ & 230 & 27 & 3 & 2 & 6 & 1,230 \\
\hline $216 b-h$ & 290 & 34 & 3 & 2 & 6 & 1,480 \\
\hline $240-h$ & 300 & 40 & 3 & 2 & 7 & 1,450 \\
\hline
\end{tabular}


Table H-09. Concentrations of SigmaVOC (i.e., sum of six target VOCs) for large-scale Experiments P-1 through P-4.

\begin{tabular}{|c|c|c|c|c|}
\hline Sample ID & Exp P-1 & \multicolumn{2}{|c|}{ Chamber Concentration, $\mu \mathrm{g} \mathrm{m}^{-3}$} & Exp P-4 \\
\hline Avg. Inlet & $<50$ & $<50$ & $<50$ & $<50$ \\
\hline Background & $<50$ & $<50$ & $<50$ & $<50$ \\
\hline $1-h$ & 9,100 & 24,300 & 26,900 & 15,200 \\
\hline $3 a-h$ & 20,000 & 28,400 & 21,600 & 16,400 \\
\hline $3 b-h$ & --- & -- & 21,100 & --- \\
\hline $6-h$ & 13,100 & 17,200 & 13,600 & 10,400 \\
\hline 24-h & 4,890 & 2,660 & 4,440 & 4,280 \\
\hline $48-h$ & 4,040 & 3,180 & 2,170 & 2,580 \\
\hline $72-\mathrm{h}$ & 4,340 & 2,890 & 1,870 & 2,440 \\
\hline $96-\mathrm{h}$ & 3,240 & $3,170^{*}$ & 2,790 & 2,100 \\
\hline $120 a-h$ & 2,490 & 2,300 & 2,740 & 1,940 \\
\hline $120 b-h$ & --- & 2,290 & 2,980 & --- \\
\hline $144-h$ & 1,980 & 2,090 & 2,660 & 1,540 \\
\hline $168 a-h$ & 1,980 & 2,740 & 2,690 & 1,920 \\
\hline $168 b-h$ & --- & 2,610 & $-\cdots$ & --- \\
\hline $192-h$ & 1,970 & 2,300 & 2,280 & 1,840 \\
\hline $216 a-h$ & 1,700 & 2,140 & 2,210 & 1,500 \\
\hline $216 b-h$ & 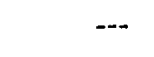 & $\cdots$ & --- & 1,810 \\
\hline $240 a-h$ & 1,240 & 1,890 & 1,970 & 1,800 \\
\hline $240 b-h$ & 1,550 & $\cdots$ & -- & --- \\
\hline 336a-h & 1,900 & 1,330 & 1,630 & $\mathrm{md}^{\star \star \star}$ \\
\hline $336 b-h$ & --- & 1,650 & --- & $\cdots$ \\
\hline
\end{tabular}

${ }^{*}$ Concentration of Texanol for this sample was estimated as the average of the concentrations for the immediately preceding and subsequent samples.

${ }^{* *} \mathrm{md}=$ Missing data; Experiment P-4 was terminated at 240-h elapsed time. 
Table H-10. Concentrations of formaldehyde for large-scale Experiments P-1 through P-4.

\begin{tabular}{|c|c|c|c|c|}
\hline Sample ID & Exp P-1 & \multicolumn{2}{|c|}{ Chamber Concentration, $\mu \mathrm{g} \mathrm{m}^{-3}$} & Exp P-4 \\
\hline Avg. Inlet ${ }^{*}$ & $<1.0$ & $1.2 \pm 0.5$ & $1.3 \pm 1.2$ & $<1.0$ \\
\hline Background & 4.8 & 1.1 & 7.0 & 6.1 \\
\hline $1-h$ & 42.9 & 104 & 25.6 & 19.0 \\
\hline 3-h & 21.0 & 62.8 & 14.0 & 10.4 \\
\hline $6-\mathrm{h}$ & 17.5 & 23.0 & 9.7 & 9.5 \\
\hline 24-h & 11.0 & $m d^{\star \star}$ & 2.9 & 7.6 \\
\hline 48-h & 9.3 & 11.2 & 1.3 & 5.3 \\
\hline 72-h & 9.0 & 10.3 & $<1.0$ & 4.8 \\
\hline $96-\mathrm{h}$ & 6.0 & 7.4 & 11.4 & 7.6 \\
\hline $120-h$ & 6.8 & 3.1 & 10.9 & 6.2 \\
\hline $144-h$ & 5.4 & 3.2 & 10.9 & 5.5 \\
\hline 168-h & 5.1 & 7.9 & 10.3 & 7.7 \\
\hline $192-h$ & 6.6 & 5.1 & 10.0 & 5.8 \\
\hline $216-h$ & 7.0 & 7.5 & 10.0 & 7.5 \\
\hline $240-h$ & 4.6 & 8.4 & 9.4 & 6.0 \\
\hline $336-h$ & 8.1 & 7.0 & 4.4 & $\mathrm{md}$ \\
\hline
\end{tabular}

*Average \pm 1 standard deviation.

${ }^{* *} \mathrm{md}=$ Missing data or invalid data; Experiment P-4 was terminated at $240-\mathrm{h}$ elapsed time. 
Table H-11. Concentrations of acetaldehyde for large-scale Experiments P-1 through P-4.

\begin{tabular}{|c|c|c|c|c|}
\hline Sample ID & \multicolumn{4}{|c|}{ Chamber Concentration, $\mu \mathrm{g} \mathrm{m-3}$} \\
\hline Avg. Inlet* & $6.8 \pm 5.3$ & $3.0 \pm 4.5$ & $<1.0$ & $1.0 \pm 0.7$ \\
\hline Background & 6.8 & 2.3 & 8.3 & 8.3 \\
\hline $1-h$ & 1,250 & 1,440 & 133 & 110 \\
\hline 3-h & 141 & 147 & 21.1 & 17.6 \\
\hline $6-h$ & 73.4 & 69.9 & 13.6 & 12.4 \\
\hline 24-h & 26.6 & $m d^{\star *}$ & 3.3 & 6.0 \\
\hline $48-h$ & 27.2 & 15.8 & 2.3 & 4.7 \\
\hline $72-h$ & 26.0 & 12.9 & 2.1 & 4.7 \\
\hline 96-h & 18.2 & 12.0 & 12.8 & 11.5 \\
\hline $120-h$ & 20.2 & 11.9 & 13.4 & 8.3 \\
\hline $144-h$ & 15.6 & 9.9 & 11.6 & 8.5 \\
\hline $168-h$ & 15.7 & 12.8 & 12.3 & 10.2 \\
\hline 192-h & 12.8 & 16.6 & 11.5 & 9.0 \\
\hline $216-h$ & 9.0 & 12.6 & 11.5 & 7.9 \\
\hline 240-h & 6.7 & 12.1 & 9.3 & 8.3 \\
\hline $336-h$ & 16.3 & 10.6 & 7.3 & md \\
\hline
\end{tabular}

${ }^{*}$ Average \pm 1 standard deviation.

${ }^{* *} \mathrm{md}=$ Missing data or invalid data; Experiment P-4 was terminated at $240-\mathrm{h}$ elapsed time. 
Table H-12. Quasi steady-state specific emission rates $\left(\mathrm{mg} \mathrm{m}^{-2} \mathrm{~h}^{-1}\right)$ of target VOCs for large-scale Experiment P-1.

\begin{tabular}{|c|c|c|c|c|c|c|}
\hline Sample ID & $E G$ & $P G$ & $\begin{array}{l}\text { Emissi } \\
\text { Butyl } \\
\text { ether } \\
\end{array}$ & $\begin{array}{c}\text { Rate, mg } \\
\text { Ethyl- } \\
\text { hexanol }\end{array}$ & $\begin{array}{l}\mathrm{h}-1 \\
\text { DEGBE }\end{array}$ & Texanol \\
\hline $1-h$ & 11.6 & 2.68 & 1.69 & 0.510 & 0.191 & 12.3 \\
\hline 3-h & 11.4 & 1.86 & 0.220 & 0.092 & 0.073 & 2.30 \\
\hline $6-h$ & 6.42 & 0.851 & 0.171 & 0.079 & 0.051 & 2.83 \\
\hline 24-h & 1.12 & 0.246 & 0.039 & 0.024 & 0.0256 & 2.45 \\
\hline $48-h$ & 0.907 & 0.151 & 0.021 & 0.012 & 0.022 & 2.11 \\
\hline $72-h$ & 0.915 & 0.118 & 0.013 & 0.007 & 0.012 & 2.39 \\
\hline $96-h$ & 0.563 & 0.077 & 0.009 & 0.005 & 0.012 & 1.92 \\
\hline $120-h$ & 0.398 & 0.029 & 0.007 & 0.004 & 0.008 & 1.54 \\
\hline $144-h$ & 0.195 & 0.032 & 0.005 & 0.002 & 0.005 & 1.34 \\
\hline $168-h$ & 0.238 & 0.021 & 0.004 & 0.002 & 0.005 & 1.31 \\
\hline $192-h$ & 0.298 & 0.022 & 0.003 & 0.002 & 0.004 & 1.24 \\
\hline 216-h & 0.234 & 0.025 & 0.003 & 0.002 & 0.004 & 1.08 \\
\hline $240-h$ & 0.168 & 0.021 & 0.003 & 0.002 & 0.003 & 0.911 \\
\hline $336-h$ & 0.252 & 0.034 & 0.002 & 0.001 & 0.004 & 1.22 \\
\hline
\end{tabular}


Table H-13. Quasi steady-state specific emission rates $\left(\mathrm{mg} \mathrm{m}^{-2} \mathrm{~h}^{-1}\right)$ of target VOCs for large-scale Experiment P-2.

\begin{tabular}{ccccccc}
\hline \hline & \multicolumn{6}{c}{ Specific Emission Rate, mg m-2 h-1 } \\
Sample ID & EG & PG & $\begin{array}{c}\text { Butyl } \\
\text { ether }\end{array}$ & $\begin{array}{c}\text { Ethyl- } \\
\text { hexanol }\end{array}$ & DEGBE & Texanol \\
\hline \hline $1-h$ & 51.5 & 12.8 & 1.77 & 0.538 & 0.269 & 10.6 \\
$3-h$ & 15.6 & 2.98 & 0.211 & 0.086 & 0.086 & 3.70 \\
$6-h$ & 8.40 & 1.80 & 0.181 & 0.072 & 0.065 & 3.17 \\
$24-h$ & 2.52 & 0.603 & 0.039 & 0.030 & 0.048 & 2.12 \\
$48-h$ & 1.26 & 0.290 & 0.019 & 0.014 & 0.033 & 2.53 \\
$72-h$ & 0.618 & 0.091 & 0.011 & 0.006 & 0.016 & 2.30 \\
$96-h$ & 0.551 & 0.071 & 0.006 & 0.004 & 0.013 & $1.88^{*}$ \\
$120-h$ & 0.272 & 0.033 & 0.004 & 0.003 & 0.008 & 1.51 \\
$144-h$ & 0.290 & 0.039 & 0.003 & 0.003 & 0.008 & 1.33 \\
$168-h$ & 0.550 & 0.066 & 0.004 & 0.002 & 0.010 & 1.50 \\
$192-h$ & 0.498 & 0.046 & 0.004 & 0.002 & 0.008 & 1.27 \\
$216-h$ & 0.459 & 0.042 & 0.005 & 0.003 & 0.008 & 1.18 \\
$240-h$ & 0.428 & 0.036 & 0.004 & 0.002 & 0.006 & 1.03 \\
$336-h$ & 0.230 & 0.022 & 0.002 & 0.002 & 0.003 & 0.928 \\
\hline
\end{tabular}

*Concentration of Texanol used in the calculation for this sample was estimated as the average of the concentrations for the immediately preceding and subsequent samples. 
Table H-14. Quasi steady-state specific emission rates $\left(\mathrm{mg} \mathrm{m}^{-2} \mathrm{~h}^{-1}\right)$ of target VOCs for large-scale Experiment P-3.

\begin{tabular}{|c|c|c|c|c|c|c|}
\hline Sample ID & $E G$ & $\begin{array}{r}\text { Spe } \\
\text { PG } \\
\end{array}$ & $\begin{array}{l}\text { Emissi } \\
\text { Butyl } \\
\text { ether } \\
\end{array}$ & $\begin{array}{c}\text { Rate, mg } \\
\text { Ethyl- } \\
\text { hexanol }\end{array}$ & $\begin{array}{l}\text { h-1 } \\
\text { DEGBE } \\
\end{array}$ & Texanol \\
\hline $1-h$ & 126.1 & 35.5 & 0.843 & 0.583 & 0.908 & 42.6 \\
\hline 3-h & 43.0 & 9.12 & 0.280 & 0.162 & 0.298 & 13.4 \\
\hline $6-h$ & 24.6 & 5.93 & 0.184 & 0.119 & 0.263 & 11.2 \\
\hline 24-h & 4.32 & 0.882 & 0.026 & 0.040 & 0.105 & 8.34 \\
\hline 48-h & 1.98 & 0.381 & 0.010 & 0.012 & 0.054 & 4.22 \\
\hline 72-h & 1.62 & 0.259 & 0.008 & 0.008 & 0.038 & 3.79 \\
\hline $96-h$ & 0.586 & 0.132 & 0.007 & 0.004 & 0.013 & 1.46 \\
\hline $120-h$ & 0.607 & 0.105 & 0.008 & 0.003 & 0.014 & 1.52 \\
\hline $144-h$ & 0.500 & 0.081 & 0.007 & 0.002 & 0.011 & 1.50 \\
\hline 168-h & 0.493 & 0.071 & 0.007 & 0.002 & 0.010 & 1.52 \\
\hline 192-h & 0.414 & 0.057 & 0.006 & 0.002 & 0.007 & 1.33 \\
\hline 216-h & 0.404 & 0.058 & 0.006 & $<0.001$ & 0.008 & 1.26 \\
\hline $240-h$ & 0.261 & 0.033 & 0.004 & $<0.001$ & 0.006 & 1.24 \\
\hline 336-h & 0.159 & $<0.008$ & 0.002 & $<0.001$ & 0.003 & 1.11 \\
\hline
\end{tabular}


Table H-15. Quasi steady-state specific emission rates $\left(\mathrm{mg} \mathrm{m}^{-2} \mathrm{~h}^{-1}\right)$ of target VOCs for large-scale Experiment P-4. Experiment was terminated at 240-h elapsed time.

\begin{tabular}{|c|c|c|c|c|c|c|}
\hline Sample ID & EG & PG & $\begin{array}{l}\text { Emissi } \\
\text { Butyl } \\
\text { ether }\end{array}$ & $\begin{array}{c}\text { Rate, mg n } \\
\text { Ethyl- } \\
\text { hexanol }\end{array}$ & $\begin{array}{l}h-1 \\
\text { DEGBE }\end{array}$ & Texanol \\
\hline $1-h$ & 62.4 & 24.1 & 0.703 & 0.461 & 0.703 & 35.4 \\
\hline 3-h & 30.2 & 7.91 & 0.245 & 0.127 & 0.317 & 10.2 \\
\hline $6-h$ & 15.6 & 4.47 & 0.158 & 0.098 & 0.230 & 10.6 \\
\hline 24-h & 5.24 & 1.11 & 0.032 & 0.029 & 0.134 & 6.22 \\
\hline 48-h & 2.57 & 0.481 & 0.014 & 0.011 & 0.069 & 4.56 \\
\hline $72-\mathrm{h}$ & 1.76 & 0.281 & 0.009 & 0.004 & 0.041 & 5.17 \\
\hline $96-h$ & 0.456 & 0.072 & 0.006 & 0.002 & 0.012 & 1.12 \\
\hline $120-h$ & 0.329 & 0.050 & 0.005 & 0.002 & 0.009 & 1.14 \\
\hline $144-h$ & 0.262 & 0.033 & 0.003 & 0.001 & 0.007 & 0.919 \\
\hline $168-h$ & 0.383 & 0.059 & 0.004 & 0.002 & 0.008 & 1.07 \\
\hline $192-h$ & 0.272 & 0.043 & 0.003 & 0.001 & 0.007 & 1.13 \\
\hline $216-h$ & 0.208 & 0.024 & 0.002 & 0.001 & 0.005 & 1.07 \\
\hline $240-h$ & 0.238 & 0.032 & 0.002 & 0.001 & 0.005 & 1.15 \\
\hline
\end{tabular}


Table H-16. Quasi steady-state specific emission rates $\left(\mathrm{mg} \mathrm{m}^{-2} \mathrm{~h}^{-1}\right)$ of SigmaVOC (i.e., sum of six target VOCs) for large-scale Experiments P-1 through P-4.

\begin{tabular}{|c|c|c|c|c|}
\hline \multirow[b]{2}{*}{ Sample ID } & \multicolumn{4}{|c|}{ Specific Emission Rate, $\mathrm{mg} \mathrm{m}^{-2} \mathrm{~h}-1$} \\
\hline & Exp P-1 & Exp P-2 & Exp P-3 & Exp P-4 \\
\hline $1-h$ & 2.90 & 77.5 & 206 & 124 \\
\hline $3-h$ & 16.0 & 22.6 & 66.3 & 49.0 \\
\hline $6-h$ & 10.4 & 13.7 & 42.3 & 31.1 \\
\hline 24-h & 3.90 & 5.36 & 13.7 & 12.8 \\
\hline $48-\mathrm{h}$ & 3.22 & 4.15 & 6.66 & 7.70 \\
\hline 72-h & 3.46 & 3.04 & 5.72 & 7.27 \\
\hline $96-h$ & 2.59 & 2.55 & 2.20 & 1.67 \\
\hline $120-h$ & 1.98 & 1.83 & 2.26 & 1.54 \\
\hline 144-h & 1.58 & 1.67 & 2.10 & 1.23 \\
\hline $168-h$ & 1.58 & 2.13 & 2.10 & 1.52 \\
\hline $192-h$ & 1.57 & 1.83 & 1.82 & 1.46 \\
\hline $216-h$ & 1.35 & 1.70 & 1.74 & 1.31 \\
\hline 240-h & 1.11 & 1.51 & 1.55 & 1.43 \\
\hline 336-h & 1.51 & 1.18 & 1.27 & $\mathrm{md}^{\star}$ \\
\hline
\end{tabular}

${ }^{*} \mathrm{md}=$ Missing data; Experiment P-4 was terminated at 240-h elapsed time. 
Table H-17. Quasi steady-state specific emission rates of formaldehyde for large-scale Experiments P-1 through P-4.

\begin{tabular}{|c|c|c|c|c|}
\hline \multirow[b]{2}{*}{ Sample ID } & \multicolumn{4}{|c|}{ Specific Emission Rate, $\mu \mathrm{g} \mathrm{m}^{-2} \mathrm{~h}^{-1}$} \\
\hline & Exp P-1 & Exp P-2 & Exp P-3 & Exp P-4 \\
\hline $1-h$ & 121 & 328 & 148 & 103 \\
\hline $3-h$ & 12.9 & 49.1 & 22.4 & 13.6 \\
\hline $6-h$ & 10.1 & 17.4 & 8.6 & 11.0 \\
\hline $24-h$ & 5.0 & $12.7^{*}$ & $<6.4$ & $<6.4$ \\
\hline $48-h$ & 3.6 & 8.0 & $<6.4$ & $<6.4$ \\
\hline $72-h$ & 3.3 & 7.3 & $<6.4$ & $<6.4$ \\
\hline $96-h$ & $<1.6$ & 5.0 & 3.6 & $<1.6$ \\
\hline $120-h$ & 1.6 & 1.6 & 3.1 & $<1.6$ \\
\hline 144-h & $<1.6$ & 1.6 & 3.1 & $<1.6$ \\
\hline $168-h$ & $<1.6$ & 5.4 & 2.7 & $<1.6$ \\
\hline 192-h & $<1.6$ & 3.2 & 2.4 & $<1.6$ \\
\hline $216-h$ & 1.7 & 5.1 & 2.4 & $<1.6$ \\
\hline 240-h & $<1.6$ & 5.8 & 1.9 & $<1.6$ \\
\hline 336-h & 2.6 & 4.6 & $<1.6$ & $m d^{\star \star}$ \\
\hline
\end{tabular}

${ }^{*}$ Concentration of formaldehyde for this sample was estimated as the average of the concentrations for the immediately preceding and subsequent samples.

${ }^{* *} \mathrm{md}=$ Missing data; Experiment P-4 was terminated at 240-h elapsed time. 
Table H-18. Quasi steady-state specific emission rates of acetaldehyde for large-scale Experiments P-1 through P-4.

\begin{tabular}{|c|c|c|c|c|}
\hline \multirow[b]{2}{*}{ Sample ID } & \multicolumn{4}{|c|}{ Specific Emission Rate, $\mu \mathrm{g} \mathrm{m}^{-2} \mathrm{~h}^{-1}$} \\
\hline & Exp P-1 & Exp P-2 & Exp P-3 & Exp P-4 \\
\hline $1-h$ & 3,970 & 4,580 & 997 & 812 \\
\hline 3-h & 107 & 114 & 40.6 & 29.6 \\
\hline $6-h$ & 53.0 & 53.3 & 16.8 & 12.9 \\
\hline 24-h & 15.7 & $31.8^{*}$ & $<6.4$ & $<6.4$ \\
\hline $48-h$ & 16.3 & 10.2 & $<6.4$ & $<6.4$ \\
\hline $72-h$ & 15.3 & 7.9 & $<6.4$ & $<6.4$ \\
\hline $96-h$ & 9.1 & 7.2 & 3.6 & 2.5 \\
\hline $120-h$ & 10.6 & 7.1 & 4.0 & $<1.6$ \\
\hline $144-h$ & 7.0 & 5.5 & 2.6 & $<1.6$ \\
\hline $168-h$ & 7.1 & 7.8 & 3.1 & $<1.6$ \\
\hline $192-h$ & 4.8 & 10.8 & 2.5 & $<1.6$ \\
\hline 216-h & 1.8 & 7.7 . & 2.5 & $<1.6$ \\
\hline $240-h$ & $<1.6$ & 7.2 & $<1.6$ & $<1.6$ \\
\hline $336-h$ & 7.6 & 6.0 & $<1.6$ & $m d^{* *}$ \\
\hline
\end{tabular}

${ }^{*}$ Concentration of acetaldehyde for this sample was estimated as the average of the concentrations for the immediately preceding and subsequent samples.

${ }^{* *} \mathrm{md}=$ Missing data; Experiment P-4 was terminated at 240-h elapsed time. 
Table H-19. Cumulative masses (milligrams) of target compounds and SigmaVOC emitted over 0 - 240 and 0 - 336 hours in large-scale Experiments P-1 through P-4.

\begin{tabular}{|c|c|c|c|c|}
\hline Compound & Exp P-1 & $\begin{array}{l}\text { Cumulati } \\
\text { Exp P-2 }\end{array}$ & $\begin{array}{r}\text { Aass, } \mathrm{mg} \\
\text { Exp P-3 } \\
\end{array}$ & Exp P-4* \\
\hline \multicolumn{5}{|l|}{0 - 240 Hour Period } \\
\hline Formaldehyde & $<13$ & 35 & $<18$ & $<11$ \\
\hline Acetaldehyde & 173 & 197 & $<47$ & $<35$ \\
\hline Ethylene glycol & 3,750 & 6,340 & 13,900 & 10,000 \\
\hline Propylene glycol & 572 & 1,300 & 3,240 & 2,620 \\
\hline n-Butyl ether & 131 & 130 & 102 & 88 \\
\hline 2-Ethyl-1-hexanol & 55 & 58 & 71 & 55 \\
\hline 2-(2-Butoxyethoxy)ethanol & 54 & 80 & 182 & 181 \\
\hline $\begin{array}{l}\text { 2,2,4-Trimethyl-1,3-pentanediol } \\
\text { monoisobutyrates (Texanol) }\end{array}$ & 6,930 & 7,130 & 13,200 & 11,800 \\
\hline SigmaVoc ${ }^{\star \star}$ & 11,500 & 15,000 & 30,700 & 24,800 \\
\hline \multicolumn{5}{|l|}{0 - 336 Hour Period } \\
\hline Formaldehyde & $<16$ & 43 & $<20$ & --- \\
\hline Acetaldehyde & 180 & 207 & $<48$ & --- \\
\hline Ethylene glycol & 4,080 & 6,840 & 14,200 & $\cdots$ \\
\hline Propylene glycol & 614 & 1,350 & 3,260 & --- \\
\hline n-Butyl ether & 134 & 136 & 107 & --- \\
\hline 2-Ethyl-1-hexanol & 57 & 62 & 71 & -- \\
\hline 2-(2-Butoxyethoxy)ethanol & 59 & 88 & 189 & -- \\
\hline $\begin{array}{l}\text { 2,2,4-Trimethyl-1,3-pentanediol } \\
\text { monoisobutyrates (Texanol) }\end{array}$ & 8,570 & 8,630 & 15,000 & -- \\
\hline SigmaVOC ${ }^{\star \star}$ & 13,500 & 17,100 & 32,900 & --- \\
\hline
\end{tabular}

*Experiment P-4 was terminated at 240-h elapsed time.

**SigmaVOC includes all target compounds except formaldehyde and acetaldehyde. 
Table H-20. Maximum exposure concentrations $(\mathrm{ppb})$ of target compounds during the first 48 hours of large-scale Experiments P-1 through P-4.

\begin{tabular}{lrrrr}
\hline & \multicolumn{4}{c}{ Maximum Concentration, ppb } \\
Compound & Exp P-1 & Exp P-2 & Exp P-3 & Exp P-4 \\
\hline Formaldehyde & 35 & 85 & 21 & 15 \\
Acetaldehyde & 696 & 800 & 74 & 61 \\
Ethylene glycol & 5,650 & 7,700 & 6,490 & 3,980 \\
Propylene glycol & 752 & 1,300 & 1,500 & 955 \\
n-Butyl ether & 100 & 104 & 21 & 16 \\
2-Ethyl-1-hexanol & 30 & 32 & 14 & 11 \\
2-(2-Butoxyethoxy)ethanol & 14 & 16 & 18 & 16 \\
2,2,4-Trimethyl-1,3-pentanediol & 438 & 525 & 633 & 494 \\
monisobutyrates (Texanol) & & & & \\
\hline
\end{tabular}

Table H-21. Cumulative exposures (ppm-hour) to target compounds during the first 48 hours of large-scale Experiments P-1 through P-4. Estimates assume 20 hours of occupancy per day.

\begin{tabular}{lcccc}
\hline & \multicolumn{5}{c}{ Cumulative Exposure, ppm-hour } \\
Compound & Exp P-1 & Exp P-2 & Exp P-3 & Exp P-4 \\
\hline Formaldehyde & 0.44 & 0.71 & 0.17 & 0.26 \\
Acetaldehyde & 1.80 & 2.01 & 0.23 & 0.24 \\
Ethylene glycol & 55.5 & 88.5 & 58.9 & 45.4 \\
Propylene glycol & 7.26 & 15.9 & 11.2 & 9.76 \\
n-Butyl ether & 0.80 & 0.81 & 0.19 & 0.18 \\
2-Ethyl-1-hexanol & 0.37 & 0.39 & 0.15 & 0.13 \\
2-(2-Butoxyethoxy)ethanol & 0.25 & 0.38 & 0.28 & 0.30 \\
2,2,4-Trimethyl-1,3-pentanediol & 13.8 & 14.4 & 12.4 & 10.8 \\
monisobutyrates (Texanol) & & & & \\
\hline
\end{tabular}


Table H-22. Cumulative exposures (ppm-hour) to target compounds over 48 - 240 and 48 - 336 hours in large-scale Experiments P-1 through P-4. Estimates assume 20 hours of occupancy per day.

\begin{tabular}{|c|c|c|c|c|}
\hline \multirow[b]{2}{*}{ Compound } & \multicolumn{4}{|c|}{ Cumulative Exposure, ppm-hour } \\
\hline & Exp P-1 & Exp P-2 & Exp P-3 & Exp P-4* \\
\hline \multicolumn{5}{|l|}{48 - 240 Hour Period } \\
\hline Formaldehyde & $<0.31$ & 0.73 & $<0.31$ & $<0.31$ \\
\hline Acetaldehyde & 0.89 & 0.87 & $<0.21$ & $<0.21$ \\
\hline Ethylene glycol & 33.5 & 40.4 & 37.7 & 28.1 \\
\hline Propylene glycol & 3.31 & 4.45 & 5.15 & 3.53 \\
\hline n-Butyl ether & 0.27 & 0.23 & 0.23 & 0.14 \\
\hline 2-Ethyl-1-hexanol & 0.14 & 0.15 & 0.08 & 0.08 \\
\hline 2-(2-Butoxyethoxy)ethanol & 0.24 & 0.35 & 0.32 & 0.27 \\
\hline $\begin{array}{l}\text { 2,2,4-Trimethyl-1,3-pentanediol } \\
\text { monoisobutyrates (Texanol) }\end{array}$ & 35.1 & 36.3 & 31.0 & 25.8 \\
\hline \multicolumn{5}{|l|}{48 - 336 Hour Period } \\
\hline Formaldehyde & $<0.47$ & 1.15 & $<0.47$ & --- \\
\hline Acetaldehyde & 1.10 & 1.24 & $<0.32$ & --- \\
\hline Ethylene glycol & 41.8 & 53.5 & 46.0 & $-\cdots$ \\
\hline Propylene glycol & 4.19 & 5.37 & 5.68 & --- \\
\hline n-Butyl ether & 0.31 & 0.29 & 0.28 & --- \\
\hline 2-Ethyl-1-hexanol & 0.17 & 0.19 & 0.08 & --- \\
\hline 2-(2-Butoxyethoxy)ethanol & 0.29 & 0.42 & 0.39 & --- \\
\hline $\begin{array}{l}\text { 2,2,4-Trimethyl-1,3-pentanediol } \\
\text { monoisobutyrates (Texanol) }\end{array}$ & 47.2 & 47.4 & 44.6 & --- \\
\hline
\end{tabular}

*Experiment P-4 was terminated at 240-h elapsed time. 


\section{APPENDIX 1}

\section{EXPOSURE REDUCTION EXPERIMENTS WITH THE CARPET ASSEMBLY}

Appendix I presents the analytical data for the three large-scale exposure reduction experiments with the carpet assembly. The experiments were conducted in $25.5 \mathrm{~m}^{3}$ chamber compartments over a period of 336 hours. The 10.4- $\mathrm{m}^{2}$ floor area was carpeted with Carpet Cushion CC4 and Carpet CP4. The concentrations of selected compounds and TVOC were measured throughout the experiments. Specific emission rates of these components were calculated. Cumulative mass emissions and cumulative exposures were estimated.

\section{List of Tables}

Table I-01. Summary of environmental parameters for large-scale Experiments C-1 through C-3 with carpet materials.

Table 1-02. Carpet materials and quantities used in large-scale Experiments C-1 through C3

Table 1-03. VOCs emitted by carpet assembly in large-scale Experiment C-1 at 24- and 240-hours

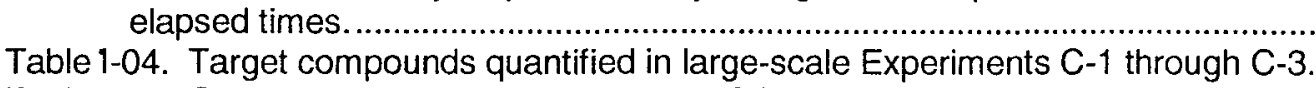

Table 1-05. Concentrations of Group 1 target VOCs for large-scale Experiment C-1

Table 1-06. Concentrations of Group 2 target VOCs for large-scale Experiment C-1

Table 1-07. Concentrations of Group 1 target VOCs for large-scale Experiment C-2

Table 1-08. Concentrations of Group 2 target VOCs for large-scale Experiment C-2

Table 1-09. Concentrations of Group 1 target VOCs for large-scale Experiment C-3

Table 1-10. Concentrations of Group 2 target VOCs for large-scale Experiment C-3

Table 1-11. Concentrations TVOC for large-scale Experiments C-1 through C-3.

Table 1-12. Concentrations of SigmaVOC for large-scale Experiments C-1 through C-3.

Table 1-13. Concentrations of formaldehyde and acetaldehyde for large-scale Experiment C-1.

Table 1-14. Quasi steady-state specific emission rates of Group 1 target VOCs for large-scale Experiment C-1.

Table 1-15. Quasi steady-state specific emission rates of Group 2 target VOCs for large-scale Experiment C-1.

Table 1-16. Quasi steady-state specific emission rates of Group 1 target VOCs for large-scale Experiment C-2.

Table 1-17. Quasi steady-state specific emission rates of Group 2 target VOCs for large-scale Experiment C-2.

Table I-18. Quasi steady-state specific emission rates of Group 1 target VOCs for large-scale Experiment C-3.

Table 1-19. Quasi steady-state specific emission rates of Group 2 target VOCs for large-scale Experiment C-3.

Table 1-20. Quasi steady-state specific emission rates of TVOC for large-scale Experiments C-1 through $\mathrm{C}-3$.

Table I-21. Cumulative masses of target VOCs and TVOC emitted over 0 - 336 hours in large-scale Experiments $\mathrm{C}-1$ through $\mathrm{C}-3$.

Table I-22. Cumulative expsoures to target VOCs during the first 48 hours of large-scale Experiments

C-1 through $\mathrm{C}-3$

Table 1-23. Cumulative exposures to target VOCs over 48 - 336 hours in large-scale Experiments C-1 through $\mathrm{C}-3$. 
Table 1-01. Summary of environmental parameters for large-scale Experiments $\mathrm{C}-1$ through $\mathrm{C}-3$ with carpet materials.

\begin{tabular}{|c|c|c|c|}
\hline Parameter & $C-1$ & $\begin{array}{c}\text { Experiment } \\
\text { C-2 } \\
\end{array}$ & $\mathrm{C}-3$ \\
\hline Starting date & $3 / 17 / 97$ & $4 / 29 / 97$ & $6 / 02 / 97$ \\
\hline Treatment & Base case & Add ventilation & Air out $\mathrm{CP}, \mathrm{CC}$ \\
\hline \multicolumn{4}{|l|}{$\begin{array}{l}\text { Ventilation rate, } \\
2-72 h\left(h^{-1}\right)\end{array}$} \\
\hline Average \pm 1 std. dev & $0.50 \pm 0.01^{*}$ & $1.91 \pm 0.01^{\dagger}$ & $0.50 \pm 0.01^{*}$ \\
\hline Range & $0.49-0.51$ & $1.87-1.94$ & $0.49-0.50$ \\
\hline \multicolumn{4}{|l|}{$\begin{array}{c}\text { Ventilation rate, } \\
72-336 \mathrm{~h}\left(\mathrm{~h}^{-1}\right)\end{array}$} \\
\hline Average \pm 1 std. dev & $0.50 \pm 0.01$ & $0.49 \pm 0.01$ & $0.50 \pm 0.01$ \\
\hline Range & $0.49-0.50$ & $0.48-0.49$ & $0.49-0.50$ \\
\hline \multicolumn{4}{|l|}{$\begin{array}{l}\text { Temperature }\left({ }^{\circ} \mathrm{C}\right) \\
2-336 \mathrm{~h}(\mathrm{~h}-1)\end{array}$} \\
\hline Average \pm 1 std. dev & $23.9 \pm 1.1$ & $23.3 \pm 1.3$ & $23.6 \pm 1.1$ \\
\hline Range & $22.1-26.6$ & $19.3-26.0$ & $21.5-25.7$ \\
\hline \multicolumn{4}{|l|}{$\begin{array}{l}\text { Relative Humidity (\%), } \\
2-336 \mathrm{~h}\left(\mathrm{~h}^{-1}\right)\end{array}$} \\
\hline Average \pm 1 std. dev. & $50 \pm 7$ & $51 \pm 4$ & $53 \pm 4$ \\
\hline Range & $34-61$ & $38-59$ & $47-61$ \\
\hline
\end{tabular}

"Ventilation rate for $0-2$ hours was $2 \mathrm{~h}^{-1}$.

tVentilation rate for $0-2$ hours was $5 h^{-1}$. 
Table 1-02. Carpet materials and quantities used in large-scale Experiments C-1 through C-3. See Appendix D, Table D-01, for complete material descriptions.

\begin{tabular}{|c|c|c|c|c|c|}
\hline Material Description & Material ID & $\begin{array}{c}\text { Unit of } \\
\text { Measure }\end{array}$ & Exp C-1 & $\begin{array}{l}\text { Quantity } \\
\text { Exp C-2 }\end{array}$ & Exp C-3 \\
\hline Commercial olefin carpet ${ }^{*}$ & $\mathrm{CP} 4$ & $m^{2}$ & 10.4 & 10.4 & 10.4 \\
\hline $\begin{array}{l}\text { Bonded urethane carpet } \\
\text { cushion }\end{array}$ & $\mathrm{CC} 4$ & $m^{2}$ & 9.43 & 9.43 & 9.43 \\
\hline Thermal seam tape & ST & $\mathrm{m}$ & 4.57 & 4.57 & 4.57 \\
\hline
\end{tabular}

*Area of carpet was used in specific emission rate calculations. 
Table 1-03. VOCs emitted by carpet assembly in large-scale Experiment C-1 at 24- and 240-hours elapsed times.

\begin{tabular}{|c|c|c|c|c|c|}
\hline COMPOUND & Code ${ }^{\star}$ & $\begin{array}{c}\mathrm{RT} \\
(\mathrm{min})\end{array}$ & $\begin{array}{l}\text { 24-h } \\
\text { ET }\end{array}$ & $\begin{array}{c}240-h \\
\text { ET }\end{array}$ & $\begin{array}{l}\text { Match } \\
\text { Quality }\end{array}$ \\
\hline \multicolumn{6}{|l|}{ Alkane Hydrocarbons } \\
\hline n-Octane & & 12.2 & + & & Confirmed \\
\hline$n$-Nonane & & 14.0 & + & & Confirmed \\
\hline Branched alkane $\mathrm{HC}$ & & 20.3 & + & & Probable \\
\hline Branched alkane $\mathrm{HC}$ & & 21.0 & + & + & Probable \\
\hline Branched alkane $\mathrm{HC}$ & & 21.6 & + & + & Probable \\
\hline Branched alkane $\mathrm{HC}$ & & 21.8 & + & + & Probable \\
\hline Branched alkane $\mathrm{HC}$ & & 23.1 & + & & Probable \\
\hline Branched alkane $\mathrm{HC}$ & & 23.4 & + & & Probable \\
\hline Branched alkane $\mathrm{HC}$ & A & 28.9 & + & + & Probable \\
\hline Branched alkane $\mathrm{HC}$ & & 29.2 & + & + & Probable \\
\hline Branched alkane $\mathrm{HC}$ & & 29.4 & + & + & Probable \\
\hline Branched alkane $\mathrm{HC}$ & & 29.6 & + & + & Probable \\
\hline Branched alkane $\mathrm{HC}$ & A & 30.3 & + & + & Probable \\
\hline Branched alkane $\mathrm{HC}$ & & 30.6 & + & + & Probable \\
\hline Branched alkane $\mathrm{HC}$ & & 30.9 & + & + & Probable \\
\hline Branched alkane $\mathrm{HC}$ & & 35.1 & + & + & Probable \\
\hline Branched alkane $\mathrm{HC}$ & & 36.3 & + & + & Probable \\
\hline \multicolumn{6}{|l|}{ Aromatic Hydrocarbons } \\
\hline Toluene & $\mathrm{T}$ & 11.9 & + & & Confirmed \\
\hline m-,p-xylene & $\mathrm{T}$ & 16.3 & + & & Confirmed \\
\hline o-Xylene & $T$ & 17.5 & + & & Confirmed \\
\hline Styrene & $T, Q$ & 17.8 & + & & Confirmed \\
\hline Isopropylbenzene & & 18.4 & + & & Confirmed \\
\hline Propylbenzene & & 19.6 & + & & Confirmed \\
\hline 4-Isopropyltoluene & B & 22.2 & + & + & Confirmed \\
\hline 4-Phenylcyclohexene & $A, Q$ & 33.2 & + & + & Confirmed \\
\hline \multicolumn{6}{|l|}{ Other Hydrocarbons } \\
\hline 4-Ethenylcyclohexene & $\mathrm{Q}$ & 13.8 & + & + & Confirmed \\
\hline alpha-Pinene & $\bar{B}$ & 17.6 & + & + & Confirmed \\
\hline Camphene & $\mathrm{B}$ & 18.5 & + & + & Confirmed \\
\hline beta-Pinene & $\mathrm{B}$ & 19.7 & + & + & Confirmed \\
\hline Alkene $\mathrm{HC}$ & & 19.8 & + & + & Probable \\
\hline Alkene $\mathrm{HC}$ & & 19.9 & + & + & Probable \\
\hline beta-Myrcene & $B$ & 20.2 & + & + & Confirmed \\
\hline Alkene $\mathrm{HC}$ & & 20.6 & + & & Probable \\
\hline Alkene $\mathrm{HC}$ & & 20.7 & + & & Probable \\
\hline Alkene $\mathrm{HC}$ & & 20.8 & + & & Probable \\
\hline 3-Carene & $B$ & 20.8 & + & + & Confirmed \\
\hline Alkene $\mathrm{HC}$ & & 21.4 & + & & Probable \\
\hline d-Limonene & $\mathrm{B}$ & 21.6 & + & + & Confirmed \\
\hline Alkene $\mathrm{HC}$ & & 21.9 & + & & Probable \\
\hline Alkene $\mathrm{HC}$ & & 22.3 & + & & Probable \\
\hline Alkene $\mathrm{HC}$ & & 22.5 & + & + & Probable \\
\hline
\end{tabular}


Table 1-03, Continued. VOCs emitted by carpet assembly in large-scale Experiment C-1 at 24- and 240-hours elapsed times.

\begin{tabular}{|c|c|c|c|c|c|}
\hline COMPOUND & Code $^{\star}$ & $\begin{array}{c}\mathrm{RT} \\
(\mathrm{min})\end{array}$ & $\begin{array}{l}\text { 24-h } \\
\text { ET }\end{array}$ & $\begin{array}{c}240-h \\
\text { ET }\end{array}$ & $\begin{array}{l}\text { Match } \\
\text { Quality }\end{array}$ \\
\hline \multicolumn{6}{|l|}{ Other Hydrocarbons, Cont. } \\
\hline Alkene $\mathrm{HC}$ & & 22.7 & + & & Probable \\
\hline Alkene $\mathrm{HC}$ & & 23.0 & + & + & Probable \\
\hline Alkene $\mathrm{HC}$ & & 23.2 & + & + & Probable \\
\hline Alkene $\mathrm{HC}$ & & 23.4 & + & + & Probable \\
\hline Alkene HC & & 23.6 & + & + & Probable \\
\hline Alkene $\mathrm{HC}$ & & 24.0 & + & & Probable \\
\hline Alkene $\mathrm{HC}$ & & 25.5 & + & & Probable \\
\hline Alkene $\mathrm{HC}$ & & 28.8 & + & + & Tentative \\
\hline Alkene $\mathrm{HC}$ & & 30.0 & + & + & Probable \\
\hline Alkene $\mathrm{HC}$ & & 30.2 & + & + & Probable \\
\hline Alkene $\mathrm{HC}$ & & 37.3 & + & + & Tentative \\
\hline $\begin{array}{l}\text { Unsaturated HCs, unresolved } \\
\text { isomers }\end{array}$ & A & $26-40$ & + & + & Probable \\
\hline \multicolumn{6}{|l|}{ Halogenated Compounds } \\
\hline 1,3-Dichloropropanol & & 22.6 & + & + & Confirmed \\
\hline 1,2-Dichlorobenzene & $\mathrm{Q}$ & 23.9 & + & + & Confirmed \\
\hline \multicolumn{6}{|l|}{ Carbonyl Compounds } \\
\hline Pentanal & $\mathrm{B}$ & 10.2 & + & + & Confirmed \\
\hline Hexanal & $\mathrm{B}$ & 14.6 & + & + & Confirmed \\
\hline 2-Furancarboxaldehyde & $\mathrm{B}$ & 18.2 & + & + & Confirmed \\
\hline Benzaldehyde & $\mathrm{B}$ & 22.6 & + & + & Confirmed \\
\hline Octanal & B & 22.7 & + & + & Confirmed \\
\hline Nonanal & $\mathrm{B}$ & 26.2 & + & + & Confirmed \\
\hline Decanal & $\mathrm{B}$ & 29.5 & & + & Confirmed \\
\hline \multicolumn{6}{|l|}{ Other Oxidized Compounds } \\
\hline 1-Butanol & $B$ & 9.9 & + & + & Confirmed \\
\hline Acetic acid & $\mathrm{B}$ & 10.1 & + & + & Confirmed \\
\hline 1-Pentanol & B & 14.3 & + & + & Confirmed \\
\hline Ethylene glycol & $\mathrm{T}, \mathrm{B}$ & 15.3 & + & + & Confirmed \\
\hline Propylene glycol & $\mathrm{B}$ & 16.6 & + & + & Confirmed \\
\hline $\begin{array}{l}\text { Di(propylene glycol)methyl ether, } \\
\text { isomer } 1\end{array}$ & $\mathrm{Q}$ & 23.1 & + & & Confirmed \\
\hline $\begin{array}{l}\text { Di(propylene glycol)methyl ether, } \\
\text { isomer } 2\end{array}$ & $\mathrm{Q}$ & 23.3 & + & & Confirmed \\
\hline $\begin{array}{l}\text { Di(propylene glycol)methyl ether, } \\
\text { isomer } 3\end{array}$ & $A, Q$ & 23.8 & + & + & Confirmed \\
\hline Phenol & $T, B, Q$ & 26.6 & + & + & Confirmed \\
\hline Phenethyl alcohol & $\mathrm{Q}$ & 28.8 & + & & Confirmed \\
\hline 1-Decanol & $\vec{Q}$ & 31.8 & + & + & Confirmed \\
\hline $\begin{array}{l}\text { 2,2,4-Trimethyl-1,3-pentanediol } \\
\text { monoisobutyrate (Texanol 1) }\end{array}$ & $B$ & 35.4 & + & + & Confirmed \\
\hline
\end{tabular}


Table 1-03, Continued. VOCs emitted by carpet assembly in large-scale Experiment C-1 at 24- and 240-hours elapsed times.

\begin{tabular}{|c|c|c|c|c|c|}
\hline COMPOUND & Code $^{*}$ & $\begin{array}{c}\text { RT } \\
(\min )\end{array}$ & $\begin{array}{c}24-h \\
E T \\
\end{array}$ & $\begin{array}{c}240-h \\
\text { ET }\end{array}$ & $\begin{array}{l}\text { Match } \\
\text { Quality } \\
\end{array}$ \\
\hline \multicolumn{6}{|l|}{ Other Oxidized Cmpds, Cont. } \\
\hline $\begin{array}{c}\text { 2,2,4-Trimethyl-1,3-pentanediol } \\
\text { monoisobutyrate (Texanol 3) }\end{array}$ & B & 35.8 & + & + & Confirmed \\
\hline $\begin{array}{l}\text { 2,6-Di-tert-butyl-4-methylphenol } \\
\text { (butylated hydroxytoluene) }\end{array}$ & $A, Q$ & 37.9 & + & + & Confirmed \\
\hline \multicolumn{6}{|l|}{ Nitrogen-Containing Compounds } \\
\hline N.N-Dimethylacetamide & $Q$ & 20.5 & + & & Confirmed \\
\hline N,N-Dimethylbenzylamine & $A, Q$ & 23.2 & + & + & Confirmed \\
\hline 2,2'-Azobisisobutyronitrile & $A, Q$ & 27.4 & + & + & Confirmed \\
\hline 2-Methyleneglutaronitrile & $\mathrm{Q}$ & 31.3 & + & + & Confirmed \\
\hline \multicolumn{6}{|l|}{ Miscellaneous Compounds } \\
\hline Hexamethylcyclotrisiloxane & B & 12.2 & + & + & Confirmed \\
\hline Octamethylcyclotetrasiloxane & B & 18.8 & + & + & Confirmed \\
\hline \multicolumn{6}{|l|}{ Unidentified Compounds } \\
\hline Unidentified compound & & 22.3 & & + & Unident. \\
\hline Unidentified glycol ether & & 26.5 & + & + & Unident. \\
\hline Unidentified glycol ether & & 27.3 & + & + & Unident. \\
\hline Unidentified glycol ether & & 27.5 & + & + & Unident. \\
\hline Unidentified glycol ether & & 27.6 & + & + & Unident. \\
\hline
\end{tabular}

* $T$ = Toxic air contaminant; $A$ = Abundant compound; $B$ = Component of chamber background; $\mathrm{Q}=$ Quantified target compound. 
Table 1-04. Target compounds quantified in large-scale Experiments C-1 through C-3. Toxic Air Contaminant (TAC) Category for June 1996 is indicated where applicable.

\begin{tabular}{|c|c|c|c|c|}
\hline Compound & TAC Cat. & Group & $\begin{array}{c}\text { Table } \\
\text { Abbrev. }\end{array}$ & Sources \\
\hline \multicolumn{5}{|l|}{ Aromatic Hydrocarbons } \\
\hline Styrene & 1 & 1 & & $\mathrm{CP} 4$ \\
\hline 4-Phenylcyclohexene & & 1 & $4-\mathrm{PCH}$ & $\mathrm{CP} 4$ \\
\hline \multicolumn{5}{|l|}{ Other Hydrocarbons } \\
\hline 4-Ethenylcyclohexene & & 1 & $4-\mathrm{VCH}$ & $\mathrm{CP} 4$ \\
\hline \multicolumn{5}{|l|}{ Halogenated Compounds } \\
\hline 1,2-Dichlorobenzene & & 1 & $1,2-\mathrm{DCB}$ & $\mathrm{CC} 4$ \\
\hline \multicolumn{5}{|l|}{ Carbonyl Compounds } \\
\hline Formaldehyde & I & Ald & & No dominant src. \\
\hline Acetaldehyde & 1 & Ald & & No dominant src. \\
\hline \multicolumn{5}{|l|}{ Other Oxidized Cmpds } \\
\hline $\begin{array}{l}\text { Di(propylene glycol)methyl } \\
\text { ethers }\end{array}$ & & 1 & DPGME & $\mathrm{CP} 4$ \\
\hline Phenol & 1 & 1 & & $\mathrm{CP} 4, \mathrm{CC} 4$ \\
\hline Phenethyl alcohol & & 1 & Phen alc & $\mathrm{CP} 4$ \\
\hline 1-Decanol & & 2 & Decanol & $\mathrm{CP} 4$ \\
\hline $\begin{array}{l}\text { 2,6-Di-tert-butyl-4- } \\
\text { methylphenol }\end{array}$ & & 2 & $\mathrm{BHT}$ & $\mathrm{CC} 4$ \\
\hline \multicolumn{5}{|l|}{ Nitrogen-Containing Cmpds } \\
\hline N,N-Dimethylacetamide & & 2 & DMA & $\mathrm{CP} 4$ \\
\hline N,N-Dimethylbenzylamine & & 2 & BDMA & $\mathrm{CC} 4$ \\
\hline 2,2'-Azobisisobutyronitrile & & 2 & AIBN & $\mathrm{CC} 4$ \\
\hline 2-Methyleneglutaronitrile & & 2 & MeGlutN & CP4 \\
\hline
\end{tabular}


Table 1-05. Concentrations of Group 1 target VOCs for large-scale Experiment C-1.

\begin{tabular}{|c|c|c|c|c|c|c|c|}
\hline \multirow{2}{*}{$\begin{array}{c}\text { Sample } \\
\text { ID }\end{array}$} & \multirow[b]{2}{*}{ Styrene } & \multirow[b]{2}{*}{ 4-PCH } & \multicolumn{3}{|c|}{ Chamber Concentration, $\mu \mathrm{g} \mathrm{m}^{-3}$} & \multirow[b]{2}{*}{ Phenol } & \multirow[b]{2}{*}{ Phen alc } \\
\hline & & & 4-VCH & 1,2-DCB & DPGME & & \\
\hline Avg. Inlet & $<1$ & $<1$ & $<1$ & $<1$ & $<3$ & $<1$ & $<1$ \\
\hline Backgrnd. & $<1$ & $<1$ & $<1$ & $<1$ & $<3$ & 1 & $<1$ \\
\hline $1-h$ & 11 & 9 & 14 & 2 & 28 & 5 & 1 \\
\hline $3-h$ & 9 & 8 & 13 & 2 & 24 & 4 & 1 \\
\hline $6-h$ & 11 & 10 & 19 & 3 & 24 & 4 & 1 \\
\hline $24-h$ & 6 & 12 & 9 & 4 & 19 & 3 & 1 \\
\hline $48 a-h$ & 3 & 12 & 5 & 4 & 10 & 3 & 1 \\
\hline $48 b-h$ & 4 & 13 & 6 & 4 & 13 & 4 & 1 \\
\hline $72-h$ & 2 & 11 & 3 & 3 & 9 & 3 & 1 \\
\hline 96-h & 2 & 10 & 2 & 3 & 9 & 3 & 1 \\
\hline $120-h$ & 1 & 9 & 2 & 3 & 6 & 2 & $<1$ \\
\hline $144-h$ & 1 & 10 & 1 & 2 & 5 & 2 & $<1$ \\
\hline $168-\mathrm{h}$ & 1 & 9 & 1 & 2 & 6 & 2 & $<1$ \\
\hline $192 a-h$ & $<1$ & 9 & 1 & 2 & 6 & 2 & $<1$ \\
\hline $192 b-h$ & 1 & 8 & 1 & 2 & 5 & 2 & $<1$ \\
\hline $216-h$ & $<1$ & 8 & 1 & 1 & 4 & 2 & $<1$ \\
\hline 240-h & $<1$ & 7 & $<1$ & 1 & 4 & 2 & $<1$ \\
\hline $336-\mathrm{h}$ & $<1$ & 6 & $<1$ & 1 & 3 & 2 & $<1$ \\
\hline
\end{tabular}


Table 1-06. Concentrations of Group 2 target VOCs for large-scale Experiment C-1.

\begin{tabular}{|c|c|c|c|c|c|c|}
\hline \multirow{2}{*}{$\begin{array}{c}\begin{array}{c}\text { Sample } \\
\text { ID }\end{array} \\
\text { Avg. Inlet }\end{array}$} & \multirow{2}{*}{$\begin{array}{c}\text { Decanol } \\
<1\end{array}$} & \multirow{2}{*}{$\begin{array}{r}\text { BHT } \\
<1\end{array}$} & \multicolumn{3}{|c|}{ Chamber Concentration, $\mu \mathrm{g} \mathrm{m}^{-3}$} & MeGlutN \\
\hline & & & $<10$ & $<1$ & $<1$ & $<1$ \\
\hline Backgrnd. & $<1$ & $<1$ & $<10$ & $<1$ & $<1$ & $<1$ \\
\hline $1-h$ & 2 & 6 & 21 & 6 & 21 & 9 \\
\hline $3-h$ & 2 & 4 & 17 & 8 & 22 & 8 \\
\hline $6-h$ & 2 & 4 & 14 & 8 & 29 & 8 \\
\hline $24-h$ & 3 & 3 & 10 & 7 & 43 & 6 \\
\hline $48 a-h$ & 2 & 3 & $<10$ & 7 & 43 & 5 \\
\hline $48 b-h$ & 3 & 3 & $<10$ & 7 & 47 & 6 \\
\hline $72-h$ & 2 & 4 & $<10$ & 8 & 39 & 5 \\
\hline $96-h$ & 3 & 4 & $<10$ & 7 & 40 & 4 \\
\hline $120-h$ & 2 & 4 & $<10$ & 4 & 32 & 2 \\
\hline $144-h$ & 2 & 4 & $<10$ & 5 & 31 & 2 \\
\hline $168-h$ & 2 & 6 & $<10$ & 7 & 30 & 2 \\
\hline $192 a-h$ & 2 & 6 & $<10$ & 5 & 27 & 2 \\
\hline $192 b-h$ & 2 & 6 & $<10$ & 5 & 25 & 2 \\
\hline 216-h & 2 & 6 & $<10$ & 5 & 22 & 1 \\
\hline $240-h$ & 1 & 7 & $<10$ & 4 & 20 & 1 \\
\hline $336-h$ & 1 & 8 & $<10$ & $<1$ & 14 & 1 \\
\hline
\end{tabular}


Table 1-07. Concentrations of Group 1 target VOCs for large-scale Experiment C-2.

\begin{tabular}{|c|c|c|c|c|c|c|c|}
\hline \multirow{2}{*}{$\begin{array}{c}\text { Sample } \\
\text { ID }\end{array}$} & \multirow[b]{2}{*}{ Styrene } & \multirow[b]{2}{*}{ 4-PCH } & \multicolumn{3}{|c|}{ Chamber Concentration, $\mu \mathrm{g} \mathrm{m}^{-3}$} & \multirow[b]{2}{*}{ Phenol } & \multirow[b]{2}{*}{ Phen alc } \\
\hline & & & 4-VCH & $1,2-\mathrm{DCB}$ & DPGME & & \\
\hline Avg. Inlet & $<1$ & $<1$ & $<1$ & $<1$ & $<3$ & $<1$ & $<1$ \\
\hline Backgrnd. & $<1$ & $<1$ & $<1$ & $<1$ & $<3$ & 1 & $<1$ \\
\hline $1-h$ & 8 & 7 & 7 & 1 & 21 & 5 & 1 \\
\hline $3-h$ & 10 & 9 & 10 & 1 & 19 & 4 & 1 \\
\hline $6-h$ & 10 & 10 & 10 & 1 & 20 & 4 & 1 \\
\hline $24-h$ & 3 & 9 & 3 & 1 & 9 & 3 & 1 \\
\hline $48 a-h$ & 1 & 6 & 2 & 1 & 4 & 2 & 1 \\
\hline $48 b-h$ & 1 & 6 & 1 & 1 & 3 & 2 & 1 \\
\hline $72-\mathrm{h}$ & 1 & 6 & 1 & 1 & 3 & 2 & $<1$ \\
\hline 96-h & 2 & 9 & 3 & 2 & 9 & 2 & $<1$ \\
\hline $120-\mathrm{h}$ & 1 & 9 & 2 & 2 & 6 & 2 & $<1$ \\
\hline 144-h & 1 & 9 & 1 & 1 & 6 & 2 & $<1$ \\
\hline $168-h$ & 1 & 9 & 1 & 1 & 6 & 3 & $<1$ \\
\hline $192-\mathrm{h}$ & 1 & 9 & 1 & 1 & 6 & 3 & $<1$ \\
\hline $216-h$ & 1 & 9 & 1 & 1 & 5 & 3 & $<1$ \\
\hline $240 a-h$ & $<1$ & 8 & 1 & 1 & 5 & 2 & $<1$ \\
\hline $240 b-h$ & 1 & 8 & 1 & 1 & 5 & 2 & $<1$ \\
\hline $336-h$ & $<1$ & 7 & $<1$ & 1 & 4 & 2 & $<1$ \\
\hline
\end{tabular}


Table 1-08. Concentrations of Group 2 target VOCs for large-scale Experiment C-2.

\begin{tabular}{|c|c|c|c|c|c|c|}
\hline \multirow{2}{*}{$\begin{array}{c}\begin{array}{c}\text { Sample } \\
\text { ID }\end{array} \\
\text { Avg. Inlet }\end{array}$} & \multirow{2}{*}{$\frac{\text { Decanol }}{<1}$} & \multirow{2}{*}{$\frac{\text { BHT }}{<1}$} & \multicolumn{3}{|c|}{ Chamber Concentration, $\mu \mathrm{g} \mathrm{m}^{-3}$} & MeGlutN \\
\hline & & & $<10$ & $<1$ & $<1$ & $<1$ \\
\hline Backgrnd. & $<1$ & $<1$ & $<10$ & $<1$ & $<1$ & $<1$ \\
\hline $1-h$ & 2 & 7 & $<10$ & 9 & 12 & 9 \\
\hline $3-h$ & 3 & 5 & $<10$ & 3 & 13 & 8 \\
\hline $6-h$ & 3 & 5 & $<10$ & 4 & 17 & 7 \\
\hline $24-h$ & 2 & 3 & $<10$ & 3 & 19 & 6 \\
\hline $48 a-h$ & 2 & 3 & $<10$ & 1 & 15 & 3 \\
\hline $48 b-h$ & 2 & 3 & $<10$ & $<1$ & 13 & 3 \\
\hline $72-h$ & 2 & 3 & $<10$ & 2 & 13 & 3 \\
\hline $96-h$ & 2 & 5 & $<10$ & 3 & 22. & 3 \\
\hline $120-h$ & 2 & 6 & $<10$ & 2 & 21 & 3 \\
\hline $144-h$ & 2 & 6 & $<10$ & 3 & 20 & 2 \\
\hline $168-h$ & 3 & 9 & $<10$ & $<1$ & 19 & 2 \\
\hline $192-h$ & 2 & 9 & $<10$ & 2 & 18 & 2 \\
\hline $216-h$ & 3 & 10 & $<10$ & $<1$ & 17 & 2 \\
\hline $240 a-h$ & 2 & 11 & $<10$ & 1 & 14 & 2 \\
\hline $240 b-h$ & 2 & 11 & $<10$ & 1 & 14 & 2 \\
\hline $336-h$ & 2 & 11 & $<10$ & $<1$ & 10 & 1 \\
\hline
\end{tabular}


Table 1-09. Concentrations of Group 1 target VOCs for large-scale Experiment C-3.

\begin{tabular}{|c|c|c|c|c|c|c|c|}
\hline $\begin{array}{c}\text { Sample } \\
\text { ID }\end{array}$ & Styrene & 4-PCH & \multicolumn{3}{|c|}{ Chamber Concentration, $\mu \mathrm{g} \mathrm{m} \cdot 3$} & Phenol & Phen alc \\
\hline Avg. Inlet & $<1$ & $<1$ & $<1$ & $<1$ & $<3$ & $<1$ & $<1$ \\
\hline Backgrnd. & $<1$ & $<1$ & $<1$ & $<1$ & $<3$ & 1 & $<1$ \\
\hline 1-h & 1 & 3 & 1 & $<1$ & 3 & 1 & $<1$ \\
\hline $3-h$ & 1 & 4 & 2 & $<1$ & 3 & 1 & $<1$ \\
\hline $6-h$ & 1 & 6 & 3 & $<1$ & 6 & 2 & $<1$ \\
\hline $24-h$ & 1 & 8 & 3 & $<1$ & 6 & 2 & $<1$ \\
\hline $48 a-h$ & 1 & 8 & 2 & $<1$ & 4 & 2 & $<1$ \\
\hline $48 b-h$ & 1 & 9 & 2 & $<1$ & 5 & 2 & $<1$ \\
\hline $72-h$ & 1 & 9 & 1 & $<1$ & 4 & 2 & $<1$ \\
\hline 96-h & 1 & 9 & 1 & $<1$ & 4 & 2 & $<1$ \\
\hline $120-h$ & $<1$ & 8 & 1 & $<1$ & 3 & 2 & $<1$ \\
\hline $144-h$ & $<1$ & 8 & 1 & $<1$ & $<3$ & 2 & $<1$ \\
\hline $168-h$ & $<1$ & 7 & $<1$ & $<1$ & $<3$ & 1 & $<1$ \\
\hline $192 a-h$ & $<1$ & 7 & $<1$ & $<1$ & $<3$ & 1 & $<1$ \\
\hline $192 b-h$ & $<1$ & 7 & $<1$ & $<1$ & $<3$ & 1 & $<1$ \\
\hline $216-h$ & $<1$ & 6 & $<1$ & $<1$ & $<3$ & 1 & $<1$ \\
\hline $240 a-h$ & $<1$ & 6 & $<1$ & $<1$ & $<3$ & 1 & $<1$ \\
\hline $240 \mathrm{~b}-\mathrm{h}$ & $<1$ & 7 & $<1$ & $<1$ & $<3$ & 2 & $<1$ \\
\hline $336-h$ & $<1$ & 5 & $<1$ & $<1$ & $<3$ & 1 & $<1$ \\
\hline
\end{tabular}


Table 1-10. Concentrations of Group 2 target VOCs for large-scale Experiment C-3.

\begin{tabular}{|c|c|c|c|c|c|c|}
\hline \multirow{2}{*}{$\begin{array}{c}\begin{array}{c}\text { Sample } \\
\text { ID }\end{array} \\
\text { Avg. Inlet }\end{array}$} & \multirow{2}{*}{$\frac{\text { Decanol }}{<1}$} & \multirow{2}{*}{$\frac{\mathrm{BHT}}{<1}$} & \multicolumn{3}{|c|}{ Chamber Concentration, $\mu \mathrm{g} \mathrm{m}^{-3}$} & MEGlutN \\
\hline & & & $<10$ & $<1$ & $<1$ & $<1$ \\
\hline Backgrnd. & $<1$ & $<1$ & $<10$ & $<1$ & $<1$ & $<1$ \\
\hline $1-h$ & 1 & 4 & $<10$ & $<1$ & $<1$ & 1 \\
\hline $3-h$ & 1 & 2 & $<10$ & $<1$ & $<1$ & 1 \\
\hline $6-h$ & 1 & 3 & $<10$ & $<1$ & 1 & 2 \\
\hline $24-h$ & 1 & 2 & $<10$ & $<1$ & 1 & 2 \\
\hline $48 a-h$ & 1 & 2 & $<10$ & $<1$ & 1 & 2 \\
\hline $48 b-h$ & 2 & 2 & $<10$ & $<1$ & 1 & 2 \\
\hline $72-h$ & 2 & 3 & $<10$ & $<1$ & 1 & 2 \\
\hline $96-h$ & 2 & 3 & $<10$ & $<1$ & 1 & 1 \\
\hline $120-\mathrm{h}$ & 1 & 3 & $<10$ & $<1$ & $<1$ & 1 \\
\hline $144-h$ & $<1$ & 4 & $<10$ & $<1$ & $<1$ & 1 \\
\hline $168-h$ & 1 & 4 & $<10$ & $<1$ & $<1$ & 1 \\
\hline $192 a-h$ & $<1$ & 5 & $<10$ & $<1$ & $<1$ & 1 \\
\hline $192 b-h$ & 1 & 5 & $<10$ & $<1$ & $<1$ & 1 \\
\hline $216-\mathrm{h}$ & 1 & 5 & $<10$ & $<1$ & $<1$ & $<1$ \\
\hline $240 a-h$ & 1 & 6 & $<10$ & $<1$ & $<1$ & $<1$ \\
\hline $240 b-h$ & $<1$ & 6 & $<10$ & $<1$ & $<1$ & $<1$ \\
\hline $336-h$ & $<1$ & 7 & $<10$ & $<1$ & $<1$ & $<1$ \\
\hline
\end{tabular}


Table 1-11. Concentrations of TVOC for large-scale Experiments C-1 through C-3.

\begin{tabular}{|c|c|c|c|}
\hline \multirow[b]{2}{*}{ Sample ID } & \multicolumn{3}{|c|}{ Chamber Concentration, $\mu \mathrm{g} \mathrm{m} \mathrm{m}^{-3}$} \\
\hline & $\operatorname{Exp} C-1$ & Exp C-2 & Exp C-3 \\
\hline Avg. Inlet ${ }^{\star}$ & $69 \pm 20$ & $67 \pm 14$ & $42 \pm 21$ \\
\hline Background & 117 & 106 & 121 \\
\hline $1-h$ & 746 & 494 & 276 \\
\hline 3-h & 700 & 537 & 264 \\
\hline $6-h$ & 741 & 574 & 349 \\
\hline 24-h & 797 & 442 & 409 \\
\hline $48 a-h$ & 781 & 369 & 415 \\
\hline $48 b-h$ & 812 & 367 & 473 \\
\hline 72-h & 781 & 349 & 420 \\
\hline $96-h$ & 753 & 593 & 368 \\
\hline $120-h$ & 631 & 622 & 335 \\
\hline 144-h & 706 & 655 & 358 \\
\hline $168-h$ & 732 & 700 & 316 \\
\hline $192 a-h$ & 666 & 680 & 286 \\
\hline 192b-h & 696 & --- & 317 \\
\hline $216-h$ & 567 & 653 & 269 \\
\hline 240a-h & 474 & 673 & 317 \\
\hline $240 b-h$ & --- & 662 & 332 \\
\hline $336-h$ & 429 & 494 & 255 \\
\hline
\end{tabular}

${ }^{\star}$ Average \pm 1 standard deviation. 
Table I-12. Concentrations of SigmaVOC (i.e., sum of 13 target VOCs) for large-scale Experiments C-1 through C-3.

\begin{tabular}{|c|c|c|c|}
\hline \multirow[b]{2}{*}{ Sample ID } & \multicolumn{3}{|c|}{ Chamber Concentration, $\mu \mathrm{g} \mathrm{m}-3$} \\
\hline & Exp C-1 & Exp C-2 & Exp C-3 \\
\hline Avg. Inlet & $<24$ & $<24$ & $<24$ \\
\hline Background & $<24$ & $<24$ & $<24$ \\
\hline $1-\mathrm{h}$ & 134 & 85 & $<24$ \\
\hline $3-h$ & 121 & 87 & $<24$ \\
\hline $6-h$ & 135 & 93 & 24 \\
\hline 24-h & 126 & 66 & 26 \\
\hline $48 a-h$ & 99 & 44 & $<24$ \\
\hline $48 b-h$ & 103 & 42 & 25 \\
\hline $72-h$ & 91 & 40 & 24 \\
\hline $96-h$ & 87 & 67 & 24 \\
\hline $120-h$ & 67 & 62 & $<24$ \\
\hline $144-h$ & 65 & 59 & $<24$ \\
\hline $168-h$ & 69 & 63 & $<24$ \\
\hline $192 a-h$ & 62 & 60 & $<24$ \\
\hline $192 b-h$ & 55 & $\ldots$ & $<24$ \\
\hline $216-h$ & 53 & 58 & $<24$ \\
\hline $240 a-h$ & 49 & 54 & $<24$ \\
\hline $240 b-h$ & -- & 54 & $<24$ \\
\hline $336-h$ & 36 & 45 & $<24$ \\
\hline
\end{tabular}


Table I-13. Concentrations of formaldehyde and acetaldehyde for large-scale Experiment C-1. Formaldehyde and acetaldehyde were not measured for Experiments C-2 and C-3.

\begin{tabular}{|c|c|c|}
\hline \multirow[b]{2}{*}{ Sample ID } & \multicolumn{2}{|c|}{ Chamber Concentration, $\mu \mathrm{g} \mathrm{m} \mathrm{m}^{-3}$} \\
\hline & Formaldehyde & Acetaldehyde \\
\hline Avg. Inlet ${ }^{*}$ & $1 \pm 1$ & $2 \pm 1$ \\
\hline Background & 5 & 8 \\
\hline $1-\mathrm{h}$ & 4 & 9 \\
\hline 3-h & $m d^{\star \star}$ & $\mathrm{md}$ \\
\hline $6-h$ & 6 & 12 \\
\hline 24-h & 4 & 9 \\
\hline $48-h$ & 7 & 12 \\
\hline 72-h & 5 & 11 \\
\hline $96-h$ & 6 & 10 \\
\hline $120-h$ & 4 & 9 \\
\hline $144-h$ & 4 & 8 \\
\hline $168-h$ & 5 & 10 \\
\hline 192-h & 5 & 10 \\
\hline $216-h$ & 4 & 8 \\
\hline 240-h & 2 & 7 \\
\hline $336-h$ & 4 & 6 \\
\hline
\end{tabular}

*Average \pm 1 standard deviation.

${ }^{\star \star} \mathrm{md}=$ Missing data; sample was not collected. 
Table I-14. Quasi steady-state specific emission rates of Group 1 target VOCs for large-scale Experiment C-1.

\begin{tabular}{|c|c|c|c|c|c|c|c|}
\hline \multirow{2}{*}{$\begin{array}{c}\text { Sample } \\
\text { ID }\end{array}$} & \multirow[b]{2}{*}{ Styrene } & \multirow[b]{2}{*}{ 4-PCH } & \multicolumn{3}{|c|}{ Specific Emission Rate, $\mu \mathrm{g} \mathrm{m}^{-2} \mathrm{~h}^{-1}$} & \multirow[b]{2}{*}{ Phenol } & \multirow[b]{2}{*}{ Phen alc } \\
\hline & & & 4-VCH & $1,2-\mathrm{DCB}$ & DPGME & & \\
\hline $1-\mathrm{h}$ & 54 & 42 & 68 & 8 & 136 & 18 & 6 \\
\hline $3-h$ & 11 & 10 & 16 & 2 & 30 & 3 & 1 \\
\hline $6-h$ & 13 & 12 & 23 & 3 & 29 & 4 & 1 \\
\hline $24-h$ & 7 & 15 & 11 & 5 & 23 & 3 & 1 \\
\hline 48-h & 4 & 16 & 7 & 5 & 14 & 3 & 1 \\
\hline $72-h$ & 2 & 14 & 4 & 4 & 11 & 3 & 1 \\
\hline $96-h$ & 2 & 12 & 3 & 4 & 11 & 2 & 1 \\
\hline $120-h$ & 1 & 11 & 2 & 3 & 7 & 1 & $<1$ \\
\hline $144-h$ & 1 & 12 & 1 & 3 & 6 & 2 & $<1$ \\
\hline $168-\mathrm{h}$ & 1 & 12 & 1 & 2 & 8 & 2 & $<1$ \\
\hline $192-h$ & $<1$ & 11 & 1 & 2 & 7 & 2 & $<1$ \\
\hline $216-h$ & $<1$ & 10 & 1 & 2 & 5 & 1 & $<1$ \\
\hline $240-h$ & $<1$ & 9 & $<1$ & 2 & 5 & 1 & $<1$ \\
\hline $336-h$ & $<1$ & 7 & $<1$ & 1 & 4 & 1 & $<1$ \\
\hline
\end{tabular}


Table I-15. Quasi steady-state specific emission rates of Group 2 target VOCs for large-scale Experiment C-1.

\begin{tabular}{|c|c|c|c|c|c|c|}
\hline $\begin{array}{c}\text { Sample } \\
\text { ID }\end{array}$ & Decanol & $\mathrm{BHT}$ & $\begin{array}{r}\text { Specific Emis } \\
\text { DMA } \\
\end{array}$ & $\begin{array}{l}\text { ate, } \mu \mathrm{g} \mathrm{m} \\
\text { BDMA } \\
\end{array}$ & AlBN & MeGlutN \\
\hline $1-h$ & 9 & 31 & 103 & 31 & 101 & 45 \\
\hline $3-h$ & 2 & 5 & 21 & 10 & 26 & 9 \\
\hline $6-h$ & 2 & 5 & 17 & 10 & 36 & 9 \\
\hline 24-h & 4 & 3 & 12 & 8 & 52 & 8 \\
\hline $48-\mathrm{h}$ & 3 & 3 & $<12$ & 9 & 55 & 7 \\
\hline $72-h$ & 3 & 4 & $<12$ & 10 & 48 & 6 \\
\hline $96-h$ & 4 & 4 & $<12$ & 9 & 49 & 5 \\
\hline $120-\mathrm{h}$ & 3 & 5 & $<12$ & 5 & 39 & 3 \\
\hline 144-h & 2 & 5 & $<12$ & 6 & 39 & 3 \\
\hline 168-h & 2 & 7 & $<12$ & 8 & 37 & 3 \\
\hline $192-\mathrm{h}$ & 3 & 8 & $<12$ & 7 & 31 & 2 \\
\hline 216-h & 2 & 7 & $<12$ & 6 & 27 & 1 \\
\hline $240-h$ & 2 & 9 & $<12$ & 5 & 24 & 1 \\
\hline $336-h$ & 1 & 10 & $<12$ & $<1$ & 17 & 1 \\
\hline
\end{tabular}


Table 1-16. Quasi steady-state specific emission rates of Group 1 target VOCs for large-scale Experiment C-2.

\begin{tabular}{|c|c|c|c|c|c|c|c|}
\hline $\begin{array}{c}\text { Sample } \\
\text { ID }\end{array}$ & Styrene & 4- $\mathrm{PCH}$ & $\begin{array}{r}\text { Speci } \\
4-\mathrm{VCH} \\
\end{array}$ & $\begin{array}{c}\text { ission Rat } \\
1,2-D C B \\
\end{array}$ & $\begin{array}{l}\mathrm{m}^{-2} \mathrm{~h}^{-1} \\
\mathrm{DPGME}\end{array}$ & Phenol & Phen alc \\
\hline $1-h$ & 103 & 89 & 83 & 9 & 262 & 47 & 15 \\
\hline $3-h$ & 49 & 40 & 48 & 5 & 87 & 13 & 5 \\
\hline $6-h$ & 46 & 47 & 47 & 6 & 94 & 13 & 6 \\
\hline $24-h$ & 14 & 44 & 16 & 6 & 44 & 9 & 4 \\
\hline $48-h$ & 6 & 30 & 7 & 4 & 16 & 4 & 3 \\
\hline $72-h$ & 4 & 30 & 5 & 3 & 16 & 3 & $<2$ \\
\hline $96-h$ & 2 & 10 & 3 & 2 & 10 & 1 & $<1$ \\
\hline $120-h$ & 1 & 10 & 2 & 2 & 8 & 1 & $<1$ \\
\hline $144-h$ & 1 & 10 & 2 & 2 & 7 & 1 & $<1$ \\
\hline $168-h$ & 1 & 11 & 1 & 2 & 7 & 2 & $<1$ \\
\hline $192-h$ & 1 & 11 & 1 & 1 & 7 & 2 & $<1$ \\
\hline $216-h$ & 1 & 11 & 1 & 1 & 6 & 2 & $<1$ \\
\hline $240-h$ & $<1$ & 10 & 1 & 1 & 5 & 1 & $<1$ \\
\hline $336-h$ & $<1$ & 8 & $<1$ & 1 & 5 & 1 & $<1$ \\
\hline
\end{tabular}


Table 1-17. Quasi steady-state specific emission rates of Group 2 target VOCs for large-scale Experiment C-2.

\begin{tabular}{|c|c|c|c|c|c|c|}
\hline $\begin{array}{c}\text { Sample } \\
\text { ID }\end{array}$ & Decanol & BHT & $\begin{array}{r}\text { Specific Emi } \\
\text { DMA } \\
\end{array}$ & $\begin{array}{c}\text { ate, } \mu \mathrm{g} n \\
\text { BDMA } \\
\end{array}$ & AIBN & MeGlutN \\
\hline $1-h$ & 31 & 87 & $<123$ & 109 & 148 & 110 \\
\hline 3-h & 12 & 22 & $<49$ & 13 & 63 & 36 \\
\hline $6-h$ & 12 & 23 & $<49$ & 18 & 79 & 35 \\
\hline $24-h$ & 11 & 16 & $<49$ & 16 & 89 & 27 \\
\hline $48-h$ & 9 & 13 & $<49$ & 3 & 66 & 15 \\
\hline $72-h$ & 10 & 15 & $<49$ & 7 & 59 & 14 \\
\hline $96-h$ & 3 & 6 & $<12$ & 4 & 26 & 3 \\
\hline $120-h$ & 3 & 7 & $<12$ & 3 & 25 & 3 \\
\hline $144-h$ & 3 & 7 & $<12$ & 3 & 24 & 3 \\
\hline $168-h$ & 4 & 10 & $<12$ & $<1$ & 23 & 3 \\
\hline $192-h$ & 3 & 11 & $<12$ & 2 & 22 & 2 \\
\hline $216-h$ & 3 & 12 & $<12$ & $<1$ & 21 & 2 \\
\hline $240-h$ & 3 & 13 & $<12$ & 1 & 17 & 2 \\
\hline $336-\mathrm{h}$ & 2 & 14 & $<12$ & $<1$ & 11 & 1 \\
\hline
\end{tabular}


Table 1-18. Quasi steady-state specific emission rates of Group 1 target VOCs for large-scale Experiment C-3.

\begin{tabular}{|c|c|c|c|c|c|c|c|}
\hline $\begin{array}{c}\text { Sample } \\
\text { ID }\end{array}$ & Styrene & 4-PCH & $\begin{array}{c}\text { Specif } \\
4-\mathrm{VCH}\end{array}$ & $\begin{array}{c}\text { ission Rat } \\
1,2-D C B\end{array}$ & $\begin{array}{l}\mathrm{m}^{-2} \mathrm{~h}^{-1} \\
\text { DPGME }\end{array}$ & Phenol & Phen alc \\
\hline $1-h$ & 5 & 15 & 5 & $<5$ & 14 & $<5$ & $<5$ \\
\hline 3-h & 1 & 5 & 2 & $<1$ & 4 & 1 & $<1$ \\
\hline $6-h$ & 1 & 8 & 4 & $<1$ & 7 & 1 & $<1$ \\
\hline 24-h & 1 & 10 & 3 & $<1$ & 7 & 1 & $<1$ \\
\hline 48-h & 1 & 11 & 2 & $<1$ & 6 & 1 & $<1$ \\
\hline $72-h$ & 1 & 11 & 2 & $<1$ & 5 & 1 & $<1$ \\
\hline $96-\mathrm{h}$ & 1 & 11 & 1 & $<1$ & 5 & 1 & $<1$ \\
\hline $120-h$ & $<1$ & 10 & 1 & $<1$ & 4 & 1 & $<1$ \\
\hline $144-\mathrm{h}$ & 1 & 9 & 1 & $<1$ & 2 & 1 & $<1$ \\
\hline $168-h$ & $<1$ & 8 & 1 & $<1$ & 2 & $<1$ & $<1$ \\
\hline $192-h$ & $<1$ & 8 & $<1$ & $<1$ & 2 & $<1$ & $<1$ \\
\hline $216-h$ & $<1$ & 8 & $<1$ & $<1$ & 2 & $<1$ & $<1$ \\
\hline $240-\mathrm{h}$ & $<1$ & 8 & $<1$ & $<1$ & 2 & 1 & $<1$ \\
\hline $336-h$ & $<1$ & 6 & $<1$ & $<1$ & 1 & $<1$ & $<1$ \\
\hline
\end{tabular}


Table I-19. Quasi steady-state specific emission rates of Group 2 target VOCs for large-scale Experiment C-3.

\begin{tabular}{ccccccc}
\hline $\begin{array}{c}\text { Sample } \\
\text { ID }\end{array}$ & Decanol & BHT & $\begin{array}{c}\text { Specific Emission Rate, } \mu \mathrm{g} \mathrm{m}^{-2} \mathbf{h}-\mathbf{1} \\
\text { DMA }\end{array}$ & BDMA & AIBN & MeGlutN \\
\hline $1-\mathrm{h}$ & 5 & 20 & $<49$ & $<5$ & $<5$ & 6 \\
$3-\mathrm{h}$ & 1 & 2 & $<12$ & $<1$ & $<1$ & 2 \\
$6-\mathrm{h}$ & 2 & 3 & $<12$ & $<1$ & 1 & 2 \\
$24-\mathrm{h}$ & 2 & 2 & $<12$ & $<1$ & 1 & 2 \\
$48-\mathrm{h}$ & 2 & 3 & $<12$ & $<1$ & 1 & 2 \\
$72-\mathrm{h}$ & 2 & 3 & $<12$ & $<1$ & 1 & 2 \\
$96-\mathrm{h}$ & 2 & 4 & $<12$ & $<1$ & 1 & 2 \\
$120-\mathrm{h}$ & 2 & 4 & $<12$ & $<1$ & $<1$ & 1 \\
$144-\mathrm{h}$ & 1 & 5 & $<12$ & $<1$ & $<1$ & 1 \\
$168-\mathrm{h}$ & 1 & 4 & $<12$ & $<1$ & $<1$ & 1 \\
$192-\mathrm{h}$ & 1 & 6 & $<12$ & $<1$ & $<1$ & 1 \\
$216-\mathrm{h}$ & 1 & 6 & $<12$ & $<1$ & $<1$ & $<1$ \\
$240-\mathrm{h}$ & 1 & 7 & $<12$ & $<1$ & $<1$ & $<1$ \\
$336-\mathrm{h}$ & $<1$ & 8 & $<12$ & $<1$ & $<1$ & $<1$ \\
\hline
\end{tabular}


Table 1-20. Quasi steady-state specific emission rates of TVOC for large-scale Experiments C-1 through C-3.

\begin{tabular}{|c|c|c|c|}
\hline \multirow[b]{2}{*}{ Sample ID } & \multicolumn{3}{|c|}{ Specific Emission Rate, $\mu \mathrm{g} \mathrm{m}^{-2} \mathrm{~h}^{-1}$} \\
\hline & Exp C-1 & Exp C-2 & Exp C-3 \\
\hline $1-h$ & 3,040 & 4,850 & 748 \\
\hline $3-h$ & 715 & 2,020 & 175 \\
\hline $6-h$ & 765 & 2,200 & 280 \\
\hline 24-h & 834 & 1,570 & 353 \\
\hline $48-h$ & 834 & 1,230 & 396 \\
\hline $72-h$ & 815 & 1,140 & 367 \\
\hline $96-h$ & 781 & 585 & 303 \\
\hline $120-h$ & 630 & 621 & 263 \\
\hline $144-h$ & 722 & 660 & 291 \\
\hline $168-h$ & 754 & 714 & 240 \\
\hline $192-h$ & 692 & 689 & 221 \\
\hline $216-h$ & 553 & 657 & 182 \\
\hline $240-h$ & 439 & 675 & 250 \\
\hline $336-h$ & 383 & 467 & 164 \\
\hline
\end{tabular}


Table I-21. Cumulative masses (milligrams) of target VOCs and TVOC emitted over 0 - 336 hours in large-scale Experiments C-1 through C-3.

\begin{tabular}{|c|c|c|c|}
\hline \multirow[b]{2}{*}{ Compound } & \multicolumn{3}{|c|}{ Cumulative Mass, $\mathrm{mg}$} \\
\hline & Exp C-1 & Exp C-2 & Exp C-3 \\
\hline \multicolumn{4}{|l|}{ Aromatic Hydrocarbons } \\
\hline Styrene & 7 & 16 & $<4$ \\
\hline 4-Phenylcyclohexene & 39 & 59 & 30 \\
\hline \multicolumn{4}{|l|}{ Other Hydrocarbons } \\
\hline 4-Ethenylcyclohexene & 12 & 18 & $<4$ \\
\hline \multicolumn{4}{|l|}{ Halogenated Compounds } \\
\hline 1,2-Dichlorobenzene & 9 & 8 & $<4$ \\
\hline \multicolumn{4}{|l|}{ Other Oxidized Cmpds } \\
\hline $\begin{array}{l}\text { Di(propylene glycol)methyl } \\
\text { ethers }\end{array}$ & 34 & 52 & 12 \\
\hline Phenol & 7 & 10 & $<4$ \\
\hline Phenethyl alcohol & $<4$ & 4 & $<4$ \\
\hline 1-Decanol & 9 & 17 & 4 \\
\hline 2,6-Di-tert-butyl-4-methylphenol & 23 & 44 & 18 \\
\hline \multicolumn{4}{|l|}{ Nitrogen-Containing Cmpds } \\
\hline N,N-Dimethylacetamide & $<43$ & $<43$ & $<43$ \\
\hline N,N-Dimethylbenzylamine & 22 & 16 & $<4$ \\
\hline 2,2'-Azobisisobutryonitrile & 123 & 115 & $<4$ \\
\hline 2-Methyleneglutaronitrile & 13 & 26 & 4 \\
\hline TVOC & 2,260 & 2,960 & 931 \\
\hline
\end{tabular}


Table 1-22. Cumulative exposures (ppb-hour) to target VOCs during the first 48 hours of large-scale Experiments C-1 through C-3. Estimates assume 20 hours of occupancy per day.

\begin{tabular}{|c|c|c|c|}
\hline \multirow[b]{2}{*}{ Compound } & \multicolumn{3}{|c|}{ Cumulative Exposure, ppb-hour } \\
\hline & Exp C-1 & $\operatorname{Exp} C-2$ & Exp C-3 \\
\hline \multicolumn{4}{|l|}{ Aromatic Hydrocarbons } \\
\hline Styrene & 64 & 43 & $<9$ \\
\hline 4-Phenylcyclohexene & 70 & 53 & 46 \\
\hline \multicolumn{4}{|l|}{ Other Hydrocarbons } \\
\hline 4-Ethenylcyclohexene & 95 & 43 & 22 \\
\hline \multicolumn{4}{|l|}{ Halogenated Compounds } \\
\hline 1,2-Dichlorobenzene & 24 & 8 & $<7$ \\
\hline \multicolumn{4}{|l|}{ Other Oxidized Cmpds } \\
\hline $\begin{array}{l}\text { Di(propylene glycol)methyl } \\
\text { ethers }\end{array}$ & 111 & 66 & 32 \\
\hline Phenol & 37 & 33 & 17 \\
\hline Phenethyl alcohol & $<8$ & $<8$ & $<8$ \\
\hline 1-Decanol & 16 & 14 & 8 \\
\hline 2,6-Di-tert-butyl-4-methylphenol & 14 & 17 & 10 \\
\hline \multicolumn{4}{|l|}{ Nitrogen-Containing Cmpds } \\
\hline N,N-Dimethylacetamide & 127 & $<112$ & $<112$ \\
\hline N,N-Dimethylbenzylamine & 45 & 21 & $<7$ \\
\hline 2,2'-Azobisisobutyronitrile & 230 & 99 & $<6$ \\
\hline 2-Methyleneglutaronitrile & 60 & 52 & 16 \\
\hline
\end{tabular}


Table 1-23. Cumulative exposures (ppb-hour) to target VOCs over 48 - 336 hours in large-scale Experiments C-1 through C-3. Estimates assume 20 hours of occupancy per day.

\begin{tabular}{|c|c|c|c|}
\hline \multirow[b]{2}{*}{ Compound } & \multicolumn{3}{|c|}{ Cumulative Exposure, ppb-hour } \\
\hline & Exp C-1 & Exp C-2 & Exp C-3 \\
\hline \multicolumn{4}{|l|}{ Aromatic Hydrocarbons } \\
\hline Styrene & $<56$ & $<56$ & $<56$ \\
\hline 4-Phenylcyclohexene & 318 & 301 & 259 \\
\hline \multicolumn{4}{|l|}{ Other Hydrocarbons } \\
\hline 4-Ethenylcyclohexene & 60 & 60 & $<54$ \\
\hline \multicolumn{4}{|l|}{ Halogenated Compounds } \\
\hline 1,2-Dichlorobenzene & 77 & 45 & $<40$ \\
\hline \multicolumn{4}{|l|}{ Other Oxidized Cmpds } \\
\hline $\begin{array}{l}\text { Di(propylene glycol)methyl } \\
\text { ethers }\end{array}$ & 198 & 187 & $<109$ \\
\hline Phenol & 137 & 139 & 94 \\
\hline Phenethyl alcohol & $<48$ & $<48$ & $<48$ \\
\hline 1-Decanol & 70 & 86 & $<37$ \\
\hline 2,6-Di-tert-butyl-4-methylphenol & 152 & 223 & 122 \\
\hline \multicolumn{4}{|l|}{ Nitrogen-Containing Cmpds } \\
\hline N,N-Dimethylacetamide & $<675$ & $<675$ & $<675$ \\
\hline N,N-Dimethylbenzylamine & 214 & 75 & $<43$ \\
\hline 2,2'-Azobisisobutryonitrile & 954 & 572 & $<36$ \\
\hline 2-Methyleneglutaronitrile & 120 & 112 & $<55$ \\
\hline
\end{tabular}




\section{APPENDIX $J$}

\section{EXPOSURE REDUCTION EXPERIMENTS WITH THE VINYL FLOORING ASSEMBLY}

Appendix $\mathrm{J}$ presents the analytical data for the four large-scale exposure reduction experiments with the vinyl flooring assembly. The experiments were conducted in $25.5 \mathrm{~m}^{3}$ chamber compartments over a period of 336 hours. The 10.4- $\mathrm{m}^{2}$ floor area was covered with Sheet Vinyl SV5. The concentrations of selected compounds and TVOC were measured throughout the experiments. Specific emission rates of these components were calculated. Cumulative mass emissions and cumulative exposures were estimated.

\section{List of Tables}

Table J-01. Summary of environmental parameters for large-scale Experiments $V-1$ through $V-4$ with sheet vinyl flooring materials.

-02. Sheet vinyl flooring materials and quantities used in large-scale Experiments V-1

Table J-02. Sheet viny
through $\mathrm{V}-4$..

24- and 240-hours elapsed times.

Table J-04. Target compounds quantified in large-scale Experiments V-1 through V-4 ................... 400

Table J-05. Concentrations of Group 1 target VOCs for large-scale Experiment V-1 ........................ 401

Table J-06. Concentrations of Group 2 target VOCs for large-scale Experiment V-1 ......................... 402

Table J-07. Concentrations of Group 1 target VOCs for large-scale Experiment V-4 ...................... 403

Table J-08. Concentrations of Group 2 target VOCs for large-scale Experiment V-4 ......................... 404

Table J-09. Concentrations of Group 1 target VOCs for large-scale Experiment V-2 ........................ 405

Table J-10. Concentrations of Group 2 target VOCs for large-scale Experiment V-2 ....................... 406

Table J-11. Concentrations of Group 1 target VOCs for large-scale Experiment V-3....................... 407

Table J-12. Concentrations of Group 2 target VOCs for large-scale Experiment V-3.......................... 408

Table J-13. Concentrations of TVOC for large-scale Experiments V-1 through V-4 .......................... 409

Table J-14. Concentrations of SigmaVOC for large-scale Experiments V-1 through V-4 ................... 410

Table J-15. Concentrations of formaldehyde for large-scale Experiments $V-1$ through $V-4 \ldots \ldots \ldots \ldots \ldots . . . .411$

Table J-16. Concentrations of acetaldehyde for large-scale Experiments V-1 through V-4 ................ 412

Table J-17. Quasi steady-state specific emission rates of Group 1 target VOCs for large-scale Experiment $\mathrm{V}-1$.

Table J-18. Quasi steady-state specific emission rates of Group 2 target VOCs for large-scale Experiment $\mathrm{V}-1$

Table J-19. Quasi steady-state specific emission rates of Group 1 target VOCs for large-scale Experiment $\mathrm{V}-4$.

Table J-20. Quasi steady-state specific emission rates of Group 2 target VOCs for large-scale

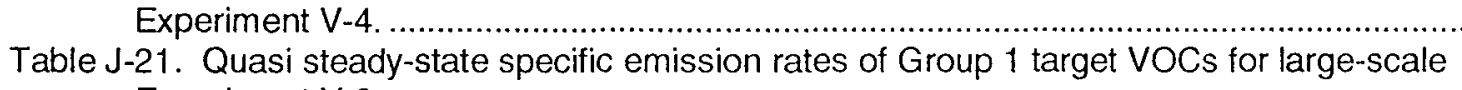

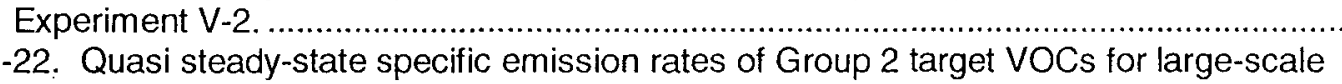

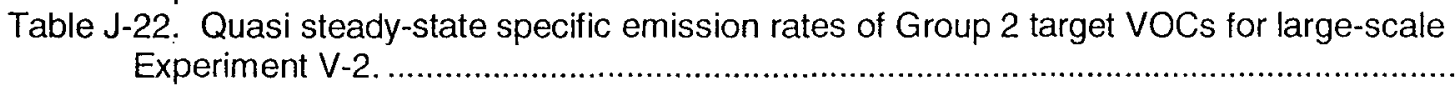

Table J-23. Quasi steady-state specific emission rates of Group 1 target VOCs for large-scale Experiment $\mathrm{V}-3$.

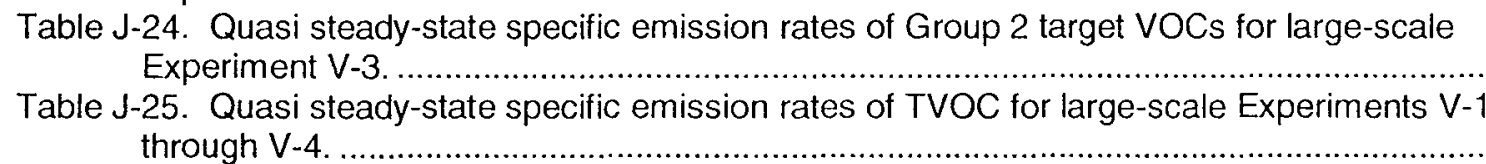

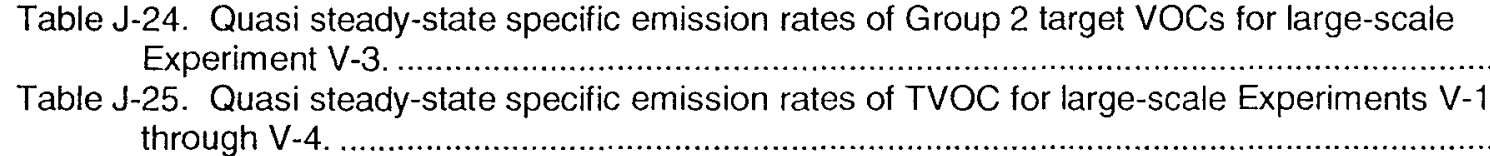

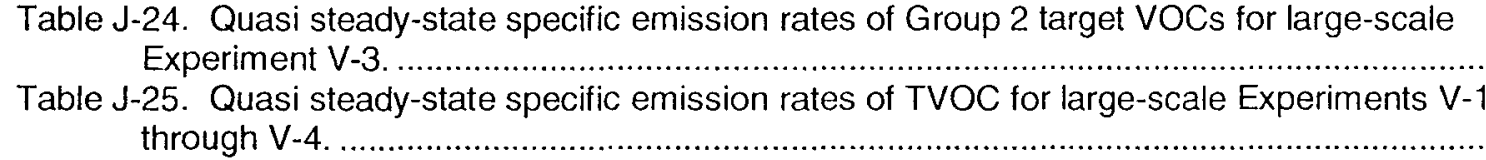

Table J-26. Quasi steady-state specific emission rates of formaldehyde for large-scale Experiments V-1 through V-4.

Table J-27. Quasi steady-state specific emission rates of acetaldehyde for large-scale

Experiments V-1 through V-4. 
Table J-28. Cumulative masses of target compounds and TVOC emitted over 0 - 336 hours in large-scale Experiments $\mathrm{V}-1$ through $\mathrm{V}-4$. 424

Table J-29. Cumulative exposures to target compounds during the first 48 hours of large-scale Experiments V-1 through V-4.

Table J-30. Cumulative exposures to target compounds over 48 - 336 hours in large-scale Experiments $\mathrm{V}-1$ through $\mathrm{V}-4$. 
Table J-01. Summary of environmental parameters for large-scale Experiments V-1 through V-4 with sheet vinyl flooring materials.

\begin{tabular}{|c|c|c|c|c|}
\hline \multirow[b]{2}{*}{ Parameter } & \multicolumn{4}{|c|}{ Experiment } \\
\hline & $V-1^{*}$ & $V-4$ & $V-2$ & $V-3$ \\
\hline Starting date & $08 / 07 / 97$ & $10 / 21 / 97$ & $08 / 14 / 97$ & $09 / 15 / 97$ \\
\hline Treatment & Base case & Base case & Add ventilation & Air out SV, CB \\
\hline \multicolumn{5}{|l|}{$\begin{array}{l}\text { Ventilation rate, } \\
2-72 h(h-1)\end{array}$} \\
\hline Average \pm 1 std. dev & $0.48 \pm 0.01 \dagger$ & $0.50 \pm 0.01 \dagger$ & $1.93 \pm 0.01 \ddagger$ & $0.49 \pm 0.01 t$ \\
\hline Range & $0.48-0.49$ & $0.49-0.51$ & $1.09-1.97$ & $0.48-0.49$ \\
\hline \multicolumn{5}{|l|}{$\begin{array}{c}\text { Ventilation rate, } \\
72-336 h(h-1)\end{array}$} \\
\hline Average \pm 1 std. dev. & $0.49 \pm 0.01$ & $0.50 \pm 0.01$ & $0.50 \pm 0.01$ & $0.48 \pm 0.01$ \\
\hline Range & $0.47-0.49$ & $0.49-0.50$ & $0.49-0.50$ & $0.48-0.49$ \\
\hline \multicolumn{5}{|l|}{ Temperature $\left({ }^{\circ} \mathrm{C}\right)$} \\
\hline Average \pm 1 std. dev & $23.5 \pm 1.0$ & $23.1 \pm 0.9$ & $24.1 \pm 0.9$ & $24.0 \pm 1.1$ \\
\hline Range & $21.7-26.8$ & $21.4-25.1$ & $22.0-26.2$ & $21.5-26.3$ \\
\hline \multicolumn{5}{|l|}{ Relative Humidity (\%) } \\
\hline Average \pm 1 std. dev & $55 \pm 2$ & $50 \pm 6$ & $56 \pm 3$ & $49 \pm 4$ \\
\hline Range & $52-61$ & $40-62$ & $49-61$ & $39-57$ \\
\hline
\end{tabular}

*Statistics were calculated for first 144 hours only.

tVentilation rate for $0-2$ hours was $2 h^{-1}$.

fVentilation rate for $0-2$ hours was $5 h^{-1}$. 
Table J-02. Sheet vinyl flooring materials and quantities used in large-scale Experiments V-1 through V-4. See Appendix E, Table E-01, for complete material descriptions.

\begin{tabular}{lcccccc}
\hline \hline & & \multicolumn{2}{c}{ Unit of } & \multicolumn{2}{c}{ Quantity } \\
Material Description & Material ID & Measure & Exp V-1 & Exp V-4 & Exp V-2 & Exp V-3 \\
\hline Particle board underlayment & $\mathrm{UL}$ & $\mathrm{m} 2$ & 10.4 & 10.4 & 10.4 & 10.4 \\
Residential sheet vinyl* & $\mathrm{SV}$ & $\mathrm{m} 2$ & 10.4 & 10.4 & 10.4 & 10.4 \\
Rubber cove base, 4" wide & $\mathrm{CB}$ & $\mathrm{m}$ & 12.5 & 12.5 & 12.5 & 12.5 \\
Sheet flooring adhesive & $\mathrm{SFA}$ & $\mathrm{kg}$ & 2.73 & 3.40 & 2.95 & 2.97 \\
Cove base adhesive & $\mathrm{CBA}$ & $\mathrm{kg}$ & 0.51 & 0.47 & 0.50 & 0.48 \\
Seam sealer & $\mathrm{SS}$ & $\mathrm{mL}$ & 15 & 15 & 15 & 15 \\
\hline
\end{tabular}

*Area of sheet vinyl was used in specific emission rate calculations. 
Table J-03. VOCs emitted by sheet vinyl flooring assembly in large-scale Experiment V-4 at 24- and 240-hours elapsed times.

\begin{tabular}{|c|c|c|c|c|c|}
\hline COMPOUND & Code $^{\star}$ & $\begin{array}{c}\mathrm{RT} \\
(\mathrm{min}) \\
\end{array}$ & $\begin{array}{c}24-h \\
\text { ET }\end{array}$ & $\begin{array}{c}240-h \\
E T\end{array}$ & $\begin{array}{l}\text { Match } \\
\text { Quality }\end{array}$ \\
\hline \multicolumn{6}{|l|}{ Alkane Hydrocarbons } \\
\hline $\mathrm{C} 7$ Branched alkane $\mathrm{HC}$ & & 9.4 & + & & Probable \\
\hline n-Octane & & 10.9 & + & + & Confirmed \\
\hline n-Nonane & & 15.2 & + & + & Confirmed \\
\hline C10 Branched alkane $\mathrm{HC}$ & & 16.6 & + & + & Probable \\
\hline C10 Branched alkane $\mathrm{HC}$ & & 17.6 & + & + & Probable \\
\hline C10 Branched alkane $\mathrm{HC}$ & & 17.7 & + & + & Probable \\
\hline C10 Branched alkane $\mathrm{HC}$ & & 18.1 & + & + & Probable \\
\hline n-Decane & $A, Q$ & 19.2 & + & + & Confirmed \\
\hline C11 Branched alkane $\mathrm{HC}$ & & 20.0 & + & + & Probable \\
\hline C11 Branched alkane $\mathrm{HC}$ & & 21.3 & + & + & Probable \\
\hline $\mathrm{C} 11$ Branched alkane $\mathrm{HC}$ & & 21.4 & + & + & Probable \\
\hline C11 Branched alkane $\mathrm{HC}$ & & 21.5 & + & + & Probable \\
\hline C11 Branched alkane $\mathrm{HC}$ & & 21.7 & + & + & Probable \\
\hline n-Undecane & & 22.8 & + & + & Confirmed \\
\hline n-Dodecane & $B, Q$ & 26.2 & + & + & Confirmed \\
\hline n-Tridecane & $A, B, Q$ & 29.3 & + & + & Confirmed \\
\hline $\mathrm{n}$-Tetradecane & $A, B, Q$ & 32.2 & + & + & Confirmed \\
\hline $\mathrm{n}$-Pentadecane & & 35.0 & + & + & Confirmed \\
\hline \multicolumn{6}{|l|}{ Aromatic Hydrocarbons } \\
\hline Toluene & $T, A, Q$ & 11.7 & + & + & Confirmed \\
\hline m-,p-Xylene & $\mathrm{T}, \mathrm{Q}$ & 16.1 & + & + & Confirmed \\
\hline o-Xylene & $\mathrm{T}$ & 17.3 & + & + & Confirmed \\
\hline Styrene & $T, Q$ & 17.6 & + & + & Confirmed \\
\hline Propylbenzene & & 19.5 & + & + & Confirmed \\
\hline Ethyltoluene isomer & & 19.7 & + & + & Probable \\
\hline 4-Ethyltoluene & & 19.8 & + & + & Confirmed \\
\hline C3 Alkylbenzene & & 20.0 & + & + & Probable \\
\hline 2-Ethyltoluene & & 20.6 & + & + & Confirmed \\
\hline $1,2,4$-Trimethylbenzene & $T, Q$ & 21.2 & + & + & Confirmed \\
\hline C4 Alkylbenzene & & 21.8 & + & + & Probable \\
\hline C4 Alkylbenzene & & 22.0 & + & + & Probable \\
\hline 1,2,3-Trimethylbenzene & & 22.4 & + & + & Confirmed \\
\hline 1,3-Diethylbenzene & & 22.9 & + & + & Confirmed \\
\hline C4 Alkylbenzene & & 23.0 & + & + & Probable \\
\hline C4 Alkylbenzene & & 23.1 & + & + & Probable \\
\hline C4 Alkylbenzene & & 23.2 & + & + & Probable \\
\hline C4 Alkylbenzene & & 23.7 & + & + & Probable \\
\hline C4 Alkylbenzene & & 24.0 & + & + & Probable \\
\hline C4 Alkylbenzene & & 24.1 & + & + & Probable \\
\hline C4 Alkylbenzene & & 24.3 & + & + & Probable \\
\hline C5 Alkylbenzene & & 24.6 & + & + & Probable \\
\hline Butenylbenzene isomer & & 24.6 & + & + & Probable \\
\hline $1,2,3,5$-Tetramethylbenzene & & 25.7 & + & + & Confirmed \\
\hline C6 Alkylbenzene & & 26.9 & + & & Probable \\
\hline
\end{tabular}


Table J-03, Continued. VOCs emitted by sheet vinyl flooring assembly in large-scale Experiment V-4 at 24- and 240-hours elapsed times.

\begin{tabular}{|c|c|c|c|c|c|}
\hline COMPOUND & Code $^{\star}$ & $\begin{array}{c}\mathrm{RT} \\
(\min )\end{array}$ & $\begin{array}{c}24-h \\
\text { ET }\end{array}$ & $\begin{array}{c}240-h \\
\text { ET }\end{array}$ & $\begin{array}{l}\text { Match } \\
\text { Quality }\end{array}$ \\
\hline $\begin{array}{l}\text { Aromatic Hydrocarbons, Cont. } \\
\text { C4 Alkylbenzene } \\
\text { C6 Alkylbenzene } \\
\text { Naphthalene } \\
\text { C2 Tetrahydronaphthalene } \\
\text { C2 Tetrahydronaphthalene } \\
\text { C2 Tetrahydronaphthalene } \\
\text { (1-Butylhexyl)benzene } \\
\text { (1-Propylheptyl)benzene } \\
\text { (1-Ethyloctyl)benzene } \\
\text { (1-Methylnonyl)benzene } \\
\text { (1-Phenylhexyl)benzene } \\
\text { (1-Butylheptyl)benzene } \\
\text { (1-Propyloctyl)benzene } \\
\text { (1-Ethylnonyl)benzene } \\
\text { (1-Methyldecyl)benzene } \\
\text { (1-Penylheptyl)benzene } \\
\text { (1-Butyloctyl)benzene } \\
\text { (1-Propylnonyl)benzene }\end{array}$ & $\mathrm{T}$ & $\begin{array}{l}27.0 \\
27.8 \\
29.5 \\
32.3 \\
32.5 \\
32.9 \\
37.1 \\
37.4 \\
37.9 \\
39.0 \\
39.4 \\
39.5 \\
39.8 \\
40.4 \\
41.5 \\
41.8 \\
41.9 \\
42.2\end{array}$ & $\begin{array}{l}+ \\
+ \\
+ \\
+ \\
+ \\
+ \\
+ \\
+ \\
+ \\
+ \\
+ \\
+ \\
+ \\
+ \\
+ \\
+ \\
+ \\
+\end{array}$ & $\begin{array}{l}+ \\
\\
+ \\
+ \\
+ \\
+ \\
+ \\
+ \\
+ \\
+ \\
+ \\
+ \\
+ \\
+ \\
+ \\
+ \\
+\end{array}$ & $\begin{array}{l}\text { Probable } \\
\text { Probable } \\
\text { Confirmed } \\
\text { Probable } \\
\text { Probable } \\
\text { Probable } \\
\text { Probable } \\
\text { Probable } \\
\text { Probable } \\
\text { Probable } \\
\text { Probable } \\
\text { Probable } \\
\text { Probable } \\
\text { Probable } \\
\text { Probable } \\
\text { Probable } \\
\text { Probable } \\
\text { Probable }\end{array}$ \\
\hline $\begin{array}{l}\text { Other Hydrocarbons } \\
\text { Dimethylcyclohexane isomer } \\
\text { Trimethylcyclohexane isomer } \\
\text { C3 Alkyl substituted cyclohexane } \\
\text { Propylcyclohexane } \\
\text { C10 Alkene or cyclic HC } \\
\text { Butylcyclohexane } \\
\text { C11 Alkene HC } \\
\text { Alkene HC } \\
\text { Alkene HC or cyclic HC } \\
\text { Alkene HC or cyclic HC } \\
\text { Alkene HC } \\
\text { Alkene HC } \\
\text { Alkene HC } \\
\text { Alkene HC }\end{array}$ & & $\begin{array}{l}10.3 \\
13.6 \\
15.1 \\
16.9 \\
18.8 \\
20.9 \\
21.0 \\
28.8 \\
28.9 \\
29.0 \\
29.4 \\
30.0 \\
30.3 \\
37.1\end{array}$ & $\begin{array}{l}+ \\
+ \\
+ \\
+ \\
+ \\
+ \\
+ \\
+ \\
+ \\
+ \\
+ \\
+ \\
+ \\
+\end{array}$ & $\begin{array}{l}+ \\
+ \\
+ \\
+ \\
+ \\
+ \\
+ \\
+ \\
+ \\
+ \\
+\end{array}$ & $\begin{array}{l}\text { Probable } \\
\text { Probable } \\
\text { Probable } \\
\text { Confirmed } \\
\text { Probable } \\
\text { Confirmed } \\
\text { Probable } \\
\text { Tentative } \\
\text { Tentative } \\
\text { Tentative } \\
\text { Tentative } \\
\text { Tentative } \\
\text { Tentative } \\
\text { Tentative }\end{array}$ \\
\hline $\begin{array}{l}\text { Carbonyl Compounds } \\
\text { Hexanal } \\
\text { Cyclohexanone } \\
\text { Benzaldehyde } \\
\text { Nonanal } \\
\text { 1-Phenylethanone }\end{array}$ & $\begin{array}{l}B \\
A, Q \\
B, Q \\
B \\
T\end{array}$ & $\begin{array}{l}14.5 \\
19.7 \\
22.5 \\
26.0 \\
26.5\end{array}$ & $\begin{array}{l}+ \\
+ \\
+ \\
+\end{array}$ & $\begin{array}{l}+ \\
+ \\
+ \\
+\end{array}$ & $\begin{array}{l}\text { Confirmed } \\
\text { Confirmed } \\
\text { Confirmed } \\
\text { Confirmed } \\
\text { Confirmed }\end{array}$ \\
\hline $\begin{array}{l}\text { Other Oxidized Compounds } \\
\text { Tetrahydrofuran } \\
\text { 1-Butanol }\end{array}$ & $A, B, Q$ & $\begin{array}{l}6.2 \\
9.9\end{array}$ & $\begin{array}{l}+ \\
+\end{array}$ & + & $\begin{array}{l}\text { Confirmed } \\
\text { Confirmed }\end{array}$ \\
\hline
\end{tabular}


Table J-03, Continued. VOCs emitted by sheet vinyl flooring assembly in large-scale Experiment V-4 at 24- and 240-hours elapsed times.

\begin{tabular}{|c|c|c|c|c|c|}
\hline COMPOUND & Code $^{*}$ & $\begin{array}{c}\mathbf{R T} \\
(\min ) \\
\end{array}$ & $\begin{array}{c}24-h \\
\text { ET }\end{array}$ & $\begin{array}{c}240-h \\
\text { ET }\end{array}$ & $\begin{array}{l}\text { Match } \\
\text { Quality } \\
\end{array}$ \\
\hline \multicolumn{6}{|l|}{ Other Oxidized Cmpds., Con't. } \\
\hline Acetic acid & B & 10.6 & + & + & Confirmed \\
\hline 1-Octanol & $Q$ & 25.4 & + & + & Confirmed \\
\hline Benzyl alcohol & $Q$ & 26.5 & + & + & Confirmed \\
\hline Phenol & $\mathrm{T}, \mathrm{A}, \mathrm{B}, \mathrm{Q}$ & 26.6 & + & + & Confirmed \\
\hline 2-Ethylhexanoic acid & & 28.4 & & + & Probable \\
\hline $\begin{array}{l}\text { 2,2,4-Trimethyl-1,3-pentanediol } \\
\text { monisobutyrate (Texanol 1) }\end{array}$ & $\mathrm{B}$ & 35.2 & + & + & Confirmed \\
\hline $\begin{array}{l}\text { 2,2,4-Trimethyl-1,3-pentanediol } \\
\text { monisobutyrate (Texanol 3) }\end{array}$ & B & 35.6 & + & + & Confirmed \\
\hline 1-Dodecanol & & 37.0 & & + & Confirmed \\
\hline $\begin{array}{l}\text { 2,2,4-Trimethyl-1,3-pentanediol } \\
\text { diisobutyrate (TXIB) }\end{array}$ & $A, B, Q$ & 40.3 & + & + & Confirmed \\
\hline \multicolumn{6}{|l|}{ Miscellaneous Compounds } \\
\hline tert-Butyl isothiocyanate & $Q$ & 16.6 & + & + & Confirmed \\
\hline Octamethylcyclotetrasiloxane & B & 18.2 & + & + & Confirmed \\
\hline Benzothiazole & $A, Q$ & 31.6 & + & + & Confirmed \\
\hline \multicolumn{6}{|l|}{ Unidentified Compounds } \\
\hline Unidentified compound & & 29.1 & + & + & Unident. \\
\hline
\end{tabular}

${ }^{\star} \mathrm{T}$ = Toxic air contaminant; $\mathrm{A}=$ Abundant compound; $\mathrm{B}=$ Component of chamber background;

$Q=$ Quantified target compound. 
Table J-04. Target compounds quantified in large-scale Experiments V-1 through V-4. Toxic Air Contaminant (TAC) Category for June 1996 is indicated where applicable.

\begin{tabular}{|c|c|c|c|c|}
\hline Compound & TAC Cat. & Group & $\begin{array}{c}\text { Table } \\
\text { Abbrev. }\end{array}$ & Source(s) \\
\hline \multicolumn{5}{|l|}{ Alkane Hydrocarbons } \\
\hline n-Decane & & 1 & $\mathrm{n}-\mathrm{C} 10$ & SV5,CBA \\
\hline n-Dodecane & & 1 & $\mathrm{n}-\mathrm{C} 12$ & SV5,CBA \\
\hline $\mathrm{n}$-Tridecane & & 1 & $\mathrm{n}-\mathrm{C} 13$ & SV5,CB \\
\hline n-Tetradecane & & 1 & $\mathrm{n}-\mathrm{C} 14$ & SV5 \\
\hline \multicolumn{5}{|l|}{ Aromatic Hydrocarbons } \\
\hline Toluene & 1 & 1 & & SV5,CB,SFA,CBA \\
\hline m-,p-Xylene & 1 & 1 & Xylenes & SV5,CBA \\
\hline Styrene & 1 & 1 & & $\mathrm{CB}, \mathrm{CBA}$ \\
\hline 1,2,4-Trimethylbenzene & III & 1 & $1,2,4-\mathrm{TMB}$ & SV5 \\
\hline \multicolumn{5}{|l|}{ Carbonyl Compounds } \\
\hline Formaldehyde & 1 & Ald & & No dominant src. \\
\hline Acetaldehyde & I & Ald & & No dominant src. \\
\hline Cyclohexanone & & 2 & C-hexone & CB,SS \\
\hline Benzaldehyde & & 2 & Benzald & SV5,CB,SFA \\
\hline \multicolumn{5}{|l|}{ Other Oxidized Cmpds. } \\
\hline Tetrahydrofuran & & 2 & THF & SS \\
\hline 1-Octanol & & 2 & Octanol & SV5 \\
\hline Benzyl alcohol & & 2 & Benz alc & SV5 \\
\hline Phenol & 1 & 2 & & SV5 \\
\hline $\begin{array}{l}\text { 2,2,4-Trimethyl-1,3- } \\
\text { pentanediol disobutyrate }\end{array}$ & & 2 & TXIB & SV5 \\
\hline \multicolumn{5}{|l|}{ Miscellaneous Cmpds. } \\
\hline tert-Butylisothiocyanate & & 2 & Butisothio & $\mathrm{CB}$ \\
\hline Benzothiazole & & 2 & Benzothiaz & $\mathrm{CB}$ \\
\hline
\end{tabular}


Table J-05. Concentrations of Group 1 target VOCs for large-scale Experiment V-1. Data subsequent to 144-hours elapsed time were invalid (see Report).

\begin{tabular}{|c|c|c|c|c|c|c|c|c|}
\hline Sample ID & $\mathrm{n}-\mathrm{C} 10$ & $\mathrm{n}-\mathrm{C} 12$ & $\mathrm{n}-\mathrm{C} 13$ & $\begin{array}{c}\text { Chamber Co } \\
n-C 14 \\
\end{array}$ & $\begin{array}{l}\text { ration, } \mu \mathrm{g} \text { r } \\
\text { Toluene } \\
\end{array}$ & Xylenes & Styrene & 1,2,4-TMB \\
\hline Avg. Inlet & $<1$ & $<1$ & $<1$ & $<1$ & 1 & $<1$ & $<1$ & $<1$ \\
\hline Background & $<1$ & $<1$ & $<1$ & $<1$ & 1 & $<1$ & $<1$ & $<1$ \\
\hline $1-h$ & 82 & 76 & 220 & 112 & 239 & 14 & 8 & 33 \\
\hline $3-h$ & 68 & 52 & 140 & 78 & 696 & 12 & 13 & 30 \\
\hline $6-h$ & 81 & 50 & 125 & 70 & 1,180 & 12 & 16 & 35 \\
\hline $24-h$ & 52 & 29 & 74 & 36 & 523 & 7 & 10 & 21 \\
\hline $43-\mathrm{h}$ & 40 & 23 & 60 & 30 & 143 & 5 & 7 & 16 \\
\hline $72 a-h$ & 39 & 23 & 63 & 30 & 130 & 5 & 6 & 17 \\
\hline$\cdots h$ & 37 & 24 & 65 & 30 & 134 & 4 & 6 & 17 \\
\hline $56-h$ & 35 & 24 & 65 & 31 & 107 & 5 & 5 & 16 \\
\hline $120-h$ & 36 & 25 & 66 & 32 & 108 & 5 & 5 & 17 \\
\hline $144-\mathrm{h}$ & 37 & 26 & 71 & 35 & 122 & 5 & 5 & 19 \\
\hline
\end{tabular}


Table J-06. Concentrations of Group 2 target VOCs for large-scale Experiment V-1. Data subsequent to 144 -hours elapsed time were invalid (see Report).

\begin{tabular}{|c|c|c|c|c|c|c|c|c|c|}
\hline Sample ID & C-hexone & Benzald & THF & $\begin{array}{c}\text { Chamb } \\
\text { Octanol } \\
\end{array}$ & $\begin{array}{c}\text { Concentrat } \\
\text { Benz alc }\end{array}$ & $\begin{array}{l}\mu \mathrm{g} \mathrm{m} \mathrm{m}^{-3} \\
\text { Phenol } \\
\end{array}$ & TXIB & Butisothio & Benzothiaz \\
\hline f.vg. Inlet & $<1$ & $<1$ & 4 & $<1$ & $<1$ & $<1$ & $<1$ & $<1$ & $<1$ \\
\hline Background & $<1$ & 1 & $<1$ & $<1$ & $<1$ & 1 & $<1$ & $<1$ & $<1$ \\
\hline $1-h$ & 3,760 & 16 & 7,020 & 12 & 29 & 108 & 8 & 6 & 25 \\
\hline $3-h$ & 1,080 & 15 & 1,850 & 13 & 30 & 97 & 31 & 8 & 28 \\
\hline $6-h$ & 853 & 15 & 1,370 & 13 & 29 & 93 & 41 & 10 & 30 \\
\hline 24-h & 137 & 11 & 196 & 10 & 24 & 70 & 28 & 6 & 29 \\
\hline 48-h & 53 & 9 & 57 & 8 & 23 & 69 & 26 & 5 & 28 \\
\hline $72 a-h$ & 39 & 9 & 49 & 8 & 23 & 72 & 28 & 3 & 28 \\
\hline $72 b-h$ & 39 & 9 & 38 & 8 & 22 & 75 & $m d^{*}$ & 3 & 29 \\
\hline $96-h$ & 31 & 9 & 35 & 8 & 26 & 75 & 31 & 3 & 29 \\
\hline $120-h$ & 29 & 9 & 43 & 8 & 25 & 74 & 32 & 3 & 27 \\
\hline $144-h$ & 29 & 10 & 44 & 10 & 26 & 79 & 38 & 3 & 29 \\
\hline
\end{tabular}

${ }^{*} \mathrm{md}=$ Missing data. 
Ta'Jle J-07. Concentrations of Group 1 target VOCs for large-scale Experiment V-4.

\begin{tabular}{|c|c|c|c|c|c|c|c|c|}
\hline Sample ID & $\mathrm{n}-\mathrm{C} 10$ & $\mathrm{n}-\mathrm{C} 12$ & $\mathrm{n}-\mathrm{C} 13$ & $\begin{array}{c}\text { Chamber Co } \\
\mathrm{n}-\mathrm{C} 14 \\
\end{array}$ & $\begin{array}{r}\text { ration, } \mu \mathrm{g} \\
\text { Toluene } \\
\end{array}$ & Xylenes & Styrene & 1,2,4-TMB \\
\hline Avg. Inlet & $<1$ & $<1$ & $<1$ & $<1$ & 1 & $<1$ & $<1$ & $<1$ \\
\hline Background & 1 & $<1$ & 1 & $<1$ & 1 & $<1$ & $<1$ & $<1$ \\
\hline $1-\mathrm{h}$ & 170 & 104 & 206 & 90 & 227 & 19 & 11 & 51 \\
\hline $3-h$ & 50 & 44 & 126 & 66 & 118 & 8 & 6 & 24 \\
\hline $6-h$ & 58 & 46 & 118 & 58 & 102 & 8 & 8 & 32 \\
\hline 24-h & 28 & 23 & 65 & 32 & 74 & 4 & 5 & 17 \\
\hline $48-h$ & 22 & 17 & 51 & 23 & 54 & 3 & 4 & 13 \\
\hline $72 a-h$ & 24 & 16 & 47 & 22 & 59 & 3 & 3 & 12 \\
\hline $72 b-h$ & 25 & 17 & 49 & 25 & 61 & 3 & 4 & 12 \\
\hline $96-h$ & 40 & 19 & 45 & 21 & 71 & 4 & 4 & 13 \\
\hline $120-h$ & 28 & 23 & 63 & 32 & 44 & 4 & 3 & 15 \\
\hline $144-h$ & 31 & 22 & 59 & 29 & 48 & 5 & 4 & 16 \\
\hline $168-h$ & 24 & 20 & 54 & 27 & 39 & 4 & 3 & 14 \\
\hline $192-h$ & 25 & 21 & 55 & 28 & 38 & 4 & 3 & 15 \\
\hline $216-h$ & 24 & 20 & 56 & 29 & 43 & 4 & 3 & 15 \\
\hline $240 a-h$ & 25 & 19 & 57 & 30 & 41 & 3 & 3 & 15 \\
\hline $240 b-h$ & 24 & 22 & 61 & 32 & 39 & 4 & 3 & 16 \\
\hline $336 a-h$ & 19 & 15 & 45 & 24 & 29 & 3 & 2 & 11 \\
\hline 336b-h & 18 & 16 & 48 & 26 & 33 & 3 & 2 & 11 \\
\hline
\end{tabular}


Table J-08. Concentrations of Group 2 target VOCs for large-scale Experiment V-4.

\begin{tabular}{|c|c|c|c|c|c|c|c|c|c|}
\hline Sample ID & C-hexone & Benzald & THF & \multicolumn{3}{|c|}{ Chamber Concentration, $\mu \mathrm{g} \mathrm{m}^{-3}$} & TXIB & Butisothio & Benzothiaz \\
\hline Avg. Inlet & $<1$ & $<1$ & 5 & $<1$ & $<1$ & $<1$ & $<1$ & $<1$ & $<1$ \\
\hline Background & $<1$ & 1 & 1 & $<1$ & $<1$ & 1 & 1 & $<1$ & $<1$ \\
\hline $1-\mathrm{h}$ & 3,640 & 18 & 7,770 & 12 & 26 & 123 & 4 & 9 & 19 \\
\hline $3-h$ & 940 & 12 & 1,840 & 8 & 24 & 114 & 18 & 8 & 24 \\
\hline $6-h$ & 530 & 16 & 680 & 10 & 24 & 108 & 22 & 12 & 24 \\
\hline $24-\mathrm{h}$ & 97 & 10 & 94 & 8 & 20 & 84 & 27 & 6 & 24 \\
\hline 48-h & 45 & 8 & 41 & 6 & 18 & 77 & 21 & 4 & 23 \\
\hline $72 a-h$ & 28 & 8 & 38 & 5 & 16 & 73 & 25 & 3 & 23 \\
\hline $72 b-h$ & 32 & 8 & 40 & 6 & 17 & 78 & $\mathrm{md}^{*}$ & 3 & 24 \\
\hline $96-h$ & 24 & 6 & 54 & 5 & 14 & 60 & 20 & 3 & 18 \\
\hline $120-\mathrm{h}$ & 25 & 10 & 30 & 8 & 23 & 91 & 38 & 3 & 27 \\
\hline 144-h & 24 & 9 & 28 & 8 & 22 & 83 & 32 & 3 & 25 \\
\hline 168-h & 18 & 8 & 21 & 7 & 20 & 76 & 29 & 3 & 22 \\
\hline 19. & 18 & 8 & 23 & 8 & 21 & 76 & 28 & 2 & 21 \\
\hline$\cdots, h$ & 16 & 8 & 20 & 7 & 19 & 71 & 35 & 2 & 19 \\
\hline $240 \mathrm{a}-\mathrm{h}$ & 15 & 9 & 16 & 7 & 19 & 77 & 37 & 2 & 19 \\
\hline $240 \mathrm{~b}-\mathrm{h}$ & 15 & 8 & 20 & 8 & 22 & 79 & 34 & 2 & 20 \\
\hline $336 a-h$ & 9 & 6 & 7 & 5 & 13 & 62 & 32 & 2 & 15 \\
\hline $336 \mathrm{~b}-\mathrm{h}$ & 9 & 5 & 9 & 5 & 16 & 68 & 31 & 1 & 15 \\
\hline
\end{tabular}

${ }^{\star} \mathrm{md}=$ Missing data. 
Table J-09. Concentrations of Group 1 target VOCs for large-scale Experiment V-2.

\begin{tabular}{|c|c|c|c|c|c|c|c|c|}
\hline Sample ID & $\mathrm{n}-\mathrm{C} 10$ & $\mathrm{n}-\mathrm{C} 12$ & $\mathrm{n}-\mathrm{C} 13$ & $\begin{array}{r}\text { Chamber Co } \\
\mathrm{n}-\mathrm{C} 14 \\
\end{array}$ & $\begin{array}{r}\text { ntration, } \mu \mathrm{g} \\
\text { Toluene } \\
\end{array}$ & Xylenes & Styrene & 1,2,4-TMB \\
\hline Avg. Inlet & $<1$ & $<1$ & $<1$ & $<1$ & 1 & $<1$ & $<1$ & $<1$ \\
\hline Background & $<1$ & $<1$ & 1 & $<1$ & 2 & $<1$ & $<1$ & $<1$ \\
\hline $1-h$ & 16 & 18 & 85 & 53 & 20 & 4 & 2 & 10 \\
\hline 3-h & 23 & 18 & 60 & 35 & 42 & 4 & 3 & 12 \\
\hline $6-h$ & 23 & 20 & 61 & 34 & 29 & 3 & 3 & 14 \\
\hline 24-h & 10 & 9 & 30 & 15 & 12 & 1 & 2 & 6 \\
\hline$\therefore h$ & 12 & 11 & 37 & 20 & 15 & 2 & 2 & 7 \\
\hline$" 2 a-h$ & 13 & 10 & 36 & 18 & 15 & 2 & 2 & 7 \\
\hline $72 b-h$ & 11 & 9 & 31 & 17 & 16 & 2 & 2 & 7 \\
\hline $96-h$ & 26 & 20 & 51 & 25 & 38 & 3 & 4 & 14 \\
\hline $120-h$ & 28 & 20 & 53 & 26 & 42 & 5 & 4 & 15 \\
\hline $144 a-h$ & 24 & 20 & 54 & 27 & 38 & 4 & 4 & 15 \\
\hline $144 b-h$ & 26 & 21 & 54 & 26 & 36 & 4 & 4 & 15 \\
\hline $168-h$ & 24 & 18 & 51 & 26 & 36 & 4 & 4 & 14 \\
\hline $192-h$ & 24 & 20 & 57 & 30 & 37 & 4 & 4 & 14 \\
\hline 216-h & 24 & 19 & 55 & 27 & 32 & 4 & 3 & 15 \\
\hline $240-\mathrm{h}$ & 26 & 19 & 54 & 29 & 34 & 3 & 3 & 15 \\
\hline $336 \mathrm{a}-\mathrm{h}$ & 25 & 20 & 54 & 30 & 31 & 3 & 3 & 14 \\
\hline $336 b-h$ & 25 & 18 & 49 & 27 & 31 & 3 & 3 & 14 \\
\hline
\end{tabular}


Table J-10. Concentrations of Group 2 target VoCs for large-scale Experiment V-2.

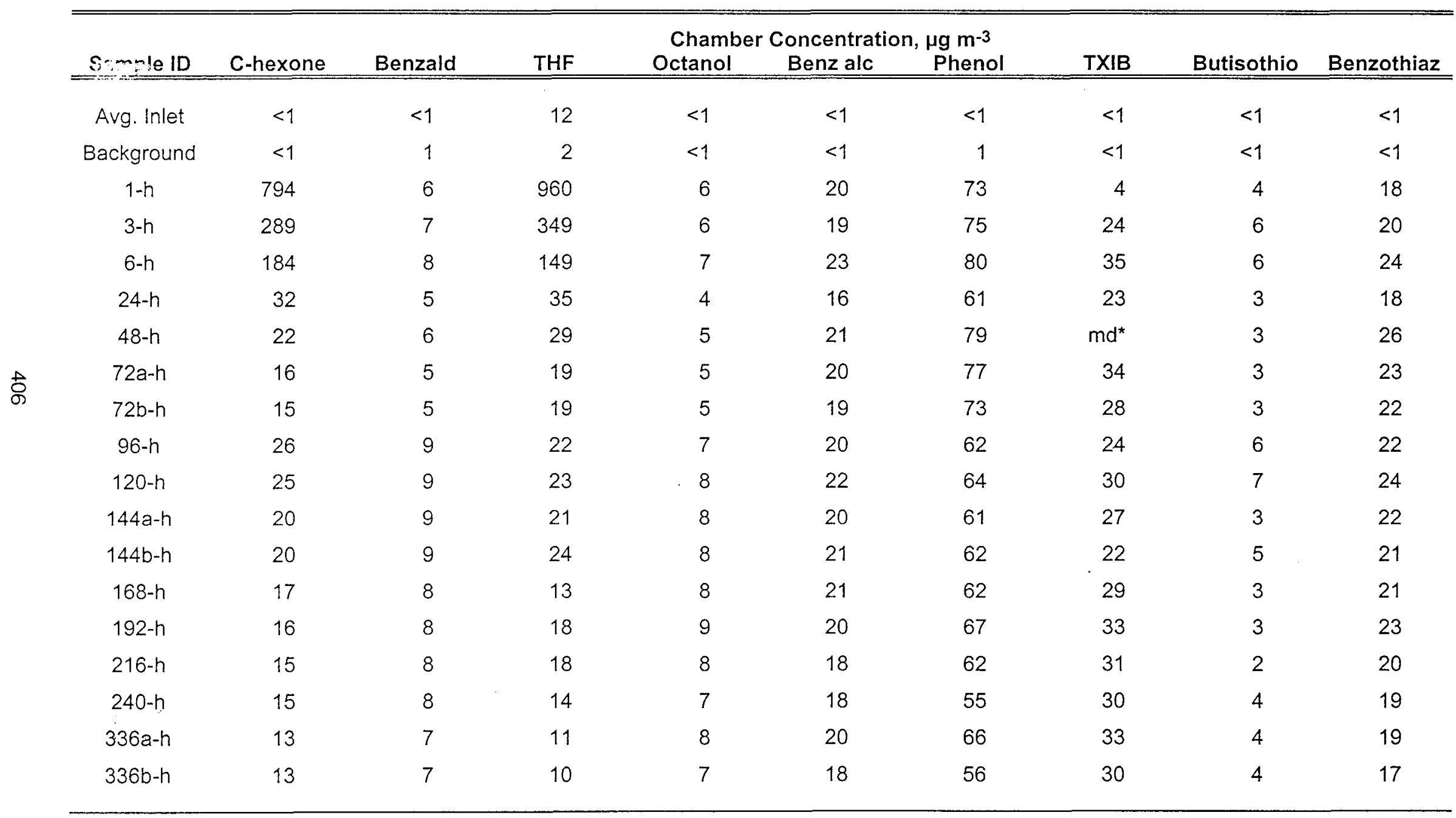

${ }^{*} \mathrm{md}=$ Missing data. 
Table J-11. Concentrations of Group 1 target VOCs for large-scale Experiment V-3.

\begin{tabular}{|c|c|c|c|c|c|c|c|c|}
\hline Sample ID & $\mathrm{n}-\mathrm{C} 10$ & $\mathrm{n}-\mathrm{C} 12$ & $\mathrm{n}-\mathrm{C} 13$ & $\begin{array}{c}\text { Chamber Co } \\
n-C 14\end{array}$ & $\begin{array}{l}\text { ation, } \mu \mathrm{g} \\
\text { Toluene }\end{array}$ & Xylenes & Styrene & 1,2,4-TMB \\
\hline Avg. Inlet & $<1$ & $<1$ & $<1$ & $<1$ & 1 & $<1$ & $<1$ & $<1$ \\
\hline Background & $<1$ & $<1$ & 1 & $<1$ & 1 & $<1$ & $<1$ & $<1$ \\
\hline $1-\mathrm{h}$ & 84 & 75 & 173 & 76 & 165 & 10 & 4 & 27 \\
\hline $3-h$ & 59 & 56 & 173 & 82 & 108 & 10 & 7 & 29 \\
\hline $6-h$ & 42 & 32 & 94 & 45 & 69 & 7 & 6 & 21 \\
\hline $24-h$ & 25 & 16 & 49 & 22 & 43 & 4 & 4 & 13 \\
\hline 48-h & 27 & 20 & 57 & 27 & 39 & 4 & 3 & 15 \\
\hline $72 a-h$ & 25 & 19 & 52 & 25 & 31 & 3 & 2 & 14 \\
\hline $72 b-h$ & 26 & 19 & 56 & 28 & 36 & 3 & 3 & 15 \\
\hline 96-h & 21 & 16 & 51 & 24 & 32 & 3 & 2 & 12 \\
\hline $120-h$ & 23 & 17 & 52 & 27 & 32 & 3 & 2 & 13 \\
\hline $144 a-h$ & 25 & 20 & 58 & 29 & 35 & 3 & 3 & 14 \\
\hline 144b-h & 25 & 18 & 53 & 26 & 33 & 4 & 3 & 14 \\
\hline $168-h$ & 25 & 18 & 53 & 27 & 31 & 4 & 3 & 14 \\
\hline $192-h$ & 24 & 20 & 58 & 29 & 30 & 4 & 3 & 14 \\
\hline $216-h$ & 29 & 21 & 61 & 31 & 36 & 5 & 3 & 16 \\
\hline$\therefore 9 a-h$ & 25 & 20 & 61 & 33 & 38 & 4 & 3 & 15 \\
\hline$\because b-h$ & 27 & 22 & 65 & 35 & 41 & 4 & 3 & 17 \\
\hline $336-h$ & 22 & 18 & 57 & 30 & 33 & 3 & 2 & 14 \\
\hline
\end{tabular}


Table J-12. Concentrations of Group 2 target VOCs for large-scale Experiment V-3.

\begin{tabular}{|c|c|c|c|c|c|c|c|c|c|}
\hline \multirow{2}{*}{ Sample ID } & \multirow{2}{*}{$\frac{\text { C-hexone }}{<1}$} & \multirow{2}{*}{$\underbrace{\text { Benzald }}_{<1}$} & \multirow{2}{*}{$\frac{\text { THF }}{4}$} & \multicolumn{3}{|c|}{$\begin{array}{l}\text { Chamber Concentration, } \mu \mathrm{g} \mathrm{m}^{-3} \\
\text { Octanol Benzalc Phenol }\end{array}$} & TXIB & Butisothio & Benzothiaz \\
\hline & & & & $<1$ & $<1$ & $<1$ & $<1$ & $<1$ & $<1$ \\
\hline Background & $<1$ & 1 & 2 & $<1$ & $<1$ & 4 & 5 & $<1$ & 1 \\
\hline $1-h$ & 2,620 & 10 & 6,450 & 6 & 10 & 41 & 4 & 2 & 10 \\
\hline $3-h$ & 863 & 13 & 2,140 & 7 & 20 & 72 & 20 & 7 & 16 \\
\hline $6-h$ & 598 & 11 & 1,230 & 7 & 13 & 48 & 20 & 7 & 11 \\
\hline $24-h$ & 88 & 8 & 130 & 6 & 13 & 43 & 18 & 6 & 13 \\
\hline 48-h & 46 & 9 & 72 & 7 & 18 & 59 & 24 & 5 & 18 \\
\hline $72 a-h$ & 29 & 9 & 53 & 7 & 19 & 59 & 21 & 5 & 16 \\
\hline $72 \mathrm{~h} h$ & 32 & 8 & 37 & 7 & 18 & 61 & $\mathrm{md}^{*}$ & 5 & 18 \\
\hline $\sin$ & 21 & 7 & 33 & 6 & 16 & 59 & 22 & 4 & 17 \\
\hline $2 \pi \cdot n$ & 20 & 7 & 32 & 7 & 18 & 63 & 22 & 4 & 18 \\
\hline $144 a-h$ & 18 & 8 & 28 & 8 & 20 & 65 & 31 & 4 & 18 \\
\hline $144 b-h$ & 19 & 7 & 30 & 7 & 19 & 59 & 29 & 4 & 17 \\
\hline $168-h$ & 18 & 8 & 29 & 7 & 18 & 63 & 29 & 4 & 17 \\
\hline $192-h$ & 16 & 8 & 25 & 7 & 19 & 66 & 31 & 3 & 18 \\
\hline $216-h$ & 17 & 9 & 25 & 8 & 18 & 68 & 37 & 4 & 17 \\
\hline $240 a-h$ & 15 & 7 & 24 & 7 & 19 & 65 & 37 & 3 & 16 \\
\hline $240 b-h$ & 16 & 9 & 27 & 8 & 19 & 70 & 40 & 3 & 18 \\
\hline $336-h$ & 11 & 7 & 17 & 6 & 14 & 61 & 31 & 2 & 14 \\
\hline
\end{tabular}

${ }^{*} \mathrm{md}=$ Missing data. 
Table J-13. Concentrations of TVOC for large-scale Experiments V-1 through V-4.

\begin{tabular}{|c|c|c|c|c|}
\hline Sample ID & \multicolumn{4}{|c|}{ Chamber Concentration, $\mu \mathrm{g} \mathrm{m}^{-3}$} \\
\hline Avg. Inlet ${ }^{\star \star}$ & $97 \pm 28$ & $31 \pm 10$ & $82 \pm 41$ & $35 \pm 12$ \\
\hline Background & 168 & 81 & 174 & 98 \\
\hline $1-h$ & 7,620 & 8,840 & 2,140 & 6,260 \\
\hline $3-h$ & 4,090 & 2,920 & 1,890 & 2,330 \\
\hline $6-h$ & 4,530 & 2,260 & 1,350 & 2,090 \\
\hline 24-h & 2,040 & 1,220 & 1,060 & 1,070 \\
\hline 48-h & 1,610 & 967 & 924 & 1,080 \\
\hline $72 a-h$ & 1,410 & 942 & 990 & 931 \\
\hline $72 b-h$ & 1,270 & 980 & 1,150 & 958 \\
\hline $96-h$ & 1,330 & 1,180 & 963 & 931 \\
\hline $120-h$ & 1,440 & 1,170 & 1,180 & 931 \\
\hline $144 a-h$ & 1,490 & 1,150 & 1,050 & 1,010 \\
\hline $144 b-h$ & --- & --- & 1,030 & 980 \\
\hline $168-h$ & -.- & 1,030 & 990 & 1,000 \\
\hline $192-h$ & --- & 997 & 960 & 1,010 \\
\hline 216-h & -.- & 1,070 & 1,000 & 1,140 \\
\hline $240 a-h$ & --- & 1,050 & 1,060 & 1,120 \\
\hline $240 b-h$ & --- & 1,050 & --- & 1,200 \\
\hline 336a-h & -.- & 850 & 1,140 & 1,000 \\
\hline 336b-h & --- & 870 & 1,060 & --- \\
\hline
\end{tabular}

*Data subsequent to 144-h elapsed time were invalid.

${ }^{* *}$ Average \pm 1 standard deviation. 
Table J-14. Concentrations of SigmaVOC (i.e., sum of 17 target VOCs) for large-scale Experiments $V-1$ through $V-4$.

\begin{tabular}{|c|c|c|c|c|}
\hline Sample ID & Exp V-1* & $\begin{array}{r}\text { Chamber Co } \\
\text { Exp V-4 } \\
\end{array}$ & $\begin{array}{r}\text { ation, } \mu g \\
\text { Exp V-2 } \\
\end{array}$ & Exp V-3 \\
\hline Avg. Inlet & $<17$ & $<17$ & $<17$ & $<17$ \\
\hline Background & $<17$ & $<17$ & $<17$ & $<17$ \\
\hline $1-h$ & 11,800 & 12,500 & 2,090 & 9,770 \\
\hline 3-h & 4,250 & 3,440 & 994 & 3,680 \\
\hline $6-h$ & 4,040 & 1,860 & 702 & 2,270 \\
\hline 24-h & 1,270 & 622 & 282 & 502 \\
\hline $48-h$ & 609 & 433 & 298 & 451 \\
\hline $72 a-h$ & 578 & 408 & 304 & 392 \\
\hline $72 b-h$ & 547 & 408 & 284 & 373 \\
\hline $96-h$ & 541 & 425 & 378 & 350 \\
\hline $120-h$ & 551 & 469 & 405 & 364 \\
\hline $144 a-h$ & 595 & 453 & 378 & 391 \\
\hline $144 b-h$ & -- & --- & 381 & 370 \\
\hline $168-h$ & --- & 390 & 362 & 373 \\
\hline $192-h$ & $\cdots$ & 396 & 386 & 376 \\
\hline $216-h$ & $\cdots$ & 395 & 362 & 409 \\
\hline $240 a-h$ & --- & 396 & 355 & 397 \\
\hline $240 b-h$ & --- & 411 & -- & 429 \\
\hline $336 a-h$ & --- & 301 & 362 & 346 \\
\hline $336 b-h$ & -- & 321 & 334 & +- \\
\hline
\end{tabular}

*Data subsequent to $144-\mathrm{h}$ elapsed time were invalid. 
Table J-15. Concentrations of formaldehyde for large-scale Experiments $V-1$ through $V-4$.

\begin{tabular}{|c|c|c|c|c|}
\hline Sample ID & Exp V-1* & \multicolumn{2}{|c|}{ Chamber Concentration, $\mu \mathrm{g} \mathrm{m}^{-3}$} & Exp V-3 \\
\hline Avg. Inlet ${ }^{\star \star}$ & $3.9 \pm 0.9$ & $2.1 \pm 0.5$ & $4.6 \pm 1.8$ & $4.1 \pm 2.6$ \\
\hline Background & 9.3 & 5.3 & 4.1 & 7.0 \\
\hline $1-\mathrm{h}$ & 14.3 & 10.1 & 5.0 & 10.5 \\
\hline $3-h$ & 19.5 & 12.0 & 7.8 & 18.5 \\
\hline $6-h$ & 21.3 & 11.4 & 7.4 & 15.1 \\
\hline 24-h & 12.2 & 6.4 & 4.4 & 9.7 \\
\hline $48-h$ & 11.6 & 5.8 & 4.4 & 7.2 \\
\hline $72-h$ & 11.2 & 5.6 & 4.5 & 10.8 \\
\hline $96-\mathrm{h}$ & 10.2 & 6.4 & 7.6 & 10.5 \\
\hline $120-h$ & 10.0 & 7.7 & 9.5 & 9.8 \\
\hline $144-h$ & 9.0 & 5.9 & 10.7 & 10.0 \\
\hline $168-h$ & $-\cdots$ & 4.5 & 10.5 & 10.4 \\
\hline $192-h$ & --- & 7.2 & 11.4 & 11.4 \\
\hline $216-h$ & $\cdots$ & 8.5 & 13.8 & 12.6 \\
\hline $240-h$ & --- & 6.1 & 16.5 & 12.4 \\
\hline $336-h$ & --- & 6.7 & 12.2 & 13.6 \\
\hline
\end{tabular}

*Data subsequent to $144-\mathrm{h}$ elapsed time were invalid.

${ }^{\star *}$ Average \pm 1 standard deviation. 
APPENDIX J - VINYL FLOORING ASSEMBLY

Table J-16. Concentrations of acetaldehyde for large-scale Experiments V-1 through V-4.

\begin{tabular}{|c|c|c|c|c|}
\hline Sample ID & $\operatorname{Exp} V-1^{*}$ & $\begin{array}{r}\text { Chamber Co } \\
\text { Exp V-4 } \\
\end{array}$ & $\begin{array}{r}\text { ration, } \mu \mathrm{g} \\
\text { Exp V-2 } \\
\end{array}$ & Exp V-3 \\
\hline Avg. Inlet** & $6.7 \pm 1.0$ & $3.0 \pm 0.7$ & $3.6 \pm 1.2$ & $3.7 \pm 1.5$ \\
\hline Background & 9.5 & 6.2 & 7.1 & 6.3 \\
\hline $1-h$ & 10.9 & 6.0 & 3.1 & 5.9 \\
\hline $3-h$ & 15.9 & 8.0 & 7.3 & 9.1 \\
\hline $6-h$ & 22.5 & 10.7 & 7.6 & 10.5 \\
\hline 24-h & 16.5 & 9.7 & 5.1 & 8.1 \\
\hline 48-h & 15.3 & 7.3 & 4.7 & 8.7 \\
\hline $72-h$ & 13.9 & 6.9 & 4.9 & 9.3 \\
\hline $96-h$ & 12.9 & 7.4 & 11.0 & 10.7 \\
\hline $120-h$ & 11.9 & 8.5 & 8.6 & 10.4 \\
\hline $144-h$ & 11.2 & 8.1 & 8.7 & 9.7 \\
\hline 168-h & --- & 6.7 & 9.4 & 10.5 \\
\hline $192-h$ & --- & 8.2 & 8.3 & 10.9 \\
\hline $216-h$ & --- & 9.5 & 8.5 & 12.0 \\
\hline $240-h$ & --- & 6.1 & 10.9 & 10.2 \\
\hline $336-h$ & -- & 7.3 & 7.7 & 11.0 \\
\hline
\end{tabular}

*Data subsequent to 144-h elapsed time were invalid.

${ }^{\star *}$ Average \pm 1 standard deviation. 
Table J-17. Quasi steady-state specific emission rates of Group 1 target VOCs for large-scale Experiment V-1. Data subsequent to 144-hours elapsed time were invalid (see Report).

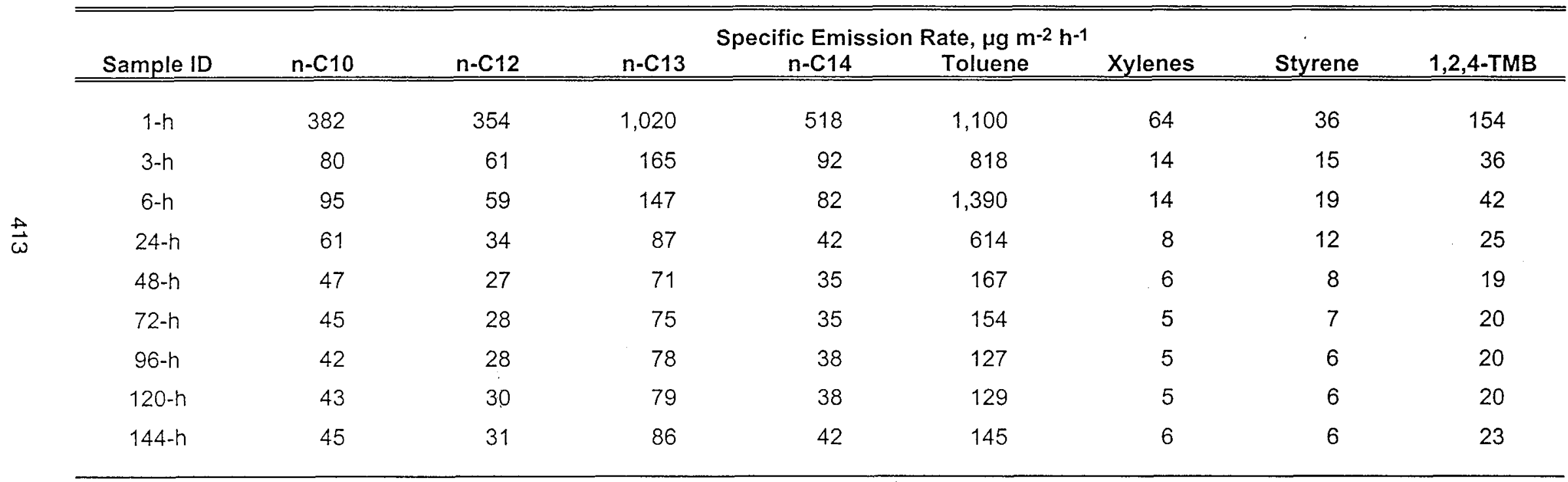


Table J-18. Quasi steady-state specific emission rates of Group 2 target VOCs for large-scale Experiment V-1. Data subsequent to 144-hours elapsed time were invalid (see Report).

\begin{tabular}{|c|c|c|c|c|c|c|c|c|c|}
\hline Sample ID & C-hexone & Benzald & THF & $\begin{array}{l}\text { Specific } \\
\text { Octanol } \\
\end{array}$ & $\begin{array}{c}\text { ission Rat } \\
\text { Benz alc } \\
\end{array}$ & $\begin{array}{r}g_{m^{-2}} \mathrm{~h}^{-1} \\
\text { Phenol } \\
\end{array}$ & TXIB & Butisothio & Benzothiaz \\
\hline $1-\mathrm{h}$ & 17,400 & 73 & 32,500 & 55 & 136 & 500 & 36 & 27 & 118 \\
\hline $3-h$ & 1,270 & 17 & 2,160 & 15 & 36 & 114 & 37 & 9 & 33 \\
\hline $6-\mathrm{h}$ & 1,000 & 18 & 1,600 & 15 & 34 & 109 & 48 & 12 & 35 \\
\hline 24-h & 162 & 13 & 227 & 12 & 28 & 83 & 33 & 7 & 34 \\
\hline $48-h$ & 62 & 11 & 63 & 9 & 27 & 82 & 31 & 5 & 32 \\
\hline $72-\mathrm{h}$ & 46 & 11 & 48 & 9 & 26 & 86 & 32 & 4 & 34 \\
\hline 96-h & 38 & 11 & 40 & 10 & 31 & 90 & 37 & 4 & 35 \\
\hline $120-h$ & 35 & 11 & 47 & 10 & 30 & 89 & 38 & 4 & 32 \\
\hline $144-h$ & 35 & 13 & 49 & 12 & 31 & 95 & 46 & 4 & 35 \\
\hline
\end{tabular}


Table J-19. Quasi steady-state specific emission rates of Group 1 target VOCs for large-scale Experiment V-4.

\begin{tabular}{|c|c|c|c|c|c|c|c|c|}
\hline Sample ID & $\mathrm{n}-\mathrm{C} 10$ & $\mathrm{n}-\mathrm{C} 12$ & $\mathrm{n}-\mathrm{C} 13$ & $\begin{array}{c}\begin{array}{c}\text { Specific Emis } \\
n-C 14\end{array} \\
\end{array}$ & $\begin{array}{l}\text { ate, } \mu \mathrm{g} \mathrm{m} \\
\text { Toluene }\end{array}$ & Xylenes & Styrene & 1,2,4-TMB \\
\hline $1-\mathrm{h}$ & 823 & 501 & 993 & 433 & 1,090 & 93 & 51 & 246 \\
\hline $3-h$ & 61 & 54 & 154 & 81 & 143 & 10 & 7 & 29 \\
\hline $6-h$ & 71 & 56 & 145 & 71 & 124 & 10 & 10 & 39 \\
\hline 24-h & 34 & 28 & 80 & 39 & 89 & 5 & 6 & 21 \\
\hline$\therefore \mathrm{h}$ & 27 & 21 & 63 & 28 & 65 & 4 & 5 & 16 \\
\hline$\because h$ & 30 & 20 & 59 & 28 & 73 & 4 & 4 & 14 \\
\hline$\cdots h$ & 50 & 23 & 56 & 25 & 86 & 5 & 5 & 16 \\
\hline $120-h$ & 34 & 28 & 78 & 39 & 53 & 5 & 4 & 18 \\
\hline $144-h$ & 38 & 26 & 73 & 36 & 58 & 6 & 5 & 19 \\
\hline $168-h$ & 29 & 24 & 66 & 34 & 46 & 5 & 4 & 17 \\
\hline $192-h$ & 31 & 25 & 67 & 35 & 46 & 4 & 4 & 18 \\
\hline $216-h$ & 29 & 25 & 69 & 36 & 51 & 5 & 3 & 19 \\
\hline $240-h$ & 30 & 25 & 72 & 38 & 48 & 4 & 3 & 19 \\
\hline $336-h$ & 23 & 19 & 57 & 31 & 37 & 3 & 2 & 14 \\
\hline
\end{tabular}


Tah!o j-20. Quasi steady-state specific emission rates of Group 2 target VOCs for large-scale Experiment V-4.

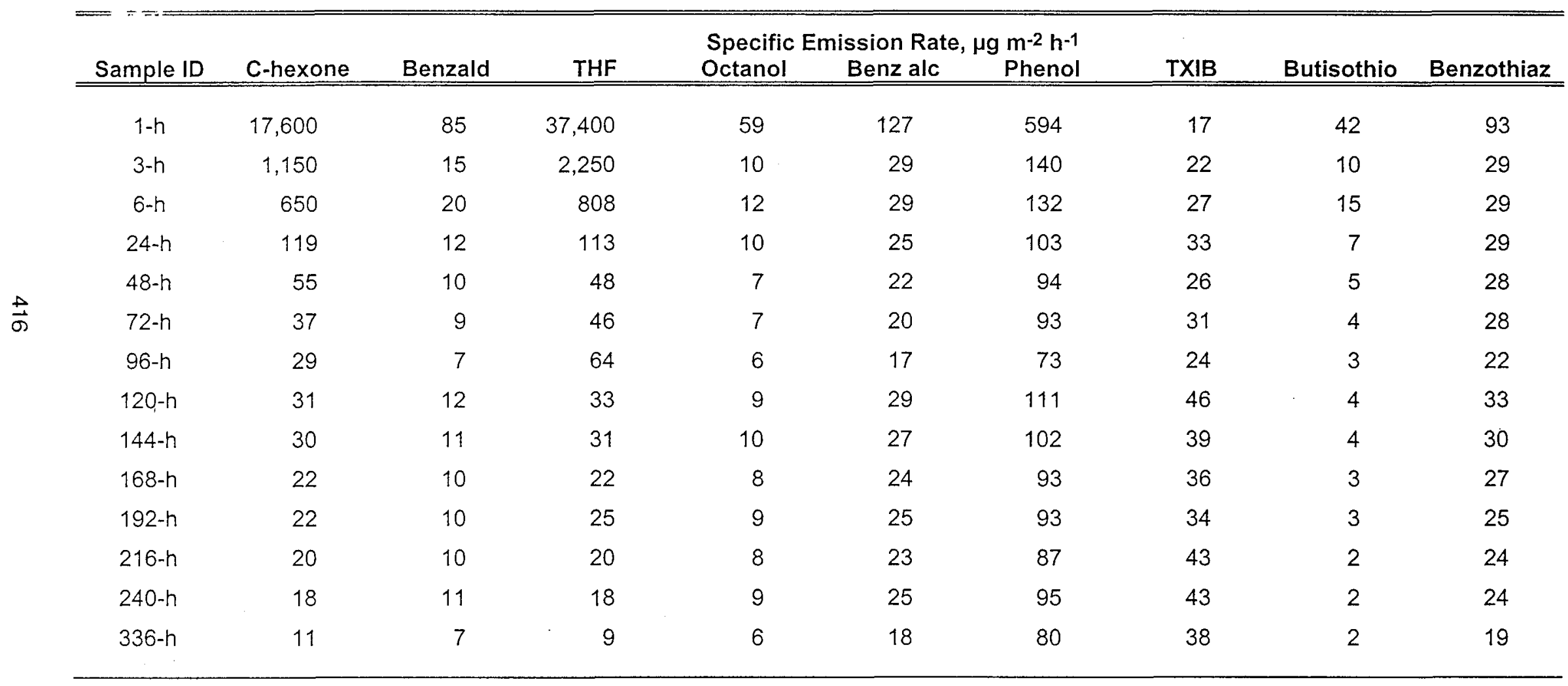


Table J-21. Quasi steady-state specific emission rates of Group 1 target VOCs for large-scale Experiment V-2.

\begin{tabular}{|c|c|c|c|c|c|c|c|c|}
\hline Sample ID & $\mathrm{n}-\mathrm{C} 10$ & $\mathrm{n}-\mathrm{C} 12$ & $\mathrm{n}-\mathrm{C} 13$ & $\begin{array}{c}\text { Specific Emis } \\
n-C_{14}\end{array}$ & $\begin{array}{c}\text { Rate, } \mu \mathrm{g} \mathrm{m} \\
\text { Toluene }\end{array}$ & Xylenes & Styrene & 1,2,4-TMB \\
\hline $1-\mathrm{h}$ & 192 & 216 & 1,010 & 623 & 228 & 48 & 24 & 120 \\
\hline 3-h & 110 & 86 & 282 & 167 & 196 & 19 & 14 & 57 \\
\hline $6-h$ & 108 & 96 & 287 & 159 & 135 & 16 & 16 & 68 \\
\hline 24-h & 48 & 41 & 140 & 73 & 53 & 6 & 10 & 29 \\
\hline 48-h & 57 & 51 & 175 & 92 & 69 & 10 & 10 & 35 \\
\hline $72-h$ & 57 & 46 & 158 & 82 & 68 & 9 & 9 & 35 \\
\hline $96-h$ & 31 & 24 & 63 & 31 & 45 & 4 & 5 & 17 \\
\hline $12.0-h$ & 34 & 25 & 64 & 32 & 51 & 6 & 5 & 19 \\
\hline 144-h & 31 & 24 & 66 & 32 & 44 & 5 & 5 & 19 \\
\hline $168-h$ & 30 & 22 & 63 & 32 & 43 & 5 & 4 & 17 \\
\hline $192-h$ & 29 & 25 & 70 & 37 & 44 & 4 & 4 & 17 \\
\hline $216-h$ & 29 & 24 & 67 & 33 & 38 & 4 & 4 & 18 \\
\hline $240-h$ & 32 & 24 & 66 & 35 & 41 & 4 & 4 & 18 \\
\hline $336-h$ & 31 & 24 & 63 & 35 & 37 & 4 & 3 & 17 \\
\hline
\end{tabular}


Table J-22. Quasi steady-state specific emission rates of Group 2 target VOCs for large-scale Experiment V-2.

\begin{tabular}{|c|c|c|c|c|c|c|c|c|c|}
\hline Sample ID & C-hexone & \multicolumn{7}{|c|}{ Specific Emission Rate, $\mu \mathrm{g} \mathrm{m}^{-2} \mathrm{~h}^{-1}$} & Benzothiaz \\
\hline $1-h$ & 9,420 & 72 & 10,400 & 72 & 240 & 863 & 48 & 24 & 216 \\
\hline $3-h$ & 1,370 & 33 & 1,270 & 29 & 91 & 354 & 115 & 14 & 96 \\
\hline $6-h$ & 868 & 40 & 326 & 32 & 108 & 378 & 163 & 16 & 112 \\
\hline $24-h$ & 150 & 25 & 142 & 19 & 76 & 287 & 108 & 6 & 86 \\
\hline $48-h$ & 105 & 29 & 118 & 22 & 99 & 373 & 134 & 10 & 124 \\
\hline $72-h$ & 74 & 25 & 76 & 22 & 91 & 355 & 147 & 6 & 106 \\
\hline $96-h$ & 31 & 11 & 24 & 8 & 25 & 76 & 30 & 4 & 26 \\
\hline ר' & 31 & 11 & 24 & 10 & 27 & 79 & 37 & 4 & 29 \\
\hline$\therefore 1$ & 25 & 11 & 19 & 10 & 24 & 75 & 30 & 3 & 26 \\
\hline .1 & 21 & 10 & 10 & 9 & 26 & 76 & 36 & 3 & 25 \\
\hline $192-h$ & 19 & 10 & 16 & 11 & 25 & 82 & 40 & 3 & 28 \\
\hline $216-h$ & 18 & 10 & 12 & 9 & 22 & 76 & 38 & 2 & 25 \\
\hline $240-h$ & 18 & 10 & 14 & 9 & 22 & 67 & 37 & 2 & 23 \\
\hline $336-h$ & 16 & 9 & 7 & 9 & 23 & 75 & 39 & 2 & 22 \\
\hline
\end{tabular}


Table J-23. Quasi steady-state specific emission rates of Group 1 target VOCs for large-scale Experiment V-3.

\begin{tabular}{|c|c|c|c|c|c|c|c|c|}
\hline Samnle ID & $\mathrm{n}-\mathrm{C} 10$ & $\mathrm{n}-\mathrm{C} 12$ & $\mathrm{n}-\mathrm{C} 13$ & $\begin{array}{c}\begin{array}{c}\text { Specific Emis } \\
n-C 14\end{array} \\
\end{array}$ & $\begin{array}{c}\text { Rate, } \mu \mathrm{g} \mathrm{m} \\
\text { Toluene } \\
\end{array}$ & Xylenes & Styrene & 1,2,4-TMB \\
\hline$\because h$ & 391 & 345 & 800 & 354 & 759 & 45 & 18 & 127 \\
\hline$\therefore 7$ & 71 & 67 & 208 & 98 & 128 & 12 & 8 & 35 \\
\hline $6-h$ & 50 & 39 & 113 & 54 & 81 & 8 & 7 & 25 \\
\hline 24-h & 29 & 19 & 59 & 26 & 51 & 5 & 5 & 15 \\
\hline 48-h & 33 & 24 & 68 & 33 & 46 & 5 & 4 & 18 \\
\hline $72-h$ & 30 & 23 & 65 & 32 & 39 & 4 & 3 & 17 \\
\hline 96-h & 25 & 19 & 60 & 28 & 36 & 4 & 3 & 14 \\
\hline $120-h$ & 27 & 20 & 62 & 32 & 37 & 4 & 3 & 15 \\
\hline $144-h$ & 29 & 22 & 65 & 33 & 39 & 4 & 3 & 17 \\
\hline $168-h$ & 29 & 21 & 62 & 32 & 35 & 5 & 3 & 17 \\
\hline $192-h$ & 28 & 23 & 68 & 34 & 34 & 5 & 3 & 17 \\
\hline $216-h$ & 35 & 25 & 72 & 37 & 41 & 5 & 3 & 18 \\
\hline $240-h$ & 31 & 25 & 75 & 40 & 45 & 5 & 3 & 19 \\
\hline 336-h & 25 & 22 & 67 & 35 & 37 & 4 & 2 & 16 \\
\hline
\end{tabular}


Table J-24. Quasi steady-state specific emission rates of Group 2 target VOCs for large-scale Experiment V-3.

\begin{tabular}{|c|c|c|c|c|c|c|c|c|c|}
\hline Sample ID & \multicolumn{9}{|c|}{ Specific Emission Rate, $\mu \mathrm{g} \mathrm{m}^{-2} \mathrm{~h}-1$} \\
\hline $1-h$ & 12,100 & 45 & 29,800 & 27 & 45 & 191 & 18 & 9 & 45 \\
\hline $3-h$ & 1,040 & 16 & 2,560 & 8 & 24 & 86 & 24 & 8 & 20 \\
\hline $6-h$ & 719 & 13 & 1,480 & 8 & 15 & 57 & 24 & 8 & 13 \\
\hline 24-h & 106 & 9 & 153 & 7 & 15 & 52 & 21 & 7 & 15 \\
\hline $48-h$ & 55 & 11 & 82 & 8 & 21 & 71 & 28 & 6 & 21 \\
\hline $72-h$ & 37 & 10 & 52 & 8 & 22 & 72 & 26 & 6 & 20 \\
\hline $96-h$ & 25 & 9 & 36 & 7 & 19 & 69 & 26 & 5 & 20 \\
\hline $120-h$ & 24 & 8 & 35 & 8 & 22 & 74 & 26 & 5 & 21 \\
\hline $144-h$ & 22 & 9 & 31 & 9 & 23 & 73 & 35 & 5 & 21 \\
\hline $168-h$ & 22 & 10 & 33 & 9 & 22 & 74 & 35 & 5 & 20 \\
\hline $192-h$ & 18 & 9 & 27 & 9 & 22 & 78 & 37 & 4 & 21 \\
\hline $216-h$ & 20 & 11 & 28 & 9 & 22 & 80 & 43 & 5 & 20 \\
\hline $240-h$ & 18 & 9 & 26 & 9 & 23 & 80 & 45 & 4 & 20 \\
\hline $336-h$ & 13 & 8 & 16 & 7 & 17 & 72 & 37 & 2 & 17 \\
\hline
\end{tabular}


Table J-25. Quasi steady-state specific emission rates of TVOC for large-scale Experiments V-1 through V-4.

\begin{tabular}{|c|c|c|c|c|}
\hline Sample ID & \multicolumn{3}{|c|}{ Specific Emission Rate, $\mu \mathrm{g} \mathrm{m}^{-2} \mathrm{~h}^{-1}$} & Exp V-3 \\
\hline $1-h$ & 34,500 & 42,300 & 23,300 & 28,600 \\
\hline 3-h & 4,620 & 3,480 & 8,120 & 2,680 \\
\hline $6-h$ & 5,130 & 2,670 & 5,560 & 2,390 \\
\hline 24-h & 2,200 & 1,400 & 4,190 & 1,170 \\
\hline $48-h$ & 1,700 & 1,090 & 3,550 & 1,180 \\
\hline 72-h & 1,380 & 1,080 & 4,240 & 1,020 \\
\hline $96-h$ & 1,400 & 1,350 & 967 & 980 \\
\hline $120-h$ & 1,530 & 1,330 & 1,230 & 980 \\
\hline $144-h$ & 1,590 & 1,310 & 1,060 & 1,060 \\
\hline $168-h$ & -- & 1,160 & 1,000 & 1,060 \\
\hline $192-h$ & -.- & 1,120 & 964 & 1,070 \\
\hline $216-h$ & --- & 1,210 & 1,010 & 1,230 \\
\hline $240-h$ & --- & 1,190 & 1,090 & 1,250 \\
\hline $336-h$ & -- & 955 & 1,130 & 1,060 \\
\hline
\end{tabular}

*Data subsequent to $144-h$ elapsed time were invalid. 
Table J-26. Quasi steady-state specific emission rates of formaldehyde for large-scale Experiments V-1 through V-4.

\begin{tabular}{|c|c|c|c|c|}
\hline Sample ID & \multicolumn{4}{|c|}{ Specific Emission Rate, $\mu \mathrm{g} \mathrm{m}^{-2} \mathrm{~h}^{-1}$} \\
\hline $1-h$ & 24.5 & 23.5 & $<25.0$ & 17.2 \\
\hline 3-h & 12.5 & 8.2 & 15.7 & 14.1 \\
\hline $6-h$ & 14.7 & 7.5 & 13.7 & 9.9 \\
\hline 24-h & 3.6 & $<2.5$ & $<10.0$ & 3.3 \\
\hline $48-h$ & 2.8 & $<2.5$ & $<10.0$ & $<2.5$ \\
\hline $72-h$ & $<2.5$ & $<2.5$ & $<10.0$ & 4.7 \\
\hline $96-\mathrm{h}$ & $<2.5$ & $<2.5$ & 3.7 & 4.3 \\
\hline $120-h$ & $<2.5$ & 2.9 & 6.0 & 3.4 \\
\hline 144-h & $<2.5$ & $<2.5$ & 7.5 & 3.7 \\
\hline $168-h$ & --- & $<2.5$ & 7.2 & 4.2 \\
\hline $192-h$ & $\cdots$ & $<2.5$ & 8.3 & 5.4 \\
\hline 216-h & --- & 3.9 & 11.3 & 6.9 \\
\hline $240-\mathrm{h}$ & --- & $<2.5$ & 14.6 & 6.6 \\
\hline 336-h & -- & $<2.5$ & 9.3 & 8.1 \\
\hline
\end{tabular}

${ }^{*}$ Data subsequent to $144-\mathrm{h}$ elapsed time were invalid. 
Table J-27. Quasi steady-state specific emission rates of acetaldehyde for large-scale Experiments V-1 through V-4.

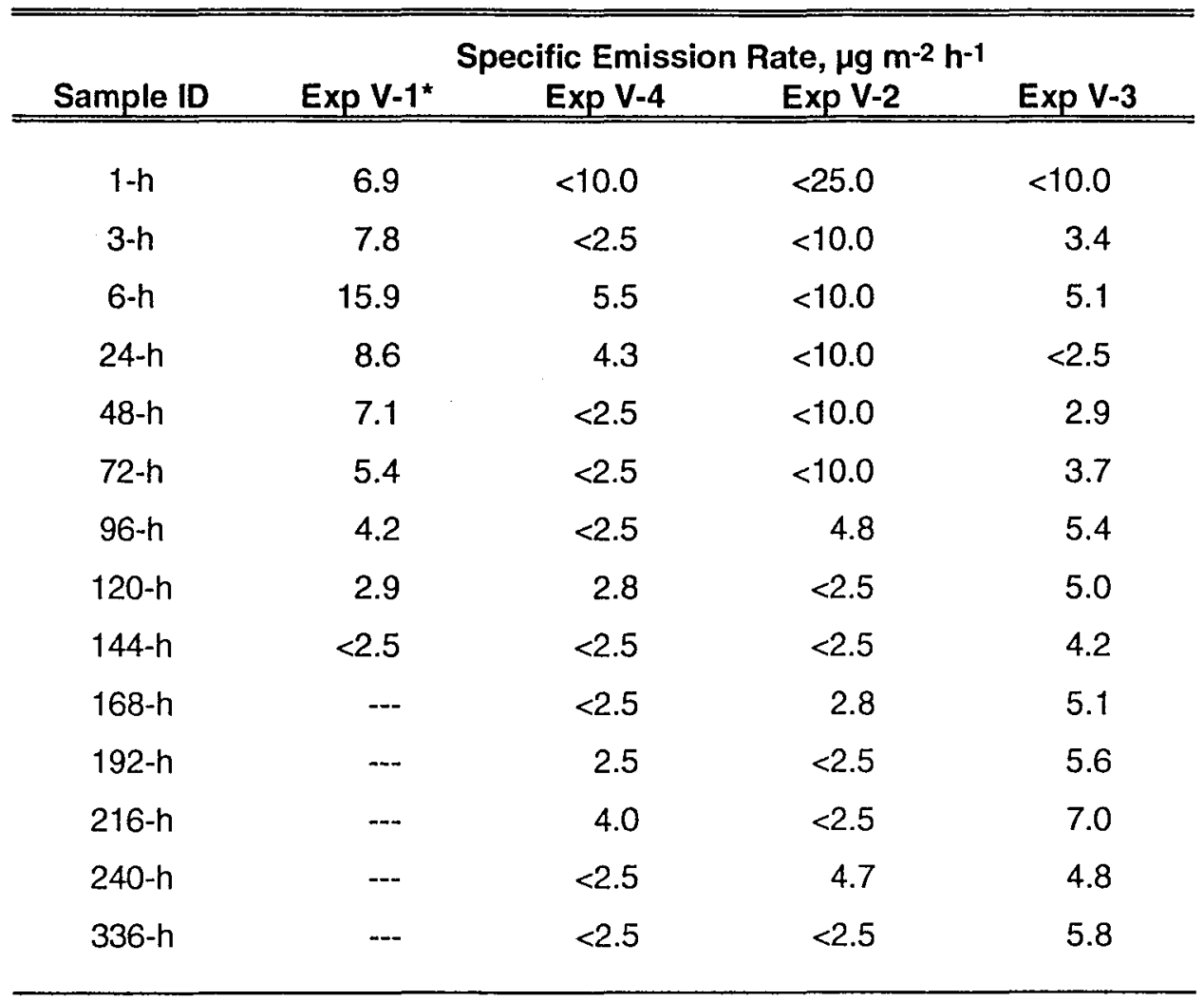

*Data subsequent to 144-h elapsed time were invalid. 
Table J-28. Cumulative masses (milligrams) of target compounds and TVOC emitted over 0 - 336 hours in large-scale Experiments V-1 through V-4.

\begin{tabular}{|c|c|c|c|c|}
\hline Compound & $\operatorname{Exp} V-1^{*}$ & $\begin{array}{l}\text { Cumulati } \\
\text { Exp V-4 }\end{array}$ & $\begin{array}{r}\text { Uass, } \mathrm{mg} \\
\text { Exp V-2 }\end{array}$ & Exp V-3 \\
\hline \multicolumn{5}{|l|}{ Alkane Hydrocarbons } \\
\hline n-Decane & 84 & 131 & 138 & 113 \\
\hline n-Dodecane & 55 & 97 & 112 & 87 \\
\hline $\mathrm{n}$-Tridecane & 147 & 264 & 337 & 257 \\
\hline n-Tetradecane & 73 & 132 & 178 & 129 \\
\hline \multicolumn{5}{|l|}{ Aromatic Hydrocarbons } \\
\hline Toluene & 492 & 227 & 179 & 162 \\
\hline m-,p-Xylene & 11 & 18 & 21 & 17 \\
\hline Styrene & 13 & 15 & 20 & 12 \\
\hline 1,2,4-Trimethylbenzene & 37 & 68 & 81 & 63 \\
\hline \multicolumn{5}{|l|}{ Carbonyl Compounds } \\
\hline Formaldehyde & $<5$ & $<7$ & $<30$ & 19 \\
\hline Acetaldehyde & 9 & $<7$ & $<11$ & $<17$ \\
\hline Cyclohexanone & 591 & 576 & 370 & 454 \\
\hline Benzaldehyde & 19 & 37 & 52 & 34 \\
\hline \multicolumn{5}{|l|}{ Other Oxidized Cmpds. } \\
\hline Tetrahydrofuran & 1,010 & 1,040 & 298 & 987 \\
\hline 1-Octanol & 17 & 30 & 45 & 29 \\
\hline Benzyl alcohol & 46 & 83 & 144 & 72 \\
\hline Phenol & 142 & 339 & 503 & 257 \\
\hline $\begin{array}{l}\text { 2,2,4-Trimethyl-1,3- } \\
\text { pentanediol diisobutyrate }\end{array}$ & 54 & 127 & 216 & 119 \\
\hline \multicolumn{5}{|l|}{ Miscellaneous Cmpds. } \\
\hline tert-Butylisothiocyanate & 8 & 14 & 16 & 16 \\
\hline Benzothiazole & 52 & 91 & 160 & 68 \\
\hline SigmaVOC & 2,850 & 3,280 & 2,860 & 2,880 \\
\hline TVOC & 3,580 & 5,200 & 6,970 & 4,630 \\
\hline
\end{tabular}

${ }^{\star}$ Cumulative masses emitted over 0 - 144 hours only. 
Table J-29. Cumulative exposures (ppb-hour) to target compounds during the first 48 hours of large-scale Experiments V-1 through V-4. Estimates assume 20 hours of occupancy per day.

\begin{tabular}{|c|c|c|c|c|}
\hline \multirow[b]{2}{*}{ Compound } & \multicolumn{4}{|c|}{ Cumulative Exposure, ppb-hour } \\
\hline & Exp V-1 & Exp V-4 & Exp V-2 & Exp V-3 \\
\hline \multicolumn{5}{|l|}{ Alkane Hydrocarbons } \\
\hline n-Decane & 338 & 264 & 97 & 224 \\
\hline n-Dodecane & 198 & 172 & 72 & 138 \\
\hline $\mathrm{n}$-Tridecane & 473 & 425 & 219 & 375 \\
\hline n-Tetradecane & 228 & 191 & 111 & 162 \\
\hline \multicolumn{5}{|l|}{ Aromatic Hydrocarbons } \\
\hline Toluene & 5,430 & 866 & 196 & 579 \\
\hline $\mathrm{m}-\mathrm{p}$-Xylene & 74 & 49 & 20 & 47 \\
\hline Styrene & 100 & 52 & 22 & 39 \\
\hline 1,2,4-Trimethylbenzene & 193 & 167 & 70 & 132 \\
\hline \multicolumn{5}{|l|}{ Carbonyl Compounds } \\
\hline Formaldehyde & 468 & 251 & 170 & 347 \\
\hline Acetaldehyde & 381 & 200 & 123 & 193 \\
\hline Cyclohexanone & 4,170 & 3,320 & 992 & 3,070 \\
\hline Benzaldehyde & 109 & 102 & 58 & 84 \\
\hline \multicolumn{5}{|l|}{ Other Oxidized Cmpds. } \\
\hline Tetrahydrofuran & 9,440 & 7,320 & 1,400 & 8,940 \\
\hline 1-Octanol & 78 & 60 & 37 & 48 \\
\hline Benzyl alcohol & 227 & 186 & 171 & 127 \\
\hline Phenol & 801 & 931 & 727 & 510 \\
\hline $\begin{array}{l}\text { 2,2,4-Trimethyl-1,3- } \\
\text { pentanediol diisobutyrate }\end{array}$ & 101 & 78 & 89 & 65 \\
\hline \multicolumn{5}{|l|}{ Miscellaneous Cmpds. } \\
\hline tert-Butylisothiocyanate & 55 & 59 & 17 & 49 \\
\hline Benzothiazole & 204 & 169 & 154 & 99 \\
\hline
\end{tabular}


Table J-30. Cumulative exposures (ppb-hour) to target compounds over 48 - 336 hours in large-scale Experiments $V-1$ through $V-4$. Estimates assume 20 hours of occupancy per day.

\begin{tabular}{|c|c|c|c|c|}
\hline \multirow[b]{2}{*}{ Compound } & \multicolumn{4}{|c|}{ Cumulative Exposure, ppb-hour } \\
\hline & Exp V-1* & Exp V-4 & Exp V-2 & Exp V-3 \\
\hline \multicolumn{5}{|l|}{ Alkane Hydrocarbons } \\
\hline n-Decane & 509 & 1,050 & 974 & 1,020 \\
\hline n-Dodecane & 277 & 665 & 631 & 657 \\
\hline n-Tridecane & 691 & 1,720 & 1,620 & 1,820 \\
\hline $\mathrm{n}$-Tetradecane & 312 & 813 & 782 & 868 \\
\hline \multicolumn{5}{|l|}{ Aromatic Hydrocarbons } \\
\hline Toluene & 2,550 & 2,830 & 2,080 & 2,190 \\
\hline m-,p-Xylene & 87 & 200 & 192 & 204 \\
\hline Styrene & 105 & 169 & 179 & 142 \\
\hline 1,2,4-Trimethylbenzene & 277 & 678 & 664 & 705 \\
\hline \multicolumn{5}{|l|}{ Carbonyl Compounds } \\
\hline Formaldehyde & $<157$ & $<470$ & 1,310 & 869 \\
\hline Acetaldehyde & 155 & $<320$ & $<320$ & 553 \\
\hline Cyclohexanone & 703 & 1,160 & 1,040 & 1,120 \\
\hline Benzaldehyde & 171 & 431 & 436 & 432 \\
\hline \multicolumn{5}{|l|}{ Other Oxidized Cmpds. } \\
\hline Tetrahydrofuran & 1,170 & 2,010 & 1,400 & 2,390 \\
\hline 1-Octanol & 127 & 296 & 329 & 312 \\
\hline Benzyl alcohol & 443 & 1,010 & 1,060 & 965 \\
\hline Phenol & 1,540 & 4,660 & 3,910 & 3,960 \\
\hline $\begin{array}{l}2,2,4-\text { Trimethyl-1,3- } \\
\text { pentanediol diisobutyrate }\end{array}$ & 209 & 630 & 609 & 623 \\
\hline \multicolumn{5}{|l|}{ Miscellaneous Cmpds. } \\
\hline tert-Butylisothiocyanate & 58 & 124 & 116 & 181 \\
\hline Benzothiazole & 407 & 894 & 903 & 730 \\
\hline
\end{tabular}

${ }^{*}$ Cumulative exposures over 48 - 144 hours only. 
APPENDIXK - COMBINED ASSEMBLIES

\title{
APPENDIX K
}

\section{EXPOSURE REDUCTION AND LONG-TERM EXPERIMENTS WITH COMBINED ASSEMBLIES}

\begin{abstract}
Appendix K presents the analytical data for the two large-scale exposure reduction experiments with the combined source assemblies. The experiments were conducted in $25.5 \mathrm{~m}^{3}$ chamber compartments over a period of 336 hours. One of the experiments was extended for a period of 2,016 hours. Paints LPS2, FLP3 and SGLP3 were applied to gypsum board and plywood panels with a total surface area of $16 \mathrm{~m}^{2}$. Two-thirds of the 10.4- $\mathrm{m}^{2}$ floor area was carpeted with Carpet CP4 and Carpet Cushion CC4. The remaining floor area was covered with Sheet Vinyl SV5. The concentrations of selected compounds and TVOC were measured throughout the experiments. Specific emission rates of these components were calculated. Cumulative mass emissions and cumulative exposures were estimated.
\end{abstract}

\section{List of Tables}

Table K-01. Summary of environmental parameters for large-scale Experiments A-1 and A-2 .................429

Table K-02. Source materials and quantities used in large-scale Experiments $A-1$ and $A-2 ~ \ldots . . . \ldots \ldots \ldots \ldots \ldots . . . . . .430$

Table K-03. VOCs emitted by combined source materials in large-scale Experiment A-2 ........................431

Table K-04. Target compounds quantified in large-scale Experiments A-1 and A-2 ..............................434

Table K-05. Concentrations of Group 1 target VOCs for large-scale Experiment A-2 . ..............................435

Table K-06. Concentrations of Group 2 target VOCs for large-scale Experiment A-2 ............................436

Table K-07. Concentrations of Group 1 target VOCs for large-scale Experiment A-1 ..........................437

Table K-08. Concentrations of Group 2 target VOCs for large-scale Experiment A-1 .............................438

Table K-09. Concentrations of SigmaVOC $P$ and $T O_{R}$ for large-scale Experiments A-1 and A-2 ...........439

Table K-10. Concentrations of formaldehyde and acetaldehyde for large-scale Experiments

A-1 and A-2

Table K-11. Quasi steady-state specific emission rates of Group 1 target VOCs for large-scale Experiment A-2

Table K-12. Quasi steady-state specific emission rates of Group 2 target VOCs for large-scale Experiment A-2.

Table K-13. Quasi steady-state specific emission rates of Group 1 target VOCs for large-scale Experiment A-1

Table K-14. Quasi steady-state specific emission rates of Group 2 target VOCs for large-scale Experiment A-1

Table K-15. Quasi steady-state specific emission rates of $T_{V O C}$ for large-scale Experiments $A-1$ and $A-2$

Table K-16. Cumulative masses of target VOCs and TVOC $_{\mathrm{R}}$ in large-scale Experiments A-1 and A-2 .......446

Table K-17. Cumulative exposures to target VOCs over $0-48$ and $48-336$ hours in large-scale Experiment A-2

Table K-18. Cumulative exposures to target VOCs over 96 - 336 hours in large-scale Experiments $A-1$ and $A-2$

Table K-19. Summary of environmental parameters over 336 - 2,016 hours in large-scale Experiment A-2.

Table K-20. Concentrations of Group 1 target VOCs over $336-2,016$ hours in large-scale Experiment A-2.

Table K-21. Concentrations of Group 2 target VOCs over 336 - 2,016 hours in large-scale Experiment A-2.

Table K-22. Concentrations of SigmaVOC $P$ and TVOC $_{R}$ over $336-2,016$ hours in large-scale Experiment A-2.

Table K-23. Quasi steady-state specific emission rates of Group 1 target VOCs over 336 - 2,015 hours in large-scale Experiment A-2. 


\section{List of Tables, Continued}

Table K-24. Quasi steady-state specific emission rates of Group 2 target VOCs over $336-2,016$ hours in large-scale Experiment A-2..

Table K-25. Quasi steady-state specific emission rates of $\mathrm{TVOC}_{\mathrm{R}}$ over $336-2,016$ hours in large-scale Experiment A-2...

Table K-26. Cumulative masses of target VOCs and TVOC $R$ emitted over $336-2,016$ hours in large-scale Experiment A-2..

Table K-27. Cumulative exposures to target VOCs over $336-2,016$ hours in large-scale Experiment A-2 
Table K-01. Summary of environmental parameters for large-scale Experiments A-1 and A-2 with combined source materials.

\begin{tabular}{|c|c|c|}
\hline \multirow[b]{2}{*}{ Parameter } & \multicolumn{2}{|c|}{ Experiment } \\
\hline & A-2 & A-1 \\
\hline Paint application date & $02 / 09 / 98$ & $01 / 20 / 98$ \\
\hline Treatment & Add ventilation & Add vent. \& heating \\
\hline \multicolumn{3}{|l|}{ Paint drying \& flooring install., $-72-0 \mathrm{~h}$} \\
\hline $\begin{array}{l}\text { Ventilation rate }\left(\mathrm{h}^{-1}\right), \text { Avg. } \pm 1 \text { std. dev. } \\
\text { (Range) }\end{array}$ & $\begin{array}{l}1.97 \pm 0.01 \\
1.94-2.00\end{array}$ & $\begin{array}{l}1.91 \pm 0.01 \\
1.88-1.94\end{array}$ \\
\hline $\begin{array}{l}\text { Temperature }\left({ }^{\circ} \mathrm{C}\right), \text { Avg. } \pm 1 \text { std. dev. } \\
\text { (Range) }\end{array}$ & $\begin{array}{l}22.1 \pm 0.3 \\
21.4-22.8\end{array}$ & $\begin{array}{l}22.3 \pm 0.3 \\
21.2-22.9\end{array}$ \\
\hline $\begin{array}{l}\text { Relative Humidity (\%), Avg. } \pm 1 \text { std. dev. } \\
\text { (Range) }\end{array}$ & $\begin{array}{l}46 \pm 1 \\
43-49\end{array}$ & $\begin{array}{l}43 \pm 2 \\
40-49\end{array}$ \\
\hline \multicolumn{3}{|l|}{ Post flooring installation, $0-6 \mathrm{~h}$} \\
\hline $\begin{array}{l}\text { Ventilation rate }\left(\mathrm{h}^{-1}\right) \text {, Avg. } \pm 1 \text { std. dev. } \\
\text { (Range) }\end{array}$ & $\begin{array}{l}1.96 \pm 0.01 \\
1.93-2.00\end{array}$ & $\begin{array}{l}1.91 \pm 0.01 \\
1.89-1.93\end{array}$ \\
\hline $\begin{array}{l}\text { Temperature }\left({ }^{\circ} \mathrm{C}\right) \text {, Avg. } \pm 1 \text { std. dev. } \\
\text { (Range) }\end{array}$ & $\begin{array}{l}23.1 \pm 0.3 \\
22.7-24.1\end{array}$ & $\begin{array}{l}23.5 \pm 0.2 \\
21.2-22.9\end{array}$ \\
\hline $\begin{array}{l}\text { Relative Humidity (\%), Avg. } \pm 1 \text { std. dev. } \\
\text { (Range) }\end{array}$ & $\begin{array}{l}47 \pm 2 \\
44-54\end{array}$ & $\begin{array}{l}44 \pm 1 \\
41-49\end{array}$ \\
\hline \multicolumn{3}{|l|}{ Heating period, $12-72 \mathrm{~h}$} \\
\hline $\begin{array}{l}\text { Ventilation rate }\left(\mathrm{h}^{-1}\right) \text {, Avg. } \pm 1 \text { std. dev. } \\
\quad \text { (Range) }\end{array}$ & $\begin{array}{l}1.96 \pm 0.01 \\
1.93-2.00\end{array}$ & $\begin{array}{l}1.91 \pm 0.01 \\
1.88-1.94\end{array}$ \\
\hline $\begin{array}{l}\text { Temperature }\left({ }^{\circ} \mathrm{C}\right), \text { Avg. } \pm 1 \text { std. dev. } \\
\text { (Range) }\end{array}$ & $\begin{array}{l}22.4 \pm 0.2 \\
21.9-23.1\end{array}$ & $\begin{array}{l}32.7 \pm 0.6 \\
31.8-33.8\end{array}$ \\
\hline $\begin{array}{l}\text { Relative Humidity (\%), Avg. } \pm 1 \text { std. dev. } \\
\text { (Range) }\end{array}$ & $\begin{array}{l}47 \pm 2 \\
44-50\end{array}$ & $\begin{array}{l}28 \pm 1 \\
24-30\end{array}$ \\
\hline \multicolumn{3}{|l|}{ Remaining period, $80-336 \mathrm{~h}$} \\
\hline $\begin{array}{l}\text { Ventilation rate }\left(h^{-1}\right), \text { Avg. } \pm 1 \text { std. dev. } \\
\text { (Range) }\end{array}$ & $\begin{array}{l}0.50 \pm 0.01 \\
0.49-0.51\end{array}$ & $\begin{array}{l}0.48 \pm 0.01 \\
0.47-0.49\end{array}$ \\
\hline $\begin{array}{l}\text { Temperature }\left({ }^{\circ} \mathrm{C}\right) \text {, Avg. } \pm 1 \text { std. dev. } \\
\text { (Range) }\end{array}$ & $\begin{array}{l}21.8 \pm 0.3 \\
21.0-22.4\end{array}$ & $\begin{array}{l}22.5 \pm 0.8 \\
21.7-27.4\end{array}$ \\
\hline $\begin{array}{l}\text { Relative Humidity (\%), Avg. } \pm 1 \text { std. dev. } \\
\text { (Range) }\end{array}$ & $\begin{array}{l}44 \pm 1 \\
41-47\end{array}$ & $\begin{array}{l}46 \pm 3 \\
35-50\end{array}$ \\
\hline
\end{tabular}


Table K-02. Source materials and quantities used in large-scale Experiments A-1 and A-2. See Appendices C, D and E (Tables C-01, D-01 and E01) for material descriptions.

\begin{tabular}{|c|c|c|c|c|}
\hline \multirow[b]{2}{*}{ Material Description } & \multirow[b]{2}{*}{ Material ID } & \multirow{2}{*}{$\begin{array}{c}\text { Unit of } \\
\text { Measure } \\
\end{array}$} & \multicolumn{2}{|c|}{ Quantity } \\
\hline & & & Exp A-2 & Exp A-1 \\
\hline \multicolumn{5}{|l|}{ Latex Paints } \\
\hline Primer Sealer & LPS2 & $g$ & 2,150 & 1,910 \\
\hline Flat & FLP3 & $g$ & 1,900 & 1,660 \\
\hline Semi-Gloss & SGLP3 & $\mathrm{g}$ & 90 & 91 \\
\hline \multicolumn{5}{|l|}{ Vinyl Flooring Materials } \\
\hline Particle board underlayment & UL & $m^{2}$ & 3.48 & 3.48 \\
\hline Residential sheet vinyl & SV5 & $m^{2}$ & 3.48 & 3.48 \\
\hline Rubber cove base, 4 " wide & $\mathrm{CB}$ & $\mathrm{m}$ & 5.33 & 5.33 \\
\hline Sheet flooring adhesive & SFA & $g$ & 910 & 800 \\
\hline Cove base adhesive & CBA & $g$ & 222 & 174 \\
\hline Seam sealer & SS & $\mathrm{mL}$ & 5 & 5 \\
\hline \multicolumn{5}{|l|}{ Carpet Materials } \\
\hline Commercial olefin carpet & $\mathrm{CP} 4$ & $m^{2}$ & 6.97 & 6.97 \\
\hline $\begin{array}{l}\text { Rebonded urethane carpet } \\
\text { cushion }\end{array}$ & $\mathrm{CC} 4$ & $m^{2}$ & 5.94 & 5.94 \\
\hline Thermal seam tape & ST & $\mathrm{m}$ & 2.29 & 3.05 \\
\hline
\end{tabular}


Table K-03. VOCs emitted by combined source materials in large-scale Experiment A-2 at 24- and 240-hours elapsed times.

\begin{tabular}{|c|c|c|c|c|c|}
\hline COMPOUND & Code $^{*}$ & $\begin{array}{c}\mathrm{RT} \\
(\mathrm{min})\end{array}$ & $\begin{array}{c}24-h \\
E T\end{array}$ & $\begin{array}{c}240-h \\
E T\end{array}$ & $\begin{array}{l}\text { Match } \\
\text { Quality }\end{array}$ \\
\hline \multicolumn{6}{|l|}{ Alkane Hydrocarbons } \\
\hline C9 Branched alkane $\mathrm{HC}$ & & 13.7 & & + & Probable \\
\hline C9 Branched alkane $\mathrm{HC}$ & & 14.1 & & + & Probable \\
\hline n-Nonane & $\mathrm{B}$ & 15.2 & + & + & Confirmed \\
\hline C10 Branched alkane $\mathrm{HC}$ & & 16.6 & & + & Probable \\
\hline C10 Branched alkane HC & & 17.7 & + & + & Probable \\
\hline C10 Branched alkane $\mathrm{HC}$ & & 17.8 & & + & Probable \\
\hline C10 Branched alkane $\mathrm{HC}$ & & 18.1 & + & + & Probable \\
\hline n-Decane & $A, Q$ & 19.1 & + & + & Confirmed \\
\hline C11 Branched alkane $\mathrm{HC}$ & $\mathrm{B}$ & 20.0 & + & + & Probable \\
\hline C11 Branched alkane $\mathrm{HC}$ & & 21.2 & + & + & Probable \\
\hline C11 Branched alkane $\mathrm{HC}$ & & 21.4 & + & + & Probable \\
\hline $\mathrm{n}$-Undecane & & 22.8 & + & + & Confirmed \\
\hline C12 Branched alkane $\mathrm{HC}$ & & 23.0 & + & + & Probable \\
\hline n-Dodecane & B & 26.0 & + & + & Confirmed \\
\hline C13 Branched alkane $\mathrm{HC}$ & & 28.5 & + & + & Probable \\
\hline $\mathrm{n}$-Tridecane & $A, B, Q$ & 29.3 & + & + & Confirmed \\
\hline C14 Branched alkane $\mathrm{HC}$ & & 29.9 & + & + & Probable \\
\hline n-Tetradecane & B & 32.2 & + & + & Confirmed \\
\hline C15 Branched alkane $\mathrm{HC}$ & & 34.6 & + & + & Probable \\
\hline \multicolumn{6}{|l|}{ Aromatic Hydrocarbons } \\
\hline Toluene & $T, B, Q$ & 11.7 & + & + & Confirmed \\
\hline$m-, p-X y l e n e$ & $\mathrm{~T}$ & 16.1 & + & + & Confirmed \\
\hline o-Xylene & $T$ & 17.3 & + & + & Confirmed \\
\hline Styrene & $\mathrm{T}, \mathrm{Q}$ & 17.6 & + & + & Confirmed \\
\hline Isopropylbenzene & & 18.2 & + & + & Confirmed \\
\hline Propylbenzene & B & 19.4 & + & + & Confirmed \\
\hline Ethyltoluene isomer & B & 19.7 & + & + & Probable \\
\hline 2-Ethyltoluene & & 20.6 & + & + & Confirmed \\
\hline 1,2,4-Trimethylbenzene & $\mathrm{T}, \mathrm{B}, \mathrm{Q}$ & 21.2 & + & + & Confirmed \\
\hline C4 Alkylbenzene & & 23.0 & + & + & Probable \\
\hline 4-Phenylcyclohexene & $Q$ & 33.0 & + & + & Confirmed \\
\hline C2 Tetrahydronaphthalene & & 32.6 & + & + & Probable \\
\hline (1-Butoxyhexyl)benzene & & 37.1 & + & + & Probable \\
\hline (1-Methylnonyl)benzene & & 39.0 & + & + & Probable \\
\hline (1-Phenylhexyl)benzene & & 39.4 & + & + & Probable \\
\hline (1-Butylheptyl)benzene & & 39.5 & + & + & Probable \\
\hline (1-Propyloctyl)benzene & & 39.9 & + & + & Probable \\
\hline (1-Ethylnonyl)benzene & & 40.4 & + & + & Probable \\
\hline (1-Methyldecyl)benzene & & 41.5 & + & + & Probable \\
\hline \multicolumn{6}{|l|}{ Other Hydrocarbons } \\
\hline 4-Ethenylcyclohexene & & 13.6 & + & + & Confirmed \\
\hline Propylcyclohexane & & 17.0 & & + & Confirmed \\
\hline alpha-Pinene & B & 17.3 & & $\perp$ & Confirmed \\
\hline
\end{tabular}


Table K-03, Continued. VOCs emitted by combined source materials in large-scale Experiment A-2 at 24- and 240-hours elapsed times.

\begin{tabular}{|c|c|c|c|c|c|}
\hline COMPOUND & Code $^{*}$ & $\begin{array}{c}\mathrm{RT} \\
(\mathrm{min})\end{array}$ & $\begin{array}{c}24-h \\
\text { ET }\end{array}$ & $\begin{array}{c}240-h \\
\text { ET }\end{array}$ & $\begin{array}{l}\text { Match } \\
\text { Quality }\end{array}$ \\
\hline \multicolumn{6}{|l|}{ Other Hydrocarbons, Con't. } \\
\hline C10 Alkene or cyclic $\mathrm{HC}$ & & 18.2 & & + & Probable \\
\hline C10 Alkene or cyclic $\mathrm{HC}$ & & 18.9 & + & + & Probable \\
\hline Butylcyclohexane & & 20.9 & + & + & Confirmed \\
\hline C11 Alkene HC & & 21.5 & + & + & Probable \\
\hline C11 Alkene HC & & 22.0 & + & + & Tentative \\
\hline C11 Alkene $\mathrm{HC}$ & & 22.1 & + & + & Probable \\
\hline C11 Alkene HC & & 22.4 & + & + & Probable \\
\hline C11 Alkene $\mathrm{HC}$ & & 22.6 & + & + & Probable \\
\hline C12 Alkene HC & & 23.0 & + & + & Probable \\
\hline C12 Alkene HC & & 23.2 & + & + & Probable \\
\hline C12 Alkene $\mathrm{HC}$ & & 23.9 & + & + & Probable \\
\hline Alkene $\mathrm{HC}$ & & 30.4 & + & & Tentative \\
\hline \multicolumn{6}{|l|}{ Carbonyl Compounds } \\
\hline Hexanal & $\mathrm{B}$ & 14.5 & & + & Confirmed \\
\hline Cyclohexanone & $Q$ & 19.8 & + & + & Confirmed \\
\hline Benzaldehyde & $\vec{B}$ & 22.5 & + & + & Confirmed \\
\hline Nonanal & $\mathrm{B}$ & 26.0 & + & + & Confirmed \\
\hline \multicolumn{6}{|l|}{ Other Oxidized Compounds } \\
\hline Tetrahydrofuran & $B, Q$ & 6.2 & + & + & Confirmed \\
\hline Acetic acid & $\mathrm{B}$ & 10.8 & + & + & Confirmed \\
\hline n-Butyl ether & & 15.3 & + & + & Confirmed \\
\hline Ethylene glycol & $T, A, Q$ & 15.6 & + & + & Confirmed \\
\hline Propylene glycol & $\mathrm{Q}$ & 16.3 & + & & Confirmed \\
\hline 2-Methylpropanoic acid & & 16.3 & + & + & Probable \\
\hline Hexylene glycol & & 23.2 & + & + & Confirmed \\
\hline Benzyl alcohol & $Q$ & 26.5 & + & + & Confirmed \\
\hline Phenol & $T, A, B, Q$ & 26.6 & + & + & Confirmed \\
\hline 1,1'-Oxybis-2-propanol & & 27.5 & + & + & Tentative \\
\hline 2-(2-Butoxyethoxy)ethanol & $T, Q$ & 29.8 & + & + & Confirmed \\
\hline $\begin{array}{l}\text { 2,2,4-Trimethyl-1,3-pentanediol } \\
\text { monisobutyrate (Texanol 1) }\end{array}$ & $A, Q$ & 35.2 & + & + & Confirmed \\
\hline $\begin{array}{c}\text { 2,2,4-Trimethyl-1,3-pentanediol } \\
\text { monisobutyrate (Texanol 3) }\end{array}$ & $A, Q$ & 35.7 & + & + & Confirmed \\
\hline 1-Dodecanol & & 37.1 & & + & Confirmed \\
\hline $\begin{array}{l}\text { 2,6-Di-tert-butyl-4-methylphenol } \\
\text { (butylated hydroxytoluene) }\end{array}$ & $Q$ & 37.7 & + & + & Confirmed \\
\hline $\begin{array}{l}\text { 2,2,4-Trimethyl-1,3-pentanediol } \\
\text { diisobutyrate (TXIB) }\end{array}$ & $A, Q$ & 40.2 & + & + & Confirmed \\
\hline \multicolumn{6}{|l|}{ Nitrogen-Containing Compounds } \\
\hline 2,2'-Azobisisobutryonitrile & $\mathrm{Q}$ & 27.2 & + & + & Confirmed \\
\hline 2-Methylenegluaronitrile & & 31.1 & + & + & Confirmed \\
\hline
\end{tabular}


Table K-03, Continued. VOCs emitted by combined source materials in large-scale Experiment A:2 at 24- and 240-hours elapsed times.

\begin{tabular}{|c|c|c|c|c|c|}
\hline COMPOUND & Code $^{\star}$ & $\begin{array}{c}\text { RT } \\
(\mathrm{min})\end{array}$ & $\begin{array}{c}\text { 24-h } \\
\text { ET }\end{array}$ & $\begin{array}{c}240-h \\
\text { ET }\end{array}$ & $\begin{array}{l}\text { Match } \\
\text { Quality }\end{array}$ \\
\hline \multicolumn{6}{|l|}{ Miscellaneous Compounds } \\
\hline Benzothiazole & $Q$ & 31.6 & + & + & Confirmed \\
\hline \multicolumn{6}{|l|}{ Unidentified Compounds } \\
\hline Unidentified glycol ether & & 23.7 & + & + & Unident. \\
\hline Unidentified glycol ether & & 26.4 & + & + & Unident. \\
\hline Unidentified glycol ether & & 28.8 & + & + & Unident. \\
\hline Unidentified compound & & 36.6 & + & + & Unident. \\
\hline
\end{tabular}

* $\mathrm{T}$ = Toxic air contaminant; $\mathrm{A}=$ Abundant compound; $\mathrm{B}=$ Component of chamber background;

$Q=$ Quantified target compound. 
Table K-04. Target compounds quantified in large-scale Experiments A-1 and A-2.

\begin{tabular}{lcccr}
\hline Compound & Group & $\begin{array}{c}\text { Table } \\
\text { Abbrev. }\end{array}$ & $\begin{array}{c}\text { Dominant } \\
\text { Source(s) }\end{array}$ & $\begin{array}{r}\text { Source } \\
\text { Area* (m }\end{array}$ \\
$\begin{array}{l}\text { Alkane Hydrocarbons } \\
\text { n-Decane }\end{array}$ & & & & \\
n-Tridecane & 1 & $n-C 10$ & SV5,CBA & 3.48 \\
& 1 & $n-C 13$ & SV5,CB & 3.48
\end{tabular}

\section{Aromatic Hydrocarbons}

Toluene

Styrene

1,2,4-Trimethylbenzene

4-Phenylcyclohexene

\section{Carbonyl Compounds}

Formaldehyde

Ald

Acetaldehyde

Cyclohexanone

Other Oxidized Cmpds.

Tetrahydrofuran
Ethylene glycol
Propylene glycol
Benzyl alcohol
Phenol
2-(2-Butoxyethoxy)ethanol
2,2,4-Trimethyl-1,3-pentane-
diol monoisobutyrates
(combined isomers)

2,2,4-Trimethyl-1,3pentanediol diisobutyrate

$$
\text { 2,6-Di-tert-butyl-4- }
$$
methylphenol

Miscellaneous Cmpds.

2,2'-Azobisisobutyronitrile Benzothiazole

1

2

1

2

Ald

1

1

1

2

2

1

1
1,2,4-TMB

4-PCH

C-hexone

THF

EG

$P G$

Benz alc

2

TXIB

BHT

AIBN

$\mathrm{CC} 4$

6.97
SV5

$\mathrm{CC} 4$

SV5,CB,SFA,CBA

3.48

CP4,CB,CBA

6.97

3.48

CP4

6.97

No dominant sre.

10.4

No dominant src.

10.4

CB,SS

3.48

3.48

16.0

16.0

3.48

3.48

16.0

16.0

LPS2,FLP3,SGLP3

3.48

6.97

${ }^{*}$ Area used for calculation of specific emission rates. 
Table K-05. Concentrations of Group 1 target VOCs for large-scale Experiment A-2.

\begin{tabular}{|c|c|c|c|c|c|c|c|c|c|c|}
\hline$\underset{\text { ID }}{\text { Sample }}$ & \multicolumn{10}{|c|}{ Chamber Concentration, $\mu \mathrm{g} \mathrm{m}^{-3}$} \\
\hline Avg. Inlet & $<1$ & $<1$ & $<1$ & $<1$ & $<1$ & 2 & $<1$ & $<1$ & $<1$ & $<1$ \\
\hline Backgrnd. & 4 & $<1$ & 2 & 1 & $<1$ & 2 & $<1$ & 3 & 0 & $<1$ \\
\hline Minus $3 \mathrm{~h}$ & $<1$ & $<1$ & $<1$ & $<1$ & $<1$ & 2 & $<1$ & 2 & 9 & $<1$ \\
\hline $1-h$ & 138 & 90 & 140 & 21 & 2,180 & 4,760 & 11 & 58 & 30 & 11 \\
\hline $3-h$ & 42 & 39 & 48 & 7 & 476 & 1,070 & 7 & 42 & 19 & 10 \\
\hline $6-h$ & 33 & 27 & 30 & 5 & 181 & 269 & 7 & 36 & 17 & 9 \\
\hline $24-h$ & 13 & 14 & 9 & 3 & 38 & 20 & 5 & 31 & 13 & 8 \\
\hline $48-h$ & 8 & 11 & 6 & 2 & 17 & 8 & 4 & 29 & 13 & 7 \\
\hline $7 ?$ & 7 & 8 & 4 & 1 & 9 & 5 & 4 & 25 & 10 & 6 \\
\hline$\because: h$ & 22 & 16 & 20 & 4 & 18 & 11 & 5 & 30 & 13 & 8 \\
\hline ). & 22 & 18 & 18 & 5 & 17 & 9 & 6 & 33 & 15 & 9 \\
\hline $144-h$ & 22 & 19 & 18 & 5 & 16 & 8 & 6 & 34 & 15 & 9 \\
\hline $168-h$ & 23 & 18 & 17 & 5 & 13 & 7 & 6 & 31 & 14 & 9 \\
\hline $192-h$ & 22 & 18 & 16 & 5 & 11 & 5 & 5 & 32 & 14 & 9 \\
\hline $216-h$ & 21 & 18 & 16 & 5 & 10 & 5 & 5 & 30 & 14 & 9 \\
\hline $240-h$ & 21 & 18 & 15 & 5 & 9 & 5 & 5 & 30 & 14 & 9 \\
\hline 336-h & 18 & 16 & 13 & 4 & 6 & 3 & 4 & 27 & 13 & 8 \\
\hline
\end{tabular}


Table K-06. Concentrations of Group 2 target VOCs for large-scale Experiment A-2.

\begin{tabular}{|c|c|c|c|c|c|c|c|c|}
\hline $\begin{array}{c}\text { Sample } \\
\text { ID }\end{array}$ & Styrene & 4-PCH & $\mathrm{BHT}$ & \multicolumn{3}{|c|}{ Chamber Concentration, $\mu \mathrm{g} \mathrm{m}^{-3}$} & DEGBE & Texanol \\
\hline Avg. niet & $<1$ & $<1$ & $<1$ & $<1$ & $<32$ & $<12$ & $<1$ & $<2$ \\
\hline$E:$ round & $<1$ & $<1$ & $<1$ & $<1$ & $<32$ & $<12$ & $<1$ & $<2$ \\
\hline l.intis $3 \mathrm{~h}$ & $<1$ & $<1$ & $<1$ & $<1$ & 567 & 36 & 21 & 2,020 \\
\hline $1-\mathrm{h}$ & 23 & 7 & 7 & 18 & 617 & 61 & 26 & 2,580 \\
\hline $3-h$ & 11 & 5 & 4 & 11 & 392 & $<35$ & 18 & 2,020 \\
\hline $6-h$ & 9 & 5 & 3 & 10 & 305 & $<35$ & 14 & 2,010 \\
\hline $24-\mathrm{h}$ & 3 & 4 & 2 & 9 & 203 & $<35$ & 10 & 1,140 \\
\hline $48-h$ & 2 & 4 & 2 & 8 & 214 & $<29$ & 7 & 1,550 \\
\hline $72-\mathrm{h}$ & 1 & 3 & 2 & 6 & 161 & $<29$ & 5 & 1,120 \\
\hline $96-h$ & 2 & 5 & 4 & 15 & 172 & $<29$ & 6 & 1,790 \\
\hline $120-h$ & 2 & 6 & 5 & 16 & 182 & $<23$ & 7 & 1,480 \\
\hline $144-h$ & 2 & 6 & 5 & 16 & 199 & $<23$ & 6 & 1,550 \\
\hline $168-h$ & 2 & 6 & 6 & 14 & 109 & $<23$ & 6 & 1,640 \\
\hline $192-h$ & 2 & 5 & 6 & 13 & 196 & $<17$ & 5 & 1,550 \\
\hline $216-h$ & 1 & 5 & 7 & 11 & 184 & $<17$ & 5 & 1,700 \\
\hline $240-h$ & 1 & 5 & 7 & 10 & 167 & $<17$ & 4 & 1,510 \\
\hline $336-h$ & 1 & 4 & 8 & 8 & 135 & $<14$ & 3 & 1,110 \\
\hline
\end{tabular}


Table K-07. Concentrations of Group 1 target VOCs for large-scale Experiment A-1:

\begin{tabular}{|c|c|c|c|c|c|c|c|c|c|c|}
\hline $\begin{array}{c}\text { Sample } \\
\text { ID }\end{array}$ & \multicolumn{10}{|c|}{ Chamber Concentration, $\mu \mathrm{g} \mathrm{m}^{-3}$} \\
\hline Avg. Inlet & $<1$ & $<1$ & 1 & $<1$ & $<1$ & 3 & $<1$ & $<1$ & $<1$ & $<1$ \\
\hline Backgrnd. & $<1$ & 1 & $<1$ & $<1$ & $<1$ & 1 & $<1$ & 2 & 1 & $<1$ \\
\hline Minus $3 \mathrm{~h}$ & 4 & 3 & 4 & $<1$ & 12 & 2 & $<1$ & 3 & 9 & $<1$ \\
\hline $1-\mathrm{h}$ & 130 & 107 & 125 & 23 & 1,520 & 3,270 & 14 & 75 & 27 & 11 \\
\hline $3-h$ & 46 & 44 & 59 & 8 & 388 & 1,080 & 8 & 50 & 21 & 7 \\
\hline $6-h$ & 33 & 30 & 43 & 6 & 147 & 285 & 7 & 43 & 18 & 7 \\
\hline $12-h$ & 36 & 45 & 23 & 8 & 125 & 160 & 10 & 81 & 32 & 10 \\
\hline $24-h$ & 25 & 39 & 14 & 7 & 55 & 61 & 12 & 82 & 41 & 10 \\
\hline $48-h$ & 19 & 28 & 10 & 5 & 23 & 20 & 9 & 57 & 33 & 7 \\
\hline $72-\mathrm{h}$ & 16 & 25 & 9 & 4 & 15 & 15 & 8 & 50 & 28 & 6 \\
\hline $78-h$ & 39 & 34 & 26 & 10 & 36 & 34 & 8 & 47 & 25 & 6 \\
\hline $96-h$ & 26 & 21 & 19 & 6 & 21 & 23 & 5 & 30 & 17 & 5 \\
\hline $120-\mathrm{h}$ & 27 & 23 & 18 & 6 & 19 & 20 & 6 & 33 & 16 & 6 \\
\hline 144-h & 24 & 21 & 16 & 5 & 16 & 17 & 6 & 31 & 14 & 5 \\
\hline $168-h$ & 21 & 18 & 14 & 5 & 13 & 14 & 5 & 28 & 12 & 5 \\
\hline $192-\mathrm{h}$ & 23 & 22 & 15 & 6 & 13 & 16 & 6 & 32 & 14 & 5 \\
\hline 216-h & 23 & 21 & 14 & 6 & 13 & 14 & 5 & 31 & 14 & 5 \\
\hline $240-h$ & 22 & 21 & 14 & 6 & 12 & 13 & 5 & 30 & 13 & 5 \\
\hline $336-h$ & 20 & 20 & 12 & 6 & 11 & 10 & 5 & 30 & 13 & 5 \\
\hline
\end{tabular}


Table K-08. Concentrations of Group 2 target VOCs for large-scale Experiment A-1.

\begin{tabular}{|c|c|c|c|c|c|c|c|c|}
\hline $\begin{array}{c}\text { Sample } \\
\text { ID }\end{array}$ & Styrene & 4-PCH & BHT & \multicolumn{3}{|c|}{ Chamber Concentration, $\mu \mathrm{g} \mathrm{m-3}$} & DEGBE & Texanol \\
\hline Avg. Inlet & $<1$ & $<1$ & $<1$ & $<1$ & $<32$ & $<12$ & $<1$ & $<2$ \\
\hline Background & $<1$ & $<1$ & 1 & $<1$ & $<32$ & $<12$ & $<1$ & $<2$ \\
\hline Minus $3 \mathrm{~h}$ & $<1$ & $<1$ & $<1$ & $<1$ & 651 & 88 & 25 & 2,020 \\
\hline $1-\mathrm{h}$ & 11 & 9 & 11 & 21 & 695 & 88 & 28 & 2,400 \\
\hline $3-h$ & 5 & 6 & 5 & 12 & 337 & $<35$ & 15 & 1,960 \\
\hline $6-h$ & 4 & 5 & 4 & 10 & 242 & $<35$ & 17 & 1,380 \\
\hline $12-h$ & 5 & 15 & 6 & 16 & 306 & $<35$ & 29 & 3,730 \\
\hline $24-h$ & 2 & 15 & 6 & 18 & 311 & $<35$ & 25 & 4,560 \\
\hline $48-h$ & 1 & 10 & 6 & 13 & 251 & $<29$ & 10 & 2,430 \\
\hline $72-h$ & 1 & 6 & 5 & 10 & 146 & $<23$ & 6 & 1,600 \\
\hline 78-h & 2 & 7 & 6 & 15 & 181 & $<23$ & 5 & 1,630 \\
\hline 96-h & 1 & 5 & 5 & 12 & 133 & $<23$ & 3 & 934 \\
\hline$? D-h$ & 1 & 5 & 6 & 14 & 146 & $<17$ & 4 & 1,070 \\
\hline $\mathrm{h}$ & 1 & 4 & 6 & 12 & 200 & $<17$ & 4 & 848 \\
\hline $108-h$ & 1 & 4 & 6 & 9 & 161 & $<17$ & 3 & 900 \\
\hline $192-h$ & 1 & 4 & 7 & 10 & 172 & $<17$ & 4 & 1,040 \\
\hline $216-h$ & 1 & 4 & 8 & 9 & 138 & $<17$ & 3 & 864 \\
\hline $240-h$ & 1 & 4 & 8 & 8 & 203 & $<17$ & 7 & 902 \\
\hline $336-\mathrm{h}$ & 1 & 3 & 10 & 6 & 159 & $<12$ & 3 & 702 \\
\hline
\end{tabular}


Table K-09. Concentrations of SigmaVOC $P$ (i.e., sum of four target VOCs for paint) and $\mathrm{TVOC}_{\mathrm{R}}$ (i.e., remainder of chromatographic response) for large-scale Experiments A-1 and $\mathrm{A}-2$.

\begin{tabular}{|c|c|c|c|c|}
\hline \multirow[b]{2}{*}{ Sample ID } & \multicolumn{4}{|c|}{ Chamber Concentration, $\mu \mathrm{g} \mathrm{m}^{-3}$} \\
\hline & \multicolumn{2}{|c|}{ SigmaVOC $_{p}$} & \multicolumn{2}{|c|}{ TVOC $_{R}$} \\
\hline Avg. Inlet ${ }^{*}$ & $<47$ & $<47$ & $35 \pm 9$ & $46 \pm 11$ \\
\hline Background & $<47$ & $<47$ & 85 & 80 \\
\hline Minus $3 \mathrm{~h}$ & 2,640 & 2,780 & 329 & 317 \\
\hline $1-h$ & 3,280 & 3,210 & 5,700 & 4,450 \\
\hline 3-h & 2,430 & 2,310 & 1,520 & 1,740 \\
\hline $6-h$ & 2,330 & 1,640 & 1,020 & 1,170 \\
\hline $12-h$ & --- & 4,060 & --- & 1,810 \\
\hline 24-h & 1,350 & 4,900 & 615 & 1,590 \\
\hline $48-h$ & 1,770 & 2,690 & 467 & 1,080 \\
\hline $72-h$ & 1,290 & 1,750 & 398 & 861 \\
\hline 78-h & -- & 1,820 & $\cdots$ & 1,230 \\
\hline $96-h$ & 1,970 & 1,070 & 781 & 943 \\
\hline $120-h$ & 1,670 & 1,220 & 761 & 876 \\
\hline $144-h$ & 1,750 & 1,050 & 800 & 767 \\
\hline $168-h$ & 1,750 & 1,060 & 749 & 712 \\
\hline $192-h$ & 1,750 & 1,220 & 695 & 780 \\
\hline $216-h$ & 1,890 & 1,000 & 665 & 748 \\
\hline $240-h$ & 1,680 & 1,110 & 690 & 728 \\
\hline $336-h$ & 1,250 & 864 & 642 & 730 \\
\hline
\end{tabular}

*Average \pm 1 standard deviation. 

Table K-10. Concentrations of formaldehyde and acetaldehyde for large-scale Experiments $A-1$ and $A-2$.

\begin{tabular}{|c|c|c|c|c|}
\hline \multirow[b]{3}{*}{ Sample ID } & \multicolumn{4}{|c|}{ Chamber Concentration, $\mu \mathrm{g} \mathrm{m}^{-3}$} \\
\hline & \multicolumn{2}{|c|}{ Formaldehyde } & \multicolumn{2}{|c|}{ Acetaldehyde } \\
\hline & Exp A-2 & $\operatorname{Exp} A-1$ & Exp A-2 & $\operatorname{Exp} A-1$ \\
\hline Avg. Inlet ${ }^{*}$ & $2 \pm 1$ & $2 \pm 1$ & $2 \pm 1$ & $2 \pm 1$ \\
\hline Background & 7 & 2 & 5 & 3 \\
\hline Minus $3 \mathrm{~h}$ & 3 & $<1$ & 2 & 2 \\
\hline $1-h$ & 6 & 1 & 5 & 5 \\
\hline 3-h & 6 & 1 & 4 & 3 \\
\hline $6-h$ & 6 & 2 & 4 & 3 \\
\hline $12-h$ & --- & 1 & $\ldots$ & 3 \\
\hline 24-h & 4 & 1 & 3 & 2 \\
\hline $48-h$ & 4 & 1 & 2 & 2 \\
\hline $72-h$ & 4 & 2 & 2 & 2 \\
\hline $78-h$ & $\cdots$ & 2 & --- & 2 \\
\hline $96-h$ & 6 & 2 & 6 & 2 \\
\hline $120-h$ & 6 & 2 & 5 & 2 \\
\hline $144-h$ & 5 & 2 & 5 & 1 \\
\hline $168-h$ & 6 & 2 & 5 & 2 \\
\hline $192-h$ & 4 & 2 & 4 & 1 \\
\hline 216-h & 6 & 2 & 5 & 1 \\
\hline $240-h$ & 6 & 3 & 4 & $<1$ \\
\hline $336-h$ & 4 & 3 & 3 & 1 \\
\hline
\end{tabular}

*Average \pm 1 standard deviation 
Table K-11. Quasi steady-state specific emission rates of Group 1 target VOCs for large-scale Experiment A-2. Source areas were taken from Table K-02.

\begin{tabular}{|c|c|c|c|c|c|c|c|c|c|c|}
\hline $\begin{array}{c}\text { Sample } \\
\text { ID }\end{array}$ & $\mathrm{n}-\mathrm{C} 10$ & $\mathrm{n}-\mathrm{C} 13$ & Toluene & $\begin{array}{r}\text { Spe } \\
1,2,4-T M B \\
\end{array}$ & $\begin{array}{l}\text { ific Emissio } \\
\text { C-hexone }\end{array}$ & $\begin{array}{c}\text { Rate, } \mu \\
\text { THF } \\
\end{array}$ & $\begin{array}{l}h-1 \\
\text { Benz alc }\end{array}$ & Phenol & TXIB & Benzothia \\
\hline Area, $m^{-2}$ & 3.48 & 3.48 & 3.48 & 3.48 & 3.48 & 3.48 & 3.48 & 3.48 & 3.48 & 3.48 \\
\hline $1-h$ & 1,980 & 1,290 & 2,010 & 298 & 31,300 & 68,200 & 157 & 840 & 426 & 164 \\
\hline $3-h$ & 600 & 558 & 690 & 98 & 6,830 & 15,300 & 98 & 607 & 272 & 139 \\
\hline $6-h$ & 474 & 390 & 425 & 70 & 2,600 & 3,810 & 98 & 523 & 251 & 132 \\
\hline $24-h$ & 181 & 195 & 125 & 42 & 544 & 264 & 70 & 446 & 181 & 112 \\
\hline $48-h$ & 122 & 157 & 81 & 29 & 238 & 87 & 64 & 413 & 180 & 99 \\
\hline $72-h$ & 102 & 116 & 60 & 19 & 125 & 55 & 51 & 353 & 144 & 84 \\
\hline $96-h$ & 80 & 59 & 73 & 16 & 67 & 35 & 18 & 108 & 49 & 31 \\
\hline $120-\mathrm{h}$ & 81 & 65 & 68 & 18 & 62 & 30 & 20 & 120 & 53 & 34 \\
\hline $144-h$ & 82 & 70 & 65 & 19 & 57 & 26 & 21 & 123 & 53 & 34 \\
\hline $168-h$ & 83 & 68 & 64 & 18 & 46 & 24 & 20 & 115 & 51 & 34 \\
\hline $192-h$ & 79 & 65 & 59 & 18 & 42 & 16 & 20 & 116 & 52 & 32 \\
\hline $216-h$ & 76 & 65 & 57 & 17 & 37 & 15 & 19 & 111 & 52 & 32 \\
\hline$? 40-h$ & 76 & 65 & 55 & 18 & 35 & 13 & 20 & 111 & 52 & 32 \\
\hline 1.8: & 66 & 58 & 47 & 16 & 23 & 8 & 16 & 100 & 47 & 28 \\
\hline
\end{tabular}


Table K-12. Quasi steady-state specific emission rates of Group 2 target VOCs for large-scale Experiment A-2. Source areas were taken from Table K-02.

\begin{tabular}{|c|c|c|c|c|c|c|c|c|}
\hline $\begin{array}{c}\text { Sample } \\
\text { ID }\end{array}$ & Styrene & 4-PCH & $\mathrm{BHT}$ & $\begin{array}{r}\text { Specific Emi } \\
\text { AlBN }\end{array}$ & $\begin{array}{c}\text { ate, } \mu \mathrm{g} \\
\mathrm{EG} \\
\end{array}$ & PG & DEGBE & Texanol \\
\hline Area, $\mathrm{m}^{-2}$ & 6.97 & 6.97 & 6.97 & 6.97 & 16.0 & 16.0 & 16.0 & 16.0 \\
\hline $1-h$ & 163 & 49 & 47 & 131 & 1,930 & 192 & 81 & 7,450 \\
\hline $3-h$ & 77 & 35 & 28 & 77 & 1,220 & $<109$ & 56 & 6,320 \\
\hline $6-\mathrm{h}$ & 63 & 35 & 21 & 73 & 952 & $<109$ & 44 & 6,280 \\
\hline $24-h$ & 21 & 28 & 14 & 63 & 634 & $<109$ & 30 & 3,550 \\
\hline$\therefore \mathrm{h}$ & 12 & 26 & 12 & 55 & 667 & $<92$ & 21 & 4,840 \\
\hline$r^{\cdots} \mathrm{h}$ & 9 & 23 & 16 & 46 & 504 & $<92$ & 16 & 3,510 \\
\hline $93-h$ & 4 & 9 & 7 & 27 & 137 & $<23$ & 5 & 1,430 \\
\hline $120-h$ & 4 & 10 & 8 & 30 & 145 & $<19$ & 6 & 1,180 \\
\hline $144-h$ & 4 & 11 & 9 & 28 & 159 & $<19$ & 5 & 1,230 \\
\hline 168-h & 4 & 10 & 10 & 25 & 151 & $<19$ & 4 & 1,310 \\
\hline $192-h$ & 4 & 10 & 11 & 24 & 156 & $<14$ & 4 & 1,230 \\
\hline $216-h$ & 3 & 9 & 13 & 20 & 147 & $<14$ & 4 & 1,360 \\
\hline $240-h$ & 3 & 9 & 13 & 19 & 133 & $<14$ & 3 & 1,200 \\
\hline $336-h$ & 2 & 8 & 15 & 14 & 108 & $<11$ & 2 & 885 \\
\hline
\end{tabular}


Table K-13. Quasi steady-state specific emission rates of Group 1 target VOCs for large-scale Experiment A-1. Source areas were taken from Table K-02.

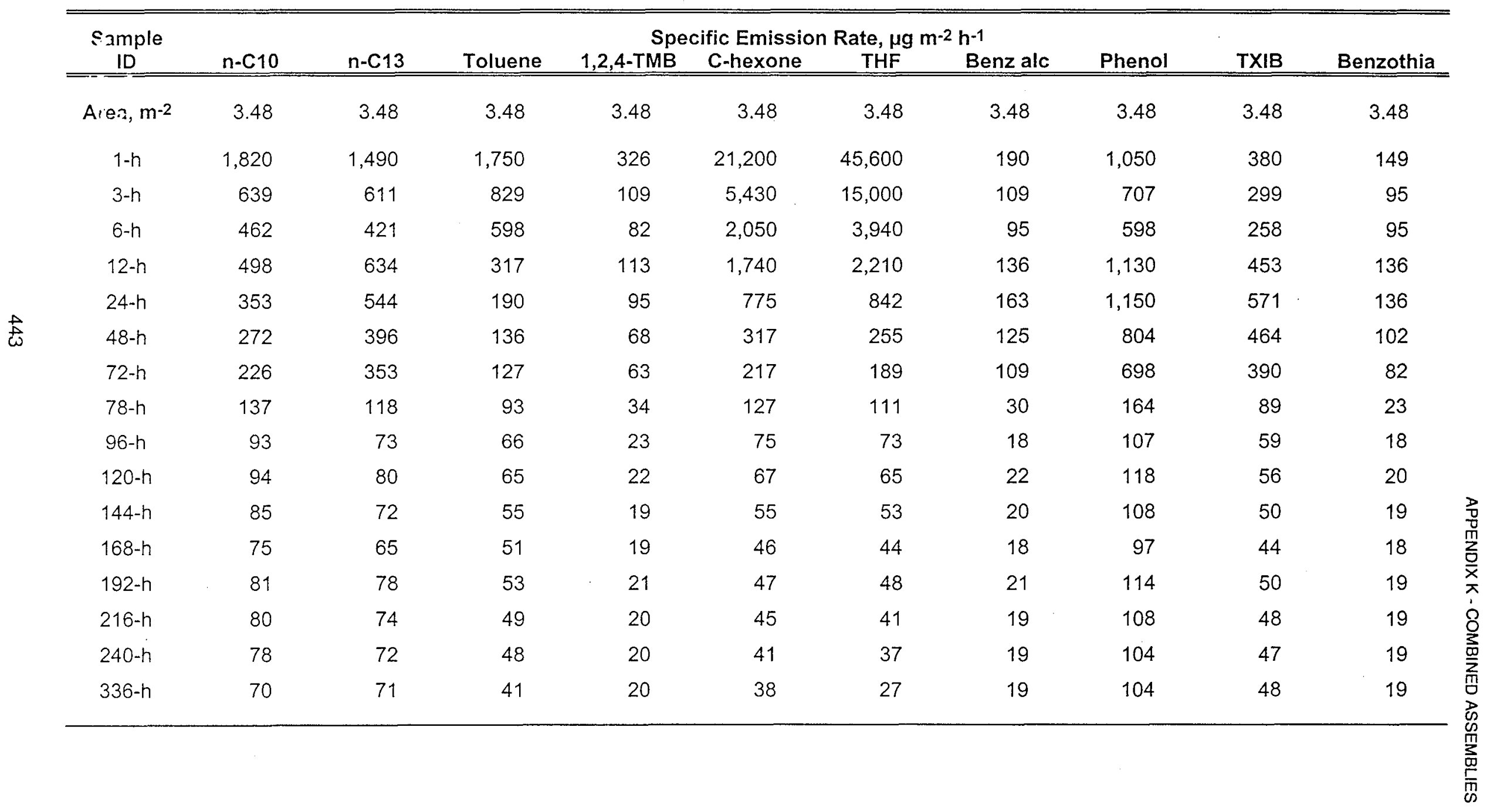


Table K-14. Quasi steady-state specific emission rates of Group 2 target VOCs for large-scale Experiment A-1. Source areas were taken from Table K-02.

\begin{tabular}{|c|c|c|c|c|c|c|c|c|}
\hline \multirow{2}{*}{$\begin{array}{c}\begin{array}{c}\text { Sample } \\
\text { ID }\end{array} \\
\text { Area, } \mathrm{m}^{-2}\end{array}$} & \multirow{2}{*}{$\frac{\text { Styrene }}{6.97}$} & \multirow{2}{*}{$\frac{\text { 4-PCH }}{6.97}$} & \multirow{2}{*}{$\frac{\text { BHT }}{6.97}$} & \multicolumn{3}{|c|}{ Specific Emission Rate, $\mu \mathrm{g} \mathrm{m}^{-2} \mathrm{~h}^{-1}$} & DEGBE & Texano \\
\hline & & & & 6.97 & 16.0 & 16.0 & 16.0 & 16.0 \\
\hline $1-\mathrm{h}$ & 75 & 61 & 75 & 149 & 2,120 & 269 & 86 & 7,300 \\
\hline $3-h$ & 34 & 41 & 34 & 81 & 1,050 & $<106$ & 47 & 5,980 \\
\hline $6-h$ & 27 & 34 & 27 & 68 & 736 & $<106$ & 53 & 4,190 \\
\hline $12-h$ & 34 & 102 & 45 & 113 & 931 & $<106$ & 89 & 11,300 \\
\hline $24-h$ & 14 & 102 & 41 & 129 & 946 & $<106$ & 77 & 13,900 \\
\hline $48-h$ & 6 & 68 & 40 & 90 & 763 & $<89$ & 30 & 7,390 \\
\hline $72-h$ & 5 & 45 & 36 & 68 & 445 & $<71$ & 18 & 4,880 \\
\hline $78-h$ & 3 & 13 & 11 & 27 & 138 & $<18$ & 4 & 1,240 \\
\hline 96-h & 2 & 9 & 9 & 22 & 102 & $<18$ & 2 & 714 \\
\hline $120-h$ & 3 & 9 & 11 & 24 & 111 & $<13$ & 3 & 820 \\
\hline 144-h & 2 & 8 & 11 & 20 & 153 & $<13$ & 3 & 649 \\
\hline 168-h & 2 & 7 & 11 & 16 & 124 & $<13$ & 2 & 688 \\
\hline $192-h$ & 2 & 8 & 13 & 17 & 131 & $<13$ & 3 & 795 \\
\hline $216-h$ & 2 & 7 & 14 & 16 & 106 & $<13$ & 3 & 661 \\
\hline $240-h$ & 2 & 7 & 15 & 13 & 156 & $<13$ & 5 & 690 \\
\hline $326-h$ & $<2$ & 6 & 18 & 10 & 122 & $<9$ & 2 & 537 \\
\hline
\end{tabular}


Table K-15. Quasi steady-state specific emission rates of $\mathrm{TVOC}_{\mathrm{R}}$ (i.e., remainder of chromatographic response after subtracting target VOCs for paint) for large-scale Experiments A-1 and A-2. Total floor area of $10.4 \mathrm{~m}^{2}$ was used to calculate values.

\begin{tabular}{|c|c|c|}
\hline $\begin{array}{c}\text { Sample } \\
\text { ID }\end{array}$ & $\begin{array}{l}\text { Specific Emission } \\
\text { Exp A-2 }\end{array}$ & $\begin{array}{c}\text { Rate, } \mu \mathrm{g} \mathrm{m}^{-2} \mathrm{~h}^{-1} \\
\operatorname{Exp} \mathrm{A}-1\end{array}$ \\
\hline $1-h$ & 27,000 & 20,500 \\
\hline 3-h & 6,900 & 7,750 \\
\hline 6-h & 4,510 & 5,120 \\
\hline $12-h$ & --- & 8,120 \\
\hline 24-h & 2,540 & 7,070 \\
\hline $48-h$ & 1,830 & 4,670 \\
\hline 72-h & 1,500 & 3,660 \\
\hline $78-h$ & --- & 1,360 \\
\hline $96-\mathrm{h}$ & 852 & 1,010 \\
\hline $120-\mathrm{h}$ & 828 & 936 \\
\hline $144-h$ & 876 & 808 \\
\hline 168-h & 813 & 744 \\
\hline $192-h$ & 747 & 824 \\
\hline 216-h & 710 & 786 \\
\hline 240-h & 741 & 762 \\
\hline $336-h$ & 682 & 765 \\
\hline
\end{tabular}


Table K-16. Cumulative masses (milligrams) of target VOCs and $T V O C_{R}$ (i.e., remainder of chromatographic response after subtracting target VOCs for paint) emitted over 0 - 336 hours in large-scale Experiments A-1 and A-2.

\begin{tabular}{|c|c|c|}
\hline \multirow[b]{2}{*}{ Compound } & \multicolumn{2}{|c|}{ Cumulative Mass, mg } \\
\hline & Exp A-2 & Exp A-1 \\
\hline \multicolumn{3}{|l|}{ Alkane Hydrocarbons } \\
\hline n-Decane & 135 & 173 \\
\hline n-Tridecane & 121 & 196 \\
\hline \multicolumn{3}{|l|}{ Aromatic Hydrocarbons } \\
\hline Toluene & 108 & 117 \\
\hline Styrene & 20 & 11 \\
\hline 1,2,4-Trimethylbenzene & 28 & 42 \\
\hline 4-Phenylcyclohexene & 32 & 51 \\
\hline \multicolumn{3}{|l|}{ Carbonyl Compounds } \\
\hline Cyclohexanone & 479 & 416 \\
\hline \multicolumn{3}{|l|}{ Other Oxidized Cmpds. } \\
\hline Tetrahydrofuran & 795 & 705 \\
\hline Ethylene glycol & 1,490 & 1,490 \\
\hline Propylene glycol & $<200$ & $<180$ \\
\hline Benzyl alcohol & 37 & 52 \\
\hline Phenol & 225 & 334 \\
\hline 2-(2-Butoxyethoxy)ethanol & 53 & 73 \\
\hline $\begin{array}{c}\text { 2,2,4-Trimethyl-1,3-pentanediol } \\
\text { monoisobutyrates (Texanol) }\end{array}$ & 10,800 & 13,400 \\
\hline $\begin{array}{l}\text { 2,2,4-Trimethyl-1,3-pentanediol } \\
\text { diisobutyrate (TXIB) }\end{array}$ & 99 & 165 \\
\hline 2,6-Di-tert-butyl-4-methylphenol & 30 & 45 \\
\hline \multicolumn{3}{|l|}{ Miscellaneous Cmpds. } \\
\hline 2,2'-Azobisisobutyronitrile & 73 & 81 \\
\hline Benzothiazole & 58 & 46 \\
\hline TVOC $_{R}$ & 4,630 & 6,960 \\
\hline
\end{tabular}


Table K-17. Cumulative exposures (ppb-hour) to target VOCs over $0-48$ and 48 - 336 hours in largescale Experiment A-2. Estimates assume 20 hours of occupancy per day. Exposure ratios were calculated by dividing the 0 - to 48-hour exposures by the total exposures over 0 - 336 hours.

\begin{tabular}{|c|c|c|c|}
\hline \multirow[b]{2}{*}{ Compound } & \multicolumn{2}{|c|}{$\begin{array}{l}\text { Cumulative Exposure, } \\
\text { ppb-hour }\end{array}$} & \multirow{2}{*}{$\begin{array}{c}\text { Exp. Ratio } \\
0-48 \mathrm{~h} / \\
\text { Total }\end{array}$} \\
\hline & $0.48 h$ & $48-336 h$ & \\
\hline \multicolumn{4}{|l|}{ Alkane Hydrocarbons } \\
\hline n-Decane & 147 & 793 & 0.16 \\
\hline $\mathrm{n}$-Tridecane & 103 & 523 & 0.16 \\
\hline \multicolumn{4}{|l|}{ Aromatic Hydrocarbons } \\
\hline Toluene & 198 & 933 & 0.18 \\
\hline Styrene & 46 & 94 & 0.33 \\
\hline 1,2,4-Trimethylbenzene & 31 & 211 & 0.13 \\
\hline 4-Phenylcyclohexene & 26 & 178 & 0.13 \\
\hline \multicolumn{4}{|l|}{ Carbonyl Compounds } \\
\hline Cyclohexanone & 1,530 & 690 & 0.69 \\
\hline \multicolumn{4}{|l|}{ Other Oxidized Cmpds. } \\
\hline Tetrahydrofuran & 3,730 & 491 & 0.88 \\
\hline Ethylene glycol & 3,920 & 16,400 & 0.19 \\
\hline Propylene glycol & $<450$ & $<1,600$ & --- \\
\hline Benzyl alcohol & 49 & 273 & 0.15 \\
\hline Phenol & 341 & 1,860 & 0.15 \\
\hline 2-(2-Butoxyethoxy)ethanol & 65 & 181 & 0.26 \\
\hline $\begin{array}{l}\text { 2,2,4-Trimethyl-1,3-pentanediol } \\
\text { monoisobutyrates (Texanol) }\end{array}$ & 6,810 & 39,900 & 0.15 \\
\hline $\begin{array}{l}\text { 2,2,4-Trimethyl-1,3-pentanediol } \\
\text { diisobutyrate (TXIB) }\end{array}$ & 49 & 277 & 0.15 \\
\hline 2,6-Di-tert-butyl-4-methylphenol & 11 & 164 & 0.06 \\
\hline \multicolumn{4}{|l|}{ Miscellaneous Cmpds. } \\
\hline 2,2'-Azobisisobutyronitrile & 54 & 405 & 0.12 \\
\hline Benzothiazole & 58 & 364 & 0.14 \\
\hline
\end{tabular}


Table K-18. Cumulative exposures (ppb-hour) to target VOCs over 96 - 336 hours in large-scale Experiments A-1 and A-2. Estimates assume 20 hours of occupancy per day.

\begin{tabular}{|c|c|c|}
\hline \multirow[b]{2}{*}{ Compound } & \multicolumn{2}{|c|}{ Cumulative Exposure, ppb-h } \\
\hline & Exp A-2 & $\operatorname{Exp} A-1$ \\
\hline \multicolumn{3}{|l|}{ Alkane Hydrocarbons } \\
\hline n-Decane & 716 & 779 \\
\hline n-Tridecane & 465 & 550 \\
\hline \multicolumn{3}{|l|}{ Aromatic Hydrocarbons } \\
\hline Toluene & 842 & 767 \\
\hline Styrene & 79 & 46 \\
\hline 1,2,4-Trimethylbenzene & 193 & 233 \\
\hline 4-Phenylcyclohexene & 155 & 125 \\
\hline \multicolumn{3}{|l|}{ Carbonyl Compounds } \\
\hline Cyclohexanone & 560 & 678 \\
\hline \multicolumn{3}{|l|}{ Other Oxidized Cmpds. } \\
\hline Tetrahydrofuran & 394 & 973 \\
\hline Ethylene glycol & 13,600 & 12,600 \\
\hline Propylene glycol & $<1,200$ & $<1,400$ \\
\hline Benzyl alcohol & 235 & 249 \\
\hline Phenol & 1,580 & 1,580 \\
\hline 2-(2-Butoxyethoxy)ethanol & 145 & 131 \\
\hline $\begin{array}{l}\text { 2,2,4-Trimethyl-1,3-pentanediol } \\
\text { monoisobutyrates (Texanol) }\end{array}$ & 33,600 & 14,600 \\
\hline $\begin{array}{l}\text { 2,2,4-Trimethyl-1,3-pentanediol } \\
\text { diisobutyrate (TXIB) }\end{array}$ & 237 & 239 \\
\hline 2,6-Di-tert-butyl-4-methylphenol & 153 & 186 \\
\hline \multicolumn{3}{|l|}{ Miscellaneous Cmpds. } \\
\hline 2,2'-Azobisisobutyronitrile & 352 & 268 \\
\hline Benzothiazole & 315 & 195 \\
\hline
\end{tabular}


Table K-19. Summary of environmental parameters over $336-2,016$ hours in large-scale Experiment A-2.

\begin{tabular}{|c|c|c|c|}
\hline Time Interval & $\begin{array}{c}\text { Ventilation Rate } \\
(\mathrm{h}-1)\end{array}$ & $\begin{array}{c}\text { Temperature } \\
\text { ( } \mathrm{C} C \\
\end{array}$ & $\begin{array}{c}\text { Relative Humidity } \\
(\%)\end{array}$ \\
\hline $\begin{array}{l}336-504 \mathrm{~h} \\
\text { Avg. } \pm 1 \text { Std. Dev. } \\
\text { Range }\end{array}$ & $\begin{array}{l}0.50 \pm 0.01 \\
0.50-0.51\end{array}$ & $\begin{array}{l}21.9 \pm 0.3 \\
21.0-22.6\end{array}$ & $\begin{array}{r}44 \pm 3 \\
38-48\end{array}$ \\
\hline $\begin{array}{l}504-672 \mathrm{~h} \\
\text { Avg. } \pm 1 \text { Std. Dev. } \\
\text { Range }\end{array}$ & $\begin{array}{l}0.50 \pm 0.01 \\
0.49-0.51\end{array}$ & $\begin{array}{l}22.0 \pm 0.7 \\
20.9-24.5\end{array}$ & $\begin{array}{r}43 \pm 3 \\
39-49\end{array}$ \\
\hline $\begin{array}{l}672-840 \mathrm{~h} \\
\text { Avg. } \pm 1 \text { Std. Dev. } \\
\text { Range }\end{array}$ & $\begin{array}{l}0.50 \pm 0.01 \\
0.49-0.50\end{array}$ & $\begin{array}{l}22.6 \pm 0.7 \\
21.5-24.5\end{array}$ & $\begin{array}{l}50 \pm 1 \\
47-52\end{array}$ \\
\hline $\begin{array}{l}840-1,008 \text { h } \\
\text { Avg. } \pm 1 \text { Std. Dev. } \\
\text { Range }\end{array}$ & $\begin{array}{l}0.50 \pm 0.01 \\
0.49-0.50\end{array}$ & $\begin{array}{l}22.5 \pm 0.5 \\
21.6-24.4\end{array}$ & $\begin{array}{l}51 \pm 2 \\
48-56\end{array}$ \\
\hline $\begin{array}{l}1,008-1,176 \mathrm{~h} \\
\text { Avg. } \pm 1 \text { Std. Dev. } \\
\text { Range }\end{array}$ & $\begin{array}{l}0.50 \pm 0.01 \\
0.48-0.50\end{array}$ & $\begin{array}{l}22.0 \pm 0.4 \\
21.2-23.3\end{array}$ & $\begin{array}{l}44 \pm 2 \\
40-48\end{array}$ \\
\hline $\begin{array}{l}1,176-1,344 \mathrm{~h} \\
\text { Avg. } \pm 1 \text { Std. Dev. } \\
\text { Range }\end{array}$ & $\begin{array}{l}0.50 \pm 0.01 \\
0.49-0.50\end{array}$ & $\begin{array}{l}22.0 \pm 0.2 \\
21.3-22.5\end{array}$ & $\begin{array}{l}44 \pm 1 \\
42-46\end{array}$ \\
\hline $\begin{array}{l}1,344-1,512 h \\
\text { Avg. } \pm 1 \text { Std. Dev. } \\
\text { Range }\end{array}$ & $\begin{array}{l}0.50 \pm 0.01 \\
0.49-0.50\end{array}$ & $\begin{array}{l}22.0 \pm 0.4 \\
21.2-23.5\end{array}$ & $\begin{array}{l}43 \pm 2 \\
40-47\end{array}$ \\
\hline $\begin{array}{l}1,512-1,680 \mathrm{~h} \\
\text { Avg. } \pm 1 \text { Std. Dev. } \\
\text { Range }\end{array}$ & $\begin{array}{l}0.50 \pm 0.01 \\
0.49-0.50\end{array}$ & $\begin{array}{l}23.0 \pm 1.0 \\
21.5-25.4\end{array}$ & $\begin{array}{l}47 \pm 3 \\
42-53\end{array}$ \\
\hline $\begin{array}{l}1,680-1,848 \mathrm{~h} \\
\text { Avg. } \pm 1 \text { Std. Dev. } \\
\text { Range }\end{array}$ & $\begin{array}{l}0.50 \pm 0.01 \\
0.49-0.50\end{array}$ & $\begin{array}{l}22.9 \pm 1.0 \\
21.5-25.3\end{array}$ & $\begin{array}{l}49 \pm 2 \\
45-53\end{array}$ \\
\hline $\begin{array}{l}1,848-2,016 \mathrm{~h} \\
\text { Avg. } \pm 1 \text { Std. Dev. } \\
\text { Range }\end{array}$ & $\begin{array}{l}0.49 \pm 0.01 \\
0.49-0.50\end{array}$ & $\begin{array}{l}22.6 \pm 0.6 \\
21.8-24.8\end{array}$ & $\begin{array}{l}51 \pm 1 \\
48-54\end{array}$ \\
\hline
\end{tabular}


$T_{8}: ! 3$ K-20. Concentrations of Group 1 target VOCs over $336-2,016$ hours in large-scale Experiment A-2.

\begin{tabular}{|c|c|c|c|c|c|c|c|c|c|c|}
\hline \multirow{2}{*}{$\begin{array}{c}\text { Sample } \\
\text { ID }\end{array}$} & \multicolumn{10}{|c|}{ Chamber Concentration, $\mu \mathrm{g} \mathrm{m}^{-3}$} \\
\hline & $\mathrm{n}-\mathrm{C} 10$ & $\mathrm{n}-\mathrm{C} 13$ & Toluene & $1,2,4-\mathrm{TMB}$ & C-hexone & THF & Benz alc & Phenol & TXIB & Benzothia \\
\hline Avg. Inlet & $<1$ & $<1$ & $<1$ & $<1$ & $<1$ & 2 & $<1$ & $<1$ & $<1$ & $<1$ \\
\hline $336-h$ & 18 & 16 & 13 & 4 & 6 & 3 & 4 & 27 & 13 & 8 \\
\hline $504-h$ & 17 & 15 & 11 & 4 & 5 & 2 & 4 & 25 & 12 & 6 \\
\hline $672-h$ & 20 & 23 & 12 & 5 & 6 & 3 & 6 & 30 & 16 & 6 \\
\hline $840-h$ & 17 & 20 & 9 & 4 & 5 & 2 & 5 & 27 & 15 & 5 \\
\hline 1,008-h & 16 & 18 & 8 & 4 & 4 & 2 & 4 & 24 & 14 & 4 \\
\hline 1,176-h & 18 & 22 & 7 & 4 & 4 & 2 & 3 & 24 & 15 & 5 \\
\hline $1,344-h$ & 16 & 21 & 7 & 4 & 4 & 1 & 3 & 21 & 14 & 4 \\
\hline $1,512-h$ & 15 & 21 & 6 & 4 & 4 & 1 & 3 & 20 & 14 & 4 \\
\hline $1,680-h$ & 15 & 19 & 6 & 4 & 4 & 1 & 3 & 21 & 14 & 3 \\
\hline $1,848-h$ & 14 & 20 & 5 & 4 & 4 & 1 & 3 & 21 & 15 & 4 \\
\hline $2,016-h$ & 11 & 17 & 4 & 3 & 3 & 1 & 2 & 17 & 13 & 3 \\
\hline
\end{tabular}


Table K-21. Concentrations of Group 2 target VOCs over 336 - 2,016 hours in large-scale Experiment A-2.

\begin{tabular}{|c|c|c|c|c|c|c|c|c|}
\hline $\begin{array}{c}\text { Sample } \\
\text { ID }\end{array}$ & Styrene & 4-PCH & $\mathrm{BHT}$ & $\begin{array}{r}\text { Chamber C } \\
\text { AlBN } \\
\end{array}$ & $\begin{array}{c}\text { ion, } \mu \\
E G \\
\end{array}$ & $P G$ & DEGBE & Texanol \\
\hline Avg. Inlet & $<1$ & $<1$ & $<1$ & $<1$ & $<32$ & $<12$ & $<1$ & $<2$ \\
\hline $336-h$ & 1 & 4 & 8 & 8 & 135 & $<14$ & 3 & 1,110 \\
\hline $504-h$ & 1 & 4 & 10 & 5 & 108 & $<14$ & 2 & 825 \\
\hline $672-h$ & 1 & 4 & 12 & 4 & 178 & $<14$ & 3 & 1,050 \\
\hline $840-h$ & 1 & 3 & 12 & 2 & 146 & $<14$ & 2 & 813 \\
\hline $1,008-h$ & $<1$ & 3 & 11 & 1 & 131 & $<14$ & 2 & 625 \\
\hline $1,176-h$ & $<1$ & 3 & 13 & 1 & 122 & $<14$ & 2 & 605 \\
\hline $1,344-h$ & $<1$ & 2 & 15 & 1 & 111 & $<14$ & 1 & 438 \\
\hline $1,512-h$ & $<1$ & 2 & 14 & 1 & 92 & $<14$ & 1 & 498 \\
\hline 1,680-h & $<1$ & 2 & 13 & 1 & 73 & $<14$ & 1 & 385 \\
\hline 1,848-h & $<1$ & 2 & 15 & $<1$ & 54 & $<14$ & 1 & 350 \\
\hline $2,016-h$ & $<1$ & 2 & 13 & $<1$ & 98 & $<14$ & 1 & 294 \\
\hline
\end{tabular}


Table K-22. Concentrations of SigmaVOC $P$ (i.e., sum of four target VOCs for paint) and TVOC $_{R}$ (i.e., remainder of chromatographic response) over $336-2,016$ hours in largescale Experiment A-2.

\begin{tabular}{|c|c|c|}
\hline Sample ID & $\begin{array}{l}\text { Chamber Co } \\
\text { SigmaVOC } \\
\end{array}$ & $\begin{array}{l}\text { tion, } \mu \mathrm{g} \mathrm{m}^{-3} \\
\text { TVOC }_{\mathrm{R}} \\
\end{array}$ \\
\hline Avg. Inlet ${ }^{*}$ & $<47$ & $31 \pm 11$ \\
\hline 336-h & 1,250 & 642 \\
\hline 504-h & 936 & 580 \\
\hline $672-h$ & 1,230 & 768 \\
\hline $840-h$ & 962 & 596 \\
\hline $1,008-h$ & 758 & 528 \\
\hline $1,176-h$ & 730 & 645 \\
\hline $1,344-h$ & 550 & 560 \\
\hline $1,512-h$ & 591 & 532 \\
\hline $1,680-h$ & 459 & 536 \\
\hline $1,848-h$ & 404 & 579 \\
\hline $2,016-h$ & 393 & 479 \\
\hline
\end{tabular}

*Average \pm 1 standard deviation 
Table K-23. Quasi steady-state specific emission rates of Group 1 target VOCs over $336-2,016$ hours in large-scale Experiment A-2. Source areas were taken from Table K-02.

\begin{tabular}{|c|c|c|c|c|c|c|c|c|c|c|}
\hline Sample & $\mathrm{n}-\mathrm{C} 10$ & $\mathrm{n}-\mathrm{C} 13$ & Toluene & \multicolumn{4}{|c|}{ Specific Emission Rate, $\mu \mathrm{g} \mathrm{m}^{-2} \mathrm{~h}^{-1}$} & Phenol & TXIB & Benzothia \\
\hline Area, $\mathrm{m}^{-2}$ & 3.48 & 3.48 & 3.48 & 3.48 & 3.48 & 3.48 & 3.48 & 3.48 & 3.48 & 3.48 \\
\hline $336-h$ & 66 & 58 & 47 & 16 & 23 & 4 & 16 & 100 & 47 & 28 \\
\hline $504-h$ & 60 & 56 & 39 & 15 & 17 & $<4$ & 14 & 90 & 44 & 24 \\
\hline $672-h$ & 75 & 84 & 44 & 19 & 23 & $<4$ & 20 & 111 & 59 & 24 \\
\hline $840-h$ & 62 & 72 & 33 & 16 & 17 & $<4$ & 17 & 98 & 54 & 20 \\
\hline $1,008-h$ & 58 & 67 & 28 & 14 & 16 & $<4$ & 13 & 88 & 50 & 16 \\
\hline $1,176-h$ & 66 & 82 & 27 & 15 & 15 & $<4$ & 12 & 89 & 56 & 17 \\
\hline $1,344-h$ & 59 & 75 & 25 & 14 & 14 & $<4$ & 11 & 78 & 50 & 15 \\
\hline $1,512-h$ & 56 & 76 & 22 & 14 & 13 & $<4$ & 10 & 73 & 50 & 13 \\
\hline $1,680-h$ & 54 & 71 & 21 & 13 & 13 & $<4$ & 9 & 76 & 52 & 12 \\
\hline $1,848-h$ & 52 & 74 & 20 & 14 & 13 & $<4$ & 10 & 78 & 55 & 13 \\
\hline $2,016-h$ & 40 & 62 & 16 & 12 & 10 & $<4$ & 8 & 63 & 47 & 11 \\
\hline
\end{tabular}


Table K-24. Quasi steady-state specific emission rates of Group 2 target VOCs over 336 - 2,016 hours in large-scale Experiment A-2. Source areas were taken from Table K-02.

\begin{tabular}{|c|c|c|c|c|c|c|c|c|}
\hline $\begin{array}{c}\text { Sample } \\
\text { ID }\end{array}$ & Styrene & 4-PCH & $\mathrm{BHT}$ & $\begin{array}{r}\text { Specific Emis } \\
\text { AlBN } \\
\end{array}$ & $\begin{array}{l}\mathrm{e}, \mu \mathrm{g} \\
\mathrm{EG} \\
\end{array}$ & $P G$ & DEGBE & Texanol \\
\hline Area, $\mathrm{m}^{-2}$ & 6.97 & 6.97 & 6.97 & 6.97 & 16.0 & 16.0 & 16.0 & 16.0 \\
\hline $336-h$ & 2 & 8 & 15 & 14 & 108 & $<11$ & 2 & 885 \\
\hline $504-h$ & 1 & 7 & 18 & 8 & 86 & $<11$ & 2 & 658 \\
\hline $672-h$ & 1 & 8 & 22 & 7 & 142 & $<11$ & 2 & 836 \\
\hline $840-h$ & 1 & 6 & 22 & 4 & 117 & $<11$ & 2 & 648 \\
\hline $1,008-h$ & $<1$ & 5 & 21 & 3 & 105 & $<11$ & 2 & 498 \\
\hline $1,176-h$ & $<1$ & 5 & 25 & 2 & 98 & $<11$ & 1 & 482 \\
\hline $1,344-h$ & $<1$ & 4 & 27 & 1 & 89 & $<11$ & 1 & 349 \\
\hline $1,512-h$ & $<1$ & 4 & 25 & 1 & 73 & $<11$ & 1 & 397 \\
\hline $1,680-\mathrm{h}$ & $<1$ & 4 & 24 & 1 & 58 & $<11$ & 1 & 307 \\
\hline 1,848-h & $<1$ & 4 & 27 & $<1$ & 43 & $<11$ & 1 & 279 \\
\hline $2,016-h$ & $<1$ & 3 & 25 & $<1$ & 78 & $<11$ & 1 & 234 \\
\hline
\end{tabular}


Table K-25. Quasi steady-state specific emission rates of $\mathrm{TVOC}_{R}$ (i.e., remainder of chromatographic response after subtracting target VOCs for paint) over 336 - 2,016 hours in large-scale Experiment A-2. Total floor area of $10.4 \mathrm{~m}^{2}$ was used to calculate values.

\begin{tabular}{cc}
\hline $\begin{array}{c}\text { Sample } \\
\text { ID }\end{array}$ & $\begin{array}{c}\text { Specific Emission } \\
\text { Rate } \\
\mu \mathbf{m ~ m}^{-2} \mathbf{h}^{-1}\end{array}$ \\
\hline $336-\mathrm{h}$ & 682 \\
$504-\mathrm{h}$ & 607 \\
$672-\mathrm{h}$ & 838 \\
$840-\mathrm{h}$ & 626 \\
$1,008-\mathrm{h}$ & 543 \\
$1,176-\mathrm{h}$ & 687 \\
$1,344-\mathrm{h}$ & 582 \\
$1,512-\mathrm{h}$ & 548 \\
$1,680-\mathrm{h}$ & 553 \\
$1,848-\mathrm{h}$ & 606 \\
$2,016-\mathrm{h}$ & 483 \\
\hline
\end{tabular}


Table K-26. Cumulative masses (milligrams) of target VOCs and $\mathrm{TVOC}_{R}$ (i.e., remainder of chromatographic response after subtracting target VOCs for paint) emitted over 336 2,016 hours in large-scale Experiment A-2.

\begin{tabular}{|c|c|}
\hline Compound & $\begin{array}{c}\text { Cumulative Mass } \\
\text { mg }\end{array}$ \\
\hline \multicolumn{2}{|l|}{ Alkane Hydrocarbons } \\
\hline n-Decane & 348 \\
\hline $\mathrm{n}$-Tridecane & 420 \\
\hline \multicolumn{2}{|l|}{ Aromatic Hydrocarbons } \\
\hline Toluene & 169 \\
\hline Styrene & $<10$ \\
\hline 1,2,4-Trimethylbenzene & 86 \\
\hline 4-Phenylcyclohexene & 61 \\
\hline \multicolumn{2}{|l|}{ Carbonyl Compounds } \\
\hline Cyclohexanone & 92 \\
\hline \multicolumn{2}{|l|}{ Other Oxidized Cmpds. } \\
\hline Tetrahydrofuran & $<23$ \\
\hline Ethylene glycol & 2,430 \\
\hline Propylene glycol & $<300$ \\
\hline Benzyl alcohol & 75 \\
\hline Phenol & 505 \\
\hline 2-(2-Butoxyethoxy)ethanol & 38 \\
\hline $\begin{array}{l}\text { 2,2,4-Trimethyl-1,3-pentanediol } \\
\text { monoisobutyrates (Texanol) }\end{array}$ & 13,500 \\
\hline $\begin{array}{l}\text { 2,2,4-Trimethyl-1,3-pentanediol } \\
\text { diisobutyrate (TXIB) }\end{array}$ & 302 \\
\hline 2,6-Di-tert-butyl-4-methylphenol & 270 \\
\hline \multicolumn{2}{|l|}{ Miscellaneous Cmpds. } \\
\hline 2,2'-Azobisisobutyronitrile & 40 \\
\hline Benzothiazole & 101 \\
\hline TVOC $_{R}$ & 10,800 \\
\hline
\end{tabular}


Table K-27. Cumulative exposures (ppb-hour) to target VOCs over $336-2,016$ hours in large-scale Experiment A-2. Estimates assume 20 hours of occupancy per day.

\begin{tabular}{|c|c|}
\hline Compound & $\begin{array}{c}\text { Cumulative Exposure } \\
\text { ppb-hour }\end{array}$ \\
\hline \multicolumn{2}{|l|}{ Alkane Hydrocarbons } \\
\hline n-Decane & 3,910 \\
\hline n-Tridecane & 3,650 \\
\hline \multicolumn{2}{|l|}{ Aromatic Hydrocarbons } \\
\hline Toluene & 2,940 \\
\hline Styrene & $<100$ \\
\hline 1,2,4-Trimethylbenzene & 1,150 \\
\hline 4-Phenylcyclohexene & 618 \\
\hline \multicolumn{2}{|l|}{ Carbonyl Compounds } \\
\hline Cyclohexanone & 1,500 \\
\hline \multicolumn{2}{|l|}{ Other Oxidized Cmpds. } \\
\hline Tetrahydrofuran & 834 \\
\hline Ethylene glycol & 62,600 \\
\hline Propylene glycol & $<6,300$ \\
\hline Benzyl alcohol & 1,110 \\
\hline Phenol & 8,580 \\
\hline 2-(2-Butoxyethoxy)ethanol & 378 \\
\hline $\begin{array}{l}\text { 2,2,4-Trimethyl-1,3-pentanediol } \\
\text { monoisobutyrates (Texanol) }\end{array}$ & 99,700 \\
\hline $\begin{array}{l}\text { 2,2,4-Trimethyl-1,3-pentanediol } \\
\text { diisobutyrate (TXIB) }\end{array}$ & 1,690 \\
\hline 2,6-Di-tert-butyl-4-methylphenol & 2,110 \\
\hline \multicolumn{2}{|l|}{ Miscellaneous Cmpds. } \\
\hline 2,2'-Azobisisobutyronitrile & 394 \\
\hline Benzothiazole & 1,200 \\
\hline
\end{tabular}




\section{DISCLAIMER}

This document was prepared as an account of work sponsored by the United States Government. While this document is believed to contain correct information, neither the United States Government nor any agency thereof, nor the Regents of the University of California, nor any of their employees, makes any warranty, express or implied, or assumes any legal responsibility for the accuracy, completeness, or usefulness of any information, apparatus, product, or process disclosed, or represents that its use would not infringe privately owned rights. Reference herein to any specific commercial product, process, or service by its trade name, trademark, manufacturer, or otherwise, does not necessarily constitute or imply its endorsement, recommendation, or favoring by the United States Government or any agency thereof, or the Regents of the University of California. The views and opinions of authors expressed herein do not necessarily state or reflect those of the United States Government or any agency thereof or the Regents of the University of California. 